\title{
GRAMMAR OF
}

\section{TEXTILE DESIGN}

H. NISB ET

SECOND REVISED EDITION

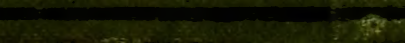





\section{GR A M MA R}

\section{or \\ TEXTILE DESIGN}

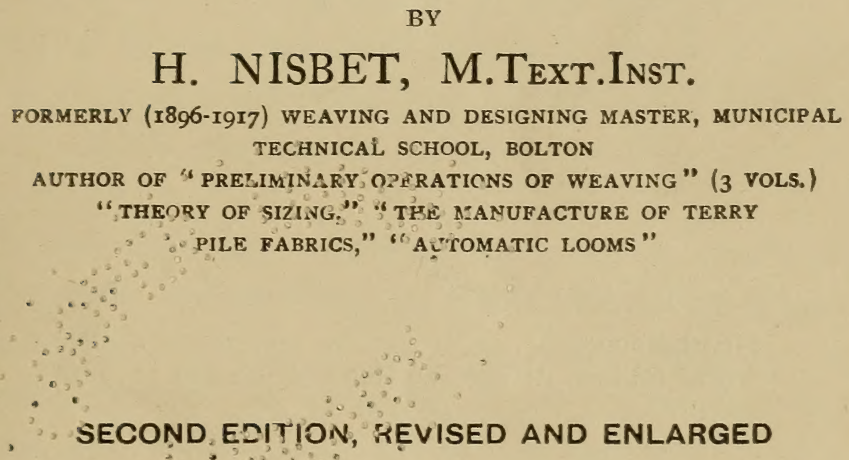

BY

H. NISBET, M.Text.Inst.

PORMERLY (1896-I9I7) WEAVING AND DESIGNING MASTER, MUNICIPAL TECHNICAL SCHOOL, BOLTON AUTHOR OF "PRELIMINARY OPERATICNS OF WEAVING" (3 VOLS.)

"THEORY OF SIZING", "THE MANUFACTURE OF TERRY " "PILE FABRICS," "At'TOMATIC LOOMS"

SECOND EOITION, REVISED AND ENLARGED

WITH $6_{35}$ ILLUSTRATIONS

\section{LONDON}

SCOTT, GREENWOOD \& SON

(E. GREENWOOD)

8 BROADWAY, LUDGATE, E.C. 4

I9I9.

[All rights reserved]

D. VAN NOSTRAND COMPANY NAW YORS 

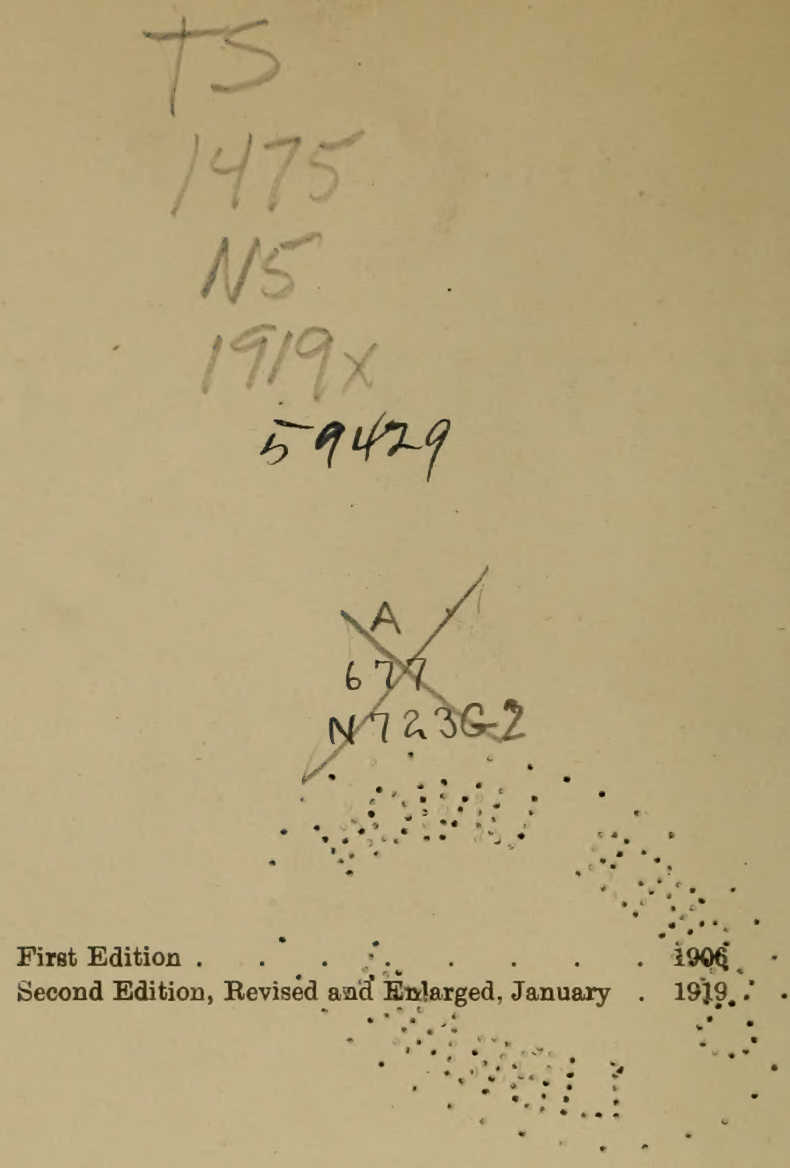


\section{PREFACE TO THE SECOND EDITION.}

In responding to the demand for a second edition of this treatise, the author has taken the opportunity to extend several chapters of the previous edition, and also to enlarge its scope by the addition of eight new chapters.

The chapter treating of terry pile fabrics is augmented by descriptions of several distinct modifications of terry pile motions; a description of figured terry weaving by means of the Jacquard machine; and also of a method of weaving terry pile fabrics in an ordinary "fast-reed" loom without the aid of a "terry motion" for that purpose.

In the eight new chapters, there are given detailed descriptions, accompanied by much useful and practical information, relating to the structure, designing, and manufacture of the principal types of textile fabrics comprising the following commercial varieties : leno brocade; brocade; damask; Alhambra ; piqué or toilet welt; matelasse; toilet quilting; patent satin or Mitcheline; tapestry; and Scotch or Kidderminster carpet.

Embracing, as it does, a much wider range than that of its predecessor, the present volume will prove even more helpful as a text-book for students of weaving and designing, and also as a work of reference for all who are interested in, or desire information relating to, the construction, production, and use of textile fabrics.

HARRY NISBET,

Mancengther, January, 1919. 



\section{PREFACE TO THE FIRST EDITION.}

THE Grammar of Textile Design is a treatise upon the Fundamental Principles of Structural Design in Woven Fabrics, and the application of those principles in the production of various types of cloth.

It has been chiefly prepared as a text-book for students of weaving and designing in all branches of the weaving trade, and contains much information of practical utility to designers, salesmen, manufacturers, and others, to whom a knowledge of the construction, characteristic features, and uses of textile fabrics will be helpful.

In the descriptions of fabrics that are produced by the aid of special mechanical devices, these are briefly described and illustrated by scale diagrams, and include descriptions of three types of steel-wire doup harnesses for crossweaving; a loom for weaving leno fabrics in which warp ends are crossed by a system of douping in front of the reed; and a loom for weaving ondulé fabrics in which warp ends, and sometimes picks of weft, are caused to assume undulating or wavy lines in the direction of warp, or weft, respectively.

HARRY NISBET.

Bozton, October, 1906. 



\title{
CONTENTS.
}

\author{
CHAPTER I.
}

INTRODUCTION.

General Principle of Fabric Structure (\$\$ 1-4) and the use of Design Paper $(\S 5)$

\section{CHAPTER II.}

The Plain Weave and its Modifications.

The Plain, Calico, or Tabby Weave (\$6). Firmness of Texture ( $(7)$. Variety of Texture (\$8). Variety of Form: Ribbed Fabrics (\$§ 9-13). Corded Fabrics (§ 14). Matt Weaves $(\$ \S 15,16)$ - _ _ pages 6-23

\section{CHAPTER III.}

\section{Twill and Kindred Weaves.}

Classification of Twill Weaves $(\S 17)$. I. Continuous Twills: $(a)$ Warpface Twills (\$ 18). (b) Weft-face Twills (\$ 19). (c) Warp and Weftface Twills (\$ 20). The Angle of Twill (\$ 21). Influences affecting the Prominence of Twill and Kindred Weaves: (a) Character of Weave, (b) Character of Yarn, (c) Number of Threads per Inch, (d) Direction of Twill in Relation to the Direction of Twist in Yarn (\$\$ 22-26).

2. Zigzag or Wavy Twills (\$§ 27-29). 3. Rearranged Twills : Satin Weaves (\$30-32). Table of Intervals of Selection for the Construction of Satin Weaves (\$ 32). Corkscrew Twills (\$§ 33, 34). Rearrangement of Twill Weaves on Satin and other Bases (\$§ 35,36). 4. Combined Twills ( $(37)$. 5. Broken Twills (§38). 6. Figured or Ornamented Twills (\$39) - _ _ _ _ _ - pages 24-77

\section{CHAPTER IV.}

\section{Diamond and Kindred Weaves.}

Diamond Weaves ( $\$ 40)$. Honeycomb and Kindred Weaves ( $\$ 41)$. Brighton Weaves $(\S 42)$. Sponge Weaves (\$ 43). Huck-a-Back and Kindred Weaves (\$ 44$)$. Grecian Weaves (\$ 45). Linear Zigzag or Spider Weaves (§ 46 ). Crêpe or Oatmeal Weaves (\$§ 47,48 ) 


\section{CHAPTER V.}

\section{BEDFORd CoRds.}

Plain Calico-ribbed Bedford Cords; also Plain Twill-ribbed Bedford Cords ( $\$ \S 49,51)$. Figured Bedford Cords ( $\$ \S 52,53)$. Tabulated Data of Particulars relating to the Manufacture of Seventeen Varieties of Bedford Cord Fabrics described in this Chapter (\$ 54) - pages 110-125

\section{CHAPTER VI.}

BACKED FABRICS.

Weft-backed Fabrics ( $\$ 55)$. Warp-backed Fabrics (§ 56). Reversible or Double-faced Fabrics (\$ 57)

- pages 126-132

\section{CHAPTER VII.}

Fustians.

Varieties of Fustians ( $\$ 58)$. Imperial or Swansdown ( 59 ). Cantoon or Diagonal (\$60). Moleskin (\$61). Beaverteen $(\$ 62)$. Velveteen and Velveteen Cutting ( $\$ \S 63-71)$. Ribbed or Corded Velveteen (§ 72). Figured Velveteon $(\$ \S 73,74)$. Corduroy $(\$ \unlhd 75,76)$. Figured Corduroy (\$ 77). Corduroy Cutting Machines (\$\$ 78, 79)

pages $133-168$

\section{CHAPTER VIII.}

\section{Terry and Loop Pile Fabrics.}

Methods of Producing Terry Pile on Textile Fabrics (\$\$ 80, 81). Terry Pile Motions ( $\S \S 82,83)$. Holden's Terry Motion ( $\$ 84)$. Lister and Carter's Terry Motion ( $\S 85)$. Dugdale's Terry Motion ( $\$ 86)$. Smith's Terry Motion ( $\$ 87)$. Hacking's Terry Motion ( $\$ 88)$. Varieties of Plain Terry Pile Fabrics (§ 89). Three-pick Terry Fabric (\$ 90). Fourpick and Five-pick Terry Fabrics (\$ 91). Six-pick Terry H'abric (§ 92). Figured Terry Pile Fabrics (\$93). Terry or Twin Jacquard Machines (\$94). Draft and Shedding Plans for Three-, Four-, and Fivepick Figured Terry Fabrics (§ 95). Methods of Preparing Applied Designs for Figured Terry Fabrics (§ 96). Fast Reed Terry Pile Weaving ( $\$$ 97, 98). Practical Details of Terry Pile Weaving (\$99). Loop Pile Fabrics $(\S 100)$ - _ _ _ _ _ _ - pages $169-210$

\section{CHAPTER IX.}

Gauze and Net Leno Fabrics.

Gauze, Net Leno, and Leno Brocade Varieties of Cross=Weaving (§ 101). Plain Gauze and a Heald Gauze or Leno Harness $(\$ \S 102,103)$. Cellular Gauze Fabrics (§ 104). Net Leno Fabrics (\$§ 105-107). Gauze 
and Net Leno Figuring by means of several Back Standard Healds to each Doup Heald ( $\S$ 103-116). Leno Specialities Produced by a System of Crossing Warp Ends in front of the Reed ( $\$ 117$ ). A Device for the Production of Special Leno Effects (§ 118). Fullcross Leno Fabrics (\$ 119). Relative Merits of a Top and a Bottomdoup Harness (\$120). Relative Merits of Different Types of Dobbies for Gauze and Leno Fabrics (§ 121). Shaking Devices for Leno Weaving (\$§ 122-126). Practical Details of Leno Weaving (\$§ 127-130). Tempered Steel=wire Doup Harnesses for Cross=weaving (\$ 131). Mock or Imitation Leno Fabrics (\$132) - pages 211-271

\section{CHAPTER $\mathrm{X}$.}

\section{Leno Brocade Fabrics.}

Varieties of Leno Brocade Fabrics (§ 133). Essential Features of a Leno Brocade Jacquard Harness (\$134). Formation of " Neutral," "Open," and "Cross" Warp-Sheds (§ 135). Different Schemes of Crossing Warp Threads (\$§ 136-137). Various Types and Modifications of Lieno Jacquard Machines and Harnesses ( $\$$ 138-142). Devoge’s Special Modifications ( $\S$ 141-142). Various Methods of Preparing Leno Brocade Designs and also of Cutting the Pattern Cards therefrom (\$§ 143-148) .

- pages 272-305

\section{CHAPTER XI.}

Tissue, Lappet, and Swivel Figured Fabrics: also Ondulé Fabrics.

Tissue Figured Fabrics (§ 149). Madras Muslin Fabrics (\$§ 150-151). Lappet Figured Fabrics ( $§ 15$ 2-154). Cross-thread Lappet Figuring (§ 155). Spot Lappet Figuring $(\S 156)$. Swivel Figured Fabrics (§ 157). Warp Ondule Fabrics; also Special Types of Reeds for their Production (\$ 158). Special Loom Attachments for the Development of Warp Ondulé Effects (\$ 159). Weft Ondulé Fabrics and Methods of Producing Them (§§ 160-161) _ _ - pages 306-337

\section{CHAPTER XII.}

\section{Brocade Fabrics.}

Weft Brocade Figuring ( $\$ 162$ ). Method of Preparing Applied Designs for Weft-figured Brocade Fabrics (§ 163). "Split-shed " Jacquard Figuring Harness for Brocade Fabrics (\$ 16t). Warp and Weft-figured Brocade Fabrics; also Method of Preparing Applied Designs for these Fabrics (\$§ 165-167). Brocade Figuring with Extra Warp Threads and Extra Picks of Weft (§§ 168-170) - - _ - - - pages 338-361 


\section{CHAPTER XIII}

DAMASK FABRICS.

Varieties and Essential Features of Damask Fabrics (\$§ 171-172). The Compound Presser Harness (\$§ 173.175). Damask or Twilling Jacquard Machines (§ 176). The Bessbrook Damask or Twilling Machine (§ 177). Method of Preparing Applied Damask Designs (\$§ 178-180). Compound Damask Fabrics (\$§ 181-182)

pages $362-389$

\section{CHAPTER XIV.}

\section{ALHAMBra aND KindRed Fabrics.}

Varieties and Characteristic Features of Alhambra Fabrics; also the Preparation of Applied Alhambra Designs (\$§ 183-184). "Trellis" Counterpanes (\$§ 185-186). Alhambra Fabrics Devoid of Binding Weaves (§ 187). Figured Repp Fabrics (\$ 188) - pages $390-402$

\section{CHAPTER XV.}

Piqués or Toilet Welts: also Matelasse Fabrics.

Varieties and Characteristic Features of Piqués or Toilet Welts (§ 189). Methods of Embellishing Piqué Fabrics (\$ 190). The Structure of Piqué Fabrics (\$ 191). Plain Piqué Fabrics (\$\$ 192-194). Backed Piqué Fabrics (§ 195). Decorative Effects in Piqué Fabrics (\$ 196). The "Float" Harness Mounting (\$ 197). Method of Preparing an Applied Design for a "Float" Harness (\$198). Piqué Fabrics with Net Leno Stripes ( $\$ 199)$. Data Relating to the Manufacture of Piqué Fabrics (§200). Matelasse Fabrics and their Characteristic Features (§ 201). The Development of Applied Designs for Matelasse Fabrics (\$ 202)

pages $403-435$

\section{CHAPTER XVI.}

TOILET QUILTING FABrics.

Distinctive Features and Modifications of Toilet Quilting Fabrics (\$\$ 203206). “Dandy" Toileting (\$ 207). “Run-up" Toileting (\$ 208). Varieties of Toilet Fabrics (\$ 209). Jacquard Harness for Toilet Quilting Fabrics (\$ 210). Two-pick, One-Shuttle Toilet Quilting (\$211). Three-pick Toilet Quilting (§ 212). Essential Equipment of a Loom. for Weaving the "Half-fast" or "Stocking-back" and "Fast-back" Varieties of Toilet Quilting Fabrics; also a Method of Operating the Comber-boards (§ 213). Four-pick Toilet Quilting and its Various Modifications (\$ 214). Five-pick Toilet Quilting (§ 215). Six-pick Toilet Quilting ( $\$ 216$ ). The Additional Embellishment of Toilet Quilting Fabrics by means of Extra Coloured Warp Threads (\$ 217). Preparation of Applied Toilet Quilting Designs (\$§ 218-219). Data Relating to Ten Examples of Toilet Quilting Fabrics (\$ 220) 


\section{CHAPTER XVII.}

Patent Satin or Mitcheline Fabrics.

Origin and Essential Features of the "Patent Satin" Type of Fabric (\$ 221). Essential Equipment of a Patent Satin Loom: also the Construction of the Jacquard Harness; the Method of Drawing-in the Warp Threads; and the Shedding Plan for Patent Satin Fabric (§ 222). A Modified Structure of the Patent Satin Fabric (\$ 223). Method of Preparing an Applied Patent Satin Design (\$ 224). Data Relating to the Manufacture of Ten Examples of Patent Satin Fabrics (§ 225) . - pages $464-470$

\section{CHAPTER XVIII.}

TApestri Fabrios: also Kidderminster or Scotch CaRpet Fabrics.

Varieties of Tapestry Fabrics (\$§ 226-227). Tapestry Fabrics with One Series of Warp and Two Series of Weft, with All-weft Surfaces (\$228). Tapestry Fabrics with Two Warps and One Series of Weft, with Allwarp Surfaces (\$ 229). Tapestry Fabrics with Two Series Each of Warp and Weft Threads (\$ 230). Construction of a Jacquard Harness for Tapestry Fabrics (§ 231). Method of Preparing Tapestry Designs (§232). Instructions for Card Cutting (§ 233). Data Relating to the Construction of T'wo Examples of Tapestry Fabrics of Coarse and Fine Textures respectively (\$ 234). Kidderminster or Scotch Carpet Fabrics (\$ 235). Drafting and Shedding Plans for Producing Scotch Carpet Fabrics (\$236). Special Type of Jacquard Machine and Harness Employed for Weaving Scotch Carpet Fabrics (\$237). Operation of the Jacquard Machine and Harness in a Scotch Carpet Loom

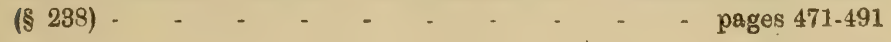





\section{CHAPTER I.}

\section{INTRODUCTION.}

Geveral Princtple of Fabric Structure and the Use of Design PAPER.

\$1. All woven fabrics are composed of at least two distinct series of threads termed "warp" and "weft" respectively. Warp threads lie lengthwise of the fabric, parallel with the selvedges (self edges), and weft threads, also termed "picks" of weft, traverse at right angles to warp threads. During the operation of weaving, warp threads are withdrawn simultaneously from their source as a continuous sheet or layer of evenly distributed threads that are spread out to the required width of cloth; whereas weft is (with exceptional instances) inserted only one thread or "pick" at a time, by means of a shuttle which places a continuous thread in successive parallel lengths, extending across the entire width of cloth between the selvedges, around which the weft returns for each successive "pick".

$\$ 2$. The interlacement of warp and weft threads is effected by dividing or separating (in a prearranged order for each pick of weft) the sheet of warp threads into two separate and distinct layers, one above the other (seen thus viewed from the end of a loom) to form an opening or division termed a "warp shed". A shuttle, carrying a supply of weft in the form of a "cop," or on a bobbin or quill, is then projected through the "warp shed" to Teave a trail of weft between the two layers of warp threads. These subsequently close upon the "pick" of weft which is "beaten up" or pushed by the reed to its ultimate place in the fabric, of which it becomes an integral part. Successive picks of weft are similarly inserted in successive "warp sheds" of different formation produced by 
separating warp threads in different orders, and according to a prearranged scheme of interweaving warp and weft, as represented by the design. After each pick is inserted, it is beaten up to its appointed place close to its predecessors, and thus is a textile fabric evolved in accordance with some definite scheme or plan of interweaving which constitutes the structural or woven design.

$\$ 3$. This mode of interlacing two distinct series of threads constitutes the chief and essential principle governing the construction of every type and variety of woven fabrics irrespective of the special division to which they belong. The particular order or scheme of interweaving those threads constitutes a minor principle of fabric structure that becomes the distinctive and characteristic feature of the class of fabrics embodying it. It is the diversity of schemes of interweaving warp and weft that gives rise to an almost infinite variety of textile fabrics, an exact classification of which would be an extremely difficult, if not impossible, task. This difficulty is greater from the circumstance of many fabrics being each known by a variety of trade names not only in different parts of the same country, but even in the same locality.

Under these circumstances the value of such a classification would be nullified, inasmuch as it could not be of general application; otherwise it would greatly facilitate reference by employing a definite term to signify a particular type or variety of fabric. All these varieties, however, may be classified under comparatively few chief divisions, each representing distinct types of fabrics known by technical descriptions and names more or less universally recognised. Each type is characterised by some special constructive element which distinguishes it from other types, and, as a rule, specially adapts it for particular uses. The present treatise is devoted to describing the characteristics of the principal types of fabrics and of their chief varieties and modifications; also the principles governing their construction, and the preparation of designs for them; the chief purposes for which they are employed, and other helpful information of a practical character relating to their manufacture. 
\$. Textile design is of two kinds, namely (1) structural, and (2) decorative. The first relates to the specific manner in which warp and weft threads are interlaced, by which is evolved woven design, which constitutes the technique of fabric structure. The second relates to the scheme of ornamentation by which a textile fabric is decorated, which is also more or less dependent upon woven design. It is the chief object of this book, however, to demonstrate the fundamental principles of structural or woven design, which may be aptly described as the "Grammar of Textile Design ".

A textile fabric may contain only one element of woven design and yet be profusely embellished without having recourse to colour. Many white and grey linen and cotton, and also white silk, damask, and brocade fabrics, are good examples of that class. The construction of such fabrics, and of damasks in particular, is frequently based upon some simple elementary weave _which is simply reversed to develop the figure and ground portions, thereby causing warp to preponderate on the surface in some parts, and weft in others; hence the contrasting tones of light which enable the figure and ground portions to be distinguished. The most elaborately decorated fabrics will oftentimes be found to contain not more than three or four different varieties of simple weaves effectively introduced in the scheme of decoration; whilst most fabrics employed for domestic purposes, and many others, contain but one element of design of a simple character and specially suited for a specific purpose. An examination of such fabrics will show that warp and weft are interwoven in some simple definite order or sequence that occurs with perfect regularity throughout the entire fabric.

Excepting by the employment of colour, it would frequently be impossible to clearly distinguish the various forms and details of an elaborate design, were it not that a designer has at his command an almost unlimited choice of fundamental weaves by which he may-develop its various parts to obtain contrast and variety of effect. Hence, it is equally if not more important that a textile designer should be conversant with the principles of fabric structure, as that he should be an artist and expert draughtsman. 
It is important to observe that in all examples of designs contained in this book, filled and shaded squares, or other symbols, on design or point paper, signify that warp threads are raised over weft, wherever those marks are placed, and blank squares signify weft passing over warp. This observation is made in consequence of some writers adopting the reverse practice of indicating warp and weft by blank and filled squares, respectively. But it is quite immaterial whichever method is adopted, so long as it is specified.

\section{Use of Design Paper,}

$\S 5$. It is also expedient, at this stage, to explain the use of squared paper on which textile designs are prepared. This is ruled with two sets of lines crossing at right angles to form a series of rectangular spaces. At regular intervals apart thicker lines, called "bar lines," are ruled in both directions to form large squares termed "bars". These large squares, or "bars," enclose a number of smaller rectangular spaces which may be either in equal numbers in both directions, or there may be more or less divisions in either direction, uniformly, according to the ratio of warp threads and picks per inch in cloth. In one direction, however, the thicker lines are ruled at regular intervals of either eight or else twelve spaces, according to the established practice in the construction of Jacquard machines, in which needles and hooks are (with exceptions) arranged in rows of either eight or else twelve. The thicker lines also incidentally facilitate the counting of spaces when setting out a Jacquard design; and they also serve as an index or guide to a cardcutter, as he "reads off" a design and records it by punching holes in the pattern cards. For the present purpose, however, it is sufficient to state that, excepting when employed for designs for certain compound fabrics, the narrow divisions on design paper correspond, in one direction, to warp threads, and in the other direction to picks of weft. If the number of spaces between two bar lines are alike in both directions, as $8 \times 8$ or $12 \times 12$, either series of divisions may represent either series of threads; but if the number of divisions are not alike each way, those of which there are either eight or twelve spaces in a 
"bar" must represent warp threads, according to the index of the Jacquard machine for which the design is intended. The ratio of warp and weft spaces in a bar should, however, correspond as nearly as practicable to the ratio of warp threads and picks per inch in the finished fabric, in order to ensure the correct shape of figures in a pattern. For example, if a design is to be prepared for a fabric that will contain eighty warp threads and sixty picks per inch, to be produced by means of a 400 's Jacquard machine, the proper counts of design paper for it is that ruled $8 \times 6$ divisions to each "bar": and for a 600 's Jacquard machine, that ruled $12 \times 9$ is the proper counts. By placing a dot in a small square, it signifies that the corresponding warp thread must be raised above the corresponding pick of weft in cloth; and a blank square signifies that weft must pass over warp at the parts indicated. 


\section{CHAPTER II.}

THE PLAIN OR CALICO WEAVE AND ITS MODIFICATIONS.

§6. The "plain," "calico" or "tabby" weave, as it is variously described, is the most simple and elementary combination of two series of threads employed in the construction of textile fabrics; albeit, it produces a relatively stronger fabric than is obtained by any other simple combination of threads, excepting that of "gauze" or "cross-weaving".

The minor principle observed in the construction of plain cloth is the interlacement of any two contiguous threads of either warp or weft in an exactly contrary manner to each other, with every thread in each series passing alternately under and over consecutive threads of the other series uniformly throughout the fabric. By this plan of interlacement, every thread in each series interweaves with every thread in the other series to the uttermost extent, thereby producing a comparatively firm and strong texture of cloth. Also, a complete unit of the plain weave occupies only two warp threads and two picks of weft, as represented in Fig. 1, which is the design (as indicated on design paper) for that weave. The rectangle enclosing two shaded and two white squares indicates one unit of the design, which is repeated sixteen times. The construction of plain cloth is clearly demonstrated by means of Figs. 2, $2 \mathrm{~A}$ and $2 \mathrm{~B}$, which are diagrams representing a plan, a transverse section (crosswise), and a longitudinal section (lengthwise) respectively of that cloth.

\section{Firmness of Texture.}

$\$ 7$. The degree of firmness of texture in woven fabrics is largely determined by the manner of interweaving warp and weft, and will be greater or less according as the two series 
of threads interlace more frequently or less frequently, respectively. Thus, if two pieces of cloth are woven from similar warp and weft, and with the same number of warp threads and picks per inch-(a) with the plain weave, in which all threads interlace to the uttermost extent, and $(b)$ with any other weave -the latter would be less firm, and therefore of weaker texture than the former, because the threads composing it would be bent in a lesser degree than those of the plain weave, thereb causing them to be less firmly compacted. For this reason it is important that the counts of warp and weft, the number of warp threads and picks per inch, and the weave, should be properly proportioned, in order to obtain the best results. This
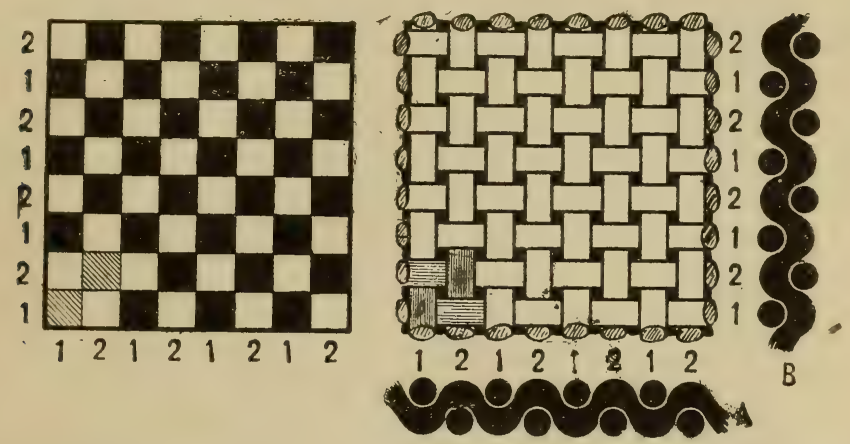

FIG. 1.-Plain or tabby weave.

FIG. 2.-Plan and sections of the tabby weave.

phase of fabric structure, however, does not come within the scope of this treatise.

Notwithstanding the very simple character of the plain weave, it is produced in a great variety of forms and textures, possessing totally different characteristics, which adapt it for specific purposes. Apart from those arising from the employment of different textile materials, and also without in any way departing from the true principle of the plain weave, as defined in $\$ 6$, the varieties of texture and form are produced $(a)$ by the employment of different counts of yarn for different fabrics; (b) by the employment of warp of one counts and weft of another counts in the same fabric; (c) by the employment of warp or weft, or both warp and weft, of different counts in the same 
fabric; and (d) by subjecting some warp threads to a greater degree of tension than others during weaving.

\section{Variety of Texture.}

§. The term "texture" is here used to signify the general qualities of a fabric as regards material, counts of yarn, relative density of threads, weight, bulk, how it feels when handled,

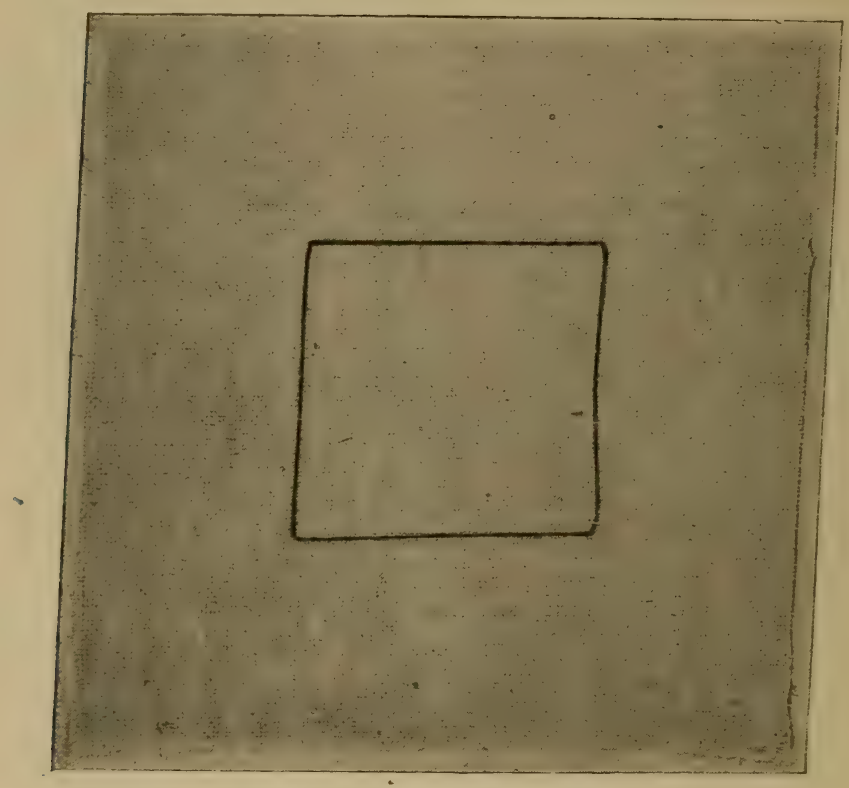

FIG, 3,-Plain calico cloth.

and other properties peculiar to woven fabrics. Plain cloth is produced in a greater variety of textures than perhaps any other weave, and varies from the fine, light, open and airy texture of muslin, to that of coarse and heavy hempen sackcloth. When produced from cotton yarn of counts ranging in different fabrics from about 20 's to 160 's for both warp and weft, and containing from about 40 to 160 warp threads and picks per inch, it is known as calico-a fabric produced in great quantities, and extensively used in both a grey (i.e., of the natural colour of 
cotton) and a bleached state, for a variety of domestic purposes. A true plain cloth is one in which the counts and qualities of both warp and weft, and also the number of warp threads and picks per inch, are similar. When these conditions exist either precisely or approximately, whether the texture is fine or coarse, light or heavy, it will produce a general evenness of surface, resulting from warp threads and picks each bending or yielding

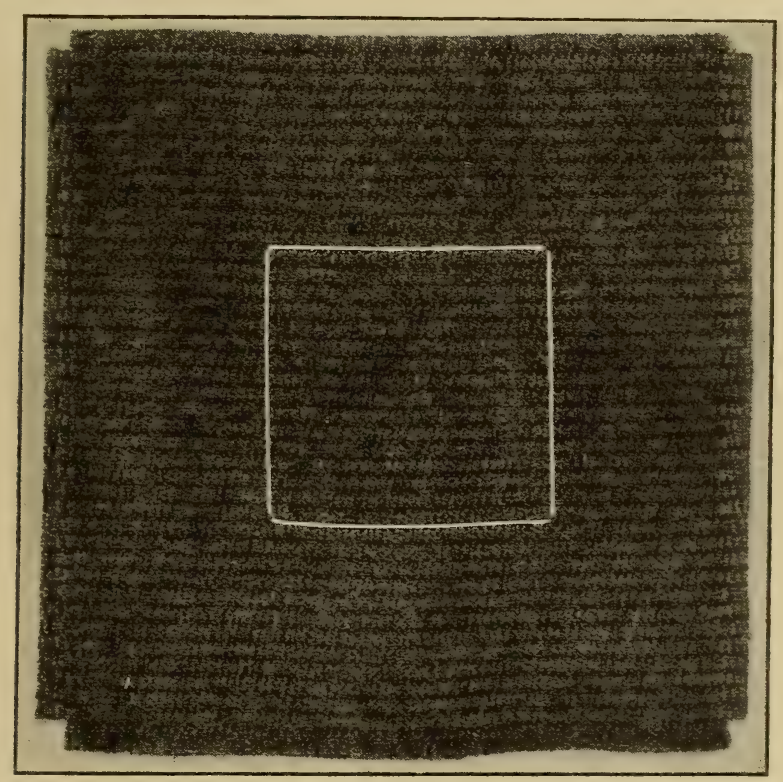

FIG. 4.-Simple warp-ribbed cloth.

to each other's influence in a corresponding degree. Fig. 3 illustrates an example of grey "calico" of medium quality, containing seventy-two warp threads of 36 's T., and sixty-two picks of 30's W., per inch. (The rectangle encloses one square inch of -cloth.)

\section{Variety of Form: Ribbed Fabrics.}

9. The least variation of form in the plain weave is effected by employing warp and weft of sufficient difference of counts 
and density of threads to produce a ribbed or corded effect throughout the fabric. The ribs or cords will lie in the direction of the coarsest threads, and will be more or less prominent according as the difference in counts between the two series of threads is greater or less respectively. Fig. 4 illustrates an example of cloth woven from comparatively fine warp and coarse
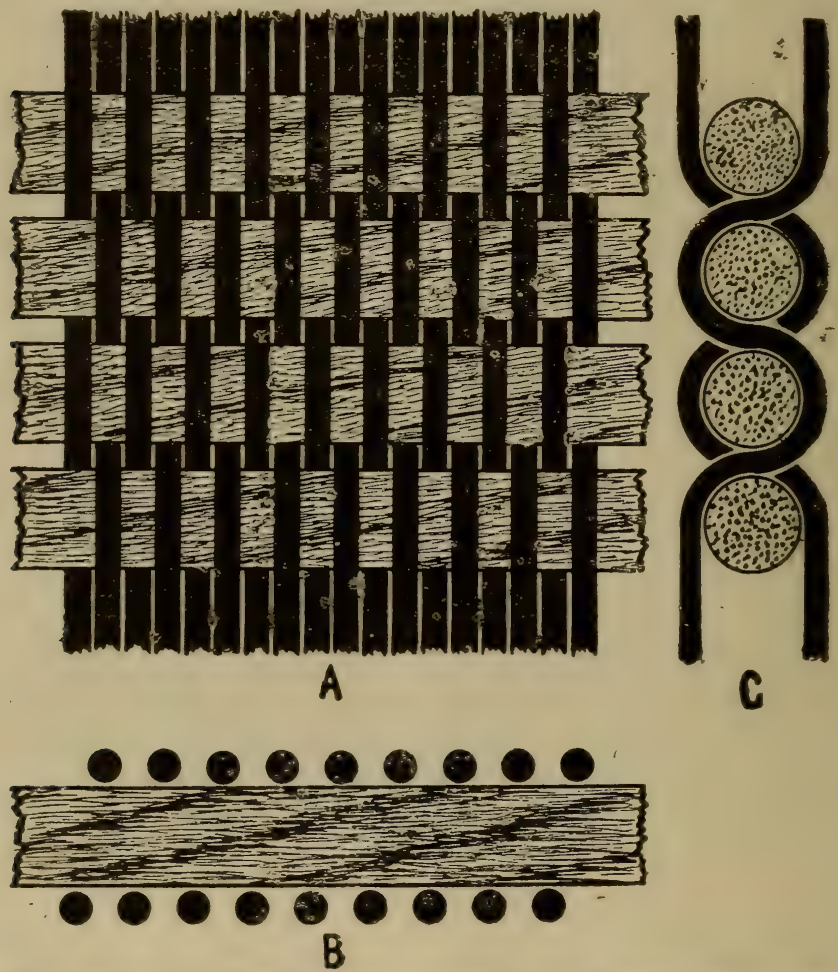

FIG. 5A, B, C.-Plan and sections of simple warp-ribbed cloth.

weft, which develop a series of ribs lying in the direction of weft, and known as warp ribs. By reversing these conditions, the ribs would lie in the direction of warp, in which case they would be termed cords, or weft ribs. In either case the rib formation is entirely due to the non-yielding quality of the coarser and stronger threads, and the yielding quality of the finer and weaker threads, which perform all the bending under 
and over the coarser threads. This is clearly illustrated by diagrams, Figs. 5A, 5B and 5c, which are a plan, transverse section and longitudinal section, respectively, of the warpribbed cloth, Fig. 4, which contains sixty-eight warp threads and sixteen picks per inch. (The rectangle encloses one square inch of cloth.)

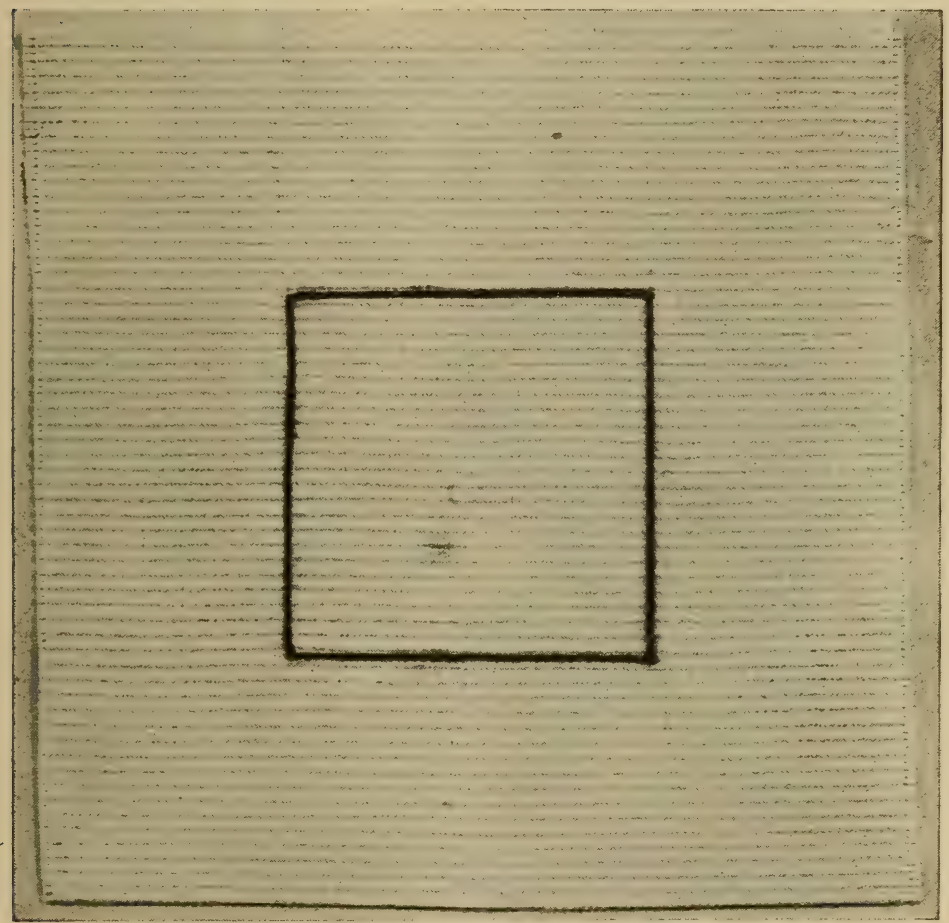

FIG. 6.-A third example of warp-ribbed cloth.

Another variety of the plain weave is illustrated in Fig. 6. In this example the rib formation results not entirely from any great difference between the counts of warp and counts of weft, as in the previous examples, but only partly from that circumstance, and, in a greater measure, in consequence of alternate warp threads being held at a considerably greater degree of tension than intermediate warp threads, during the operation of 
weaving. This difference of tension between what are virtually two series of warp threads-although each series is of exactly the same kind of yarn-necessitates the use of two warp beams -one containing, say, all odd-numbered warp threads, and the
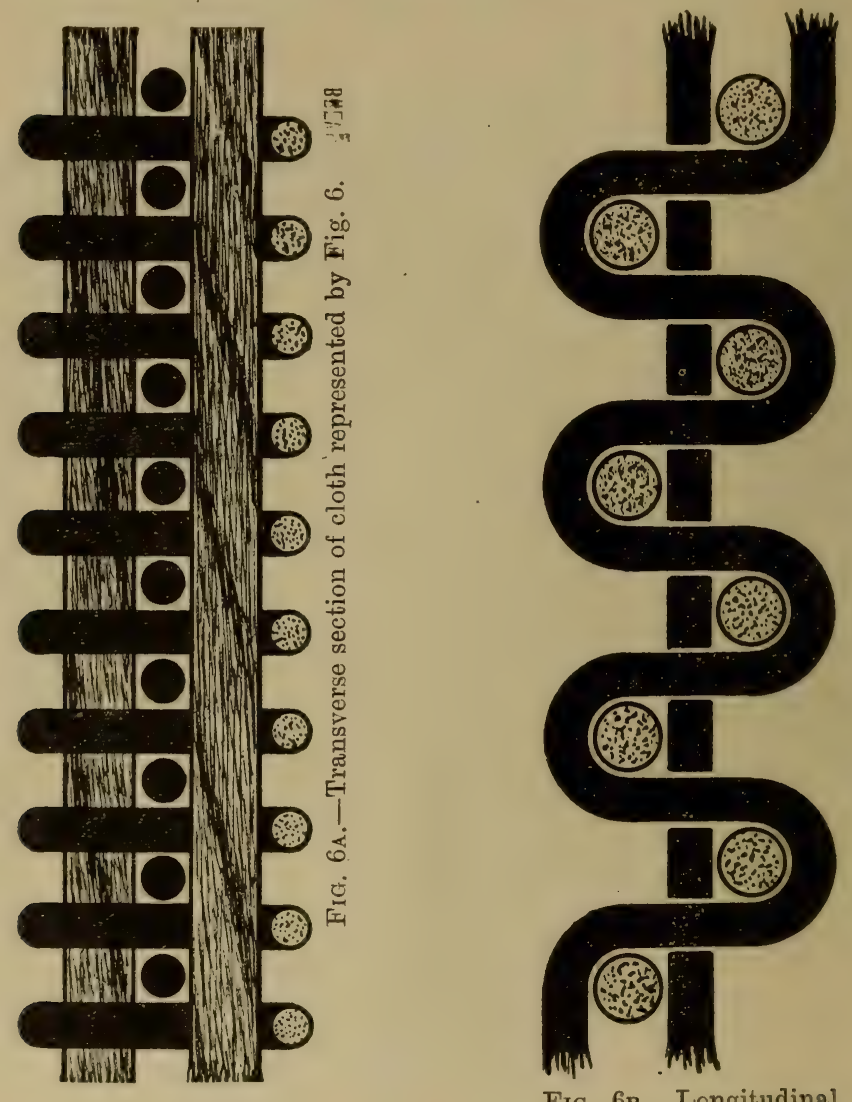

FiG. 6B.-Longitudinal section of cloth represented by Fig. 6 .

other all even-numbered warp threads. Only one system of weft, which is of coarser counts and much softer than the warp, is employed. In consequence of some warp threads being held taut during weaving they are prerented from bending, and therefore lie in an almost perfectly straight line throughout the 
length of cloth. This causes picks of weft to lie perfectly straight and in two planes, above and below taut warp threads, and so form prominent ribs on both sides of the cloth; whilst slack warp threads freely bend over and under picks of weft, to bind them firmly in position, as indicated in transverse and longitudinal sections, diagrams Figs. $6 \mathrm{~A}$ and $6 \mathrm{~B}$.

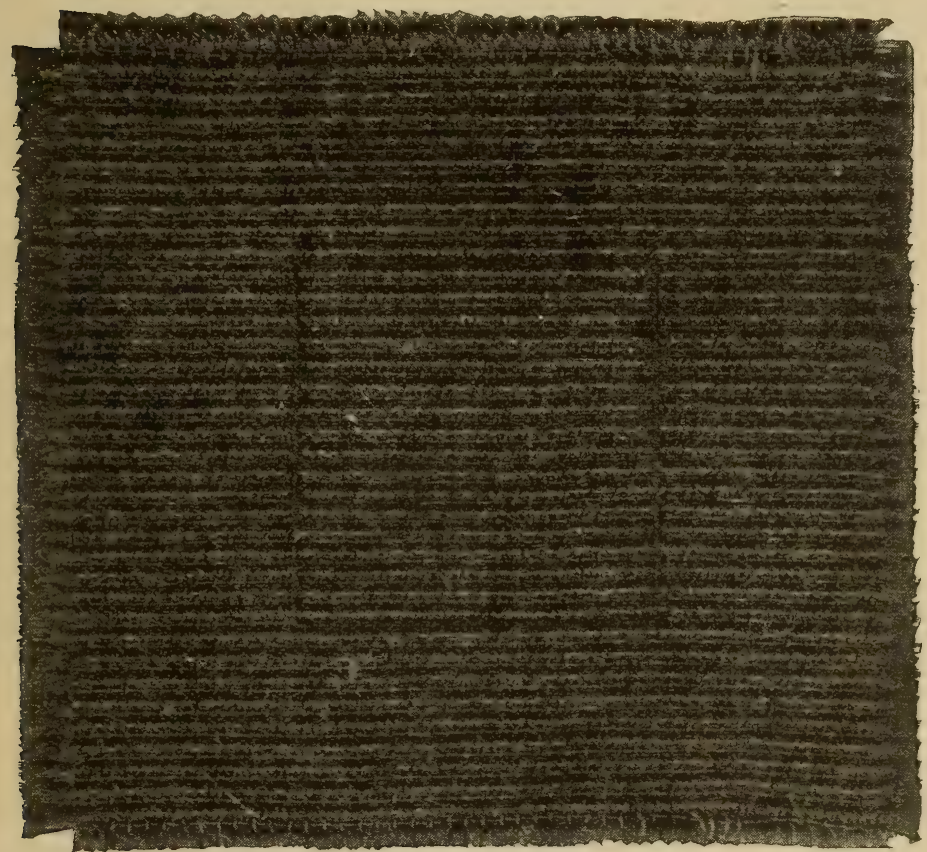

FrG. 7.-Repp (warp-ribbed) cloth.

The amount of contraction of warp during weaving, in cloth of this description, will vary according to the counts of weft and number of picks per inch, as well as according to the degree of tension upon the warp during weaving. The contraction will be greater or less in proportion to the thickness of weft and the number of picks per inch in cloth. In the example, Fig. 6, 
which contains eighty-four warp threads of 30 's T. and forty-six picks of 16 's W. per inch, the amount of contraction is equal to $2 \frac{1}{3}$ per cent. for taut, and $33 \frac{1}{3}$ per cent. for slack warp threads. (The rectangle encloses one square inch of cloth.)

10. One more variety of ribbed cloth, based on the principle of the plain weave, will serve, along with the two previous examples, to demonstrate its variation of form in the development of ribbed fabrics. This is the well-known "repp" cloth, illustrated in Fig. 7, so extensively employed for window blinds in railway carriages and other vehicles, and for the upholstering of furniture. In general appearance it closely resembles the ribbed cloth illustrated in Fig. 4 ; but a close inspection of both examples will reveal a great difference in their construction and texture-the repp cloth being much firmer and stronger than the ribbed cloth.

In the production of repp cloth, as illustrated in Fig. 7, two distinct series of both warp and weft threads are employed-the counts and character of each series being such as to develop a series of very prominent and sharply defined ribs in the direction of weft. The warp series comprise two counts of yarn-one fine and strong, which is held at greater tension during weaving, and the other coarse and soft, which is held at a lesser degree of tension, to enable it to easily yield and bend over coarse and under fine picks of weft. Each series of warp threads is wound upon a separate warp beam to allow of a different rate of contraction during weaving. They may be arranged in the harness and reed in the order of one fine and one coarse warp thread alternately; but a superior rib will be produced by running two medium warp threads together, as in the example, Fig. 7, and as indicated in plan, Fig. 8A. The weft series also comprise two counts of yarn-one fine and strong, similar to the fine warp, and the other very coarse and strong. These are inserted, one fine and one coarse pick alternately, thereby requiring a loom with two shuttle boxes at each end of the sley, and a "pickand-pick" picking motion, i.e., one capable of picking twice, or more than twice, in succession, from each side of a loom. When coarse picks are inserted, all medium warp threads only are raised to form ribs; and when fine picks are inserted, all 
fine warp threads only are raised, thereby forming deep furrows by binding down all medium warp threads between the coarse picks, as seen in longitudinal section, diagram, Fig. 8в. The sample of repp cloth illustrated in Fig. 7 contains $21 \times 2=42$ medium, and 21 fine warp threads per inch; and 17 coarse and 17 fine picks per inch. (The rectangle encloses one square inch of eloth.)
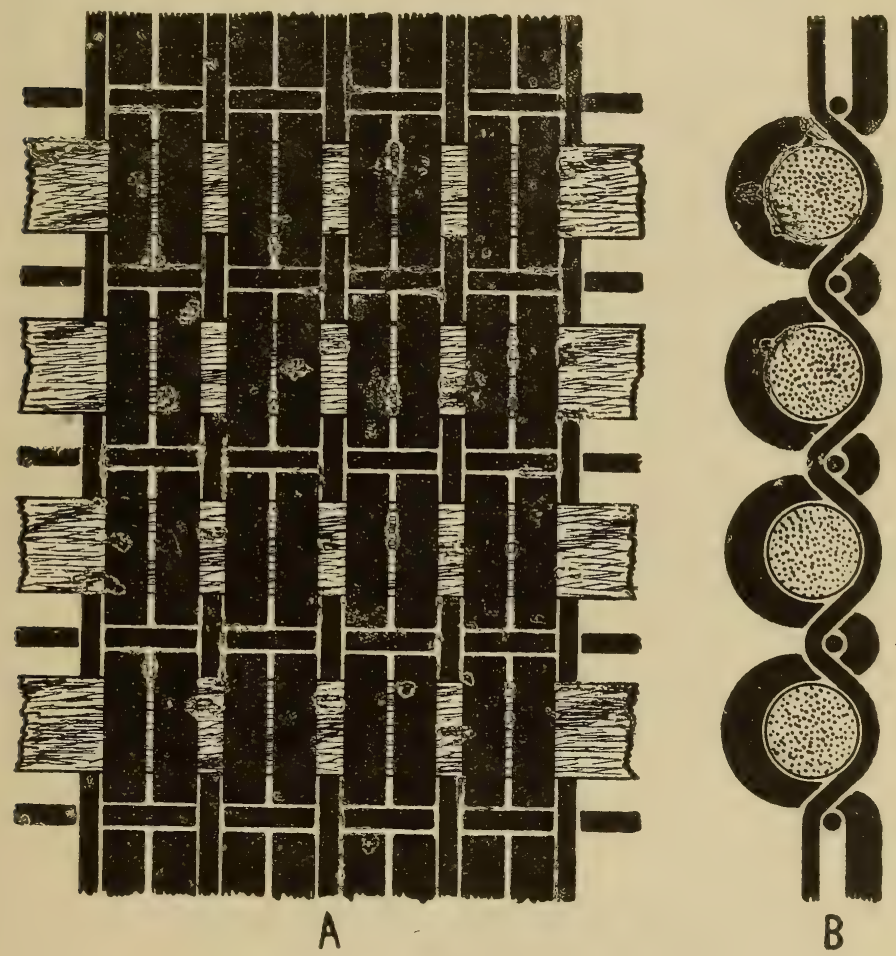

FIG. 8A, B.-Plan and longitudinal section of repp cloth.

$\S 11$. In the foregoing examples of ribbed fabrics, the ribs of the respective pieces are of uniform size, and occur in immediate succession, thereby producing a general evenness of effect and uniformity of texture throughout the entire piece of cloth. Cords or ribs are, however, frequently employed as a simple means of ornamenting what would otherwise have been 
entirely plain fabrics, but which are made to assume a variety of decorative effects of a very pleasing character. Such effects are, of course, confined to stripes, running either up or across the cloth, and to checks. Stripes may be formed in an upward direction in a plain calico fabric by disposing comparatively coarse warp threads or else groups of warp threads either at regular or irregular intervals apart, according to the effect desired. Such threads may be either of uniform counts, to produce plain ribs, or of different counts, to produce variegated ribs. By inserting coarse picks of weft instead of coarse warp threads in the manner just described, stripes would be formed across the piece; and by introducing coarse threads in both series, checks

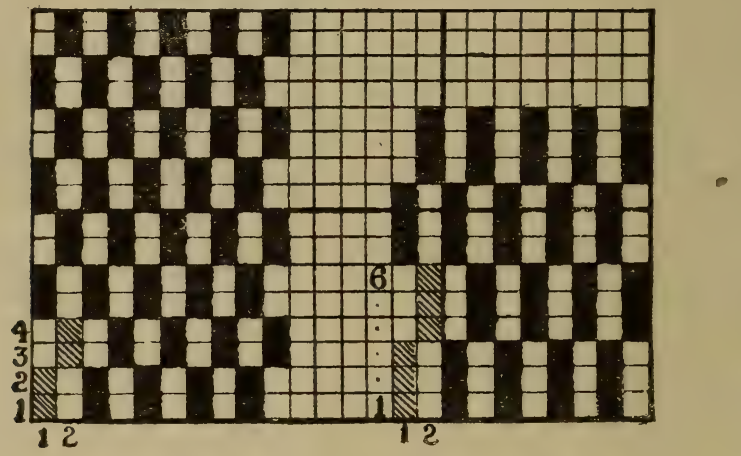

Fig. 9.

Fig. 10.

of great variety may be formed. A familiar example of this method of embellishing a plain fabric is that of a cambric pocket handkerchief, bordered either by a series of thick threads or by placing two or more fine threads together side by side to form cords.

$\S 12$. The development of ribs and cords is not dependent upon the employment of coarser threads in one series than in the other. They may be formed in fabrics composed of warp and weft of uniform counts, by causing two or more threads of one series (according to the required prominence of rib) to lie closely side by side, so as to virtually constitute a coarse thread composed of several strands not twisted together, and 
interweaving such groups of threads with separate threads of the other series. If threads are grouped in uniform quantities throughout, the ribs will be of uniform size; but if grouped in irregular quantities, a series of variegated ribs will be produced.

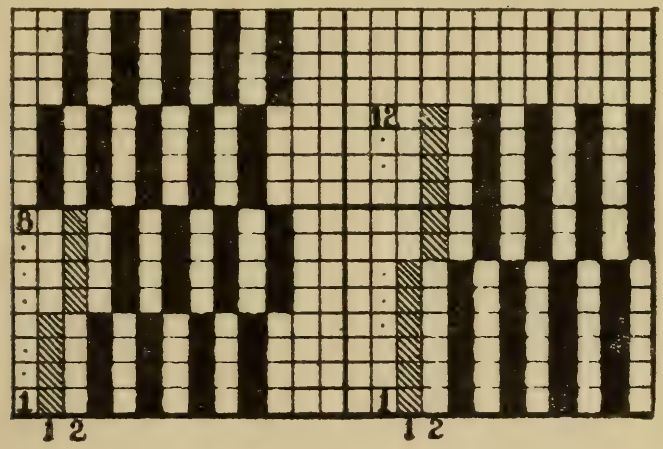

Fig. 11.

FIG. 12.

By this method the rib formation is caused by the combined resistance of the grouped threads, which lie straight, thereby compelling the separate threads of the other series to yield and bend under and over them, in accordance with the principle of fabric structure which determines that the relative

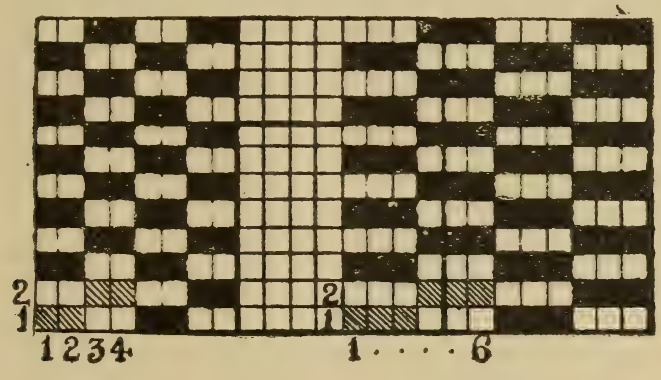

FIG. 13.

Fig. 14.

prominence of threads diminishes in proportion to the amount of bending performed by them in cloth.

$\S 13$. Simple ribs of various sizes may be formed in the direction of weft by separating alternate warp threads (as in the plain calico weave) and inserting two or more picks of weft in the 
same warp sheds. Figs. 9 to 12 are designs for this class of rib weaves containing two, three, four, and six picks respectively, in each warp shed. Each design repeats on two warp threads, and such number of picks as are contained in two ribs, namely,

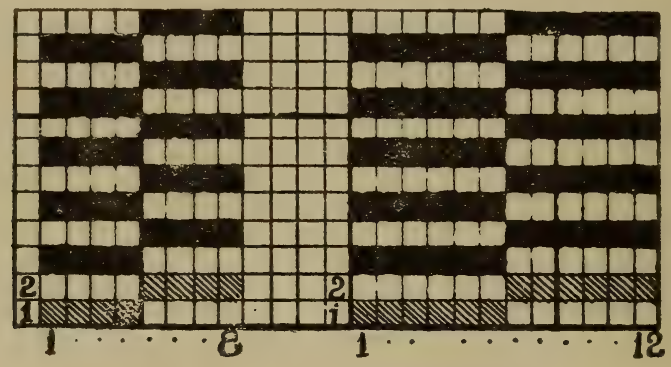

FIG. 15.

FIG. 16.

four, six, eight and twelve respectively. In the production of these or other weaves, in which several successive picks are inserted in the same shed, it is necessary to furnish a loom with a selvedge motion, to operate selvedge warp threads in a different order from that of warp threads forming the body of the fabric

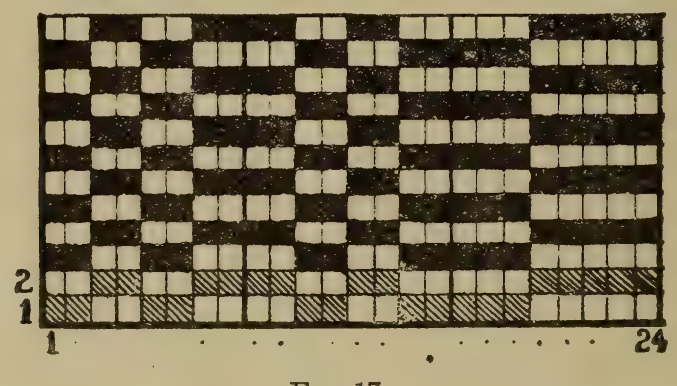

FIG. 17.

and thereby prevent picks of weft from being pulled backward into the shed when a shuttle passes through the same shed for several picks in succession.

$\S 14$. Simple cords may be formed in the direction of warp by raising warp threads in uniform groups of two or more threads which may pass through heald eyes either separately or in groups. Figs. 13 to 16 are designs for cords in which two, 
three, four, and six warp threads respectively are grouped together. Each design repeats on as many warp threads as are contained in two cords, and two picks of weft. Since each pick is contained in a separate warp shed, it is unnecessary to employ a special selvedge motion when weaving those designs.

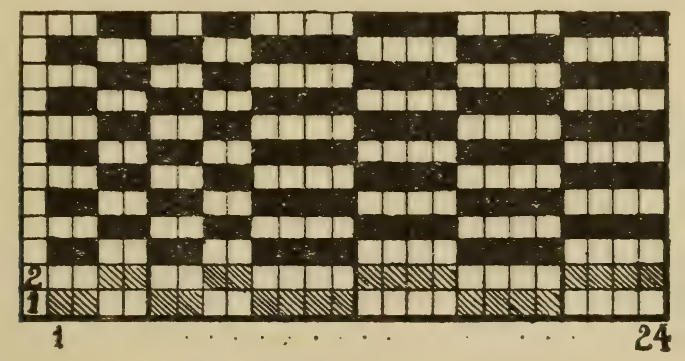

FIg. 18.

It was stated in $\$ 12$ that variegated cords or ribs may be formed by an irregular system of grouping threads either warp way or weft way respectively. It should be observed, however, that much greater scope is afforded in this respect by grouping warp threads, than by grouping picks of weft; also that varie-

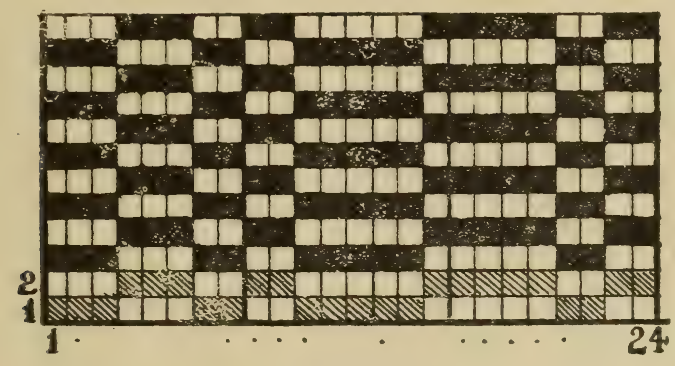

FIG. 19.

gated cords (warp way) may be produced more economically than variegated ribs (weft way). This arises from the fact that cords of any variety may be formed in a plain loom simply by varying the drafting of warp threads through the healds; whereas variegated ribs would require to be woven in a loom mounted with a dobby or even a small Jacquard machine (for large 
patterns) and furnished with a selvedge motion. Also, in addition to the extra cost of such looms, the weavers would require to be paid a higher rate of wages. Figs. 17 to 20 are designs for variegated cords, each repeating on twenty-four warp threads

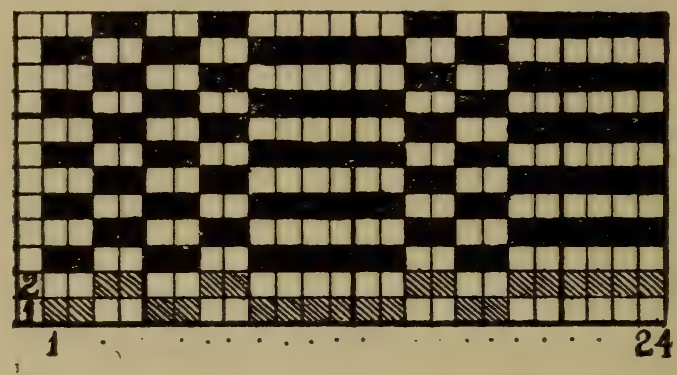

Fra. 20.

and two picks. By turning those designs on their side they become variegated ribs, repeating on two warp threads and twentyfour picks.

\section{Matt Weaves.}

$\S 15$. Simple matt weaves are those in which groups of two or more contiguous warp threads and picks interlace with each

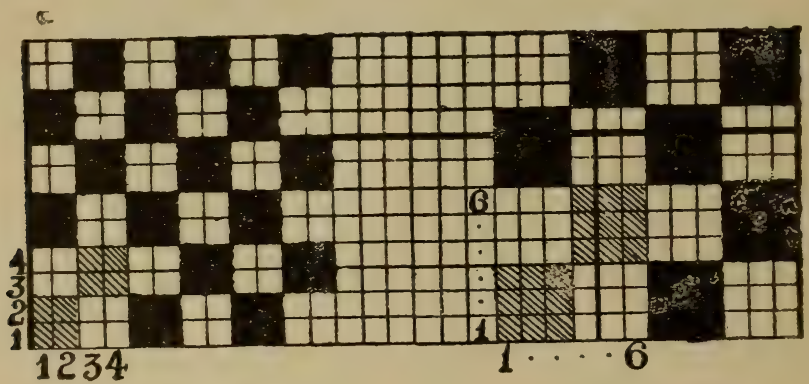

FIG. 21.

FIG. 22.

other so as to produce a chequered or dice effect, as represented in designs, Figs. 21, 22 and 23. The simplest of these weaves is that known as a two-and-two or four-end matt weave, indieated in Fig. 21, in which warp threads and picks interweave in pairs throughout the fabric, on the princip le of the plain weave. 
This matt weave is extensively adopted for a great variety of fabrics, of which dress materials, shirtings, sailcloth (for ships' sails), and "duck" cloth are, perhaps, the more notable examples. Figs. 22 and 23 are designs for three-and-three (six-

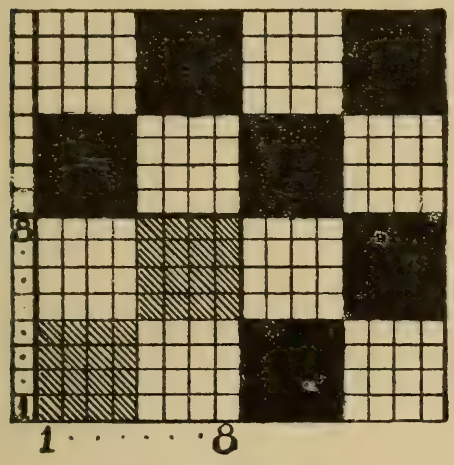

Fig. 23.

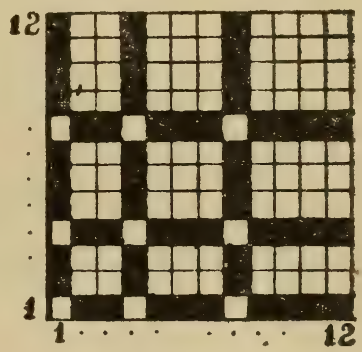

FIG. 24.

end) and four-and-four (eight-end) matt weaves respectively. When these and larger matt weaves are employed, the number of warp threads and picks per inch in cloth should be propor-

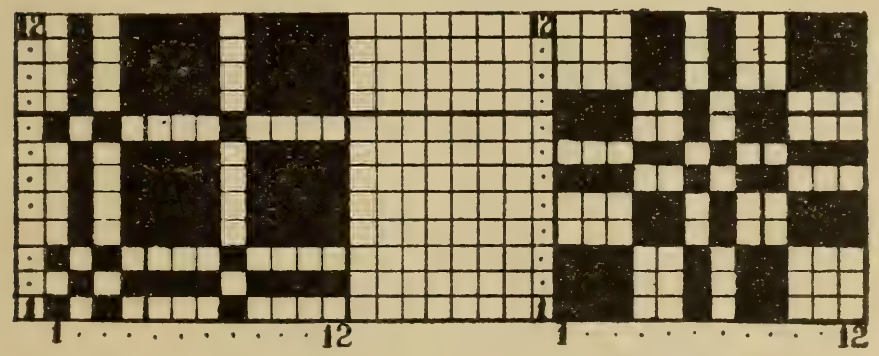

FIG. 25.

Fig. 26.

tionately increased, otherwise they would produce fabrics of an open and flimsy texture, in which the threads would become easily displaced, in consequence of the very few intersections made by them.

$\S 16$. Variegated matt weaves are developed by combining irregular groups of warp and weft threads, after the manner 
indicated in Figs. 24 to 29, of which the first three are designs repeating on twelve, and the last three, on sixteen warp threads and picks. They may be formed with weft preponderating on

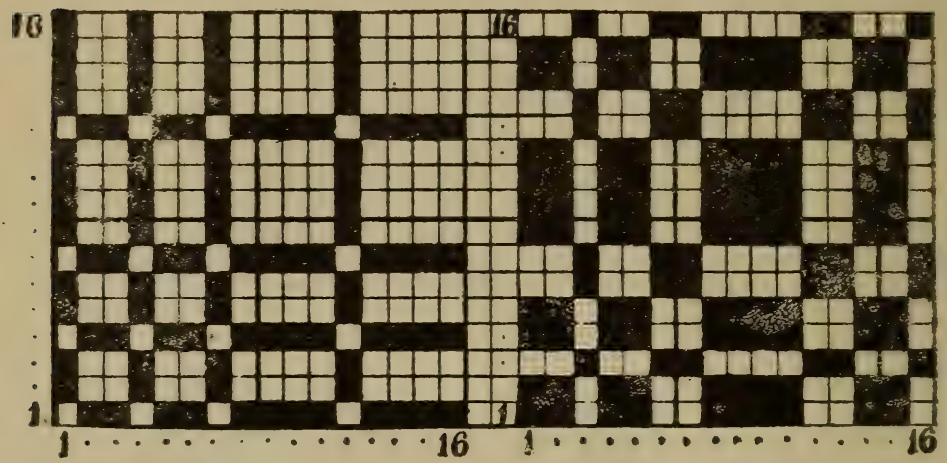

FIg. 27.

FIG. 28.

the face, as Figs. 24 and 27 ; with warp preponderating on the face, as Figs. 25 and 28 ; or they may be designed as true counterchange or diaper patterns, as Figs. 26 and 29, in which

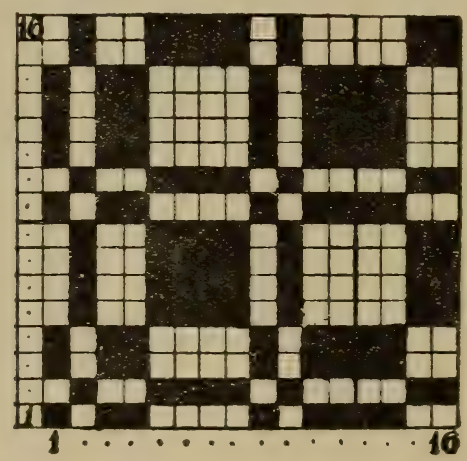

FIG. 29.

warp and weft are exactly counter to each other and in equal quantities on both the face and back of the fabric. It will be observed that in these designs, as in all others of the same class, there are only two orders in which warp threads interweave with 
PLAIN OR CALICO WEAVE AND ITS MODIFICATIONS. 23

weft, thereby requiring not more than two healds for their production in cloth, although the healds would require to be operated by a dobby or other shedding device, for designs repeating on such number of picks as are beyond the reasonable scope of tappets. 


\section{CHAPTER III.}

\section{TWILL AND KINDRED WEAVES.}

17. Twill weaves form a distinct departure from any of the foregoing, and they constitute a most useful variety of weaves extensively employed in the construction of numerous classes of fabrics. They exist in endless varieties of form, and are based on a simple principle of design; but whatever particular appearance they assume, they are generally characterised by a series of more or less pronounced diagonal wales or ridges and furrows, with either warp or weft preponderating, or in equal quantities, on the face of the fabric. The twill may be produced continuously either from right to left (i.e., sinistrally), as in Fig. 30 ; or from left to right (i.e., dextrally); or again, it may be produced in reverse directions in the same fabric, as desired. The variety of twill weaves is so considerable as to render an exact classification of them impossible. For the present purpose, however, they may be broadly divided into six chief varieties, namely: (1) continuous twills; (2) zigzag or wavy twills ; (3) rearranged twills, including satin weaves and "corkscrew" twills; (4) combined twills; (5) broken twills; (6) figured and other twill weaves of an indefinable character. Each of these divisions may be subdivided into $(a)$ warp-face twills; $(b)$ weft-face twills; and $(c)$ warp and weft-face twills, in which warp and weft are in either equal or unequal quantities on the face of the fabric.

\section{Continuous Twills.}

\$18. (a) Warp-face Twills.-These are formed by raising all warp threads, excepting one, in each repeat of the pattern, for each pick, and stepping one warp thread in consecutive rotation (to the right or left, according to the required direction of twill) 
as successive picks are inserted. These will develop a series of diagonal wales or ridges of warp, separated by furrows formed by single stitches of weft. Twill weaves may be formed on any number of warp threads and picks, from three upwards. Figs.

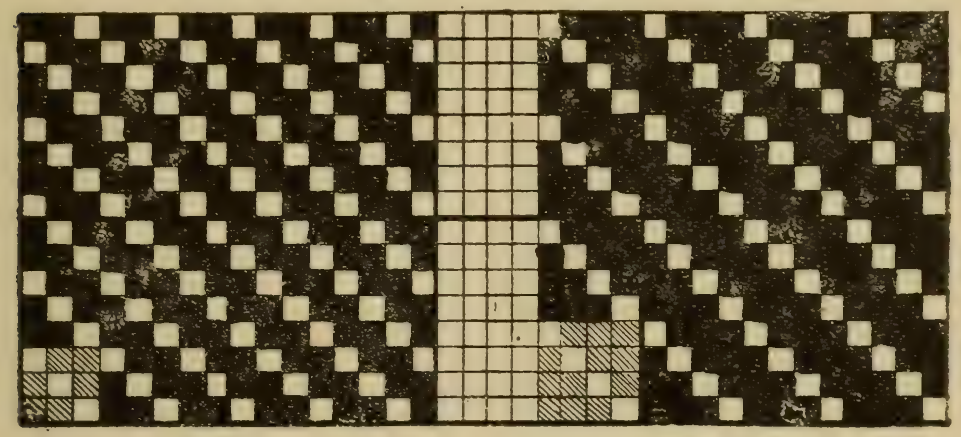

F'IG. 3U.

FIG. 31.

30 to 35 are designs for warp twills repeating on three to eight warp threads and picks respectively, and will be sufficient to indicate the principle of their construction.

$\$ 19$. (b) Weft-face Twills.-These are produced by reversing

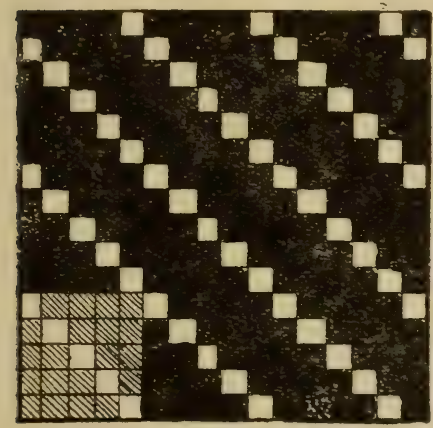

FIG. 32.

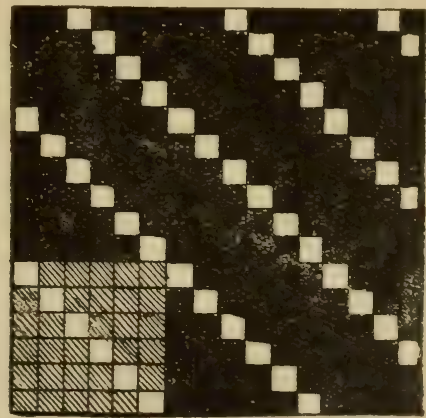

FIG. 33.

the conditions stated in $\$ 18$, by raising one warp thread only, in each repeat of the pattern, for each pick, and proceeding in a similar manner to that described for warp-face twills. This will produce a series of diagonal ridges of weft separated by single stitches of warp, as indicated in designs, Figs. 36 to 41, which 
are for weft twills repeating on three to eight warp threads and picks respectively.

$\S$ 20. (c) Warp and Weft-face Twills.-These may be formed with either equal or unequal wales of warp and weft arranged

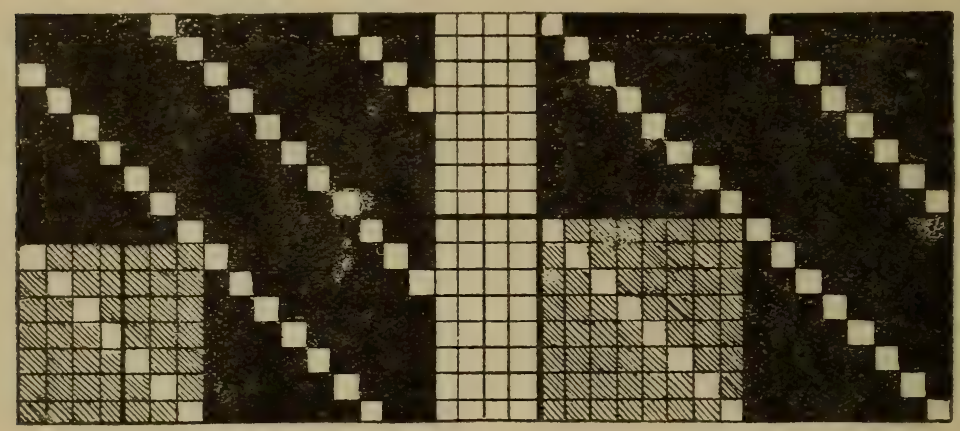

FIG. 34.

FIG. 35.

alternately. If the wales are equal, that is, if both warp threads and picks pass over and under the same number of threads uniformly, warp and weft will necessarily be in equal quantities on both the face and back of the fabric; but if the wales are

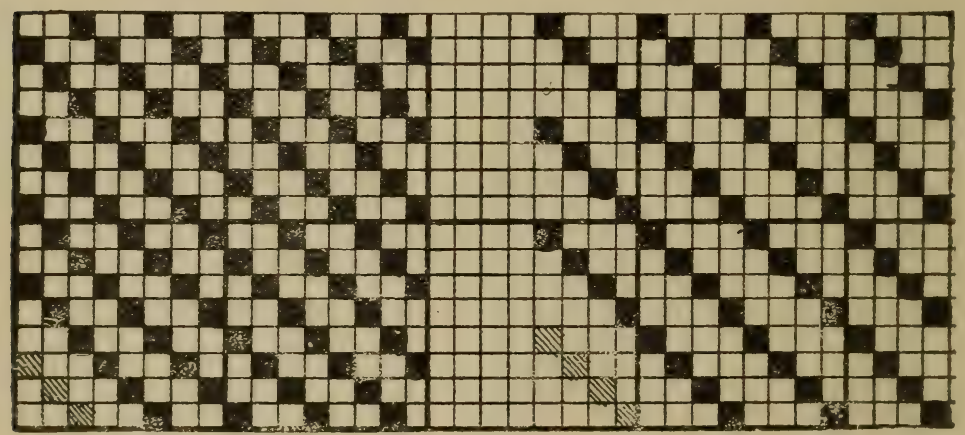

FIG. 36.

FIg. 37.

unequal, warp and weft may be either in equal or unequal quantities on the face and back of cloth. Equal wales are formed by alternately raising and leaving down equal groups of two or more warp threads for each pick, and stepping one warp 
thread in consecutive rotation as successive picks are inserted. The least of this class is that variously known as the "two-andtwo" $\left(\frac{2}{2}\right)$, the "Harvard " and the "Cassimere" twill given in Fig. 42, which repeats on four warp threads and picks. This is

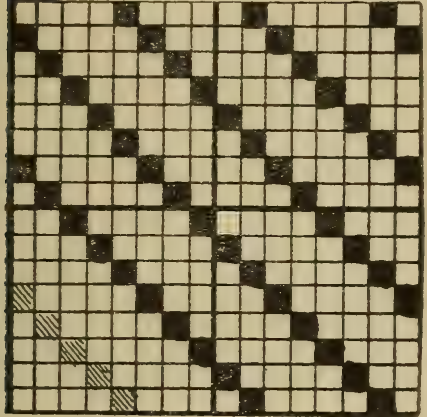

FIG. 38.

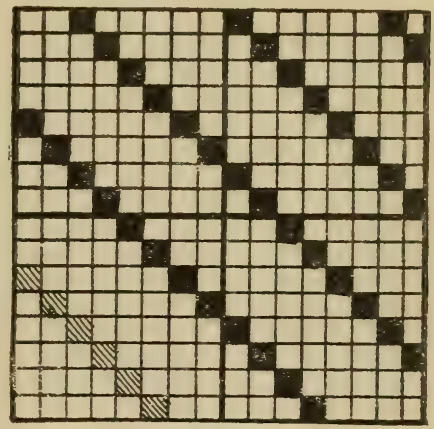

FIG. 39.

a very useful weave, and one that is perhaps more extensively employed than any other of its class. The principle on which it is constructed is conducive to the production of firm and strong cloth of comparatively light, texture. These qualities

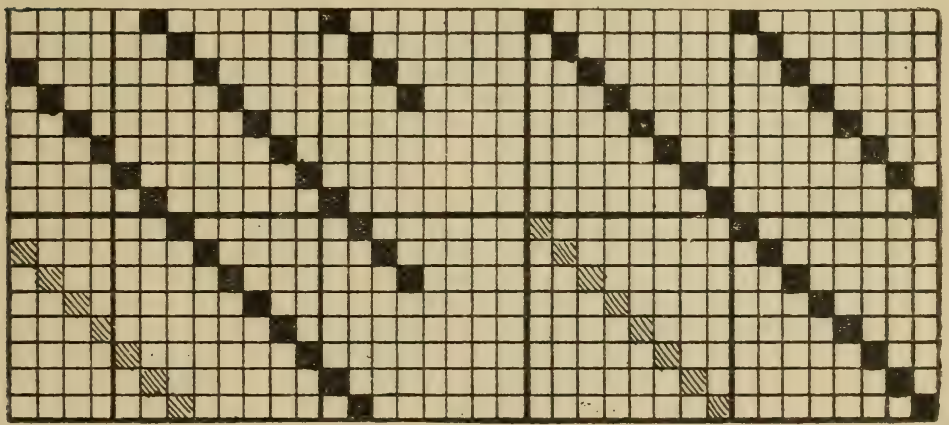

Fig. 40.

FIG. 41.

arise from warp and weft interlacing with such frequency and in such a manner as to permit of the threads of each series lying close together. On examining this weave, it will be seen that alternate threads of warp or weft interweave in an opposite 
manner at the same time; that is, when one is above, the other is below the same threads of the other series, although all threads in both series interweave in a precisely similar manner to each other. For these reasons, this simple twill weave is

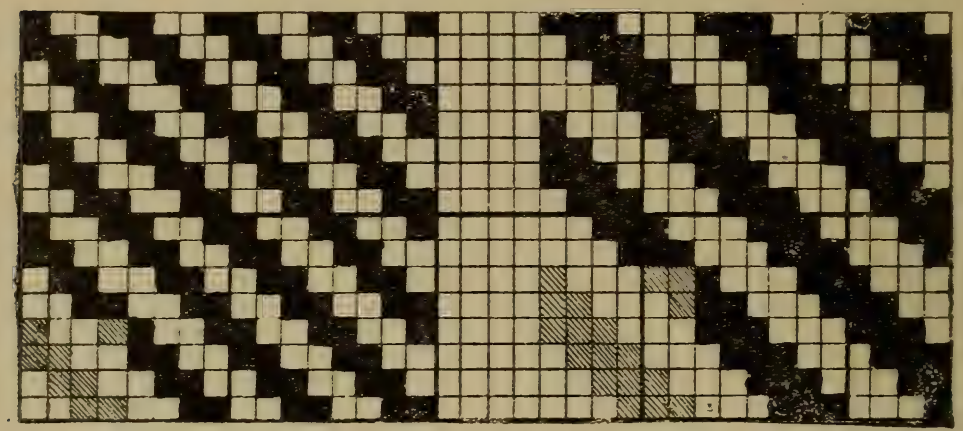

FIG. 42.

FIG. 43.

capable'of producing a firm, close and compact texture, and is one of the most useful weaves to a textile designer. Figs. 43 and 44 are two other examples of twill weaves having warp and weft in equal quantities on both the face and back of cloth.

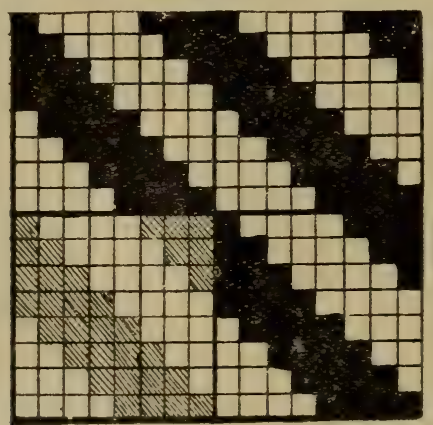

FIG. 44.

Fig. 43 is a six-end $\left(\frac{3}{3}\right)$ twill, and Fig. 44 an eight-end $\left(\frac{4}{4}\right)$ twill. It will be observed in Fig. 43 that the first and fourth threads in either series, counting from any thread, interweave in an opposite manner to each other at the same time. Like- 
wise with the first and fifth threads in Fig. 44. Knowledge of these features is valuable to a designer in the development of broken twills, and other designs having a twill foundation.

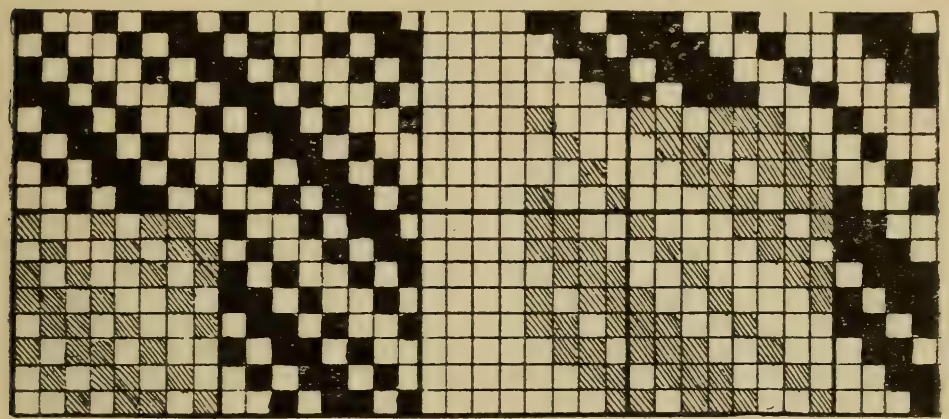

FIG. 45 .

FIG. 46.

Figs. 45,46 and 47 are designs for twill weaves to produce unequal wales of warp and weft in equal quantities on both face'and back; whilst Figs. 48,49 and 50 are designs for twills

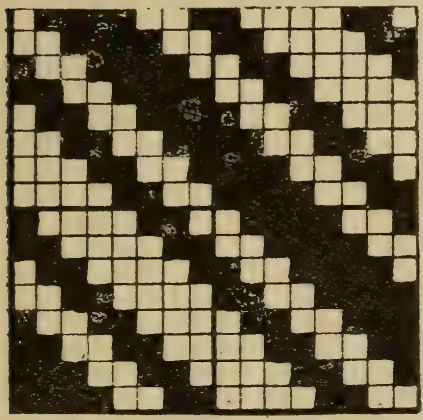

FIG. 47.

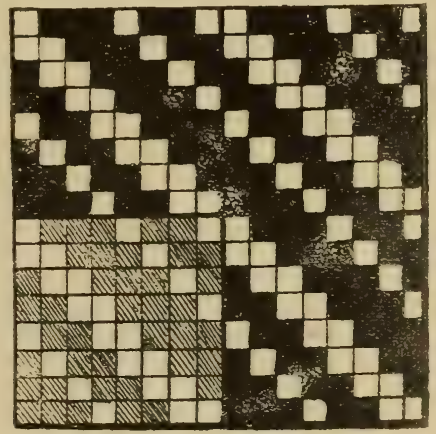

Frg. 48.

having unequal wales of warp and weft, but with warp preponderating on the face. The three designs of each of these latter varieties repeat on eight, twelve and sixteen warp threads and picks respectively.

Before proceeding to describe the second class of twill weaves, as enumerated in $\S 17$, it will be both interesting and instructive 
to indicate the main influences affecting the angle, and also the relative prominence, of twills in cloth.

\section{The Angle of Twill.}

$\S 21$. The angle of twill in any continuous twill weave in which the progression is accomplished by advancing one thread

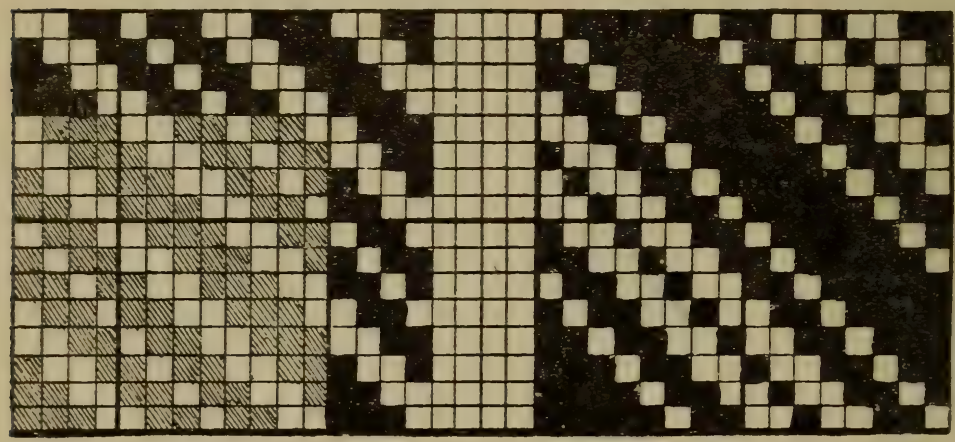

Fig. 49.

Frg. 50.

only at a time, with both warp and weft, is determined by the ratio existing between the number of warp threads and picks in a given measurement, say, one inch. If warp threads and picks

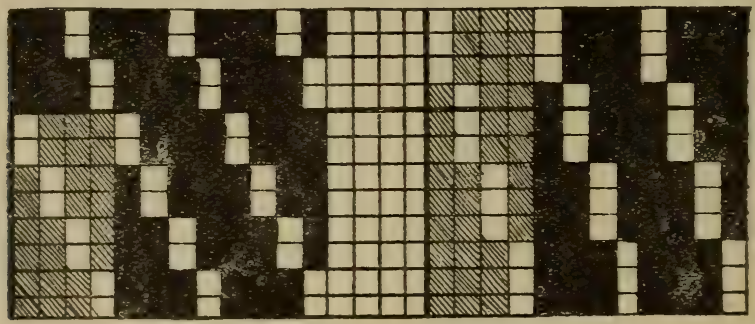

FIG. 51.

FIG. 52.

are in equal numbers per inch, the angle of twill must necessarily be one of forty-five degrees, irrespective of any difference that may exist between the counts of warp and weft; but if the threads of one series are more numerous than those of the other, the angle of twill will assume an inclination towards those threads 
in greater number. Thus, if there are more warp threads than picks per inch, the angle of twill will incline in the direction of warp threads in proportion to the excess of warp threads over picks; but if there are more picks than warp threads per inch, the angle of twill will incline more in the direction of weft. High-angle or low-angle twills may also be formed by advancing two or more threads together in one series, and one thread only in the other series, as in Figs. 51 to 54. If a high-angle twill is produced by this method, or if warp threads exceed picks per inch, the twill should be developed with warp, as in Figs. 51 and 52. If a low-angle twill is required, or if picks exceed

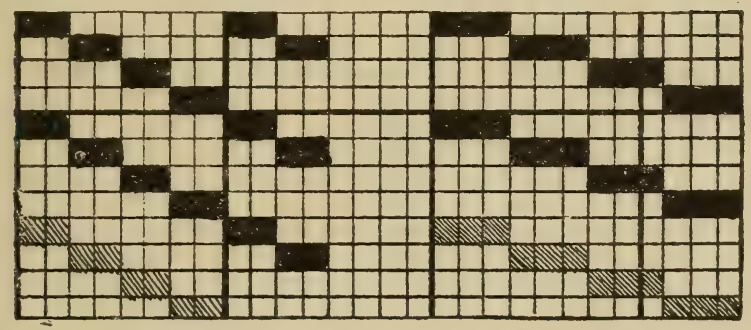

FIG. 53.

FIG. 54.

warp threads per inch, the twill should be developed with weft, as in Figs. 53 and 54.

\section{Influences Affecting the Prominence of Twills and Kindred Weaves.}

$\$ 22$. A twill weave will assume either a more or a less pronounced character in cloth, according to different circumstances. The relative prominence of twills is chiefly determined by $(a)$ the character of weave; $(b)$ the character of yarn; $(c)$ the number of warp threads and picks per inch; and $(d)$ the direction of twill in relation to the direction of twist imparted to yarn during spinning.

(a) Character of Weave.-A twill weave will be relatively more pronounced if developed from longer than from shorter floats of yarn; but unless the freer interlacement of threads is counterbalanced by a proportionate increase in their number per 
inch, the fabric will be relatively weaker, for reasons stated in $\S 7$. It is to obtain longer floats of yarn that high-angle twills should be developed with warp, and low-angle twills with weft, as explained in $\$ 21$. If those conditions were reversed, the twill would lack fulness owing to the short flushes of yarn, as may be readily observed on examining the reversed side of a fabric of this class.

(b) Character of Yarn.-A more pronounced twill will result either from coarse-spun or soft-spun yarn than from fine-spun or hard-spun yarn; also from folded yarn (i.e., a thread consisting of two or more single strands of yarn twisted together) than from single yarn.

(c) Number of Threads per Inch.-A twill will be relatively more or less pronounced in proportion to the number of warp threads and picks of weft per inch.

(d) Direction of Twill in Relation to the Direction of Twist in Yarn.-If the same twill weave is produced to the left in one fabric, and to the right in another fabric of exactly similar texture, and woven from similar yarn, or (which amounts to the same) if the same twill is produced in both directions in different parts of the same fabric, it will appear to be more pronounced in one direction than in the other, according to the direction of bwill in relation to the direction of twist in the yarn composing it. This difference is also observable between the obverse and reverse sides of the same fabric, especially if warp and weft are in equal or in nearly equal quantities on both sides. For example, if a twill inclines to the left (thus $\backslash$ ) when viewed obversely, it will incline to the right (thus/) when viewed on the reverse side, albeit the direction of twist in both warp and weft remains the same. Therefore the direction of twill in relation to the direction of yarn twist is different on each side of the fabric, with the result that the twill appears to be more prominent on one side than on the other. In this case, however, the influence exerted by the deflection of the warp line out of a straight course between the breast beam and back rest of a loom (to spread the warp threads and thereby obtain what is termed "cover" in cloth) will be a contributory factor affecting the relative prominence of twill on both sides of a fabric. This 
circumstance, however, does not entirely account for the difference between the obverse and reverse sides of a twill cloth, otherwise no difference would be manifest between the same twill produced -to the right and to the left in different parts of the same fabric.

What actually occurs, is that the series of ridges and furrows in a tivill fabric are more sharply defined and pronounced if they incline in the opposite direction to the twist in yarn with which the ridges of twill are formed; and per contra, the twill will be less prominent if the twill and yarn twist lie in the same direction.

$\S 23$. This peculiar and interesting phenomenon in twill and allied weaves has engaged the attention of several textile experts who have sought to discover the cause; and although various theories have been suggested as probable explanations of its true cause, this is still a matter for conjecture, and cannot, therefore be stated positively.

According to one theory this phenomenon is attributed to the effect produced by the reflection of light at different angles from the fibres composing the threads, according to the direction in which the fibres lie in relation to the direction of twill. This may partly account for the different effects, but it is apparently not the chief factor, as may be easily demonstrated by taking: a piece of cloth in which the same twill is produced in both directions, in different parts, and viewing it in a neutral or well-diffused light, when a decided difference will be observed between the twill inclined to the right, and that inclined to the left. The twill in the opposite direction to the twist of yarn will be more distinct than that in the same direction as yarn twist. It would appear, therefore, that the difference is caused either partly or entirely by some influence exerted by the direction of twill upon the twist of yarn. This preconception forms the essence of another theory based on the assumption that since the spirality of a spun thread is artificial, and not a natural feature of such a thread, the fibres composing it subsequently tend, under favourable conditions, to recover their original straight and free condition, thereby causing the thread to untwist, especially when it is subjected to tensile strain. Hence 


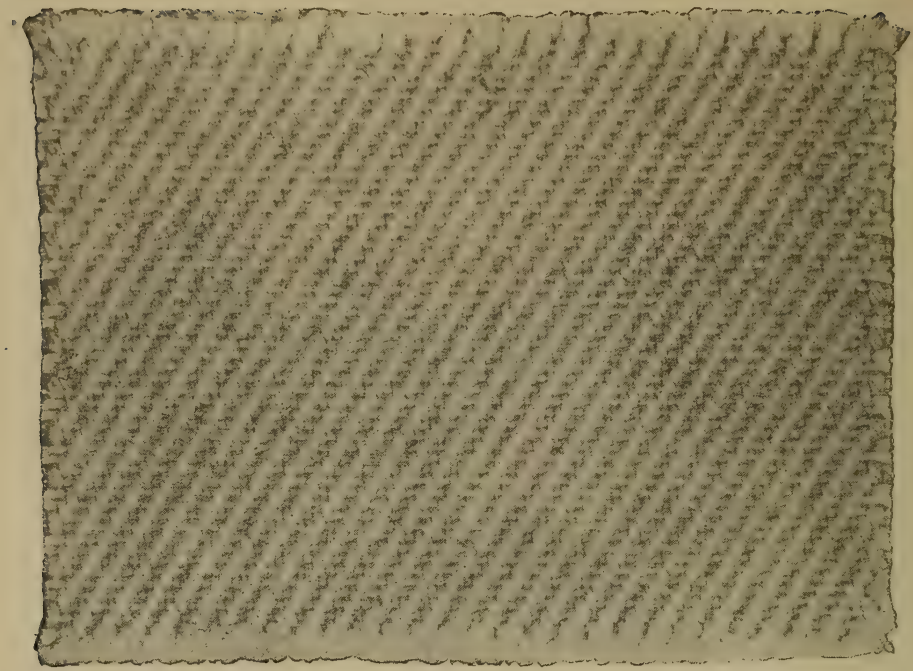

FrG. 55.-Showing the obverse side of $\frac{2}{2^{2}}$ twill cloth of coarse texture, produced from folded warp and weft twisted sinistrally, or weft-way (when folded), and with the twill produced upward from left to right, or dextrally.' (Note the prominence of twill.)

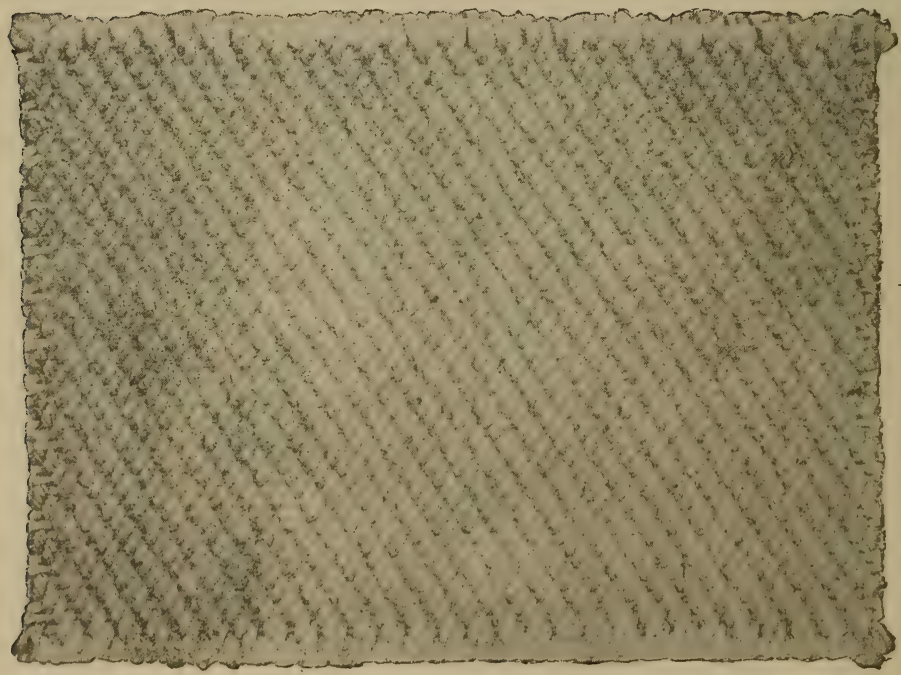

FIG. 56.-Showing the reverse side of the piece of twill cloth represented in Fig. 55. (Note the peculiar inclination of warp threads from a straight course, and its effect in subduing the twill.) 
it is suggested that, during weaving, when the respective threads are under tension, they tend to untwist in cloth, and consequently roll slightly out of their original perfectly straight course, and assume a more or less oblique inclination between the points where they intersect with other threads, unless some means are adopted to prevent or check such tendency by producing the twill in the reverse direction to that of the yarn twist.

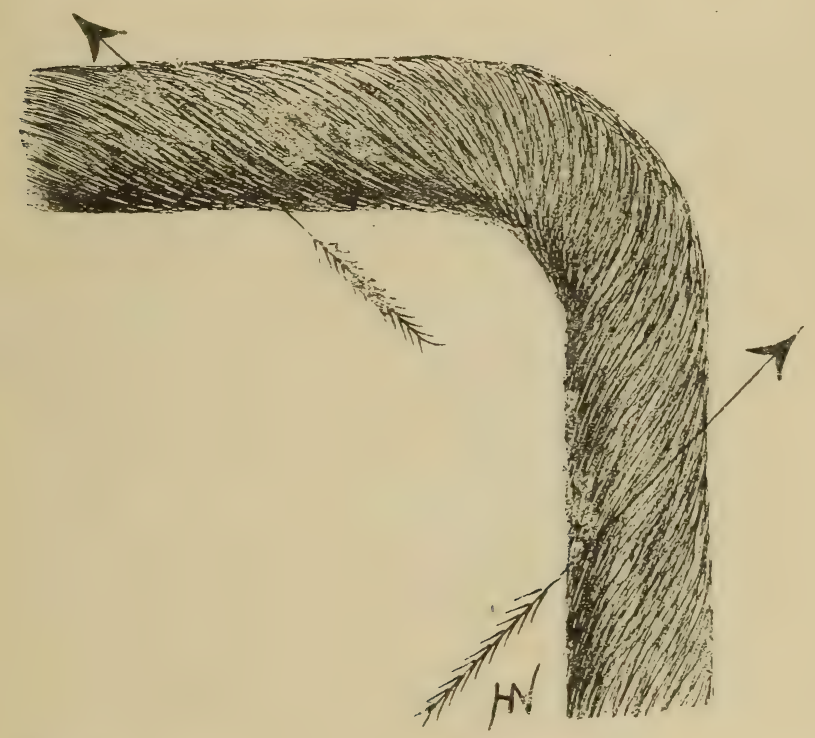

FrG. 57.-Showing a thread spun with a right-hand twist, or "twist-way".

$\$ 24$. The different effects of the same twill weave produced in reverse directions in the same fabric are exemplified in a very striking manner by Figs. 55 and 56, which represent portions of the obverse and reverse sides respectively, of an actual example of grey cotton two-and-two twill cloth, containing thirtyfive warp threads per inch of $4 / 6$ 's yarn; and twenty-two picks per inch of $4 / 10$ 's yarn. The single strands of yarn composing the folded threads of both warp and weft are spun "twist" way, i.e., dextrally, with the twist or spirality extending upward 
from left to right, thus / (when the thread is viewed either suspended vertically, or extending away from the observer) and as indicated in Fig. 57; but the doubling twist of the folded thread is in the reverse direction to that of the single-yarn twist (in accordance with usual practice in doubling spinning), namely, "weft" way, i.e., sinistrally, with the twist inclining from right

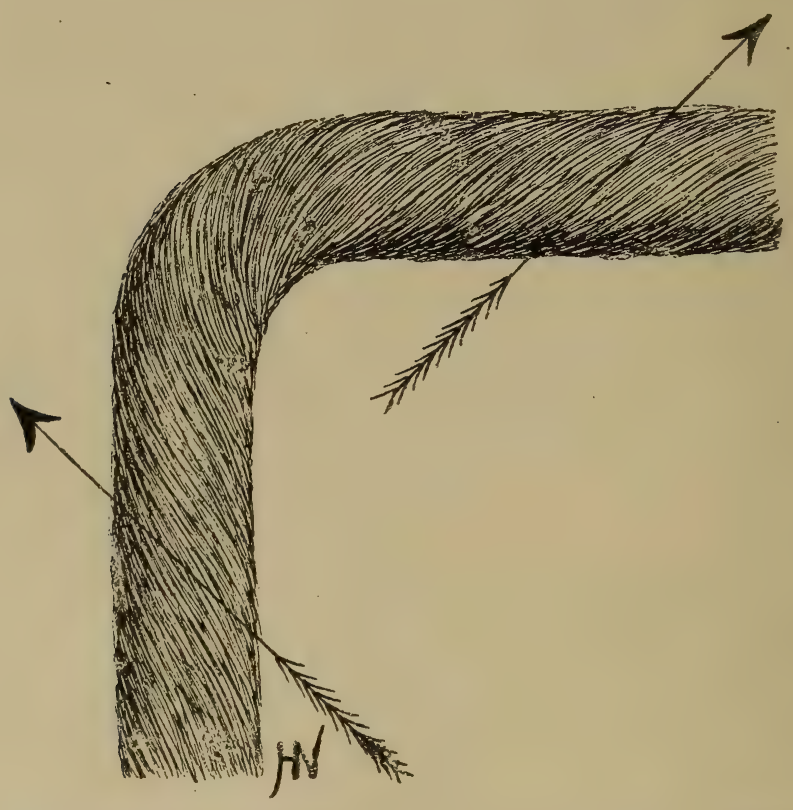

FIG. 58.-Thread spun with left-hand twist, or "weft-way". (Right-hand -American style.)

to' left, as indicated in Fig. 58. The twill on the obverse or face side of cloth inclines to the right, and therefore opposes the direction of fwist in the warp threads, which are both coarser and more numerous than picks of weft, and are consequently more pronounced than these. Hence, the twill is much more prominent on the obverse than on the reverse side of the cloth, where it inclines in the same direction as the warp twist.

$\$ 25$. According to the second theory explained in $\$ 23$, a 
twill will be more pronounced if produced in such a direction that the tendency of threads to untwist and roll out of their straight course will be prevented or checked. Thus, if the untwisting action of threads that form the ridges of the twill causes them to incline to the left the direction of the twill also should be produced to the left, whereby the threads will support each other, at the extremities of the float, on those sides towards which they tend to roll. If, however, the threads are left unsupported at those parts, as would occur if the twill were pro-

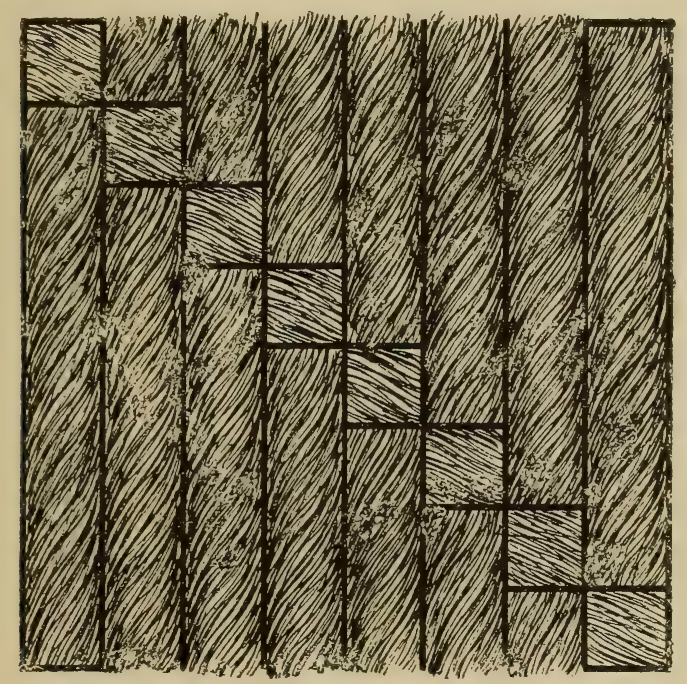

'FrG.[59.-Showing the direction of twill for a warp-face twill fabric produced with warp yarn spun "twist-way," to develop a bold twill rib.

duced in the same direction as the angle of twist, their tendency to untwist and roll would be unchecked, and the floats would therefore assume a slight inclination in the reverse direction to the twill, as clearly observed in Fig. 56.

$\$ 26$. But whatever may be the dominant factor determining the relative prominence of twills, it may be repeated that if they are produced in the reverse direction to that of the twist in the yarn, they will be more pronounced than if both the angle of twist and that of the twill are both inclined in the same direction. 
And so long as this dictum is observed, it is immaterial in whichever direction a twill may incline, or in which direction the yarn is twisted during spinning. Therefore, if a bold warp twill is produced from yarn spun "twist-way," as indicated in Fig. 57, the twill should incline upward from right to left, as represented in Fig. 59. Likewise, if a weft twill is produced from yarn spun "weft-way," as indicated in Fig. 58, the twill also should incline upward from right to left, as represented in Fig. 60. (This may at first appear inconsistent, until it is observed that the direction or spirality of twist in a spun thread

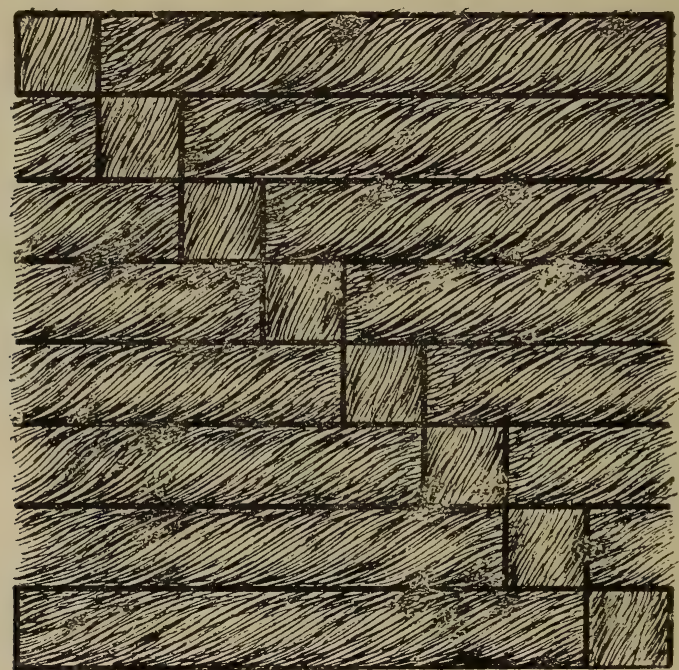

Fig. 60.-Showing the direction of twill for a weft-face twill fabric produced with weft yarn spun "weft-way," to develop a bold twill rib.

inclines in reverse directions when placed at right angles to itself, as indicated by arrows in Figs. 57 and 58.) Again, if a twill weave having warp and weft displayed in equal or nearly equal quantities on both sides of cloth is produced from warp spun "twist-way" and weft spun "weft-way," the twill should, in this case also, incline upward from right to left, as indicated in Fig. 61. If, however, a weft twill is produced from yarn spun "twist-way," or a warp twill from yarn spun "weft-way," the twill should incline upward from left to right, as indicated in Fig. 62. 


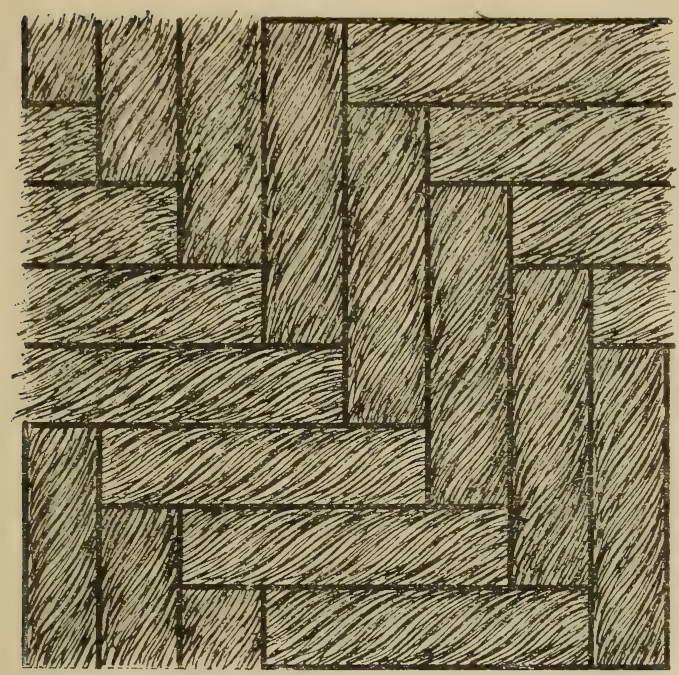

FIG. 61.-Showing the direction of twill in a fabric having a warp and weft face, and produced from warp yarn spun "twist-way," and weft yarn spun "weft-way," to develop a bold twill rib.

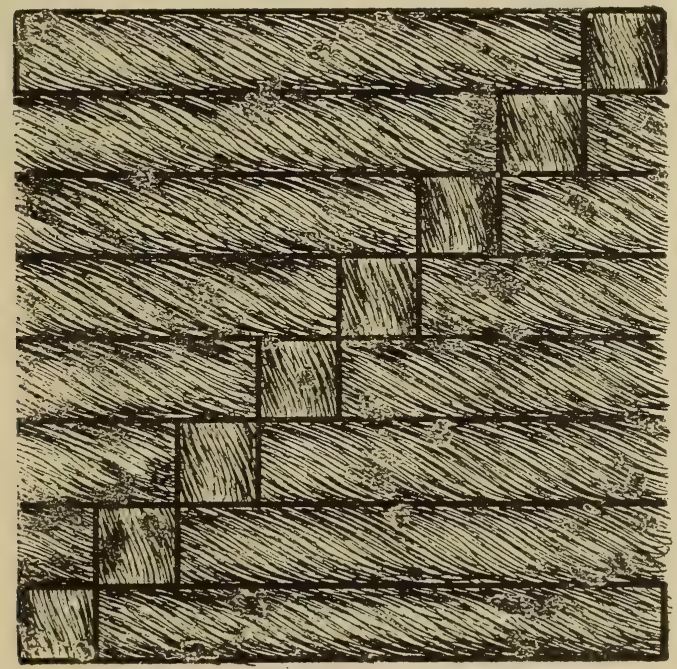

FIG. 62.-Showing the direction of twill in a weft-face twill fabric produced from yarn spun "twist-way" to develop a bold twill rib. 


\section{Zigzag or Wavy Twills.}

$\$ 27$. This subdivision of twill weares comprises those in which the direction of twill is frequently reversed, to produce a series of waves running horizontally, obliquely or vertically, according to the particular manner in which the reversals are made. Any regular twill weave may be employed in the development of wavy twills; also the twill may be reversed at regular or irregular intervals on either warp threads or picks, according to the effect desired. It should be observed, however, that, as a rule, the best results will be obtained by reversing the twill on that series of threads which predominate on the face of

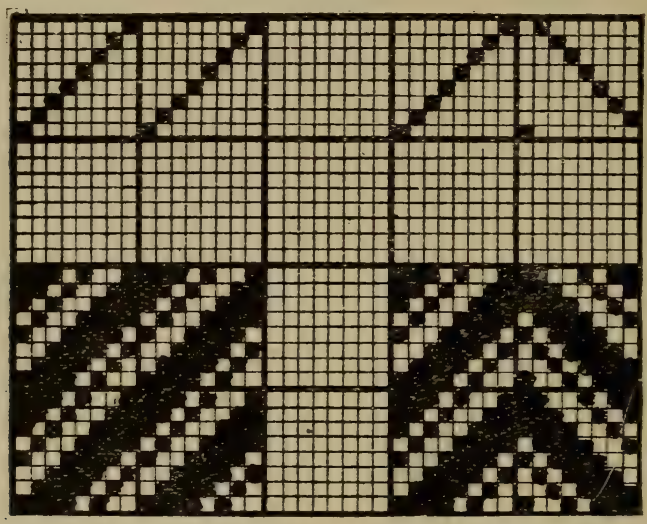

Fig. 63.

Fig. 64.

the fabric. Thus, if the warp preponderates orer weft, the waves should reverse on warp threads; and if the weft preponderates over warp, the waves should reverse on picks of weft provided, of course, that the preponderating threads are not inferior either in number or quality. By adopting this course, long floats, which would otherwise occur at all points where the twill is reversed, and which look like imperfections in cloth, are aroided, and sharper wave crests and furrows are produced. The accompanying examples of wary twills are uniformly based on the regular twill weare represented in Fig. 63, which repeats on eight warp threads and picks, and requires eight shafts of healds to weare it, with warp threads drawn through them with 
a "straight-over" draft, as indicated above the design. This twill weave has warp preponderating over weft in the ratio of five of warp threads to three picks of weft, thus $\frac{4}{1} \frac{1}{12}=\frac{5}{3}$.

Figs. 64, 65 and 66 are horizontal wavy twills produced by rerersing the weave (Fig. 63) at regular intervals of eight, twelve and sixteen warp threads, thereby causing them to repeat on sixteen, twenty-four, and thirty-two warp threads, and only eight picks, respectively. As indicated by the drafts immediately above the designs, each design requires only eight shafts of healds (as does the original weave) for its production; but they would each require a different set of healds in consequence of

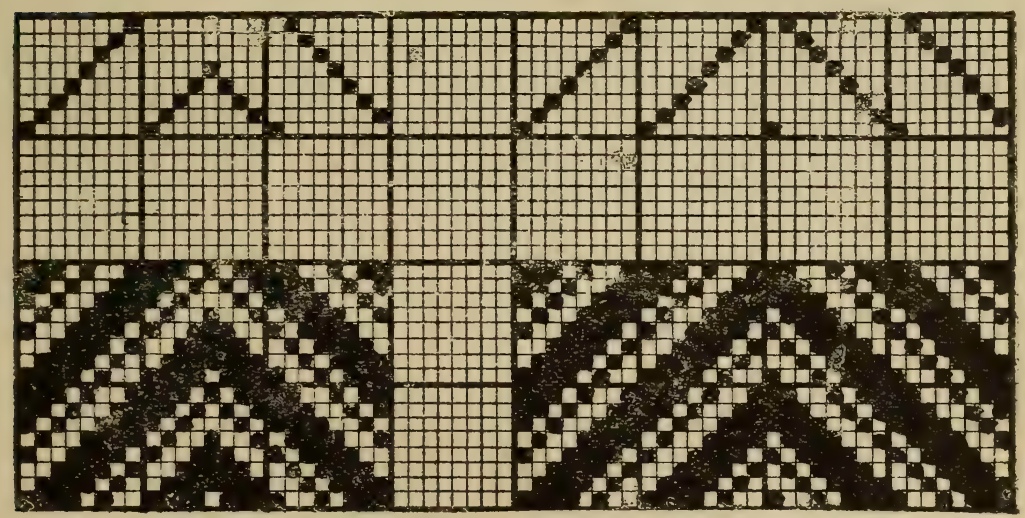

FIG. 65.

Fig. 66.

the different methods of drafting the warp threads through them. If the same weave (Fig. 63) were employed to produce similar wavy effects to those of Figs. 64,65 and 66 , but vertically instead of horizontally, the draft shown above (Fig. 63) would answer, and the healds would be raised in consecutive order, forward and backward alternately, for eight, twelve, and sixteen picks respectively; thereby causing the designs to repeat on twice that number of picks. This latter course would inrolve the use of dobbies or other shedding devices capable of weaving designs repeating on a large number of picks ; whereas, in the former case, the designs could be woven by means of eight-pick tappets. 
28. Figs. 67, 68 and 69 are variegated wave effects produced by reversing the twill at irregular intervals of warp threads, so as to produce large and small waves in a horizontal

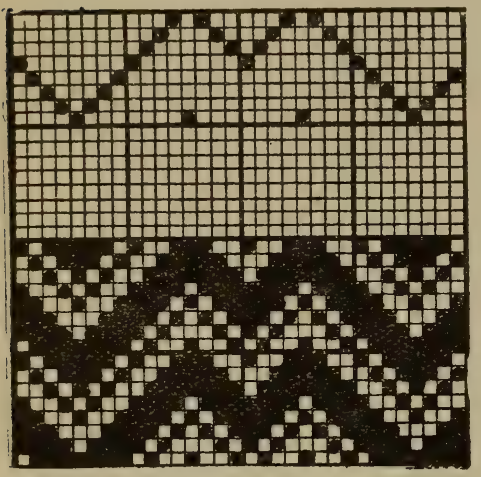

FIG. 67.

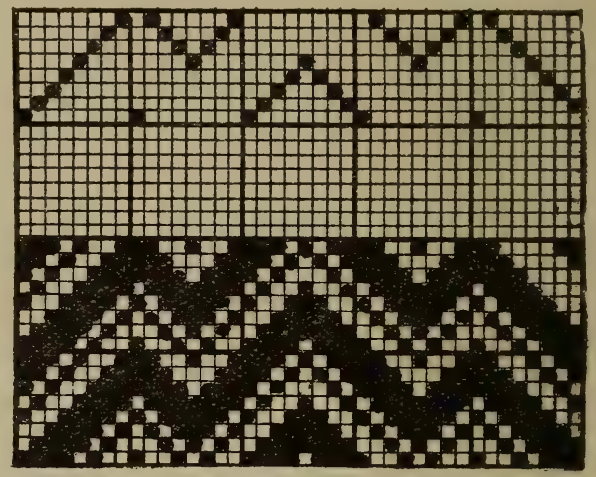

Fig. 68.

direction. In Fig. 67 the twill is reversed at intervals of four, eight, and four warp threads continuously. In Fig. 68 the intervals are eight, four, and eight warp threads continuously : and in Fig. 69 they are four, eight, four, eight, and four warp

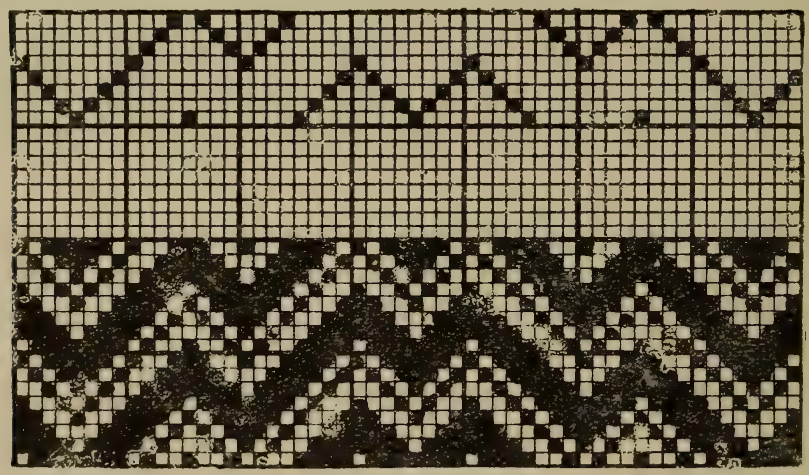

Fig. 69.

threads continuously. By thus reversing for an equal number of warp threads in both directions, the waves assume a horizontal course so far as one or more than one repeat of the pattern is concerned. Only eight healds are required to pro- 
duce these designs; but the drafting of warp threads through them must be as indicated above the respective designs. This causes the patterns to repeat on thirty-two, forty, and fifty-six warp threads, and eight picks respectively.

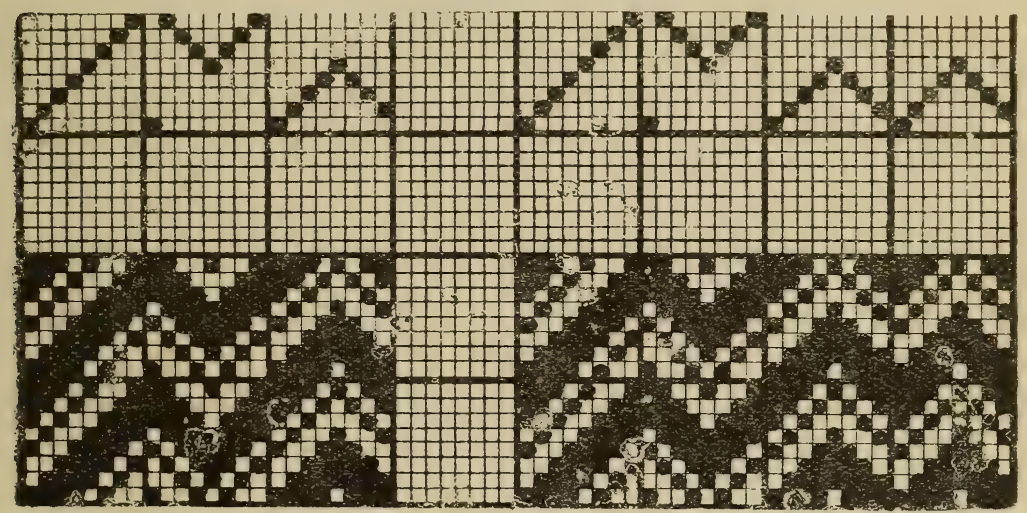

FIG. 70.

FIG. 71.

\$29. Figs. 70, 71 and 72 are wavy effects in which the waves are produced obliquely by reversing the twill uniformly

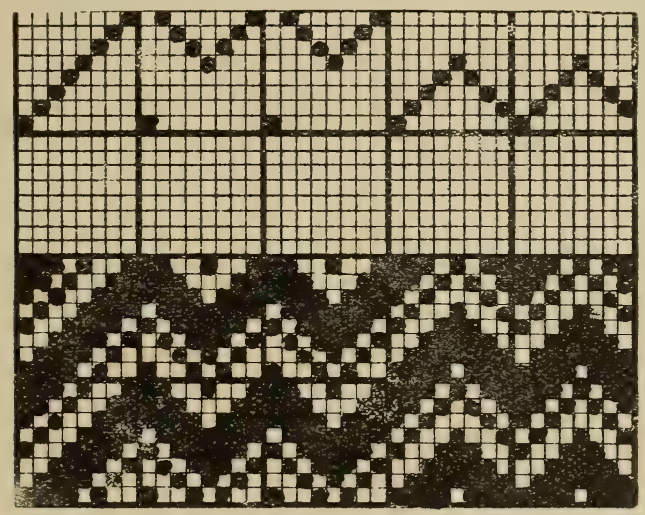

FIG. 72.

at shorter intervals in one direction than in the other. The obliquity of the waves may be more or less acute according to the system of reversing, and the intervals at which the reversals 
occur, as seen in the examples given. In Fig. 70 the intervals are eight and four warp threads alternately, throughout. In Fig. 71 a more acute obliquity is obtained by reversing the twill at intervals of eight, four, eight, four, and four warp threads continuously; and in Fig. 72 a still more acute slant is produced by reversing the twill at intervals of eight, four, four, and four warp threads continuously. These designs repeat on eight picks, and require eight shafts of healds, with warp threads drafted as shown, to produce them. In the development of wavy twill designs, the relative sizes of waves are determined by the number of threads on which the twill is produced in any direction.

\section{Rearranged Twills.}

$\$ 30$. Rearranged twills are those evolved by the rearrangement of either warp threads or picks of any regular or continuous twill weave, according to some definite plan. For example, consecutive threads of a given weave may be redistributed at regular intervals of two or more threads apart, as required; or, as an alternative method, threads of a given weave may be taken at intervals of two or more, and arranged consecutively to form a new design.

\section{Satin Weaves.}

The simplest application of this system of rearranging twill weaves obtains in the development of what are known as "satin" weaves, produced by rearranging simple continuous warp-face or weft-face twills (as represented in Figs. 31 to 35 , and 37 to 41 respectively), according as warp-face or weft-face satin weaves are required. Satin weaves are characterised by an even and smooth surface, of either warp or weft, resulting from a perfectly regular distribution of intersections of those threads. They constitute one of the most useful varieties of weaves and are extensively employed, in conjunction with other weaves, as an element or component part of elaborately decorated fabrics, as well as in the production of piece-good fabrics constructed entirely on the basis of one of such weaves. Although satin weaves are (for convenience of classification) generally regarded 
as derivations or rearrangements of simple continuous twill weaves, it will be seen that they bear no resemblance whatever to that class, but are entirely different in respect of the distribution of intersections.

$\S 31$. In the production of satin weaves, the intersections or binding points of warp and weft should be distributed as freely and far apart as possible, on such number of threads as are to constitute one repeat of the pattern. The more perfectly such distribution is accomplished, the more perfect will be the evenness and smoothness of cloth. The rearrangement of any continuous twill weave, to produce either a simple satin weave, or other design having a satin basis, may be made in accordance with an arithmetical formula to obtain the "interval of selection" which determines the positions of intersections or binding points on consecutive threads of either series for any size of satin weave, excepting those contained on four and six threads (which are imperfect satin weaves). Having decided upon the number of threads on which to construct a satin weave, the "interval of selection" may be either of the reciprocals of that number and which have no common measure.

Example: It is required to construct a ten-end satin weave The only two reciprocals of ten, which have no common measure, are three and seven; therefore either three or seven may be taken as the "interval of selection," and the intersections disposed at intervals of three or seven threads of either series, on consecutive threads of the other series.

The application of this formula will be easily understood by reference to Fig. 73, where a ten-end weft-face satin (B) is produced by transposing the threads of a ten-end weft-face twill (A) in the manner indicated; namely, by disposing say every third warp thread $\mathrm{A}$, in consecutive rotation to produce $\mathrm{B}$. Or the same result is virtually attained by the method shown at Fig. 74 , where consecutive warp threads A are redisposed at intervals of three threads to produce B. The only difference between Figs. 73 and 74 is in the reversed sequence of intersections. Again, similar results would obtain by rearranging picks instead of warp threads, and also by adopting the reciprocal number, seven, as the "interval of selection". 

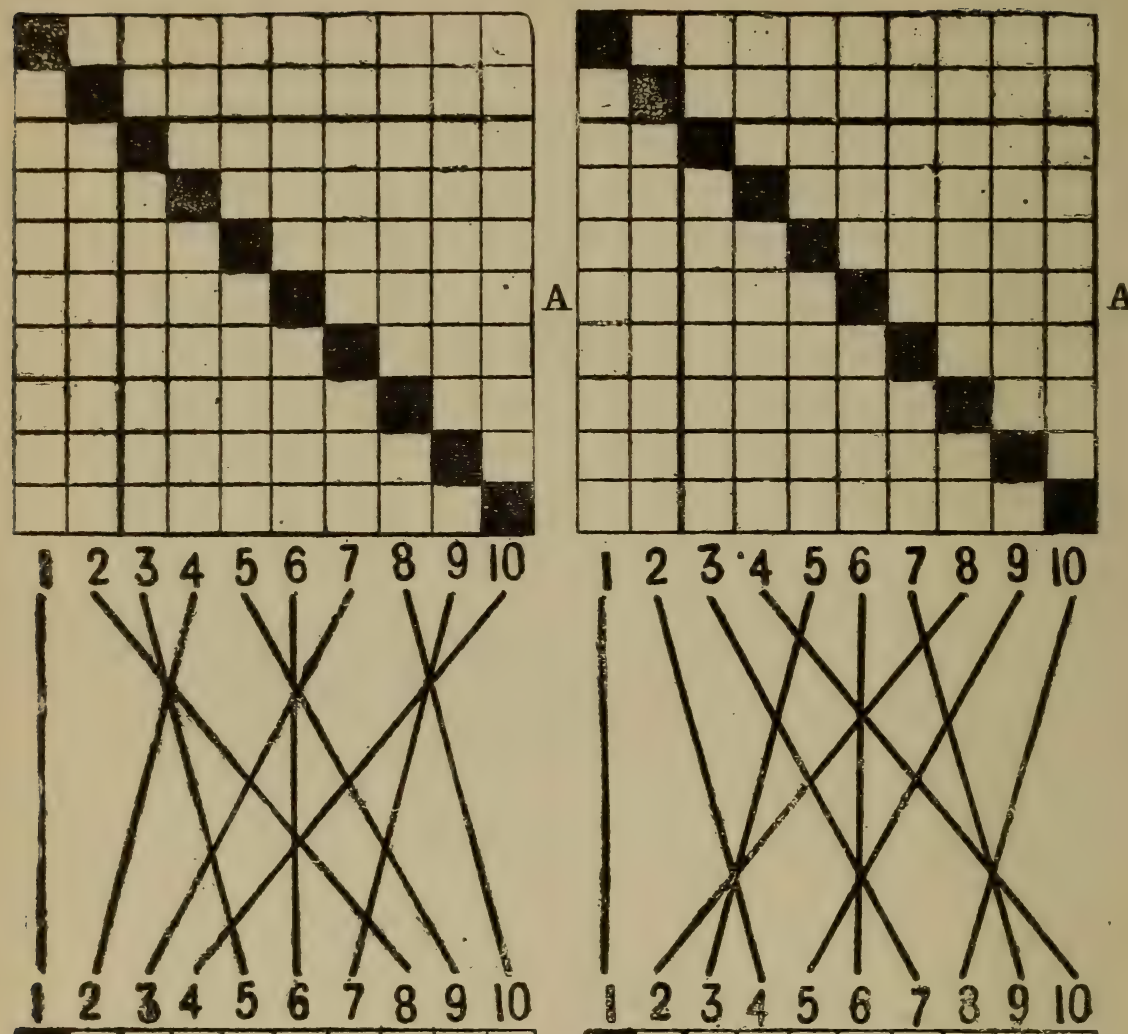

A

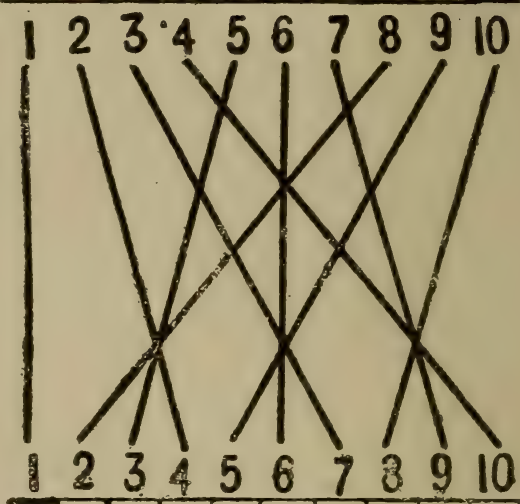

a

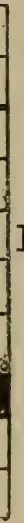

B

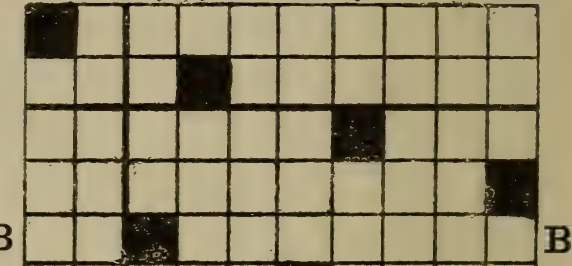

FIG. 73.--Showing the construction of satin weaves.

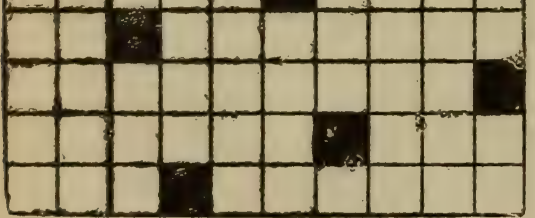

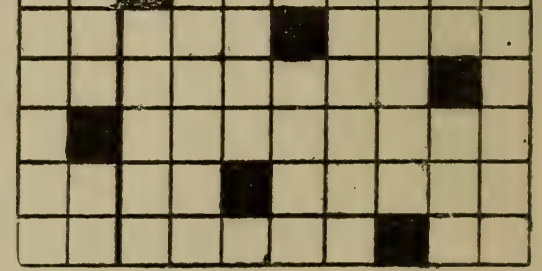

FIG. 74.- Showing an alternative method of constructing satin weaves. 
Some numbers, as five, eight, ten, and twelve, each permit of only two reciprocals which have no common measure; whilst some have four, and others more than four reciprocal divisions which have no common measure. As regards those numbers which have four minor reciprocal divisions, a similar distribution of intersections will result whichever one of the four divisions is

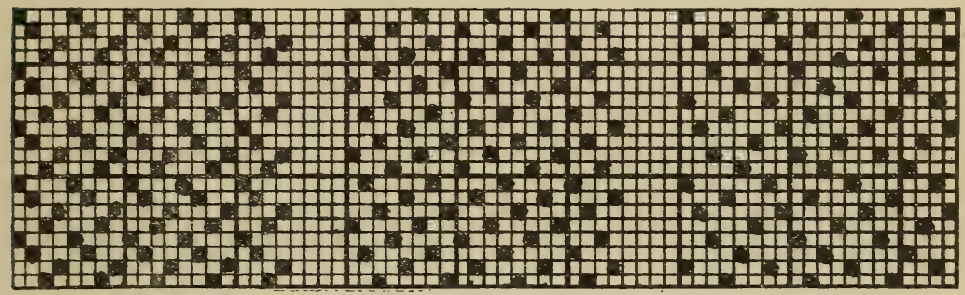

FIG. 75 .

FIG. 76.

Fig. 77.

selected as the interval; but as regards those numbers which offer a greater choice of intervals, the choice of the best "interval of selection " is entirely a matter of judgment and not of rule. In such cases it is advisable to construct weaves based on each of the respective reciprocals, and then choose that "interval of selection " which gives the most perfect and regular distribution of intersections.

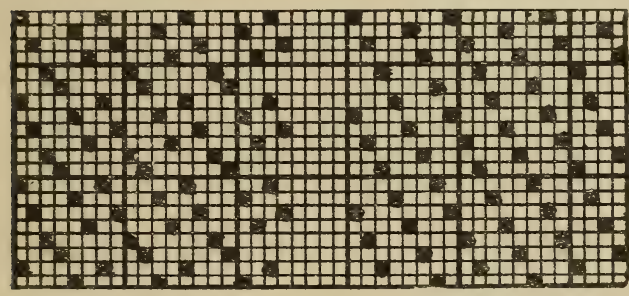

FIG. 78 .
FIG. 79.

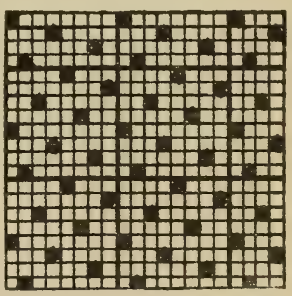

FIG. 80 .

$\$ 32$. The following table shows the intervals of selection for the construction of satin weaves on five, and seven to twentytwo threads. Instead of the numbers given, their reciprocals may be taken. Where two intervals are given, each of these or their reciprocals will produce similar results. Where more than two intervals are given, the number or numbers shown in 
heavy type (or their reciprocals) will give the most perfect distribution of intersections; and those weaves indicated in italics are the only satin weaves (included in the following table) in which the distribution of intersections is geometrically perfect:-

Table of Intervals of Selection for the Construction of Satin Weaves.

5-end satin-2.
7-end satin-2, 3 .
8-end satin-3.
9-end satin-2, 4 .
10-end satin-3.
11-end satin-2, 3, 4, 5.
12-end satin-5.
13-end satin-2, 3, 4, 5, 6 .
14-end satin-3, 5.

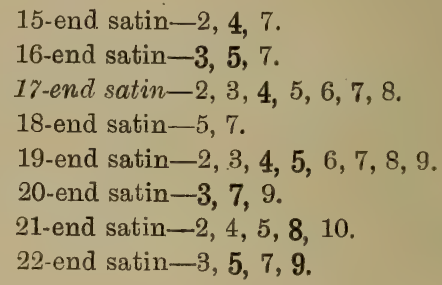

\section{"Corkscrew" Twills.}

$\$ 33$. Corkscrew twills constitute a variety of rearranged twills largely employed in the production of worsted garment fabrics, for which they are eminently suited, as they are capable of producing firm and compact textures of great strength, warmth

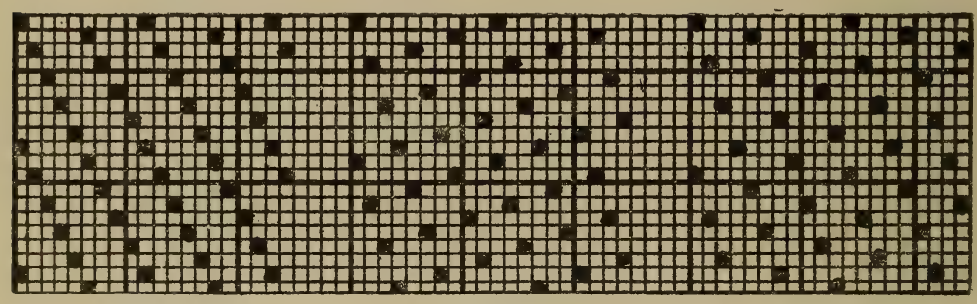

Fig. 81.

Fig. 82.

Fig. 83.

Figs. 76 and 79 to 94 are weft-satin weaves constructed in accordance with the above table. Fig. 75 is the so-called four-end "satinette" weave; and Figs. 77 and 78 are alternative arrangements of the six-end satin weave. The arrangement shown in Fig. 77 is preferable to that shown in Fig. 78, as it gives a more perfect distribution of intersections.

and durability. Perfect corkscrew weaves are characterised by a somewhat subdued twill formation, with either warp or weft only visible on the face of the fabric, and are usually constructed on an odd number of warp threads and picks. The latter 


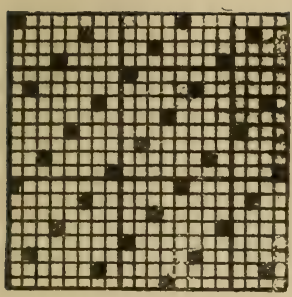

FIG. 84.

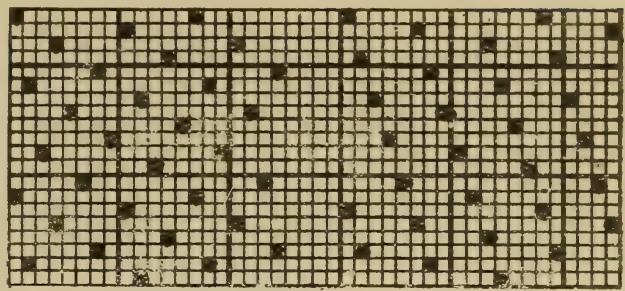

FIG. 85.
FIG. 86.

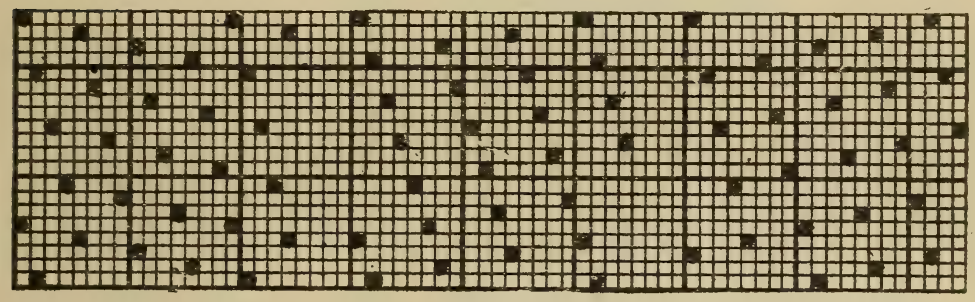

Fig. 87.

FIG. 88.

FIG. 89.

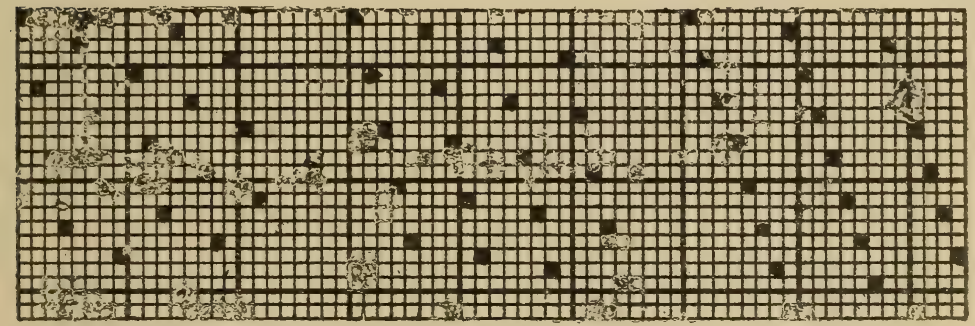
FIG. 90.

FIG. 91.

F.G. 92.

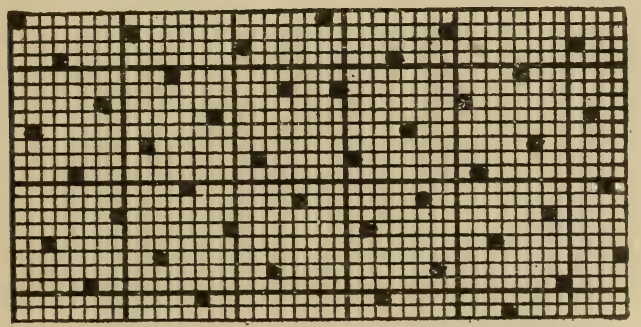

FIG. 93.

FIG. 94. 
circumstance arises from the particular method of constructing these weaves, namely, by rearranging either series of threads of any suitable continuous twill weave at intervals of tro, or

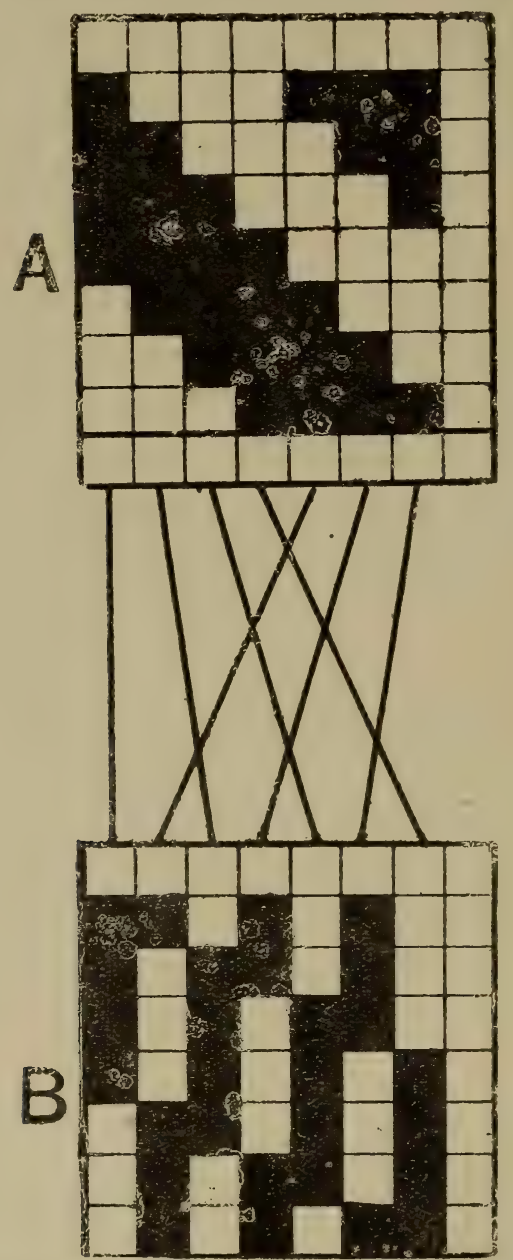

FIG. 95, - Showing the construction of warp-face corkscrew weaves.

alternately; and since two is not a measure of odd numbers, an odd number of threads is, therefore, required for one repeat of the pattern, in accordance with the principle governing the construction of satin weaves, as explained in $\$ 31$. 
Warp-face corkscrew weaves may be produced by rearranging, in the manner described, the warp threads of any continuous twill that repeats on an odd number of threads, and in which

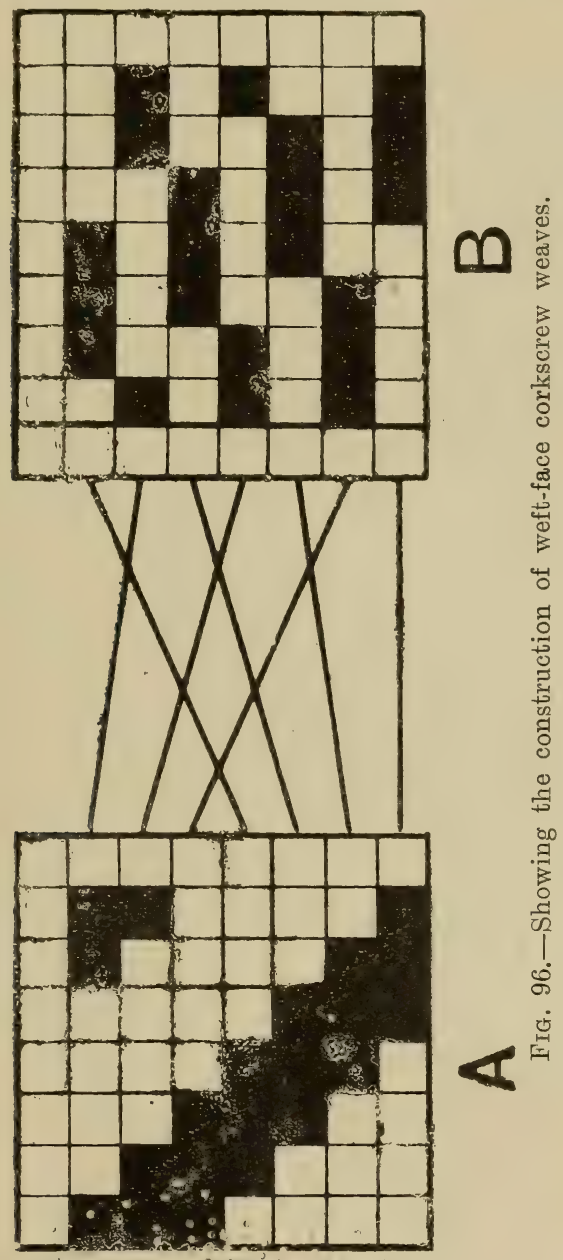

warp floats ano one thread only longer than weft floats. For weft-face $30 r^{\prime}$ sscrews, the base pattern must have weft floating one thread more than warp; but whichever series of threads is 
required on the face, they should be of better quality and in greater abundance than the other series.

Fig. 95 shows the method of constructing a warp-face corkscrew weave $B$, by rearranging warp threads of the seven-end $\left(3^{\frac{1}{}}\right)$ continuous twill weave $\mathrm{A}$, in the manner indicated. It will be

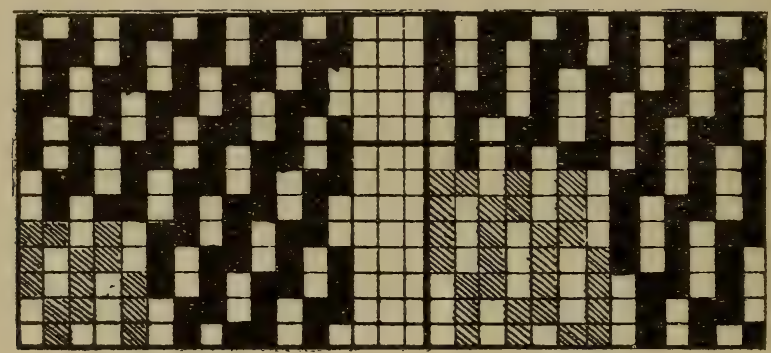

FIG. 97.

Fig. 98.

seen that $B$ is produced by rearranging consecutive warp threads in $A$ at intervals of two threads, or alternately, on the same number of warp threads. In like manner, a weft-face corkscrew B (Fig. 96) is produced by rearranging picks of the seven-end $\left(\frac{3}{4}\right)$ continuous twill A. Figs. 97 to 101 are examples of perfect

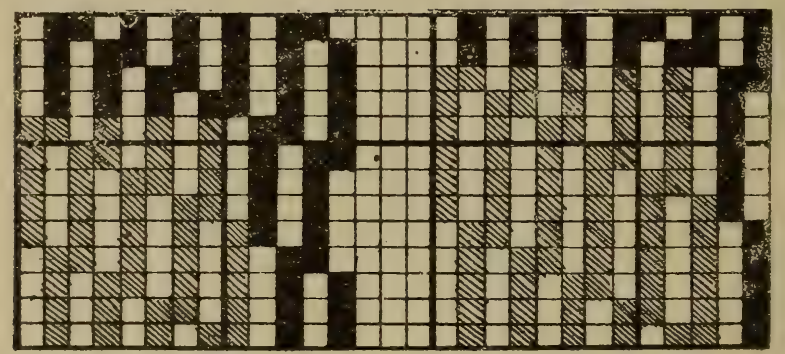

Fig. 99.

FIG. 100.

warp-face corkscrew weaves, and Figs. 102 to 106 of weft-face corkscrew weaves, repeating on five, seven, nine, eleven, and thirteen threads respectiveiy. .'udging "from these weaves as indicated on design paper, in which the theads are represented as if spread out and lyirg parallel side by sice without compression, it would appear that weft would be visible on the face of the fabric in warp-face corkscrews, and warp in weft-face 
corkscrews. This would actually occur if the floating threads were not in considerably greater numbers and therefore more densely crowded than the other series of threads. But by

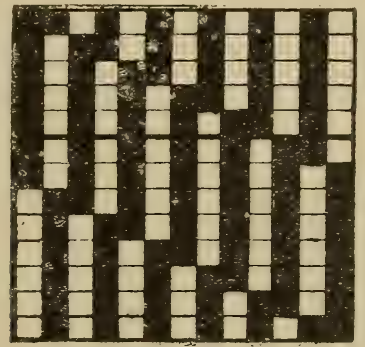

Fig. 101 .

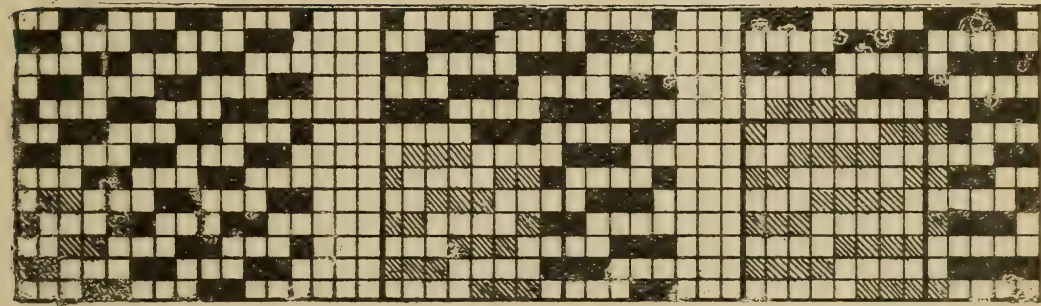

FIG. 102.

FIG. 103.

FIg. 104.

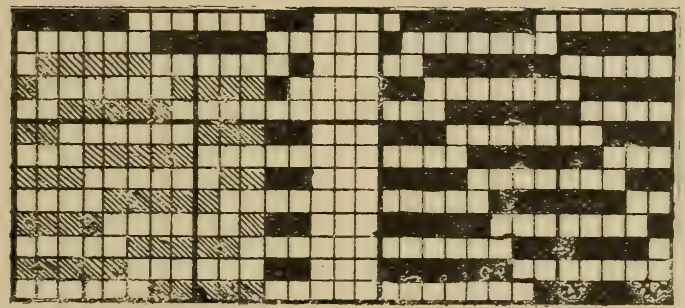

FIG. 105.

FIG. 106.

increasing the numerical density of floating threads, orer corered threads, the latter will be quite obscured by the former closing over and entirely covering them.

$\$ 34$. Corkscrew weaves may be modified to a considerable extent without departing from the general principle governing 
their construction. They may also be made to assume variegated and other decorative effects, as horizontal and oblique waves, and many others; but the necessity of having one series of threads greatly in excess of the other series prevents the successful employment, in bulk, of the sparse threads. Simple corkscrews may also be constructed on an even number of threads; but these will lack the perfect uniformity of surface

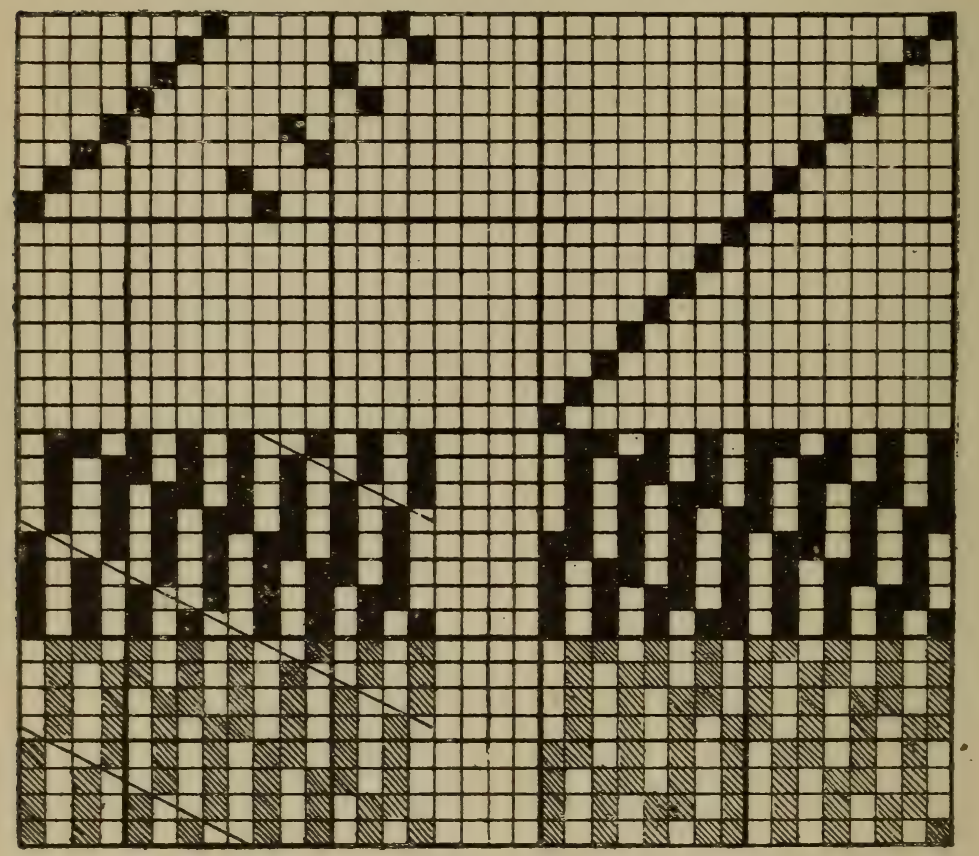

FIG. 107.-Even-thread corkscrew weave.
FIG. 108.-Even-thread corkscrew weave.

possessed by those constructed on an odd number of threads; yet, on the other hand, it opens out unlimited scope to a designer in the production of new and varied effects. It should be pointed out, however, that odd-thread warp-face corkscrews repeat on the same number of threads as their base weares, and require only that number of healds to weave them; whereas even-thread warp-face corkscrews occupy twice as many threads as their base 
weares, and sometimes, require twice that number of healds to weave them.

Fig. 107 is an example of an even-thread corkscrew weave, based on an eight-end four-and-four twill, and requiring sixteen war'p threads and eight picks to complete the pattern. It is but slightly removed from a perfect corkscrew weave, and virtually consists of a double diagonal warp rib, separated by a single diagonal cutting of weft which emphasises the twill formation in cloth. Fig. 108 is another example of a corkscrew weave on eight threads, but without a definite twill formation. It is produced by causing alternate warp threads to float over one pick more than the others, thereby preventing weft from passing over more than one warp thread, as in perfect corkscrews. This unequal floating of warp threads will, of course, produce diagonal ribs of different widths; but that feature will be scarcely, if at all, discernible in the larger weaves, excepting where the variation in the length of float is considerable. It is worthy of note, also, from an economical point of vierv, that the slight departure in the construction of Fig. 108 involves the use of sixteen shafts of healds, with a straight-over draft, whereas Fig. 107 could be woven with only eight shafts of healds, with a broken draft, as indicated above the respective designs.

$\$ 35$. It was explained in $\$ 31$ that satin weaves were evolved by rearranging threads of warp-face or weft-face continuous twill weaves in a prescribed manner. That system of rearrangement is equally applicable to other forms of continuous twills, and is one that offers considerable scope to a designer in the production of fancy weaves of great utility. Whatever form of twill weave may be selected, its rearrangement on a satin basis is governed by the same principle as that which operates in the construction of simple satin reaves. The weave to be rearranged must, of course, repeat on the same number of threads as that of the satin weave which forms the basis of rearrangement; otherwise the new design could not be completed on that number. The rearrangement may also be made in respect of either warp threads or picks of weft, with oftentimes very different results, as will be seen in some of the following examples. The best course to adopt, in the rearrangement of 
twills, on a satin basis, is to indicate on design paper the particular satin weave required to form the basis of rearrangement, and then proceed to rearrange either warp threads or picks of

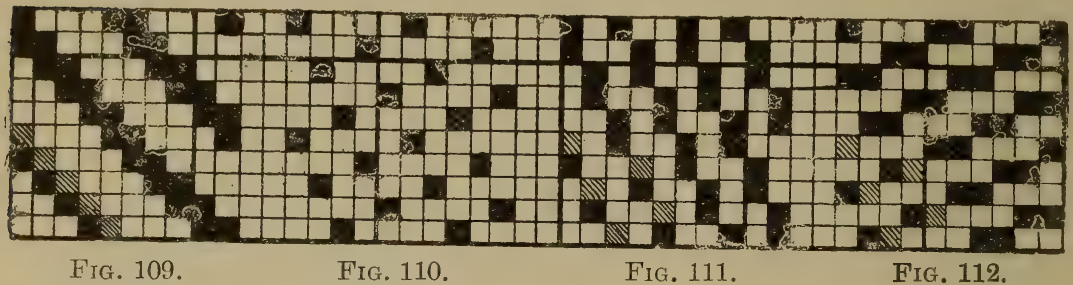

the base twill weave, according to the disposition of the binding points indicated, which serve as starting-points. In the following examples, illustrating the development of designs by this

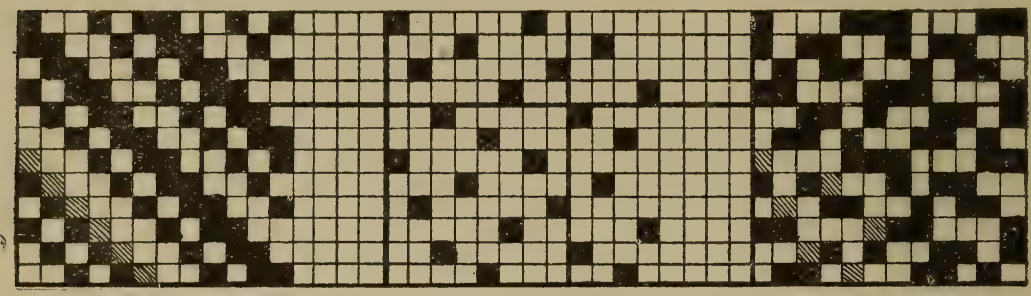

FIG. 113.

FIG. 114.

FIG. 115.

method, shaded squares in the base weaves indicate the twill basis; whilst in the re-formed designs, shaded squares indicate the satin basis on which they are rearranged.

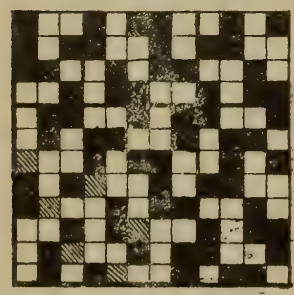

Fig. 116 .

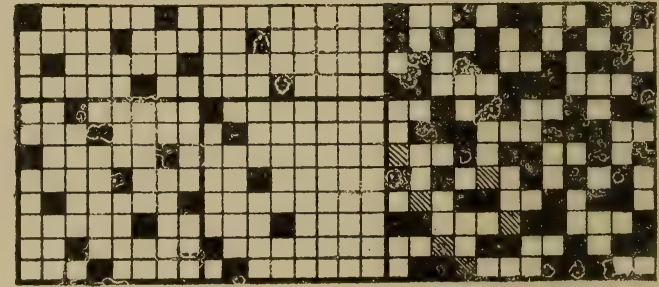

FIG. 117.
FIG, 118.

Iig. 109 is a twill weave contained on five threads, and constitutes the base weares for designs Figs. 111 and 112. Fig. 
111 is produced by rearranging warp threads of Fig. 109 on a five-end satin basis, as indicated in Fig. 110. Fig. 112 is another weare produced by rearranging, in the same order, picks of weft of the same base weave. Figs. 115 and 116 are rearrangements of warp and weft threads respectively of a six-end twill (Fig. 113) on the six-end satin basis indicated in Fig. 114;

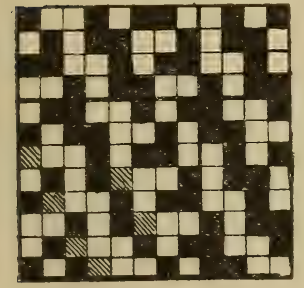

FIG. 119.

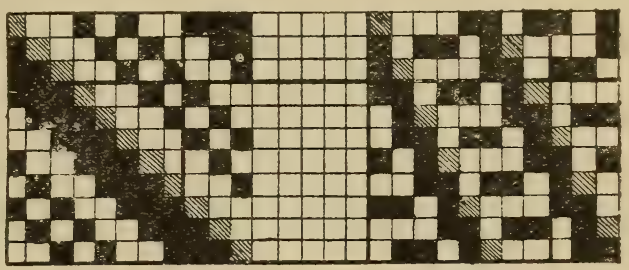

Fig. 120.

FIG. 121.

whilst Figs. 118 and 119 are rearrangements of warp and weft threads respectively of the same base weave (Fig. 113), but on the six-end satin basis indicated in Fig. 117.

When a base weave repeats on such a number of threads as will permit of two or more "intervals of selection" that are not reciprocal numbers, a proportionately greater diversity of new

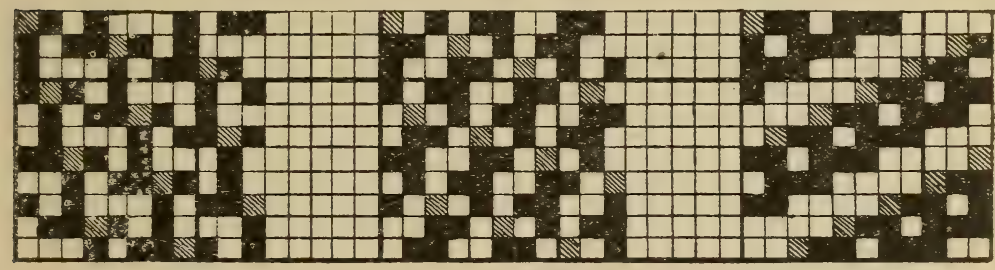

FIG. 122.

FIG. 123.

FIG. 124.

weares may be produced from that base weave by rearranging the threads on the respective intervals afforded by the original number. For example, a base twill weave (Fig. 120) repeating on eleven threads, may be rearranged so as to produce a range of eight different weaves constructed on a satin basis, because eleven is a number which gives eight intervals of selection, namely, two, three, four, and five, and their reciprocals nine 
eight, seven, and six respectively. Therefore, by rearranging both warp and weft threads on each of these intervals, eight different designs are obtained. Figs. 121 to 124 are produced by rearranging warp threads, and Figs. 125 to 128 are produced by rearranging picks of weft, at intervals of two, three, four, and five threads respectively.

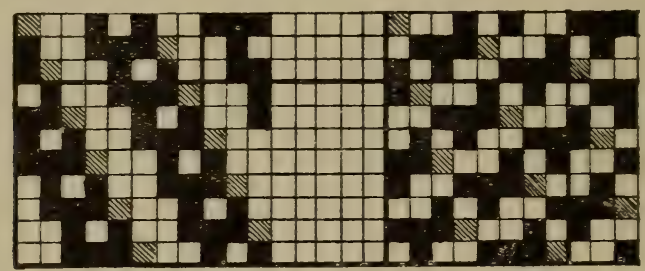

FIG. 125.

FrG. 126.

$\$ 36$. Another method of rearranging either warp or weft threads of a base pattern, to create new designs, is by adopting a uniform interval of two threads irrespective of the number of threads on which the original weare is contained. This system, however, offers considerably less scope to a designer than the foregoing, and should only be employed for the production of

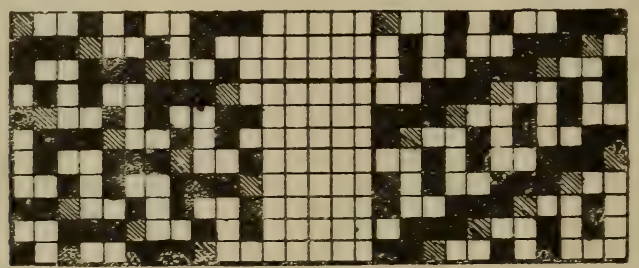

Fig. 127 .

FIG. 128.

weares in which a decided twill formation will not be displeasing. By this system, a twill formation will almost inevitably result in all cases, in consequence of laying alternate threads of a base weave in consecutive rotation, or vice verst. If warp threads are rearranged, the twill will approach the vertical, and if weft threads are rearranged, the twill will approach the horizontal.

It should be observed that by this system, rearranged weaves 
based on odd-thread weaves will repeat on the same number of warp threads and picks as that of their base weaves; whilst those based on even-thread weaves will repeat on only one-half the number of threads in one direction, as that of their base

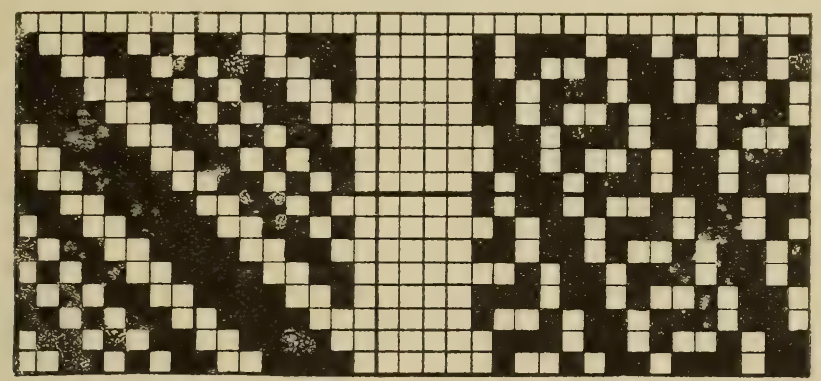

FIG. 129.

Fig. 130.

weaves. This is explained by the fact that two (the interval employed) is a measure of eren numbers, but not of odd numbers. Therefore, designs repeating on an odd number of threads require them all to be employed in order to complete the nerv design; whilst only one half are necessary in respect of eventhread designs.

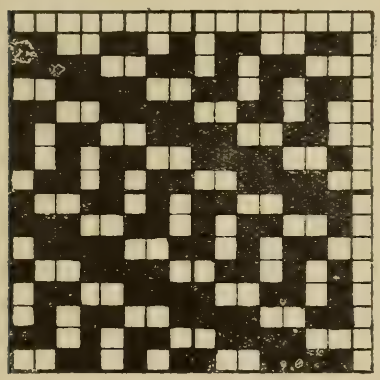

FIG. 131.

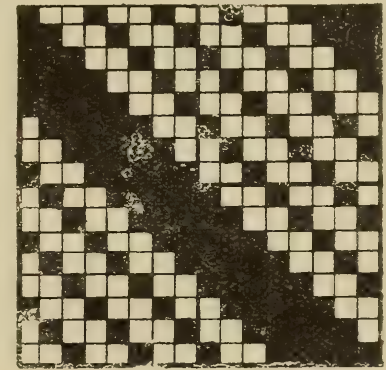

FIG. 132.

Figs. 129 to 134 will serve to demonstrate the application of this principle of rearrangement in the creation of new designs. Fig. 129, a twill weave contained on fifteen warp threads and picks, is selected as the base weave. By placing alternate warp threads of the base weave in consecutive rotation until the 
pattern is complete, a new design repeating on fifteen warp threads and picks, as indicated in Fig. 130, is obtained. In like manner, if alternate picks of weft of the base weave are placed in consecutive rotation, the weare indicated in Fig. 131 is obtained. The only difference between the two new designs is in respect of the angle of twill, as just explained.

Figs. 133 and 134 are produced by rearranging, in a similar manner, warp threads and picks of weft respectively, of a base weare (Fig. 132) contained on an even number of threads, namely, sixteen. Since only one half the number of warp threads in the base weave are required to produce Fig. 133, the latter is complete on eight warp threads and sixteen picks. Also, for a similar reason, but in respect of picks, Figs. 134 is complete on sixteen warp threads and eight picks, as indicated in both cases by shaded squares.

\section{Combined Twills.}

$\$ 37$. Combined twills are those produced by arranging the threads of two continuous twill weaves alternately with each other. Either warp threads or picks of weft of the two base weares may be alternated. If warp threads are combined, the

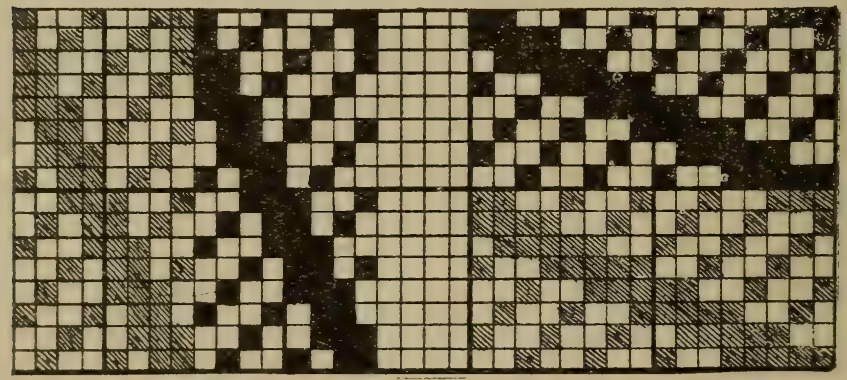

FIG. 133.

FIG. 134.

angle of twill in the resultant weave will be less than forty-five degrees; but if picks are combined, the angle of twill will be greater than forty-five degrees to picks of weft. If it is desired to produce a low-angle twill by this method, the best results will be attained by selecting two base weares in which weft prepon- 
derates over warp. For high-angle twills, the base weaves should have warp preponderating over weft.

By this system of combination, there is practically no limita-

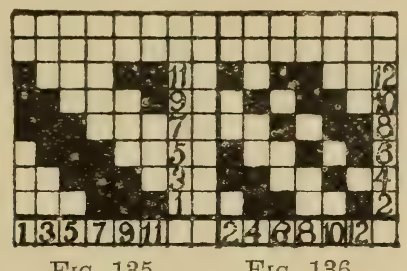

tion to the production of new weaves of great variety and interest, and of great value to the textile designer. It obtains

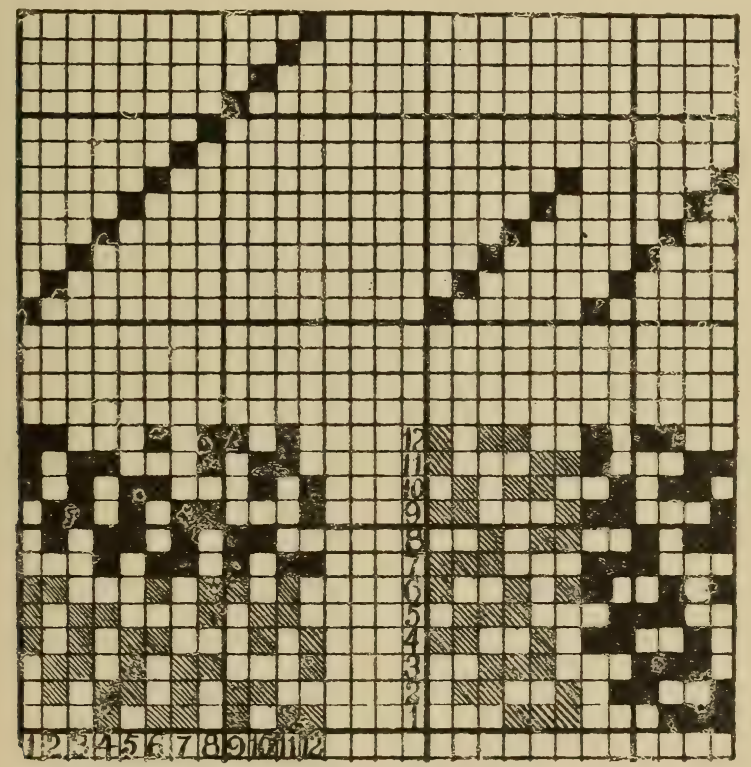

FIG. 137.-Design evolved by an end-and-end combination of Figs. 135 and 136.

Frg. 138.-Design evolved by a pick-and-pick combination of Figs. 135 and 136.

almost exclusively in the worsted industry in the production of garment fabrics, as it is capable of producing compact and firm textures. 
Any two weaves may be combined in the manner described, irrespective of their relative sizes. The size of the resultant weare, however, depends upon the number of threads occupied by the respective base weaves employed. Thus, if two base weares, each occupying the same number of threads, are combined end and end (i.e., a warp thread from one weave, and a warp thread from the other alternately), one repeat of the combined twill weave will occupy twice as many warp threads, and the same number of picks, as those of the respective base weaves.

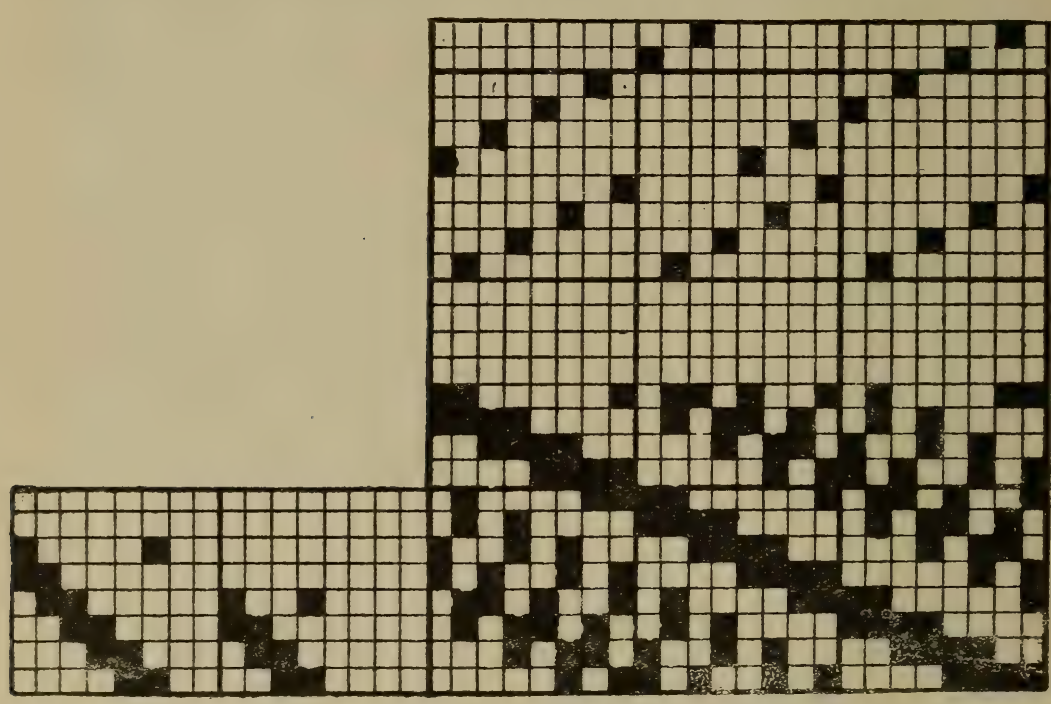

FIG. 139.

Fig. 140.

FIG. 141.-Design evolved by an end-andend combination of Figs. 139 and 140.

If, on the other hand, the base weares are combined pick and pick, the combined twill would occupy twice as many picks, and the same number of warp threads, as either of the base weaves. For example, Figs. 135 and 136 are two continuous twill weaves, each repeating on six warp threads and picks. By combining them end and end a new design is produced, repeating on $6 \times 2=12$ warp threads and six picks, as shown in Fig. 137 . If picks instead of warp threads of the same base weaves are alternated, a new weave is produced, repeating on six warp threads, and $6 \times 2=12$ picks, as shown in Fig. 138 . 
If two weares, each repeating on a different number of threads, are combined end and end, the resultant weave will repeat on such number of warp threads as equals the least common multiple of those numbers, multiplied by 2 ; and on such

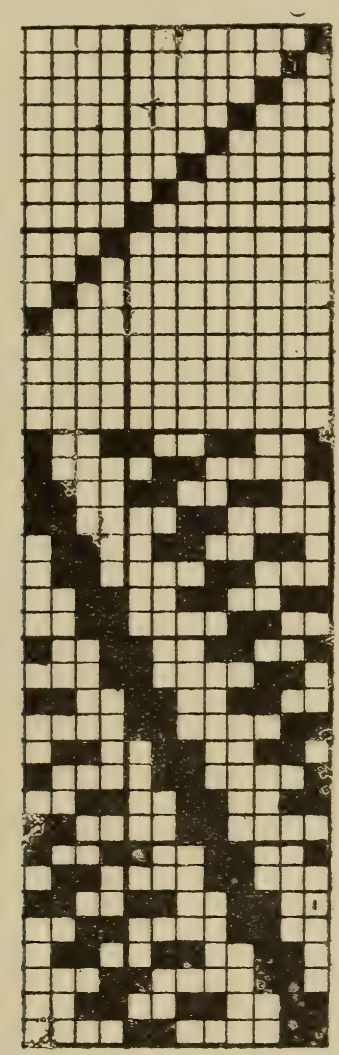

FIG. 142.-Design evolved by a pick-and-pick combination of Figs. 139 and 140 .

number of $i$ picks as equals the least common multiple only of those numbers. This rule also applies in a corresponding manner if picks of weft are combined. Example: two weaves, Figs. 139 and 140, repeat on four and six warp threads and picks respectively. If combined end and end, the resultant weare, Fig. 141, will repeat on twenty-four warp threads and 


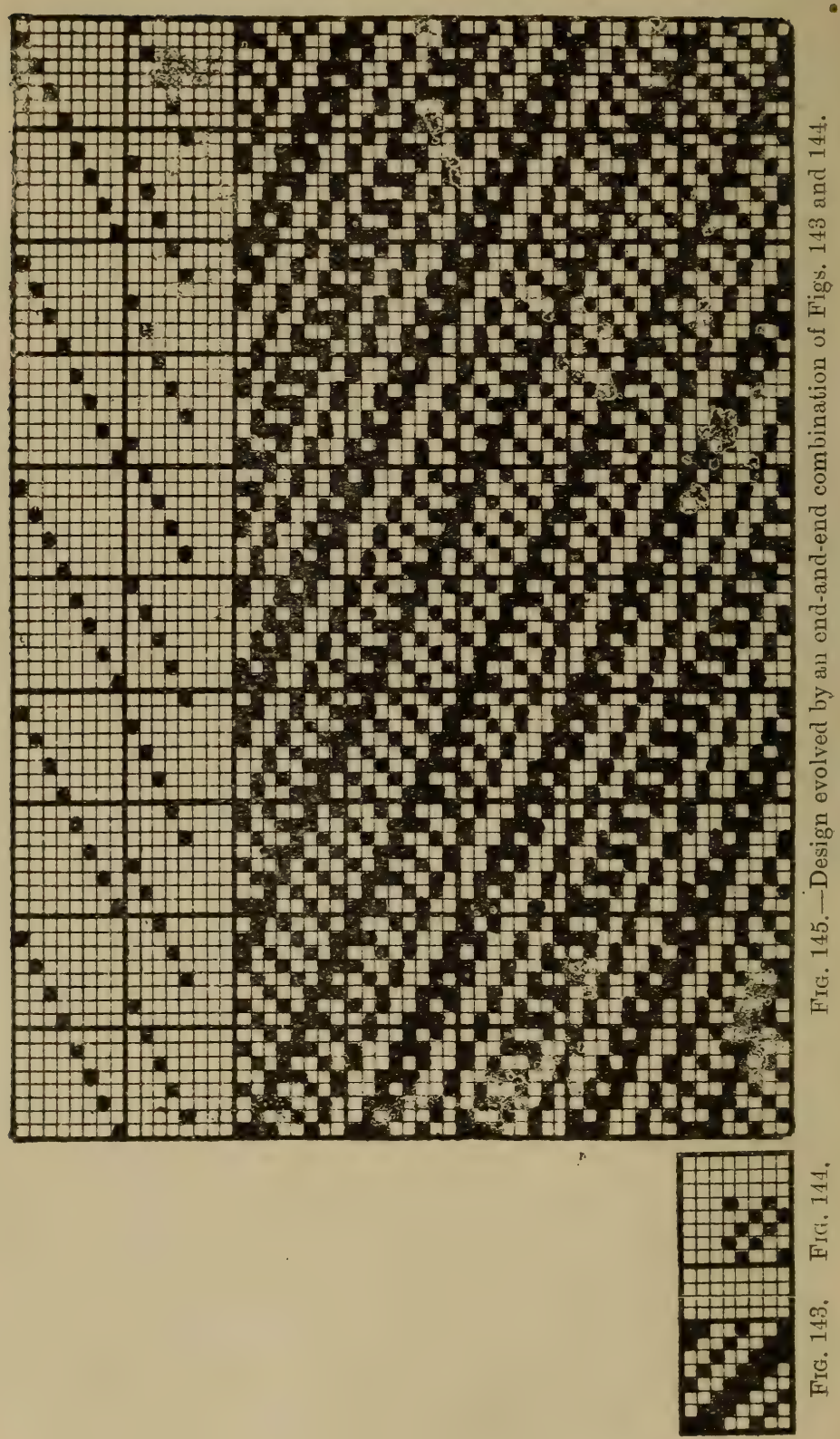


twelve picks, because twelve is the least common multiple of the numbers four and six. By combining the same base weaves pick and pick, a design is produced, repeating on twelve warp threads and twenty-four picks, as shown in Fig. 142. If two weaves repeating on eight and five threads respectively are

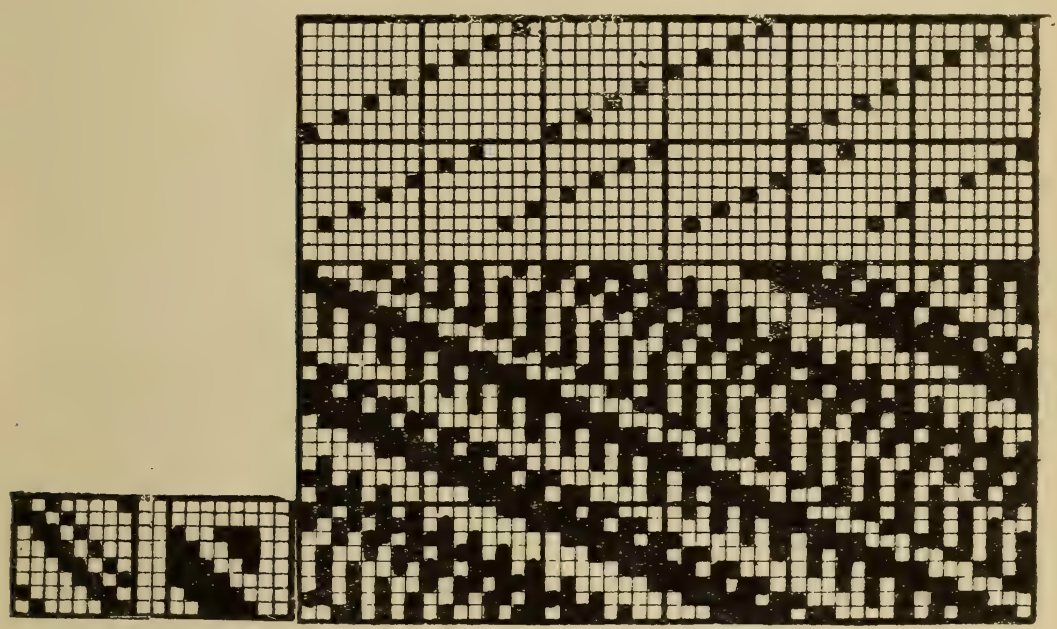

FIG. 146. Frg. 147. FiG. 148.-Design evolved by an end-and-end combination of Figs. 146 and 147.

combined, the resultant weave will occupy forty threads in one direction, and eighty in the other direction, according to which series of threads are combined. This is exemplified by Fig. 145, which is produced by combining end-and-end the base weaves, Figs. 143 and 144, repeating on eight and five threads respectively.

It should be observed that although a combined twill weave obtained by alternating warp threads of two base weaves may occupy many times more warp threads than either of its base weaves, it will only require, for its production, such number of healds as equals the sum of those required to weave the respective base weaves; whereas, if picks of weft of two base weaves are alternated to produce a combined twill weave, the number of healds required to weave it corresponds with the number of warp threads occupied by the resultant weave. This is 
indicated by the drafts placed above the respective designs. It will be seen that a design produced by combining picks may be woren with a "straight," i.e., continuous, drafting; whereas, one produced by combining warp threads will require what may be termed a "compound" draft, i.e., in which the healds are

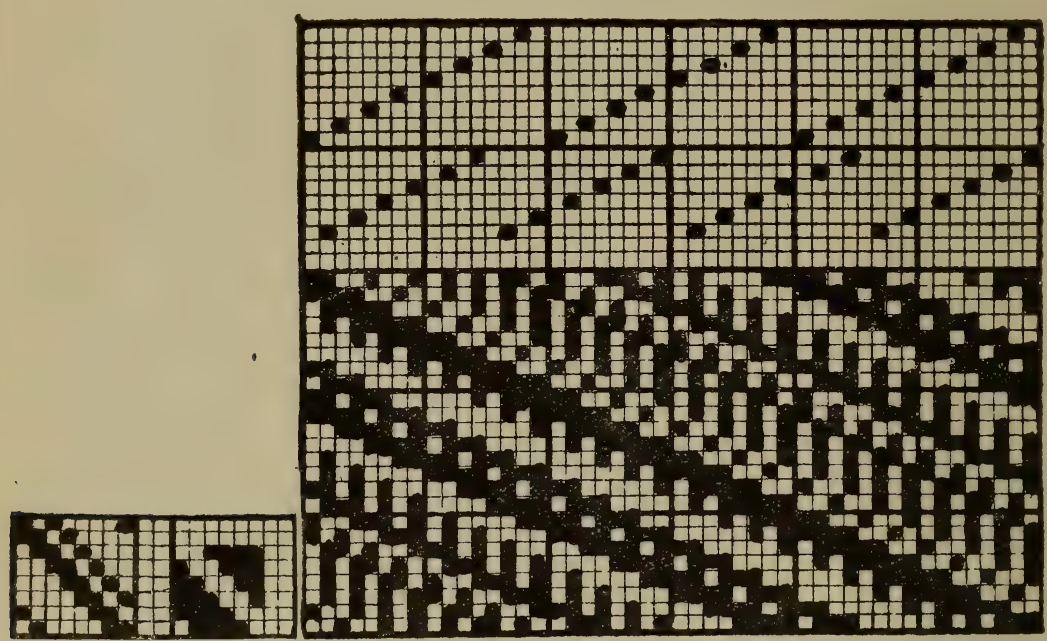

FIG. 149. FIG. 150. FIG. 151.-Design evolved by an end-and-end combination of Figs. 149 and 150.

virtually in two sets-one set governing alternate warp threads in accordance with one base weave, and the other set governing the remaining warp threads in accordance with the other base weave, and with each division of threads drawn "straight through" on their respective sets of healds, as shown.

$\mathrm{By}$ this system of combining twill weaves, additional scope is sometimes offered a designer in the production of varied effects, even from the same base weaves, by the simple expedient of placing the latter in different relative positions; that is, by placing the threads of one weave one thread, or more than one thread, in advance or in rear of those of the other weave. This is exemplified by Figs. 146 to 151. Figs. 146 and 147 are two base weaves, repeating on eight and six threads respectively, and combined end and end to produce design Fig. 148. By 
combining the same base weaves, but in the relative positions shown in Figs. 149 and 150, a new design, Fig. 151, is produced.

\section{Broken Twills.}

$\$ 38$. Broken twills comprise that class of weaves produced by breaking the continuity of any continuous twill weave at either regular or irregular intervals of threads. Such a course somewhat tends to the formation of stripes, either in the direction of warp or of weft, according as the twill is broken lengthwise or crosswise respectively. If the twill is broken at regular intervals of threads the stripes will be of uniform width; but if broken at irregular intervals the stripes will be variegated. In either case the stripes will be more or less pronounced according to the character of twill employed, and the manner in which it is broken. The twill may incline in one direction throughout, or it may be reversed! in alternatel stripes, or in any other

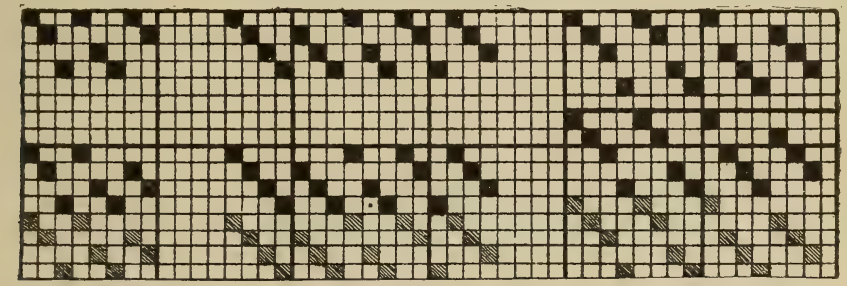

FIG. 152.

FIG. 153.

Fig. 154.

manner, to emphasise the striped effect. The stripe formation may also be emphasised by the principle of counterchange effected by reversing the weave after each break, as in Figs. 155 to 162 , and 165 and 166 . The reversal of weave may also be made in conjunction with a reversal of the direction of twill (to obtain a still more pronounced stripe formation) as exemplified in Figs. 170 to 176.

Fig. 152 is one of the simplest examples of a broken twill weave in which the twill inclines in the same direction throughout. It is based on the four-end weft-face twill, broken at intervals of two warp threads, and advanced one pick at a time after each break. The pattern is complete on eight warp threads and four picks, and may be woven with only four healds, drafted 
in the manner indicated above the design. In the following examples, shaded squares indicate one repeat of the pattern, and the drafts are indicated above their respective designs.

Figs. 153 to 166 are other examples of broken twill weaves, based upon (a) simple, $(b)$ even-sided, and $(c)$ uneven-sided

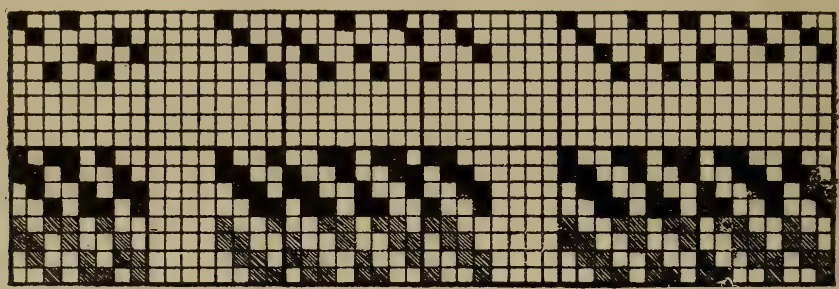

FIG. 155.

Fig. 156.

FIG. 157.

twills, in all of which the twill inclines in the same direction throughout. Fig. 153 is also based on the four-end weft-face twill, broken at intervals of four warp threads, and advanced one pick after each break; whilst Fig. 154 is produced by breaking a five-end weft-face twill at intervals of three warp threads, with a very different result.

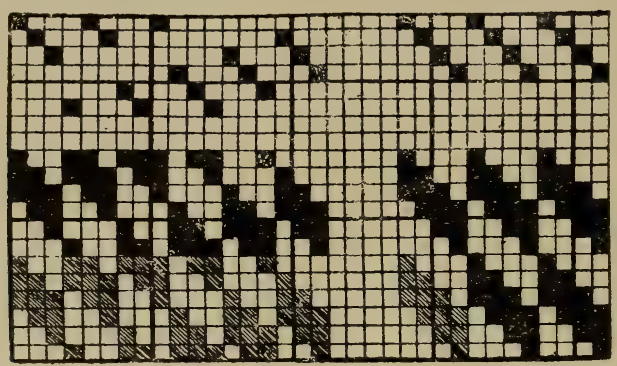

FIG. 158.

Fig. 159.

Figs. 155 and 156 are produced by breaking a four-end twoand-two twill at intervals of two and four warp threads respectively; whilst Fig. 157 is based on the same weave broken at irregular intervals of five and three warp threads alternately.

Figs. 158 to 160 are all based on a six-end three-and-three twill, broken at intervals of three, four, and five warp threads 
respectively. Fig. 161 is based on the same weave, broken at irregular intervals of eight, four, two, four, two, and four warp threads.

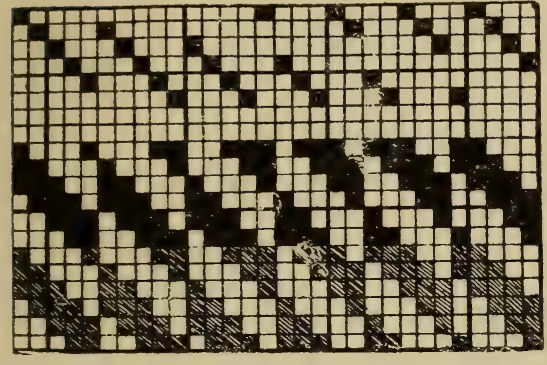

FIG. 160.

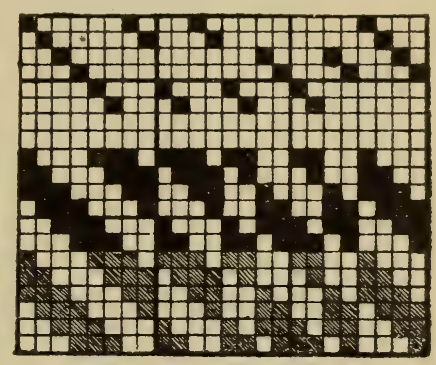

FrG. 161.

Fig. 162 is also based on an even-sided twill, but of different construction to that employed for the last four designs. The base weave is an eight-end $\frac{31}{2} \frac{1}{2}$ twill, broken at intervals of five threads, with the.weave entirely reversed, but with the twill

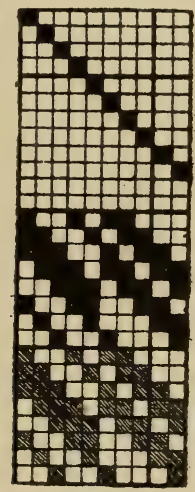

Fig. 162.

continued in the same direction. By reason of the foregoing even-sided twills being broken in the manner indicated, they are counterchanged in respect of the weave only, and not in respect of the direction of twill, which remains the same throughout.

Figs. 163 and 164 are based on the uneven-sided five-end twoand-three twill, broken at intervals of three and five threads 
respectively; whilst Fig. 165 is based on the same weave, broken and counterchanged at irregular intervals of ten, two, four, and two warp threads.

Fig. 166 is based on the six-end four-and-two twill, broken and counterchanged at intervals of eight, four, two, four, two, and four warp threads.

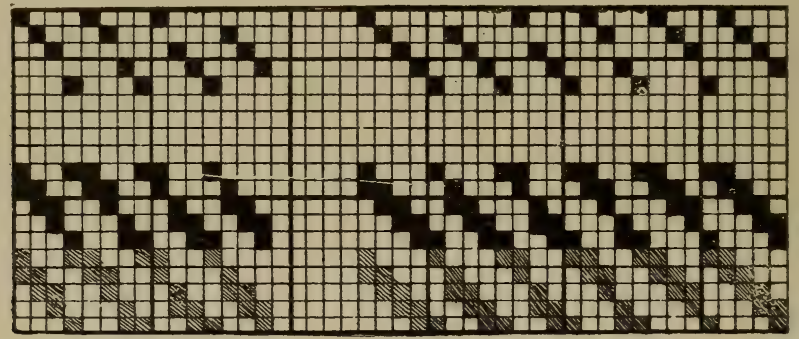

FIG. 163.

FIG.'164.

Although the foregoing examples illustrate the development of broken twill weaves having the twill in one direction only, they serve equally well to demonstrate the formation of those in which the twill is reversed, i.e., produced to the right and left alternately, or otherwise. The simplest example of this

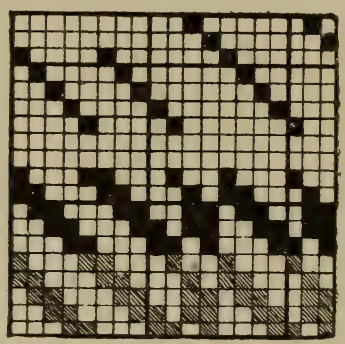

FIG. 165.

variety is that shown in Fig 167, based on the simple four-end weft twill, and well known as the four-end satin or satinette weave. It is produced by breaking and reversing either warp threads or picks at intervals of two threads. Figs. 168 and 169, known as "rice" weaves, are produced in a similar manner by breaking and reversing simple six-end and eight-end weft twills at intervals of three and four threads respectively. 


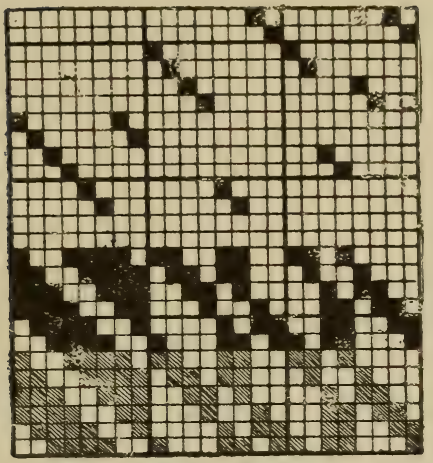

Fig. 166.

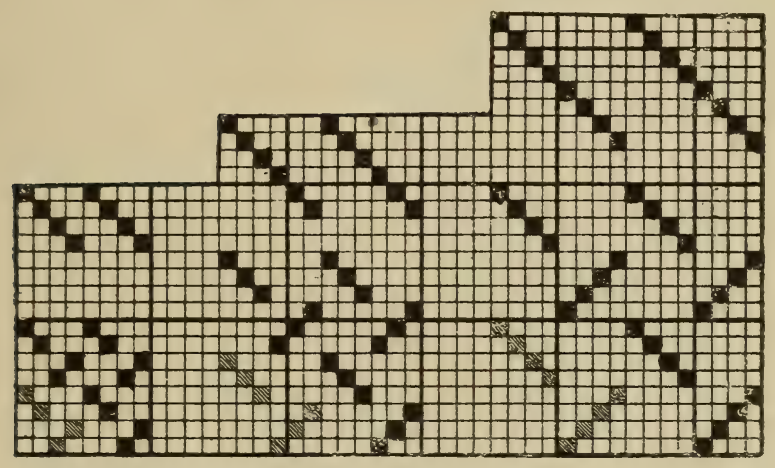

FIG. 167.

FIG. 168.

Frg. 169.

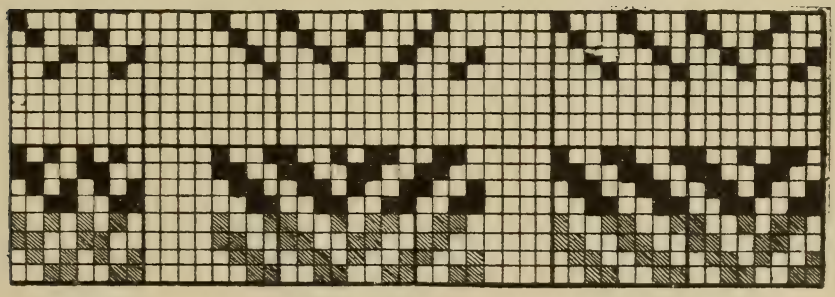

FIg. 170.

FIG. 171.

FiG. 172 
The next and last variety of weaves to be described under this division comprises those in which the twill is broken and reversed in counterchange-a plan largely adopted by designers in the construction of garment and other fabrics requiring good

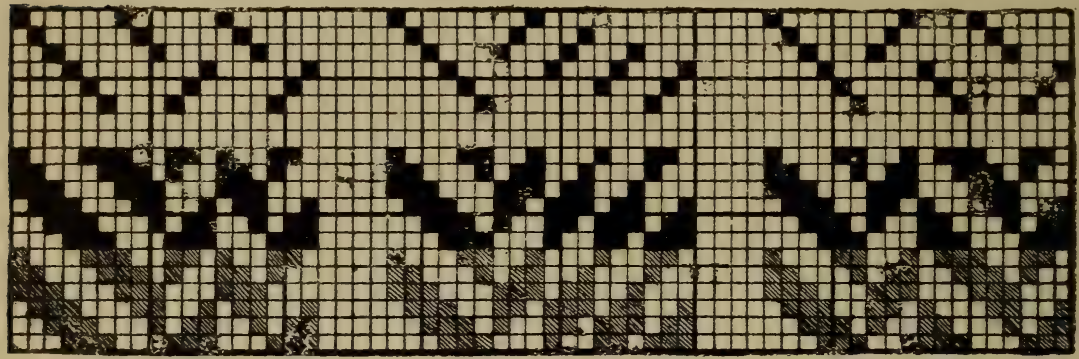

FiG. 173.

FIG. 174.

FIG. 175.

wearing properties, and capable of resisting tensile strain in all directions:

One of the simplest examples of this variety is that shown in Fig. 170, produced by breaking and reversing, in counter-

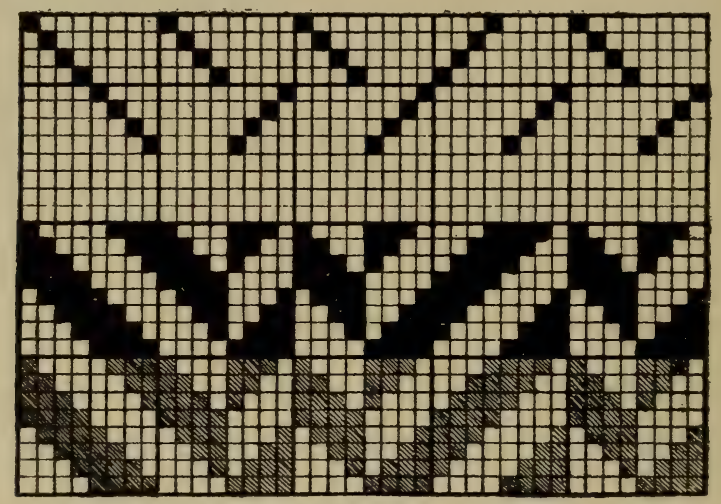

FIG. 176

change, the four-end two-and-two (also known as the "Halvard ") twill, at intervals of two warp threads. This weave is extensively employed in all classes of fabrics, as it produces a comparatively firmfand compact texture. As a neutral ground filling of a subdued character, for light or medium brocade 
Labrics figured by a Jacquard machine, it cannot be excelled. If the twill of this or similar twill weaves is continued for a number of threads and then reversed, as in Fig. 171, it produces the well-known "herring-bone" effect, repeating, in this case, on sixteen warp threads and four picks. Other examples of this class of weaves are given in Figs. 172 to 176, which are sufficient to indicate the general lines on which they are constructed, and the immense scope which this plan offers in the development of new weaves of great variety and utility.

\section{Figured or Ornamented Twills،}

39. This division of twills comprises that variety of weaves produced by combining simple figuring, with a twill weave, as a means of embellishment. The amount of embellishment may vary from the least departure from a normal twill, to a degree when there is little to identify it, as a twill weave, beyond its diagonal formation. It must not be assumed from this that all patterns having a diagonal formation may be classed as figured twills; but only such as conform, in some measure, to the general principles governing the construction of twill weaves.

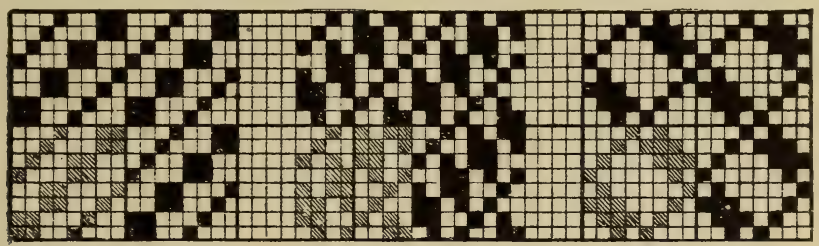

Fig. 177.

Frg. 178.

Fig. 179.

The term "figured twills" is here used to signify only such weaves of that class as may be produced by the aid of tappets and dobbies. The present examples will, therefore, be confined to such as will require not more than twenty healds to weave them. Notwithstanding this limitation, the construction of figured twills virtually affords illimitable scope to a designer, since he is no longer restricted to twilled effects pure and simple, but may call to his aid all the elements of fabric structure.

The additional scope which this class of design offers to a 
designer demands both greater technical and artistic ability than is required for the simpler varieties of twill weaves. In the latter, the perfect regularity of weave causes uniform tension upon all warp threads, thereby producing a general evenness of texture throughout; whereas, in the former class, care must he taken to ensure sufficient and uniform interlacement of threads;

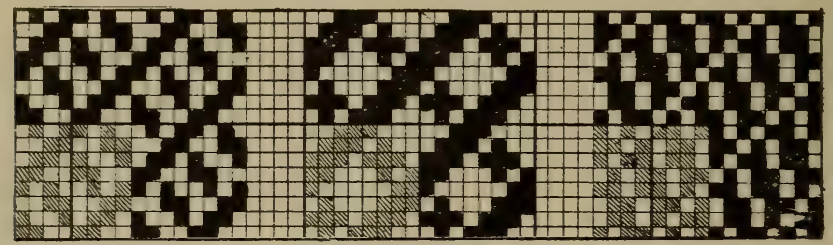

FIG. 180.

Fig. 181.

FIG. 182.

otherwise some portions of the fabric will be flimsy and weak, and some threads will be subjected to greater strain than other's, thereby causing a crimped or wrinkled appearance in cloth. These precautions must be taken when producing designs for any class of fabric in which warp threads, during weaving, are delivered from one warp beam only.

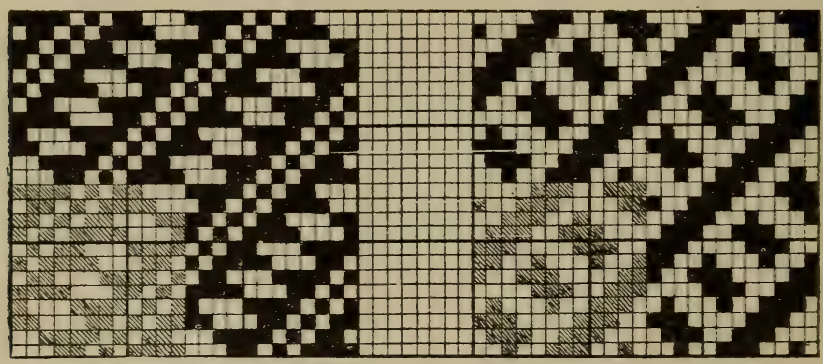

FIG. 183.

FIG. 184.

The present examples are illustrative of figured twill weaves, repeating on eight, twelve, sixteen, and twenty warp threads and picks. Little can be said regarding their construction, as they are not conformable to any definite rules, but depend wholly upon the imagination and technical ability of the designer. It should be observed, however, that whatever is introduced or combined with a twill weave, as a recurring element, it must 
recur on such number of threads (counting diagonally) as constitutes a measure of the total number of threads on which the pattern is required to repeat, otherwise the continuity of the

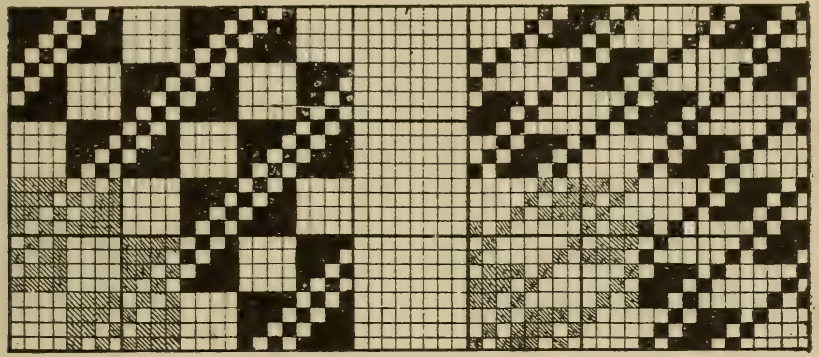

FIG. 185.

FIG. 186.

pattern will be broken. For example, if a pattern is required to repeat on twenty warp threads and picks, figuring elements may

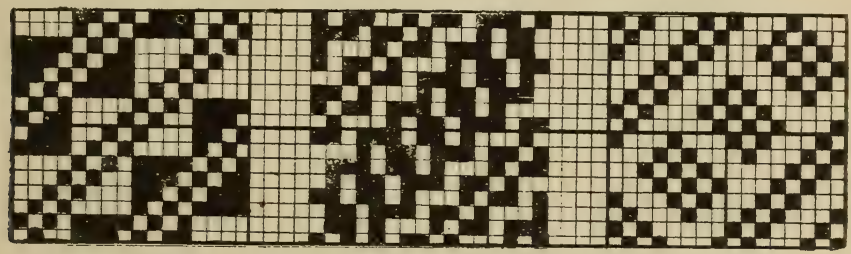

FIG. 187.

FIG. 188.

FIG. 189.

recur at intervals of two, four, five, or ten threads diagonally. This will be better understood by reference to the accompanying

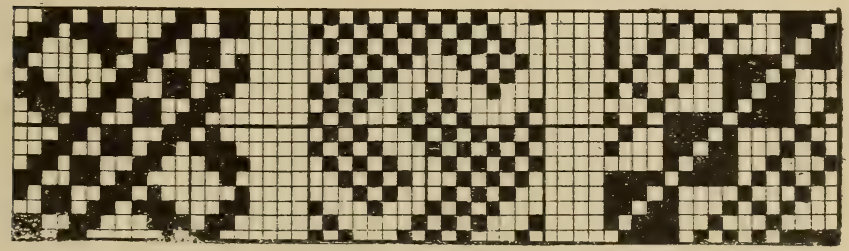

FIG. 190.

FIG. 191.

FIG. 192.

designs. Figs. 177 to 182 are various forms of twill weaves, repeating on eight warp threads and picks, to which are added simple figuring devices, disposed at intervals of two threads diagonally, in Figs. 177, 178, 180 and 182; whilst in Figs. 179 
and 181 the devices are four threads apart, counting from and to corresponding points.

Figs. 183 to 186 are designs repeating on twelve warp threads

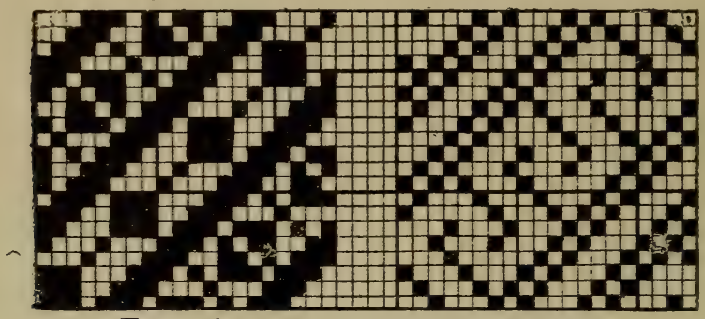

$$
\text { FIG. 193. FIG. } 194 .
$$

and picks, with the figuring devices recurring at intervals of two, three, four, and six threads respectively.

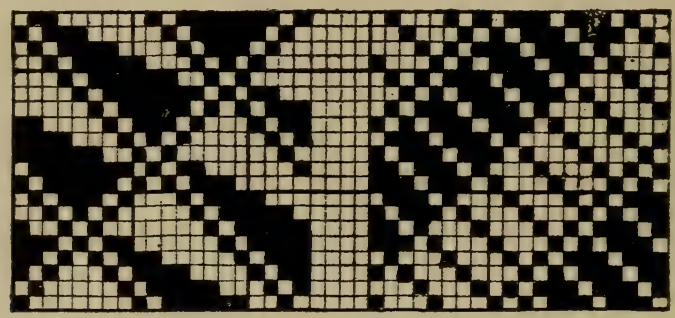

FIG. 195.

FIG. 196.

Figs. 187 to 192 are designs repeating on sixteen threads each way. In Fig. 188 the figuring units recur at intervals of two

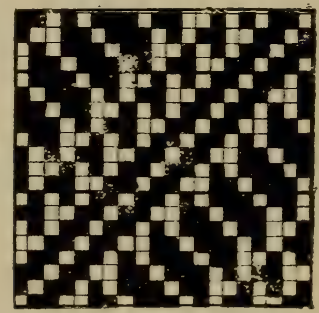

FIG. 197.

threads ; in Figs. 187 and 189, at intervals of four ; in Fig. 191, at intervals of eight; whilst in Figs. 190 and 192, some devices 
recur at intervals of two, and others at intervals of four threads.

Figs. 193 to 197 are designs repeating on twenty threads each way, with the figuring devices recurring at intervals of five threads in Figs. 193, 194 and 195, and at intervals of two threads in Figs. 196 and 197.

Although examples of figured twills could be multiplied act libitum, the foregoing are sufficient, to indicate the immense scope they offer for the production of varied effects suitable for all classes of fabrics. 


\section{CHAPTER IT.}

\section{DIAMOND AND KINDRED WEAVES.}

$\$ 40$. Diamond weaves comprise all such weaves as are characterised by a diamond or a lozenge general formation. They may be produced in infinite variety, and constitute one of the most valuable classes of weaves for almost any type of fabric. They may be constructed with either warp or weft preponderating on the face of the fabric, or with each developed in equal quantities as required, and may be made to yield either a comparatively smooth or else a very rough and open texture, as exemplified in honeycomb and similar weaves. Their diamond or lozenge formation may be more or less pronounced, or even entirely obscured, as in honeycomb and similar weaves, which are characterised by a more or less distinct rectangular cellular formation.

The simplest example of a diamond weave is that contained on four warp threads and picks, as shown in Fig. 198, formed by producing a four-end weft twill to both the right and left. Larger weft diamond weaves of a simple character are formed by crossing larger twill weaves in a similar manner; thus Figs. 199 and 200 are produced from six-end and eight-end weft twill weaves respectively.

Although diamond weaves may be made on any number of threads, those contained on an even number may be produced with sharper definition than those based on an odd number, providing the opposing twills cross on a single warp thread and pick, as in Figs. 198 to 200, and not on two contiguous threads, as in Figs. 201 to 203, which are contained on five, seven, and nine threads respectively.

Figs. 204 to 213 are a few examples of diamond weaves repeating on twelve warp threads and picks uniformly, and are 
giren merely as suggestions indicating general methods of procedure in their development. In all cases their diamond formation is apparent, although they are not all actually based upon twill weaves produced in opposite directions. A careful

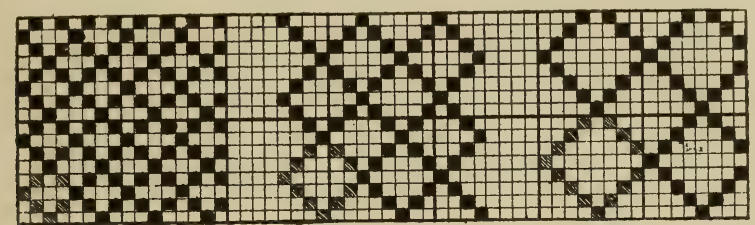

FIG. 198.

FIG. 199.

FIG. 200.

examination will rereal the means by which the different results are attained, and students are urged to make original designs of a similar character and so develop the faculty of invention in constructive textile design.

\section{Honeycomb and Kindred Weaves.}

$\$ 41$. One of the most interesting varieties of weaves based, with few exceptions, on the diamond, are what are technically termed "honeycomb" weaves, from their partial resemblance to the hexagonal honeycomb cells of wax in which bees store their honey. As preriously stated, these weaves are characterised by a more or less distinct cellular formation, which imparts to cloth a

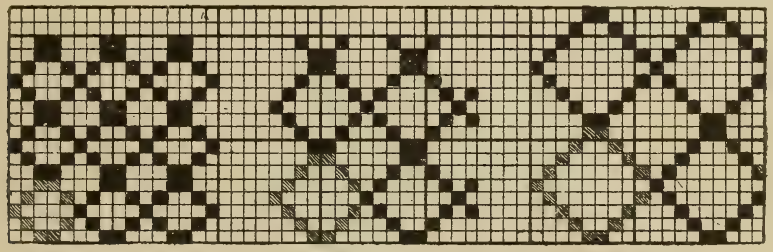

FIG: 201.

Frg. 202.

FIG. 203.

somewhat rough and rugged appearance, as seen in Figs. 214 and 215, which are photographs of actual examples of cloth, representing two of the best-known varieties of this class of weaves, namely, "honeycomb" proper, and "Brighton "weaves respectively. The peculiar character of texture resulting from honeycomb weaves in general, and particularly from those just 
named, renders them eminently suited for use as bathroom towels, which require to be soft and absorbent, and for which

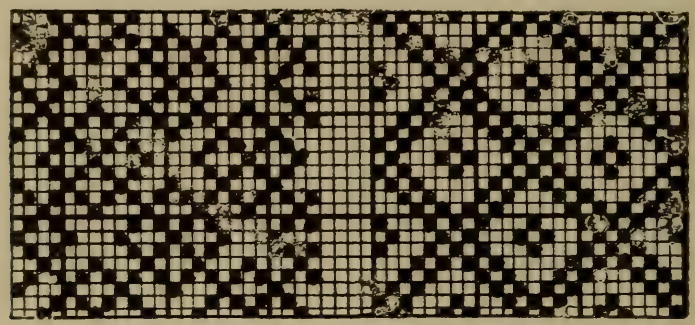

FIg. 204.

FIG. 205.

use they are extensively employed. They are also very largely used in the production of heavy cotton and woollen textures for

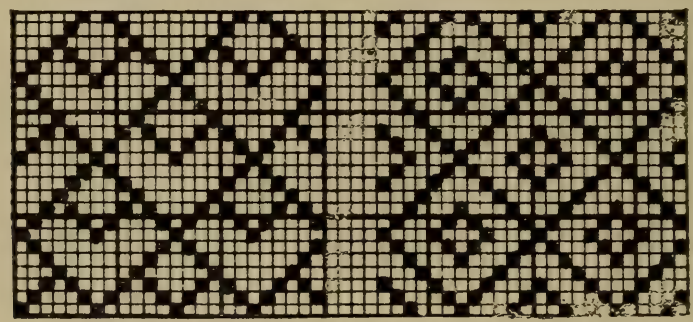

FIG. 206.

FIG. 207.

ladies' winter garments for both under and outer wear, and for many domestic purposes. The well-known honeycomb counter-

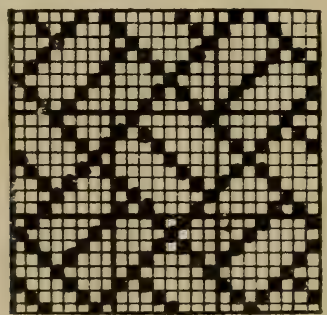

FIG. 208.

panes and toilet covers are so named from the almost exclusive adoption of honeycomb weaves, as elements with which is developed the ornamentation peculiar to those fabrics, which 
ornamentation is usually of an angular and linear geometrical character.

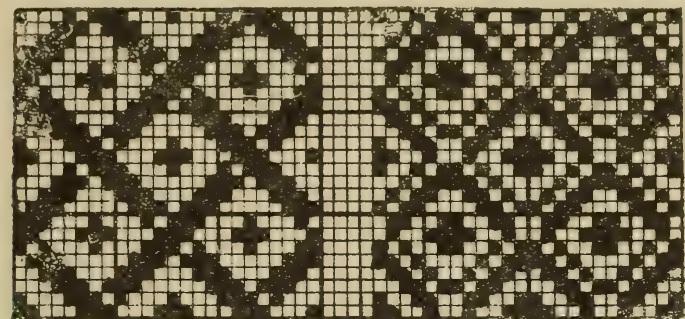

FIG. 209.

FIG. 210.

Although the peculiar effects of honeycomb weaves are primarily due to the particular methods of interlacement of

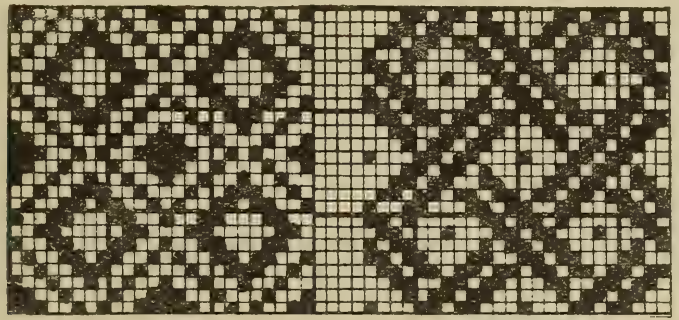

EIG. 211.

FIG. 212.

warp and weft, those effects are relatively more pronounced in compact textures produced from coarse and folded yarns, than in

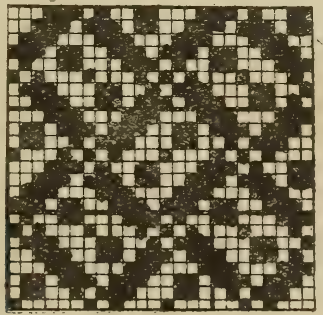

FIG. 213.

loose textures produced from fine and single yarns. For this reason it is usual to weave them from folded yarn for warp, and 
frequently for both warp and weft, with a high number of threads per inch. The samples of cloth illustrated in Figs. 214 and 215 are very heavy textures made from threefold 8's (equal to $8 \div 3=2 \frac{2}{3}$ 's single) cotton yarn for both warp and weft. The honeycomb sample contains 32 warp threads and 36 picks per inch, and the "Brighton" sample 44 threads of each series per inch.

Whilst conformable to the same general conditions, true honeycomb weaves may vary in detail of construction, but all

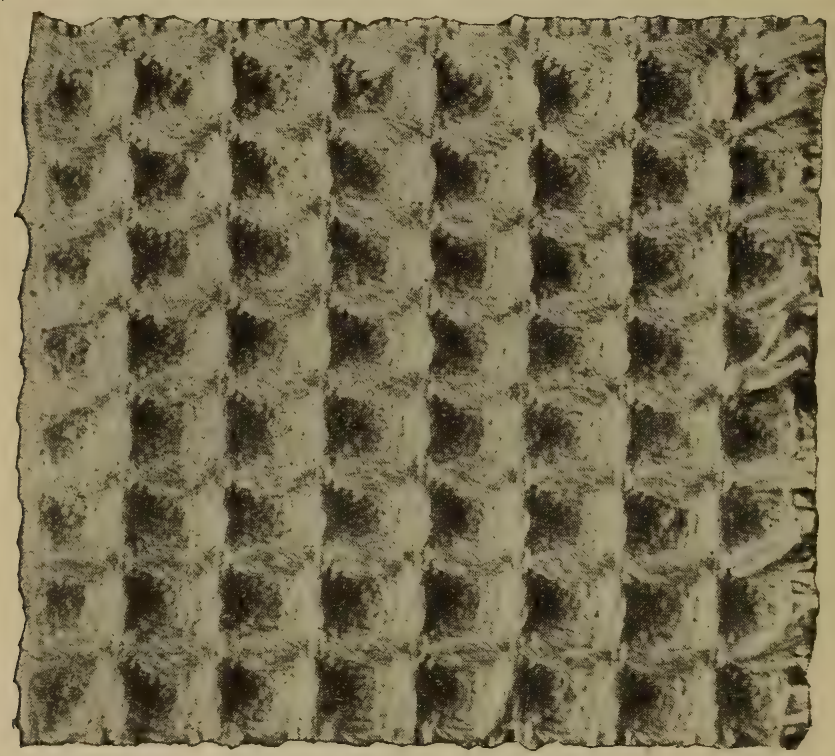

Fig. 214.--Simple honeycomb weave in fabric of coarse texture.

must repeat on an even number of both warp threads and picks. Sometimes they are made to repeat on the same number of threads each way, and sometimes on a less number one way than the other, according to the ratio of warp threads and picks required in the fabric. If warp and weft are to be in equal quantities, the weave selected should repeat on the same number of threads of each series; but if one series of threads is in excess of the other, the pattern should repeat on such number of threads each way as most nearly corresponds to the ratio of 
warp threads and picks per inch, otherwise the honeycomb cells would not be square in cloth.

Fig. 216 is the simplest example of a true honeycomb weave. The pattern repeats on six warp threads and four picks, with warp and weft on the face, and therefore at the back, in equal quantities, thereby causing both sides of cloth to be exactly alike-a feature not attainable in honeycomb weaves repeating on the same number of threads each way, in which either warp or weft must slightly preponderate. In, the larger weaves, however, the difference is scarcely, if at all, perceptible.

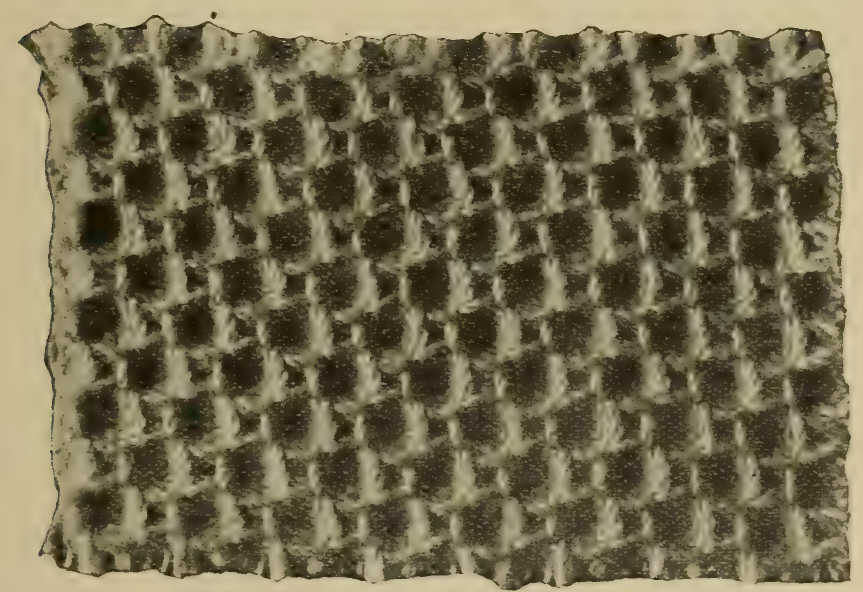

FrG. 215.- "Brighton" honeycomb weave in fabric of coarse texture.

Figs. $217 \mathrm{~A}$ and $217 \mathrm{~B}$ are designs showing the obverse and reverse sides respectively of the six-end honeycomb weave. As seen at $217 \mathrm{~A}$ it appears as a simple six-end diamond weare, with alternate divisions, diagonally, filled in with a small warp diamond. The pattern is contained on $6 \times 6=36$ small squares, fifteen of which represent warp, and twenty-one represent weft. At $217 \mathrm{~B}$, however, which is the reverse of $217 \mathrm{~A}$, the ratio of visible warp and weft is also reversed. This variation will cause the cellular formation to be more pronounced, and therefore superior, on one side, according to the respective counts of warp and weft and the ratio of warp threads and picks per inch, which data should be known to a designer to enable him to 
decide how to display the weave to the best advantage. For example, if a fabric were to be made with the same number of warp threads and picks per inch, from warp of slightly finer counts

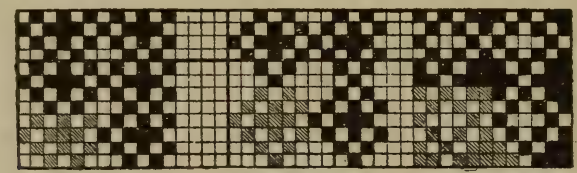

FIG. 216. FIG. 217A. FIG. 217B.

than that of weft, the best results would obtain from the weave shown at $217 \mathrm{~B}$, by reason of the longer float of finer warp compensating for the shorter float of coarser weft. If, on the other

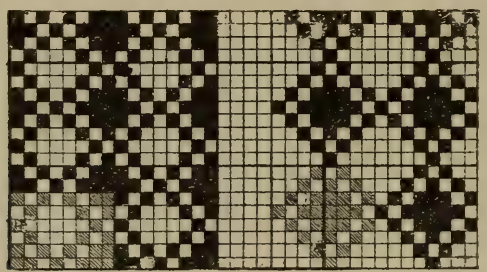

FIG. 218.

FIG. 219.

hand, weft is to be a little finer than warp, the weave as given at $217 \mathrm{~A}$ would give the best results, for similar reasons.

All other varieties of simple honeycomb weaves are constructed

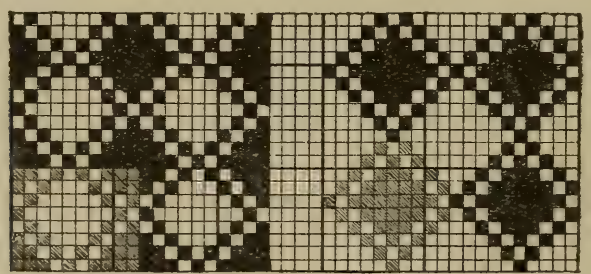

FIG. 220.

FIG. 221.

in a similar manner to those given in Figs. 216, 217 $\mathrm{A}$ and 217B, the difference being one of magnitude only. For example, Figs. 218 to 223 are honeycomb weaves of all sizes, from that contained on eight by six, to that contained on twelve by twelve threads. It is rarely that the latter size of honeycomb weave is 
exceeded, excepting in fine textures containing a comparatively high number of warp threads and picks per inch. As the weaves increase in size, the threads are proportionately less frequently interlaced, thereby producing a weaker texture. It is advisable, therefore, to construct the larger weaves on the basis of what is termed a double-stitch diamond, as shown in Fig. 224, which

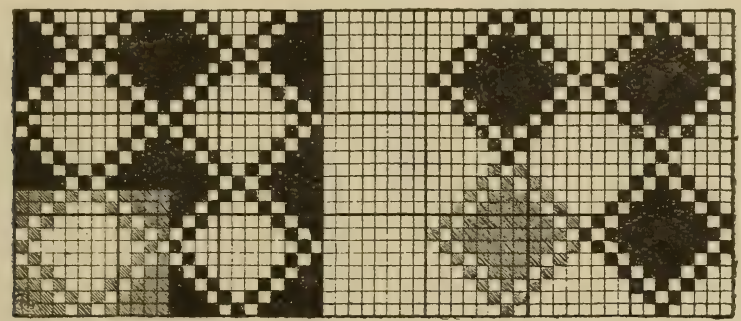

FrG. 222.

Fig. 223.

forms the basis of the sixteen-end honeycomb weave given in Fig. 225. By thus increasing the degree of interlacement of threads, a fabric of firmer texture is produced without destroying the salient features of the honeycomb weave.

At the outset of these observations on the construction of diamond weaves, it was stated that their diamond formation

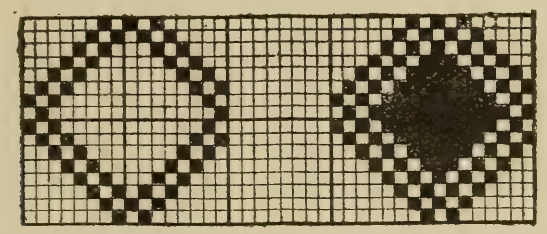

FIG. 224.

FIG. 225.

may be more or less pronounced, or even entirely obscured, as in honeycomb and similar weaves, which are characterised by a more or less distinct cellular formation. This is easily observed in Figs. 214 and 215. In the former, which is a twelve-end honeycomb weave proper, the rectangles are of uniform size, whilst in thelatter, which is a twelve-end "Brighton " weave, the rectangles are of two sizes, a large and small one alternately, both longitudinally and transversely. In neither case is their diamond 
origin apparent, nor is there any resemblance between the woven effects and their respective designs (as represented on design paper), as is usual with most weaves. The cause of this phenomenon will be understood on reflecting that warp and weft cross at right angles to each other, and that threads are more or less conspicuous according as they are interlaced in a lesser or greater degree respectively. Thus it comes about that the ridges of honeycomb cells are formed by the longer floats of warp and weft, which lie uppermost, whilst the recesses are caused by threads interlacing to a greater degree in those parts. By reference to the eight-end honeycomb weave, Fig. 226, it will

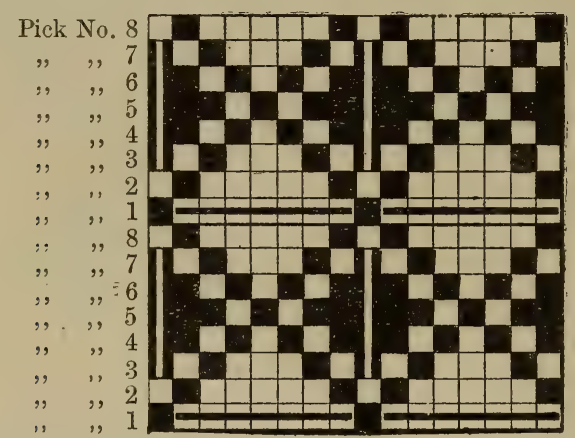

Warp-thread No. 1234567812345678

FIG. 226.-Demonstrating the cellular formation in honeycomb weaves.

be seen that the ridges of the cells are formed by the first warp thread and first pick of weft-in each repeat of the pattern-which are least interlaced, and therefore lie in a higher plane than the threads on each side of them. The threads lie in a lower plane, as their length of float diminishes, up to the fifth warp thread and pick, which lie in the lowest plane and form the recesses of the cells.

\section{"Brighton" Weaves.}

$\S 42$. "Brighton" weaves are also constructed on a diamond basis, yet they are quite different from simple honeycombs, and more complex in structure. They are characterised by a cellular formation comprising two sizes of cells, as seen in Fig. 215. Unlike simple honeycomb weaves, "Brighton" weaves are not 
reversible, but have a totally different appearance on each side of the fabric. Instead of clearly defined cells, as on the face of the fabric, the reverse side presents a rough, indefinite appearance, the cause of which will be presently explained. This circumstance requires to be carefully borne in mind by designers when employing "Brighton" weaves as elements in the development of ornamental designs, otherwise the designs are liable to be spoiled.

Examples of "Brighton" weaves are given in Figs. 228, 229 and 230. It is noteworthy that they may only be constructed on a multiple of four threads of warp and weft (the least size being contained on eight warp threads and eight picks, as shown in Fig. 228). They are constructed on a diamond formed by producing a simple weft twill to the right or left (for such number of

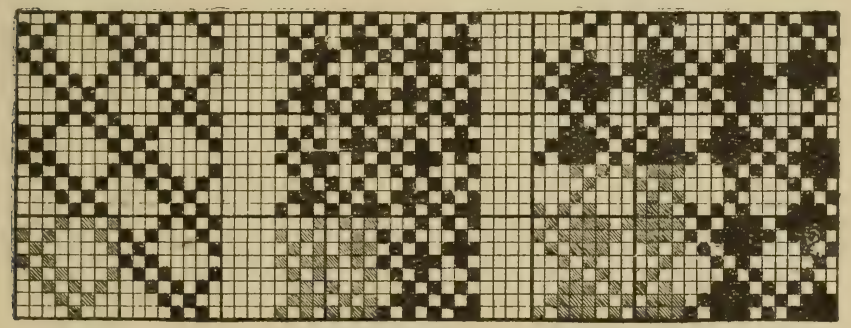

FIG: 227.

FIG. 228.

FIG. 229.

Construction of "Brighton" weaves.

threads as the weave is required to repeat on, say eight), and then crossing it by a double-stitch weft twill, as in Fig. 227. The next step is to put in a warp diamond spot in the right and left corners of each diamond, so as to form a weft diamond spot in the upper and lower corners. The longer floats of warp at the sides, and the longer floats of weft at the top and bottom of each diamond, form the ridges of the cells as explained in the last paragraph of $\$ 41$. The two sizes of cells are caused by the intervals between the ridges being greater and less, thereby enclosing larger and smaller areas alternately. If the relative positions of the warp and weft diamond spots are reversed, the weave will be the wrong side up by reason of the longer floats forming a cross, instead of a square, within the diamonds. A parallel reference to Figs. 230 and 231 will enable this to be easily 
understood. Fig. 230 is a perfect sixteen-end "Brighton" weave with the warp and weft spots in their proper relative positions, showing the squares formed by their longer floats. Fig. 231 has

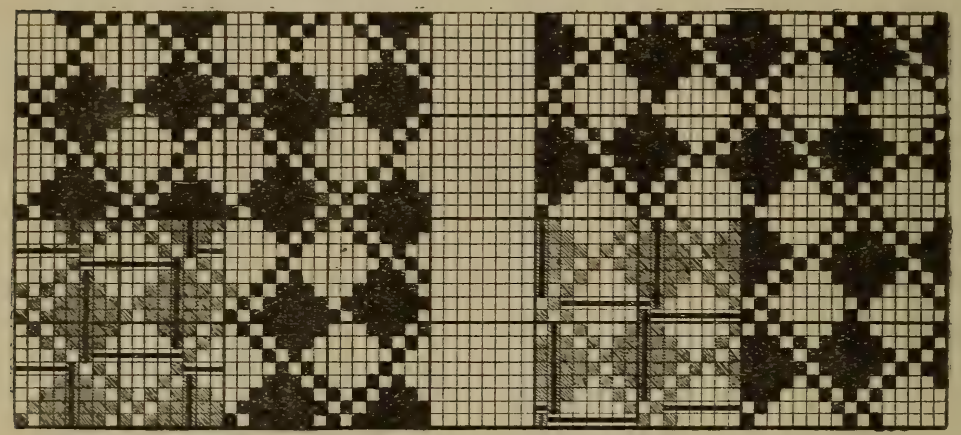

FIG. 230.-Right method.

Fig. 231.-Wrong method.

Construction of "Brighton" weaves.

the same diamond foundation, but the warp and weft diamond spots are placed in the wrong relative positions, thereby causing their longer floats to form a cross within each diamond, as shown.

\section{"Sponge" Weaves.}

$\$ 43$. In addition to the true honeycomb and "Brighton" weaves there is a great variety of weaves, termed "honeycomb effects," that are closely allied to those weaves, and which possess, in varying degrees, many of their characteristics. They

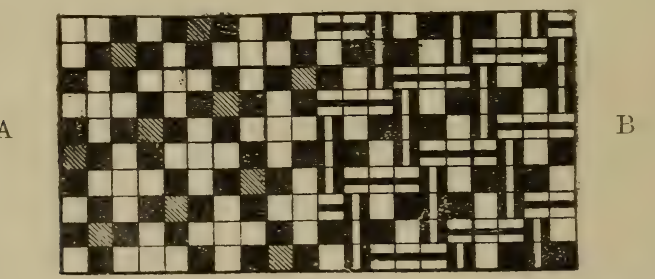

FrG. 232.-Demonstrating the cellular formation in "sponge" weares.

are extensively employed as constructive elements in the development of Jacquard designs for honeycomb counterpanes, toilet covers and fancy woollen shawls, as well as in the production of piece-goods of comparatively heavy and thick textures 
for use as ladies' winter underclothing, towels, and many other' domestic purposes. They are usually characterised by a cellular formation, but of a less pronounced character than that of a perfect honeycomb. This arises chiefly from adopting a less restricted basis than that of a diamond on which to construct

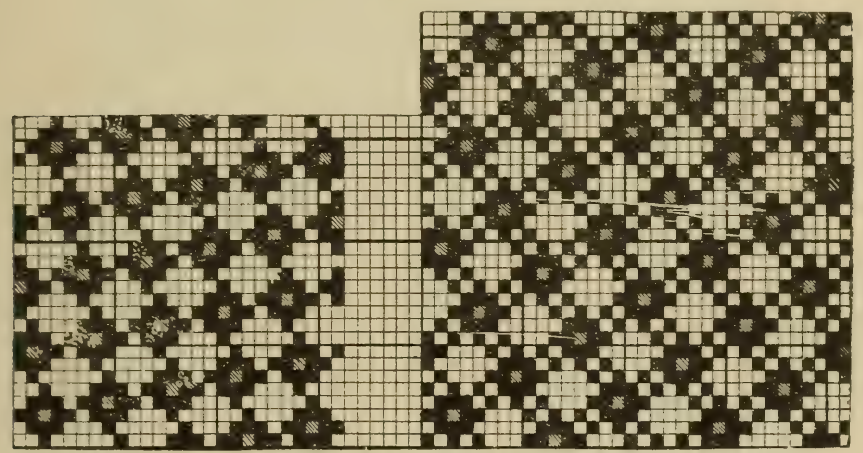

FiG. 233.

FIG. 234.

them, as, for example, a satin-weave basis, which gives a free distribution; broken diamonds; and irregular bases that cannot be exactly defined.

One of the commonest examples of these honeycomb effects is that known as the "sponge" weave (Fig. 232), contained on

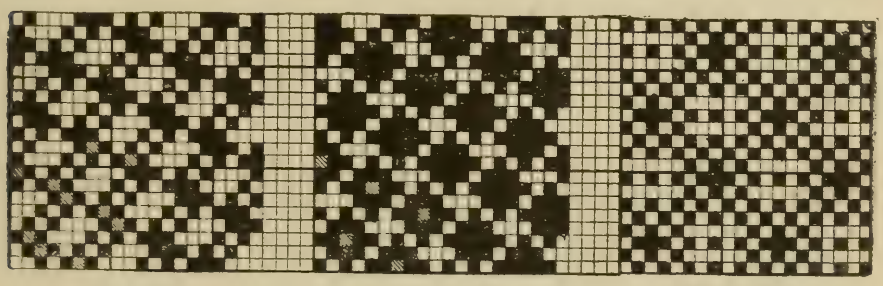

FiG. 235.

FIG. 236.

FIG. 237.

ten warp threads and ten picks. It is produced by disposing a small diamond figure or spot on a ten-end satin basis, as indicated by the shaded squares in the portion lettered $A$. This causes the woven fabric to assume a very neat cellular formation consisting of minute cells, the ridges of which are formed by floats of warp and weft, whilst the recesses are formed where 
the threads are most interlaced. This effect is illustrated in the portion lettered $B$, where the warp and weft ridges are repre-

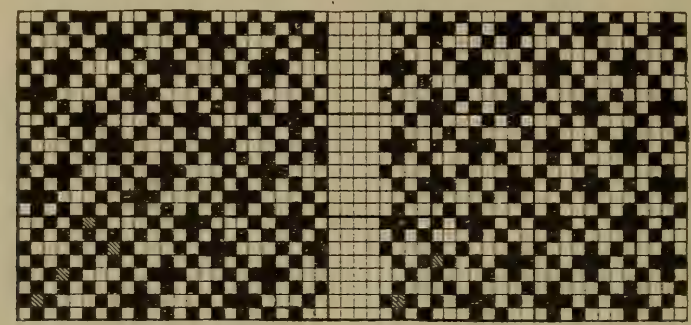

FIG. 238.

Fig. 239.

sented by white and black lines respectively, and the recesses by the enclosed spaces.

Figs. 233 and 234 are other varieties of sponge weares on a

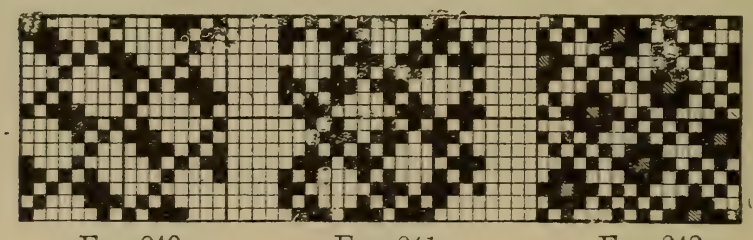

Fig. 240.

Fig. 241.

FIG. 242.

larger'scale than the previous one. They are produced by arranging larger diamond: spots on the basis of a twenty-six shaft, and a thirty-four shaft satin weave, respectively, as irdicated by the shaded squares. Their effect in cloth is similar to that of Fig.

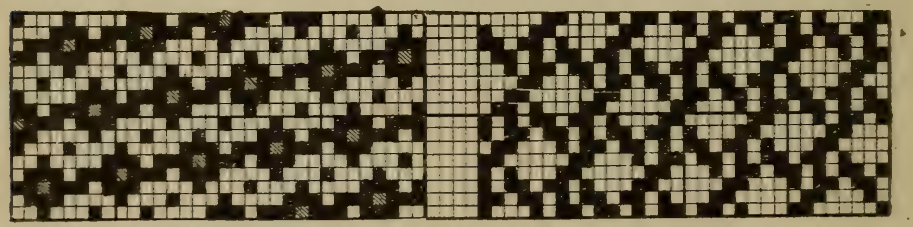

FIG. 243.

FIG. 244.

232 , but with a more pronounced cellular formation, resulting from longer floats and less frequent interlacing of yarn, which enable heavier and taicker fabrics to be made by them. Figs. 235 to 244 are other examples of sponge and honeyco mb effects, 
and are but a few of many varieties of similar character to indicate the general principles governing the construction of that class of weaves.

\section{"Huck-a-Back" and Kindred Weaves,}

$\S 44$. There are many other varieties of weaves which, whilst not bearing even the slightest resemblance to true honeycomb weaves, are generally associated with honeycomb fabrics, and

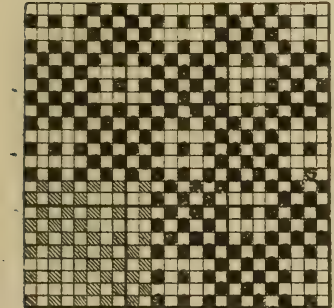

FIG. 245.—"Huck-a-back" weave.

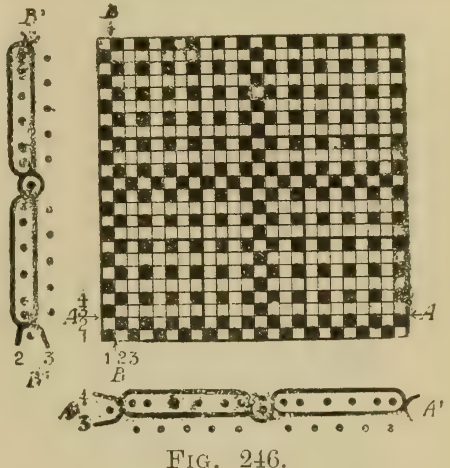

FIG, 246.

are, therefore, classed as honeycomb effects. Of this variety that known as the "huck-a-back" weave, shown in Fig. 245, and contained on ten warp threads and picks, is an example. This

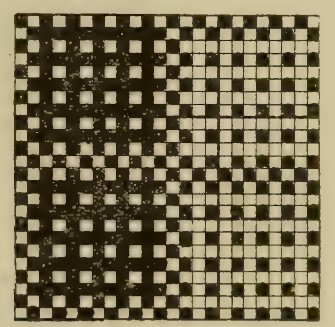

FIG. 24i.-Desizn for "huck-a-back" cloth, Fig. 248.

familiar weave is also very largely employed in the manufacture. both of linen and cotton towels for bathrooms, and also linen towels for use as glass-cloths. The principle of fabric structure embodied in the huck-a-back weave forms the nucleus of a wide 
range of interesting weaves capable of producing extremely thick and heavy textures. For this reason, such weaves are generally employed as constructive elements in the manufacture of the class of heary counterpanes commercially known as "Grecians," usually woven from bleached twofold and threefold yarn of coarse counts for both warp and weft, and ornamented with designs of a strictly geometrical diaper character. Figs. 246 to

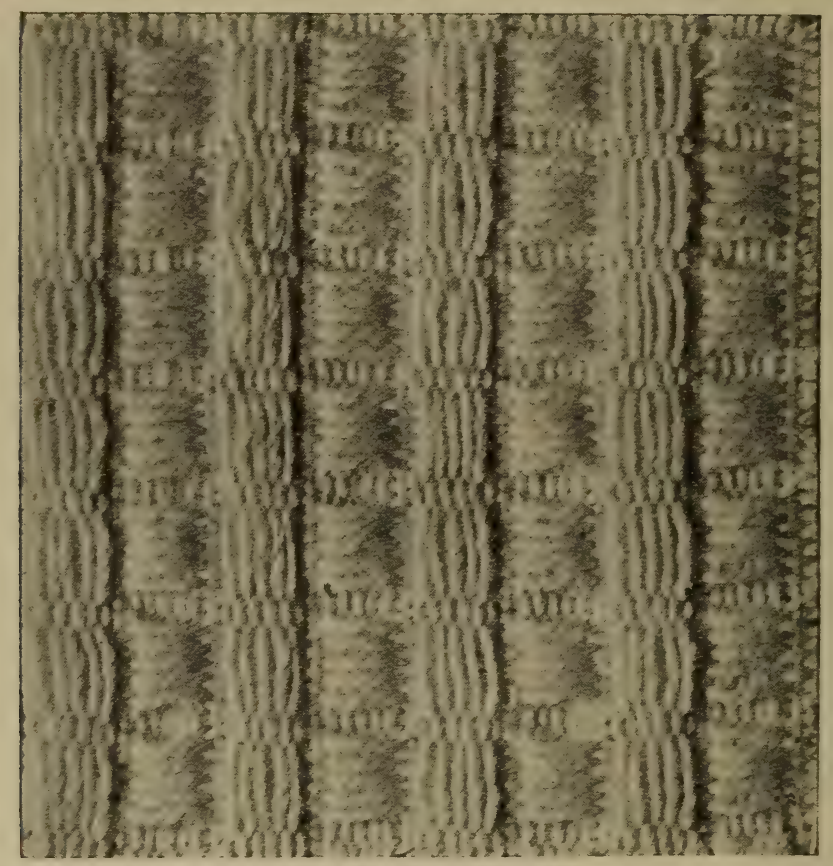

FIG. 248.- "Huck-a-back" fabric of coarse texture, from design Fig. 247

249 are three examples of weares shorring developments of the "huck-a-back" principle to form plain, striped, and chequered effects respectively. In a fabric produced from Fig. 246, which repeats on twelve warp threads and picks, weft preponderates on the face and warp at the back. On examining this weave it will be seen that on the third and following odd-numbered picks to the eleventh, only the first warp thread in each repeat of the pattern is raised; also that the third and following odd-numbered 


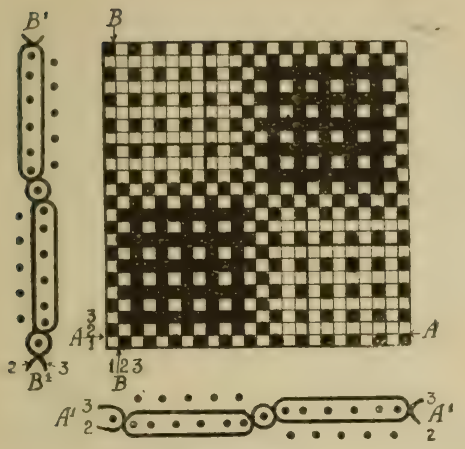

FIG. 249 .

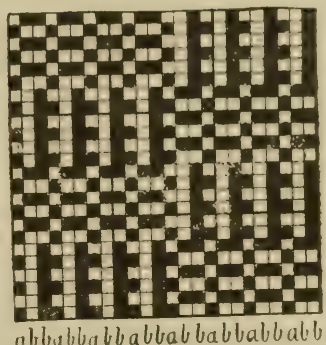

FiG. 250.

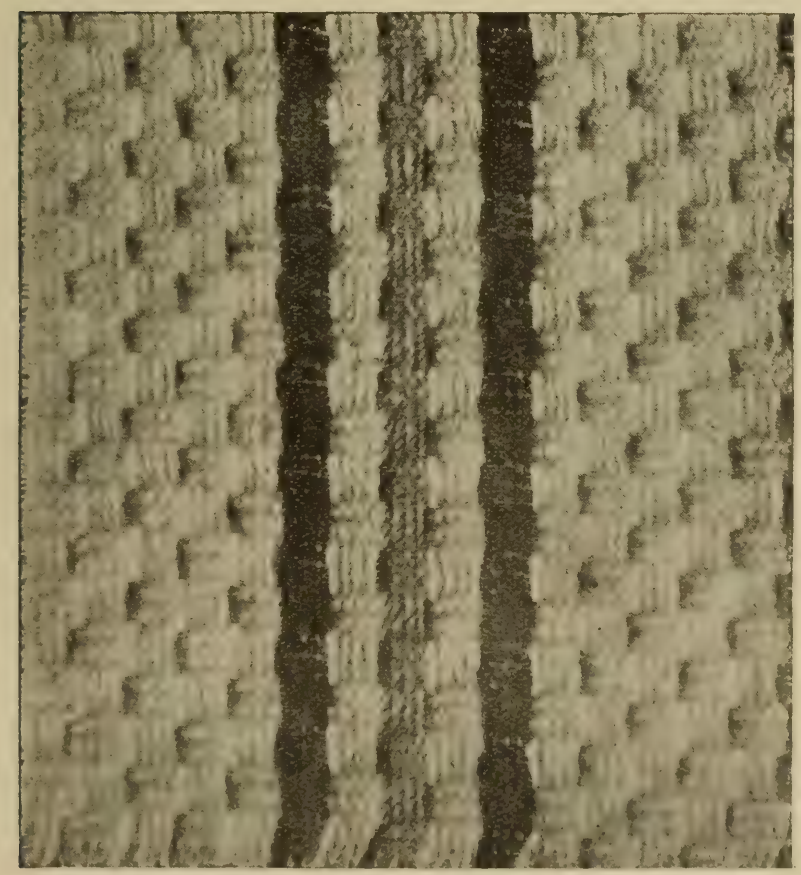

FIG. 251.- "Huck-a-ba:k" fabric, from design Fig. 250. 
warp threads to the eleventh, are raised for the first pick only in each repeat of the pattern. This has the peculiar effect of causing warp and weft threads (excepting the first of each series) to occupy four distinct planes or strata without any interlacement whatever, after the manner indicated in the accompanying diagrams, which show transverse and longitudinal sections of the weave at $\mathrm{A}, \mathrm{A}$, and $\mathrm{B}, \mathrm{B}$, respectively. It is in consequence of causing: such disposition of warp and weft that weaves of this class produce bulky fabrics of great weight and warmth. Fig. 247 is a design contained on twenty-four warp threads and twelve picks, produced by counterchanging the warp and weft effects of Fig. 246 to form stripes; whilst Fig. 248 is a photograph of a woven example of the same weave produced from $3 / 12$ 's warp and weft, and containing thirty-two threads of each series per inch. Fig. 249 is a design produced by counterchanging warp and weft effects of the same weave to produce a check pattern repeating on twenty-four warp threads and picks. The diagrams accompanying Fig. 249 show transverse and longitudinal sections at A, A, and B, B, of that design as it would appear in cloth. Fig. 250 is another good example of the huck-a-back variety of designs, repeating on'twenty-four warp threads and twelve picks. The photograph, Fig. 251, showing the woven effect of that design, is taken from cloth containing forty warp threads of $3 / 12$ 's, and twenty of $2 / 14$ 's cotton, and forty picks of 18 's single cotton weft per inch. The different counts of warp are contained on separate warp beams, with the $2 / 14$ 's (lettered $a$ on the design) held at greater tension, during weaving, than the $3 / 12$ 's (lettered b). Taut warp threads interweave with weft in plain or tabby order throughout; whilst slack warp threads are more loosely interwoven to form the figured effect.

\section{"Grecian" Weaves.}

$\$ 45$. Another useful variety of weaves that are frequently associated with honeycomb and "Grecian" counterpanes, and also largely employed in the manufacture of piece-goods, are exemplified in Figs. 252 to 265 . The most suitable designs for such fabrics are those based on the diaper or counterchange principle, to produce chequered effects in which both warp and 


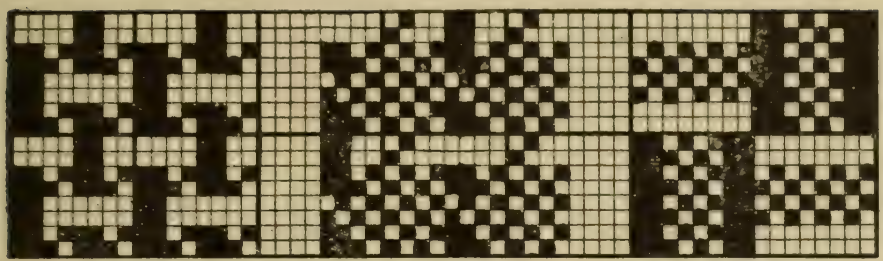

FIG. 252.

Fig. 253.

FI(i. 254.

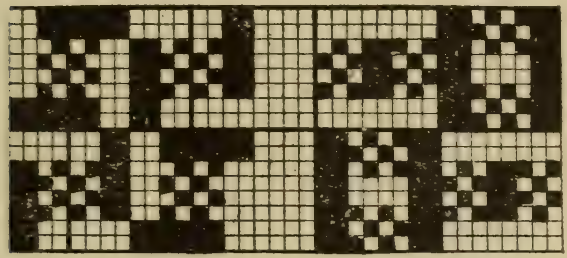

FIG. 255.

FIG. 256.

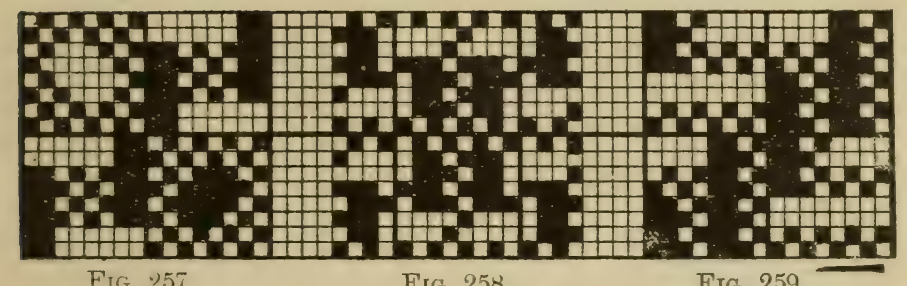

FIG. 257.

FIG. 258.

FIG. 259.

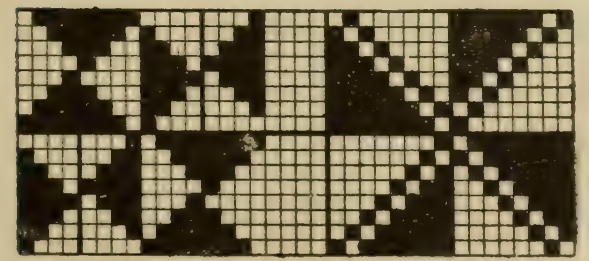

FIG. 260. FIG. 261. 
weft are freely displayed on the face side of cloth. The construction of weaves of this variety affords considerable scope for the exercise of a student's ability in fabric structure, which he should put into practical effect, and carefully note the results.

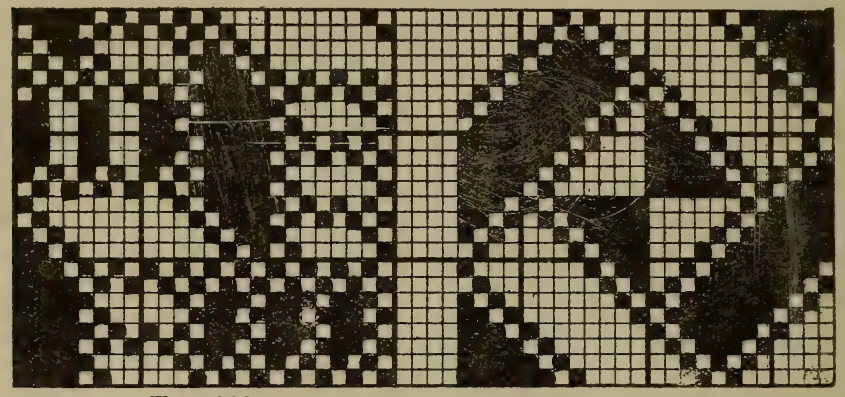

FrG. 262.

F1G. 203.

Little can be said respecting their construction, since they are conformable to no special conditions of fabric structure; but, by carefully analysing them, the means by which they are obtained will become manifest. For the general guidance of students,

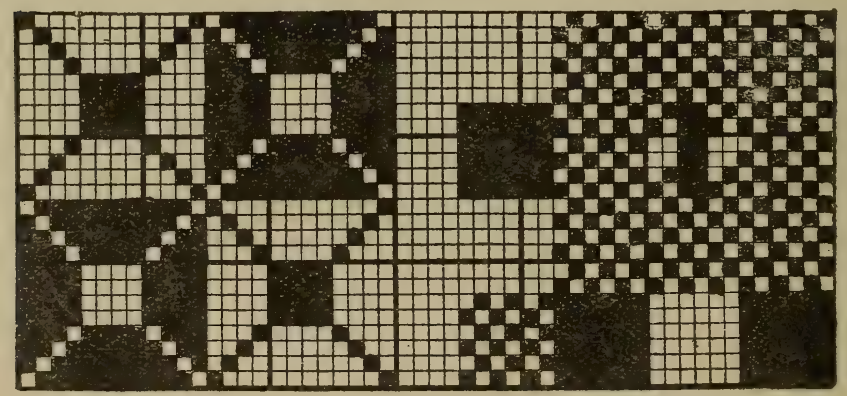

FIG. 264.

FIG. 265.

however, it should be observed that these effects are chiefly dependent either upon a suitable combination of extreme degrees of interlacement of threads, or else by causing warp threads to float over a comparatively large number of picks; and picks of weft over a large number of warp threads; otherwise, unsatisfactory results would obtain. If, for example, the 
present weaves (excepting Fig. 255) were counterchanged, their effects would be lost by reason of an insufficiency of floating threads. Fig. 255 is an exception to this condition, as that weave would be equally effective whether counterchanged or not, and would, therefore, appear the same on hoth sides of the cloth.

\section{Linear Zigzag or Spider Weaves.}

$\$ 46$. An interesting variety of weaves of a totally different character from any described previously under "honeycomb effects," but closely related to them, are those in which some threads, usually of weft, are pulled in opposite directions at different points, thereby causing them to deviate from their

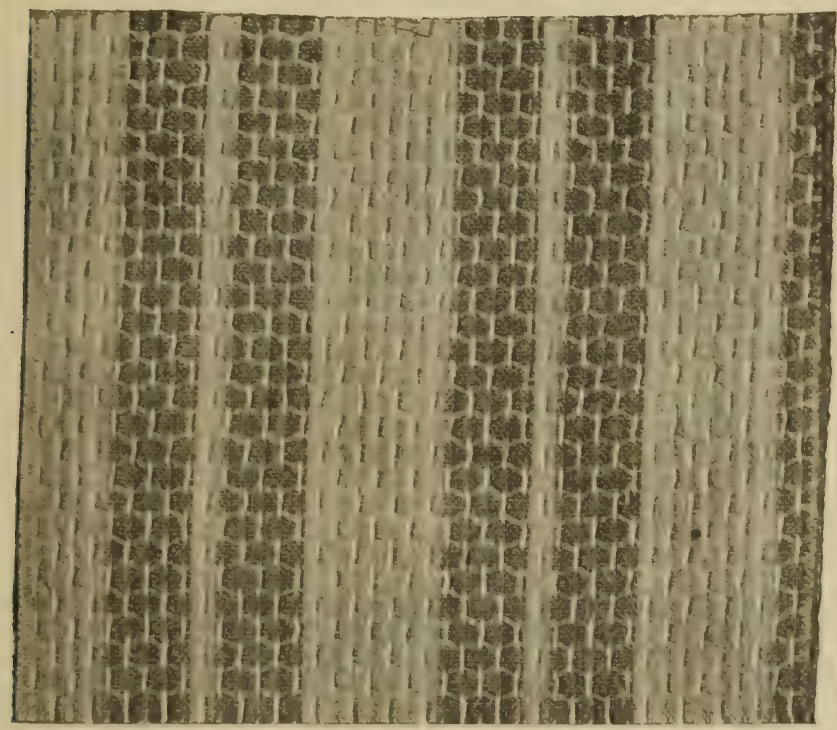

FIG. 266.- "Linear zigzag" effect, from design Fig. 267.

original straight line, and to assume sinuous lines of a more of less iwavy or zigzag character, not unlike that of a "net" leno effect, produced by means of a "doup" or "leno" harness. The threads required to perform that peculiar function may be wared in the same direction uniformly, to produce a series of parallel 
wares, or they may be waved in opposite directions to produce diamond, lozenge, ogee, and other simple linear effects, as seen

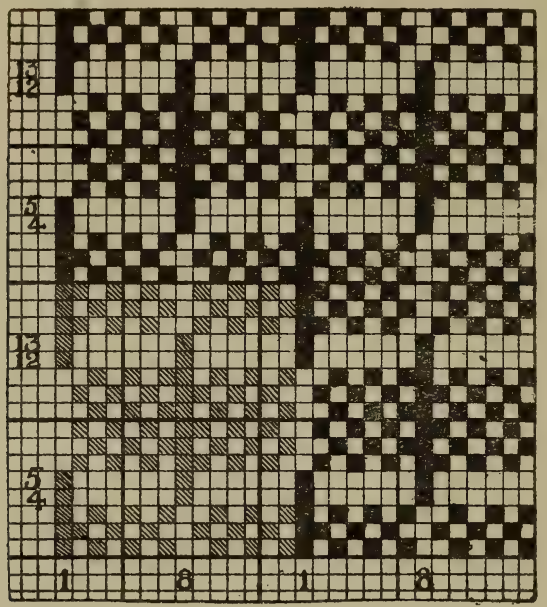

FIG. 267.--Design for "linear zigzag" effect in Fig. 266.

in Figs. 266 and 274, which are reproduced from actual examples of cloth. This phenomenon of fabric structure occurs in obedi-

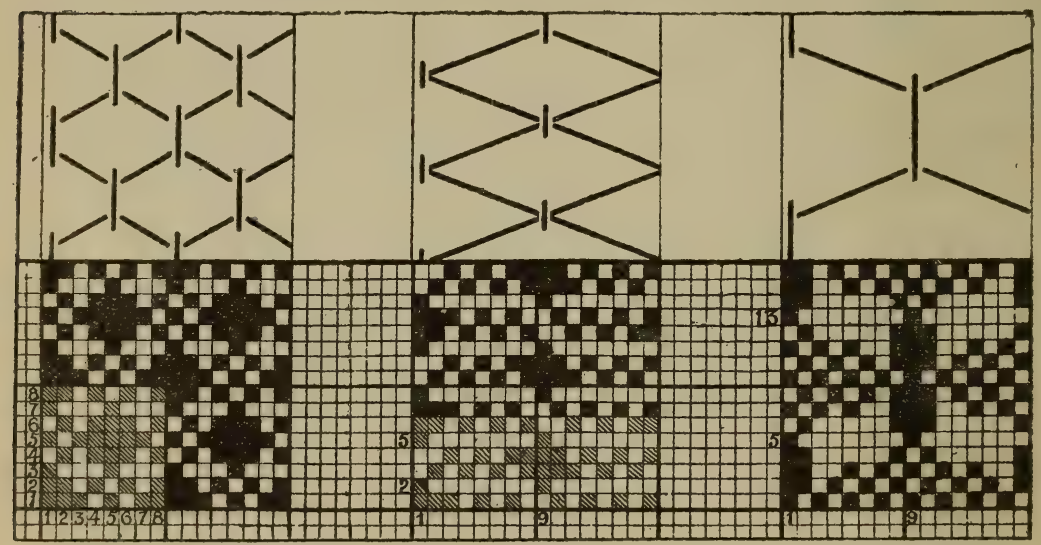

FIG. 268.

FIG. 269.

FIG. 270.

ence to the law of bodies yielding in the direction of least resistance, and forms an interesting and instructive study of the 
behariour of threads in textile fabrics. By taking advantage of the opportunities it affords, a great variety of very pleasing decorative effects may be developed in cloth, the character of which effects is chiefly dependent upon the relative density or compactness of different parts of a weave, and upon the particular manner of interlacing the threads. Thus, by so developing a weave that warp and weft are more thoroughly interwoven, and therefore more compacted, in some parts than in others (and by observing such other conditions as to the manner of interweaving as will contribute to the desired effect), it will cause some threads to pass from the denser towards the less compact

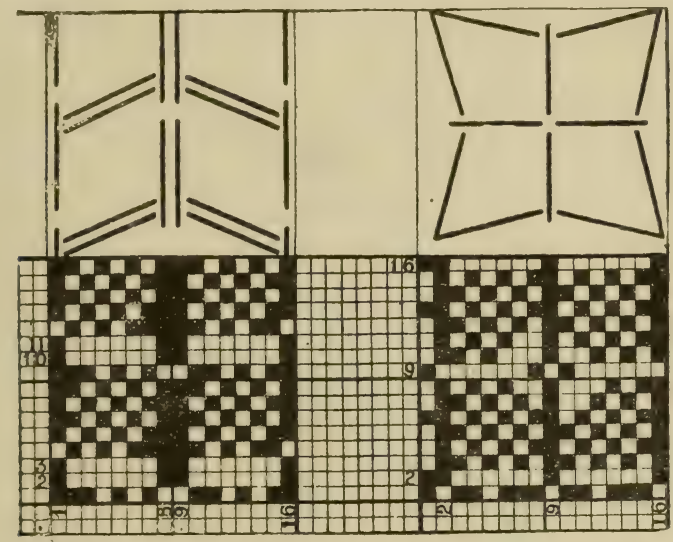

FIG. 271.

FIg. 272.

portions, and so become more or less diverted from a straight line, in proportion to the relative density of threads in those parts. These remarks will be easily comprehended after carefully studying the present examples of these weaves, in conjunction with their accompanying diagrams illustrating their effects in cloth. Weaves of this character (which, as a means of identification, may be aptly described as "linear zigzag" or "spider" weaves) are sometimes produced on a small scale in light cotton and silk textures for ladies' summer attire. They assume a more vigorous character, however, when developed with coarser material to produce heavier textures (as honeycomb and "Grecian" counterpane, and similar fabrics), and by densely 
crowding that series of threads which are required to perform the bending. Thus, if weft threads are required to assume a zigzag course, a more pronounced effect will result from a high ratio of picks per inch, and from a high degree of tension upon warp threads during weaving. If, on the contrary, warp threads are required to bend out of a straight course, they should be more numerous than picks, and held at less tension; whilst the tension of weft, as it leaves the shuttle, should be

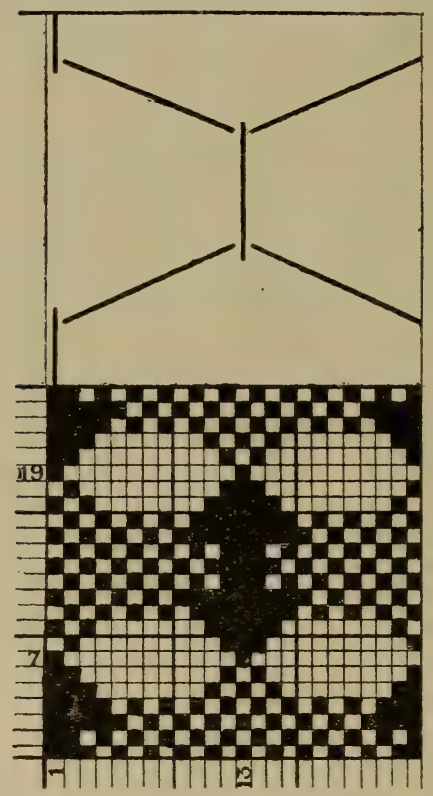

FIG. 273.-Design for fabric, Fig. 274.

increased. It should be observed, however, that better zigzag effects are produced with'weft than with warp, as warp threads may be held at greater tension during weaving, which enables a relatively greater number of picks to be inserted in cloth.

Figs. 266 to 276 are examples of "linear zigzag" weaves, with diagrams showing their woven effects placed immediately above them to facilitate comparison. Fig. 266 is a full-scale photogiaph of a sample of light zephyr cotton dress fabric of the plain or calico weave, on which are developed a series of

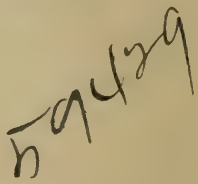


linear figures of hexagonal formation similar to that above Fig. 268. The cloth is woven from the design Fig. 267, contained on fourteen warp threads and sixteen picks, and has ninety warp threads and serenty-six picks per inch. The floating warp threads, numbered one and eight on the design, which pull at opposite sides of the floating picks numbered four, five, twelve, and thirteen, are of stronger yarn than the other warp threads; the counts being

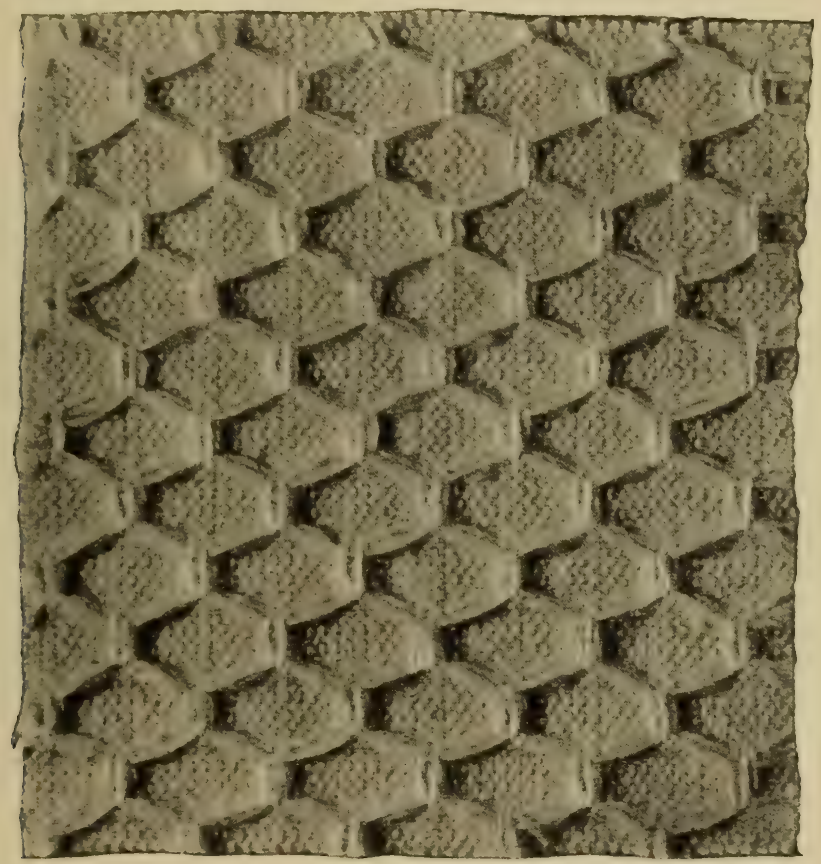

FIG. 274._-"Linear zigzag" effect, from design Fig. 273.

$2 / 60$ 's and 40 's T. respectively; whilst the weft is 40 's counts throughout. There is iittle or nc resarnblance between the design and its wuvєu oifect; but a little consideration will enable those previously linacquainted with this class of weaves to understand the cause of that difference. It will be observed that picks numbered four, five, twelve, and thirteen do not interweave with warp threads, but sicupiy lie above them all, excepting those numbered one anil eigint, which always overlap those picks 
from opposite sides. The floating picks, therefore, form no integral part of the fabric ; for during weaving, the adjacent picks close in, so that warp threads which appear to float over ten picks in the design, float over only six picks in cloth. Thus, in consequence of being overlapped by those warp threads, the floating picks are pulled in opposite directions out of their previous

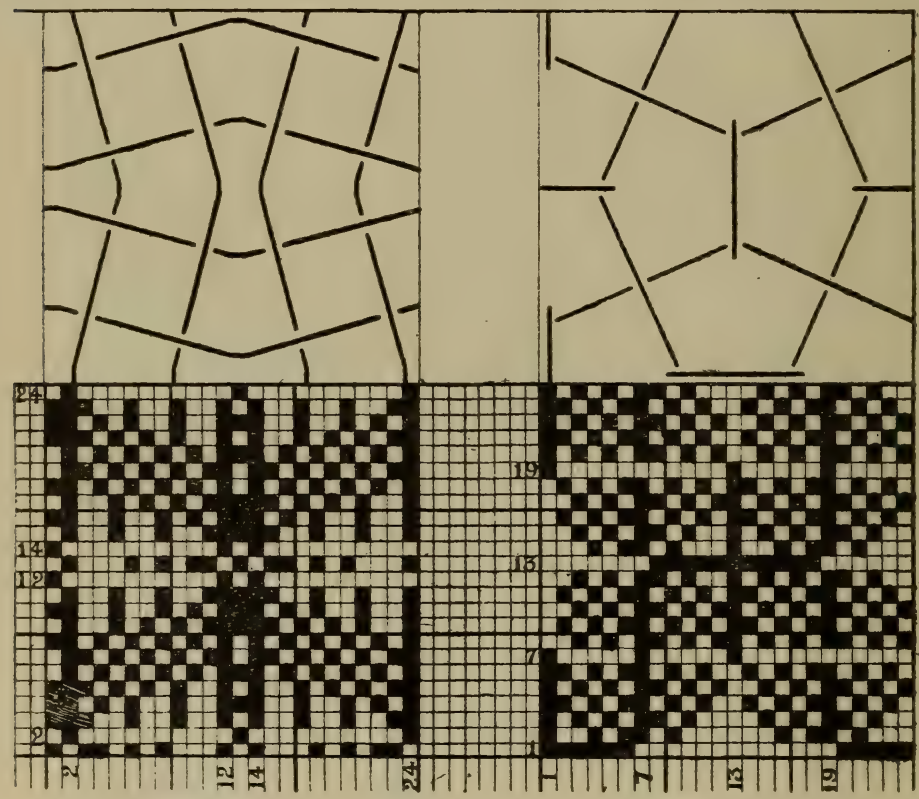

FIG. 275.

FIG. 276.

straight line, and produce the linear zigzag effect seen in the cloth.

Figs. 268 to 276 are examples of linear zigzag weaves based on this principle of wearing, and are sufficient to indicate the variety of effects which it afiorus : Fig: 274 is a full-scale photograph of cloth woven from the design, Fig. 273, which repeats on twenty-four warp threads and picks. The olotin contains thirtysix warp threads and picks per inch of $3 / 16^{\prime}$ s. yarn throughout, which produces $a_{1}$ somew bot bold effect. 


\section{Crêpe or Oatmeal Weaves.}

$\$ 47$. A very useful variety of simple weaves are those embodied in a certain type of cotton textures known in the tracle as "crêpe" or "crape," and sometimes described as "oatmeal " fabrics, so called from their somewhat rough and speckled surface, as illustrated in Fig. 277, which represents a typical example of a cotton crêpe fabric of good quality and medium weight and containing fifty-six warp threads and fifty-six picks of weft per inch, of 18 's counts of yarn for both warp and weft, but with the warp yarn spun with a little more twist than the weft, to

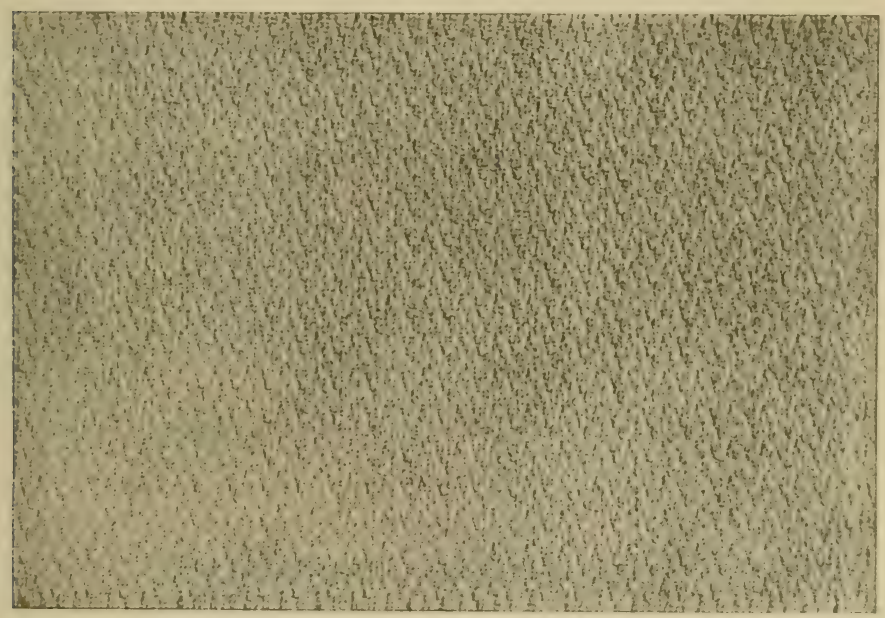

FIG. 277.-Crêpe effect, from design Fig. 278.

increase its strength. Crêpe weares are frequently employed in conjunction with other elementary weaves, in order to produce a variety of different and contrasting effects in elaborate Jacquard designs for brocade and similar fabrics. They are also employed in the production of cotton piece-goods that are usually woven in the grey state, to be afterwards bleached and used for a variety of domestic purposes. Crêpe falorics are also sometimes printed with decorative designs, and sold as a light and cheap material known as "cretonne," which is employed 
extensively as loose coverings for furniture; also antimacassars, covers, curtains, hangings, and for many other similar household articles. Cretonnes are usually printed on both sides of the fabric, with a design and colour scheme of a different character on each side, to make them quite reversible.

The manufacture of crêpe or oatmeal fabrics permits of a wide scope of variation according to the particular character of texture required, and also the special uses for which they are intended. Thus, they are produced in relatively light, medium, and heavy textures from single and folded yarn, either for warp threads only or for both warp and weft. In some, the warp threads only are taped in pairs, whilst in others both warp and weft threads interweave in pairs uniformly. Also, both the warp and weft in some crêpe fabrics are of the same counts of yarn, but with the warp threads a little harder spun than the weft, and with the same number of warp threads and picks of weft per inch. The majority of these fabrics, however, are produced from warp yarn of finer counts than the weft, which is usually soft spun to produce a roving or a condenser weft to serve as a better filling material, but they usually contain about the same number of warp threads and picks of weft per inch.

The development of crêpe weaves affords a fair amount of scope in the creation of textural effects in cloth, but their construction, however, is not based upon any definite scheme of interlacing warp and weft threads, nor are they governed by any systematic distribution of the intersections of those threads such as those governing the construction of satin, diamond, honeycomb, and almost every other variety of elementary weaves. In fact, the more effectually the scheme of interlacement is obscured, the more completely successful will be the crêpe or oatmeal effect produced. This object is attained by an irregular distribution of the floats and intersections of the warp and weft threads in such a manner as will prevent the occurrence of stripes and lines in any direction, and also by interweaving all warp threads in an equal degree in order to ensure the same amount of tensile strain upon all those threads, uniformly, during weaving. Otherwise if warp threads are of unequal tension, they will tend to create more or less conspicuous 


$$
\text { - CRÊPE OR OATMEAL WEAVES. }
$$
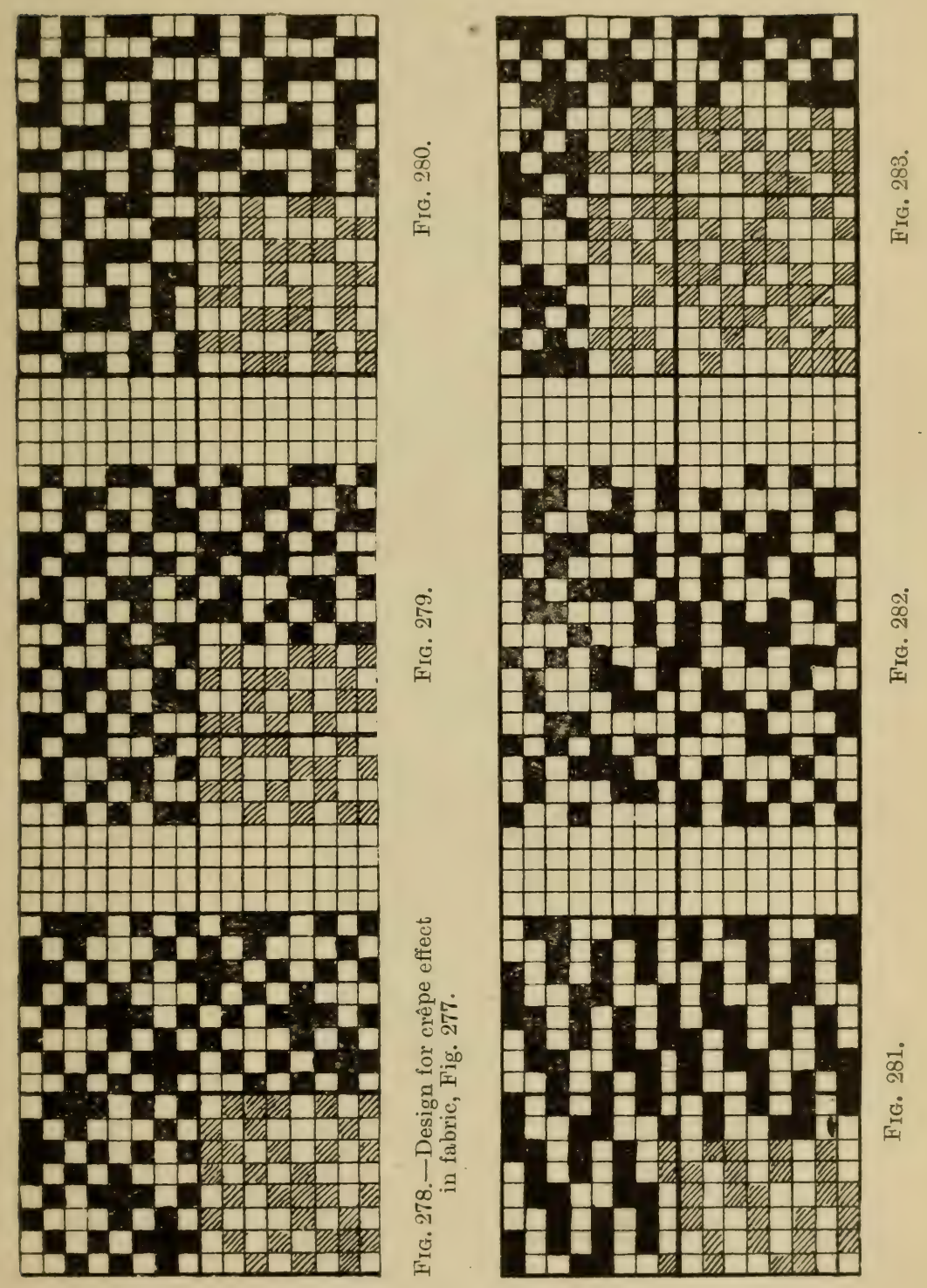
blemishes in the cloth produced, in consequence of the taut and the slack threads pulling unequally. Also it is usual, when evolving crêpe designs, to indicate the warp and weft in equal parts, on the design or point paper, in order to develop a fabric with both sides exactly alike and therefore reversible. But since these fabrics are usually produced from weft which is both softer spun and also of coarser counts than the warp yarn, the weft series of threads will, in these circumstances, predominate over, and almost obscure the finer warp threads, and thereby produce what is virtually a weft surface on both sides of the cloth.

$\S 48$. Examples of seven typical crêpe weaves from actual specimens of cloth are indicated in Figs. 278 to 284, which will serve to demonstrate the general character of these weaves and also the method of their construction. Thus, Fig. 278 is the design for the sample of crêpe fabric illustrated in Fig. 277, repeating on eight warp threads and eight picks of weft. This is a very popular example of a crêpe weave which gives a good effect in cloth; and although it is precisely the same design as the "sponge" weave indicated in Fig. 238, if it is woven with warp and weft of single yarn, as in the example of cloth here illustrated, it produces a more subdued effect in cloth, without the cellular formation which characterises "sponge" effects, which may only be developed successfully by employing folded yarn for both warp and weft. The crêpe weave indicated in Fig. 278 also bears a close resemblance to the eight-end "Brighton" weave indicated in Fig. 228; but a comparison of these two weaves will reveal a difference between them, and one which, to a textile designer, will explain the very different results which those weaves produce in cloth. Thus, in the "Brighton" weave, the disposition of warp and weft floats are such as to develop a distinct cellular formation of a honeycomb character, as explained in $\$ 42$; whereas the disposition of the warp and weft floats in the crêpe weave indicated in Fig. 278 is such as to produce a more even surface without the cellular appearance referred to.

The crêpe design indicated in Fig. 279 is another good example of this class of weaves also repeating on eight warp 
threads and picks. In this example, the intersections of warp and weft threads are of a more irregular and indefinite character than in the previous design, of which the specimen of cloth contains forty-four taped or double warp threads (i.e., eighty-eight single threads) of $2 / 40$ 's yarn, and forty-four double (eighty-eight

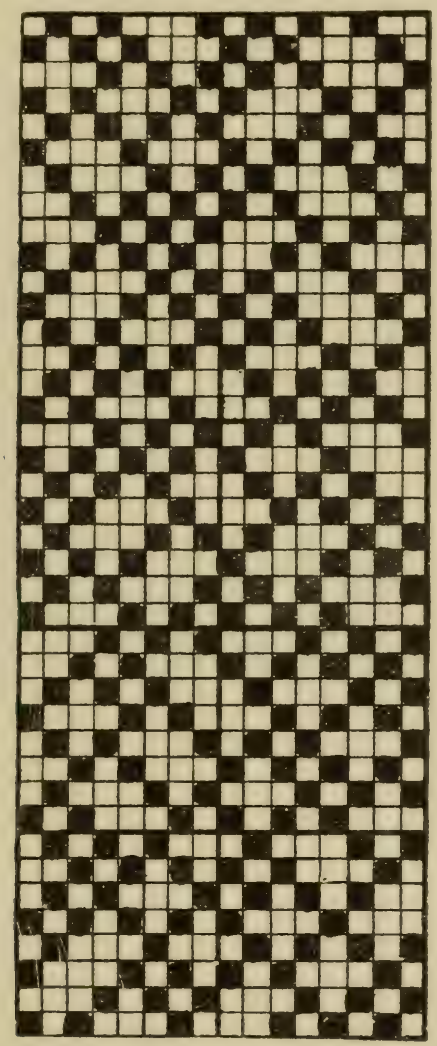

Fig. 284.-Design for "oatmeal " crêpe effect, Fig. 285.

single) picks of 20 's soft weft per inch. Also, on inspecting the design given in Fig. 279, it will be seen that the first four and the last four picks are an exactly reverse counterchange of each other.

A third example of a crêpe or "oatmeal" weave is that indicated in Fig. 280, repeating on eight warp threads and picks 
of weft. This is a very good weave which produces an excellent "oatmeal" effect with a somewhat rough or broken surface, especially if produced from both warp and weft of folded yarn.

Another good example of a crêpe weave is that given in Fig. 281 , repeating on only six warp threads and nine picks. If produced from folded warp and weft with a high rate of warp. threads and picks per inch, this weave yields a very strong and firm texture, which is employed in the corset trade.

The crêpe weave indicated in Fig. 282, which repeats on sixteen warp threads and picks, is one that is adopted for printed "cretonne" cloth, containing forty-four warp threads of 32's T. and thirty-six picks of 10's weft per inch. This fabric is of medium texture and quality, and is printed on both sides of the cloth with different patterns and schemes of colouring.

The design given in Fig. 283 is from a crêpe fabric of similar texture to the specimen woven from the design indicated in Fig. 279, and is produced from similar warp and weft of the same counts of yarn with the same number of warp threads and picks of weft per inch. This design, like that indicated in Fig. 278 , is also of the character of a "sponge" weave, but it produces a more even surface without developing the cellular formation which characterises true "sponge" weaves as described in $\$ 43$.

The seventh and last example of a design for a crêpe weave is that given in Fig. 284, which is of a distinctly different character from any of the previous examples and one that produces a very pronounced "oatmeal" effect in the cloth woven from it, as illustrated in Fig. 285. The "oatmeal" or speckled appearance is still more pronounced by the use of soft spun weft of coarse counts and irregular diameter to resemble "spun flake" weft, of which a short strand is seen below the sample of cloth, Fig. 285, which also exposes a few loose strands of warp and weft threads. On examining the design for this fabric it will be observed that warp and weft threads interweave on the "tab" or "shed" by raising and leaving down warp threads on the "tabby" or plain weave principle of fabric structure, whereby, say, odd-numbered warp threads only are raised above alternate picks of weft, and even-numbered threads only above inter- 
mediate picks. Also, all warp threads make the same number of intersections with the picks of weft uniformly, namely, thirty; and the picks make the same number of intersections with warp threads, uniformly, namely, twelve. Further, picks

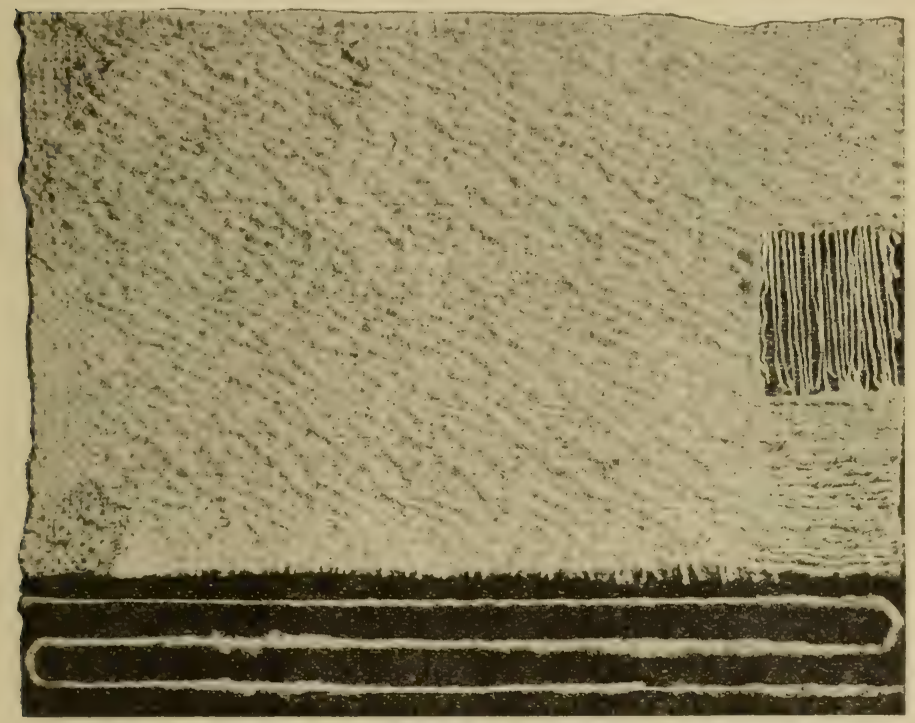

Fig. 285.- "Oatmeal" crêpe effect, from design Fig. 284.

of weft never float over more than three warp threads in succession, with weft preponderating over warp in the proportion of 5 to 3 respectively, on the surface, thereby producing a onesided and not a reversible fabric such as result from the six previous examples of crêpe weaves. 


\section{CHAPTER T.}

\section{BEDFORD CORDS.}

$\$ 49$. Bedford cords are a variety of fabrics characterised by a series of more or less pronounced plain or twilled ribs or cords, lying in the same direction as warp threads, with weft floating somewhat freely at the back of the ribs, and usually with one, two or more wadding threads (according to width of ribs) lying loosely between. They are developed by causing either alternate picks of weft, or alternate pairs of picks, to interweave with the warp threads of one rib and then pass underneath those of the next rib alternately; whilst the intermediate picks or pairs of picks pass under the first rib, and interweave with the second rib alternately. Consequently, odd-numbered picks or pairs of picks always interweave with warp threads of the same (say odd) series of cords throughout, whilst the other picks always interweave with the even series of cords. This circumstance is helpful for the purpose of producing stripes of solid colours by picking with corresponding colours of weft in such manner that they only interweave with warp threads of the same colour, and float underneath those of the other colour. These features are clearly discernible in the photograph (Fig. 286), which shows the obverse and reverse sides of the same cloth.

Bedford cords are produced in a variety of both cotton and worsted textures, varying from light to relatively heavy cloths, according to the particular use for which they are intended. The lighter and medium fabrics are chiefly used as ladies' dress materials; whilst the heavier and coarser fabrics are generally made up into men's clothing of a special character, as fancy vests, breeches, military, sporting and riding suits, and such like. The lighter cotton textures are usually bleached, or else dyed in tints of some light and bright hue, for ladies' light summer and 
holidat clothing. (ienerally speaking, Bedford cords afford little scope for variation of structure. This, howerer, is compensated for by the fair scope they offer to simple decorative effects, either by means of rariegated cords, coloured threads of warp, or Jacquard weft figuring of an elementary and bold character, and consisting preferably of small detached sprigs or simple geometrical forms evenly distributed in such manner as to ensure that

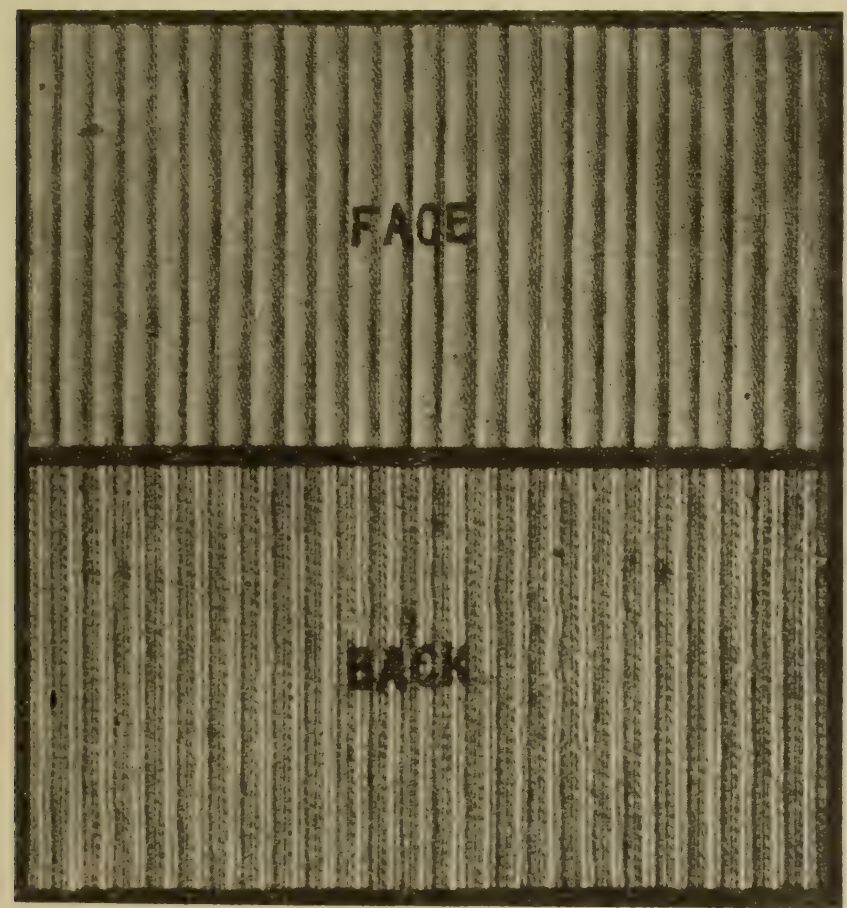

FIG. 286.—Bedford cord fabric, woven from design Fig. 291.

all warp threads shall bear the same degree of tension. Coloured threads may he introduced either as extra or crammed warp threads for figuring purposes, or in substitution for ordinary warp threads for coloured effects only. When Jacquard figuring is adopted in Bedford cords, it is virtually a system of brocade weft figuring with a Bedford cord for a ground filling.

For the purpose of giving the ribs or cords greater prominence 
and also to increase the weight, bulk and strength of the fabric, one, two or more extra warp threads are sometimes introduced in each cord to serve as wadding. These extra threads never interlace with weft, but lie perfectly straight between the ridges of their respective cords and the floating weft at the back. In addition to wadding threads, some of the heavier fabrics for men's clothing contain backing warp threads that interweave with weft at the back of the cloth only, thus forming a series of tubes along which wadding threads lie straight, and which considerably increase the stability and warmth of the fabric. With few exceptions wadding threads are of considerably coarser counts of yarn than the principal or face warp threads, and since they never interlace with weft, but remain straight, their contraction during weaving is nil. This circumstance necessitates their being wound upon a separate warp beam, and held at greater tension than face warp threads during weaving.

It may be observed, at this juncture, that Bedford cords of low quality and somewhat open texture are usually woven face downwards, so that fewer healds require to be raised; thereby requiring less motive power to drive the loom, and reducing the wear and tear of healds and shedding mechanism. These considerations, however, are sacrificed in the production of superior qualities which are woven face side upward to permit of the readier detection of broken warp threads, and other faults liable to occur during weaving.

The present examples of Bedford cord weaves are of fabrics selected as typical specimens of their class from those of ordinary commerce. In all cases, both heald and reed drafting are indicated above their respective designs, with such other information as will be helpful to students; and the present chapter will conclude with an instructive table giving complete data of the manufacture of each example.

$\$ 50$. Fig. 287 is a design of a light Bedford cord of the most elementary character devoid of wadding threads. Each rib contains eight warp threads, which interweave on the plain or calico principle with one-half of the picks of weft, thereby causing the complete design (consisting of two cords) to repeat on sixteen warp threads and four picks. The first and last warp threads 
of each cord, termed "cutting" threads, interweave on the calico principle with all picks of weft, thereby forming a furrow or " cutting," which sharply divides the cords; whilst the intervening warp threads, termed "face" threads, interweave on the calico principle with alternate pairs of picks only, and lie completely above the intermediate pairs of picks, as clearly indicated in the diagram, Fig. 288, showing a transverse section of cloth woven from the design, Fig. 287. The production of this cloth involves the employment of six heald shafts, namely, four in the rear for face threads, and two at the front for cutting threads. Warp threads are drafted in the manner indicated above the design, with four threads in each dent of the reed,

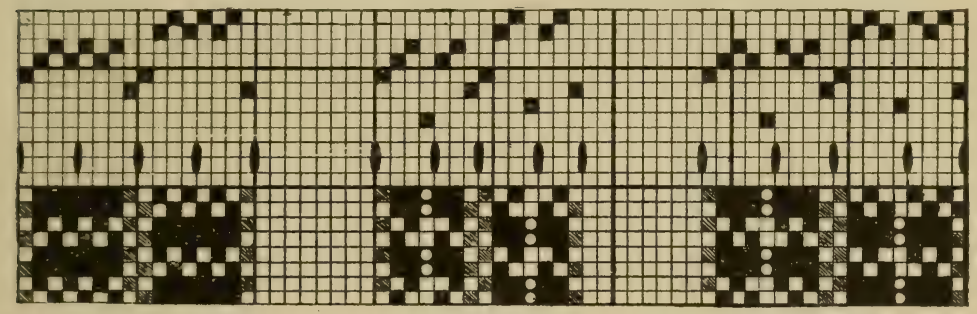

FIG. 287.--Design for

Fig. 289.

FIG. 290.

Bedford cord fabric

indicated in Fig. 288.

and a reed wire separating the cutting threads. Each cord, therefore, occupies two dents of the reed.

Fig. 289 differs in construction from Fig. 287 chiefly by the introduction of a wadding thread (indicated by white dots) in each cord. Wadding threads are drawn through two healds placed immediately in front of those governing cutting and face threads respectively, in accordance with usual practice. Sometimes the healds governing cutting threads are placed in front, followed by those governing wadding and face threads respectively; but this is quite optional. It will be seen that wadding threads are always raised along with all face threads of the same cords when it is required to place weft at the back; but they remain down when weft interweaves with face threads, to form the ridge of a cord, whereby they lie between the face of a cord and the floating weft. 


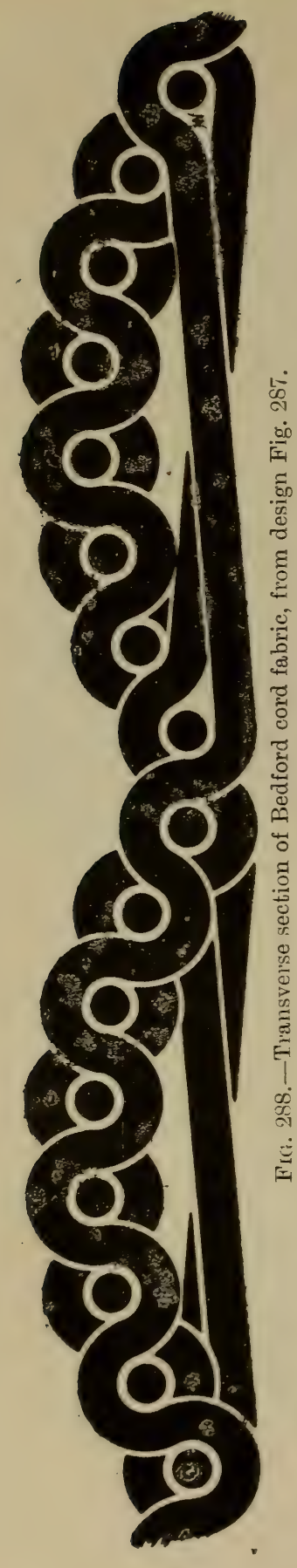

\section{GRAMMAR OF TEXTILE DESIGN.}

Fig. 290 is similar to Fig. 289, with two additional face threads per cord, and each cord occupying two dents of the reed. Figs. 291 and 292 have two and four wadding threads in each cord, and occupy four and three dents per cord respectively. Fig. 293 is a Bedford cord occupying twenty warp threads, including eight wadding and two cutting threads drawn through five dents of the reed. Fig. $2 \varepsilon_{4}$ is a variegated cord with one broad and two narrow cords alternately. The broad cord occupies twenty-six warp threads, including four of wadding, drawn through seven dents; whilst the narrow cords each occupy eleven warp threads, including one of wadding, drawn through three dents, making a total of forty-eight warp threads for the series. Since three cords constitute an odd series, the drafting of warp threads for this design requires to be extended to include two series of cords to make an even number, and so conform to the practice, common to Bedford cords, of causing weft to interweave with the warp threads of alternate cords, and float under those of intermediate cords.

Figs. 295 and 297 are slight deviations from the previous examples, in that alternate picks of weft interweave with face warp threads of alternate cords, and float behind the intermediate cords; whereas, in the former examples, two contiguous picks either interweave or float at the same time. There is little difference between the two systems, but slightly superior results obtain with, an alternate 
arrangement of picks, as these are more perfectly distributed in cloth. It is also capable of producing a closer texture, and forms a clearer cutting between the cords, which appear more distinct. Fig. 295 is an uncommon variety of Bedford cord, inasmuch as there are no cutting warp threads. The development

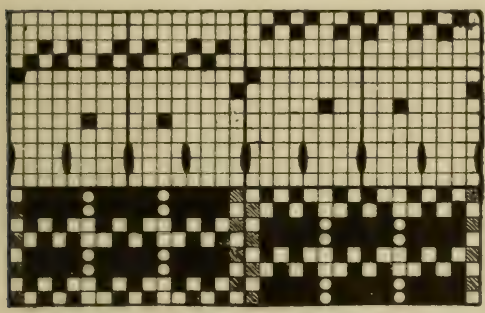

FIG. 291.-Design for Bedford cord fabric, Fig. 286.

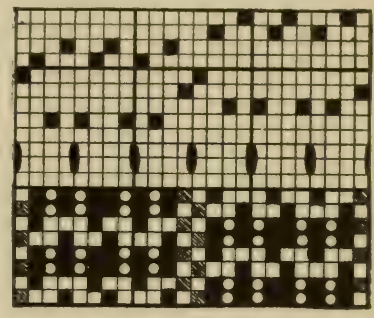

FIG. 292.

of the ribs is, therefore, entirely dependent upon each pick of weft interweaving with and floating under alternate groups of warp threads. In the present example there are eight face and two wadding threads per cord drawn through three dents of the reed. Fig. 296 is a transverse section of cloth woren from the

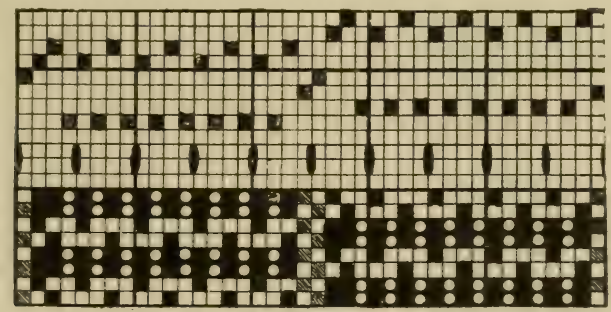

FIG. 293.

design Fig. 295. Fig. 297 is a cord similar to the previous one, but having cutting threads and a greater number of face threads in each cord, which occupies sixteen warp threads drawn through four dents of the reed.

Figs. 298 to 301 are examples of Bedford cord weares in which the ribs or cords are developed with a three-end $\left(\ddot{z}_{1}\right)$ twill, with alternate picks of weft interweaving with alternate cords 
and then floating beneath intermediate cords; whilst the intermediate picks interweave with and then float beneath the inter-

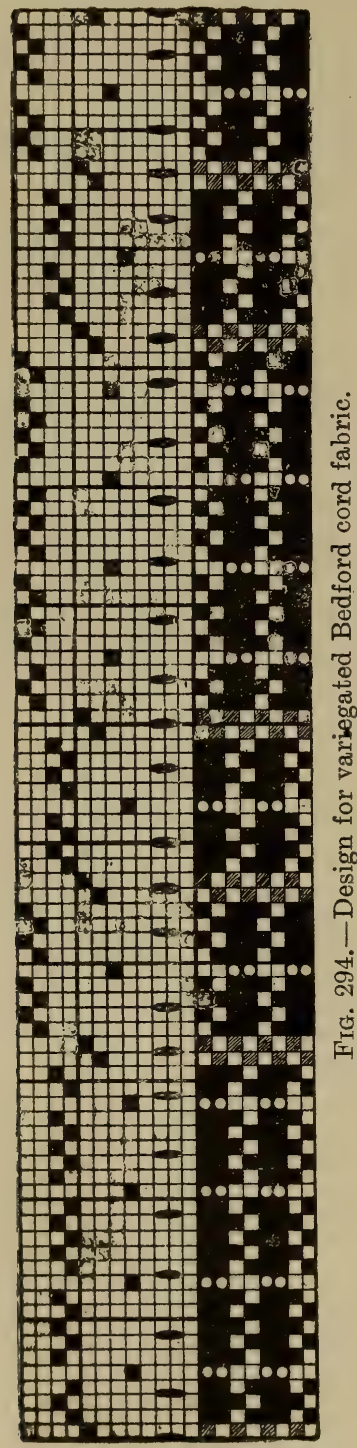
mediate series of cords. This alternate disposition of picks appears to be uniformly observed in the production of twilled Bedford cords, whereas it obtains in a lesser degree than the two-and-two disposition of picks in the production of Bedford cords having the ribs developed with the plain or calico weave, as exemplified in Figs. 287 to 293. It is in respect of the twill weave and the uniform alternate disposition of picks that the present examples of Bedford cords differ from those previously described. These, like those, may or may not be devoid of wadding warp threads, according to the weight and character of texture required. As a rule, twilled Bedford cords are more compact and comparatively softer and more supple than the calico-ribbed variety, consequent upon a lesser degree of interlacement of warp and weft.

Fig. 298 is an example of a twilled Bedford cord devoid of wadding warp threads. Each cord occupies nine warp threads, including two cutting threads, drafted on eight healds and drawn through three dents of the reed, as indicated above the design. Fig. 299 is a twilled cord occupying six face, two'wadding and two cutting warp threads per cord, drafted on twelve healds and drawn through three 


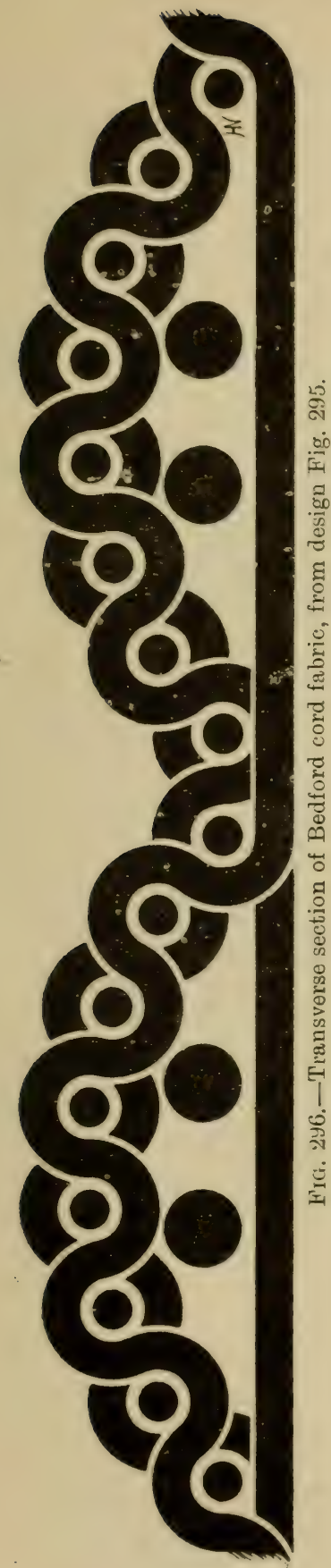

BEIFIRT ('ORIS. 117

dents of the reed. Fig. 300 is a twilled cord occupying eighteen face, five wadding and two cutting warp threads drawn through six dents of the reed. The fabric from which this design was obtained contained six cords per inch (when in the loom), equal to 120 warp threads (excluding wadding threads) per inch. This comparatively high number of threads produces somewhat flattened ribs resembling tucks, which slightly overlap each other.

$\S 51$. All the foregoing examples of Bedford cords are of light and

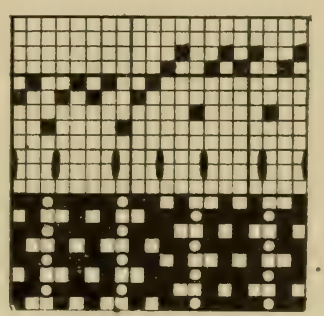

FIG. 295.-Design for Bedford cord, Fig. 296.

medium textures suitable for ladies' dress material. The two following examples, Figs. 301 and 302, are of heavy textures such as are employed for men's sporting suits. Fig. 301 occupies thirteen face, seven wadding and two cutting warp threads drawn through five dents of the reed. Fig. 302 is a full scale photograph of an interesting variety of Bedford cord of a specially heavy 
and strong texture, and with wide prominent ribs. Its $/$ construction is very different in many respects to any of the previous examples. In addition to wadding threads, it contains "backing" warp threads; also picks of weft comprise two distinct series, namely $(a)$ face and $(b)$ back picks, inserted in the proportion

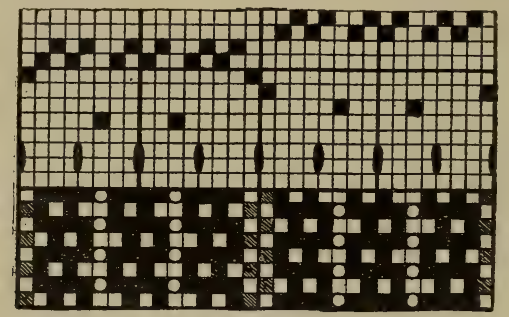

- $\quad$ Frg. 297.

of one face and two back picks alternately. Face picks interweave with face warp threads of successive cords, whilst back picks interweave with back warp threads of successive cords, thus forming a series of tubes along which wadding threads lie straight. As indicated in the design, Fig. 303, each cord occu-

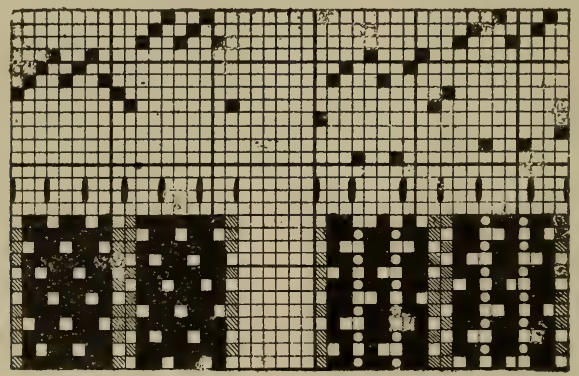

FIG. 298.

FIG. 299.

pies a total of twenty-nine warp threads, of which eighteen are "face," three "cutting," four "wadding" and four "backing" warp threads. Only two warp beams are necessary to contain the four series of warp threads, namely, one for face and cutting threads (whose rate of contraction during weaving is equal), and one for wadding and back warp threads, which are held at greater tension than face and cutting threads. Two 
counts of yarn are employed in the production of this example, namely, $2 / 60$ 's for cutting, and $2 / 16$ 's for face, wadding and back war'p threads (the latter being sized); also $2 / 16$ 's wett of similar yarn to the warp threads for both fice and hack picks. Cutting

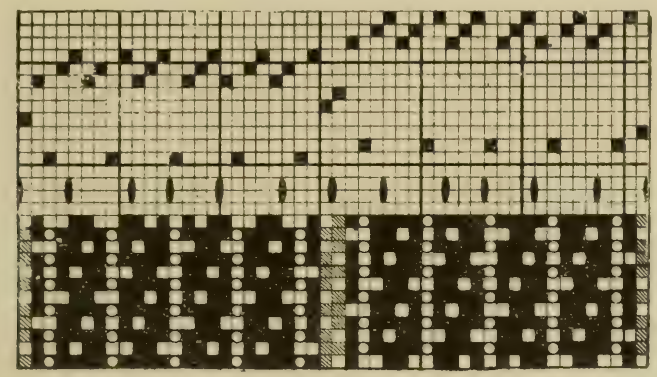

FIG. 300.

threads (represented by shaded squares) interweare in the plain or calico order with successive picks of weft. Face warp threads (filled squares) interweave with face picks only (every third pick) to produce a three-end $\left(\ddot{\sim}_{1}\right)$ twill, and are raised when

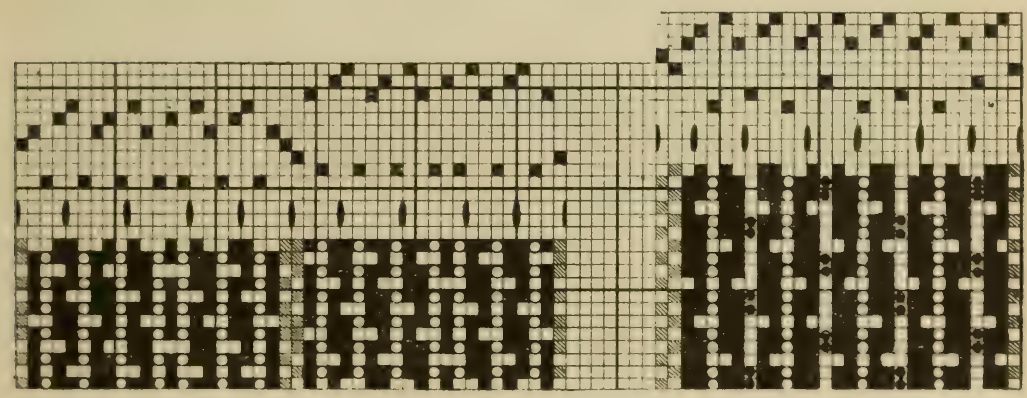

Fig. 301.

Fig. 303.-Design for Bedford cord fabric, Fig. 302 .

back picks are inserted. Back warp threads (round black lots) interweave with back picks (two out of three) on the calico principle, but with the picks running together in pairs instead of separately. IVadding threads (round white dots) never interweare with weft, but are simply raised when back picks are 
inserted, and depressed when face picks are inserted, to cause them to lie loosely between the face and back of the fabric.

$\$ 52$. All the examples of Bedford cords described above are of a strictly plain or unfigured character. It now only remains, to make their description more complete, to describe the usual methods adopted for their embellishment. At the outset of these observations, it was stated that Bedford cords were capable of decorative treatment by means of coloured threads and by

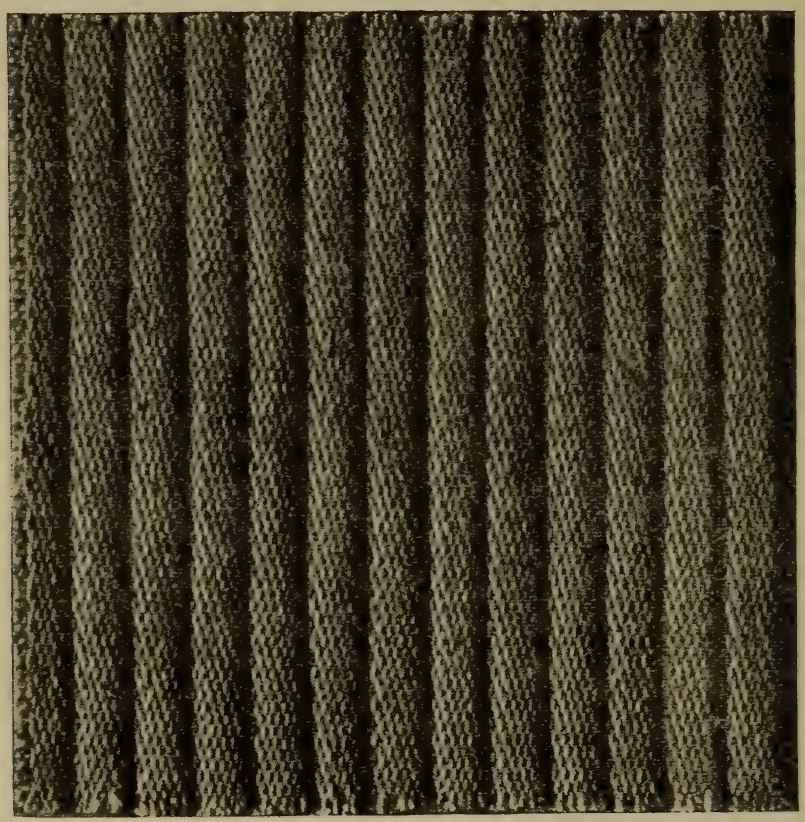

FIG. 302.-Bedford cord of heavy texture, from design Fig. 303.

simple Jacquard figuring. If coloured threads are merely substituted for undyed threads, other conditions remain unchanged; but if they are additional threads for figuring purposes, they require to be governed by extra healds. Coloured threads are sometimes substituted for wadding threads in certain cords at required intervals, and employed for the development of simple figured effects. In such instances, figuring threads do duty for wadding threads when not required on the face for figuring 
purposes, with the result that they tend to impart a tinge of colour to those cords containing them. A more satisfactory method of introducing coloured threads is exemplified in Figs. 304 and 306. In Fig. 304 a neat wave stripe is developed at intervals by means of four coloured additional warp threads, represented in the design (Fig. 305) by crosses. One unit of the pattern comprises five cords, namely, a broad one containing the extra figuring warp threads, and four narrow plain ones,

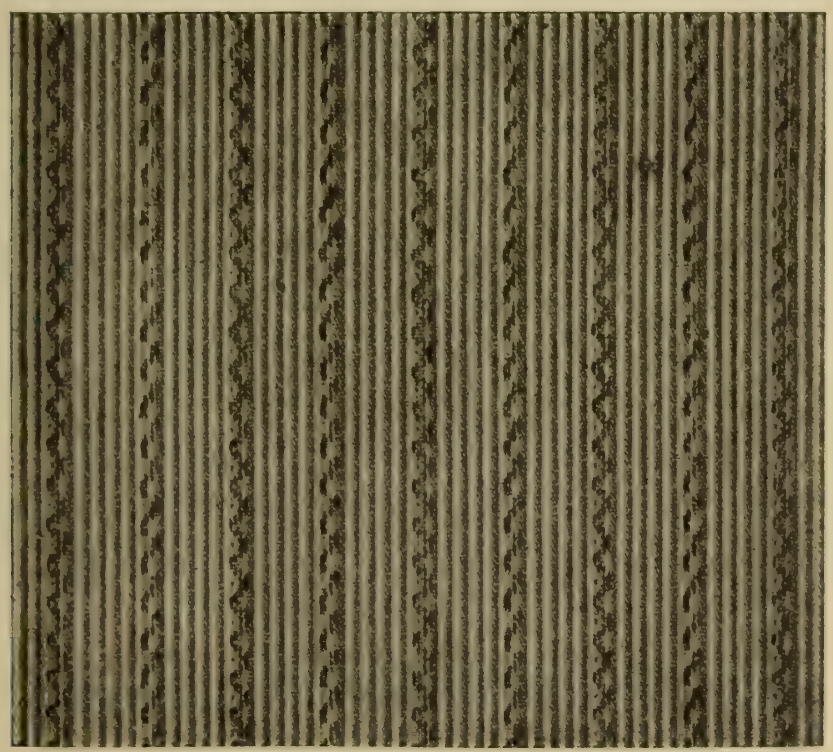

FrG. 304.--Embellishment of Bedford cord fabric by coloured warp threads, as indicated in design Fig. 305.

thereby requiring two units of the pattern to complete one repeat of the design, which must occupy an eren number of cords. This circumstance does not prevent figuring threads in each cord from being governed by the same healds. In the present example, the extra figuring threads are governed by four healds, making a total of twelve healds disposed as follows: Four at the front governing extra figuring threads, followed by two governing wadding, two governing cutting, and four in the rear governing face threads. By drawing figuring threacls 
through healds placed in front, they are subjected to less strain due to shedding, since each successive heald from the front requires to be moved through a greater distance in order to maintain the proper angle of the warp shed. Warp threads are contained on three separate warp beams containing figuring, face and wadding threads respectively, with figuring threads lightly tensioned to permit of their being easily withdrawn during weaving. Another example of simple figuring by the employment of extra warp threads is illustrated in Fig. 306, showing the face and back of the same cloth. The extra threads are employed at intervals of eight cords for the development of small spots arranged alternately. To prevent figuring threads

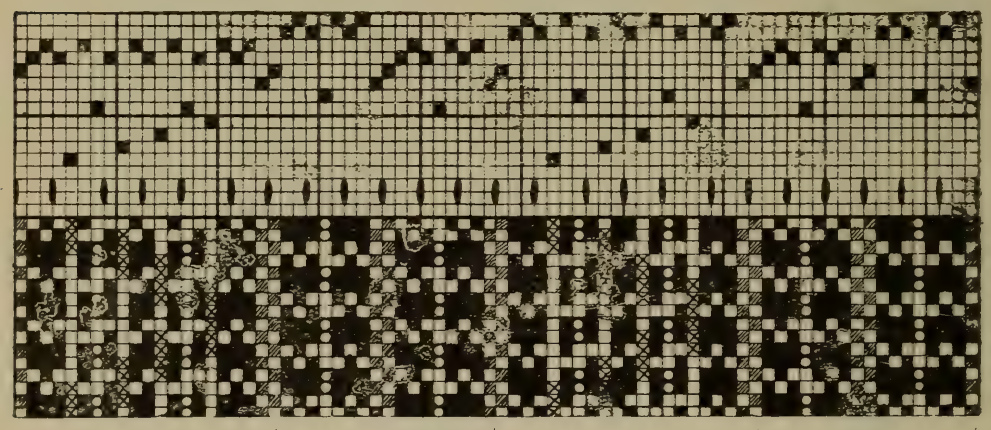

Twice

Twice

Fig. 305.-Design for Bedford cord fabric, Fig. 304.

from floating too far at the back of cloth, between any two spots, they are raised over every twelfth floating pick as indicated in the portion of design (Fig. 307).

Instead of lying at the back of cloth, as in the last two examples, figuring threads may, as an alternative method, lie with wadding threads, between the face of cloth and floating picks at the back; but if face and figuring threads are of contrasting colour, the latter will tend to impart a tinge of their colour to the whole of the cord, as stated previously.

5 53. Fig. 308 is an example of figured Bedford cord having a simple floating weft figure developed by means of a Jacquard machine. Surrounding the figures is a ground filling of an ordinary plain-ribbed Bedford cord, each rib of which comprises 
four face, two cutting and two wadding warp threads drawn through two dents of the reed. All warp threads are controlled

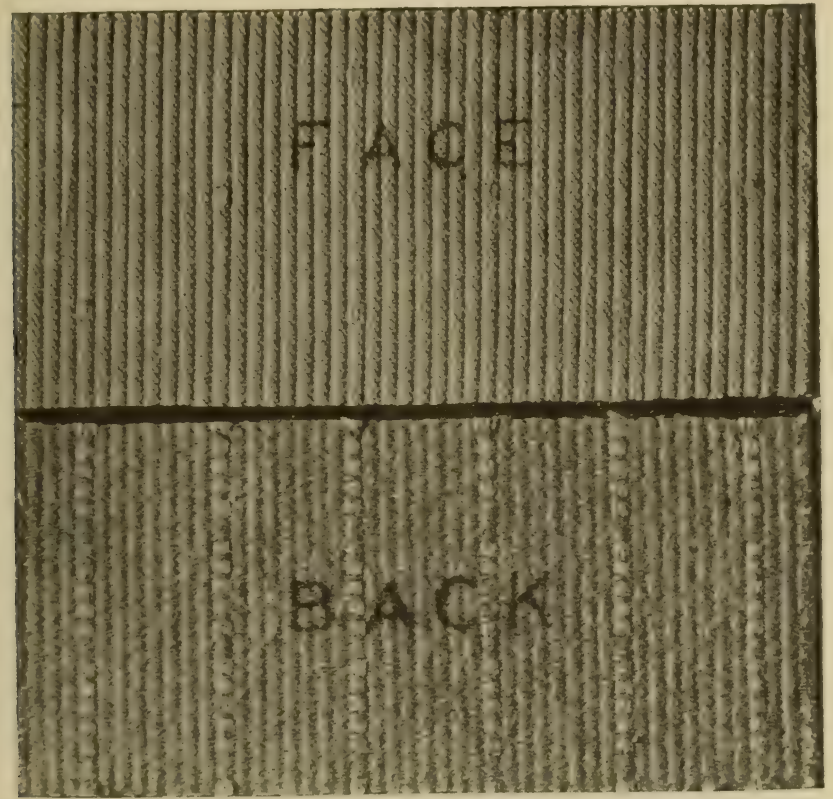

FIG. 306.-Embellishment of Bedford cord fabric by coloured warp threads, as indicated in design Fig. 307.

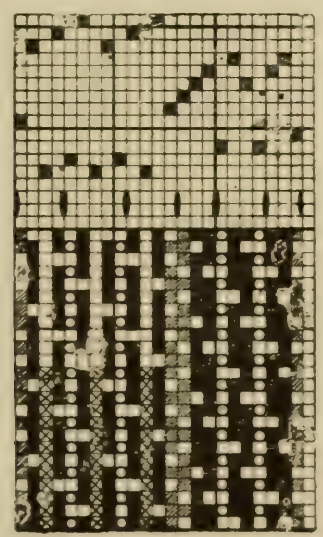

FIG. 307.-Design for Bedford cord fabric, Fig. 306. 
by a Jacquard machine, but only face and cutting warp threads are utilised for the purpose of binding weft floats in the figure portions; whilst wadding threads are kept entirely at the back in those parts. This necessitates an applied design being prepared in two stages as follows: The design is first set out on design paper of the proper counts (according to the ratio of face and cutting threads and picks per inch) without regard to wadding threads, and afterwards transferred to another sheet of design paper on which wadding threads are indicated at their

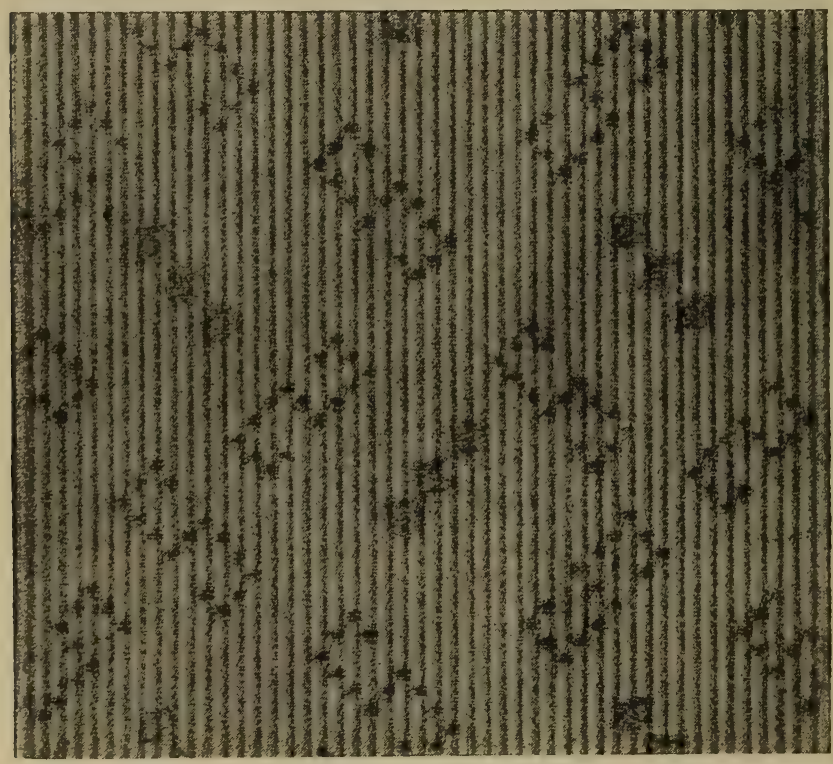

FrG. 308.-Embellishment of Bedford cord fabric by Jacquard figuring.

proper intervals. In the present case the third and sixth vertical divisions would be marked in each bar of design paper (assuming eight divisions in a bar) corresponding to the third and sixth long rows of hooks in the Jacquard machine.

$\$ 54$. The following table gives data of the manufacture of all examples of Bedford cord fabrics described in the present chapter. The character of warp and weft yarn employed in their production is, in general, normal as regards both the amount of twist and quality of yarn:- 


\begin{tabular}{|c|c|}
\hline 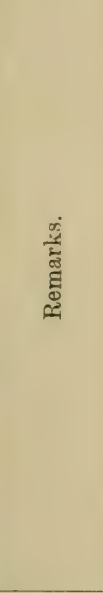 & 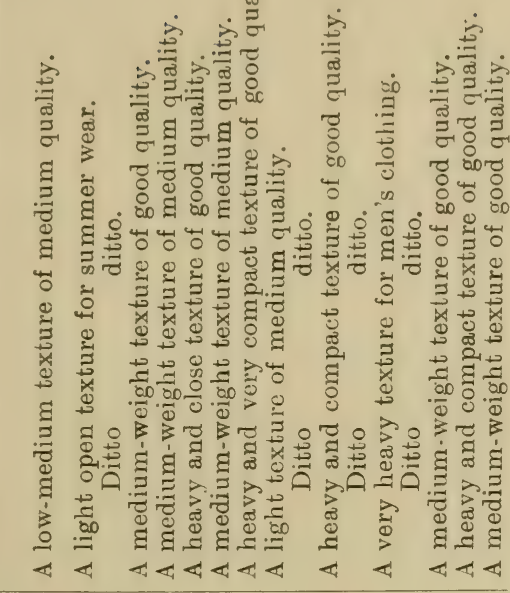 \\
\hline 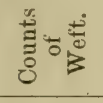 & 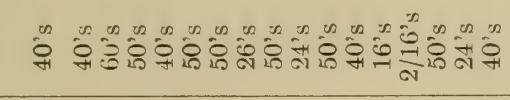 \\
\hline 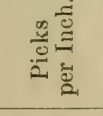 & 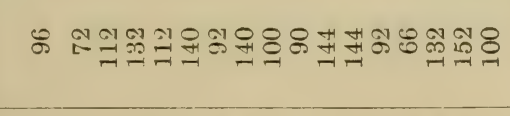 \\
\hline 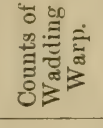 & 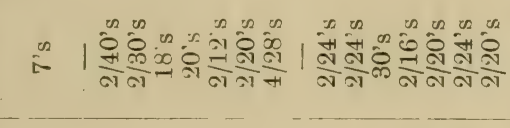 \\
\hline 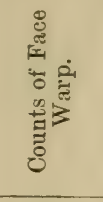 & 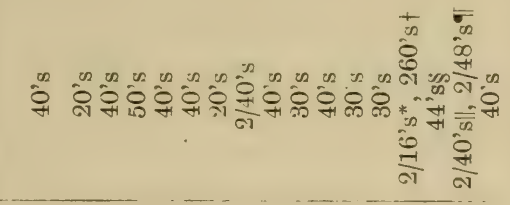 \\
\hline 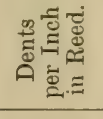 & 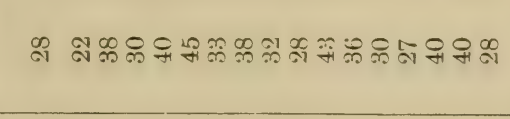 \\
\hline 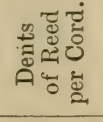 & H बलM \\
\hline 离 & 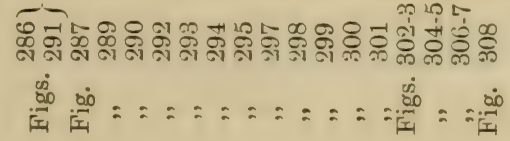 \\
\hline
\end{tabular}




\section{CHAPTER TI.}

\section{BACKED FABRICS.}

55. "Backed" fabrics are characterised by an additional series either of warp or weft threads employed for the purpose of increasing their strength, weight, bulk and warmth, or any one of those properties, without affecting their surface appearance. They are largely produced in worsted textures and fustians intended for boy's' and men's clothing, for which purpose they are eminently adapted, as they are capable of yielding firm and compact though soft and warm textures. Backed fabrics occupy a position midway between "simple" textures, which are composed of one series each of warp and weft threads, and "compound" textures, which contain two or more series each of warp and weft, as exemplified in all double cloths. When properly constructed, they bear no indication whatever on their surface of these additional threads, which lie entirely at the back and are, therefore, completely obscured by the surface texture. The extra series of threads introduced for "backing" may be either of warp or weft, with "face" and "back" threads arranged either alternately or in the proportion of two "face" threads to one "back" thread. If "backing" threads are of weft, it is generally coarser and of inferior quality to that employed for "face" picks, thereby requiring a loom provided with a checking motion for at least two shuttles, and a picking motion that will' permit of picking (i.e., propelling a shuttle) twice in succession from either shuttle-box of the loom sley : or in such other manner as is determined by the particular disposition of "face" and "back" picks. If the extra series of "backing" threads are of warp, a loom of ordinary construction without any special device will serve all requirements; also production will be increased by reason of inserting fewer picks 
per inch, and a weaver will be paid a lower rate of wages than if employed on a check loom. Against these advantages, however, the extra warp threads will necessitate the use of a greater number of healds, thereby impeding the progress of a weaver when "drawing in" warp threads through healds and reed also, in some circumstances it may be desirable to wind "backing" warp threads upon a separate beam to permit of the tension of each series of warp threads to be independently regulated to suit their different rates of contraction during weaving.

Whether "backing" threads are of warp or weft, the chief aim of a designer should be directed towards interweaving them with the face texture so as to effectually conceal their attachment when the fabric is viewed obversely. This may only be

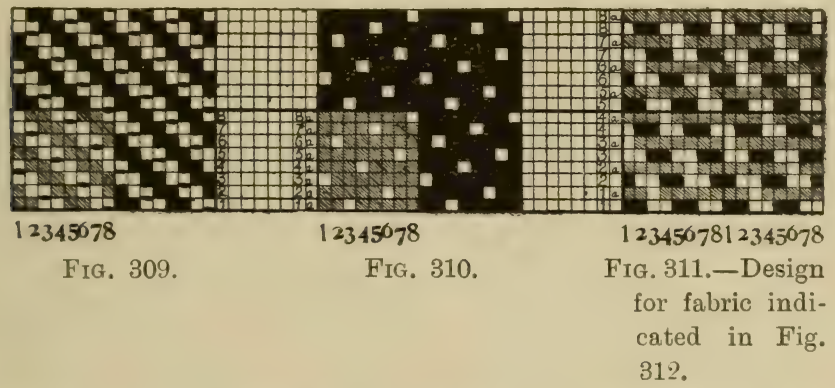

successfully accomplished when due regard is paid to the character of weare constituting the face texture, which should, so far as is compatible with other requirements, conform to the conditions imposed by this principle of fabric structure. If it is desired to "back" a fabric with extra weft, a design should be selected in which each warp thread passes beneath not less than tuo contiguous picks of weft (but with each warp thread under different picks) at least once in each repeat of the design. If extra warp threads are to constitute the "backing," the design should be one in which each pick of weft passes beneath not less than two contiguous warp) threads (but with each pick under different warp threads) at least once in each repeat of the design. By adopting this expedient, suitable places are prorided at which to bind or "tie" the extra series of threads to the face texture 
so that the "ties" or binding points will be effectually obscured. These remarks will be easily understood on consulting Figs. 309 to 312 .

Fig. 309 is a design for a four-end $\left(\frac{2}{2}\right)$ twill, to constitute the face weave of a fabric to be "backed" with weft in the proportion of one "face" pick to one "back" pick-the "back" picks to interweave on the eight-end satin basis, Fig. 310. When the two weaves are combined pick and pick, they produce a design repeating on eight warp threads and sixteen picks, as shown in Fig. 311, in which filled squares represent "face" picks, and shaded squares "back" picks. The points selected for binding "back" picks into the face cloth are where a warp thread passes beneath two contiguous picks (as indicated in Fig. 309), which by lying close together above those points, entirely obscure the

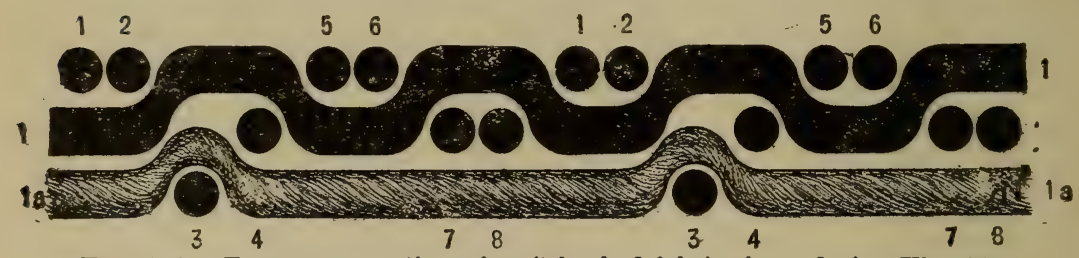

FIG. 312.-Transverse section of weft-backed fabric, from design Fig. 311.

intersections from view. It will now be perfectly clear that suitable binding places (in weft-backed fabrics) occur only at such points where the binding thread passes beneath at least two contiguous face picks which serve to cover those points, and that if a design is of such character that warp threads pass beneath only one face pick at a time, the binding points are liable to show on the face, in consequence of imperfect covering by face picks. It should be observed that when a back pick is inserted, all warp threads are raised excepting those which are required to pass under it for the purpose of binding it to the fabric, as seen in Figs. 311 and 312.

Fig. 312 represents the "face" and "back" picks (Nos. 1 and $1 a$ respectively) of design Fig. 311 as they would appear in cloth when viewed transversely, and shows the "back" pick passing over the third warp thread as the latter passes beneath 
the first and second "face" picks which close over and completely cover the binding or "tie".

In order to obtain the best results, all war'p threads should, if

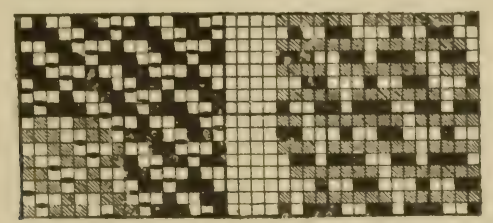

FIG. 313. FIG. 314.

possible, be utilised for binding "back" picks to the face texture, and binding points should be uniformly distributed; therefore, the nearer such distribution approaches to that of a satin'basis

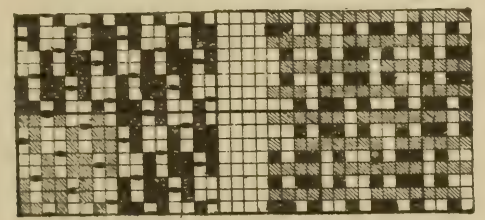

FIG. 315 .

FIG. 316.

the better. Many designs, however, will not permit of the employment of every warp thread for binding purposes; nor of the distribution of binding points on a satin basis. In such cases,

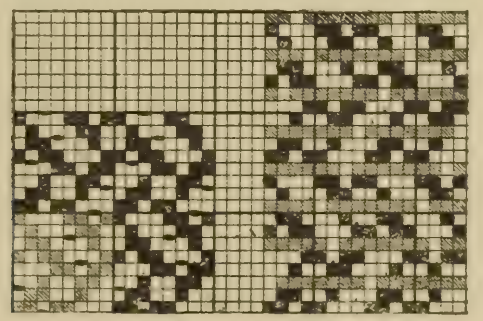

- FIG. $317 . \quad$ FIG. 318.

a little skill is oftentimes required to make a selection of binding places that will give the best results. Hence the necessity of avoiding such impediments when preparing designs intended for this class of fabrics. 
Figs. 313 and 314 are examples of small figured weaves for "backing" with extra weft, and show the face weaves or plans; and completed designs, separately, with the most suitable places for binding "back" picks to the face texture indicated upon each plan. Figs. 313 and 315 have the binding points arranged for

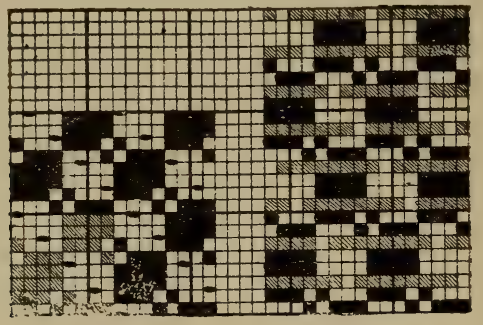

FIG. 319.

FIG. 320 .

an alternate disposition of "face" and "back" picks, as observed in the completed designs, Figs. 314 and 316 respectively. The weave plans shown in Figs. 317, 319 and 321 have the binding places indicated for the picks to be disposed in the order. of two "face" picks and "one" back pick alternately, as exemplified in their respective completed designs, Figs. 318, 320 and 322.

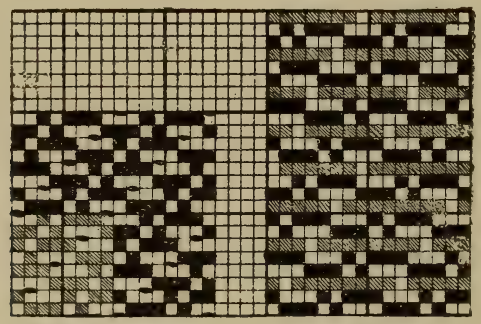

FIG. 321.

FrG. 322.

$\$ 56$. The construction of warp-backed fabrics is governed by the same principles as those which govern the construction of weft-backed fabrics, as regards the method of securing the extra series of threads to the face texture. Therefore, the binding of "back" wrap threads must occur at such places as will ensure the binding points being properly covered by "face" warp threads. 
In other words, a "back" war'p thread should only be raised over a pick when the latter is passing beneath two or more "face" warp threads; otherwise the binding points will be liable to show on the face of the fabric. Fig. 323 represents the face weave of a fabric to be backed with warp on the one-and-one or alternate disposition of threads, with the most suitable binding places indicated on the design, which permits of a distribution of binding points on an eight-end satin basis. Fig. 324 is the same design with "back" warp threads added, and with the heald drafting of warp threads shown above. Fig. 325 is another design for a warp backed fabric with warp threads disposed in the proportion of two

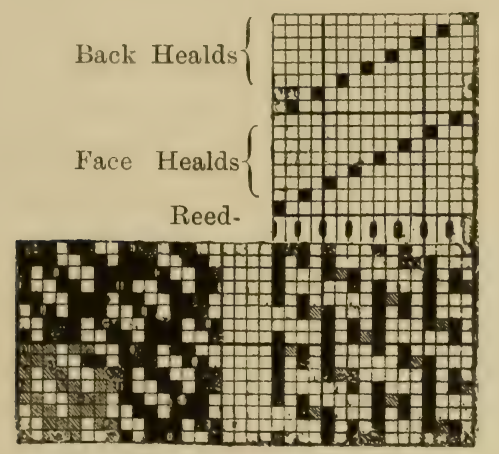

FIG. 323.

Fig. 324.

Design for warp-backed fabric.

"face" threads and one "back" thread alternately, as shown in the complete design, Fig. 326, with the draft shown above it.

Warp-backed textures are usually constructed with an alternate disposition of warp threads to produce a superior back to those constructed on the two "face " and one "back" arrangement of threads. "Back" warp threads' usually interweave with weft in a much less degree than do the "face" warp threads, thereby producing a softer hack, arising from the greater length of floats. This lesser degree of interlacement of "back" warp threads results in a consequent lesser rate of contraction than "face" threads during weaving, thereby requiring each series of warp threads to be contained on separate warp beams to permit of their independent regulation for tension. "Back" warp threads 
should be drawn through a set of healds apart from those governing "face" threads, and should preferably be placed in the rear of face healds, as indicated in the drafts above Figs. 324 and 326. Also, "back" warp threads should pass from the warp beam to healds in a slightly lower plane than "face" threads, and should not be raised quite so high as the latter during shedding. By observing these precautions, unnecessary abrasive friction and chafing of warp threads will be avoided, and any tendency of binding points to show on the face is thereby reduced.

$\$ 57$. Another variety of backed fabrics, constructed on exactly the same principles as the previous examples, are known

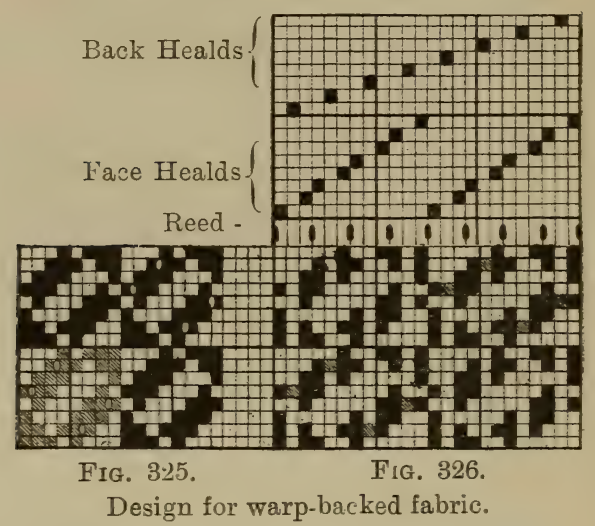

also as reversibles or double-faced fabrics, from the fact that it is quite optional which side is exposed to wear. Reversible fabrics are exemplified in some ribbons (which usually have. both sides equally exposed when in wear), shawls, travelling rugs, mantle cloths, coatings, and some fustians, which may be made to present a similar appearance on both sides; or each side may be different both in respect of weave and colouring. This opportunity is often seized upon to provide mantle cloths and coatings with self-linings of a character quite different both in colouring and texture to the face or outer texture, albeit the lining forms an integral part of the fabric, which is thereby rendered heavier, thicker and warmer. 


\section{CHAPTER VII.}

\section{FUSTIAN FABRICS.}

$\$ 58$. Fustians are a well-known type of cotton fabrics comprising several varieties, the chief of which are known as "imperial," "swansdown," "cantoon," or "diagonal," "moleskin," " beaverteen," "velveteen " or cotton velvet formed with a weft pile, and "corduroy". With the exception of velveteens, which simulate the real silk velvet formed with a warp pile, they are comparatively firm, heavy and compact textures of great strength and durability, chiefly employed in the production of clothing. The first three varieties embody no special constructive feature in their design, as they are based on some simple weave that permits of an abnormally high rate of picks being inserted so as to produce a compact fabric. Each of the remaining varieties, however, is characterised by some peculiar constructive element that distinguishes it from all other fabrics. These are virtually "backed" fabrics, since they are constructed from one series of warp threads and two series of weft threads, namely, "face" and "back" picks, although both series of picks are of the same kind of weft, thereby requiring for their production a loom with only one shuttle-box at each end of the sley.

Unlike all other varieties of fustian fabrics, velveteens and corduroys are characterised by a short and soft fur or plush pile closely resembling that of silk velvet. This fur-like effect is obtained subsequent to weaving by an operation known as "fustian cutting," in which certain floating picks of weft are cut or severed by specially constructed knives that are operated either manually or else mechanically, thereby causing those picks to become more or less erect and thereby expose their transverse sections to the surface, which gradually simulates the character of true velvet. In plain velveteens, short tufts of pile of uniform 
length are distributed uniformly over the fabric, thereby forming a perfectly level surface; but in corduroys, the tufts of pile are caused to develop a ribbed or corded formation, with the cords produced lengthwise or parallel with warp threads. These characteristics are clearly illustrated by Figs. 327 and 328, which are reproduced from actual examples of velveteen and corduroy

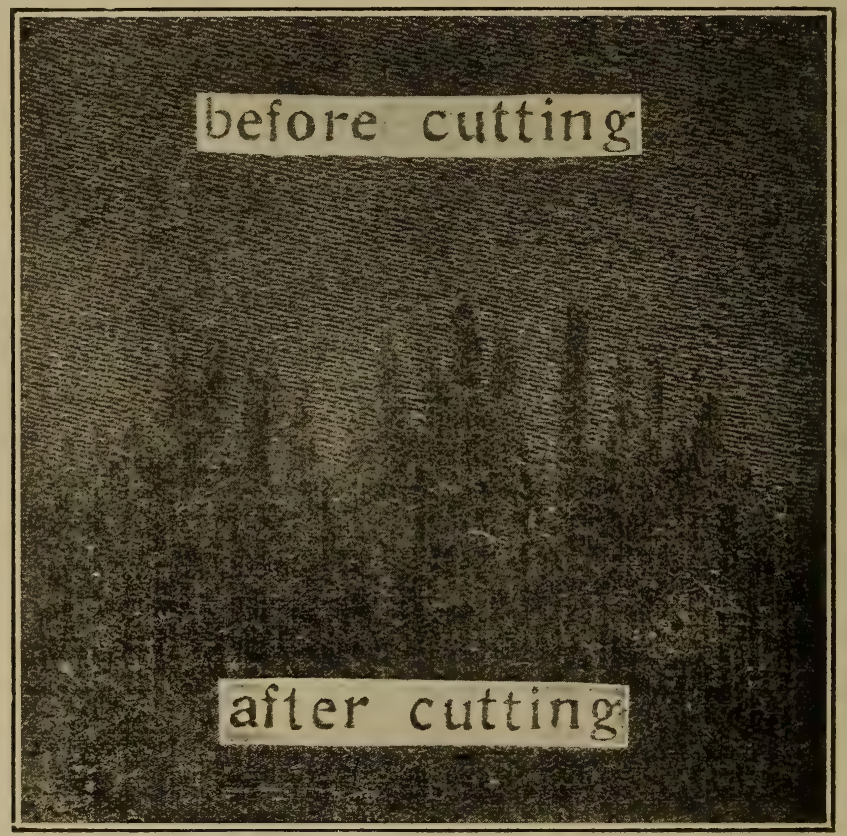

FIG. 327.-Velveteen fabric (cotton velvet), before and after the operation of tustian cutting.

fabrics respectively. Each example shows a portion of cloth both before and after the operation of fustian cutting. It should be observed, however, that velveteens are sometimes made to assume a corded appearance resembling that of corduroys; but their different texture and construction enable them to be easily distinguished from the latter when the characteristics of each are known. 


\section{Imperial,}

\$59. The variety of fustians known to the trade as " imperial" comprises several modifications of what is perhaps better known as "swansdown" cloth, so called from the soft nap or downy surface produced, after weaving, by scratching up or raising the fibres composing the threads of weft, by an operation termed "perching". The nap thus formed simulates

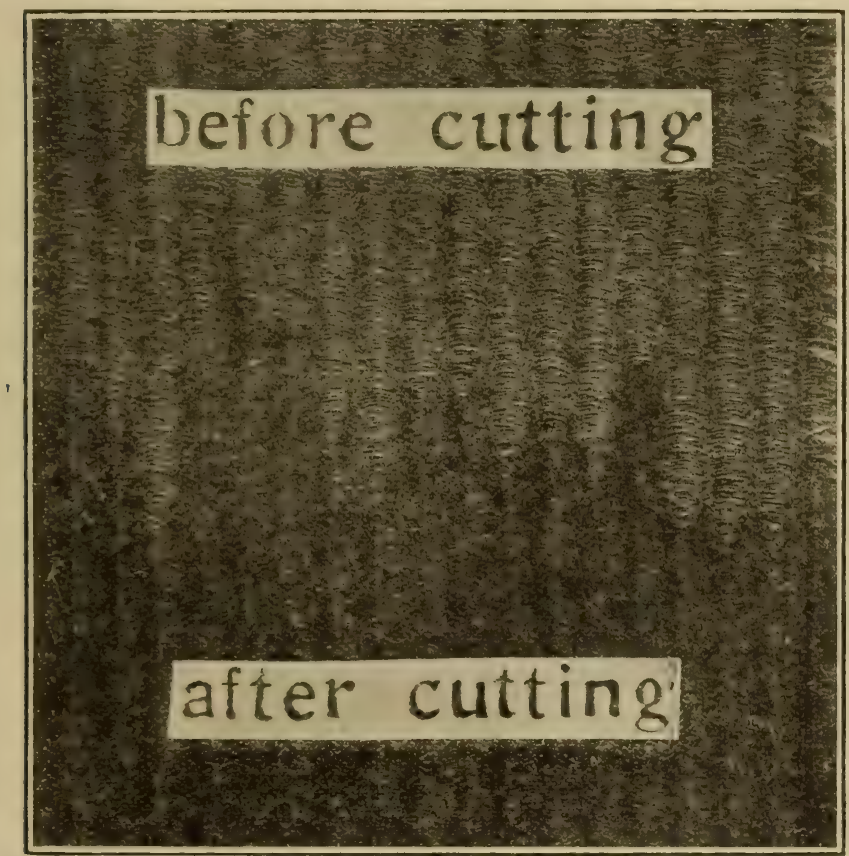

T'rg. 328, - Corduroy fabric, before and after the operation of fustian cutting.

the soft down of swans (hence its description as "swansdown!") and greatly increases the warming properties of the fabric, which for this reason is largely employed for ladies' underclothing. Fig. 329 is a design for swansdown repeating on five warp threads and five picks. From the design, it would appear that weft preponderates on the face in the ratio of three of weft to two of war'p ; but, rirtually, it gives an all-weft surface by reason of 
the much greater density of picks, as compared with warp threads, which latter are entirely obscured on the face. Also, to facilitate the development of a nap by perching, a fairly soft weft of good quality is used. A good quality of swansdown contains 60 warp threads of 18 's T. and 120 picks of 20 's soft weft per inch.

A heavier make of swansdown, known as "imperial sateen," is produced from the design Fig. 330, repeating on eight warp threads and picks, and based on an eight-end satin weave, but with two contiguous warp threads always raised together. This weave produces relatively longer floats of weft, which latter preponderates over warp in the ratio of six to two respectively.

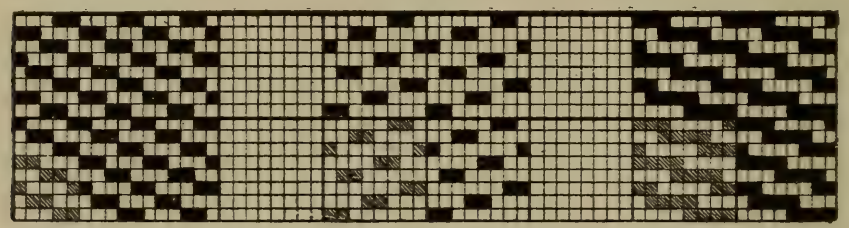

Fig. 329. - Design for "swansdown" fabric.
Fig. 330.-Design for " imperial sateen" and "lambskin" fabric.
Fig. 331.-Design for reversible sateen fabric.

Imperial sateens are sometimes dyed and finished to imitate light moleskins, with a short nap raised on the back ; but when imperials are perched on the face they are named "lambskins," from their long soft woolly nap. A medium quality of dyed imperial sateen contains sixty-eight warp threads of 16 's T. and 150 picks of 16 's weft per inch ; whilst a good quality of "lambskin" contains forty-six warp threads of $2 / 20$ 's yarn and 450 picks of 20 's weft per inch. A design for what is termed a reversible "imperial " contained on eight warp threads and picks is given in Fig. 331. By densely crowding picks of weft, this weave produces a very compact texture, with only weft visible on both surfaces of cloth. A good quality of this cloth contains sixty-two warp threads of 14 's T. and 330 picks of 30 's weft per inch. 


\section{"Cantoon" or "Diagonal" Fabrics,}

$\$ 60$. Cantoon is a variety of fustian largely employed in the production of men's riding and sporting suits, and occasionally of ladies' jackets. As with the previous examples of fustians, its construction embodies no special feature of design, but merely consists of a pick-and-pick combination of the two regular six-end twill weares, Figs. 332 and 333, to produce the design Fig. 334, which repeats on six warp threads and twelve picks. A good ex-

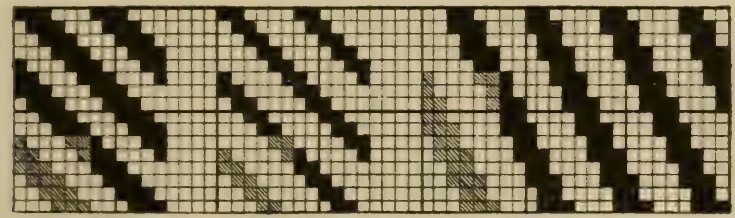

FIG. 332 .

Fr', 333.

Fig. 334.-Design for

"cantoon" or "diagonal" fabric.

ample of this cloth under present notice contains fifty-four warp threads of $2 / 20$ 's yarn and 400 picks of 20 's weft per inch. This abnormal density of picks produces a rery strong and compact fabric having a fine corded appearance, with the cords or wales rumning obliquely at an angle of $18^{\circ}$ to the picks of weft. These fabrics are usually dyed either a fawn or drab hue, and perched on the back.

\section{Moleskin Fabrics.}

\$61. Moleskin is a smooth but thick leathery variety of fustian of greater strength and weight than other varieties, and is largely employed in the production of strong suits of clothing for iron and brass moulders, narvies and other workmen engaged in rough occupations. Its thickness and compactness of texture, combined with its smooth and even surface, make it well adapted for moulders, as it is imperrious to sand, and not so easily penetrated as other fabrics by splashes of molten metal. Moleskins are produced from one series of warp threads and two series of picks (of the same kind of weft), namely, face and back picks, inserted in the proportion of two face picks to one back pick. Face picks combine with alternate warp threads 
only, to produce a modified satin weave repeating on six warp threads and three picks; whilst back picks interweave with all warp threads to produce a three-end weft twill at the back, as indicated in design Fig. 335, which repeats on six warp threads and nine picks. By causing only alternate warp threads to bind over face picks, in addition to combining with back picks, there is a slight tendency to impart a little more strain upon those threads than upon intermediate threads which combine with back picks only. The additional strain upon those warp threads tends to develop a faint stripy formation in cloth, which is, however, considered to be a point of excellence.

Moleskins permit of little or no structural modification without departing from their true character. The moleskin design given in Fig. 336 shows a slight departure from the previous

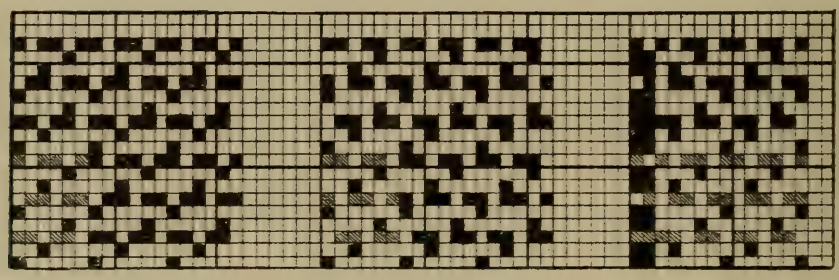

FIG 335 .

Fig. 336.

FIG. 337.

Designs for moleskin fabrics.

example, but one that would manifest no appreciable difference in cloth, excepting to an experienced person. In Fig. 335 it will be seen that at certain points alternate warp threads pass abruptly from above a face pick and underneath a back pick, whereas in Fig. 336 there is always an interval of one pick between the hindings of a face and a back pick by the same warp thread. For example, in Fig. 335 the third warp thread is orer the second face pick and under the following, which is a back pick. The passage of warp threads over a face pick and then immediately under a back pick increases their tension and thereby tends - slightly to emphasise the stripy appearance just mentioned.

Moleskins are not well adapted to decorative treatment of a structural character, but they, as well as heavy imperials, are sometimes printed with simple decorative effects to imitate worsted suitings, and employed in the production of men's 
clothing. They are also sometimes woven with a simple stripe formation, as exemplified in the design, Fig. 337, which repeats on fourteen warp threads and nine picks. A moleskin fabric of good quality contains thirty-eight warp threads of $3 / 24$ 's yarn and 400 picks of 14 's weft per inch.

\section{Beaverteen Fabrics.}

$\$ 62$. Beaverteens are virtually moleskins produced in lighter textures, that are afterwards dyed and printed and then perched on the back, to produce a short and soft nap. Fig. 338 is a design for a bearerteen contained on six warp threads and twelve picks, with three face picks to one back pick. The face weave is almost similar to the swansdown weave given in Fig. 329, and the back is a plain but not a true calico or tabby weare. All

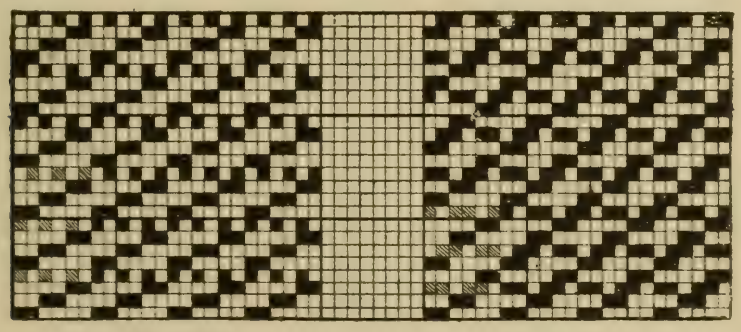

FIG. 338.

FIG. 339.

Designs for beaverteen fabrics.

back picks interweave under and over consecutive warp threads, as in the plain calico weare; but alternate warp threads are raised for trro out of three back picks, and intermediate warp threads weare in an opposite manner to those, namely, down for two picks and up for one. Fig. 339 is another design for beaverteen, contained on six warp threads and nine picks. It has the same face weare as the previous example, but is backerl with three-end weft twill and contains only two face picks to one back pick. A good quality of beaverteen contains thirtytwo warp threads of 218 's yarn and 240 picks of 18 's weft per inch. 


\section{Velveteen Fabrics,}

$\$ 63$. Velveteens constitute an important variety of fustians, generally of much lighter texture than other varieties. As previously stated in $\$ 58$, their characteristic velvet appearance is produced subsequent to weaving by an operation of fustian cutting (performed sometimes, for low qualities only, by machinery, but more frequently by hand) which is usually conducted independently as a kindred branch of fustian manufacturing. Previous to being cut, a velveteen fabric presents no unusual structural appearance, but has a smooth level surface with weft floating abundantly on the face, as in fabrics constructed on a simple satin basis.

Velveteens are produced from one kind of weft inserted so as to constitute two series of picks, namely, "pile" and "ground" picks, corresponding to face and back picks respectively. Pile picks are floated somewhat loosely on the surface, to be afterwards cut to form pile; whilst ground picks interweave more frequently with warp, to build up a firm foundation texture to sustain the pile. Indeed, the simplest definition of a velveteen fabric is: A simple texture of calico, twill or other simple weave, embodying a vast number of short tufts of weft (thus-U) evenly distributed to produce a soft velvet-pile surface. Thus, if all the tufts of pile were entirely withdrawn, there would still remain a perfect foundation texture of a plain, twill or other simple weave according to the basis adopted. For light and medium textures, the latter is usually based either on the tabby or three-end twill weave; and for heavier textures, on the four-end $\left(\frac{2}{2}\right)$ twill weave.

It is important that pile picks should be securely attached to the foundation texture to prevent the tufts of pile being accidentally withdrawn either during fustian cutting or when the fabric is in use. This may be accomplished in two ways, namely, $(a)$ by compression, caused by densely crowding picks of weft; and (b) by interweaving pile picks with several warp threads in succession, to produce what is termed a "fast" or "lashed" pile; or by adopting both of these methods. Most velveteens, however, are constructed on the former plan, in which pile picks are bound into the foundation texture by only one warp thread at regular intervals of six, eight or ten threads, according to length of pile 
required. The second plan is usually adopted when it is required to float pile picks for a greater distance, for the purpose of producing longer pile, the tufts of which would be more liable to accidental withdrawal. Whether pile picks are bound by only one or more than one warp thread in each repeat of the pattern,

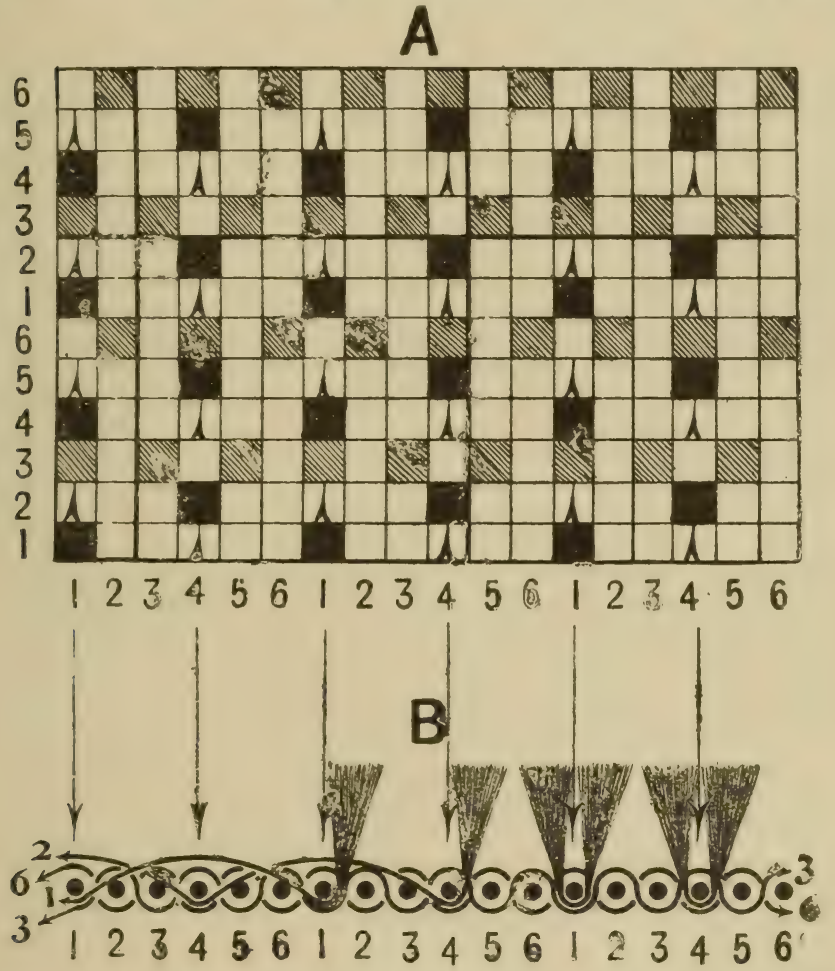

Fig. 340.-(A) Design for velveteen fabric, of which $B$ represents a transvcrse section, showing certain picks of weft standing erect, after being severed by the fustian knife to form tufts of pile.

it is imperative that the binding should occur at regular intervals on each pick, to give uniform lengths of floats, and, therefore, a uniform length of pile after cutting.

$\$ 64$. Fig. $340 \mathrm{~A}$ is a simple design, repeating on six warp threads and six picks, for a tabby back velveteen containing two pile picks to one ground pick, with pile picks bound at intervals 


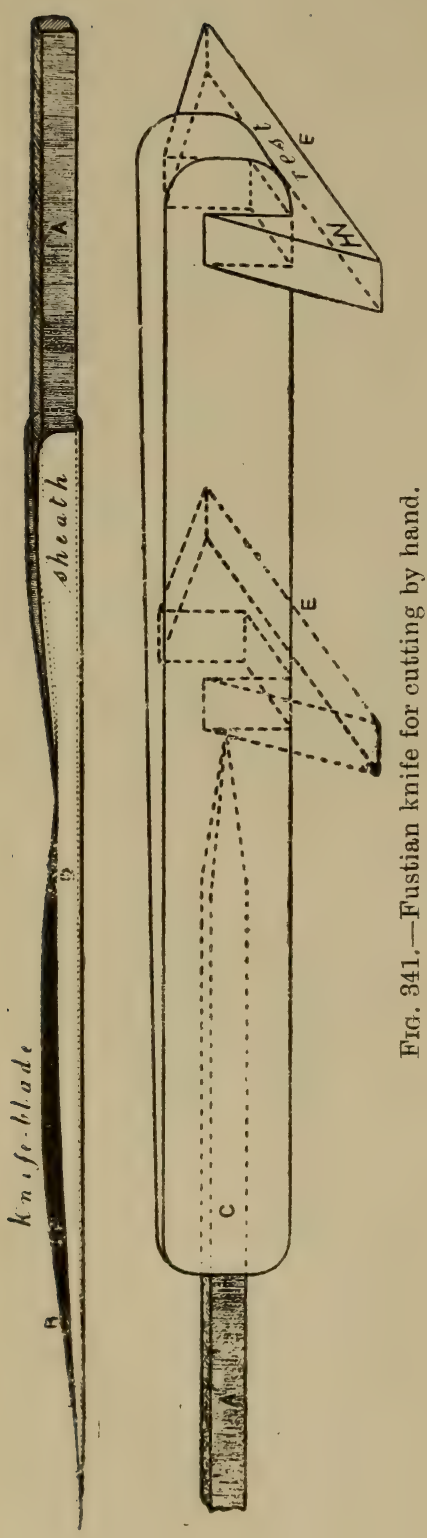

of six, to cause them to float over five, warp threads. In this example, every third warp thread only is utilised for binding pile picks to the ground texture, namely, the first and fourth in each repeat of the pattern; whilst all warp threads interweave with ground picks to produce the foundation texture. This combination of threads causes the floats of pile picks to develop a series of courses or passages running lengthwise, termed "races," which lie above the ground cloth and along each of which a fustian cutter passes the fustian knife, so that the cutting edge of the latter passes under all floats of weft forming a "race," thereby severing them in the centre and causing them to become erect on each side of a binding thread to produce the characteristic short tufts of pile. This is clearly illustrated in Fig. $340 \mathrm{~B}$, which represents a transverse section of cloth (produced from the design above it) both before and after cutting. The paths along which the fustian knife takes its course, and also the points at which pile picks are severed, occur at intervals of three warp threads, as indicated by arrows.

$\S 65$. A fustian knife for cutting by hand is illustrated in Fig. 341. It consists of a square 
steel rod $A$, beaten out at one end to form an extremely thin keen-edged blade $B$, and is provided with a handle $C$ at the other end. The knife blade is inserted in a shaped and pointerl sheath D, of sheet iron or steel, which serves the threefold purpose of $(a)$ giving tirmness to the slender blade: $(b)$ guiding the blade along its true course under the proper floats; and (c) tautening the floats of weft as it passes under and brings them up to the exposer edge of the knife to be severed. Fustian knives are made in various lengths, from about 12 ins. to 30 ins., from steel rods rarying from $\frac{1}{8}$ in. to $\frac{1}{4}$ in. square, and each is provided with a sheath or guide specially shaped and pointed to suit the particular kind of eloth for which it is intended (as velveteen or corduroy), and also the width of "race". A fustian knife handle is sometimes furnished with a piece of wood $\mathrm{E}$, to serve as a rest for the knife, and maintain it at the proper angle as it traverses a "race". The rest $\mathrm{E}$ is fixed at the rear end of the haft when cutting velveteen on a "shortrun " frame (of 2 yards in length), and in the centre of the haft (as indicated by dotted lines) when cutting corduroy.

$\$ 66$. Before being submitted to the operation of fustian cutting, velveteens are first subjected to a process of liming, in which a thin coating of lime paste is applied to the face side of cloth by passing it over a roller revolving in slaked lime. From the lime trough the cloth is immediately passed over a number of steam-heated cylinders to be dried; after which it is coated on the back with flour paste and again dried for the purpose of stiffening it and to prerent the withdrawal of tufts of pile during cutting. After this preparation, the cloth is made taut by stretching in a suitable frame of either 2 or about 10 yards in length, when the cutter passes a knife smartly along successive "races," taking each in turn from one selvedge to the other. Subsequent lengths of cloth are then stretched and cut in a similar manner until the whole piece is completed, after which it is submitted to various finishing processes. The operation of cutting velveteen by hand on short frames is illustrated in Fig. $342,{ }^{*}$ in which fustian cutters are shown standing at the side of

* The author is indebted to Messrs. Henry Bannerman \& Sons, Limiter, for their kind permission to use this illustration. 


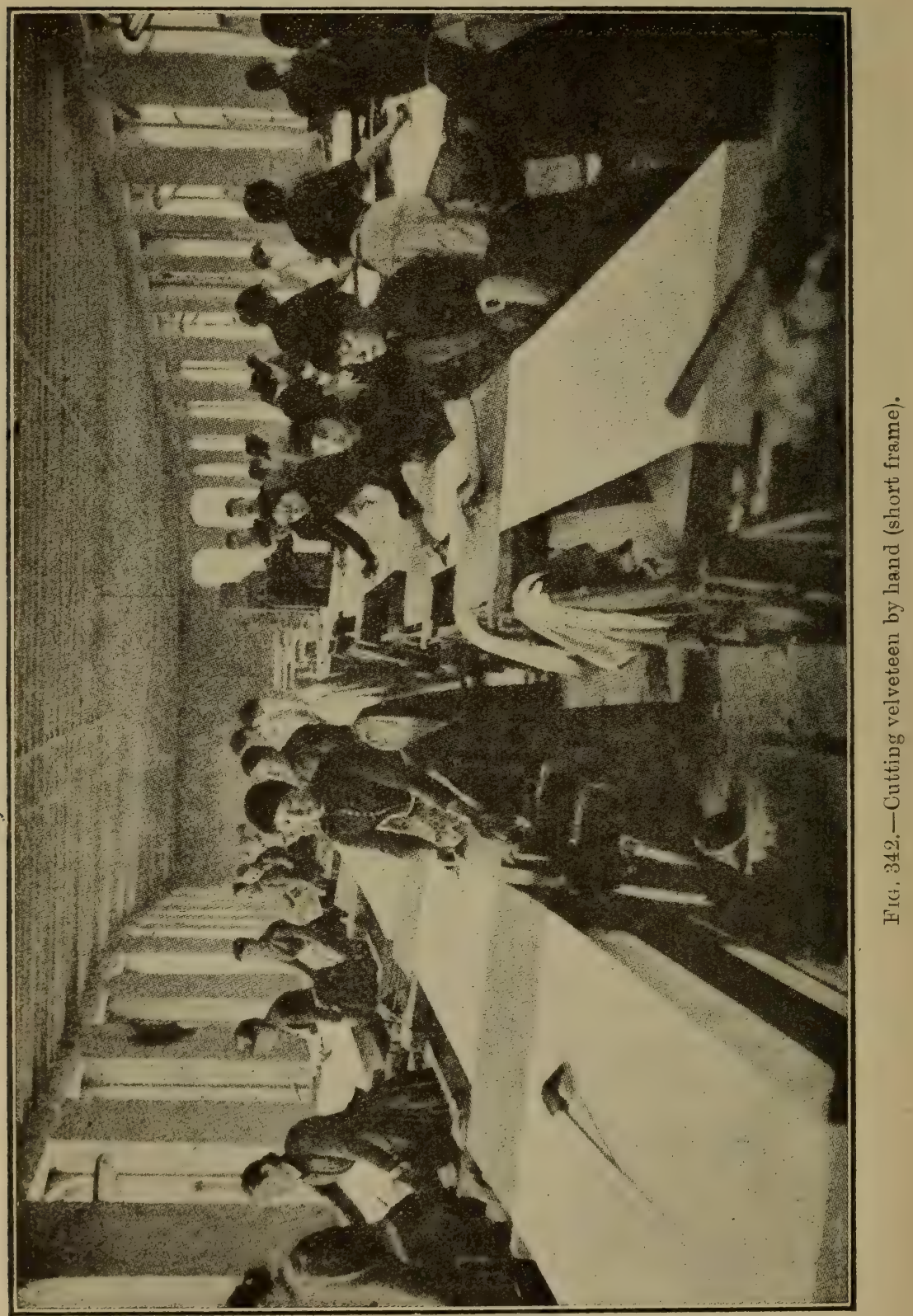


their frames; but when cutting corduroy on a short frame the cutter stands at one end.

The foregoing description of a simple example of velveteen, and of fustian cutting, will enable a student to intelligently comprehend some of the circumstances affecting the production of velveteens and corduroys, and thereby to better conform to the conditions which their construction imposes upon a designer, namely, the proper security of pile to the foundation texture, and the distribution of binding places in such manner as to provide suitable "races" or passages at regular intervals for the reception of the fustian knife.

$\$ 67$. Fig. 343 is a design in extensive use for a tabby-back velveteen repeating on six warp threads and eight picks, having three pile picks to one ground pick, with consecutive pile picks bound by alternate warp threads at intervals of six threads. It will be observed that pile picks are bound by the same alternate warp threads that are raised for the first ground pick in each repeat of the pattern ; whilst the intermediate threads are only raised for the second ground pick, thereby causing the three pile picks between each ground pick to become equivalent to, and subsequently occupy the space of, only

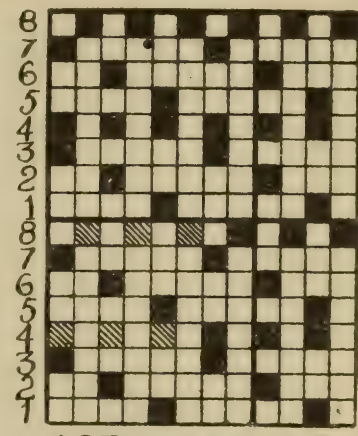

123456123456

FIG. 343.-Design for velveteen fabric. one pick of weft. Hence, the eight picks constituting one repeat of the design are equivalent to only four picks when in eloth.

The employment of alternate instead of consecutive warp threads for the purpose of binding pile picks is a practice which, for several reasons, is adopted in the construction of velveteens. In the first place, it reduces the number of "races" by onehalf, by creating them along alternate warp threads only, instead of along all warp threads, thereby requiring less time for cutting and reducing the cost of that operation. In the second place it facilitates the operation of cutting by developing more clearly 
defined "races" for the reception of a fustian knife. And, finally, by causing tufts of pile to lie along alternate warp threads, instead of being distributed on all warp threads, a more perfect simulation of real velvet is produced, and one that makes the difference between velvet and velveteen sometimes very difficult to detect. The use of alternate threads of the same warp to bind over face picks as well as under back picks tends (as ex-

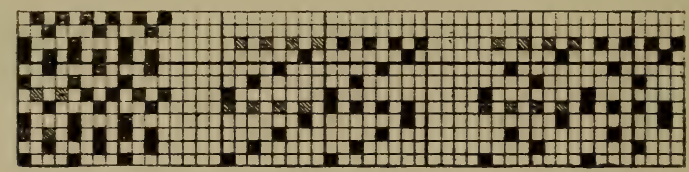

FIG. $34 \pm$.

FIG. $3 \pm 5$.

FIG. 346.

plained under the heads of backed fabrics and moleskins, in $\$$ 56 and 61 ) to impart a little greater strain upon those threads; but in consequence of the sparseness of warp threads, and the considerable degree of tension at which they are held during the weaving of fustians, the difference in tension between binding and non-binding threads is so small as to develop only the faintest stripiness in the uncut cloth, which entirely disappears after cutting; nor is the difference in tension such as to necessitate the binding and non-binding warp threads being wound

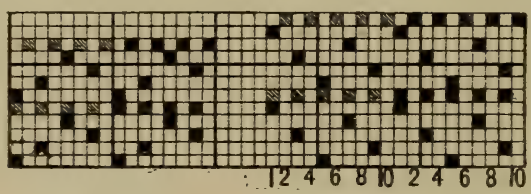

FIG. 347.

FIG. 348.

upon separate warp beams. This is explained by the circumstance of warp threads being held so taut during weaving that the softer, finer and more supple weft exerts little or no influence upon them, and so they remain perfectly straight.

3 68. Figs. 344 to 373 are examples of designs showing various modifications in the construction of velveteen fabrics. A cursory examination will show that the essential points of difference in them are in respect of their foundation weaves, and the method of securing pile picks thereto. Other points of 
difference, not of a structural character, are the ratio of pile picks to ground picks, and the length of float between the binding points of pile picks. Fig. 344 is a design for a tabby-back velveteen containing two pile picks to one ground pick, with pile weft floating orer only three warp threads between each inter-

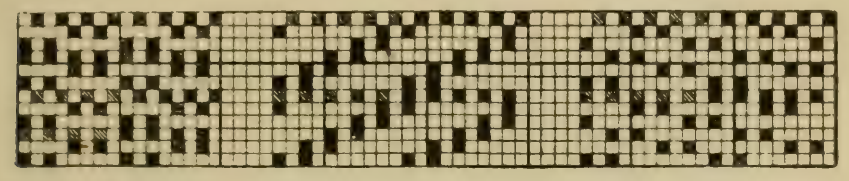

FIG. 349.

FIG. 350.-Represented by transverse section, Fig.

FIG. 351.-Represented by transverse section, Fig. 354. 353.

section, which would produce an exceedingly short and poor pile. Figs. 345, 346 and 347 are three designs for tabby-back velveteens, each containing four pile picks to one ground pick, with pile weft floating over seven warp threads. Although pile picks are bound in a different order in each design, they would produce absolutely identical results in the finished fabric: as the four pile picks between two ground picks in each design

A

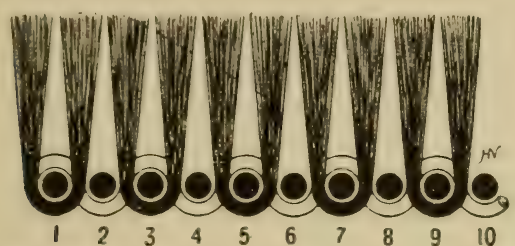

B

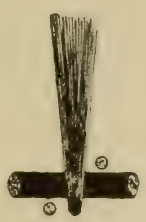

FIG. 352.-(A) Transverse section showing usual method of securing tufts of pile in the foundation texture of ordinary velveteen fabrics.

would constitute only one row of tufts disposed on alternate warp threads. Fig. 348 is a design for a tabby-back velveteen containing five pile picks to one ground pick, with pile picks floating over nine warp threads to produce a longer pile.

$\$ 69$. Figs. 349, 350 and 351 are three examples of designs for tabby-back velveteens with a "fast" or "lashed" pile, so called because the tufts of pile are more securely attached to the 
foundation texture; thus, instead of being looped under and held by only one warp thread, as in previous examples and as illustrated in Fig. 352, each tuft of pile is secured by interlacing with three consecutive warp threads, as shown in Figs. 353 and 354. Although the binding of pile picks in Figs. 350 and 351 is

A

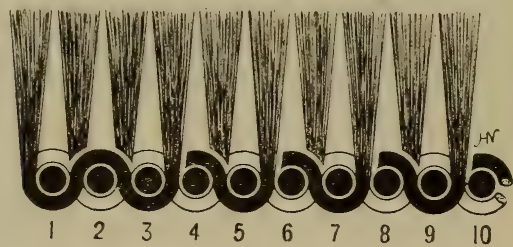

B

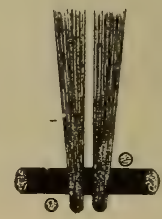

FrG. 353.-(A) Transverse section of velveteen fabric, with fast or lashed pile, from design Fig. 350.

of a different arrangement in each, they will produce no material difference in their ultimate results, as seen by comparison of Figs. $353 \mathrm{~A}$ and $354 \mathrm{~A}$, which represent transverse sections of cloth produced from designs Figs. 350 and 351 respectively. In both examples, warp threads are raised over two out of the five pile picks between two ground picks, so that the five pile picks

A

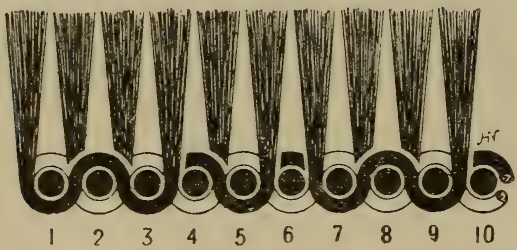

B

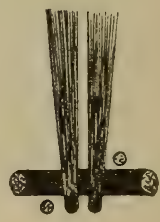

FIG. 354.-(A) Transverse section of velveteen fabric, woven with fast or lashed pile, from design Fig. 351.

will occupy the space of two picks in cloth (as seen in end views of sections at B). Hence, five tufts of pile will be formed over an interval of ten warp threads, from what virtually constitutes two picks of weft (whereas, by the method of binding shown in Fig. 352, five tufts of pile would be formed in the same interval, and from the same number of picks, which would virtually con- 
stitute only one pick of weft (as seen in end view of section at B), and which would result, for example, from the design Fig. 348. It is evident, therefore, that a "fast" pile can only be obtained in fabrics of similar quality by sacrificing the density of tufts of pile.

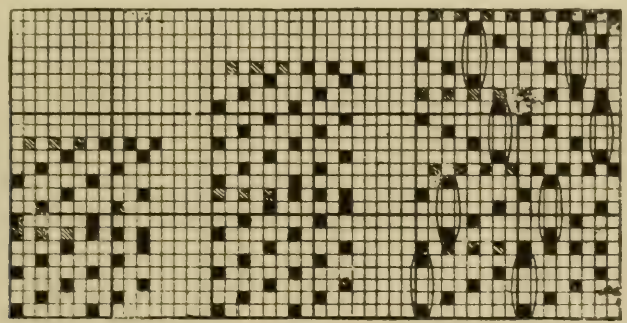

FIG. $355 . \quad$ Fig. $356 . \quad$ FIG. 357.

$\$ 70$. It may be observed at this juncture that the relative density of pile in fabrics of similar quality may only be increased by additional rows of tufts between ground picks. Thus, instead of warp threads binding over only one pile pick between two ground picks to produce only one row of tufts (as in all

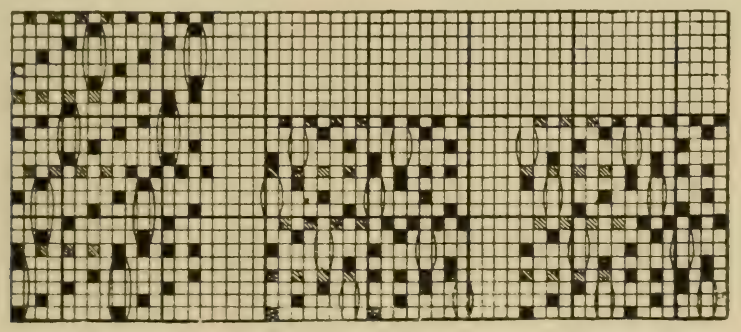

FIG. 358.

FIG. 359.

FIG. 360 .

previous examples), they may bind over two or three pile picks to produce a corresponding number of rows of tufts between two ground picks, as exemplified in Figs. 355 and 356 . Also, each binding warp thread should preferably contain the same number of tufts between ground picks to ensure a more perfect distribution of pile; though this precept is not always observed in practice, as will be seen presently. Density of pile is sometimes 
slightly increased by causing additional tufts of pile to occur in certain places only, between two ground picks. Conversely, density of pile may be slightly diminished by omitting tufts of pile in a similar order. In either case, care should be taken to dispose the additional tufts (or the spaces where they are omitted) so that they will not tend to develop lines in any direction in the finished fabric. Any such tendency is avoided in an ingenious manner in Figs. 357 and 359, which are designed to increase and diminish the density of pile respectively. In Fig. 357 the successive binding points on pile picks are produced in an opposite direction at intervals of two ground picks, thereby disposing the additional tufts of pile in the four-end satin order

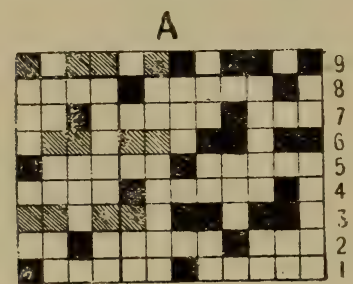

1234561234.56

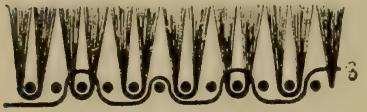

123456123456

B

FIG. 361
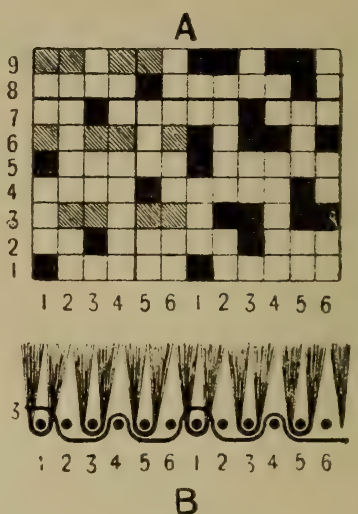

FrG. 362.

(as indicated by bracketed squares). Had the successive binding points been produced in the same direction throughout, as in Fig. 358, the regular occurrence of the extra tufts would tend to develop a series of lines running obliquely across the fabric. In Fig. 359, which will give a less dense pile, the same practice has been observed of reversing the direction in which binding points are produced at intervals of two ground picks, so as to dispose the gaps (caused by missing tufts, as indicated by bracketed squares) in a four-end satin order, for reasons just explained. Had the binding points in that design been produced. in the same direction throughout (as indicated in Fig. 360 ), the vacant places would, in consequence of their regular succession, tend to develop oblique lines across the fabric. 
$\$ 71$. Figs. 361 to 367 are designs for velveteens of a little heavier texture than the tabby-backed rariety. Their foundation texture is based on the three-end twill weave, which permits of a greater number of picks per inch being inserted to produce a more compact fabric. In other respects their construction is similar to the previous examples. Apart from the object of increasing weight, a greater degree of compactness in twillbacked relveteens is essential to hold the pile firmly; otherwise the freer character of the weave would produce a more open texture, and thereby permit of the easier withdrawal of tufts of pile. It is sometimes advocated that, when constructing twillbacked velveteens, warp bindings on pile picks that immediately either precede or succeed a ground pick should be placed counter to a weft binding on that pick, so that such warp and weft binding points will lock or check each other, and thus give

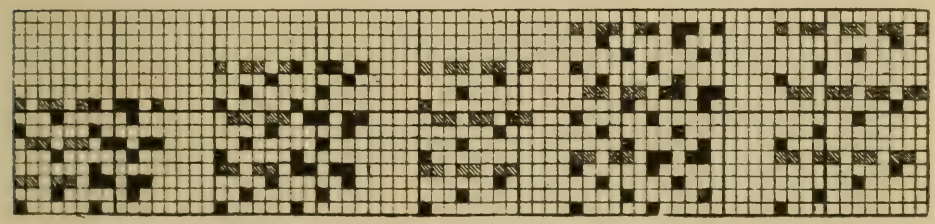

F'IG. 363.

FIG. 364.

FIG. 365.

FIG. 366.

FIG. 367.

additional firmness to the fabric. A little reflection, however, will show that whilst such precaution would conduce to better results if the weft were noi to ke sirbsequently cut to form pile, it is unnecessary to observe it in the scrstruction of a velveteen fabric in which the several picks between, two ground picks virtually ocerpy the space of only one pick, or sometimes two picks, in the tnished cloth. It may also be pointed out that, whether the warp and weft binilings are or are not placed counter to essh other, in the des:yn, they automatically become so in the finished fabic ; so that precisely similar results obtain whichever practice is arlopted. This is clearly demonstrated by means of diagrams Figs. $361 \mathrm{~B}$ and $362 \mathrm{~B}$, representing transrerse sections of cloth (after cutting) woven from designs Figs. $361 \mathrm{~A}$ and $362 \mathrm{~A}$ respectively, which designs are identical, excepting that warp bindings on every second pile pick in Fig. $361 \mathrm{~A}$ 
are placed counter to weft bindings of the contiguous ground pick; whereas the binding points are not so placed in Fig. $362 \mathrm{~A}$, yet their transverse sections are virtually alike. (It will be observed that these two designs are identical with the moleskin designs, Figs. 335 and 336 respectively.) Figs. 363 to

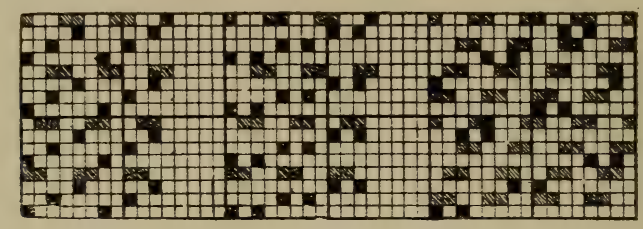

FIG. 368. FIG. $369 . \quad$ FIG. 370.

367 are other examples of designs for velveteens with three-end twill backs, showing various modifications; whilst Figs. 368 to 373 are for velveteens having a foundation texture based on the two-and-two twill, and other weaves, to produce still heavier textures.

Weft plushes are simply velveteens in which pile weft is

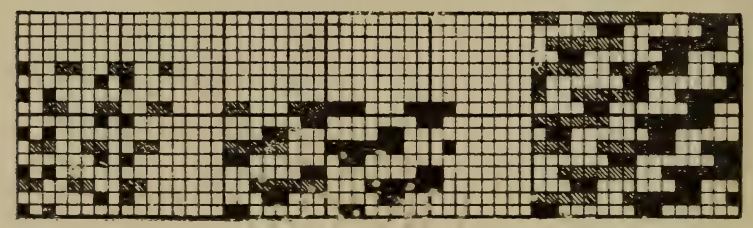

FIG. 371. . FIG. 372. ‥ 373.

allowed to float over a considerable number of wir p threads to produce a longer pile, the tufts of which are mone firmly interlaced by interweaving them under and over three or five consecutive warp threads, as a sescrined in \$ $\$ 69$. In all other respects their construction is similar to hat of ordinary velveteen fabrics having short pile.

\section{Ribbed or Corded Velveteen.}

372. Velveteen is sometimes made to assume a ribbed or corded formation, resembling that of corduroys, with the ribs or 
cords produced lengthwise or parallel with warp. There are, however, socalled "velvet cords" that are not true velveteens, but simply lighter textures of corduroy, which are described under that head in $\$ 75$ and 76 . So-called "ribbed velvet" or ribbed velreteen (also known as "hollow-cut" velveteen), however, is woven as ordinary plain velveteen, and afterwards made to assume a corded appearance by a special mode of fustian cutting, in which a cutter first passes a knife along certain "races" in each cord, with the blade vertical, as in ordinary cutting, and then along intermediate "races," with the knife blade held at different angles, to sever floats of weft out of the centre, and so form longer and shorter tufts which develop rounded ribs of pile. For some "races" the knife. is inclined towards the cutter, and for others away from the cutter; hence the terms "towart" and "frowart" used amongst that class of fustian cutters,

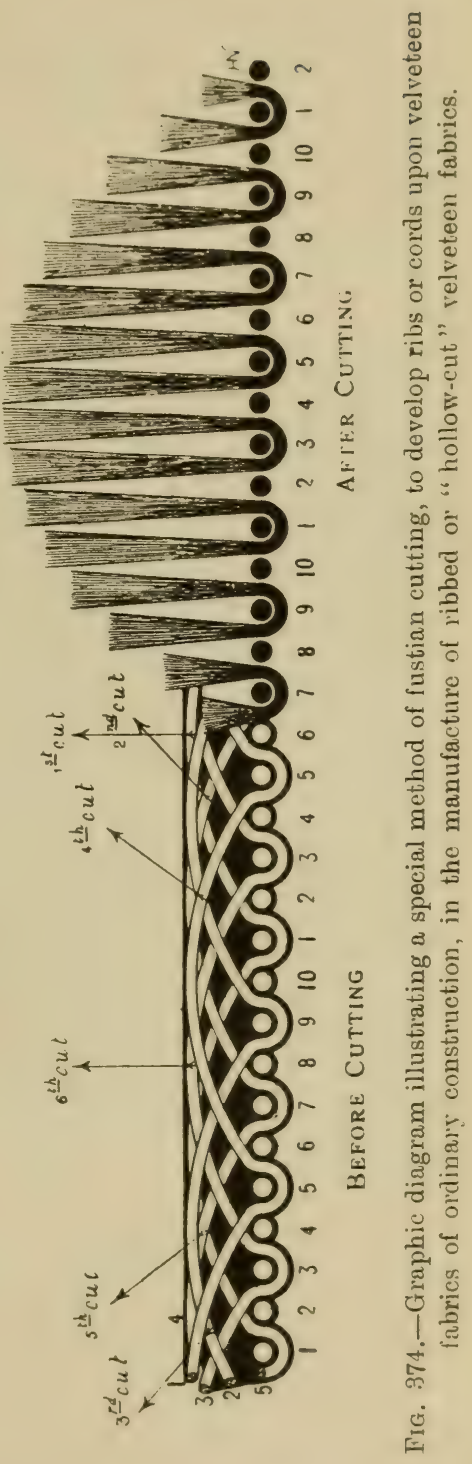
whose work is regarded as specially skilful. An example of ribbed velveteen under present notice is produced from the 
design shown in Fig. 348, containing five pile picks to one ground pick, and with pile weft floating over nine warp threads. A transverse section of this cloth (before and after cutting) is represented in Fig. 374, in which arrows indicate the various angles at which the knife blade is held as it is passed along the different "races". The width of cords is not regulated by the number of warp threads on which the woven design repeats, but is arbitrarily decided by the fustian cutter, who may produce various widths of cords from exactly similar fabrics. In the present example the ribs occur at intervals of sixteen warp threads, although the woven design repeats on only ten warp threads. The same fabric could have been cut to produce broader or narrower ribs, as desired.

\section{Figured Velveteen.}

$\$ 73$. The embellishment of velveteens is not confined to that created by a simple ribbed or corded formation. They are frequently rendered of a more or less ornate character by means of designs printed in various colours, embossed designs, and designs produced by Jacquard machines. Many woven designs are of a somewhat elaborate character, as exemplified in the specimen reproduced in Fig. 375.

The construction of Jacquard-figured velveteen is governed by the same principles as those regulating the construction of simple velveteen, so far as the development of pile in the figure portion is concerned; but in the ground portion of the pattern, which is without pile, some method must be adopted to effectually obscure pile weft from the face of the fabric. Various methods are employed to achieve that object. By one-which is perhaps the most generally satisfactory method-when pile picks are not required on the face for the development of figure, they are placed at the back in the ground portion, and interwoven with binding warp threads in an exactly opposite manner to that which obtains in the figure portion. By another method (of which the specimen illustrated is an example), surplus pile weft is allowed to float quite freely beneath the ground portion, and after the operation of cutting, it is brushed off as waste 
material. A third practice is to employ an extra fime warp to interweave loosely with surplus pile weft at the back of cloth for the purpose of producing a light gauzy and imperfect tissue, which, after the operation of cutting, is drawn bodily away from the principal texture, thereby removing all surplus pile weft. This precaution, however, is only necessary for designs containing comparatively large areas of ground filling, in which case

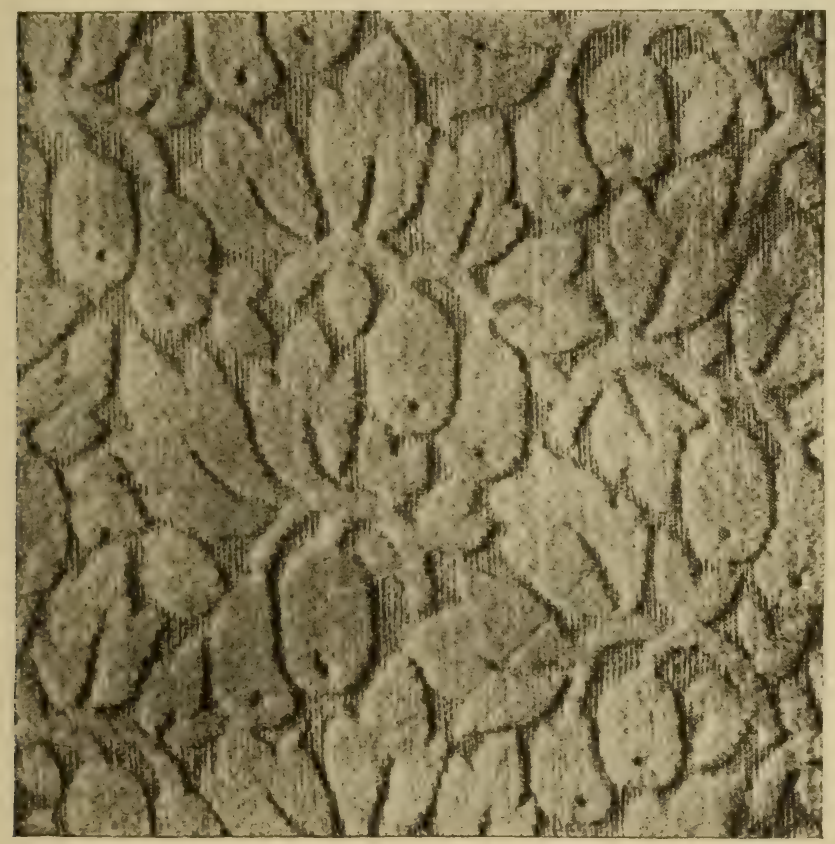

FIG. 375.-Velveteen figuring by Jacquard machine.

the resulting masses of floating weft at the back would be liable to be caught and pulled both during and subsequent to weaving, thereby involving risk of injury to the fabric and impeding the operation of cutting.

$\$ 74$. When preparing an applied or working design for a figured velveteen fabric, it must be first drawn to the required dimensions on the proper counts of squared or point paper, and painted in en bloc, as in Fig. 376. It may then be transposer 
to point paper of any other counts to be "read off" by the card cutter. The proper counts of design paper for the block design is determined according to the number of rows of tufts to be contained in one inch, both horizontally and vertically, because the margin of figure steps in intervals of tufts of pile each way; thus each small square of the block design corresponds to one tuft. If, therefore, a design is to be prepared for a fabric containing 88 warp threads and 420 picks per inch, with pile weft bound by alternate warp threads, and having three pile picks (which constitute only one horizontal row of tufts) to one ground pick (as in the present example), the proper counts of point

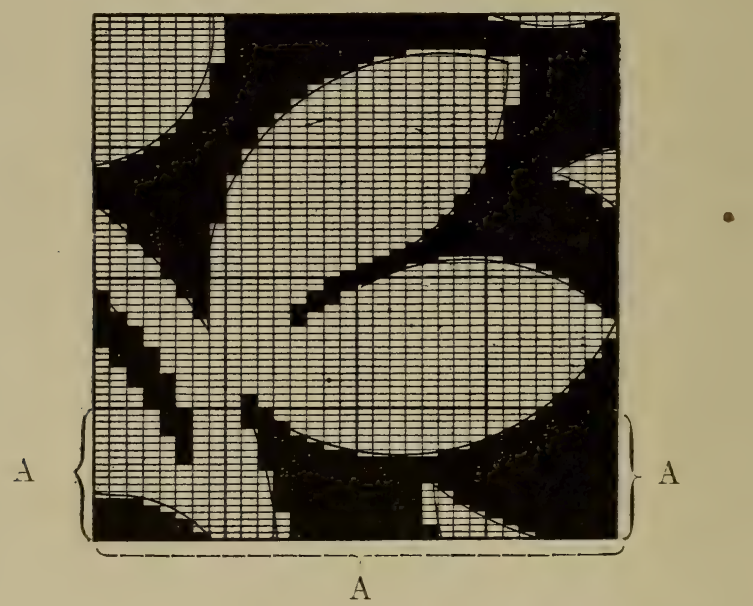

Frg. 376.-Block sketch-design for figured velveteen fabric.

paper for the block design is in the ratio of $(88 \div 2=44)$ to $(420 \div 4=105)$; or (assuming a Jacquard machine has eight long rows of hooks) the point paper should contain eight squares hy nineteen squares in each bar. If a fabric contains four pile picks, constituting only one row of tufts, between two ground picks, the number of horizontal rows of tufts per inch will be one-fifth of the actual number of picks inserted. Again, if a fabric contains six pile picks constituting two rows of tufts between two ground picks, the number of horizontal rows of tufts per inch will be two-serenths of the actual number of picks inserted, and so on. 
It is necessary to prepare the pattern en bloc on design paper of the proper counts in order to ensure that the correct forms and shapes of its component parts will be preserved when reproduced in cloth; but the counts of paper employed for the working design, and from which pattern cards are read off by the card-cutter, is quite immaterial. When preparing an applied design from a block pattern, it must be remembered that all warp threads are controlled by a Jacquard machine, and that a pattern card is required for each pick of weft inserted. Therefore, since each small square of the block pattern corresponds to one tuft of pile, each vertical space on the former represents two, three, or four spaces on the working design (according as tufts of pile are contained on alternate warp threads, or on every third

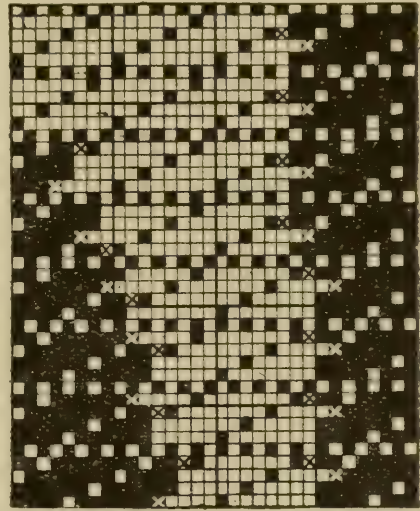

Fig. 377.-Portion of applied design (for figured velveteen fabric), demonstrating the development of the block sketch-design (part A) Fig. 376.

or fourth warp thread), and each horizontal space in the block pattern represents the number of pile picks inserted for each ground pick, plus one ground pick. For these reasons, when setting out the pattern for the working design, the margin where figure and ground meet must change or step at intervals of two or more warp threads in a horizontal direction, and at intervals of two, three or more picks in a vertical direction, according to the weave selected.

Fig. 376 is a portion of the pattern, en bloc, of the cloth represented in Fig. 375. A small portion of the block pattern (bracketed) is shown transposed in Fig. 377, and is developed for a tabby-back velveteen containing four pile picks to one ground pick, with pile weft bound by alternate warp threads, and in accordance with the first-named method as described in $\leqslant 73$. By this method pile picks are placed at the back in the ground 
portion and interwoven with binding warp threads in an opposite manner to that of the figure portion. The margin of figure in a vertical direction in velveteens constructed in accordance with this method is always formed with a half-tuft, i.e., one-half of a complete loop (thus $\mathrm{J}$ ). For this reason it is advisable to continue some short marginal floats of weft to their full extent, and to stop others that are very short (as indicated by white and black crosses respectively), and thus prevent short marginal tufts of pile. This object will be more easily achieved by forming the margin of ground (in a vertical direction) with the binding warp ends only, as observed in Fig. 377.

Fig. 378 is a portion of the same block pattern showing the development of a working design for figured velveteen, in accordance with the second method as described in $\$ 73$, by which surplus pile weft floats loosely beneath the ground portion of the fabric, to enable it to be readily brushed away after the operation of cutting, and is a reproduction of a portion of the fabric represented in Fig. 375, which is a tabby-back velveteen containing three pile picks to one ground pick, with alternate warp threads employed for binding pile weft. The scheme of binding pile picks in this example is the same as that given in Fig. 359, in which tufts of pile are omitted in a certain order, for the twofold purpose of increasing the length, but reducing the density, of pile. It should be observed that in velveteens constructed in accordance with this method the margin of figure in a vertical direction is formed with entire tufts, as any half-tufts that may be formed during the operation of cutting are withdrawn on removing surplus pile weft from the back. For this reason, greater care is required on the part of a designer in order to preserve a good margin of figure and ground. This may be accomplished by extending certain marginal floats into the ground portion, of sufficient length to enable the fustian knife to pass underneath and cut them, and also by filling in the spaces to stop all floats of weft that are too short to be cut, as indicated on the working design by white and black crosses respectively. By carefully studying this design, the method of preparing designs on this principle will become manifest.

As observed in $\$ 73$ velveteens are sometimes ornamented by 
an operation of stamping, whereby plain velveteens are furnished with embossed designs which closely simulate those produced by Jacquard machines. The fabrics to be ornamented in this way are subjected to considerable pressure against a roller matrix of the required pattern, which depresses the pile to form the

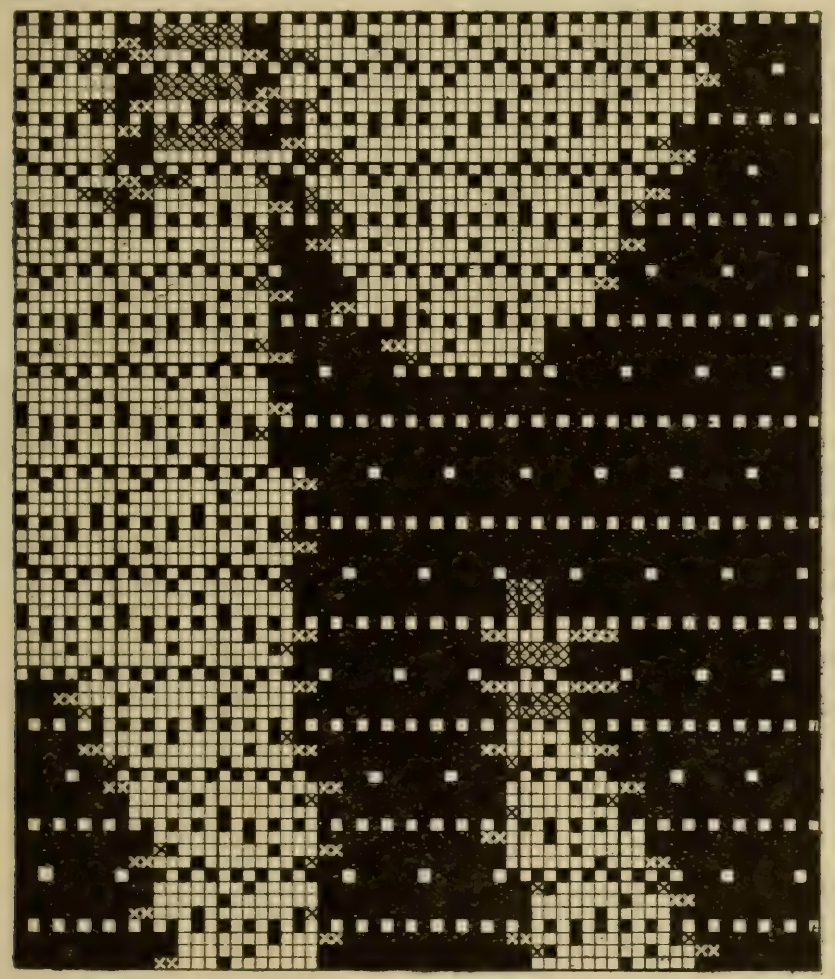

FIG. 378. -An alternative method of developing an applied design for a figured velveteen fabric.

ground portion, and leares the pile erect in the figure portion, which stands out in sharp relief as an embossed design. When put into use, the pattern of an embossed velveteen becomes indistinct and finally obliterated, in consequence of the depressed tufts of pile in the ground portion being disturbed by friction, and thereby becoming partially erect. Thus, the difference 
between woven and stamped figured velveteen may be easily detected by scratching the ground portion with a pointed instrument, which will raise the depressed pile in that part of the counterfeit fabric.

\section{Corduroy Fabrics.}

$\$ 75$. Corduroy fabrics âre constructed on similar principles to those governing the construction of velveteens, and, like those, are submitted to an operation of fustian cutting for the development of a pile surface. They are, however, produced in much heavier and more durable textures than velveteens, in view of the greater wearing properties required of them. They consist of a foundation texture, usually based upon a three-end or four-end twill or other simple weave, containing tufts of pile disposed at regular intervals on from two to six contiguous, warp threads (according to the width and character of cord required) in such manner as to develop a series of rounded pile ribs or cords in the same direction as warp threads. The ribs are usually of uni-

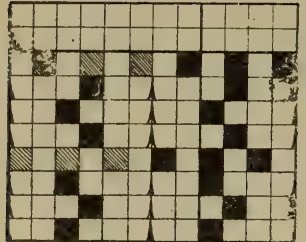

Fig. 379.-Design for corduroy fabric.

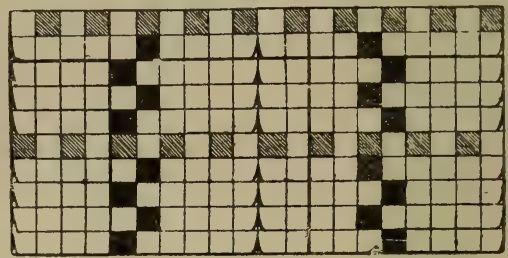

FIG. 380,-Design for corduroy fabric.

form width in the same fabric, but sometimes they are variegated. Most corduroy fabrics have pile and ground picks in the ratio of two to one respectively, with a twill foundation weave; but, as stated previously, in $\S 72$, some varieties known as "velvet cords " are produced in comparatively light textures based on the plain or tabby weave, and containing three, four and five pile picks to each ground pick, to produce a denser pile. Fabrics of this description are usually employed in the production of boys' and ladies' clothing. Figs. 379 and 380 are two designs for "velvet cords," each having a foundation texture of plain cloth and containing three and four pile picks to each ground pick respectively. A transverse section of cloth (before and after 
cutting) produced from design Fig. 380 is represented in a graphic manner in Fig. 381. As a fustian knife is thrust along each successive "race," the floats of weft are severed at or near the centre, thereby producing tufts of pile, which rise on each side of binding warp threads and form the characteristic rounded ribs of pile.

The rounded or convex formation of cords in corduroys is entirely due to floating weft being cut at unequal distances on each side of binding points, thereby causing each complete tuft to be formed with a long and short tuft (thus J). This will be easily understood on examining Figs. 379 and 380 , in which are indicated the points at which floats of weft are severed by the fustian knife. In Fig. 379 the binding points of pile picks occur in the same order for each rib, thereby producing all floats of the same uniform length, and causing each cord to constitute one repeat of the design: whereas in Fig. 380 the binding points of

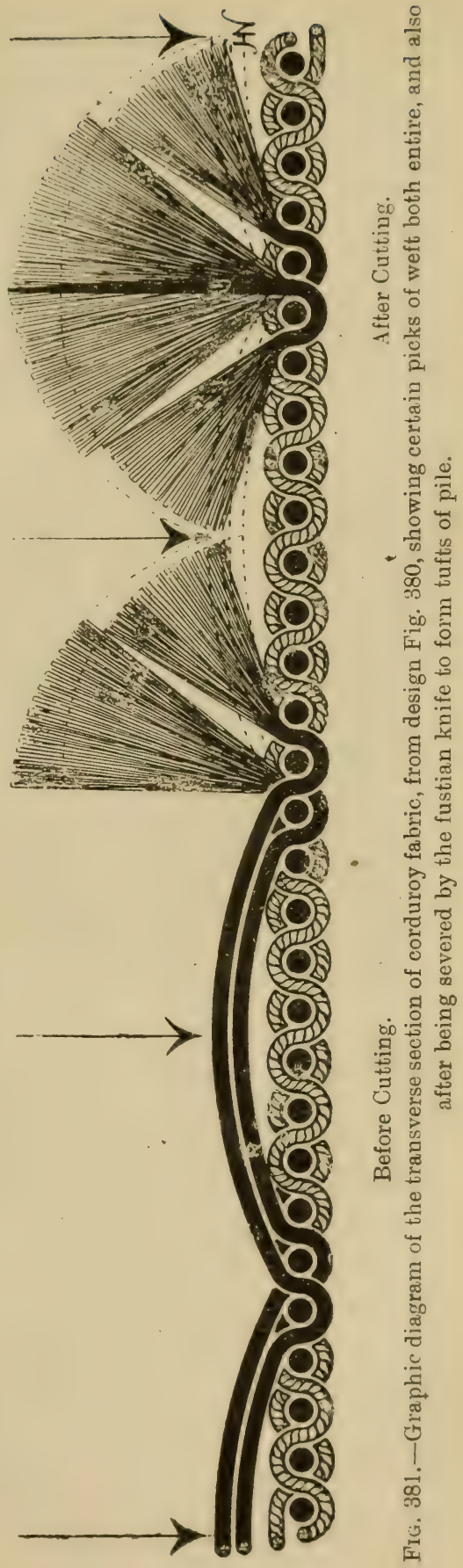


any two contiguous ribs are in reverse order, thereby producing floats of two unequal lenyths and causing two cords to constitute one repeat of the design. In the first example all floats will be cut a little out of the centre, and in the second example they

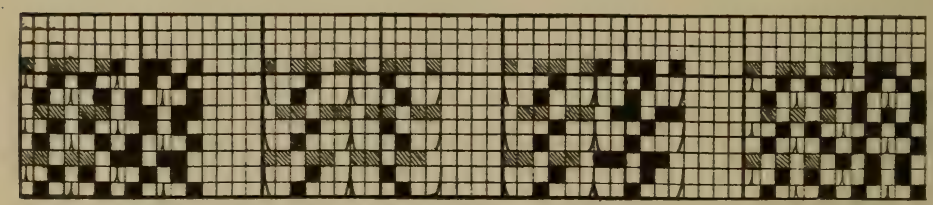

FIG. 382.

FiG. 383.

Frg. 384.

FIG. 385.

will all be cut cxactly in the centre; ret, in both instances, each complete tuft will be formed with a long and short tuft with precisely similar results, notwithstanding the two different methods of binding pile weft.

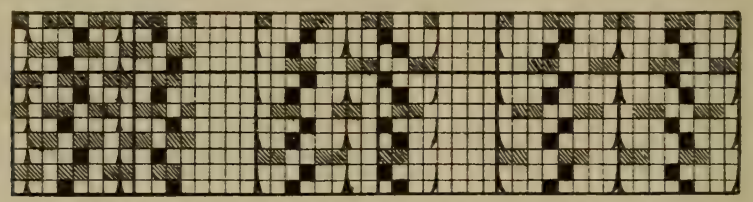

FIG. 386.

FIG. 387.

FIG. 388.

$\$ 76$. Fig. 382 is the simplest and smallest design for a corduroy fabric, commonly termed " thickset" cord. It repeats on six warp threads and nine picks, and has a foundation texture based on the three-end twill weare, with two pile picks to one

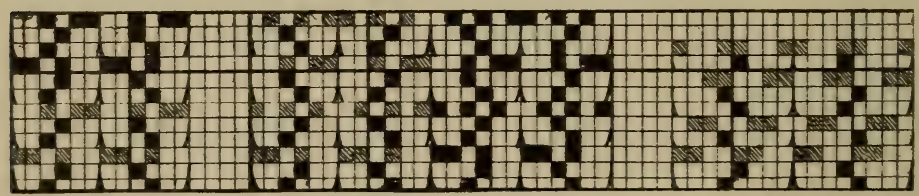

FrG. 389.

Fig. 390.

Fig. 391.

ground pick. The floats of weft are very short--being over only three warp threads - thereby producing a short stubby pile, the tufts of which are firmly bound in the ground cloth, after the manner of "lashed" pile, described under the head of relveteens in $\$ 69$. Figs. 383 to 397 are other designs for corduroys showing 
various modifications in their construction as regards their foundation weares, widths of cords, ratios of pile and ground

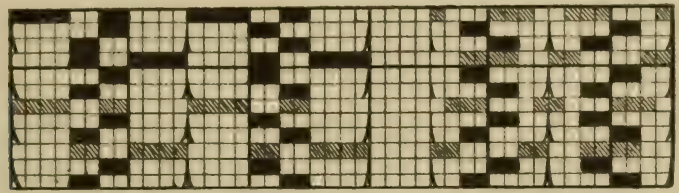

Fic. 392.

Fig. 393.

picks, methods of binding pile weft, and many other interesting features that will become manifest to observant students and

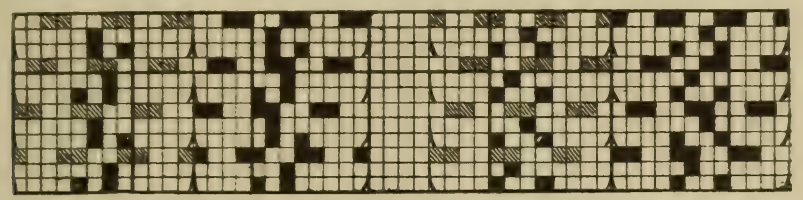

Fig. 394.

FIG. 395.

which it will well repay them to investigate. The irregular method of binding, exemplified in Figs. 394 to 397, is for the

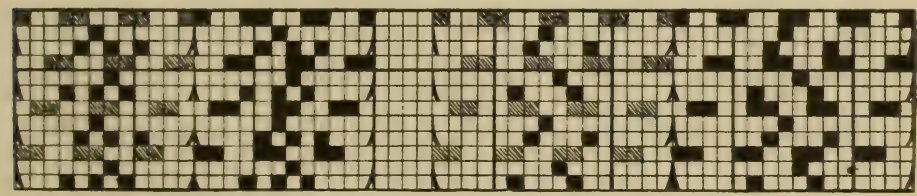

FIG. 396.

FIG. 397.

purpose of producing a variety of different lengths of floats, which, after cutting, will produce various lengths of pile, and thereby derelop cords having a much rounder formation.

\section{Figured Corduroy Fabrics.}

$\$ 77$. Fabrics of the variety of corduroys known as "velvet cords" are sometimes figured on a similar principle to that which obtains in some figured velveteens, namely, by causing pile weft to float on the face in the usual manner where it is required to form figure, and to interweave it at the back, in the ground portion, in an opposite manner. An example of figured 
"velvet cord" constructed on this principle is reproduced in Fig. 398, with the method of preparing a design for the same, shown (in part) in Fig. 399. This example has a foundation texture of plain cloth, with four pile picks to one ground pick, and with each rib extending over six warp threads. In conse-

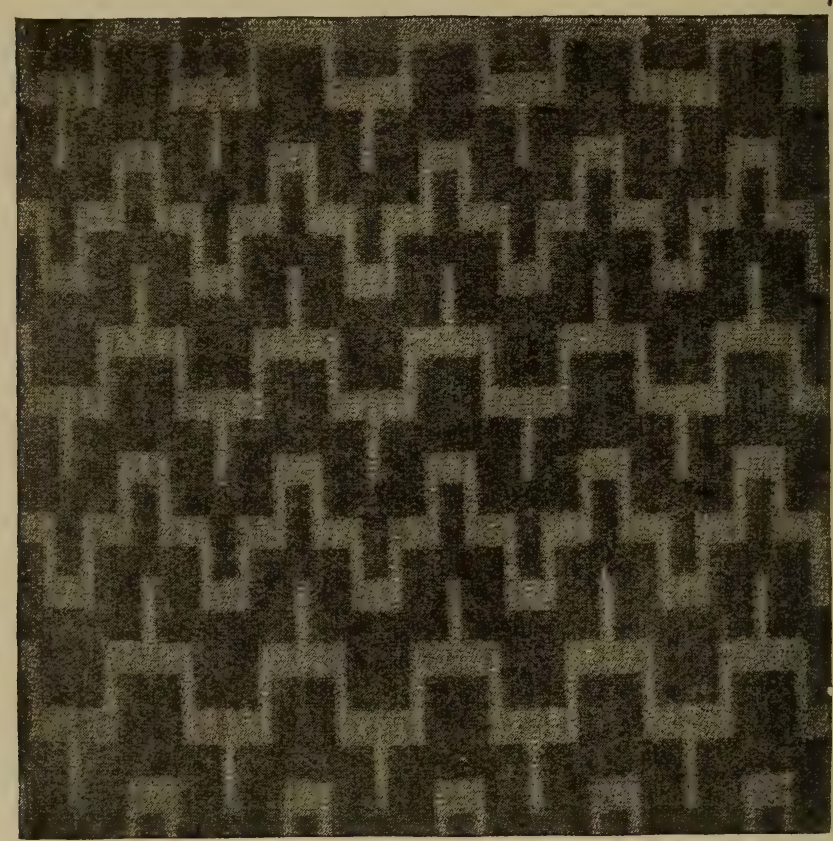

FIG. 398,-Figured corduroy, from design Fig. 399.

quence of the ornamentation of these fabrics being developed by a series of straight ribs occurring at regular intervals, it should be of a very simple character, consisting entirely of straight lines, as it would be impossible to satisfactorily develop curved lines upon them.

\section{Corduroy Cutting.}

$\S 78$. The cutting of corduroy fabrics is performed, as previously stated, sometimes by hand, similarly to that employed for velveteens (as described in $\$ 65$ and 66 ); but perhaps more 
extensively by machinery, as their coarser and stronger texture renders them better adapted than velveteens to mechanical cutting. Fustian-cutting machines comprise various modifications of two distinct types, known as "circular-knife" and "straightknife" machines. A graphic full-scale diagram illustrating the

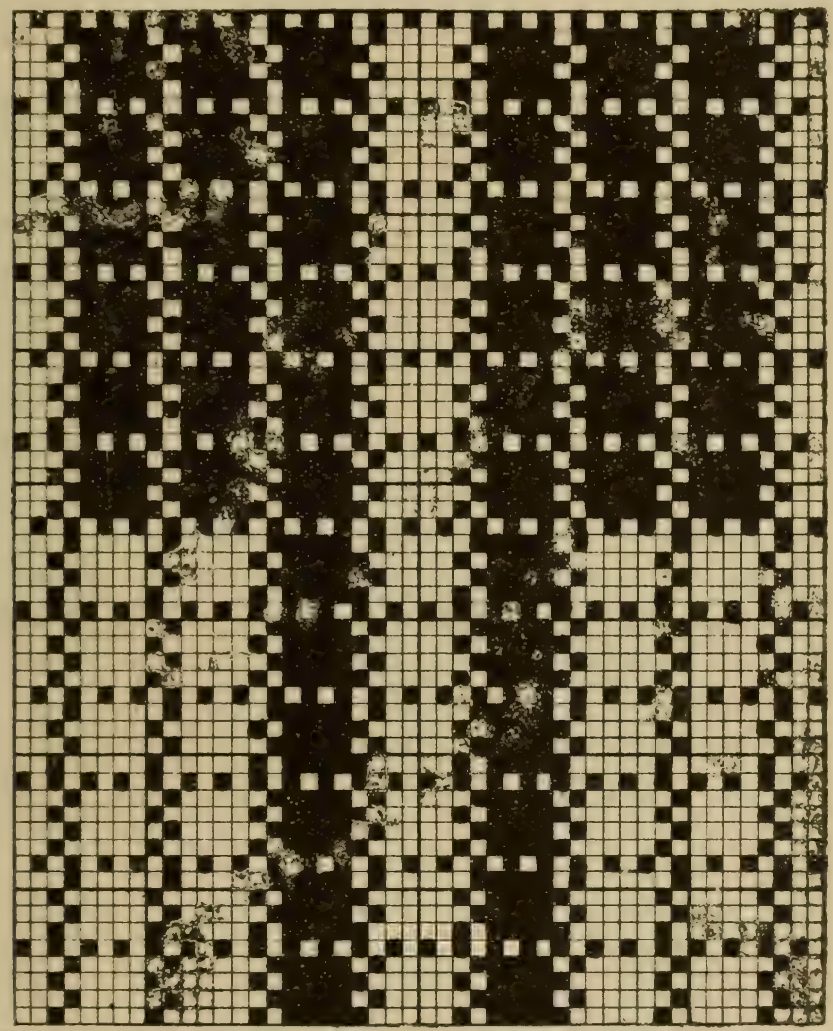

Fig. 399.-Design for figured corduroy fabric, Fig. 398.

operation of a "circular-knife" machine is shown in Fig. 400. In this machine all cords across the entire width of cloth are cut simultaneously by means of a corresponding number of thin sharp-edged steel discs B, placed at regular intervals (coinciding with the width of cords) upon a mandrel A, which extends across the machine and revolves with considerable velocity in 
the direction indicated by an arrow $\mathrm{D}$. As the knives revolve, cloth advances towards them in the direction indicated by an arrow $\mathrm{F}$, to a point $\mathrm{H}$, where it is sharply deflected over the bevelled edge of a cross-rail G. At this point, floats of weft forming each "race" are directed and presented by means of

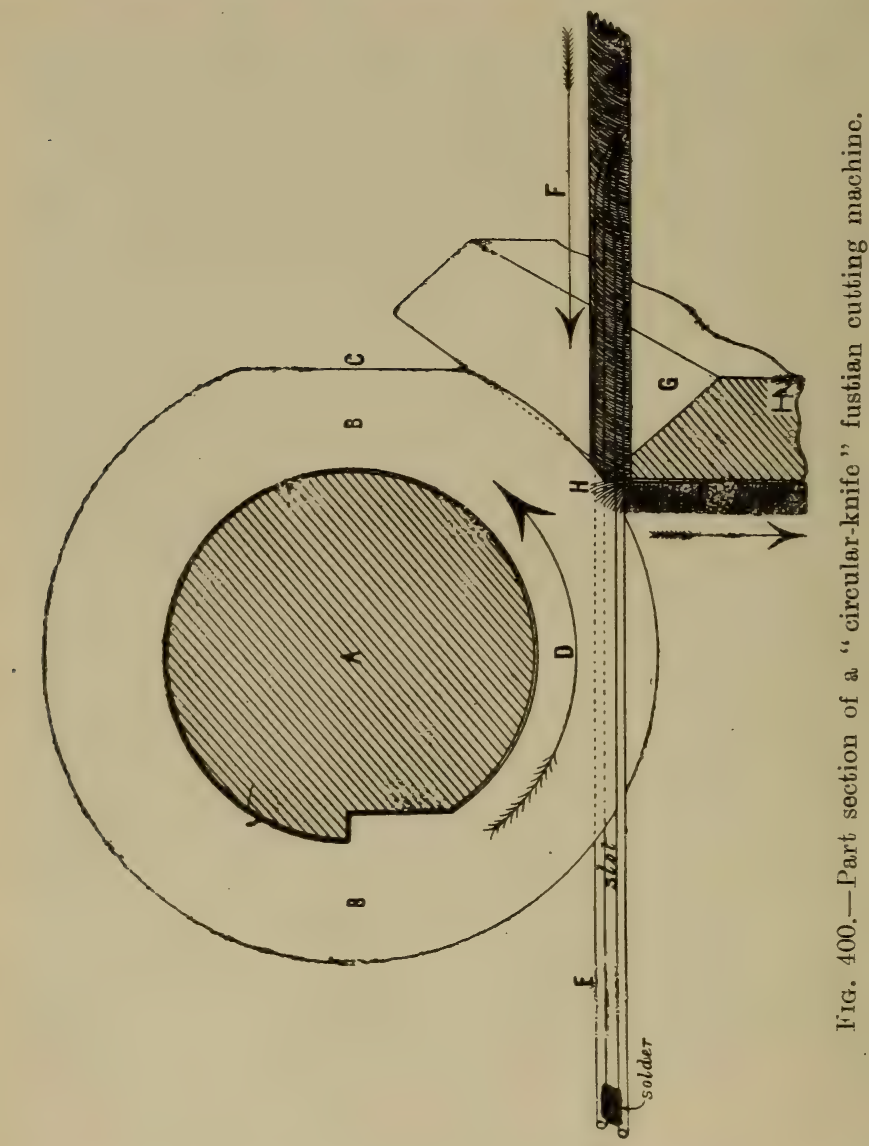

guide wires $\mathrm{E}$ to their respective knives to be cut. A guide wire $\mathrm{E}$ is inserted in each "race," with the lower portion of a knife partially entering its long narrow slot. Guide wires $\mathrm{E}$ are pieces of steel wire bent acutely to form a long loop. The extremities of the wire are soldered together where they meet, whilst the 
curved end is thattened and slightly bent as shown. They are inserted in the "races" of cloth with the point downward and pointing in the opposite direction to that in which cloth approaches the knives. Guide wires serve the functions of (a) guiding floats of weft forming a "race" to the knives, and tautening them as they are cut; and (b) keeping the knives (which are not fixed rigidly, but we placed somewhat freely upon the mandrel) in the centre of each "race". A small segment is cut off each knife, as shown at C', to reduce their diameter at that part. Thus, by turning the mandrel until the straight edges $\mathrm{C}$ of the knives are at the cutting point $\mathrm{H}$, it allows a little greater space between the knives and the rail edge, thereby facilitating the insertion of guide wires in the "races" at the commencement of cutting, or subsequently for the replacement of wires that may become injured.

As the uncut cloth approaches the knives, guide wires are conveyed along by it, and consequently require to be pushed forward again intermittently. This is accomplished by means of pushers actuated at frequent and regular intervals by a series of spirally arranged rotary cams. Each pusher acts upon three or more guide wires (according to the width of cords) at their soldered ends, so as to push them forward in groups instead of collectively, thereby ensuring greater constancy of action by preventing extreme fluctuations of energy exerted by the machine, and also of motive power required to drive it.

79. Fustian-cutting machines of the second-named type are constructed either with four stationary knives or one stationary knife, to cut four cords simultaneously, or only one cord at a time respectively. In either case, the extremities of cloth to be cut are sewn together to form an endless band or web, which is passed through the machine at a rapid pace as often as is required to complete cutting. After each complete circuit of the entire length of the cloth, the machine is stopped, and the knife or knives adjusted by hand to cut the next "race" or " races". In a four-knife machine, the knives (which are similar to those employed in hand cutting) are fixed at intervals corresponding to one-quarter of the width of fabric to be cut, and operate in a similar manner to a hand fustian knife, excejting that the cloth 
adrances upon the knives in the former, whilst in hand cutting a knife is thrust along, cloth which is held stationary. The adjustment of the knives in these machines is so contrived that, on their leaving a "race," penetrating cloth, meeting with any obstruction, or from any other irregularity which constitutes an impediment, the machine stops automatically. 


\section{CHAPTER VIII.}

\section{TERRY AND LOOP PILE FABRICS.}

$\$ 80$. "Terry pile" is a term used to distinguish a variety of woren fabrics that are characterised by the formation of a series of loops (thus $n$ ) projecting from the main body of the fabric. These loops are formed by an extra series of comparatively slack warp threads, and may be distributed uniformly either on the tace side only; or on both the face and back of the fabric, to form a perfectly eren surface; or the loops of pile may be developed in such manner as to create a figured design upon a plain or simple ground. Or, again, a figured terry fabric may contain an all-orer pile surface on both the face and back, with the figure and ground portions dereloped in contrasting colours of pile warp threads.

A terry or loop pile surface may be developed, during the operation of wearing, by each of three distinct methods, namely: (1) By means of wires that are inserted in the warp sheds at interrals (as if they were picks of weft) and subsequently withdrawn, thereby causing all warp threads that passed over them to torm a corresponding number of loops; (2) By means of what are known as "terry pile motions," whereby, during weaving, sereral picks of weft are inserted a short distance from the "fell" of the cloth (or last pick inserted), to produce a short salp or "fret," after which they are all pushed forward together to take their final place in the fabric. (As each group of picks are thus pushed forward by the reed, pile warp threads buckle or loop either on one side only or on both sides of the cloth as predetermined, and so develop the characteristic loops of pile known as "terry," loop, or uncut pile); and (3) By interweaving pile warp threads in such a mamer that a looped or terry pile may be produced in an ordinary "fast-reed" loom without 
employing a special "terry pile motion," and in which each successive pick of weft is beaten-up to the "fell" of the cloth, as in the production of a fabric of ordinary construction, and in the manner described subsequently in $\$ \$ 97,98$ and 100 .

Examples of looped pile fabrics produced by the aid of wires are seen in Brussels and warp-printed tapestry pile carpets, moquette pile, mohair and other furniture upholsterings of comparatively heavy texture, as well as in silk upholsterings of much lighter texture and great beauty. In fabrics of this description the pile is formed on one side only, and (with the exception of tapestry pile carpets) pile warp threads may sometimes lie perfectly straight or interweave as ordinary warp threads, and then be required to form loops of pile, all within a short interval. Under these circumstances, pile warp threads are severally contained upon a corresponding number of bobbins that are separately weighted to permit of the independent withdrawal of their threads, and thus provide for their variable and irregular contraction. If all pile warp threads were contained on the same warp beam, they would, of necessity, either be required to form pile or else lie straight, uniformly, at the same time, in consequence of their uniform and simultaneous delivery during weaving.

$\$ 81$. Terry fabrics produced by means of terry motions are exemplified in so-called "Turkish" towels, bath mats, counterpanes, antimacassars, toilet covers and mats, and many other articles employed for domestic purposes, and in which the loops of pile occur with a more or less dense or close formation. The majority of these goods are produced entirely from cotton, although terry towels are sometimes produced either entirely or in part from linen. Terry weaving is a principle eminently adapted to the production of towels, as the loops of pile give considerable bulk and impart good absorptive properties to the fabric. The variety of terry fabrics under present notice are produced from two distinct series of warp threads, namely: $(a)$ ground warp threads, and $(b)$ pile warp threads, each of which is contained on a separate warp beam. They are usually employed in equal proportions, and arranged in the harness and reed either alternately with each other, or else in alternate pairs of ground and pile threads. 
The particular disposition of war'p threads, however, is quite optional. Some advocate an alternate distribution of ground and pile warp threads, whilst other's prefer to dispose them in alternate pairs of each series of warp threads. In both cases the ultimate results are virtually alike. During weaving ground warp threads are held taut, whereas the warp beam containing pile warp threads, is very lightly weighted to enable those threads to be easily withdrawn for the formation of loops of pile. Terry fabrics are termed three, four, five or six-pick terry fabrics, according to the number of picks inserted for successive horizontal rows of loops. Most of these fabrics are constructed with only three picks for each row of loops. The object of inserting a greater number of picks for each row of loops is to produce a superior fabric and to bind pile rarp threads more securely to the foundation texture.

\section{Terry Pile Motions.}

$\$ 82$. Before describing the construction of terry fabrics, however, it will be helpful briefly to describe the essential features of terry-forming devices, as this course will the more readily conduce to a clearer conception of the essential conditions governing the construction of this type of fabrics. Terry pile motions are usually based upon one or other of three distinct mechanical principles; but the greater number are constructed on what is known as the "loose-reed" principle, as represented in Fig. 401. If these devices are based upon this principle, they are designed to act upon the reed in such manner that, as the sley advances, the reed is caused to swing or fall backward, at the bottom, from its normal vertical position to an inclined position, for two only out of three or more picks, after which the reed is securely fastened in its normal position for the following pick (or picks), when all are thrust forward together to take their final place in the cloth. As the picks are thus thrust forward from their temporary to their final position, they slide along the tense ground warp threads; but the degree of frictional resistance between the three picks and slack pile warp threads is sufficient to draw the latter forward en masse, thereby causing them to benul and thus form a series of loops to constitute the pile surficer. 
As the reed swings backward at the bottom, for the two "loose" picks, it swivels on the upper ribs which are retained in a mortise cut into the under side of the sley cap. Therefore, since the line of contact made by the "fell" of cloth with the reed is situated approximately midway between the upper and lower ribs of the latter, it follows that the bottom of the reed will require to recede (for the two "loose" picks) for a distance of not less than twice the length of gap or "fret" necessary to yield the desired length of pile on the fabric. Such excessive backward movement of the reed creates a tendency to develop loops

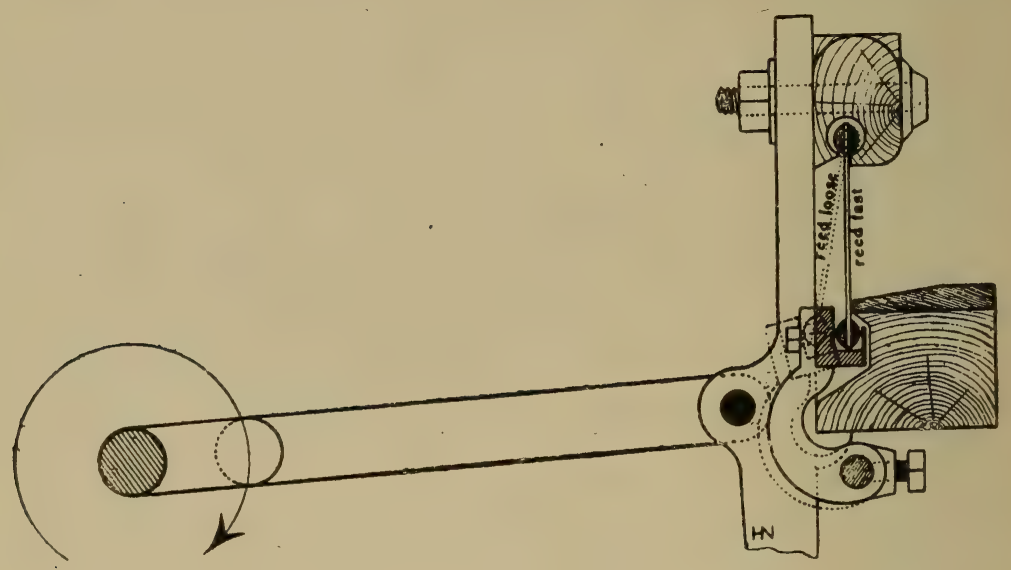

FIG. 401.-Loose reed action, for terry pile wearing.

of pile of irregular lengths in different horizontal rows of pile, but not in the.same horizontal rows. This tendency arises in consequence of the abnormal inclination of the reed from the normal vertical position, whereby it inclines forward at the top, and therefore bears downward upon the "loose" picks as it approaches the "fell" of cloth. Hence, those picks tend more or less to slide downward along the reed (according to circumstances to be stated presently), and thus produce gaps or "frets" of different lengths, and, therefore, different lengths of pile.

This evil is more liable to manifest itself in figured terry fabrics in which the number of pile warp threads either raised 
or depressed is liable to fluctuate according to the design. Thus, the "fell" of the cloth will occupy either a higher or else a

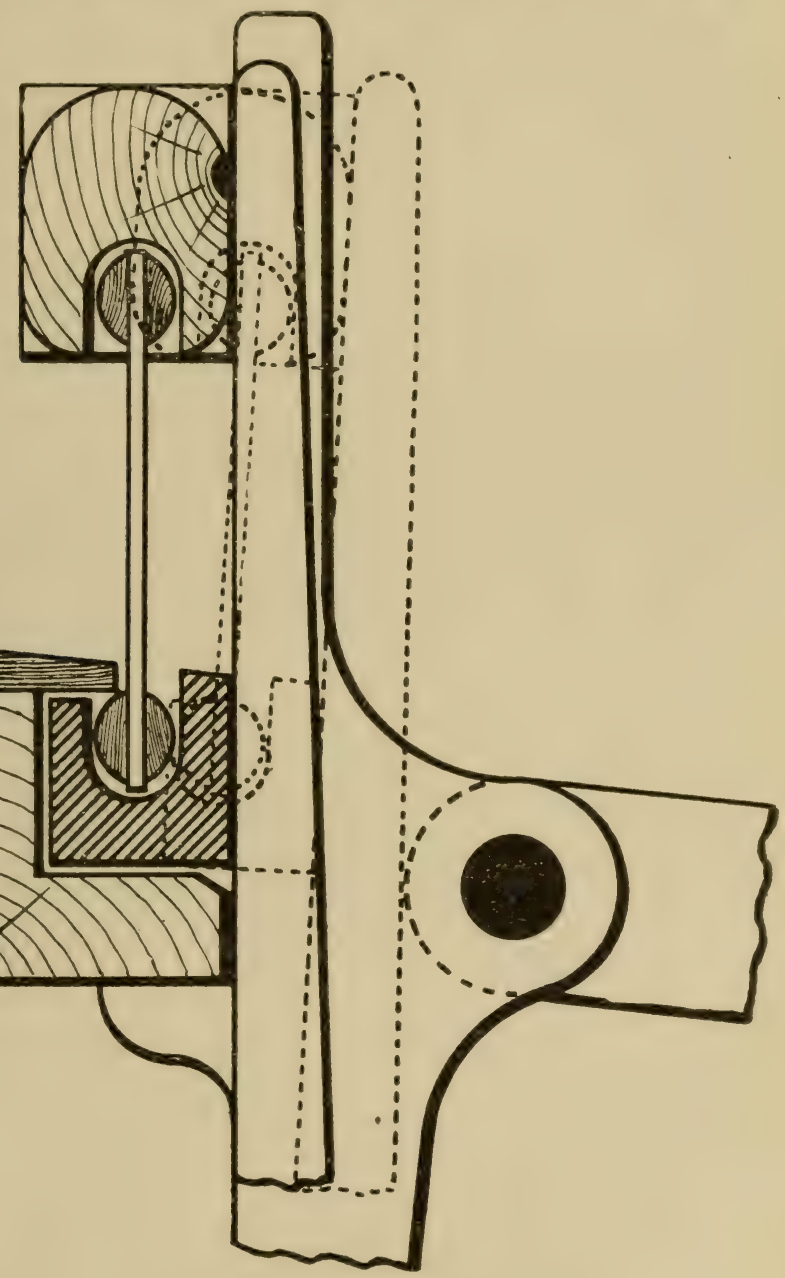

FIG. 402.-Loose reed action for terry pile weaving.

lower plane during beating up, according to the preponderance of warp threads in either the upper or lower half of the warp shed respectively; also, the strain upon the reed, as it approaches 
the "fell " of the cloth, will be greater or less in proportion to the number of warp threads forming the bottom half of the warp shed, because they bear against the reed farthest from the upper ribs on which it swivels in the sley cap.

\$ 83. A modification of the "loose-reed" principle of terry wearing, and one that is designed with the object of overcoming the disadvantages of the system just described, is effected by mounting the reed in a separate case or frame carried at the upper ends of two long rertical arms that extend downwards and are respectively fulcrumed either upon studs secured to the sley swords, or else upon the rocking shaft on which the sley oscillates, as illustrated by a graphic diagram in Fig. 402 . The said arms are analogous to auxiliary sley swords for the sole purpose of supporting the reed only, so that the latter may be carried bodily backward, with the least deviation from its normal vertical position, when beating up the two "loose" picks to produce the desired length of gap or "fret" at the "fell " of the cloth. After the two "loose" picks of weft are inserted, however, the reed-case, containing the reed, advances to its normal forward position in which it becomes locked rigidly in order to thrust the two "loose" picks, along with the first "fast" pick, forward, to take their final position right up to the "fell" of the cloth, and thus withdraw the slack pile warp threads to produce the loops of terry pile in the manner described.

Instead of supporting the reed-case at the upper ends of auxiliary arms that are hinged in the manner just described, an alternative method is to slide the reed-case and reed backward, for the two "loose" picks, and then forward, in a perfectly horizontal direction, whereby the reed is maintained always in a true vertical position instead of inclining backward either from the lower or the upper edge, as indicated in Figs. 401 and 402 respectively.

\section{Holden's Terry Motion,}

$\$$ 84. A second type of terry pile motion is designed to oscillate the sley for a shorter distance for the two consecutive "loose" picks, and for a greater distance for the third and following "fast" picks inserted for each horizontal row of loops. 
By this means, two out of three or more picks are beate'n up within a short distance from the "fell " of eloth, and then after. the third pick is inserted in the warp shed, the three picks are thrust forward together as described, although the reed remains fixed in the loom sley; as in a "fast-reed" loom. This is effected in the manner illustrated in Fig. 403, which represents Holden's terry motion designed with the object of imparting to the loom sley and reed a variable length of stroke when beating-up the "loose" and "fast" picks respectively: By this method the connecting-pins $C$ ' of the comnecting-arms B are inserted quite treely in rertically slotted ears $\mathrm{D}$ of the sley swords $\mathrm{E}$ in which

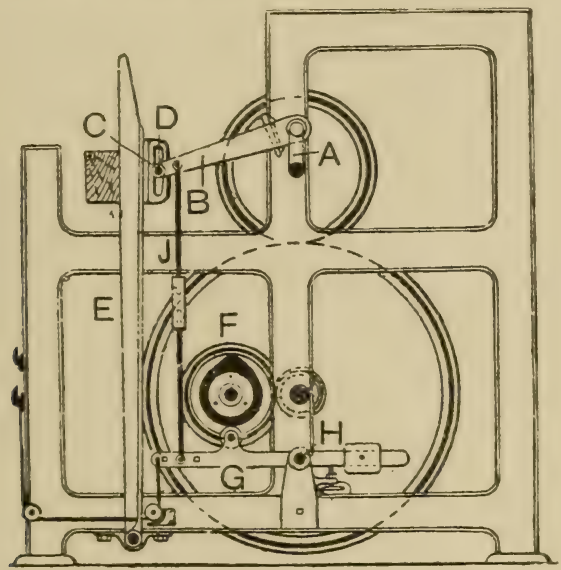

FIG. 403.-Holden's terry motion.

those pins rise and fall, and so virtually increase or decrease the length of the sley swords to impart a shorter" or a longer stroke to the loom sley when beating-up the "loose" and "fast " picks respectively. The vertical sliding movement of the connectingpins $\mathrm{C}$ is governed by means of tappets or cams $\mathrm{F}$ which act upon weighted treadle-levers $G$, fulcrumed on studs $H$, and from the forward ends of which levers' motion is transmitted through the medium of connecting link-rods $J$ to the connecting-arms $B$ of the loom cranks $A$, as indicated in the diagram. By changing the point of connection between the connecting link-rods.J and the treadle-lever $G$, the variation in the stroke of the loom sley may, within certain limits, be regulated so as to produce loops 
of terry pile of different length uniformly. Also, the movement of the loom sley may be adapted to the production of terry fabrics of any variety by employing tappets $F$ that are designed and driven according to the number of picks to be inserted for each horizontal row of loops of pile as, for example, 3-, 4-, 5and 6 -pick terry fabrics.

\section{Lister and Carter's Terry Motion.}

$\$ 85$. A third type of terry pile motion, and one that is designed to operate in an exactly contrary manner to either of the

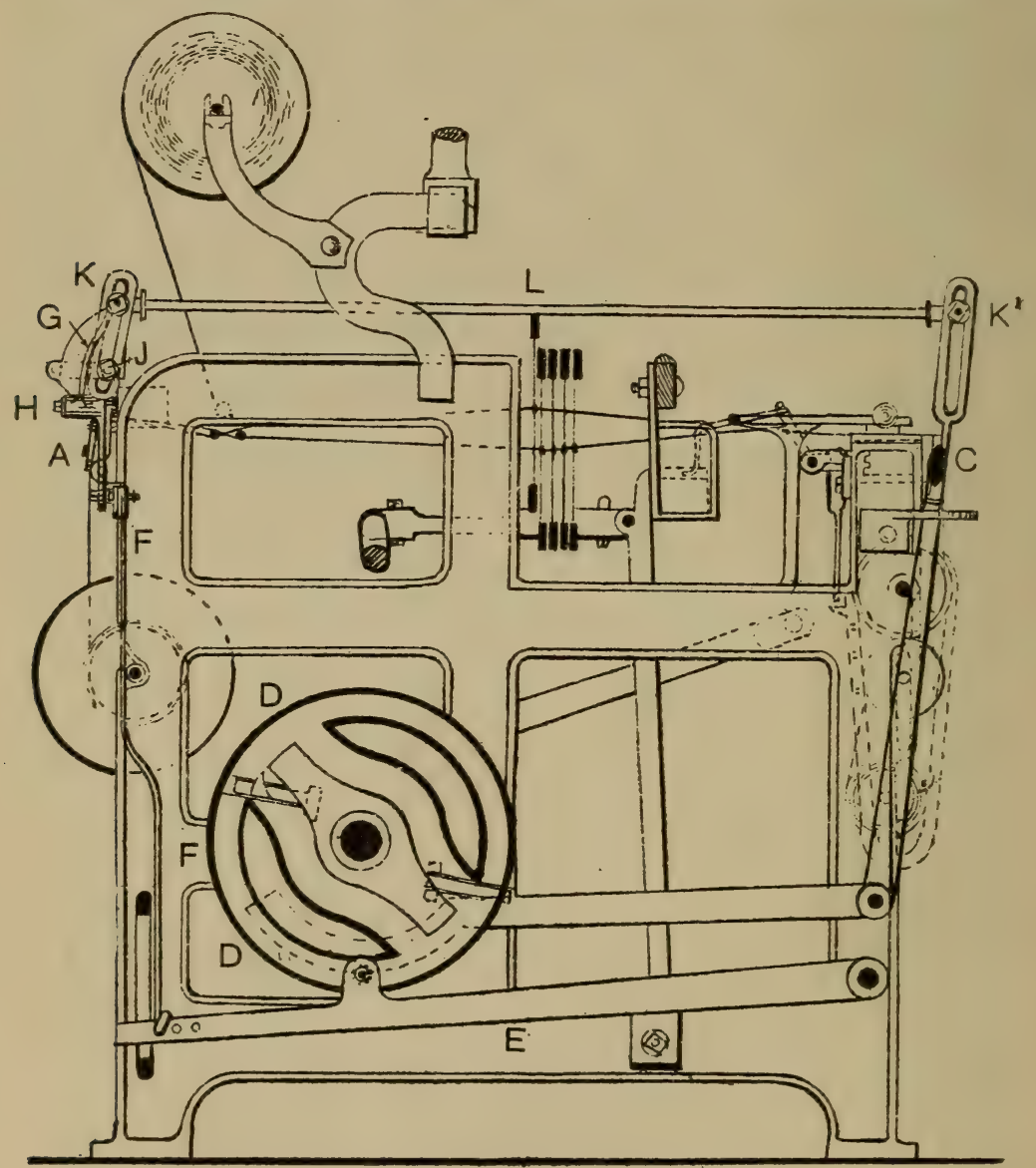

FIG. 404.--Lister and Carter's terry motion. 
two types just described, is that of Lister and Carter's, as illustrated in Figs. 404, 40:5 and 406 . Thus, instead of causing the

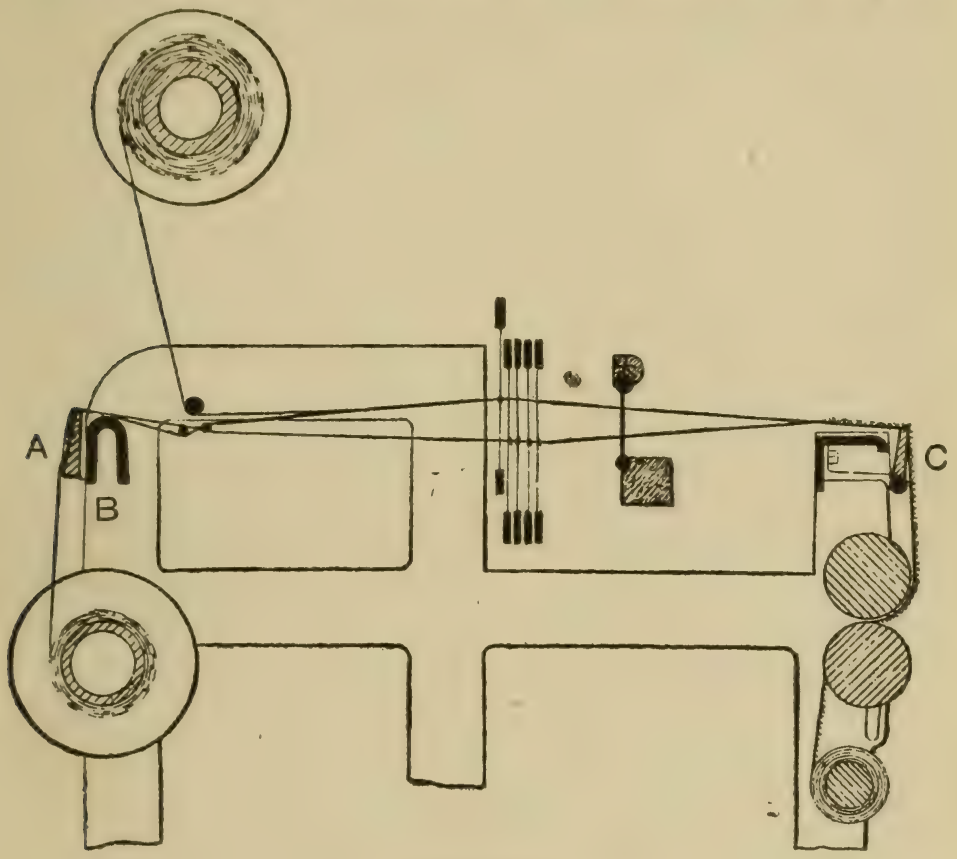

Fig. 405.

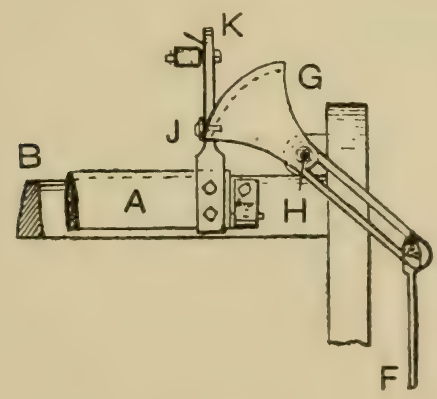

FIG. 406.

reed to swing backward for a short distance in order to produce a gap or "fret" at the "fell " of the cloth, the latter, along with 
the entire stretch both of ground and pile warp threads, is drawn forward bodily for a short distance in advance of the reed, when beating-up the two "loose" picks of weft, whilst the reed is mounted rigidly in the loom sley, as in a "fast-reed"

- loom. This object is effected by passing the warp threads over a rocking-bar A immediately behind the fixed "back-rail" B situated in the rear part of the loom, with the cloth passing over a similar rocking-bar C situated immediately in front of the front or "breast-rail," in the fore part of the loom, and also by oscillating both the front and back rocking-bars to and fro simultaneously, in order to carry the entire stretch of all the warp threads and cloth forward, bodily, for two out of three or more picks of weft, after which the two rocking-bars return to their normal position, thereby causing all the picks of weft to be thrust forward by the reed to take their final place in the cloth.

The intermittent reciprocal movement of the front and back rocking-bars $\mathrm{A}$ and $\mathrm{C}$ is governed by a grooved tappet $\mathrm{D}$ which operates through the medium of a long side lever $\mathrm{E}$ and a connecting link-rod $\mathrm{F}$, of which the upper end is connected to the end of a segment cam lever G fulcrumed on a stud $H$ and formed with an inclined beaded rim edge that bears preferably against a small anti-friction bowl or runner mounted on a stud $J$ fixed in one of two similar slotted arms $K$, secured one at each end of the rear rocking-bar A, as indicated by a rear view of these parts represented in Fig. 405. Two similar slotted arms $K, K^{\prime}$ are also secured one at each end of the front rocking-bar $\mathrm{C}$ with which the rear rocking-bar communicates by means of two long connecting link-rods $\mathrm{L}$, of which there is one at each side of the loom, to ensure a perfectly parallel movement of those rocking-bars as these advance whilst the two "loose" picks of weft are inserted, and also as they return for the "fast" pick or picks of weft, in the manner described.

\section{Dugdale's Terry Motion.}

$\$ 86$. The prevailing forms of terry pile motions, however, are based chiefly on the "loose-reed" principle, as illustrated in Fig. 401, and of which there are many modifications that are 
usually constructed so that the intermittent release of the reed may be governed optionally by means of a tappet or cam, a dobby, or a Jacquard machine, according to the equipment of the loom. One of the simplest examples of this type of terry motion is that illustrated in Fig. 407, which represents Dugdale and Talbot's derice, in which either a cam or else a crank-pin A raises a "frog-" lever B intermittently for two out of every three or. more picks of weft, uniformly, according to the particular variety of terry fabric required. The function of the "frog." lever is to

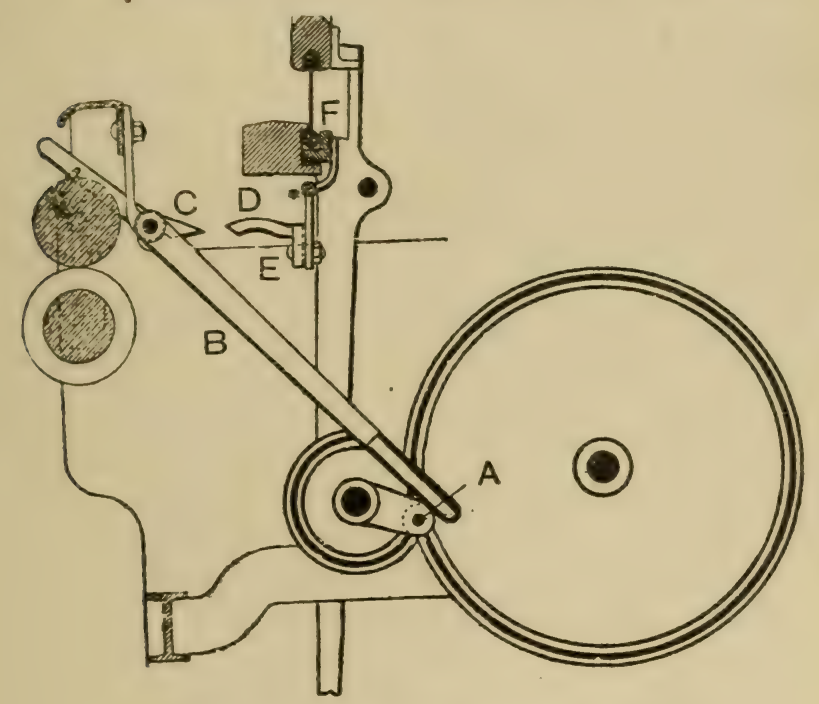

FrG. 407.-Dugdale's terry motion.

raise and depress a tapered "frog-blade" C, which is secured to that lerer near the point on which this is fulcrumed, and thus cause a "duck-bill " blade D, projecting from a bracket $\mathrm{E}$ depending from the reed-case F, either to pass above the "frog-blade" ( in order to release the reed for the two "loose "i picks of weft, or else cause the "duck-bill " D to pass underneath the "frog," and thereby lock the reed securely in position when beating-up the "fast" pick or picks of weft, to form the loops of terry pile, in the manner described in $\$ \$ 0$ and 82 .

If at any time it becomes necessary to stop the formation of 
the terry pile for the purpose of inserting "fancy headings," as in Turkish towels, or for other purpose, the terry motion is put out of operation simply by depressing the forward end of the "frog"-lever B and retaining it by means of a hook or catch fixed for that purpose. By this means the "frog"-lever B is held clear away from the lifting cam or crank-pin A whereby the "frog" remains constantly in the locking position until it is required to produce terry pile, when the "frog"-lever is again released from its retaining catch. Also, if the loom is equipped either with a dobby or a Jacquard machine, these may be employed to govern the terry motion in any manner according to the variety of terry fabric required. Or again, by an alternative method, the "frog"-lever B may be dispensed with, in which case the "frog" is fixed permanently in position on the loom framing, and the "duck-bill " $\mathrm{D}$ is then governed so as to control the action of the reed in the manner desired.

\section{Smith's Terry Motion.}

$\$ 87$. Another modification of the loose reed type of terry motion is that illustrated in Fig. 408, which represents Hobson and Smith's device in conjunction with a simple method of controlling the delivery of pile warp threads from the beam. The action of the reed may be controlled either by a spare hook of the Jacquard machine, or else by a " jack"-lever A of the dobby as indicated in the diagram. Thus, through the medium of a chain B the " jack"-lever either raises or depresses an elbow "frog "-lever $\mathrm{C}$ in a prescribed manner, in order either to release or else lock the reed when this is beating up the "loose " and "fast" picks of weft respectively. This object is effected by securing the lower reed-case to two or more bracket arms $D$ that are setscrewed to a rod $\mathrm{E}$ which extends across the loom and is supported freely in brackets that are bolted to the sley swords F. On one end of this rod $\mathrm{E}$ there is set-screwed an arm $\mathrm{G}$ formed with a slotted end in which there is fixed an adjustable stud carrying a small bowl or runner $H$. Thus, as the sley advances, the bowl passes either above or else below the horizontal tapered and curved arm of the "frog"-lever $\mathrm{C}$ and thereby either locks 
TERRY ANT LOOP PILE FABRICS.

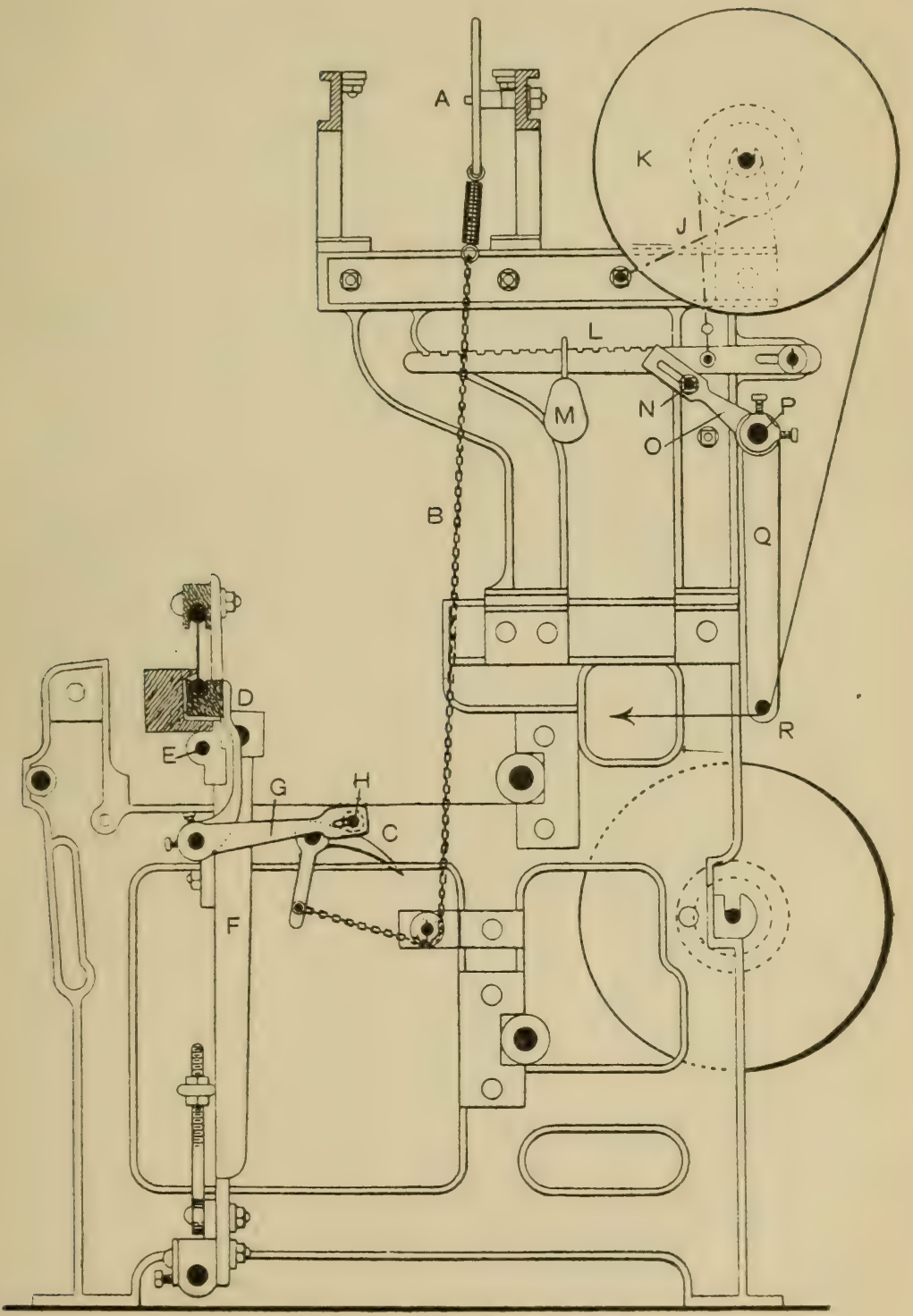

FIG. 408.-Smith's terry motion. 
the reed securely in its normal forward position, when beatingup the "fast" pick or picks of weft, or else the reed moves backward, at the bottom, when beating-up the two "loose" picks of weft respectively.

The length or depth of the loops of terry pile may be regulated by adjusting the position of the runner $\mathrm{H}$ in the slot at the end of the arm G, and also by adjusting the position of the stud on which the "frog"-lever $\mathrm{C}$ is pivoted, in order to vary the angular movement of the arm $G$, and thereby cause the reed to swing backward for a shorter or a longer distance and so produce a narrower or a wider gap or "fret" at the "fell " of the cloth, according to the depth of loops required.

The delivery of pile warp threads is controlled by passing a band-brake J around a large iron "ruffle" or pulley fixed on one end of the pile warp-beam $K$, and attaching one end of the bandbrake to a lever $\mathrm{L}$ containing an adjustable weight $\mathrm{M}$ for the purpose of regulating the degree of tension upon the pile warp threads during weaving. Bearing constantly against the lower edge of the weighted lever $\mathrm{L}$ there is preferably a small bowl, mounted on a short stud $\mathrm{N}$, fixed adjustably in the slotted end of a short arm $\mathrm{O}$, secured on one end of a strong iron bar $\mathrm{P}$ extending across the entire width of the loom. To this cross-bar there are also secured two similar long pendant arms $Q$, of which the lower extremities support a yarn tension-bar $R$, under which the pile warp threads are deflected in their progress from the beam towards the front of the loom. Hence, the vibration of the tension-bar $R$, caused by the varying tension to which the pile warp threads are subjected during weaving, releases the bandbrake $J$, and thereby liberates the warp beam automatically in order to deliver the pile warp threads more freely when the two "loose" picks and the first "fast" pick of weft are being beaten up to produce the loops of terry pile in the manner described previously.

\section{Hacking's Terry Motion.}

\$88. A third modification of the "loose-reed" type of terry pile motion is that illustrated in Figs. 409, 410 and 411, representing Hacking and Hilton's device which may be controlled 
TERRY ANT LOOP PIIE FATIRICS.

1(เ.)

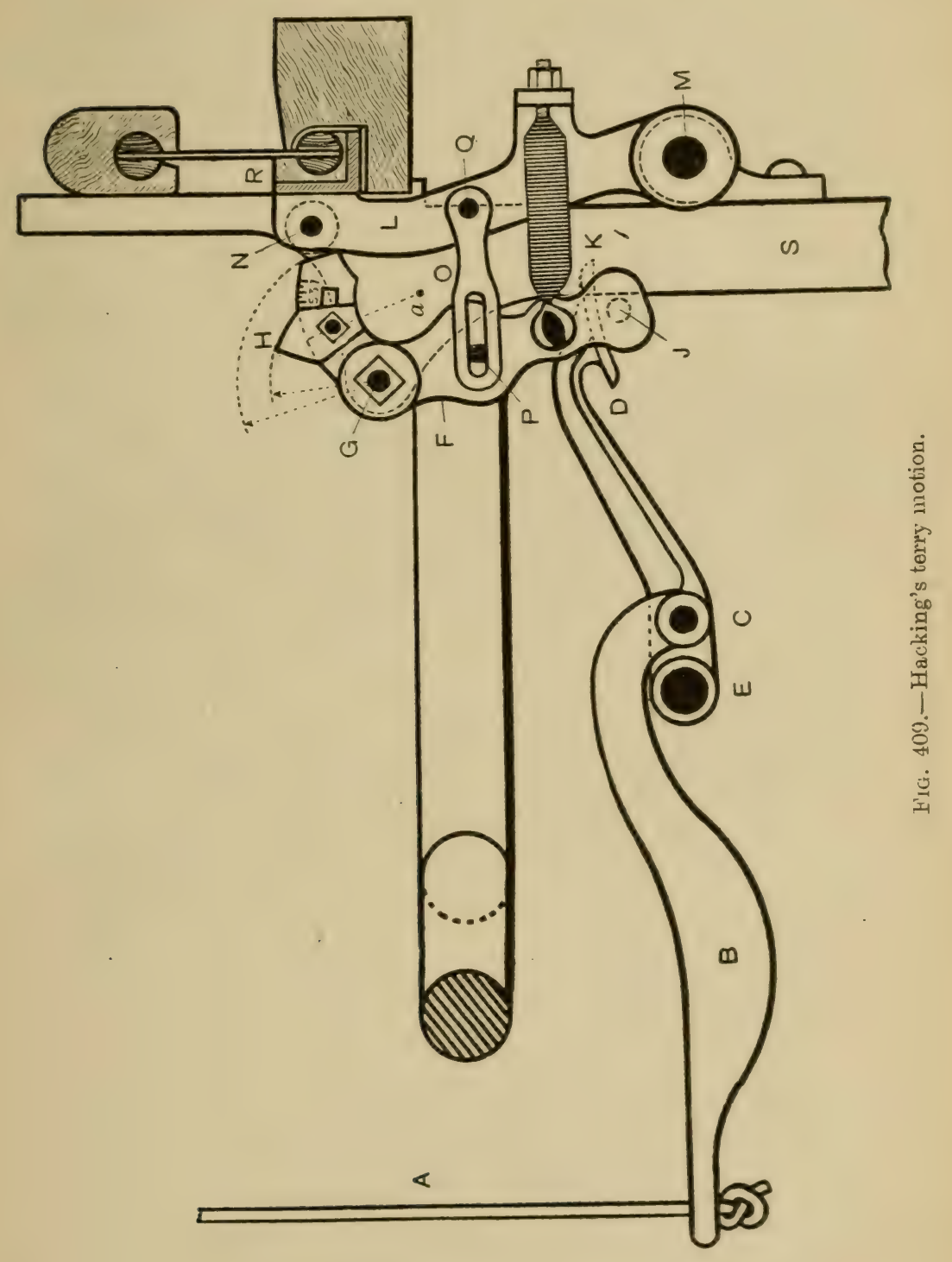


either by means of a dobby "jack"-lever or a hook of a Jacquard machine, as indicated in Fig. 409; or else by means of a simple cam or tappet, as indicated in Figs. 410 and 411, which represent the reed in the act of beating-up a "fast" and a "loose" pick of weft respectively. The cardinal features of this ingenious

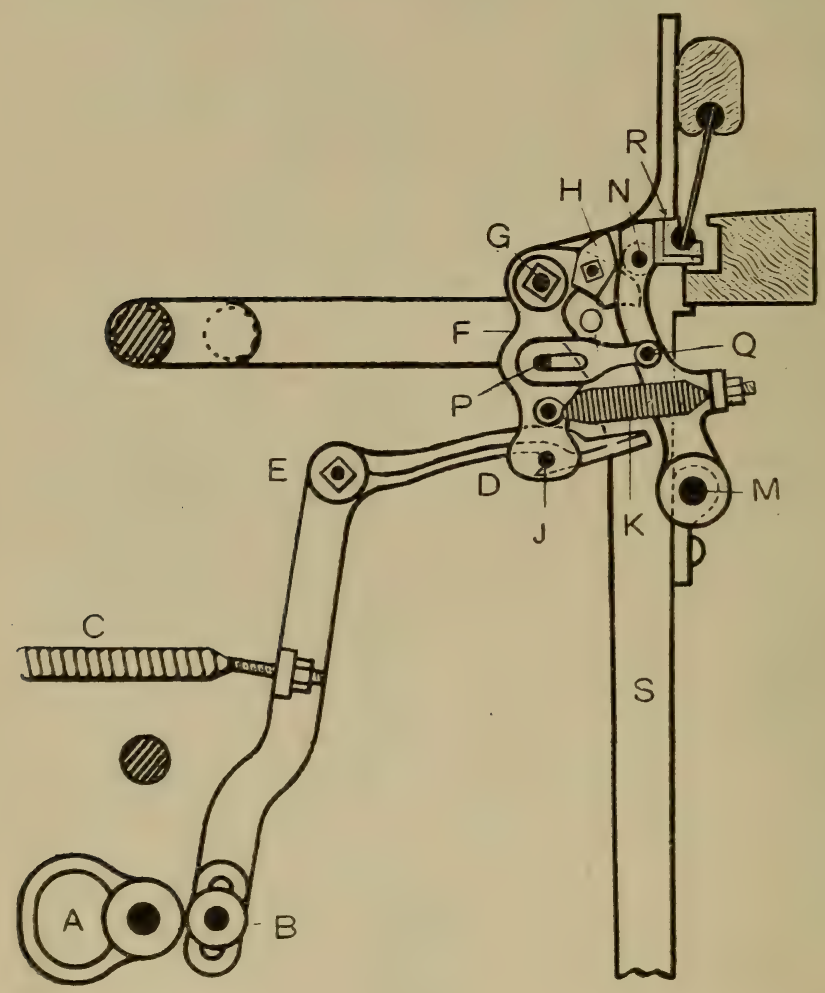

FIG. 410.

device consist essentially of an elbow cam-lever $\mathrm{F}$ fulcrumed on a stud G secured to the ear of one of the sley swords $S$, and of which the short horizontal curved arm is furnished with an adjustable stop-piece $H$. This elbow-lever, which is operated in a prescribed manner to govern the action of the reed according to the number of picks to be inserted for each horizontal row of loops in the terry fabric produced, is controlled in reverse 
directions from two independent sources, namely, $(a)$ by causing the hooked end of an arm D, which is fulcrumed on a fixed stud $\mathrm{E}$, to descend and thereby engage with a stud .J projecting on one side at the lower end of the rertical arm of the elbow-lever $\mathrm{F}$ which, as the sley adrances, is retained by that

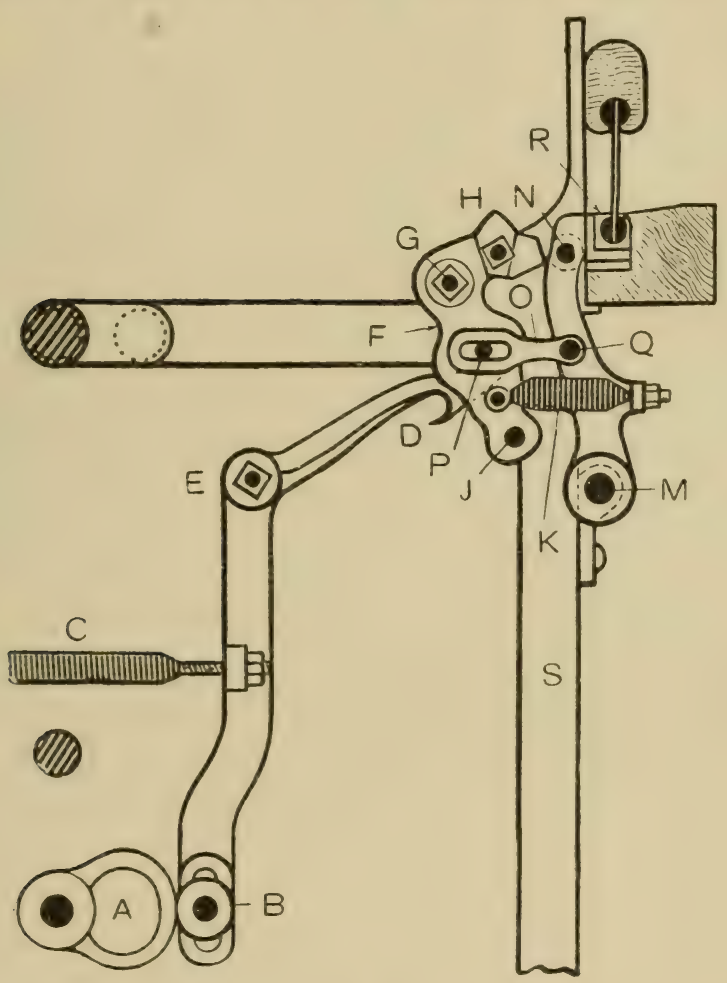

FIG. 411.

hook, thereby deflecting the horizontal curved arm of that lerer in order to liberate the reed, and, at the same time, exerting, through the medium of a spiral spring $K$, sufficient effort upon one of the arms $L$, supporting the reed-case $R$, to pull the lower edge of the reed backward, in the manner indicated in Fig. 411; and (b) by the reflex action of the spiral spring $K$ which, as the sler recedes, reacts upon the rertical arm of the elbow-lever $F$ 
and thereby causes the upper surface of the horizontal arm of that lever to bear against a small anti-frictional bowl $\mathrm{N}$ mounted on a stud secured to the lower reed-case $R$, which is thereby forced forward until the reed is in its normal position in which it becomes securely locked by reason of the end of the curved arm of the elbow-lever $\mathrm{F}$ impinging firmly against the small bowl $\mathrm{N}$ as indicated in Figs. 409 and 410 .

If the operation of this terry motion is governed either by means of a dobby or a Jacquard machine, as represented in Fig. 409, the hooked arm D constitutes one of two members of a double-jointed lever of which the other member is a weighted arm $\mathrm{B}$ hinged freely on a stud $\mathrm{C}$ passing through both arms at a point immediately in front of the stud $\mathrm{E}$ on which the hooked arm D is fulcrumed; whilst the free end of the weighted arm B is connected either to a dobby "jack"-lever, or a hook of a Jacquard machine. By hinging the two arms of the compound lever B, D on separate studs C, E, in the manner indicated, instead of casting both arms of that lever as one member, it affords greater latitude in the setting and timing of the shedding and terry motions, in relation to each other, with less risk of failure or breakage. If, however, this device is governed by means of a special tappet for that purpose, as represented in Figs. 410 and 411, the entire device is, therefore, self-contained and quite independent of the shedding motion, in which case the hooked arm D forms the horizontal arm of an elbow-lever fulcrumed on a stud $\mathrm{E}$, and of which the vertical arm is furnished with an anti-frictional bowl $\mathrm{B}$ that bears constantly, under the influence of a spring $C$, against the rim of a tappet A which performs one revolution during such number of picks as are, inserted for each horizontal row of loops of terry pile in the fabric produced.

The free movement of the reed, and therefore the length or depth of the terry loops, is determined by the position in which the adjustable stop-piece $H$ is fixed on the horizontal curved arm of the elbow-lever $\mathrm{F}$ which is graduated with the object of enabling the stopper to be adjusted with greater precision, according to the length of pile required, and which will either be longer or shorter by fixing the stopper $\mathrm{H}$ nearer to, or further 
from, the fulcrum $G$ of that lever respectively. Thus, on the deflection of the curved arm of the elbow-lever $\mathrm{F}$ to release the reed, the latter, under the influence exerted by the spring $\mathrm{K}$, immediately recedes until the small bowl $\mathrm{N}$ comes in contact with the stop-piece $\mathrm{H}$ when its further movement is arrested. The inclined surface of the stop-piece $\mathrm{H}$ against which the small bowl $\mathrm{N}$ abuts, forms a small are of a cirele which is concentric with the centre of the stud $G$ on which the elbowlever $\mathrm{F}$ is fulcrumed; whilst the curved arm of that lever forms the arc of a circle described from a point marked $a$, as indicated by dotted lines in the diagram, Fig. 409.

By producing the curvature of the stop-piece $\mathrm{H}$ so that it is concentric with the stud $G$ it thereby ensures the line of maximum resistance offered by that stopper against the small bowl $\mathrm{N}$, passing through the centres of that bowl and the stud $\mathrm{G}$ respectively. Also, as a precaution against the tendency of the bowl N, owing to the impulsive momentum exerted by the sley when beating-up the two loose picks of weft, to move forward out of contact with the stop-piece $\mathrm{H}$ and thereby involve the risk of producing loops of terry pile of irregular length, in different horizontal rows, there is provided a slotted link $\mathrm{O}$ which is hinged freely on a short stud $\mathrm{Q}$ projecting on one side of the arm L supporting the reed-case, whilst the slotted end of that link is penetrated by an adjustable stop-pin $\mathrm{P}$ fixed in the vertical arm of the elbow-lever $\mathrm{F}$ as indicated in the diagrams. Thus, by adjusting the stop-pin $\mathrm{P}$ to cause it to bear lightly against the extreme rear end of the slotted link when the small bowl $\mathrm{N}$ is in contact with the stopper $\mathrm{H}$, as indicated in Fig. 411, the stop-pin $\mathrm{P}$ will thereby prevent the reed from advancing until it is forced forward by the curved arm of the elbow-lever $\mathrm{F}$ only when this is released by raising the retaining hook $\mathrm{D}$, in order to lock the reed in its normal position, when beating-up the "fast" pick or picks, as described previously.

\section{Varieties of Terry Pile Fabrics.}

$\$ 89$. Fig. 412 is the design for a plain "three-pick" terry fabric with the pile formed on both sides, and in which the war'p 
threads are arranged in the order of one pile and one ground warp thread alternately, as represented in the design. In the production of terry piece-goods, such as roller towelling, not more than four healds are necessary, namely, two for each series of warp threads; but in the production of separate towels, with "headings" or borders, it is usual to employ five healds, namely, two (at the front) for pile warp threads, and three (in the rear) for ground warp threads, drafted in the manner indicated above the design. When pile and ground warp threads are disposed alternately, one thread from each series is passed between each successive dent of the reed; but when the warp

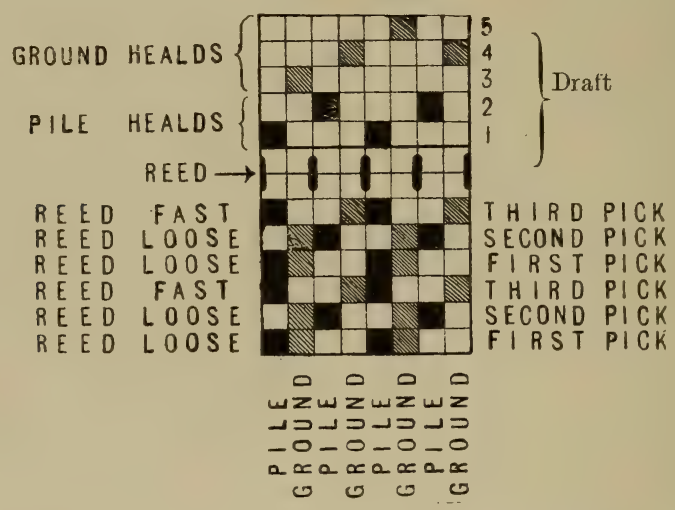

FIG. 412.-Design for three-pick terry pile fabric

threads from both series are drafted in pairs, they are also passed through the reed in pairs, whereby two warp threads of the same series are contained in the same dent of the reed. The relative merits of each system of drafting, and other practical considerations, are stated in $\$ 94$. On examining the design, Fig. 412, it will be seen that consecutive ground warp threads interweave in a contrary manner to each other, as do also consecutive pile warp threads. Pile warp threads that are over two picks and under one pick form pile on the face, whilst. those that are under two picks and over one pick will form pile at the back of cloth when in the loom.

$\$ 90$. In the production of terry fabrics, without the aid of wires, it is absolutely essential to observe a specific order of 
shedding in relation to the action of the reed as governed by the terry motion, otherwise the loops of pile will either be imperfectly developed, or they may not appear at all. This order is clearly indicated in the design (Fig. 412) and also in the graphic

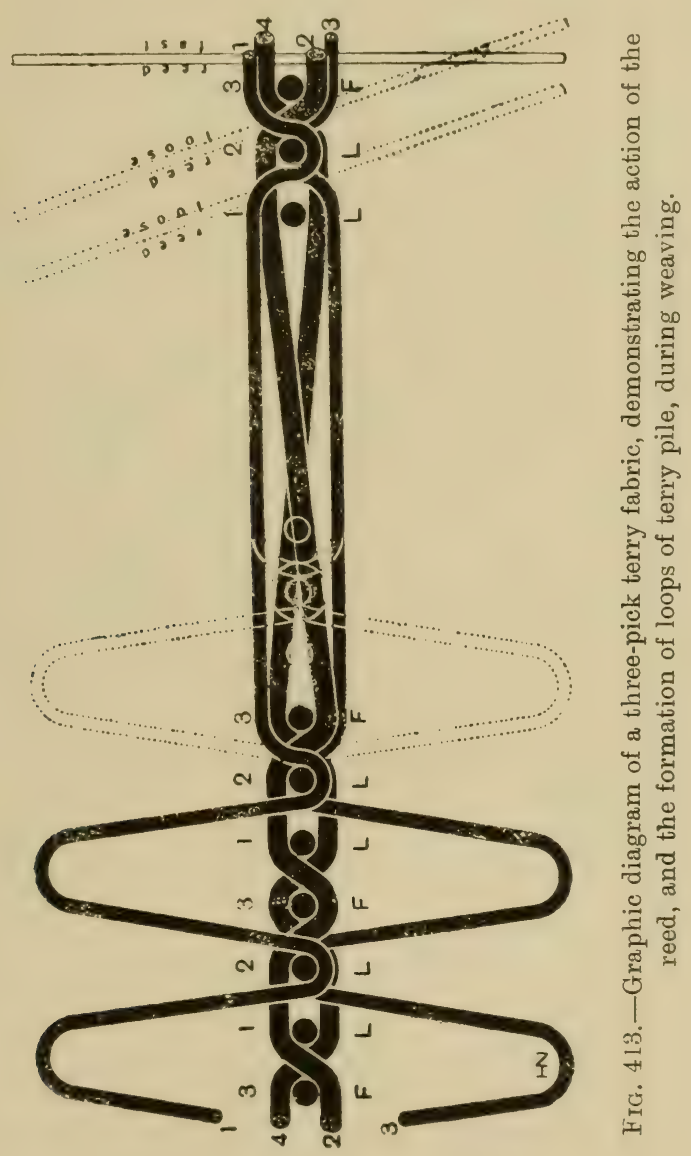

diagram (Fig. 413) which represents a longitudinal section of a three-pick terry fabric. On examining these figures it will be seen that the reed is "loose" for the first and second picks, termed "loose" picks, and "fast" for the third pick, termed 
the "fast" pick. It will also be observed that the "loose" picks are always inserted in reverse pile warp sheds, but in the same ground warp shèds. With this order of shedding in relation to the reed motion, pile warp threads make two intersections, and ground warp threads only one intersection with the picks, before the latter are beaten up to the "fell" of cloth; hence, the picks easily slide along ground warp threads which are held taut, whereas their better grip of pile warp threads, which are slack, thereby causes the latter to be drawn forward and thus form loops of pile.

$\S 91$. As stated in $\$ 81$, the number of picks inserted for each horizontal row of loops, in terry pile fabrics produced by

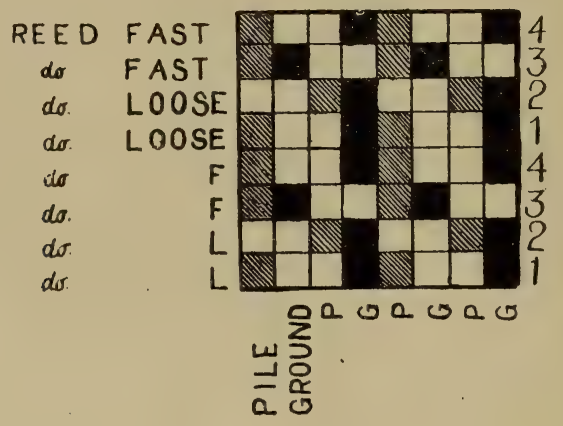

FIG. 414.-Design for a four-pick terry pile fabric.

the aid of terry motions, varies in different fabrics from three to six picks. The number of picks selected, however, does not, in the very least degree, affect the essential factor governing the construction of these fabrics, as regards the order of shedding in relation to the timing of the reed motion, as described in $\$ 90$. This fact is specially emphasised because it constitutes the cardinal and most essential condition in the production of terry pile fabrics, and the lack of such information sometimes proves a hindrance to their successful manufacture. It should also be observed that the relative density of loops of pile is either greater or less in a measure which is inversely proportional to the number of picks inserted for each horizontal row of loops.

The design for a four-pick terry fabric in which the loops of 
TERTL AND TOOP PIIE FALIRICS.

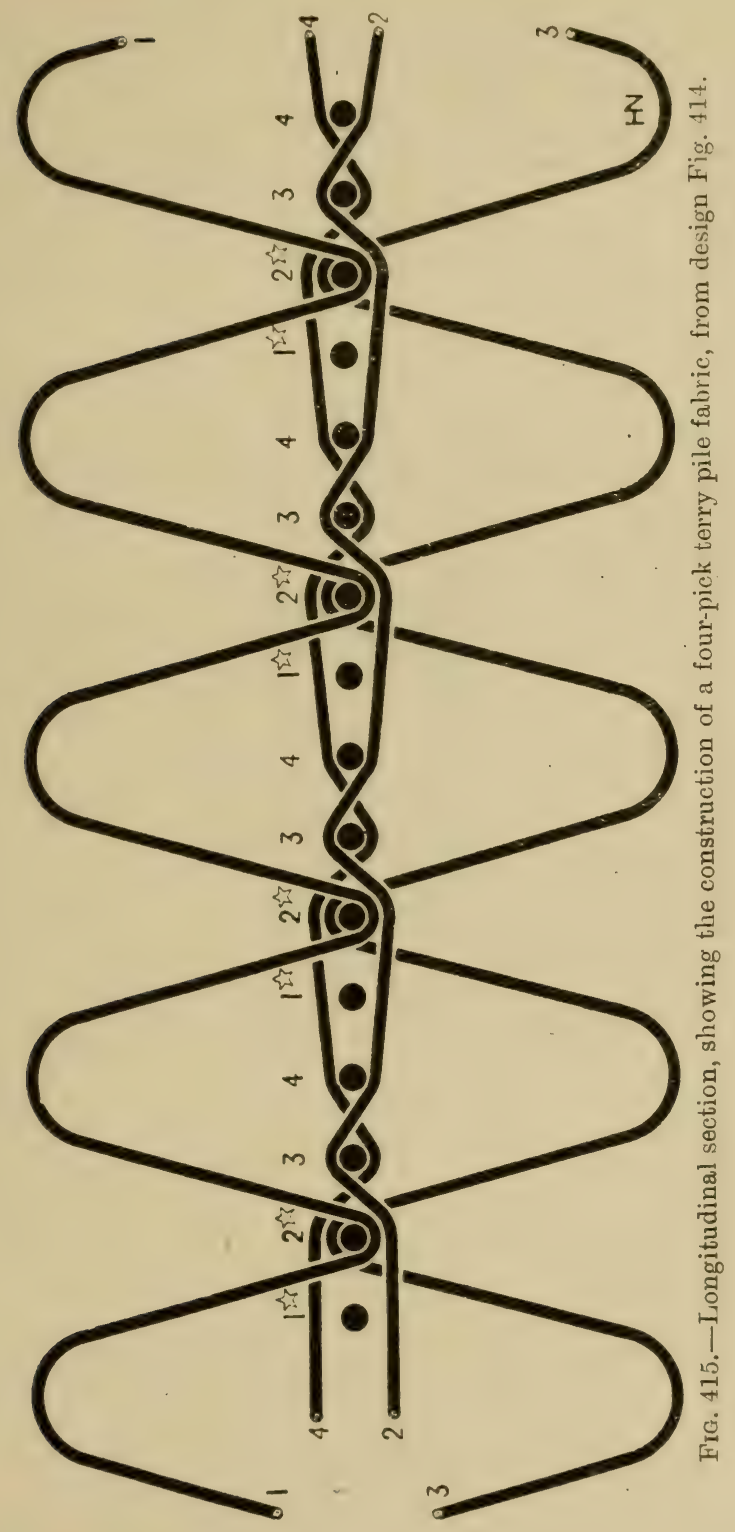


pile are distributed equally on both surfaces, is indicated in Fig. 414, whilst a longitudinal section of the cloth produced from that design is represented graphically in Fig. 415. This variety differs from a three-pick terry fabric by having two picks, namely, the first and fourth picks, which are contiguous, inserted in the same warp shed, a circumstance which necessitates the use of a "catch-cord" for one of the selvedges. (A "catch-cord" is a device to govern extreme outer selvedge warp threads, so that the weft will be caught by those threads and thereby prevented from being pulled backward into the warp shed in the event of a shuttle passing through the same shed for two or more picks in succession.) By inserting four instead

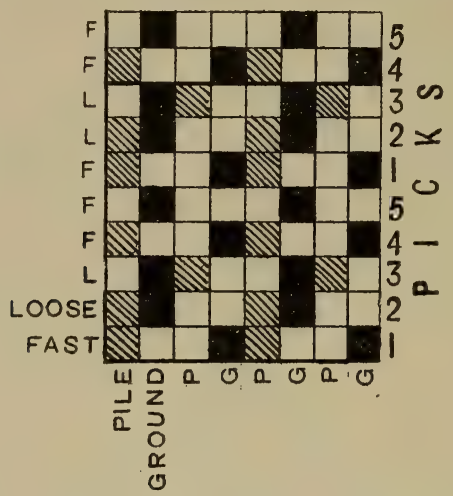

FIG. 416.-Design for a five-pick terry pile fabric.

of three picks for each horizontal row of loops, a firmer and heavier texture is produced. Also in the manufacture of those fabrics in which differently coloured threads are employed to produce simple counterchange effects (as exemplified in many terry towels and bath mats), that course enables a sharper and more perfect definition to be made at the horizontal edges of figure, where pile warp threads pass from face to back, and vice versâ. The starred numbers 1 and 2 in Fig. 415 and the subsequent diagrams of terry fabrics signify that the reed is loose for the picks indicated, and fast for the intermediate picks during weaving.

The construction of a "five-pick" plain terry fabric is 
TERRE ANT) LOOP PIIJ: FABRICS.

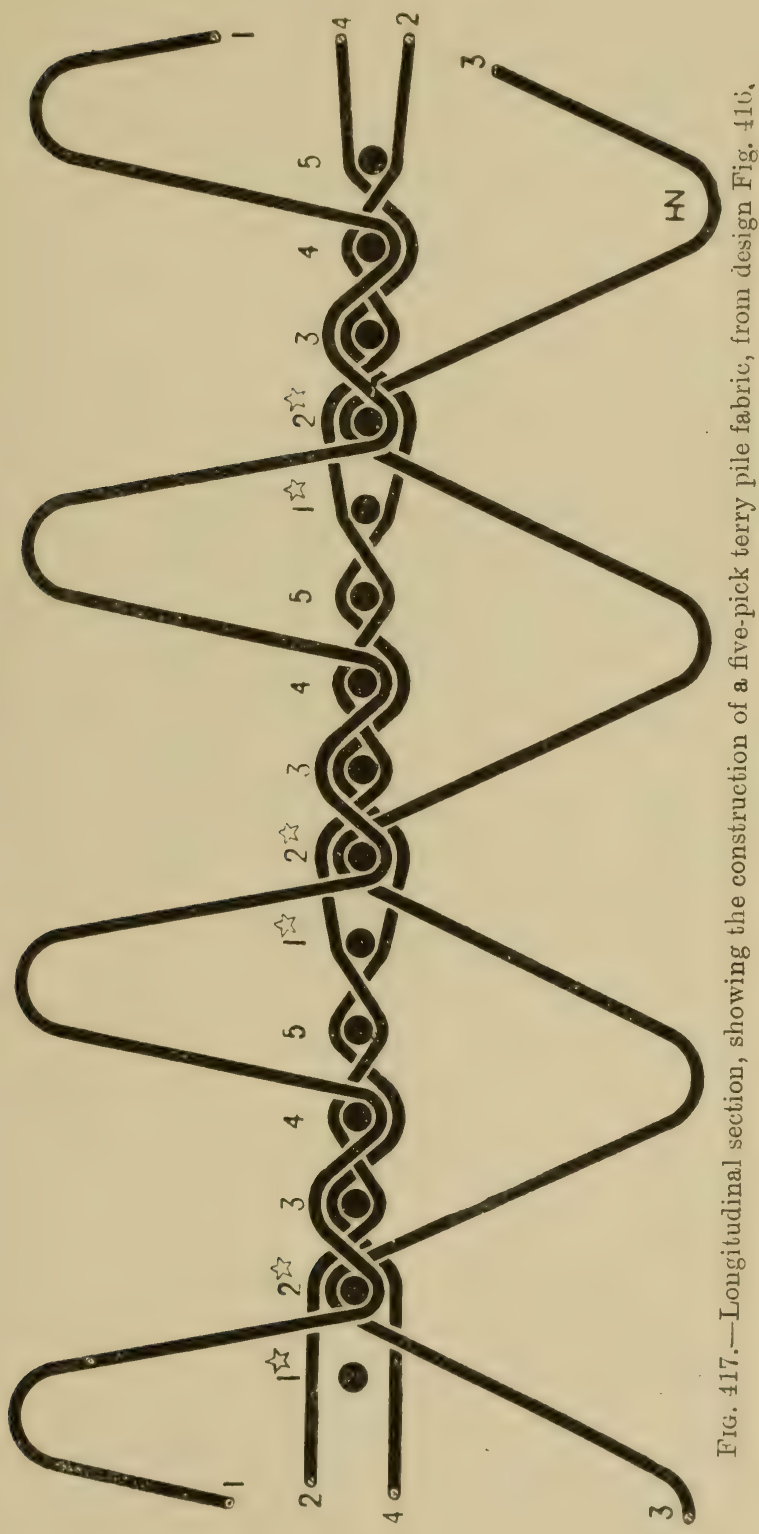


indicated in Figs. 416 and 417 which represent the design and sectional diagram, respectively; of that variety, in which it will be observed that the pile warp threads do not interweare in an exactly contrary manner on the face and back of the cloth, as in the three-pick and four-pick varieties. Thus, whereas face pile warp threads loop over two consecutive picks of weft and intersect with three picks, the back pile threads loop under four consecutive picks of weft and pass over only one pick, as indicated in the sectional diagram. By adopting this course, it permitsi of a relatively greater density of picks of weft than would be possible if the back pile warp threads also intersected

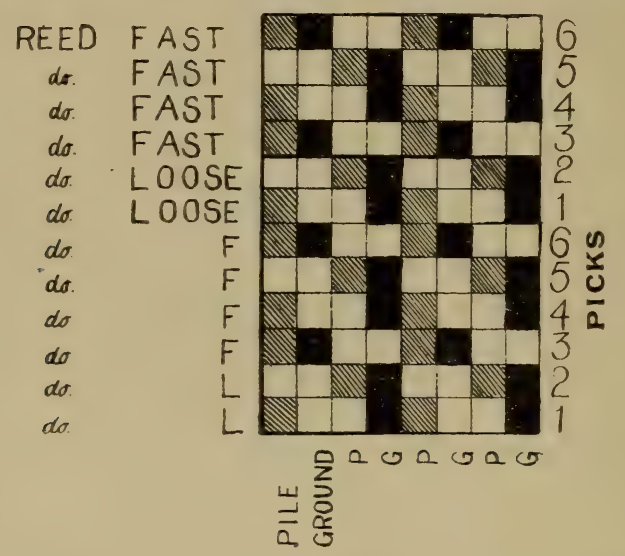

FIG. 418.-Design for a six-pick ("Osman ") terry pile fabric.

with the same three picks of weft. Five-pick terry pile weaveing, however, is usually restricted to the production of Jacquard figured terry pile fabrics, as described in $\$ \$ 93$ to 96 .

$\$ 92$. Another modification in the construction of terry pile fabrics is exemplified in the well-known Turkish towel, sold under the trade name of "Osman," of which the design and longitudinal section are giren in Figs. 418 and 419 respectively. So far as the structural design of this fabric is concerned, it is identical with that of an ordinary three-pick terry pile fabric (Fig. 412), 'which repeats on three picks. In the production of "Osman "Turkish towels, however, the reed is governed so as to be loose for two out of six picks (instead of for two out of 
'TERRY AN1) LOOP PILE FAJRICS.

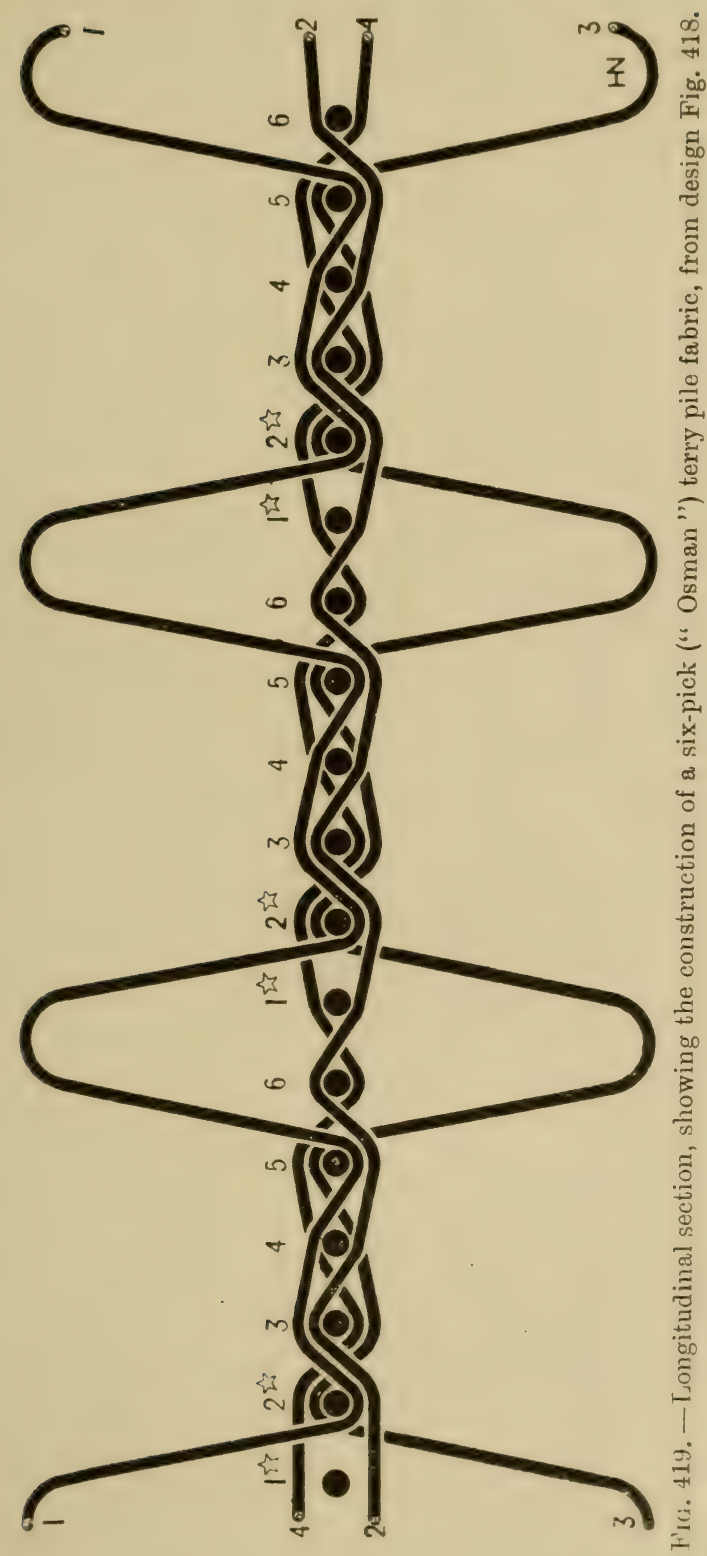


three picks, as in a three-pick terry fabric), thereby causing six picks to be inserted for each horizontal row of loops, as shown in diagram, Fig. 419. Terry pile fabrics constructed on this principle are of relatively stronger and firmer texture than either of the previous examples; also pile warp threads are more firmly interwoven with the foundation texture, and therefore less liable to be accidentally or otherwise withdrawn when the fabric is in use. In the production of six-pick terry fabrics, the reed is loose for the first and second picks only, and fast for the four remaining picks.

\section{Figured Terry Pile Fabrics.}

$\$ 93$. The principle of terry weaving is not restricted to fabrics that are usually produced with an entire surface of terry pile, as exemplified in Turkish towels, bath mats and similar articles in which coloured threads are sometimes introduced simply for decorative effect; but this principle of weaving is also employed as a means of embellishment by Jacquard figuring of a more or less elaborate character, as exemplified in bed counterpanes, dressing-table covers, toilet covers and mats, curtains or hangings, furniture antimacassars and many other articles both of utility and adornment. Figured terry fabrics are usually constructed on the principle of the "three-pick" variety of terry weaving, as described in $\$ 89$, and of which the design and longitudinal section of that variety are represented in Figs. 412 and 413 respectively; whereas the "five-pick" variety, which yields a firmer and more durable texture and also affords greater security to pile warp threads, is usually restricted to fabrics of superior quality and heavier textures, for use as bed counterpanes.

The structure of a "three-pick" and a "five-pick" figured terry pile fabric is indicated graphically by sectional diagrams, Figs. 420 and 421 respectively. As a general rule these fabrics are produced with terry pile figuring on a simple foundation texture, which is quite bare or plain in consequence of the pile warp threads developing the loops of terry pile on the reverse side of cloth when they are not required on the obverse side for figuring purposes, as represented in the sectional diagrams. 
TERRY AND LOOP PILE FABRICS.
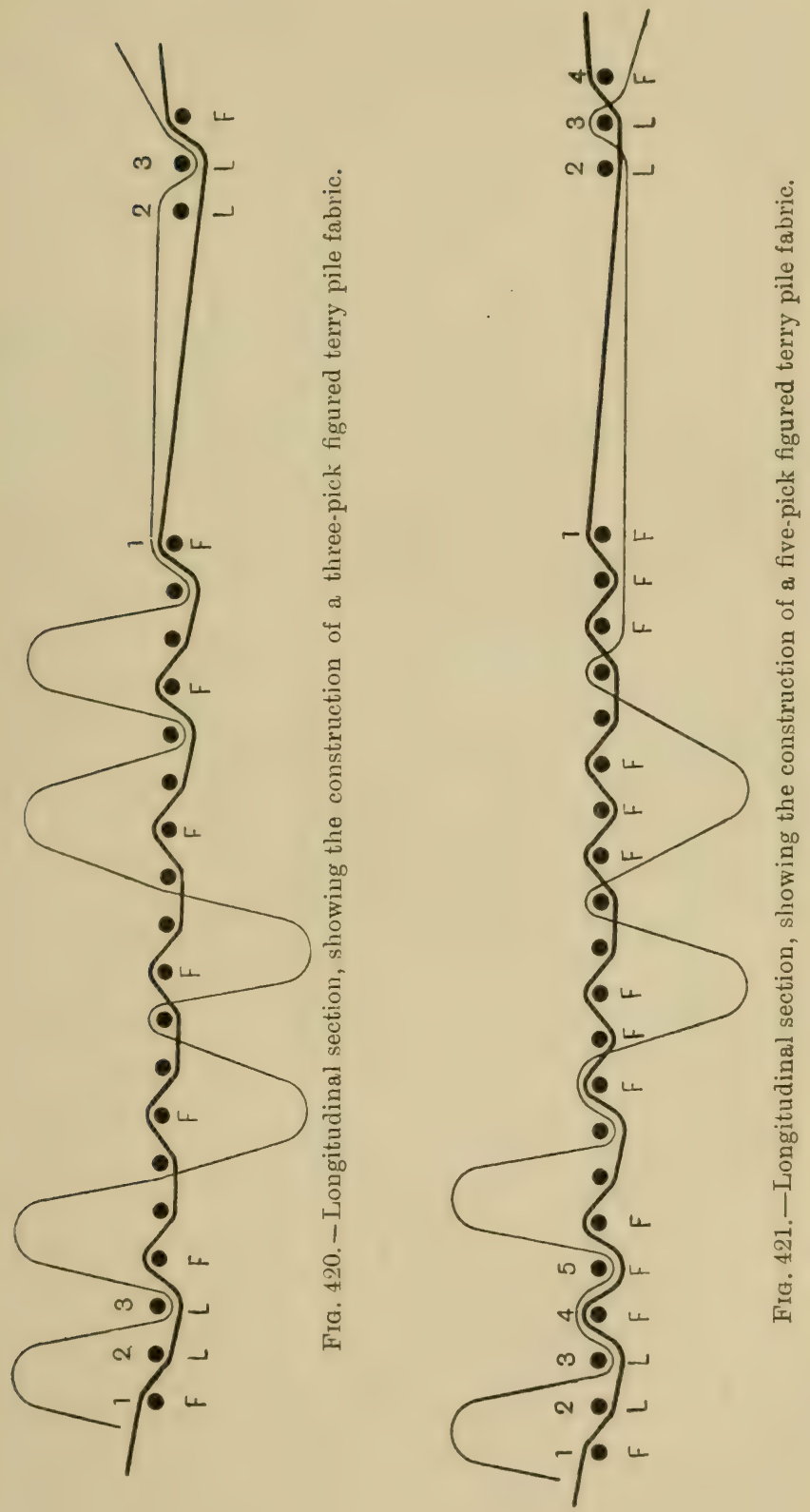
It will be observed in Figs. 413, 415 and 419 that in the three, four and six-pick terry fabrics consecutive pile warp threads always intersect with weft in an entirely reverse manner to each other; whereas, in the present example of a five-pick terry fabric, when pile warp threads are forming pile on the face they interweave in a different manner from that when forming pile at the back. Thus, when forming pile on the face, pile warp threads interweave over two picks, under one, over one, and under one pick; whereas, when forming pile at the back, they interweave under four picks and over one pick. The object of this arrangement is to cause pile warp threads to intersect less frequently with picks of weft, and so permit of a greater number of picks per inch to be inserted in cloth. Such a course, however, involves a less'secure interlacement of the pile warp threads with the foundation texture, from which they are more liable to be withdrawn accidentally.

\section{Terry or Twin Jacquard Machines.}

$\S 94$. In the production of figured terry fabrics, as in that of the plain varieties, the ground warp threads are controlled by means of healds, whereas the pile or figuring warp threads are controlled by a Jacquard harness which, from considerations of economy, is usually operated by means of a special type of terry or "twin" machine whereby each pattern card serves for three or five picks of weft in succession, according to the number of picks inserted for each horizontal row of loops in the fabric; also, the preparation of applied terry designs and the operation of card-cutting is thereby greatly facilitated and simplified:

A special terry machine of this type, as represented in Fig. 422 , is designed to control the same pile warp thread or threads either by means of two contiguous reversed hooks C, $\mathrm{C}^{1}$ (or else by a "twin" or double hook, as represented at F, detached) that are connected by their "tail" or "neck" cords D, and which are contained in two contiguous rows (as the first and second, third and fourth, and so on) as indicated in the diagram. The two hooks constituting either a pair or a "twin" hook are both controlled by the same needle B, but they are raised independently by two distinct sets of griffe-blades $\mathrm{E}, \mathrm{E}^{1}$ that are 


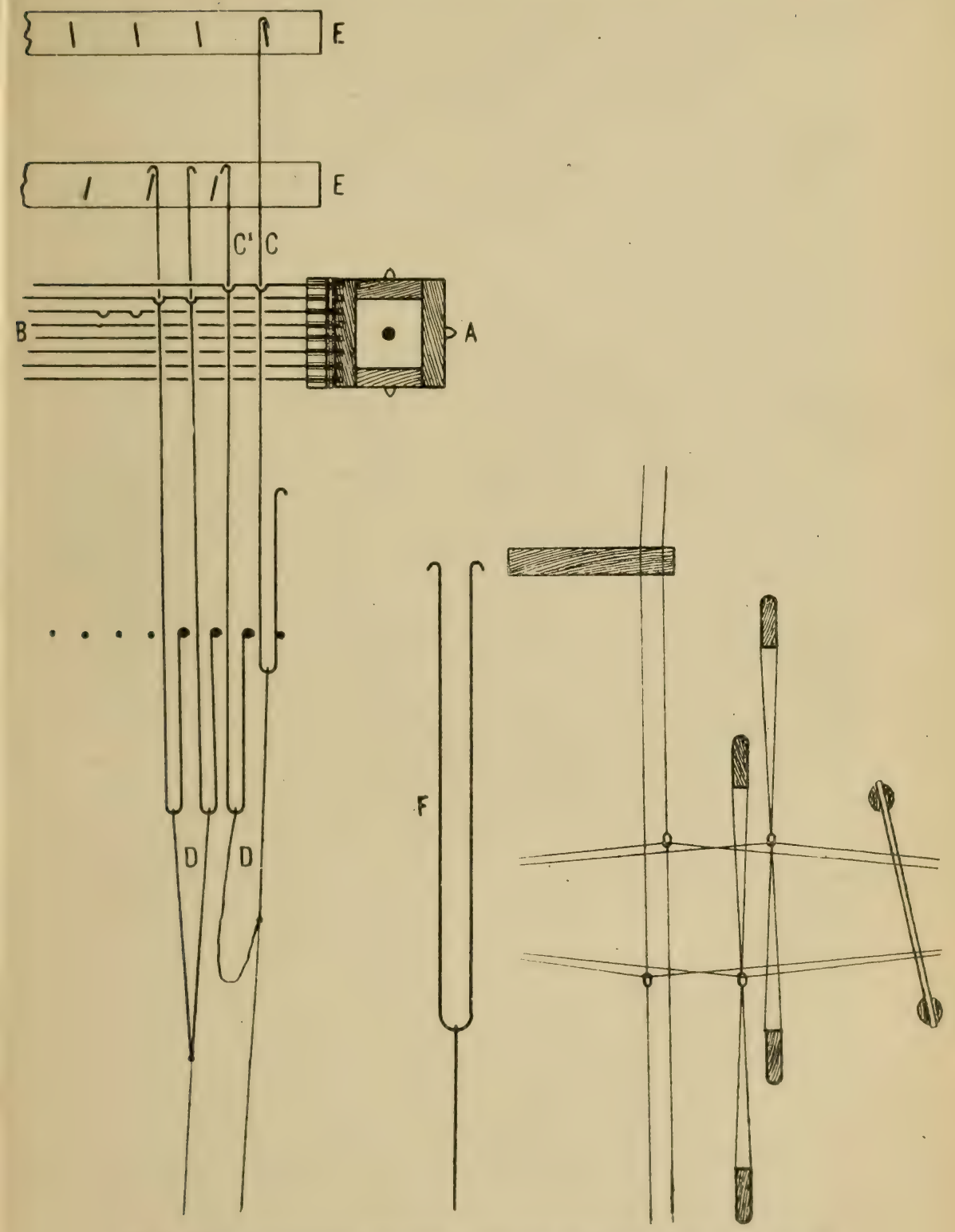

FIG. 422.-Compound Jacquard figuring and heald binding harness for figured terry pile fabrics. 
operated independently of each other and also of the card cylinder $\mathrm{A}$, and in a prescribed manner, according to the particular variety of terry fabric to be produced, to be described presently. All hooks in the first and alternate rows face the card cylinder and, in their normal position, remain over their respective griffe-blades $\mathrm{E}$, by which they are raised whenever a hole in a pattern card is presented to the needle controlling that pair of hooks; whereas all hooks in the second and intermediate rows are reversed and therefore point away from the card cylinder and, in their normal position, remain off their respective griffe-blades $\mathrm{E}^{1}$, by which they are raised only when they are pushed over those griffe-blades by presenting to the corresponding needles B a blank place in the pattern cards.

$\$ 95$. The drafting chart and shedding plans, of which the latter also indicate the operation of the card cylinder, griffeblades, healds and reed, for the production of three-pick and five-pick figured terry fabrics, as represented by the sectional diagrams, Figs. 420 and 421, are indicated in Fig. 423, A, B and $\mathrm{D}$ respectively, whilst the shedding plan for a four-pick variety is indicated at C. Thus, when producing a three-pick figured terry fabric, the card cylinder retains each pattern card against the needle-board for three picks in succession, during which the leading set of griffe-blades, 1 , ascends and remains up for the first two picks of weft, to develop terry pile figuring on the face of the fabric; whilst the second set of griffe-blades remains down, in order to develop the loops of pile on the reverse side of the cloth with all the pile warp threads that are, in respect of that same horizontal row of loops, not required on the obverse for figuring purposes. Then after the first two picks are inserted, the two sets of griffe-blades reverse their relative positions for the insertion of the third pick of weft, during which the card cylinder moves outward to change the pattern cards for the next following series of three picks, and so on for each pattern card in succession, corresponding to one horizontal. row of loops. At the same time, the first heald is raised for the first pick and depressed for the second and third picks of weft, whilst the second heald operates in an exactly contrary manner to that of the first heald, and the reed is "fast" for the first 


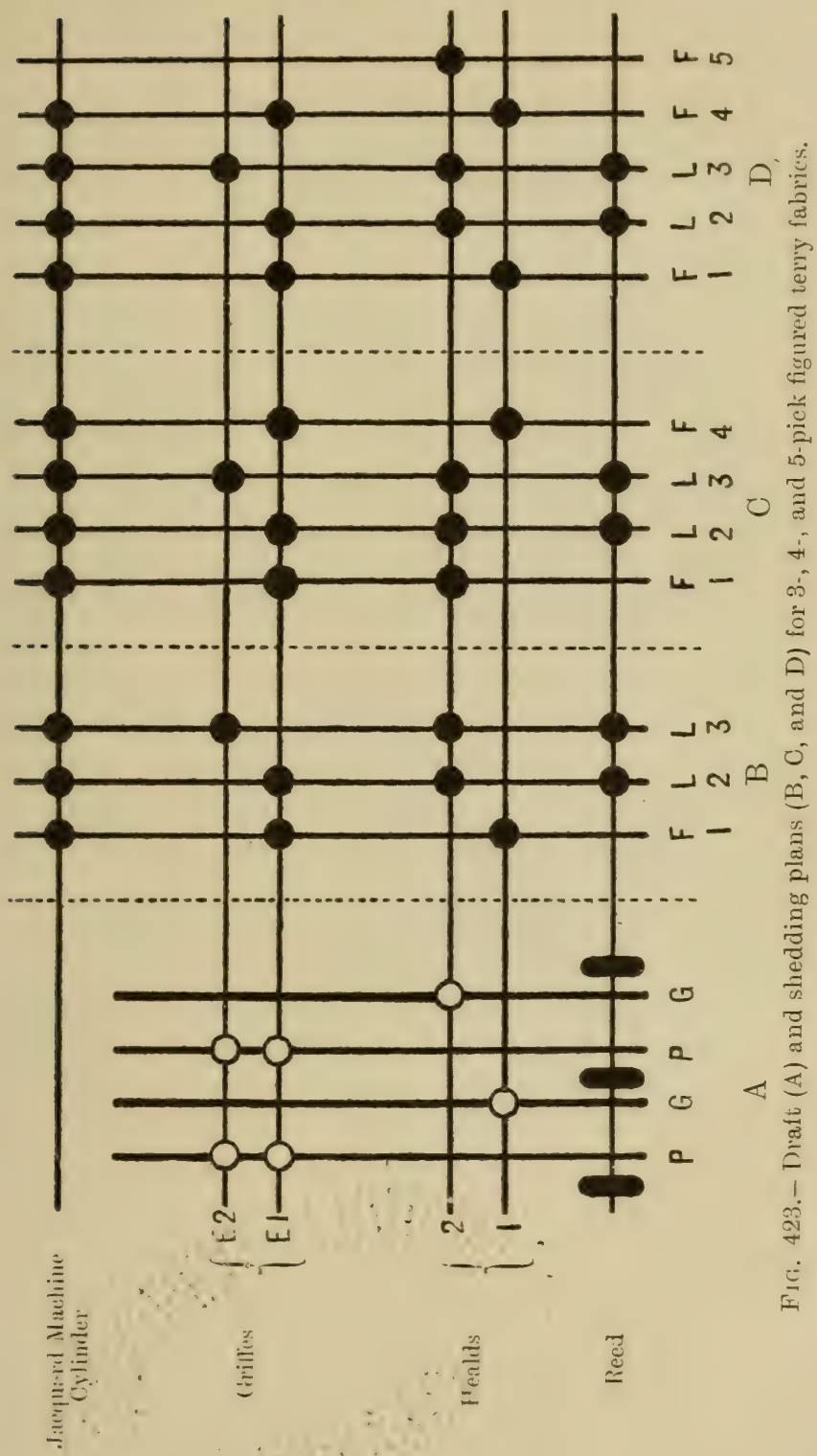


pick and "loose" for the second and third picks, as indicated in the shedding plan, Fig. 423, B.

The shedding plan for a four-pick figured terry fabric, which is of rare occurrence, is virtually the same as that for the threepick variety, in respect of the first three picks of weft; whilst the third and fourth picks are inserted in reverse warp sheds, as indicated in Fig. 423, C.

Also the shedding plan for a five-pick figured terry fabric is identical with that of the four-pick variety in all respects, excepting for the fifth pick when both sets of griffe-blades remain down, and thereby leave down all pile warp threads for that pick, and the card cylinder moves outward, in order to change the pattern cards, after the fourth pick, and rremains out during the fifth pick, thereby allowing a longer period for changing the pattern cards. Also the second heald is raised, and the reed remains "fast" for that pick, as indicated in the shedding plan for this variety, Fig. 423, D.

\section{Method of Preparing Applied Designs for Figured Terry Pile Fabrics.}

$\S 96$. The preparation of an applied design for a figured terry fabric, of any variety to be produced by means of a special terry machine of the type described in the previous sections, is a comparatively simple procedure, as also is the operation of cutting the pattern cards from such a design. This is due to the fact that a design of this character is developed en bloc, with the figure portion painted up solidly, and the ground portion left blank on the design or point paper, of which the small vertical and horizontal divisions represent rows of loops of terry pile in each direction respectively, 'ard in which each small filled square represents one luop of pile on the face side, and a blank square a loop of pile on the reverse side of the cloth, quite irrespective of the number of picks to be iuserited for each pattern card of which the entire set may, therefore; 'be' employed optionally for any variety of these fabrics, since the particular character they assume is determined mechanically by tho shedding plan. The counts 'of äsigr paper required, therefore, is determined by the ratio of loops of pile contained in an equal 
space horizontally and vertically, and not by the number or ratio of warp threads and picks of weft contained in the fabric.

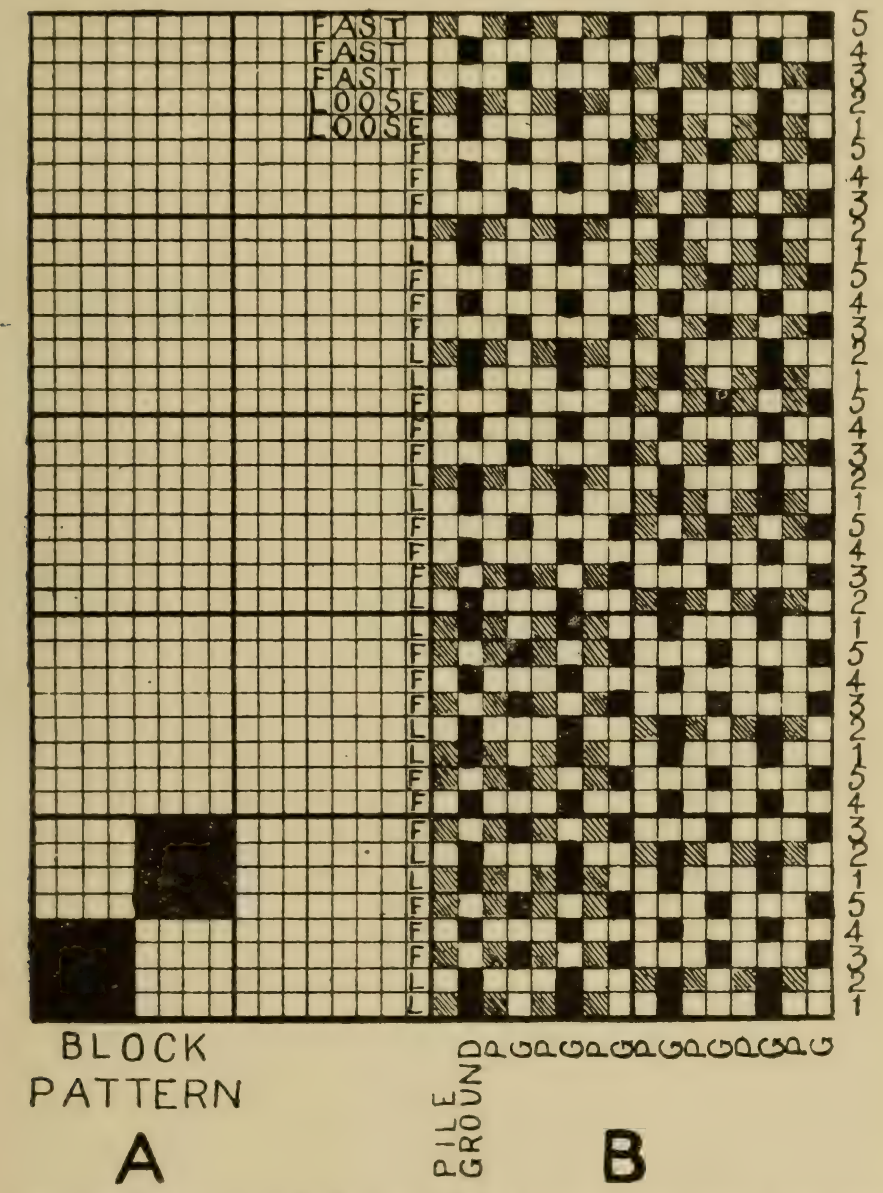

FIG. 424.-Applied design (B) for a 5 pick figured terry pile fabric embellished with pattern indicated en bloc at A.

The method of indicating, on point paper, a figured terry design, on a small scale, is represented at A, Fig. 1424, consisting of a simple four by four dice pattern of which the actual 
interlacement of the pile and ground warp threads with the picks of weft, in a five-pick variety of terry fabric, is indicated at $B$ of the same figure.

\section{Fast-Reed Terry Pile Weaving,}

$\$ 97$. As stated in $\$ 80$, terry pile fabrics may be produced in an ordinary fast-reed loom which is not equipped with a special terry motion for that purpose, and in which each pick of weft is beaten up immediately to the full extent, close to its predecessors, and without the necessity of creating temporary gaps or "frets," without weft, as is usual in ordinary terry pile weaving. The method here indicated, however, is quite distinct from the improvised methods that are sometimes employed for the purpose of developing a series of loops that are usually disposed at moderately wide intervals apart, and as briefly described in $\$ 100$ under the sub-heading "Loop Pile Fabrics" .

By conforming to certain conditions which constitute the essential features of a method of terry weaving, patented by Taylor and Dawson, terry pile fabrics which, in their general outrard appearance, bear a very close resemblance to those of the usual type, may be produced in an ordinary "fast-reed" loom that beats up erery pick of weft in the usual manner, and without the aid of a special terry motion. By this means, the patentees claim that terry pile fabrics are thereby not only produced both more expeditiously and economically, but also that the distribution of the loops of pile is more perfect than that obtained by the usual methods of terry weaving.

The method under present consideration consists essentially of conforming to the conditions specified as follows, and as indicated in the accompanying Figs. 425 to 431 :-

1. By drawing-in the ground and pile warp threads through the shedding harness and reed with a "two-and-two" disposition in the specific order of one ground thread, two pile threads and one ground thread drawn through each dent of the reed, in regular succession, and with a reed wire separating the two adjacent ground threads, as indicated in the "drawing-in" chart, or " draft," Fig. 425.

2. By interlacing pile warp threads, with the picks of weft, in a specified manner so that pile threads in alternate dents 
only will form pile (either on the face or back of the cloth), simultaneously, on the same pile pick or picks of weft; and pile threads in intermediate dents only will form pile, simultaneously, on the next pile pick or picks of weft, and so on in alternate succession, and as indicated in the designs, Figs. 426,428 and 430 , and also in the graphic diagrams, Figs. 427 , 429 and 431, which represent longitudinal sections of the fabrics produced from those designs respectively.

3. By regulating the delivery of pile warp threads in a manner that will permit of these being drawn forward either by each successive pick of weft, or at any other prescribed interval, in order to form loops of pile of the required depth; and

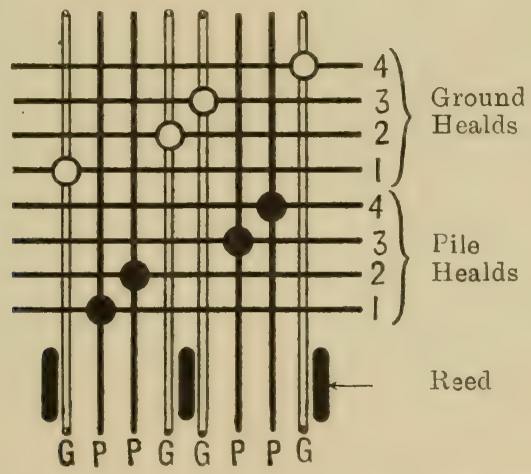

FIG. 425.-Draft of warp threads for " fast-reed "terry pile fabrics.

4. By beating-up each successive pick of weft, immediately, to its final position in the cloth, instead of leaving a short gap or "fret" for three picks and then beating them up together, as when a special "terry motion" is employed.

$\$ 98$. [Although it is not absolutely essential for the development of a terry pile surface, by this method, that object is facilitated by delivering the pile warp threads in a positive and prescribed manner either by passing those threads between a pair of nip or tension rollers to which there is imparted an intermittent movement at regular intervals of one or more than one pick of weft, as required, and as actually recommended by the patentees of this method.] 


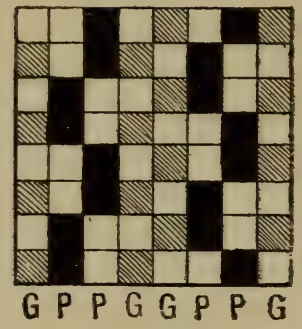

Fig. 426.

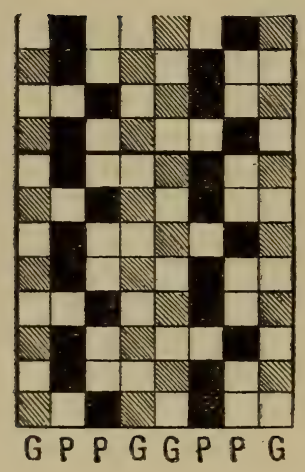

Fig. 428.

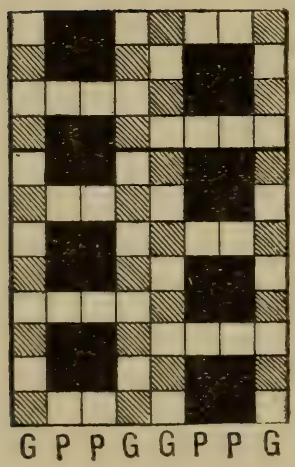

FIG. 430.

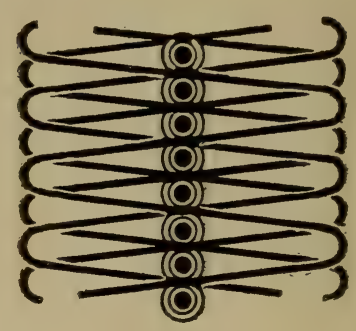

FIG. 427.

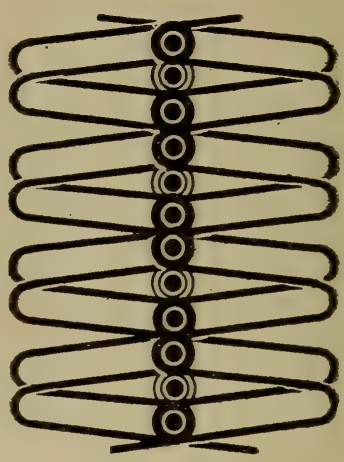

FIG. 429.

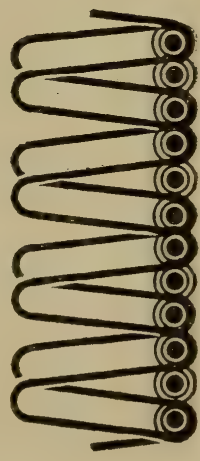

FIG. 431. 
The design indicated in Fig. 426 repeats on four picks, and is adapted for a reversible terry fabric in which pile threads are raised for two and depressed for two picks in alternate succession, and in a reverse manner (in respect of contiguous pile threads), without interlacing those threads, with any intermediate pick or picks of weft for the purpose of binding them more securely to the foundation texture. These threads, therefore, are liable to be accidentally withdrawn very easily in fabrics constructed in accordance with this design. A preterable method of interweaving pile threads is that indicated in the design, Fig. 429, which repeats on six picks, and is also for a reversible pile fabric in which pile threads are raised for two picks and depressed for one pick, or vice versâ, thereby securing those threads more firmly to the foundation texture, as indicated in the diagram, Fig. 429. The third design, Fig. 430, also repeating on six picks, is for a fabric in which pile threads are raised for two picks and depressed for one pick uniformly, thereby producing a terry pile surface on one side of the cloth only, as indicated in the diagram, Fig. 431.

The draft, Fig. 425, indicates pile warp threads drawn through four healds, at the front, and ground warp threads through four healds, in the rear, although only two healds are necessary to gorern ground threads. Also, both the number and proportion of ground and pile threads in each dent of the reed and also the number of picks inserted between horizontal rows of loops mar be raried according to the relative density of pile required, as well as to give additional firmness both to the pile threads and to the foundation texture. Further, decorative effects in the form of check patterns, stripes, and many other simple figured effects may be developed by the use of "fancy" drafts and spaced healds; or more elaborately figured designs may be produced by employing a Jacquard machine and figuring harness.

\section{Practical Details of Terry Pile Weaving.}

\$99. In $\$ 89$, reference was made to different optional systems of drafting warp threads through the harness and reed for the production of terry tabrics. In that section, it was also stated 
that pile and ground warp threads were sometimes disposed in the harness and reed alternately, and sometimes in alternate pairs, with practically similar results. Theoretically, it would appear that an alternate disposition would ensure a more uniform distribution of each series of warp threads; but on this point there is little, if any, appreciable difference between the two arrangements. In practice, however, an advantage is afforded by the two-and-two over the alternate disposition of warp threads. This arises from the fact that, with such a disposition of the tro series of threads, either consecutive pile warp threads or consecutive ground warp threads always interweave in an opposite manner to each other (excepting in the example of five-pick terry fabric just described). Therefore, by placing two warp threads of the same series in the same dent of the reed, they are never either up or down together for the same picks of weft, but always occupy opposite positions; whilst a reed wire separates a pile and ground warp thread that are contiguous, and that are sometimes up or down together at the same time. Hence, these tro circumstances conduce to the formation of a clearer warp shed for the passage of a shuttle during weaving.

In the production of terry pile fabrics of strong and heary textures, such as linen Turkish towels, it is a common practice to employ what is termed a double reed. Such a reed virtually consists of two reeds placed one behind the other, with a space of about $\frac{1}{8}$ in. between them, and constructed in such manner that the wires of one reed are placed exactly midway between those of the other, thereby constituting a compound reed of which the practical counts or sett is equal to twice that of the single reeds. The objects of such a reed are to obtain greater resistance when beating-up weft, and also to gain a little more space for yarn and thereby diminish the abrasive frictional action of the reed upon the warp threads during weaving.

Another practical point in terry weaving relates to the manufacture of those fabrics in which coloured threads are employed for the production of striped counterchange effects, as seen in roller towelling and bordered towels. It is well known to all practical men that however near to the reed the temples are placed, there is a greater or lesser degree of contraction in width 
at the fell of the cloth. It follows, therefore, that the inward pull of cloth will cause the warp threads to bear on the outer sides of the reed wires around which they bend. For this reason it is found advantageous (when pile and ground warp threads are alternated with each other) to place pile warp threads on the left, and ground warp threads on the right, between the dents towards the left-hand selvedge, and in the reverse order between the dents towards the right-hand selvedge, when facing the loom. The object of this arrangement (which is particularly desirable where figured counterchange stripes occur) is to cause ground warp threads, which are taut, to bear against the reed wires, and to allow pile warp threads, which are slack, to lie on that side of ground warp threads nearest the selvedges, and so aroid the risk of pile warp threads being impeded in their passage between the reed wires and ground warp threads.

\section{Loop Pile Fabrics.}

$\$ 100$. A variety of looped pile fabrics that are in vogue as ladies' dress materials are formed with a series of loops either sparsely distributed, or arranged in stripes, on the obverse side of the cloth only. These loops are developed by means of extra warp threads, upon a foundation texture of simple construction. The extra warp threads require to be wound upon a separate warp beam, which is very lightly weighted, to permit those threads being freely withdrawn when required to form loops. Fabrics of this class are but very remotely, if at all, related to the more typical varieties of terry pile fabrics described in the previous sections of this chapter, as they neither embody the same principles of construction, nor is it essential to employ a terry pile motion to produce the loops-although loops are sometimes formed in these fabrics by causing the reed to recede for certain picks, and to be held fast in its normal position for others, in a manner similar to that which obtains in terry pile weaving, and as described in $\$ 82$, excepting that the liberation of the reed is effected by means of the dobby (if such is employed), or by other improvised contrivance, to save the expense of a loom equipped with a special terry pile motion. The prevailing method, however, of forming loops in this variety of fabrics is 
to pass the required warp threads between two cloth-covered tension or delivery rollers which are moved intermittently, in order to deliver uniform lengths of warp according to the size of loops required and to weave without allowing the reed to swing backward at the bottom, from its normal position, as the sley advances to beat up p:cks of weft, as in ordinary terry weaving by means of a special terry pile motion. In some respects, therefore, this method of producing terry or loop pile in a "fast-reed" loom by the beating-up of each pick of weft in immediate succession, to the "fell" of cloth, possesses certain features in common with Taylor and Dawson's method of producing ordinary terry pile fabrics in "fast-reed" looms, as described in the previous sections, $\$ 97$ and 98 , of this chapter. 


\section{CHAPTER IX.}

\section{GAUZE AND NET LENO FABRICS.}

$\$ 101$. "Gauze" and "leno" are terms which designate different varieties of one of the most interesting types of woven fabrics comprised under the generic term of "cross-weaving". The distinctive characteristic feature of this type of fabrics is the peculiar crossing of warp threads with each other, caused by pulling them out of their normal straight and parallel course, first to one side and then to the other side, of other warp threads, which cross and re-cross in some definite order.

Cross-weaving is a useful principle of fabric structure which is adopted extensively in the production of silk, cotton, worsted and linen textures, for almost every variety of purpose, such as garments that are employed as underclothing, curtains, antimacassars, and many other domestic articles. It is also frequently employed in combination with tissue, lappet and swivel figuring (described in Chapter XI.) piqués, and many other types of fabrics. When it is applied to fabrics of an extremely light, open and Himsy texture, and especially if produced from silk, they are usually described as "gauze" fabrics ; but if applied decoratively to heavier textures of cotton and linen, they are usually termed "leno" fabrics, of which there are two distinct classes, namely, (1) "net leno," and (2) "leno brocade" fabrics, each comprising several different varieties of texture.

In the weaving trade, howerer, the terms "gauze," "leno" and "net leno" each denote a distinctive woven effect produced by the application of the principle of cross-weaving. For example, a "gauze" effect is developed by causing one series of warp threads, termed "doup" threads, to form more or less zigzag or wary lines, whilst another series of warp threads, termed 
"regular " or " standard " threads, remain comparatively straight, thereby requiring a separate warp beam for each series of threads.

A "leno" effect, however, is developed by causing both "standard" and "doup" warp threads to bend equally, as in "leno brocade" fabrics, therefore requiring only one warp beam to contain both series of threads. Also, in some instances, both "gauze" and "leno" effects are developed in the same fabric, in which case the warp threads require to be wound on at least two separate warp beams to allow for the different rates of contraction during weaving.

"Net leno" fabrics are chiefly produced by means of healds, and are usually characterised by a more or less pronounced striped formation, developed by the introduction of comparatively coarse warp threads, termed "net" warp threads, which usually assume a more or less wavy or zigzag course, and produce a series of "net" leno stripes. Sometimes the vertical stripes are crossed by a series of horizontal stripes to produce "check" patterns. Net leno weaving offers unlimited scope to a capable designer in the production of decorative leno effects, which are frequently of a most interesting and sometimes of a very beautiful and attractive character. And it is, perhaps, not too much to say that no other principle of weaving is capable of yielding such variety of exquisite decorative effects for so small an expenditure of artistic or technical effort.

"Leno brocade" fabrics are those in which the cross or leno principle of weaving is employed as an additional means of embellishment, as described in Chapter $\mathrm{X}$. They are produced by means of Jacquard machines, and generally consist either of a gauze or leno figure surrounded by a ground of the plain or calico weave, or vice versâ; and' sometimes either the warp, weft, or both are allowed to float where required for the purpose of developing ordinary brocade figuring. An interesting variety of leno brocade fabrics are those in which both leno brocade and also net leno figuring are employed in combination for the development of stripes of each kind arranged either alternately or otherwise. In the production of this variety of fabrics it is usual 
to employ a compound harness consisting of a Jacquard mounting for the leno brocade stripes, and a heald harness for the net leno stripes.

\section{Plain Gauze,}

$\$ 102$. The simplest example of gauze or cross-wearing is that in which one or two warp threads cross each other on successive picks, or pairs of picks, in regular succession so as to produce an open net-like structure of uniform texture, as represented graphically at C, Fig. 432. This diagram indicates the method of drafting war'p threads through the healds and reed at $\mathrm{A}$, and the order of shedding at $\mathrm{B}$, to produce the gauze structure represented at C. By studying that diagram in conjunction with those given in Figs. 433, 434 and 435, the principles of crossweaving, which are generally so puzzling to students, will be easily understood. A shedding harness for cross-weaving, whether it consists of healds or Jacquard mountings, may be constructed optionally on either of two alternative schemes known as a "bottom-doup" or a "top-doup" harness, according to whether the "doup slips" or healds are situated below or above the warp threads respectively, to be explained presently. A heald harness constructed with a "bottom-doup " arrangement, and also the formation of the sheds for the production of plain gauze, are represented graphically in diagrams Figs. 433, 434 and 435. In those diagrams the healds are shown perspectively, and with the object of making their functions clear and distinct only one heald eye is shown on each heald. Fig. 433 shows all parts in their normal or inoperative position. Warp threads, all of which come from the same warp beam, are separated into two divisions of alternate threads, respectively termed "standard" or "regular" warp threads S, and doup warp threads D.

The essential features of this harness are-a heald $G$, termed the "front standard," situated immediately in front of all other healds, and a half-heald, termed the "doup heald" $\mathrm{H}$, placed in front of the front standard, and consisting of a number of loops or slips .J, termed "doups," of which the upper parts pass orer the eyes of the "front standard," and return 
through them, to prevent their withdrawal. The doups are attached to a single heald-stave $\mathrm{H}$, situated below warp

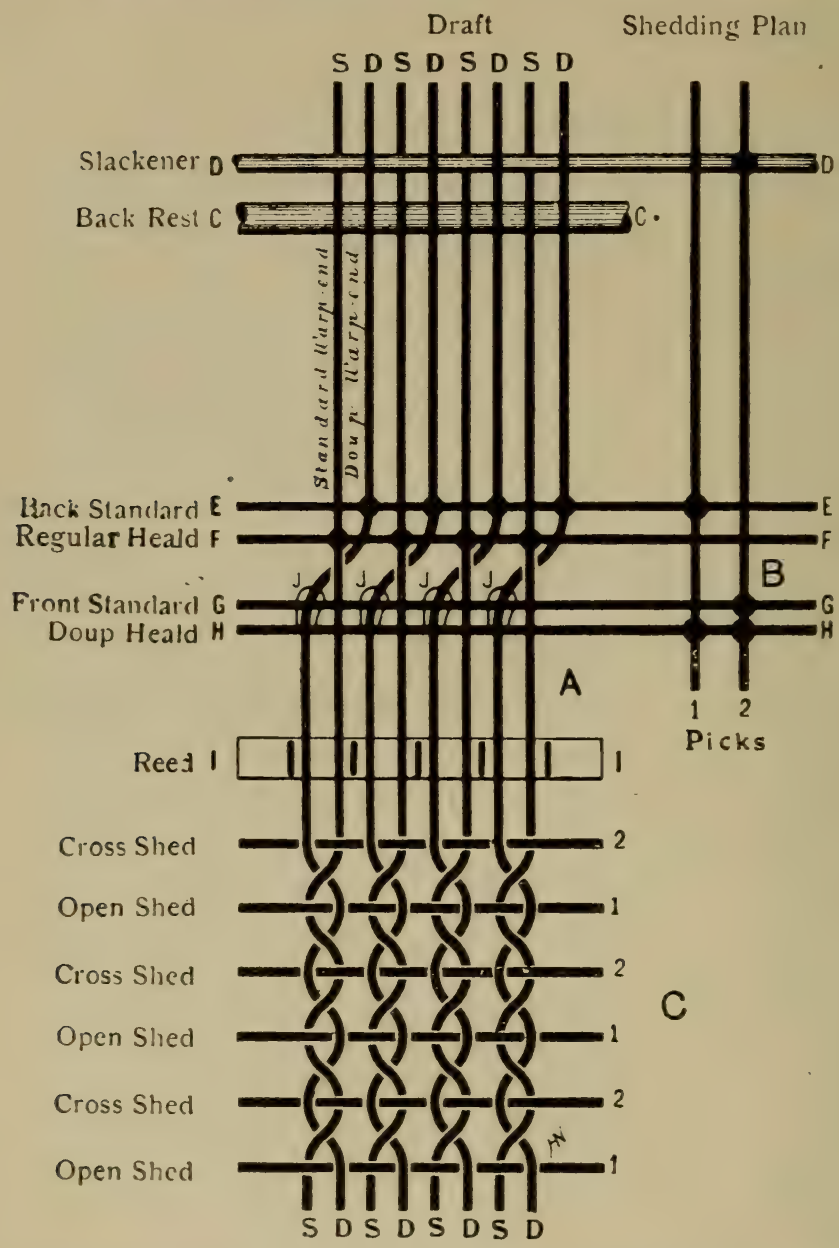

Fig. 432.-Draft (A) and shedding plan (B) for simple gauze (leno) fabric of which a plan is shown at $A$.

threads; hence the term "bottom-doup" as distinguished from a "top-doup" harness, in which the doups are attached to a stave situated above warp threads. 


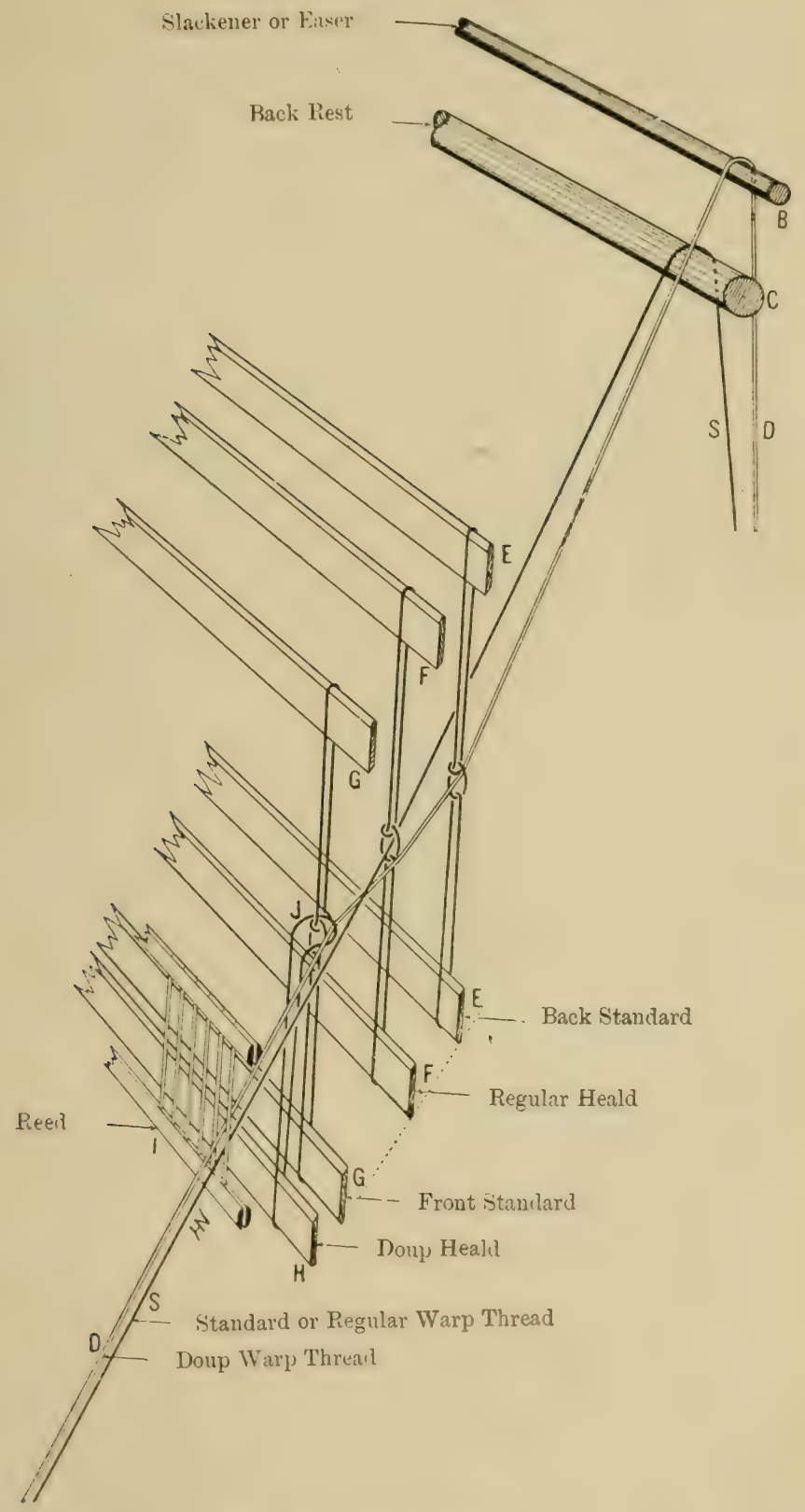

Fig. 133.- - Warp shed closed. 


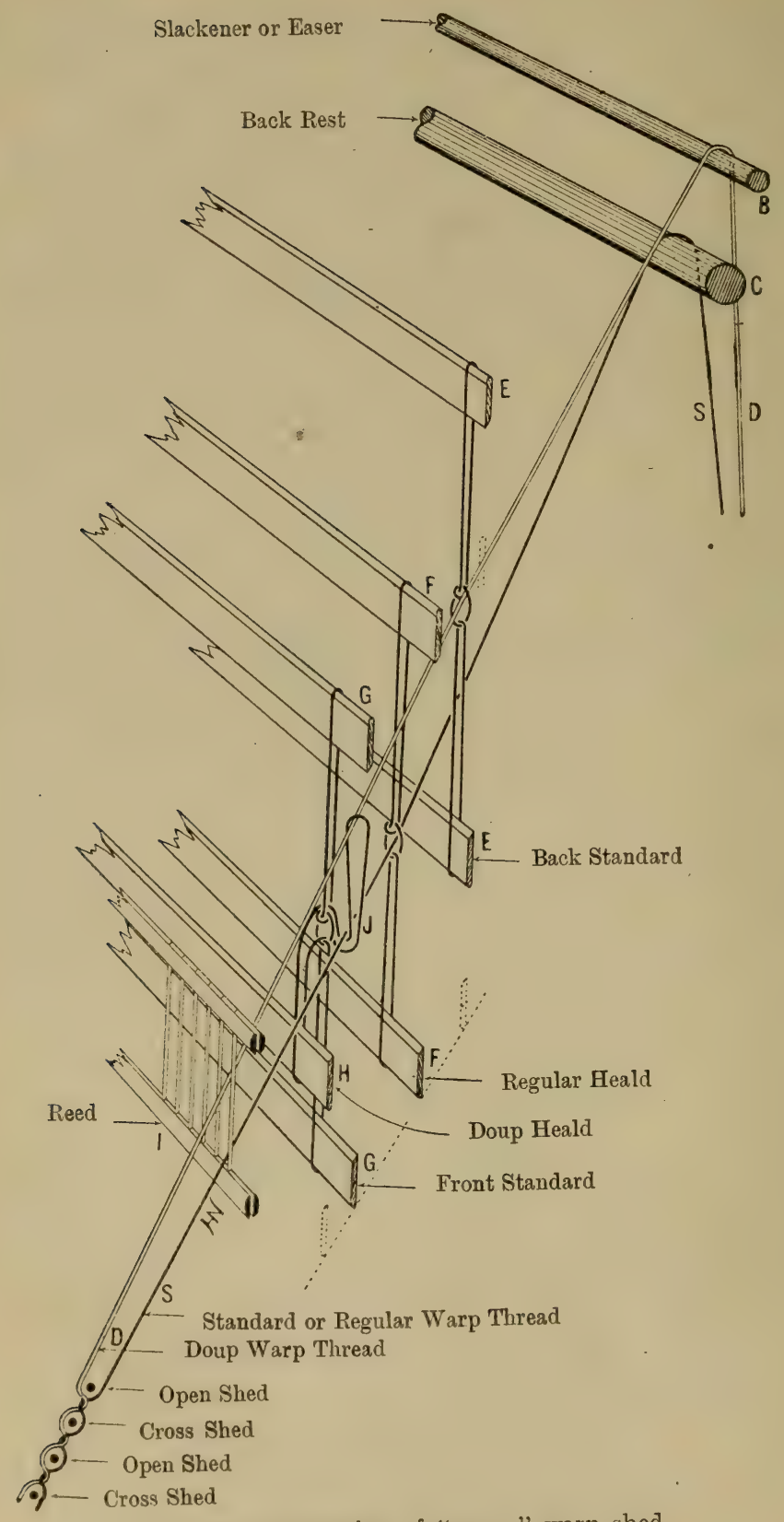

FIG. 434.-Formation of "open" warp shed. 


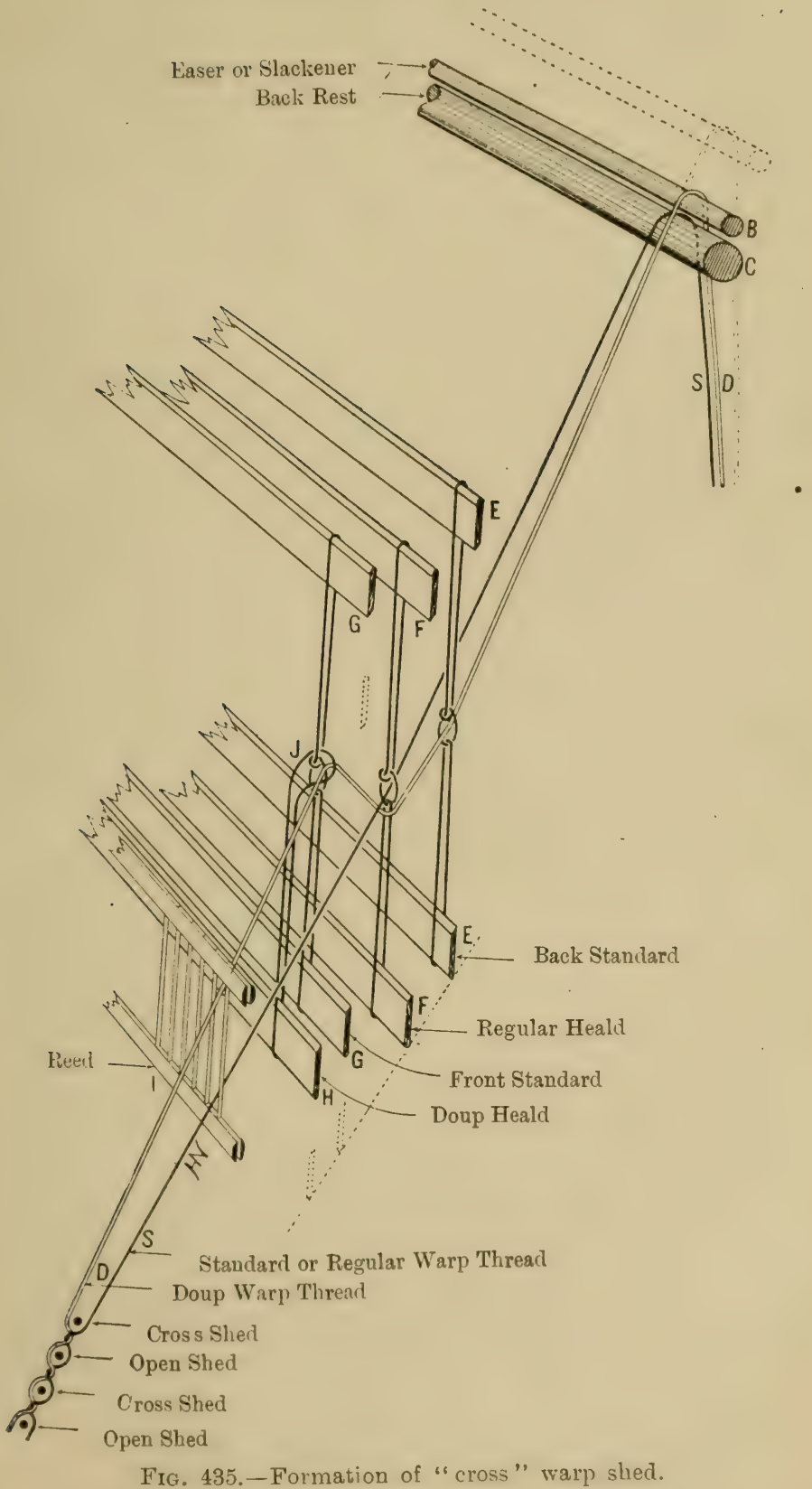


Standard warp threads pass from the warp beam to cloth in a perfectly straight course, first over the back rest $\mathrm{C}$, thence through the respective eyes of a regular heald $\mathrm{F}$, and through a dent of the reed I. Doup warp threads pass over a bar variously termed the "easer," "vibrator" or "slackener" B, situated a little to the rear of, and in a little higher plane than, the back rest $\mathrm{C}$; thence through eyes of a heald $\mathrm{E}$ termed the "back standard". From here they pass in front of heald eyes governing standard warp threads, and then cross underneath the latter from right to left, after which they pass through a loop $\mathrm{J}$ attached to a single heald-stave $\mathrm{H}$ (situated below warp threads), and finally they pass between the same dents of the reed (1) as their fellow standard warp threads.

(Note.-In order to prevent confusion of terms, the attention of students is specially directed to the apparent inconsistency in the use of the term "back standard" to describe those healds $\mathrm{E}$ which govern doup warp threads when the latter are raised on the normal side of their respective standard warp threads (to form "open" sheds), instead of that term being used to describe the regular healds $\mathrm{F}$ which govern standard or regular warp threads. The term "back standard," however, is that established by custom to distinguish the healds that are complementary to, and which govern doup threads in conjunction with, "front standard" healds.)

$\$ 103$. In the production of a simple gauze texture entirely devoid of figuring, two distinct forms of shedding are required, namely, a straight or "open" shed, and a "cross" shed. A straight or open shed is one in which warp threads are separated without deviating from their normal parallel course. Its formation with a bottom-doup harness (as illustrated in Fig. 434 and indicated in the shedding plan of Fig. 432) is effected by raising. both the back standard heald E (which controls "doup" warp threads) and the "doup" heald stave H. By raising the halfheald stave, "doups" J become slackened, and thereby release their control of "doup" warp threads, which are quite free to return from their crossed position on the left, to their open or parallel position on the right of standard warp threads. Thus by raising the "back standard" E, "doup" warp threads are 
raised on that side of "standard" warp threads which they occupy before being crossed underneath those threads, without being impeded by the "doup" slips, which, being slack, are taken up by their respective "doup" warp threads.

A "cross" shed is one in which "doup" warp threads are raised on the opposite side of "standard" warp threads to that which they occupy in the healds before being crossed-that is, on their crossed side. Its formation, as illustrated in Fig. 435, is accomplished by raising both the "front standard" heald $G$ and the "doup" heald $H$ together. Unless means were adopted for its prevention, the formation of a "cross" shed would impart abnormal tension to "doup" warp threads, in consequence of the short interval or " stretch" of warp between the "fell" of the cloth and the eyes of heald F, around which "doup" warp threads bend on being raised. Such undue strain is prevented by passing "doup" warp threads over an easing bar or "slackener" B, situated in the rear of the back rest C. By that means "doup" warp threads are allowed a little longer course or "stretch" between the rarp beam and "fell" of the cloth. Thus, when a "cross" shed is formed the "easer" or "slackener" is brought forward from its normal position (indicated by dotted lines) to slacken the "doup" warp threads, and thereby prevent undue tension being imparted to those threads. This function is variously described as "easing," "slackening" and "vibrating".

An examination of the gauze structure represented in Fig. 432, and also of Figs. 434 and 435 , will show that "standard" warp threads are never raised, and that "doup" warp threads are raised for every pick of weft inserted-first on the right and then on the left of "standard" warp threads alternately. That peculiarity, however, is characteristic of plain gauze-weaving only, and not of cross-weaving generally; otherwise the development of figuring (as exemplified in "net leno" and "leno brocade" fabrics) could not be accomplished. In those fabrics all warp threads, whether "standard" or "doup" threads, may be raised as desired to produce any ordinary woven effect in combination with cross-weaving, and their construction is governed by the same general principles as those underlying the construction of simple gauze. 


\section{"Cellular" Gauze Fabrics.}

$\$$ 104. An example of gauze cloth, which is sold under the trade name of "cellular" cloth and employed extensively in the production of light summer garments for underclothing, is that represented in Fig. 436, and of which the plan of cloth drafting chart and shedding plan are indicated at $\mathrm{A}, \mathrm{B}$ and $\mathrm{C}$ respectively, Fig. 437. The distinctive feature of fabrics of this class, of which there are several modifications, consists essentially of a more or less pronounced cellular diamond formation which

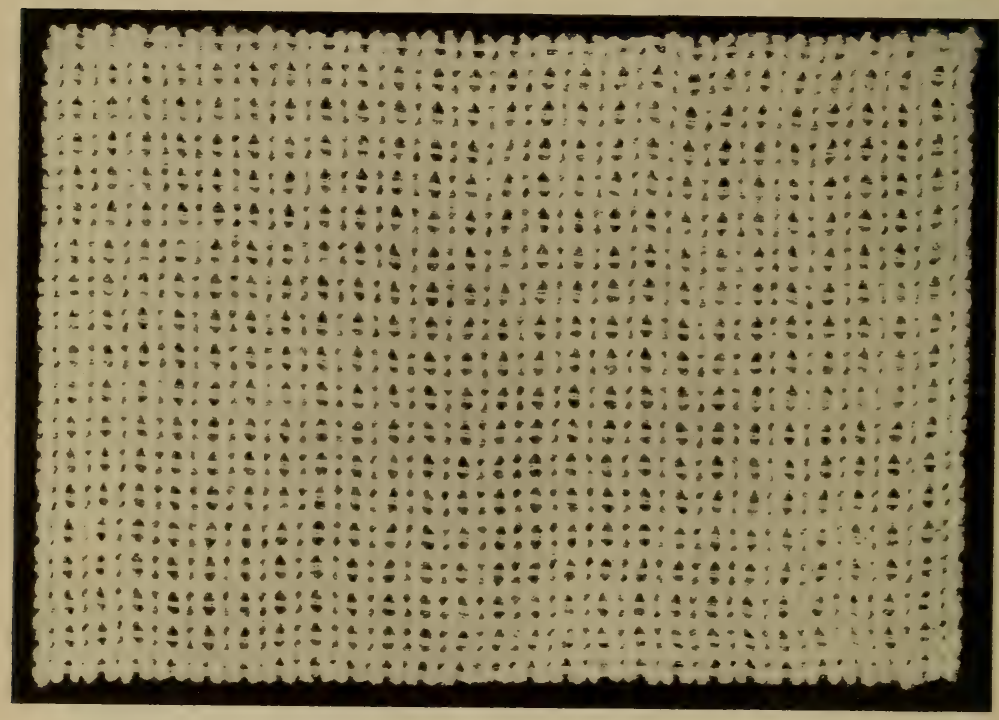

FIG. 436.-"Cellular" gauze or leno fabric.

results chiefly from the difference of tension between the "standard" and "doup" series of warp threads during weaving in conjunction with the principle of cross-weaving, thereby necessitating the employment of a separate warp beam for each series of threads. Thus, standard warp threads are taut and therefore lie in comparatively straight and parallel lines, whereas doup warp threads are slack and assume more or less zigzag or wavy and opposing lines, thereby producing in the fabric the characteristic cellular formation as described. 
A Plan of Clotli.

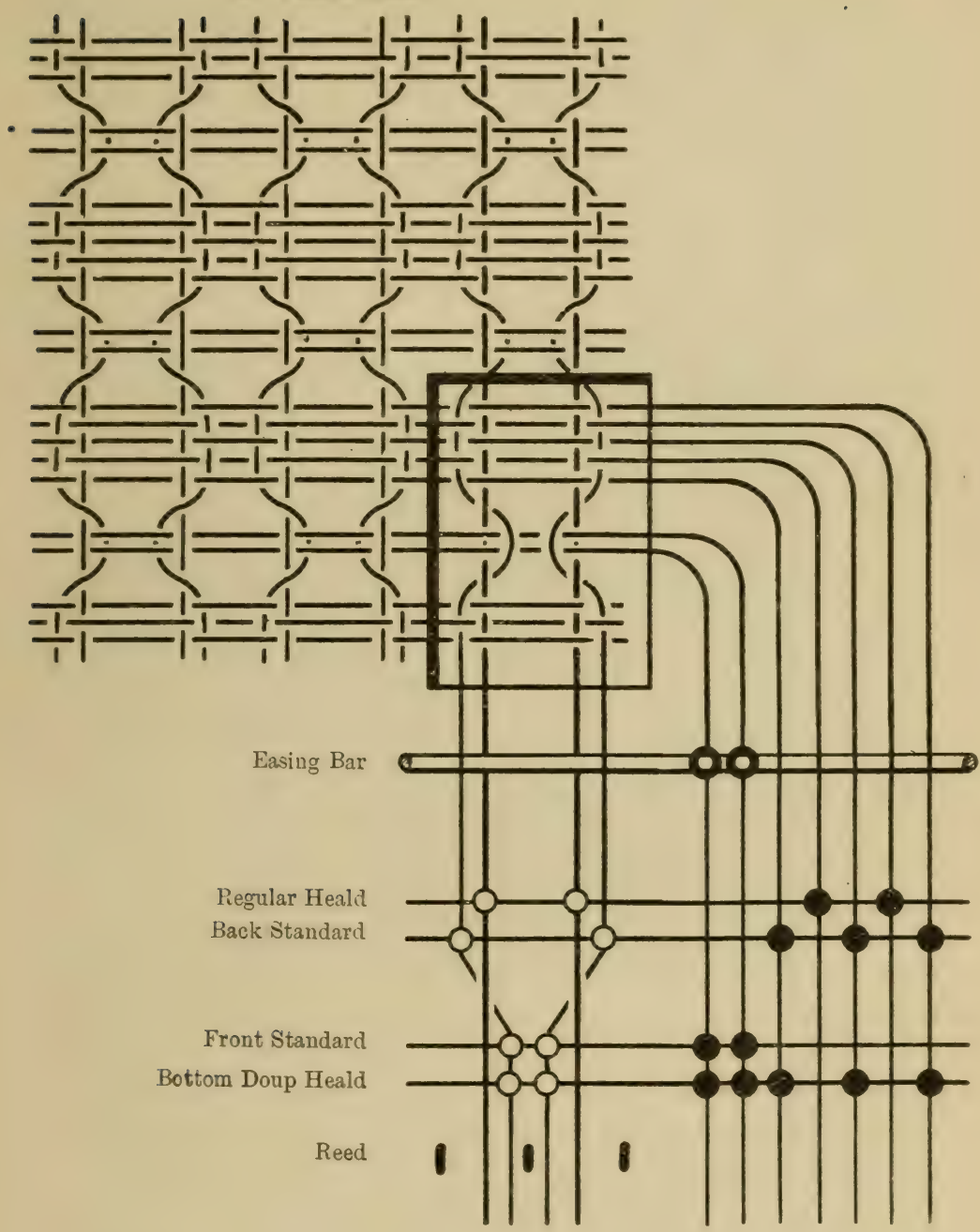

B

Dratt

Shedding Plan

FIc 437.-Plan, Draft and Shedding Plan of "Cellular" Cloth, Fig $\neq$ : 
For practical considerations, it is more expedient to weave fabrics of this character with the face side downward, in order to minimise the effort of shedding during weaving. This course involves the use of a bottom-doup harness, as indicated in drafting chart B and shedding plan C, Fig. 437, which has the disadvantage of presenting the reverse side of the cloth to the weaver, whereby faults are more liable to escape observation during weaving.

The example of cellular gauze cloth illustrated in Fig. 436, and also the plan of that cloth, A, Fig. 437, are both represented obversely, but with the lower right-hand corner of the plan reversed, as seen in the loom. This sample, which is of a fairly good quality of texture, contains fifteen of each series of warp threads, per inch, of $2 / 16$ 's and $2 / 18$ 's counts of yarn for standard and doup threads respectively; and fifty picks, per inch, of $2 / 24$ 's weft. The rate of contraction, during weaving, by standard and doup warp threads, is, in the present example, equal to fifteen and thirty-three per cent respectively of the original warp length on the beam.

\section{Net Leno Fabrics.}

$\$ 105$. In the production of typical net leno fabrics (as exemplified in the accompanying photographic reproductions), the number of doup healds, front standard healds, back standard healds, easers or slackeners, and extra warp beams containing the net doup threads, must sererally correspond with the number of different schemes of douping in the same fabric. Thus, if all doup warp threads in a piece of cloth cross their standard threads either in the same direction or in reverse directions simultaneously, only one doup heald and one each of the other several parts just enumerated would be required for its production. The direction in which doup threads cross is quite optional. They may cross uniformly either in the same direction, or in reverse directions simultaneously; as predetermined by the manner in which they are crossed in the shedding harness before being passed through their respective doup slips. If they are crossed over (in the harness) in the same direction uniformly, they will all cross in the same direction simultane- 
ously, in cloth, as exemplified in the second net leno stripe 13, Fig. 442 ; but if some doup threads are crossed orer to the right, and others to the left, of their respective standard warp threads, they will always cross in reverse directions in cloth. Thus, by drafting alternate doup threads in one direction, and intermediate threads in the opposite direction, a neat diamond formation may be produced, as exemplified in the net leno stripe A, Fig. 438, which illustrates an example of net leno

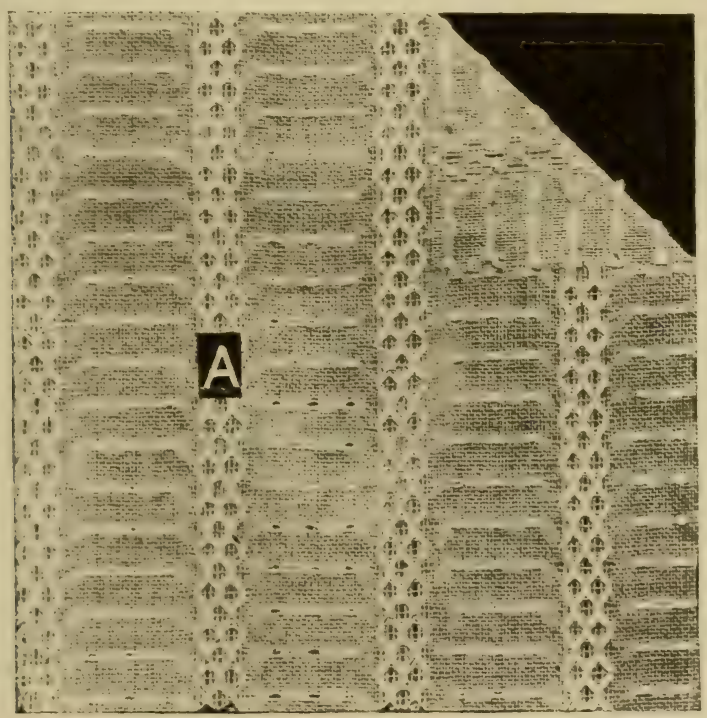

Fic. 438,-One-doup net leno fabric, for which the design, draft and shedding plan are given in Fig. 439.

wearing produced by means of only one set of doups operating in conjunction with one back standard heald.

For reasons stated subsequently in $\$ 120$ (in which the relative merits of a top and a bottom-doup harness are compared), it is usual to weave net leno fabrics with a top-doup harness. For this reason, the "drafts" and shedding plans, for the samples of leno fabrics represented in the accompanying illustrations, ale adapted for top-doup harnesses. With such a harness, the conditions of shedding which obtain in respect of a bottom-doup harness, as 
explained in $\$ 103$, are simply reversed, just as if a bottom-doup harness were inverted. By inverting the diagrams representing a bottom-doup harness, given in Figs. 433, 434 and 435, and also by reversing the shedding plan given in Fig. 432 (except that representing the easer), the conditions of a top-doup harness will be faithfully represented (excepting that, being ink lines drawn on paper, and not actual threads, the relative positions of standard and doup warp threads remain the same, whereas doup warp threads should cross over the top of standard warp threads). Thus, an open shed is formed with

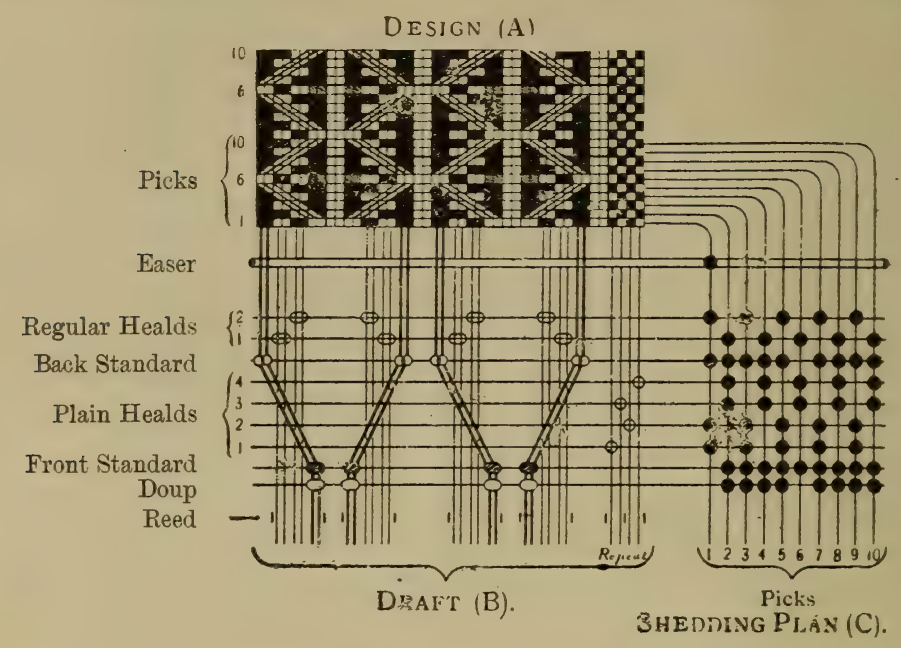

Fig. 439.-Design, draft and shedding plan for net leno fabric, Fig. 438.

a top-doup harness by raising the heald governing standard warp threads, and also the front standard; and a cross shed is formed by raising the back standard controlling doup warp threads, and also the heald governing standard warp threads, and, at the same time, slackening doup warp threads to prevent excessive strain upon them.

$\$ 106$. Fig. 438 represents an example of net leno weaving by means of only one set of doups. In this example, a net leno stripe is developed at regular intervals, from four pairs of white doup warp threads drafted to the right and left alternately, of 
their respective standard warp threads, which latter consist of tine threads taped in pairs. The intervening stripes consist of the plain or calico weave, on which a spotted effect is developed by means of extra picks of coarse white weft. These float loosely underneath the leno stripes, whence they are subsequently cut away, as seen in the corner turned down. The design, with the draft and shedding plan for that cloth, are respectively indicated at A, B and C, in Fig. 439. (Horizontal lines in the dralts and

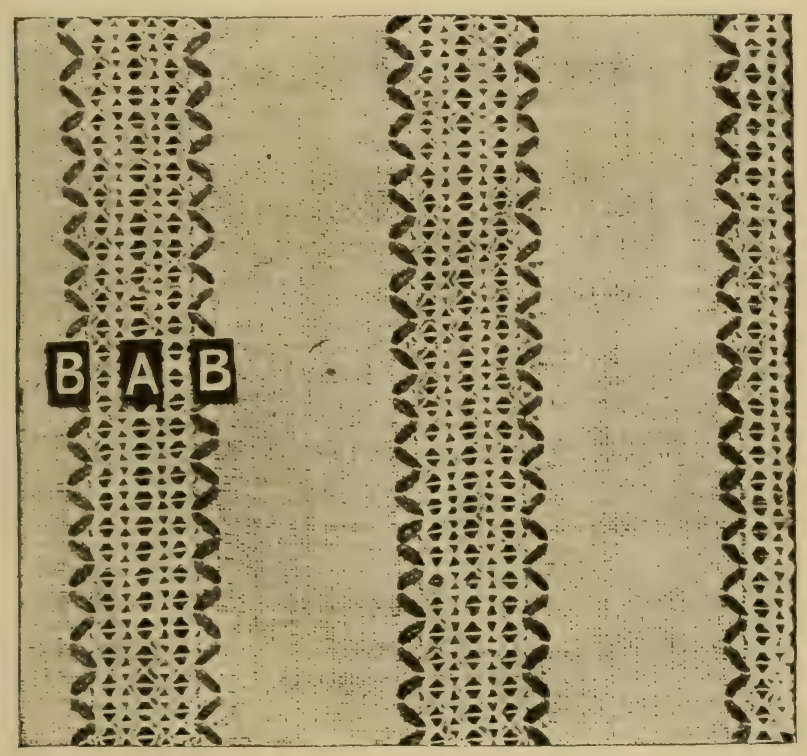

Fic. 440.- Two-doup net leno fabric, for which the design, draft and shedding plan are given in Fig. 441.

shedding plans represent healds; rertical lines in the drafts represent warp threads; and a circle placed on an intersection of a warp thread and heald indicates that such warp thread is drawn through such heald. Vertical lines in the shedding plan represent picks of weft ; and a black spot placed on an intersection of a heald and pick signifies that such heald is raised for such pick.) For simplification of the shedding plan, the spotting with extra weft, in the present example, is left out of consideration. By carefully studying the design, dratt and 
shedding plan of each of the accompanying examples of leno fabrics their construction will not be very difficult to under-

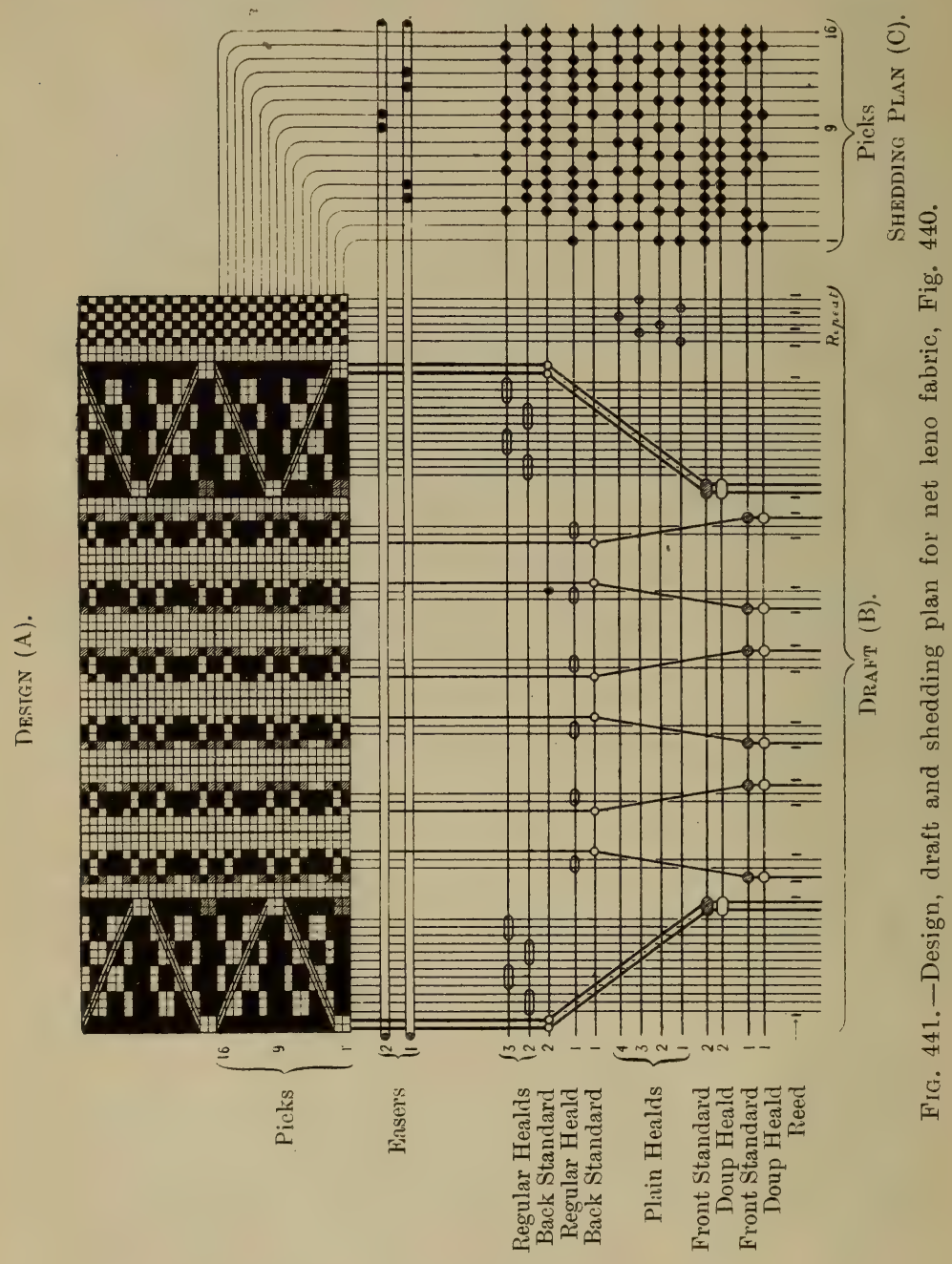

stand. The operation of the healds for each pick of weft may be seen by tracing them individually from the design to the shedding plan. 
For the general guidance of students it may be stated that with a top-doup heald harness, a cross shed is formed (in respect of any one series of doup threads) by raising standard and doup warp threads together, by means of their respective regular healds and back standards, whilst the same doup threads are held down in front by means of their front standard and doup healds; at the same time, doup warp threads are slackened by

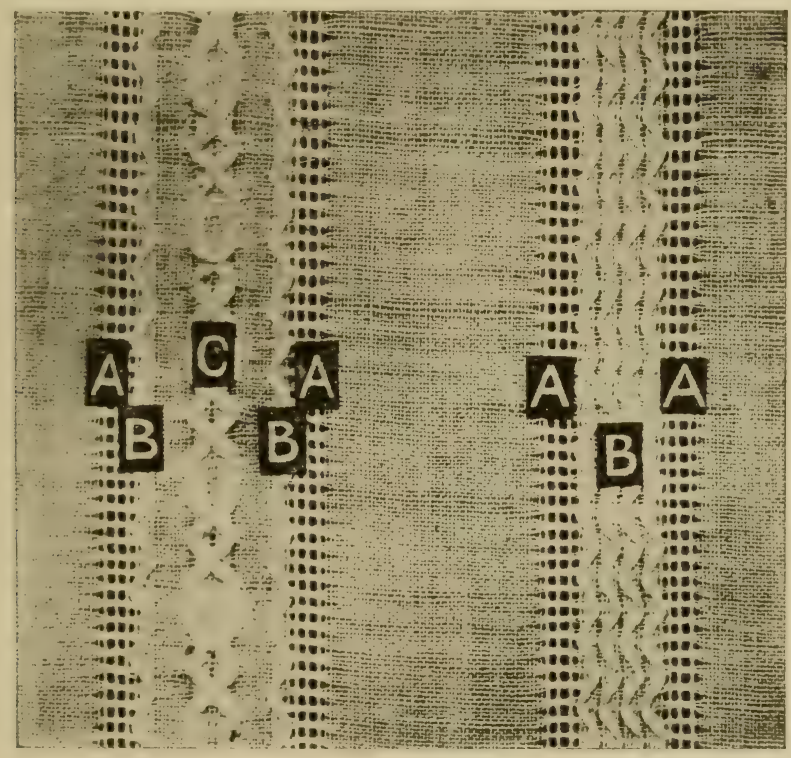

FIG. 442.-Three-doup net leno fabric, for which the design, draft and shedding plan are given in Fig. 443.

means of their easers, to prevent undue strain upon them whilst making a cross shed. An open shed is formed by raising front standards (as indicated by shaded squares in the designs), with such standard warp threads as are required, and, at the same time, learing doup healds down. (The object of raising a front standard heald without its corresponding doup heald is to liberate doup warp threads so that they may return to the normal side of their respective standard warp threads.) When a doup thread is required at any time to pass over one or more than one pick 
of weft, it must be raised for such pick or picks by means of the front standard and doup healds, as well as by the back standard heald, both at the same time.

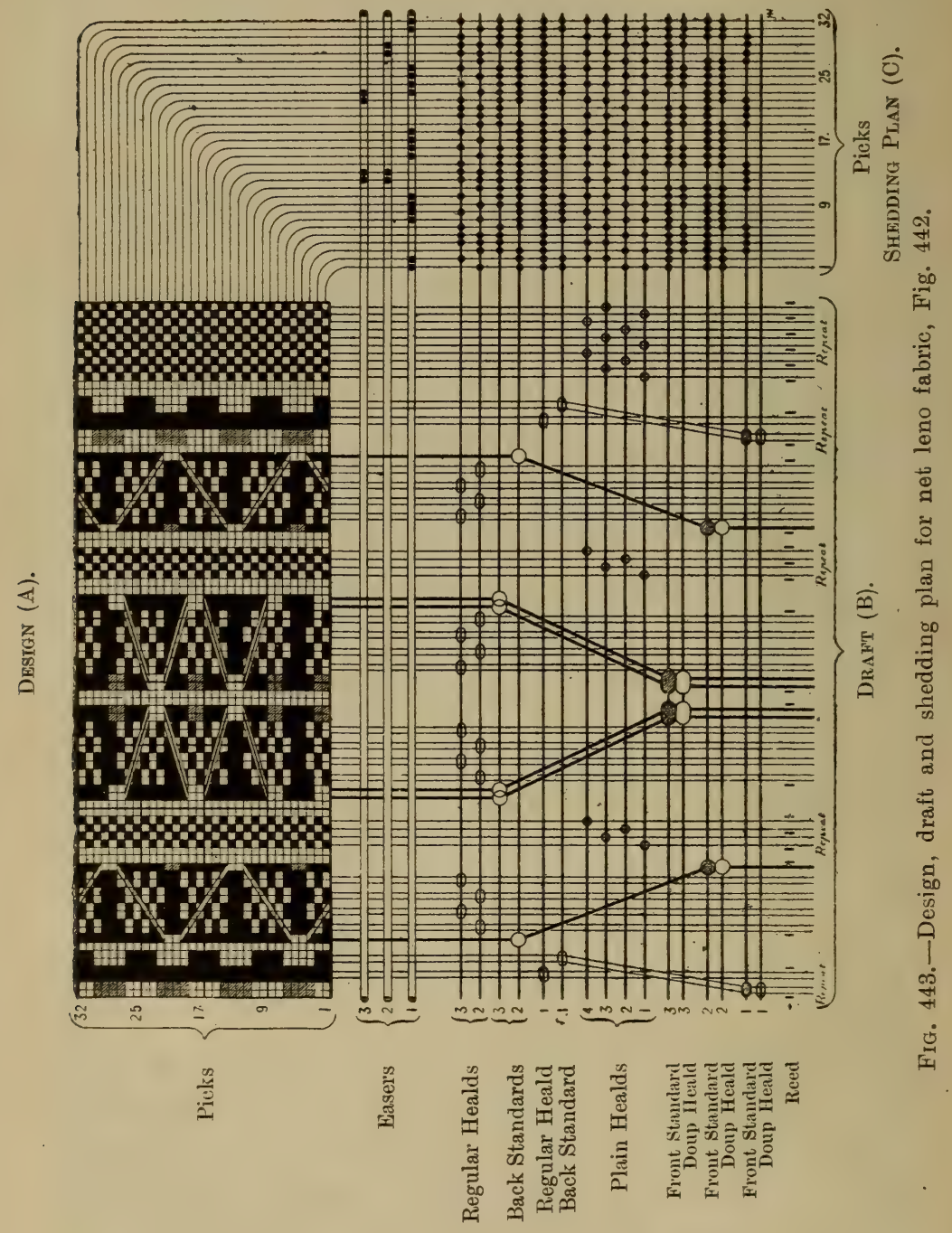


$\$ 107$. On examining the accompanying drafts it will be observed that the dents of the reed are not of uniform width, and also that some dents are left empty. It frequently becomes necessary to remore reed wires in order to obtain wider dents to receire doup and standard warp threads-when these are in such quantity as to prevent their free movement or passage (during shedding) within a dent of normal width, which would chafe and break them as the sley oscillates to and fro. Also,

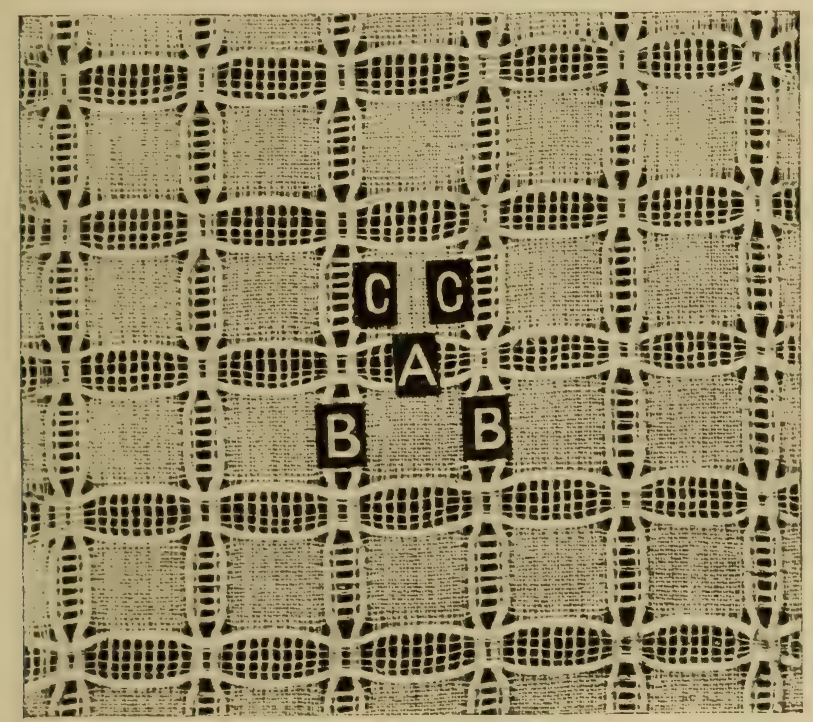

FIG. 441.-Three-doup compound net leno fabric, for which the design, draft and shedding plan are given in Fig. 445.

dents of the reed are sometimes left empty to permit of net doup threads spreading in cloth, and also to accentuate the perforations characteristic of many leno effects, as exemplified in stripes A, Fig. 440, and stripes B, Fig. 444.

Fig. 440 illustrates an example of a two-doup net leno fabric, of which the design, draft and shedding plan are given in Fig. 441. One set of doups are required to produce stripes $\mathrm{A}$, and another set to produce stripes B. Stripes A consist of a neat open network, caused by the reverse drafting of six doup threads, 
each of which crosses to taped standard warp threads. Stripes B consist of a pair of doup threads, which cross over twelve standard threads (taped in threes) to produce a continuous wave line.

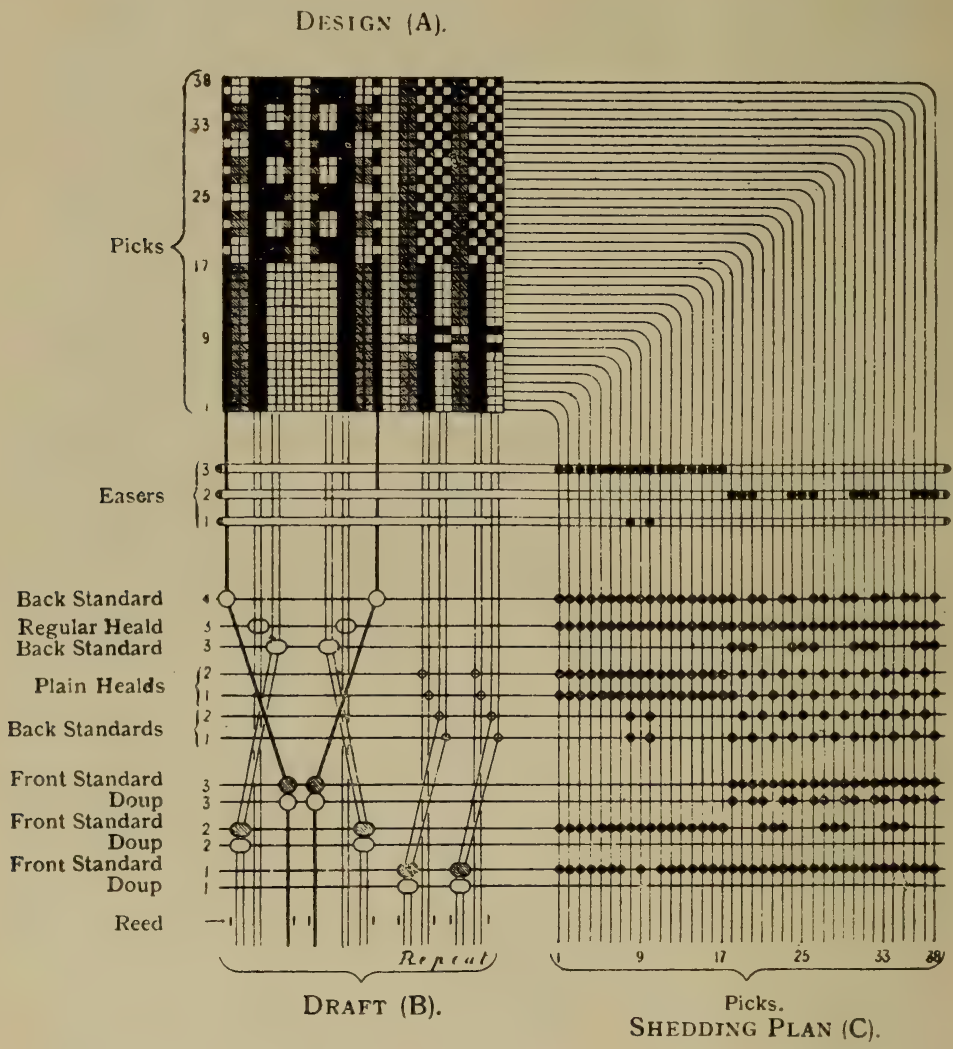

FIG. 445.-Design, draft and shedding plan for compound net leno fabric, Fig. 444.

Figs. 442 and 444 illustrate examples of leno fabrics, each requiring the use of three sets of doups to produce net stripes A, $\mathrm{B}$ and $\mathrm{C}$ respectively. That number of doups is rarely exceeded in one loom, owing to the complications to which they give rise by the addition of numerous accessories, and the difficulty of 
obtaining grood and clear warp sheds during weaving. The construction of the fabric represented in Fig. 442 is clearly indicated in the accompanying design, draft and shedding plan (Fig. $443)$, which it will well repay a student to carefully investigate.

Fig. 444 is a check leno fabric of special interest, inasmuch as it embodies an uncommon feature in leno weaving. This consists of a compound leno effect produced by causing a thick net doup thread to cross from side to side of other doup and standard threads, which, combined, constitute the standard threads for that net doup thread. By carefully examining the design, draft and shedding plan (Fig. 445) for that cloth, and following the operation of the healds for each pick of weft, its construction will be easily understood. The same course should be pursued with each of the preceding examples, paying special attention to the method of drafting, and the operation of doup healds, front and back standard healds, and easers.

\section{Cauze and Net Leno Figuring by Means of Several Back Standard Healds to each Doup Heald.}

$\$ 108$. In $\$ 105$ it was stated that, for the production of typical net leno fabrics, as represented by the foregoing examples, the number of doup healds, front standards and certain other essential equipments of a leno loom, must necessarily correspond with the number of different schemes of interweaving the respective doup threads in the same fabric; and, also, that the number of doup healds in one loom rarely, if ever, exceeds three. Under certain conditions, however, it is possible to develop gauze and leno effects of a more or less ornate character by means of only one doup heald, and one front standard that operate in conjunction with any practicable number of back standard healds to govern doup warp threads, and with a corresponding number of regular healds to govern standard or regular warp threads. Or, the scope of this type of leno harness may be increased by employing more than one doup heald, each to operate in conjunction with a distinct set of back standard healds of any practicable number. It is doubtful, however, if more than two doup healds could be satisfactorily 
employed with this arrangement of doup harness. Patterns developed by this system are frequently so elaborate as to give the impression that they have been produced either by a Jacquard machine, or else by quite an impracticable number of doup healds.

Of course this system of leno weaving imposes certain limitations in respect of the style or character of "douping" that are not existent with the use of independent doup healds. For example, all doup waip threads must necessarily cross from the normal or open side to the crossed side of their respective standard warp threads simultaneously, since they are all controlled by the same doup heald; but they may be raised either on the normal or open side of standard threads, or left down (on certain picks only) in practically any pre-determined manner. The system also virtually demands the crossing of warp threads in some definite and uniform manner at regular intervals of picks. Indeed, in one variety of this class, in which single threads cross each other to form a true gauze figure on a ground of the plain weave, or vice versâ, such conditions are inevitable ; but in another variety, in which comparatively thick net doup threads are introduced solely as a means of embellishment, the restrictions are not quite so limited.

Leno fabrics of the class under present notice are of three distinct varieties, namely: (1) those in which a gauze figure is surrounded by the plain or tabby weave (or vice versâ), and in which warp threads cross each other as single threads, with one pick in each shed, as illustrated at C, Fig. 446 ; (2) those in which either warp or weft is allowed to float freely (for the development of brocade figuring), but which in all other respects are like (1); and (3) those in which net leno figuring is developed by means of thick net doup threads upon a comparatively light ground texture, preferably of the plain calico weave.

Although it is for many reasons (as explained subsequently in $\$ 120$ ) more expedient to weave net leno fabrics of the ordinary type by means of a top-doup harness, that arrangement is perhaps not so well suited as a bottom-doup harness to the production of the class of leno fabrics under present consideration, 


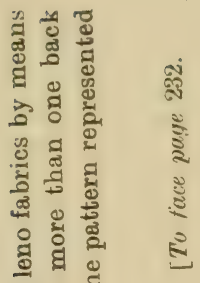

r) $\frac{5}{5}$

तิ है

\& 9 व

क्ष

ס 으 토

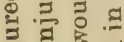

30 \%

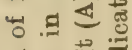

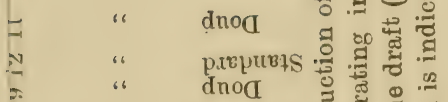

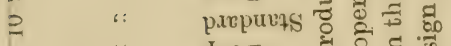

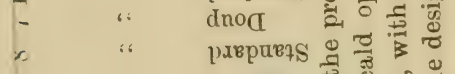

- $\quad \therefore \quad$ pxepurats

- " "Inod

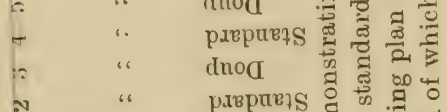

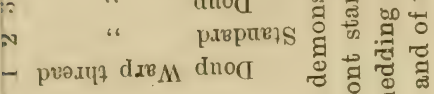

च

용 \&

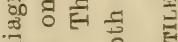

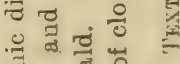

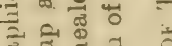

ज्ञ

万ण

!. ฮึ

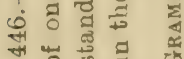

ఏ 



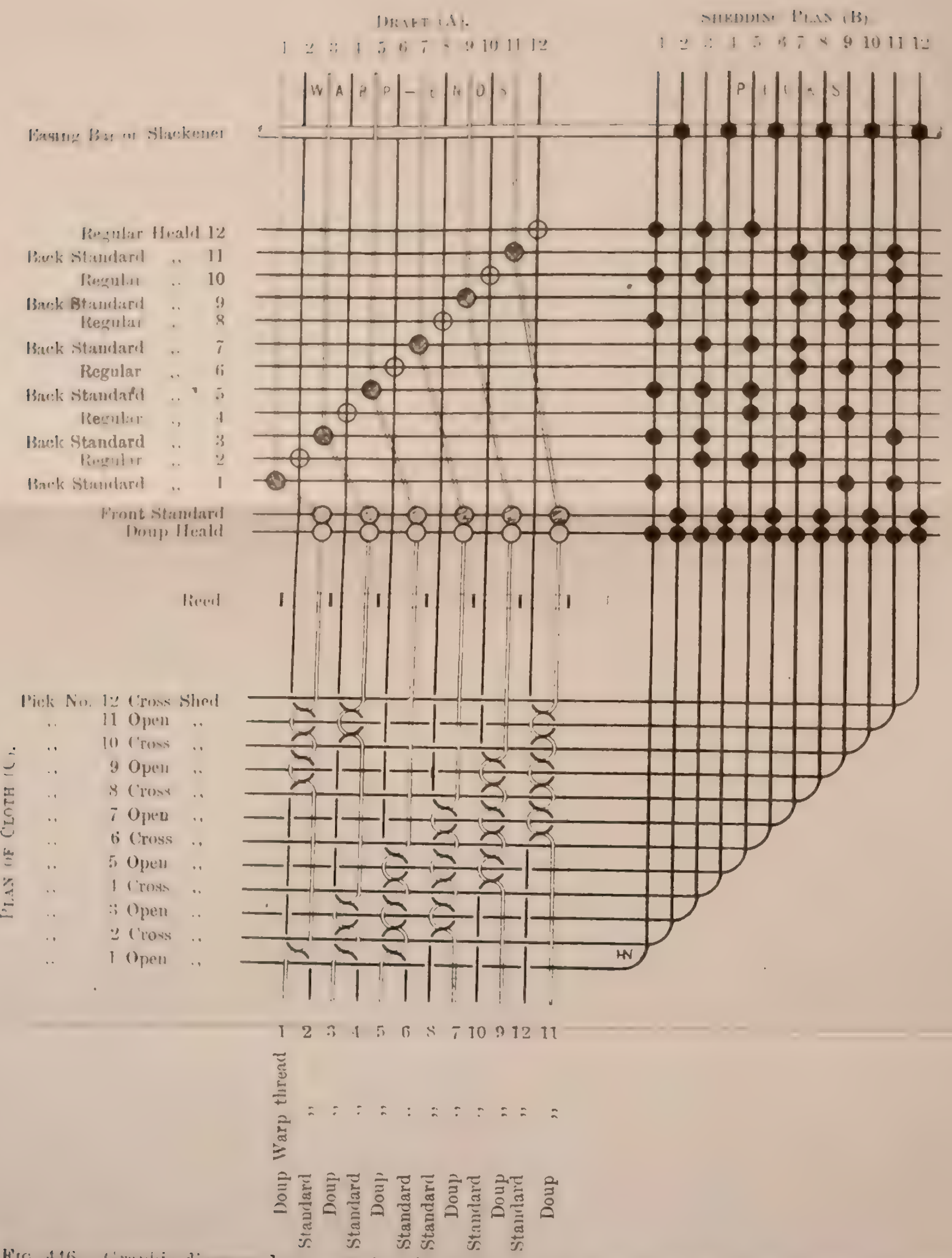

H'tc. $\$ 16$. - - iruphic diagram demoustrating the production of tigured gauze and leno fabrics by means of oue doup and one front standard heald operating in conjunction with more than one back stundard hoald. The shedding plau (B), with the draft (A), rould develop the pattern represented in the plan of cloth $(C)$, and of which the design is indicated in Fig. 447.

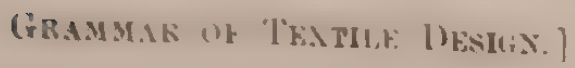

[To face paye 232 . 

chiefly because the formation of a cross shed with a harness of this type would require all healds (excepting the front standard and doup healds) to be raised. Still, when that course would not involve excessive straining and risk of breakage of mechanical parts, nor absorb an abnormal degree of motive power, it would be adrisable (if other circumstances were favourable) to employ a top-doup harness in preference to a bottom-doup harness. It should be carefully observed, however, that the accompanying drafts and shedding plans for this variety of leno fabrics are all arranged for bottom-doup harnesses.

$\leqslant 109$. The construction of the first-named variety of this class of fabrics is illustrated in Fig. 446, where A and B respectively show the method of drafting and shedding to produce cloth represented at $\mathrm{C}$, in accordance with the design given in Fig. 447. This is a simple pattern, repeating on twelve warp threads and picks, developed by alternating diagonal bands of the gauze and calico iveaves, and will serve to demonstrate the principles on which they are designed and woven.

As indicated in the draft at A (Fig. 446) warp threads are drawn through twelve healds with a straight-over draft. After passing through those healds in regular succession, alternate warp thread;, that are passed over an easer, are taken as doup threads (represented by white lines), which, after crossing underneath from left to right of the intermediate warp threads (which become standard threads, represented by black lines), are passed through the loose slips of the doup or half-heald which hang underneath warp threads, as previously described and illustrated in \$ 102. Thus: healds Nos. 1, 3, 5, 7, 9, 11 become back standards to govern doup threads; whilst healds Nos. 2, 4, 6, 8, 10, 12 are regular healds to govern standard war'p threads.

$\leqslant$ 110. It is characteristic of this particular variety (1) of leno fabrics that all doup threads, and those only; are invariably raised by the front standard and doup healds for alternate (say even-numbered) picks to form cross sheds; but both doup and standard threads may be raised for intermediate (or odd-numbered) picks to form open sheds, according to the pattern required; care being taken not to raise, at the same time, fellow doup and standard threads that cross each other, and pass through the 
same dent of the reed. Thus, where it is required to form gauze, doup threads are raised by their respective back standards; whilst in the calico portion standard threads are raised by their respective healds for odd numbered picks only. The operation of healds in this manner gives rise to a peculiar phenomenon in cloth which is, at first, very puzzling to those who are more or less intimately acquainted with leno fabrics constructed in the usual manner. An examination of the plan of cloth will show that doup threads appear to form an open shed when raised on the right of their respective standard threads, whereas they are actually forming a cross shed, and vice versci. This apparent anomaly arises in consequence of employing only one doup heald which must rise for alternate picks to develop the plain weave in observance of the principles governing the construction of these fabrics. It should also be observed $(a)$ that the easer must slacken doup threads whenever both front standard and doup healds are raised together to form a cross shed - that is, on alternate picks; and (b) that the doup healds must lift without the front standard on intermediate picks. The reason for raising the doup heald without the front standard, when an open shed is formed, is to liberate all doup threads, and permit of the required doup threads being raised on the normal side of their respective standard threads by means of their back standard healds. The reason will now be manifest why a bottom-doup harness is preferable to a top-doup harness for weaving these fabrics. If the latter were employed to weave the design indicated in Fig. 447, the shedding plan B (Fig. 446) would require to be entirely reversed in all parts excepting the easer, and such a course would involve much greater power for shedding.

The chief considerations affecting the construction of these fabrics are clearly indicated in the plan of cloth at $\mathrm{C}$, which should be carefully studied in conjunction with the design (Fig. 447), draft at $A$, and shedding plan at $B$. This may best be accomplished by comparing each pick of weft, in the design, with the corresponding picks in the shedding plan; and by tracing the lines (representing picks) from the shedding plan to the corresponding picks of weft, in cloth, and thereby trace the 
cause to the effect. The preparation of designs for these fabrics will be greatly facilitated by using design or point paper on which the narrow divisions, in one direction, are ruled off in pairs, with 1 lines of medium thickness, as seen in Fig. 447. The two narrow divisions between two medium lines correspond to a doup warp thread and its fellow standard warp thread that cross with each other and pass through the same dent of the reed. Such a course will reduce the risk of a designer inadvertently raising

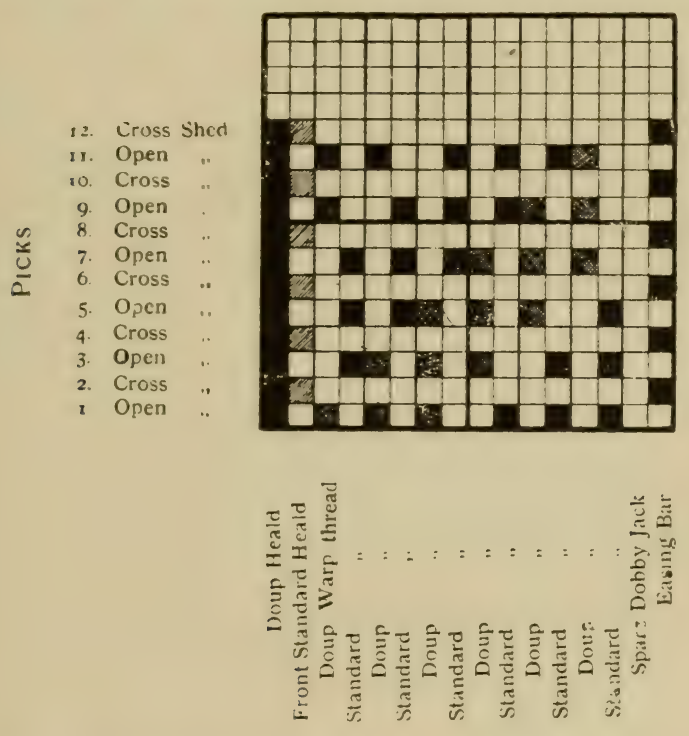

FIG. 447.-Design for pattern represented in graphic plan of cloth (C), Fig. 446.

two fellow warp threads at the same time, which would not be in strict accordance with the principles governing this particular variety (1) of the class of fabrics under present consideration.

$\$ 111$. In $\$ 108$ and 109 it was described how the simple gauze and plain calico weaves could be combined to develop simple figured effects by means of only one doup heald and a front standard heald, operating in conjunction with several back standard and regular healds, to produce the first-named rariety (1) 
of this class of leno fabrics, as illustrated in Fig. 446. The second variety (2) of this class is characterised by warp-float figures on the obverse side, and weft-float figures on the reverse side of cloth (when in the loom), either in combination with the simple gauze weave only, or with both that and the plain calico weave. It should be noted, however, that in observance of the principles governing the construction of these fabrics, doup warp threads may only be allowed to float on that side of their fellow standard warp threads to which they have been crossed in the shedding harness, and not on the normal or open side of those threads. The reason for this will be understood when it is remembered that all doup warp threads must necessarily be raised on their crossed side of standard warp threads by means of the front standard and doup healds, for alternate picks of weft, as required for the development of the gauze and plain weaves ; but where warp figure is required, standard warp threads also may be raised in those parts for the same picks. For the intermediate picks, therefore, doup warp threads must be raised in the float or brocade figure and gauze portions only, by means of their respective back standard healds; but they must be left down in the plain weave; also, standard warp threads must be raised in the brocade figure and plain weave portions only, but left down in the gauze portion, for the same picks. When those picks are inserted the doup heald is raised, in order to liberate all doup warp threads, and permit such as are required up to be raised by their respectire back standard healds. It should be explained that, although doup warp threads may be raised (for the intermediate picks) on the normal side of standard warp threads by means of their back standard healds, in order to develop brocade figuring, they will not remain on that side in the cloth, but will be pulled to the crossed side (when raised for the alternate picks) by means of the front standard and doup healds, and permanently remain there, as described in $\$ 110$. Doup threads are enabled to return to their crossed side in the brocade figure portions, in consequence of both standard and doup warp threads being raised together in those portions, and not intersecting with weft. When preparing designs for this variety of leno fabrics, it is adrisable, in order to obtain the best results, 
to always separate warp figure from gauze by a margin of the plain or calico weave, as illustrated in the design, Fig. 448 , in which shaded squares represent the gauze weave. The shedding plan for that design, given in Fig. 449, is adapted for a bottom-doup harness similar to that represented in diagram, Fin. 446 , but consisting of eight back standard and eight regular healds. with a front standard and a doup heald, and an easing bar. By studying Figs. 448 and 449 in conjunction with that diagram, the foregoing description will be more easily comprehended. It should be observed that these two varieties of leno fabrics impose certain restrictions with regard to the method of drafting warp

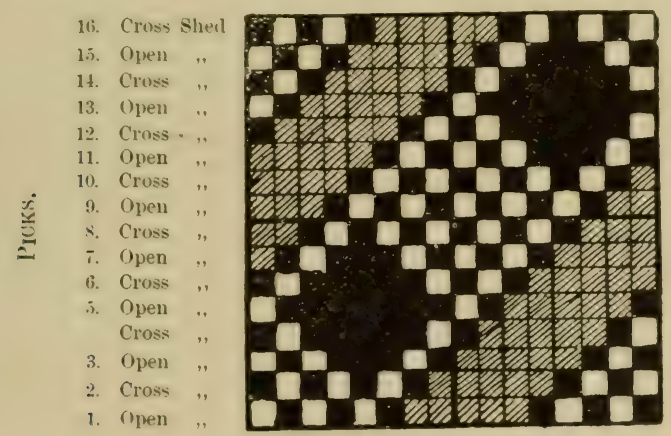

$123+5$ i $\tau$, ! 1 10 111213141510

FIG. 448.-Design for figured gauze fabric for which the shedding plan is given in Fig. 449 .

threads through the healds, namely: Doup and standard threads must be arranged in the harness alternately with each other, and with doup threads crossing their fellow standard threads uniformly in the same direction, when passing from their respective eyes in the back standard healds to their appointed doup slips in the doup heald. These conditions are necessary in order to effect the combination of true gauze with the plain or tabby weave, which characterises these fabrics. Also, their construction does not permit of the employment of "pointed" or "centred" drafts that are obtained by simply reversing in the usual manner. This arises in consequence of warp threads being in pairs, each of which consists of a doup and a standard 
thread that are complementary to each other and operate in conjunction in the gauze and calico portions of the fabric, and not as independent threads.

112. By far the most useful and interesting variety of this type of figured leno fabrics is that constituting the variety (3), in which " net leno" figuring is developed upon a comparatively light muslin texture, as exemplified in the accompanying reproductions of cloth (Figs. 450, 452, 454). This variety (3) of leno fabrics is of a distinctly different character from any
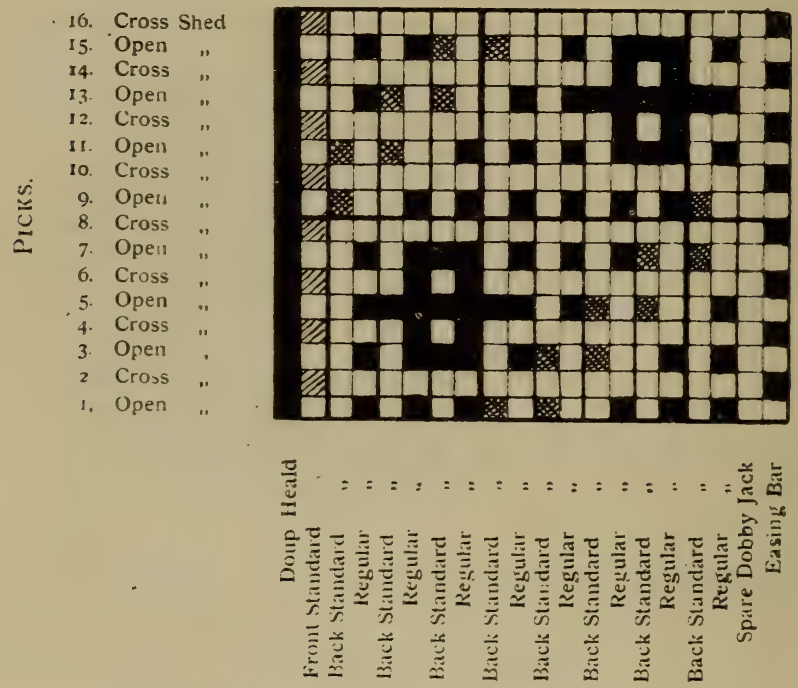

FIG. 449.-Shedding plan for design Fig. 448.

that have been previously described, and affords much greater scope to a designer in the production of decorative effects. Also, it imposes fewer restrictions in the construction of designs than are imposed by the varieties of one-doup leno fabrics (1 and 2). This arises from the employment of comparatively thick "net" doup warp threads for figuring purposes only. These work quite independently from the fine ground warp threads, and play no part whatever in the construction of the foundation texture. They may, therefore, be employed in greater or lesser numbers in relation to ground warp threads, and may be dis- 
posed either in groups to produce stripes (as in Fig. 450), or at regular intervals and short distances apart, for the development of all-over patterns. All doup warp threads are controlled by one doup heald and a front standard heald (for the formation of cross sheds) and such number of back standard healds (to form open sheds) as corresponds with the different orders of interwearing doup threads with the ground texture. It is imperative, therefore, that doup warp threads must "doup," i.e., be raised

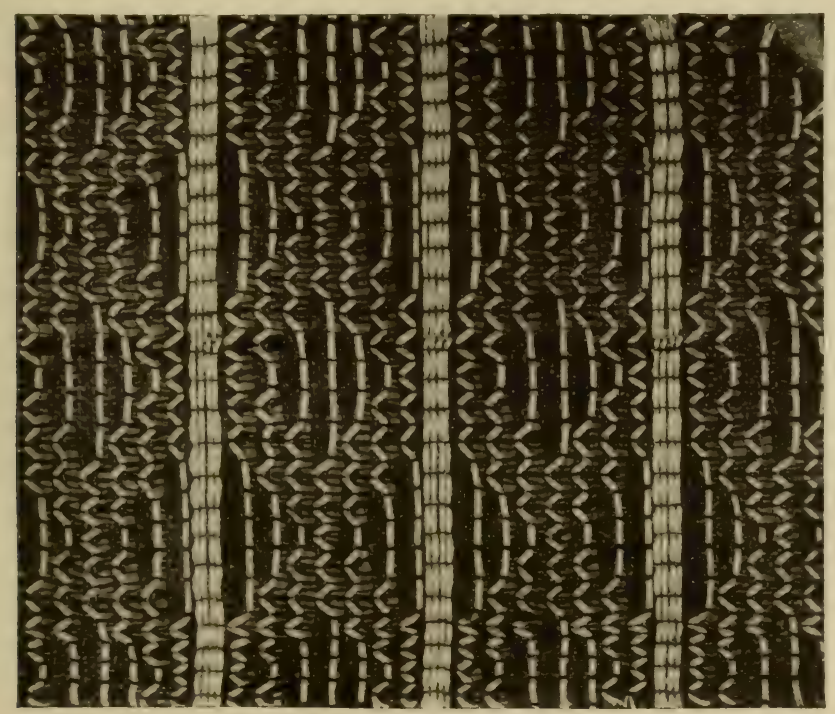

Fic. 450. - Yet leno fabric woren by means of one doup and one front standard heald, and four back standard healds, for which the design, draft and shedding plan are given in Fig. 451.

on their crossed side to form cross sheds simultaneously, although they may be either raised or left down by their respective back standards, for the open sheds; hence, only one easing bar is required to slacken all doup warp threads simultaneously during the formation of cross sheds. Provided designs are constructed to ensure a uniform rate of contraction by all doup threads, the latter may be contained on one warp beam: but if their rate of contraction varies, they will require to be wound upon separate beams, according to the different rates of 
contraction. Ground warp threads are governed by healds placed in the rear of back standard healds, and are contained upon a separate warp beam.

Doup threads may be crossed under their respective standard or ground warp threads (in the shedding harness) in almost any desired manner, either in the same direction, uniformly, or in reverse directions, as required. In the example of cloth represented in Fig. 450, white mercerised doup threads of twofold yarns are arranged in groups to form stripes. Each group consists of seven pairs of threads, all of which cross their standard warp threads in the same direction in the harness, with each pair of doup threads crossing three pairs of black ground warp threads that are intersected with black weft to produce a light muslin foundation texture. The leno stripes are separated by a white stripe composed of four pairs of white mercerised threads of the same material as doup threads.

The method of designing and drafting for this variety of leno fabrics is demonstrated in Fig. 451, which shows the design, draft and shedding plan at A, B and $\mathrm{C}$ respectively, for the production of the example of cloth represented in Fig. 450. The pattern repeats on sixty-three warp threads (counting each pair of white mercerised threads as one) and sixty picks of weft. Warp threads would require to be wound upon three warp beams, namely, one for ground threads, one for doup threads and one for the white threads to form the narrow stripes. The shedding harness comprises a doup and a front standard heald, four back standard healds, two healds to govern ground warp threads, two healds to govern black warp threads to weave plain cloth in the narrow stripes, and one heald to control the threads forming the narrow white stripes.

$\$ 113$. It is important at this particular stage to inform readers that if net leno fabrics are produced by means of a bottom-doup harness, they will be woven face downward. This warning is necessary to prevent confusion by the apparent inconsistency between the specimens of cloths, as here represented, and their respective designs, which latter, being prepared for bottom-doup harnesses, represent the reverse side of those cloths. The dotted lines on the design (Fig. 451) are not essen- 
tial to its construction, but are merely introduced to indicate doup warp threads as they will appear on the face side of cloth, so that their working may be easily followed. On examining that design and shedding plan, it will be seen that a cross shed is formed for two consecutive picks out of every six, by raising

$$
\text { Design (A). } \quad \begin{aligned}
& \text { Sheding } \\
& \operatorname{PlaAN}(\mathrm{C}) \text {. }
\end{aligned}
$$
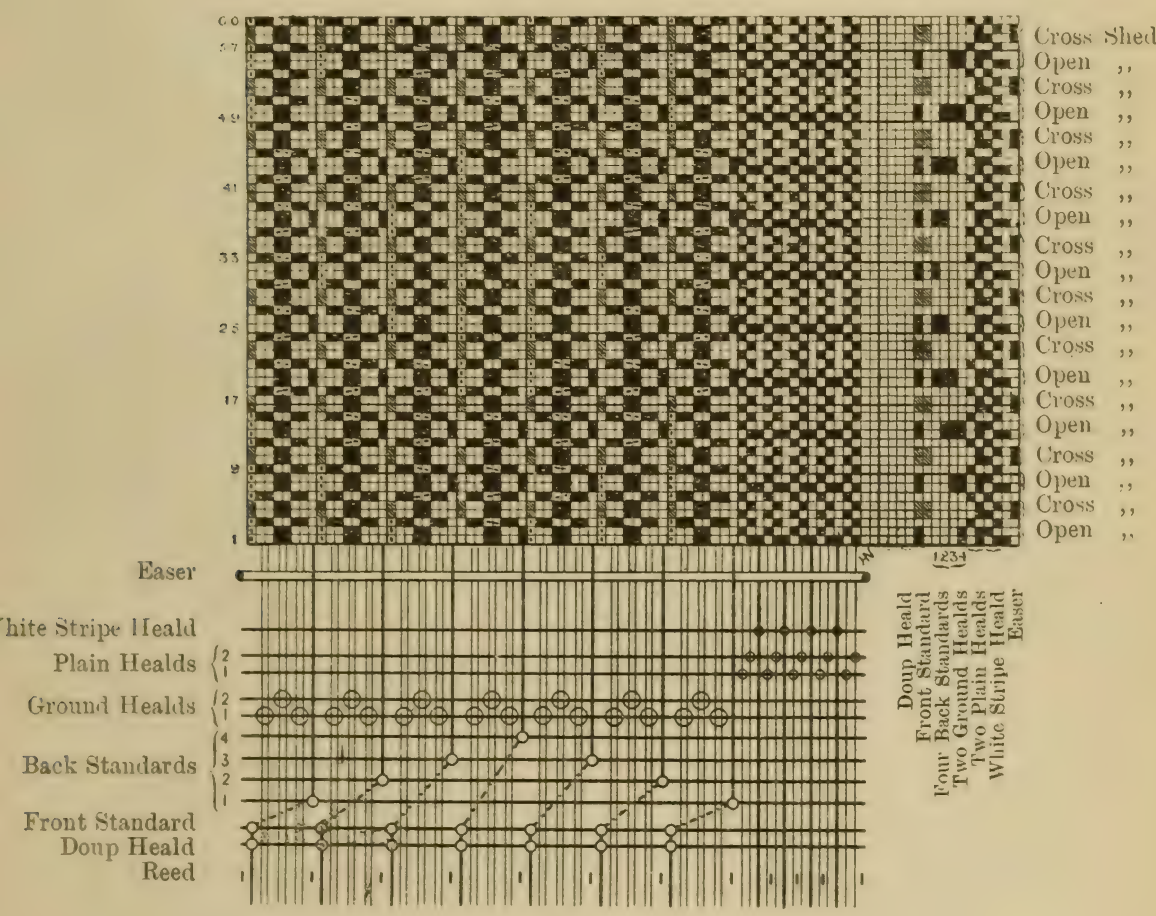

\section{Draft (Bi.}

FIG. 451.-Design, draft and shedding plan for net leno fabric, Fig. 4.50.

the front standard and doup healds, and slackening the easer; whilst the ground healds operate as indicated. The two consecutive picks midway between those in the cross sheds are inserted in open sheds, for which doup warp threads may be either raised or left down by their respective back standard healds, as required. When, however, the single picks of weft, 
that intervene between those in the cross and open sheds, are inserted, all doup warp threads must remain down. The peculiarity of these fabrics (that was referred to in $\$ 110$ and 111) of doup threads appearing to be on the normal side of standard warp threads, when they are actually on the crossed side of those threads, and vice versâ, is also observable in this variety of leno fabrics.

114. Figs. 4503 and 455 are reproductions of other examples of figured "net leno" fabrics in which the figuring is developed by means of only one doup and one front standard heald,

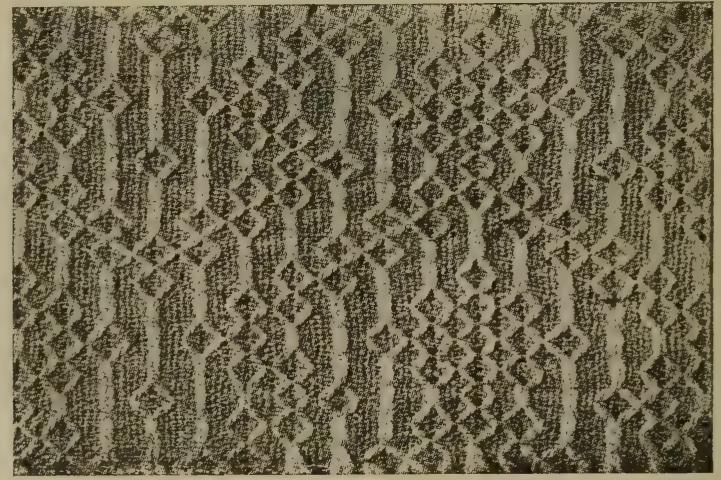

FiG. 452.-Net leno fabric, woven by means of one doup and one front standard and five back standards for which the design, draft and shedding plan are given in Fig. 453.

operating in conjunction with several back standard healds, and two or more healds to govern ground warp threads to produce the foundation texture. In these examples doup warp threads are arranged in pairs disposed at regular intervals apart, for the production of all-over designs. The threads of each pair cross their respective standard ground warp threads in reverse directions, so as to develop a neat diamond formation, excepting where doup threads lie straight and parallel on their crossed side of standard warp threads. The present examples will serve to indicate the general character of designs suitable for these fabrics, and also the fair scope they offer to a designer in the creation of effective patterns. The example of cloth 


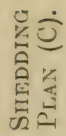

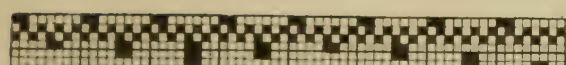

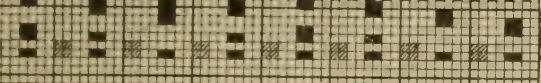

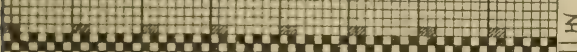

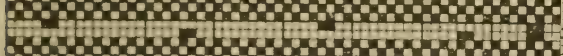

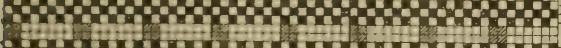

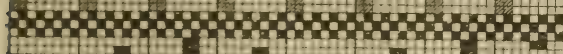

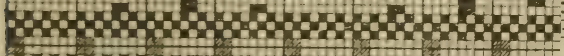

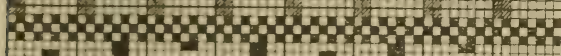

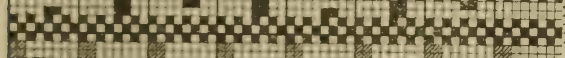

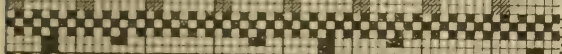

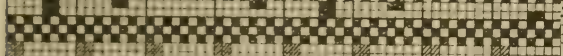
3.

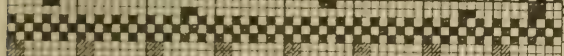

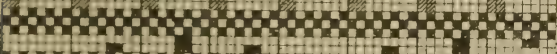
xmom कx.m. xhom xomosom $x$

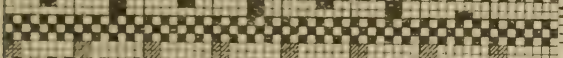

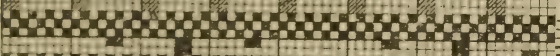
monkmonkm

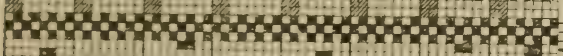
$2 \cos x \times m \times$

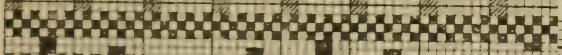
$2 \operatorname{mon} \times x \times m$ smonkmon $x \times m$ $x \times m$ maxh $x \times m \times x$

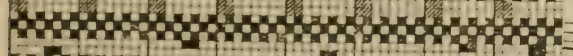
mankm
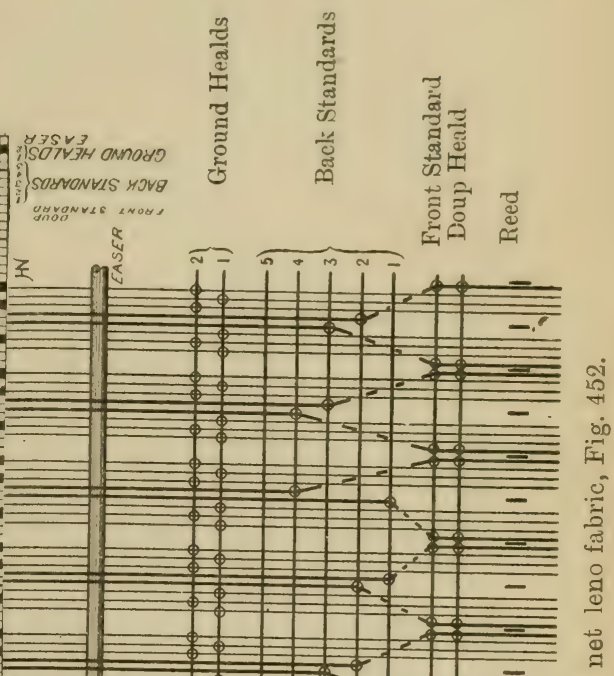
represented in Fig. 452 has a foundation texture of the plain weave, consisting of fine ground warp threads (taped in pairs) picked with fine weft. Doup warp threads, of two-fold yarn, each cross three pairs of standard threads, and are governed by five back standard healds, in addition to a front standard and a doup heald. It has required a different drafting of doup threads through the back standards, but not of ground warp threads, which are governed by two healds that rise and fall alternately for consecutive picks throughout.

The design, draft and shedding plan (arranged for a bottomdoup harness) required to weave the example of cloth (Fig. 452) are given at A, B and C respectively (Fig. 453). The pattern repeats on eighty-four pairs of ground warp threads (represented in the design and draft as single threads) and twenty-eight doup warp threads and sixty-four picks of weft. Doup warp threads are drawn through the back standards so as to form a reversed pointed draft. The method of drafting doup warp threads for these examples of cloth marks a distinctive and important feature of interest in their construction. As will be seen, on consulting the draft (B, Fig. 453), doup warp threads are disposed in pairs, with the two threads constituting a pair crossing from their normal to their crossed side in reverse directions simultaneously, and drawn through heald eyes in the same back standard. For example, the central pair of doup threads are drawn through the fifth back standard heald, and constitute one extreme point of the draft; whilst the first and last doup threads in the pattern, which cross in reverse directions, both pass through the second back standard, and constitute a pair forming another point in the draft.

This arrangement of the draft causes the threads of each pair to converge and lie side by side, quite straight and parallel with each other, when on their crossed side, but to diverge when raised by their back standard healds. Thus, by forming cross and open sheds at regular intervals (of picks) apart, the neat net leno diamond formation, characteristic of the present examples, is produced. On examining the design and shedding plan, it will be observed that an open shed is formed for two contiguous picks (the third and fourth) out of every eight picks, 
and a cross shed for the intermediate pairs of picks (the seventh and eighth), whilst the ground healds rise alternately for consecutive picks throughout, to produce the foundation texture. Owing to the different rates of contraction of doup warp threads with this design, those threads will require to be contained upon three separate warp beams, in addition to one containing ground warp threads.

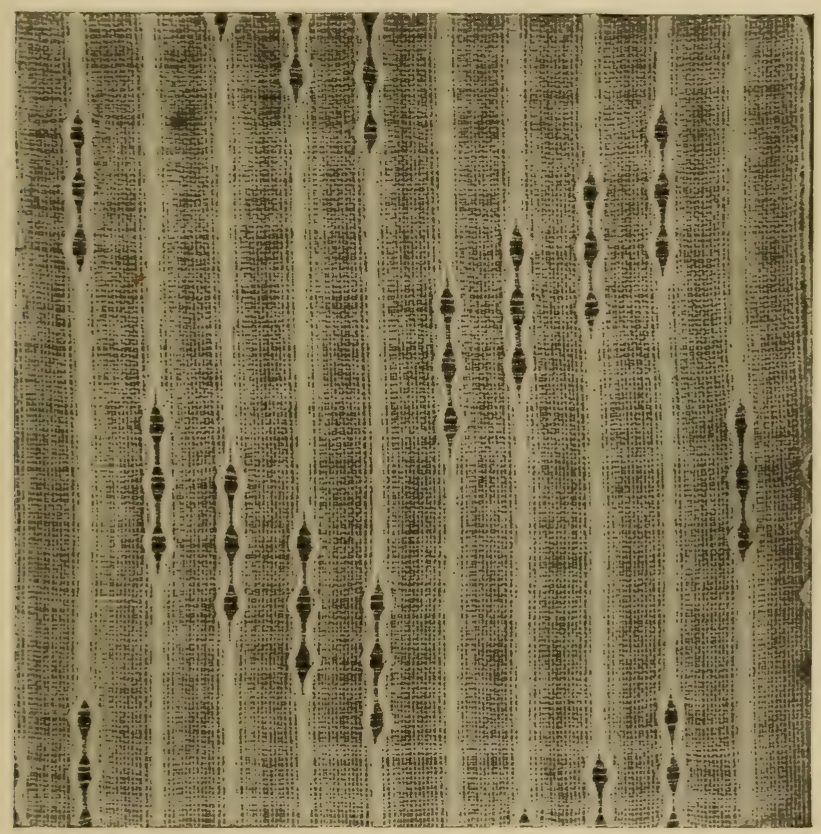

Frg. 454. -Net leno fabric, woren by means of one doup and one front standard, and eight back standards.

$\leqslant$ 115. The cloth represented in Fig. 454 shows a slight variation from the previous examples in having doup warp threads more widely dispersed upon a muslin ground texture of plain cloth. In this example, each set of doup warp threads consists of two pairs of threads of two-fold yarn, crossing their respectire standard warp threads in reverse directions. The ground warp threads, which serve as standard threads for each doup 
warp thread, consist of four threads taped in pairs, whilst the intervening stripes of plain cloth consist of eleven single warp threads. This example has required eight back standard healds to produce the pattern which it contains, with consecutive pairs of doup threads drawn through them in regular| succession to form a straight-through draft. The leno effect is developed by forming two cross sheds in succession, for single picks, at intervals of six picks, and by forming an open shed, where re-

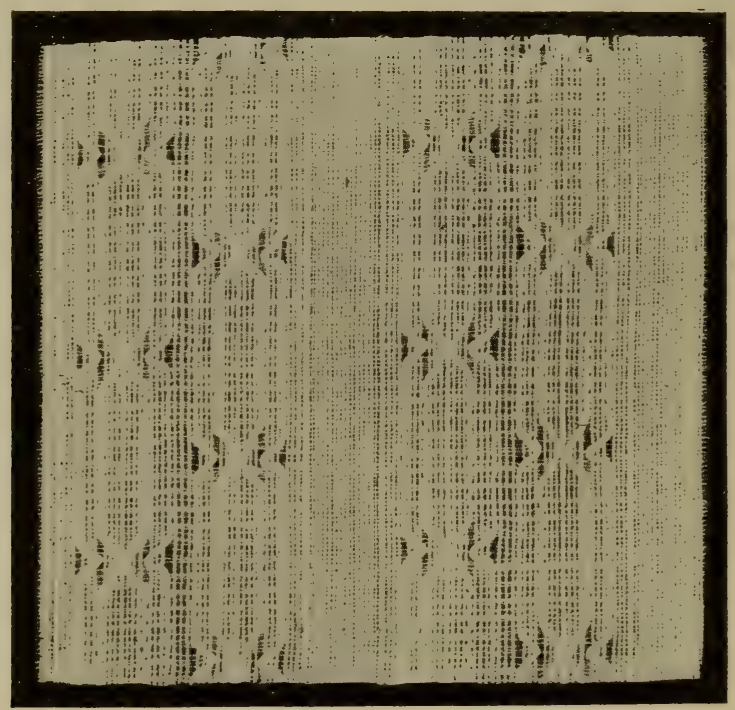

Frg. 455.-Net leno fabric, woven by means of two doups and two front standards, with two back standards operating in conjunction with each doup heald, and its front standard.

quired, also for single picks, midway between two cross sheds, but only at intervals of twelve picks; thus: 1 (cross shed), 2, 3, 4, 5, 6, $\mathbf{7}$ (cross shed), 8, 9, 10 (open shed), 11, 12, and so on. It is imperative, in order to create harmonious leno effects in fabrics of this variety, that cross and open sheds should be formed in a rhythmical order, and not at irregular intervals of picks apart. It may also be observed that if doup warp threads cross their standard threads in the same direction uniformly (as in Fig. 451), it is advisable to draw them 
consecutively through successive back standard healds; but if they cross in reverse directions (as in Fig. 453), they should be drawn through the back standards in pairs, as indicated in the draft $B$.

$\$ 116$. It is stated in $\$ 108$ that more than one doup heald, each to operate in conjunction with several back standard healds, may be employed to increase the scope of the type of leno harness under present notice. The example of cloth represented by Fig. 455 has required two doup healds, each operating with two back standards. In this example doup threads lie straight when on the normal side of their standard threads, as in ordinary net leno fabrics.

\section{Leno Specialities.}

$\$$ 117. In the production of gauze and leno fabrics by the methods previously described in this chapter, it is impossible to effect a crossing of any two or more warp threads with each other, unless the crossing threads are severally contained in the same dent of the reed: hence, a crossing may not be made with warp threads that are separated by a reed wire. This restriction, however, may be avoided by the use of specially. adapted leno weaving devices, whereby the crossing of warp threads is accomplished by means of doup healds situated betwcen a disappearing beating-up half-reed, or comb, and an ordinary deep stationary reed, situated between the doup healds and regular healds, as clearly represented in Fig. 463. Such arrangement of healds and reeds enables warp threads to be crossed either separately, or in groups, in almost any conceivable manner, irrespective of the order in which they pass through the dents of the stationary reed, during weaving. Thus, it is possible to effect a crossing of threads, en masse, that extend over several dents, without those threads crossing or douping with the intermediate warp threads which they simply pass over. The doup harness may be either a top or bottomdoup harness, or it may be a combination of both these arrangeinents. Also, warp threads may be passed through and governed by two separate and distinct doup slips of the same or different lengths, to effect a crossing to the right or left over 
a smaller or a greater number of threads, as required. This system of leno weaving affords almost illimitable opportunities to a capable designer in the creation of decorative effects of a very ingenious and pleasing character, as exemplified in Figs. 456 to 462 , which are full-size photographic reproductions from actual pieces of cloth representing a few typical examples of this particular variety of leno fabrics.

$\$ 118$. The construction of these fabrics will be better un-

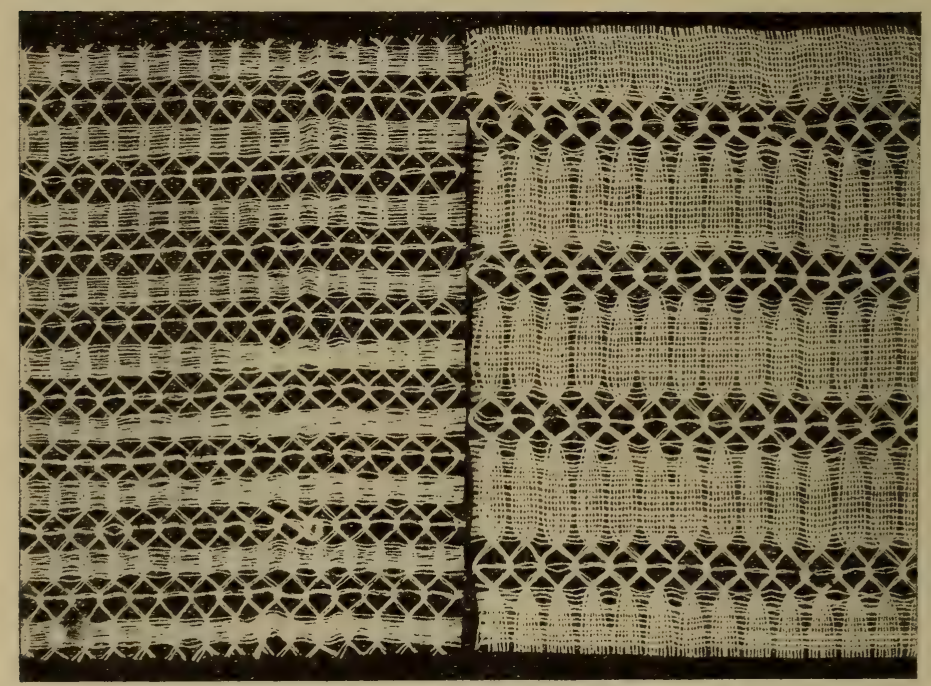

Fig. 456.

Fig. 457.

Special leno effe:ts produced by a system of crossing warp threads in front of the reed.

derstood if the reader is informed of the mechanical devices employed in their manufacture, of which there are several modifications that differ chiefly in details of construction and operation. With the object of conveying that information to students, a diagram representing a part sectional elevation of a loom equipped with Whitehead and Wood's modification of a special leno weaving device is given in Fig. 463. With a view to better demonstrating the operation of this device, the sley and its appurtenances are represented both at the backward 
and forward extremities of their movement by full lines and dotted lines respectively. As indicated in the diagram, instead of fixing a reed in its usual place in the sley, a reed $\mathrm{O}$, of unusual depth, is placed between the regular healds $\mathrm{N}$, and the doup healds $\mathrm{P}$, and permanently fixed to brackets secured to the loom framing. The function of the stationary reed is simply to effect an even distribution of warp ends over the required

FIG. 458.

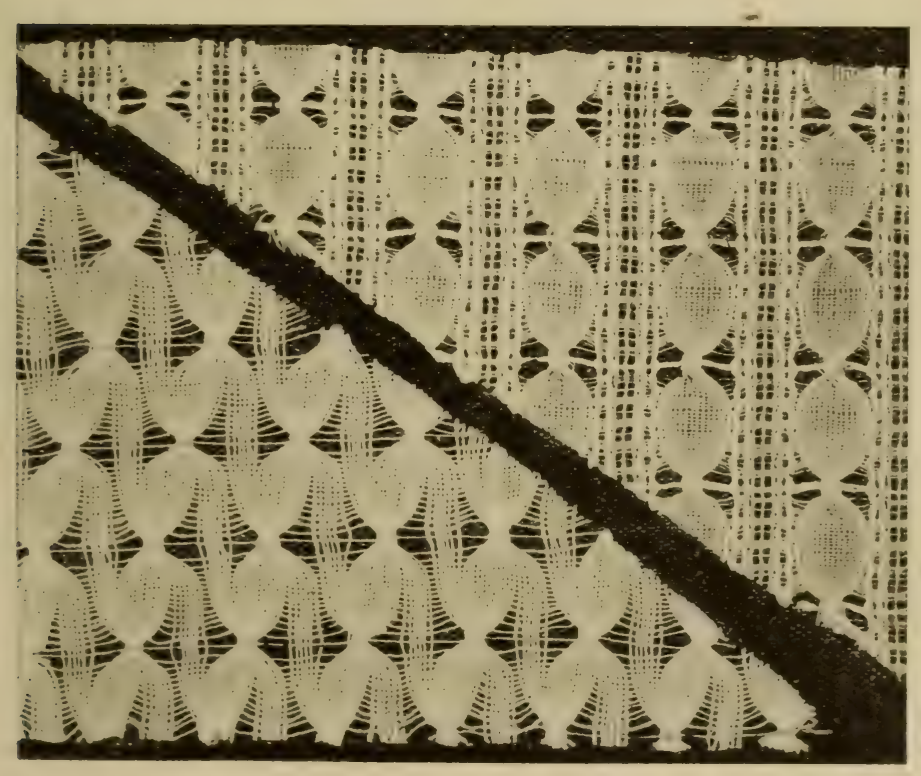

FIG. 45!.

Figs. 458 and 459.-Special leno effects produced by a system of crossing warp threads in front of the reed.

width of cloth, and to retain them at that width. Fixed at each end, and in the rear of the sley, are two iron brackets, each formed with three vertical slots to freely receive the ends of three staves extending from end to end of the sley. The foremost slot in each bracket contains a coarsely-pitched half-reed or pin-stave $G$, situated immediately behind the shuttle raceboard, in place of the usual reed, for the purpose of guiding a 


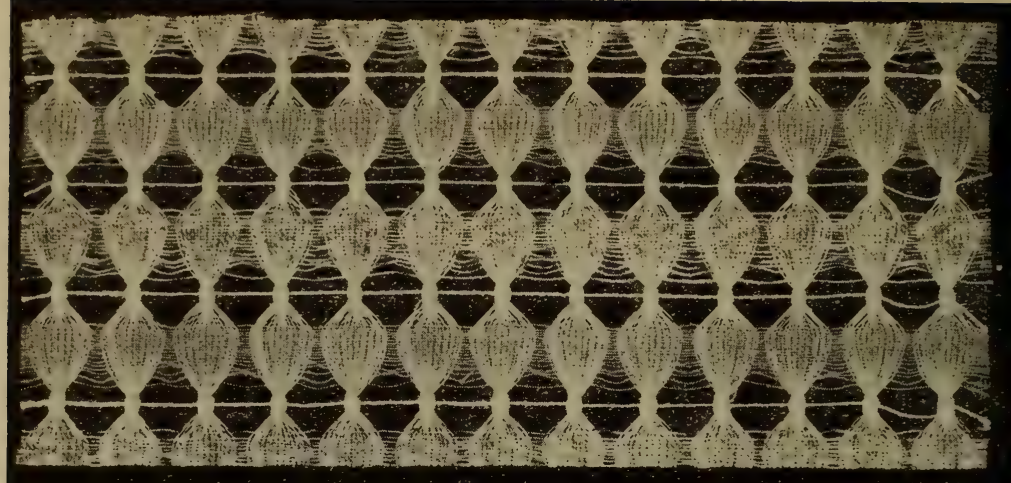

FIG.

460.

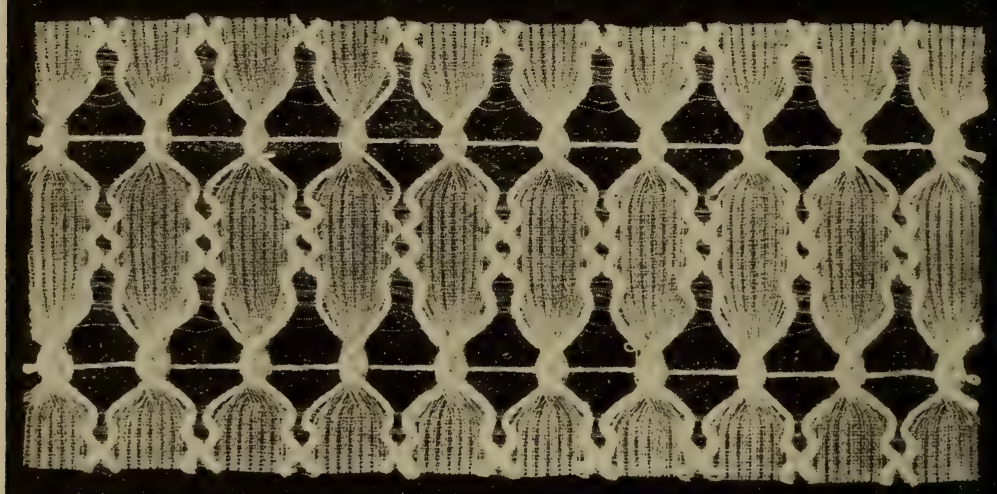

Fig.

461.

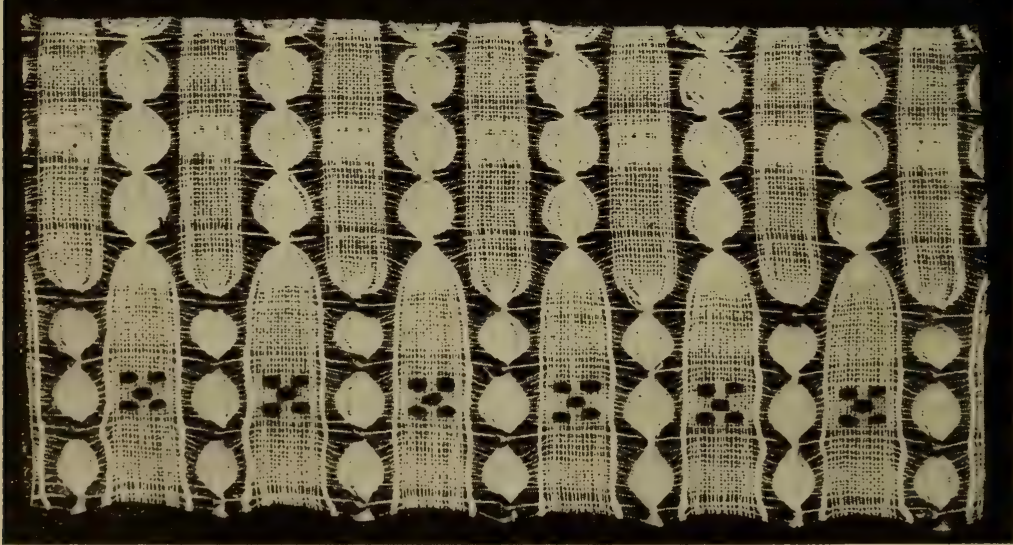

EIG.

462.

Figs. 460 to 462 . - Special leno effects produced by a system of crossing warp threads in front of the reed. 
shuttle in its transit through the warp sheds. To the rear of the pin-stave is a half-reed or comb $F$, for the purpose of beating up the picks of weft; whilst above the half-reed is an iron locking bar J, to securely lock the half-reed, and make it rigid whilst beating up. All these parts alternately rise and fall in unison with the movement of the sley, and in the following manner, namely: As the sley advances, the half-reed rises to beat up weft, and the locking bar falls, thereby passing immediately behind the upper ends of the reed wires, to give them firmness whilst beating up. As the sley recedes, the half-reed falls, and the locking bar rises, so that both move clear of warp threads during the formation of a shed, and warp threads are thereby free to be crossed as required between the stationary reed and the "fell" of cloth. The base of the half-reed is connected by rods $\mathrm{E}$, to the ends of levers $\mathrm{C}$, and its upward and downward motion is effected by means of two eams B, fixed one near each end of the crank shaft $\mathrm{A}$, of the loom. The cams act upon the levers $C$, which are each fulcrumed upon studs D fixed in the ends of arms that project from the sley swords, and, therefore, oscillate with the sley. The motion of the pin-stave $G$ is contrary to that of the half-reed F. Thus, as the sley recedes, the pin-stave rises, to act as a guide for the shuttle as it passes through the warp sheds, but falls clear of the warp threads, cloth and loom temples as the sley advances to beat up weft. The contrary action of the pin-stave and halfreed enables the first to be actuatec by the scrond, by attaching them to opposite ends of "curds $\mathrm{H}$; which puss over pulleys I. The upward and downvard motion of the locking bar J is obtained by means of "onräs $K$, which pass over pulleys $L$, and have each one end attached to fixed poinus. M, on the loom framing. Thus, as the sley advances: the cords diminish in length between the pilleys $L$ ard the fixed ends M, thereby allowing the bar io fall by giavitition; but as the sley recedes, the cords increase in length hetween the fixed points and pulleys, thereby raising the locking bar clear of warp threads. A similarity exists between the sley of this type of loom and that of a lappet loom, as represented in Fig. 501 (\$136). Both are provided with pin-staves for the guidance of a shuttle during 


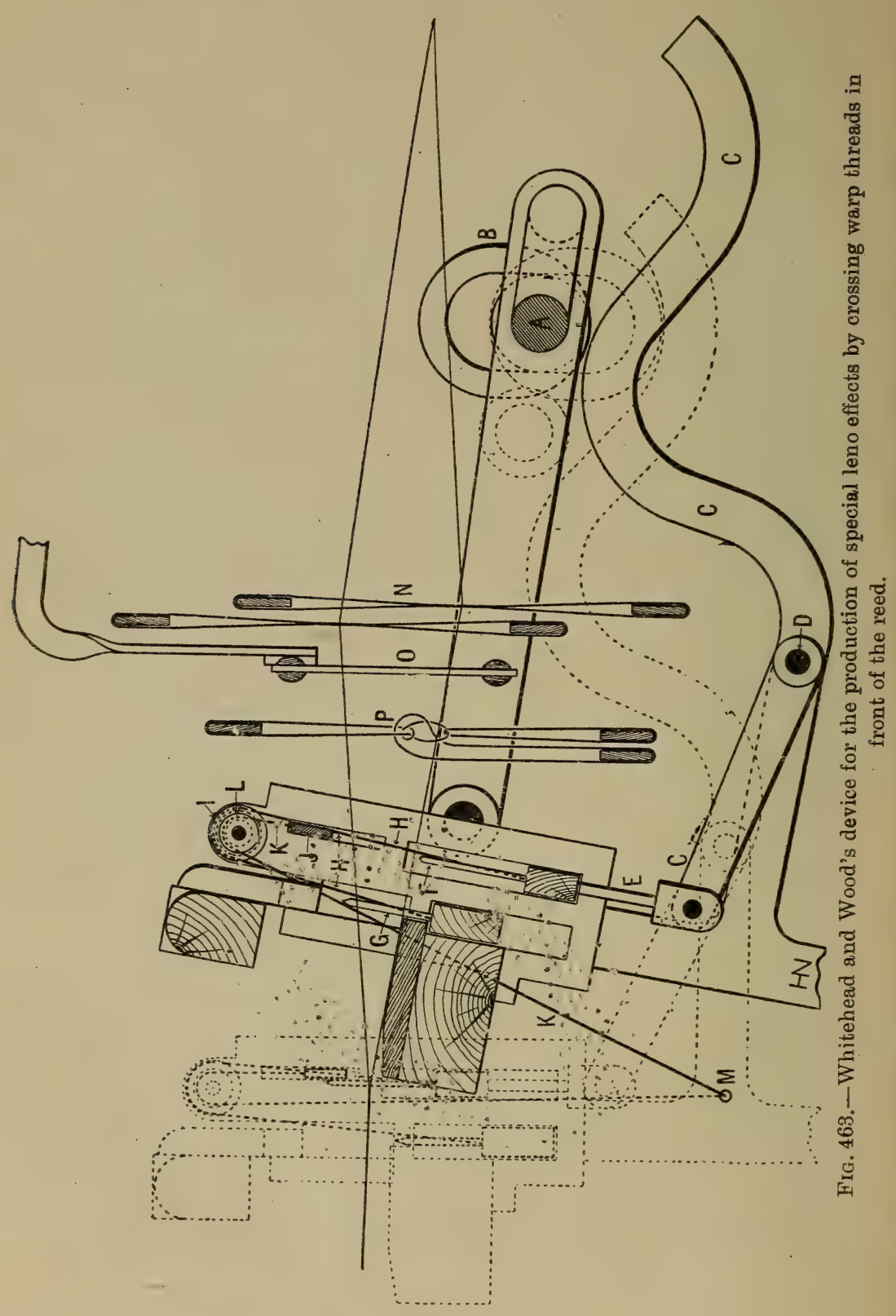


picking; but, instead of a half-reed, a lappet loom is furnished with one or more needle-bars to control figuring or "whip" threads, and these bars are moved laterally for figuring purposes, as well as vertically, to insert figuring threads into the warp sheds.

\section{Full-cross Leno Fabrics.}

$\$ 119$. In all the examples of leno fabrics herein described, doup threads make only a partial or half turn around their respective standard threads: that is, they pass from one side to

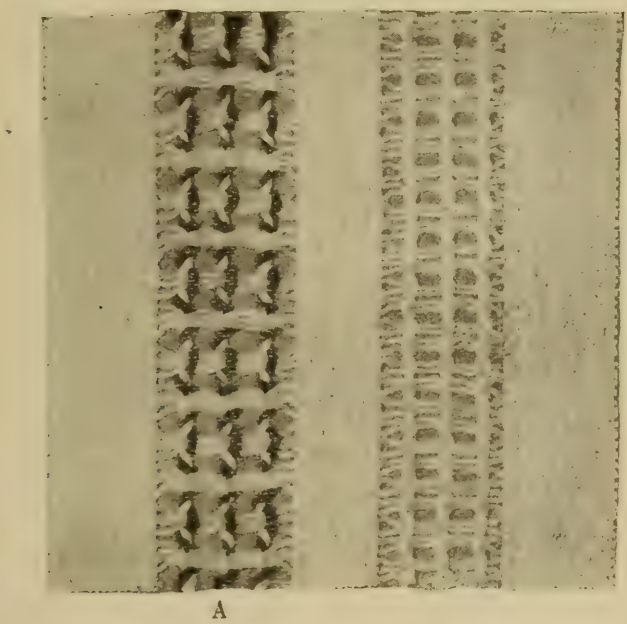

Frg. 464.-Full-cross net leno fabric in which doup warp threads are twisted completely around standard warp threads, first to the right and then to the left alternately at intervals of four picks.

the other side of those threads, and then return to the same side, on different picks of weft, but do not completely twist around them. Sometimes, however, leno fabrics are produced in which doup threads are caused to completely encircle their standard threads, and thereby produce a full crossing or twist with them, as exemplified in an actual specimen of cloth illustrated by Fig. 463, in which pairs of black doup threads cross or twist with pairs of white standard threads, to develop the striped leno effect shown at $A$. This unusual system of crossing 
is accomplished by causing the doup slips to completely wrap around the standard threads, as they pass from the doup threads to the heald stave on which they are contained. Thus, whenever a shed is formed, whether it be an open or a cross shed, the doup threads are either up or down always on the same side of their respective standard threads, thereby causing them to make a full crossing, as described.

\section{Relative Merits of a Top and a Bottom-Doup Harness.}

$\$ 120$. Throughout this chapter frequent reference has been made to the alternative methods of placing doup slips above or below warp threads, for the production of gauze and leno fabrics, both of which systems are described in $\$ 102$. Since the choice of position is quite optional, it is not surprising that the opinions of practical men, respecting the relative advantages of both systems, should vary according to their personal prejudices and practical experience, and that some advocate one system and some the other. From this circumstance, it is quite evident that each method possesses some peculiar advantage over the other, at least for certain classes of fabrics; otherwise, one of the two would long since have been discarded in favour of the superior system. It will, therefore, be both interesting and profitable to briefly compare the relative merits of each system, and to state which it may be more expedient to adopt, under different circumstances.

For the production of net leno and similar fabrics containing thick net doup threads or cords, that are chiefly displayed on one side of cloth, it is more expedient to employ a top-doup harness. By that arrangement, such fabrics are woven face side upward, thereby enabling a weaver to more readily detect imperfections in cloth during weaving. Another great advantage to a weaver, of doups being placed above warp threads, is that they are more accessible and therefore more easily repaired, or else replaced by new ones, which frequently becomes necessary, in consequence of doup slips rapidly wearing out. In the event of breakages, however, top-doup slips are liable to prove a source of serious trouble to a weaver by hanging down and becoming entangled with warp threads, thereby involving the risk of 
breaking them, and causing faults in cloth. Another disadvantage of top doups is in respect of shedding. If a negative acting dobby and a spring under motion are employed to operate a top-cloup harness, it is mole difficult to obtain a good lower half of the warp shed, in consequence of healds being depressed and held down negatively, by means of springs. This arises in consequence of the abnormal tension of doup rarp threads during the formation of both cross and open sheds, whereby they tend to rise a little abore the surface of

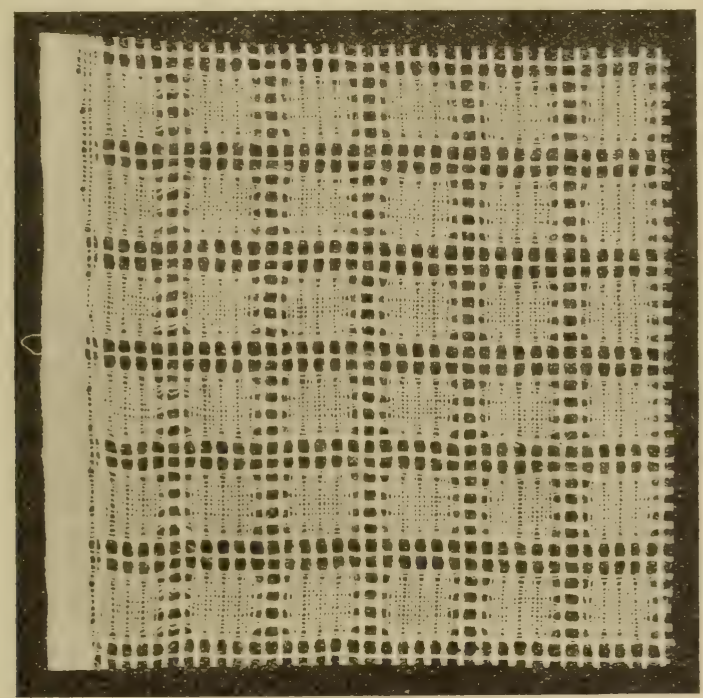

FIG. 465.-Check gauze fabric.

the shuttle race-board, instead of lying well down upon it, as a shuttle is transmitted through the warp shed. If bottom doups are employed, cross sheds are formed by raising front standard and doup healds positively by the dobby, whereby better and clearer warp sheds are formed. In consequence, howerer, of the cloth being woven face downward by them, as previously described in $\$ 113$, they are not generally used for net leno and similar fabrics, but are chiefly confined to the production of those fabrics not containing thick net doup 
threads, and of which both sides are exactly similar, such as that illustrated in Fig. 465. Also, the renewal of bottom-doup slips is much more difficult than the renewal of top doups; but if bottom doups break, they fall away from warp threads, and do not, therefore, become entangled with them.

\section{Relative Merits of Different Types of Dobbies for Gauze and Leno Fabrics.}

$\$ 121$. In the production of gauze or leno fabrics it is desirable to effect a crossing of warp threads with the least possible straining or chafing of those threads. This desideratum is the principal stumbling-block to the successful adoption of dobbies that are unprovided with auxiliary attachments which specially adapt them for leno weaving. To avoid excessive straining and chafing of warp threads whilst in the act of crossing each other, one of two conditions must exist, namely, either the crossing threads must be quite level at the commencement of crossing, either at the bottom, or in the centre of the warp shed; or else doup threads must pass either from the upper or else the lower part of the warp shed, when the crossing takes place, according to whether a top or a bottom-doup harness, respectively, is employed. It will now be manifest, therefore, that either a "closed-shed" dobby, or one that will produce the conditions just described, is better adapted than an "openshed" dobby for leno weaving. By reason, however, of openshed double-acting dobbies enabling a loom to be worked at a greater speed than is possible with closed-shed dobbies (which are necessarily single-acting), it is a common practice to employ an open-shed dobby for leno weaving. In such cases it is expedient to equip either the dobby or else the loom with a suitable auxiliary attachment known as a "shaking" device, to enable the crossing of warp threads to take place as freely as possible.

\section{Shaking Devices.}

$\$ 122$. The function of a shaking device is to facilitate the crossing of warp threads when forming both cross and open sheds; but since it is, under certain conditions, unnecessary to 
employ a shaking motion for the reproduction of some leno designs, it will be useful to indicate when shaking is, and when it is not, necessary. If either an open-shed dobby or a semiopen-shed dobby is employed for leno weaving, either with a top or a bottom-doup harness, it will be expedient to employ a shaking motion for designs that require a cross shed to inmediately succeed an open shed, and vice versit; but such a motion is not required for designs in which one or more than one pick intervenes between cross and open sheds as exemplified in the net leno stripes A, Fig. 438; B, Fig. 439 ; and B, C, Fig. 442 ; because for those picks, doup threads would be raised by a top-doup harness, and depressed by a bottom-doup harness, and would therefore pass either from the upper or lower part of the warp shed respectively, when required to form both cross and open sheds as described in $\$ 121$. The reason for this will be manifest after a little reflection upon the circumstances. With a top-doup harness, cross sheds are formed by depressing doup threads on the crossed side of their respective standard threads, which are raised; and open sheds are formed by depressing doup threads on their normal side. Therefore, either standard threads should be lowered, or doup threads raised, at least half-way, to prevent excessive chafing of the crossing threads during the formation of cross and open sheds. This function is described as "shaking". With a bottom-doup harness the conditions of shaking are exactly contrary to those which obtain with a top-doup harness.

$\$ 123$. "Shaking" is effected in a variety of ways, either by auxiliary attachments fixed either to the loom, or else to the dobby; and, as just indicated, it may be accomplished with a top-doup harness either by raising doup healds, or else by depressing the healds governing standard or regular warp threads: half-way; and with a bottom-doup harness in a contrary manner, by depressing doup healds, or else raising standard threads half-way-the choice being frequently quite optional. One very simple and common method of shaking depressed healds, without employing a special leno dobby, is to connect the required heald stave or staves to one of the loom-crank connecting arms by means of a suitable arrangement of levers. 
and connecting rods, as represented. in Fig. 466, in which $\mathrm{A}$ represents the loom cranks; $\mathrm{B}$ the connecting arms; and $\mathrm{C}$ a connecting rod to oscillate an arm $\mathrm{D}$ secured to the end of. a square cross bar E, placed above, and in the rear of the

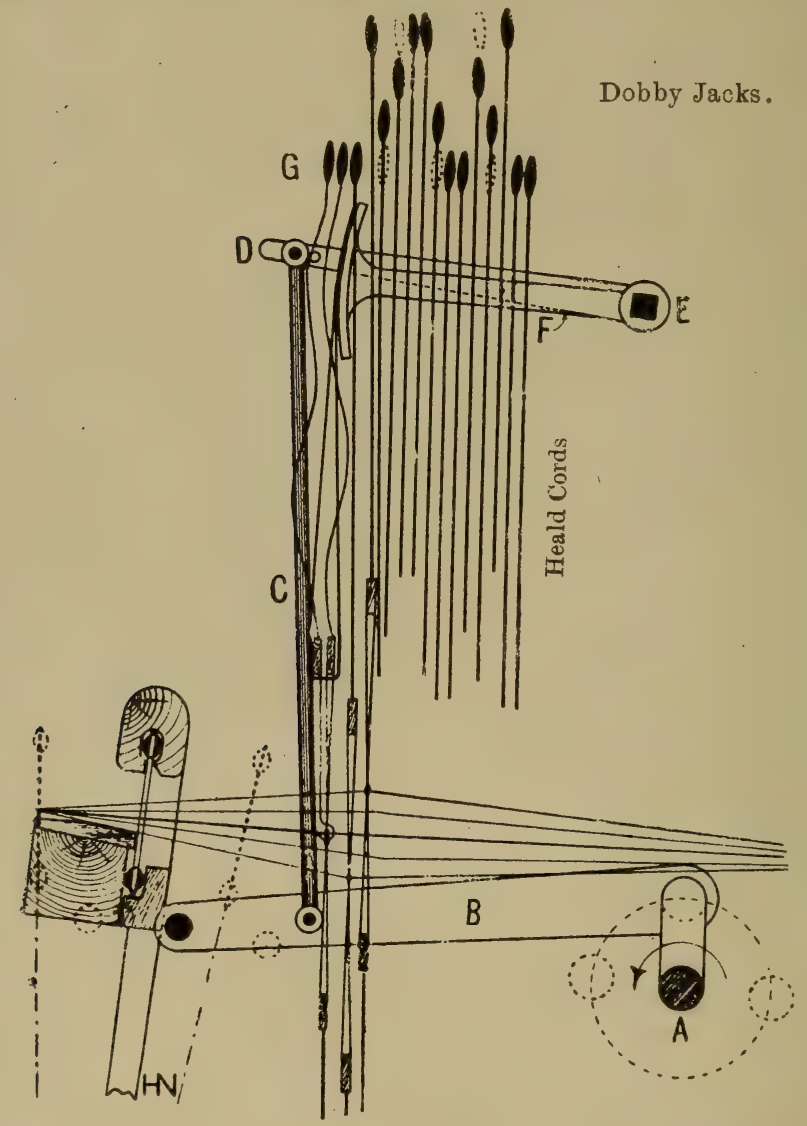

Fra, 466.- "Shaking" device to operate in conjunction with open shed dobbies employed for cross weaving, to facilitate the crossing of warp threads.

healds, and upon which are also mounted half-moon quadrant arms $\mathrm{F}$, to which the required healds are connected. The same healds are also attached to separate dobby jacks G. The disadvantage of this arrangement, however, is that it does not 
permit of readjusting the timing of its movement in relation to shedding, if such a course were necessary. Thus, the front standard and doup healds, which are being shaked, are raised to the centre of the warp shed by the time the loom cranks arrive at the top centre of their circuit. This is one-eighth of a revolution sooner than the time usually selected for rising and falling healds to meet in the centre of the warp shed, when doup and standard warp threads commence to cross each other. This is indicated in the diagram by representing rising and falling dobby jacks $G$, a short distance from the centre of their complete morement. Still it has the advantage of being simple and is found to give satisfaction under some conditions.

$\$ 124$. Another method of shaking depressed healds from the loom is represented in Fig. 467. By this method, in addition to being connected to separate dobby jack-levers $G$, the healds required to be shaked are also attached to half-moon quadrant arms $F$, that are secured to a cross-bar E, placed above and in the rear of the healds. This is caused to oscillate by connecting it, by means of an arm $\mathrm{D}$, and connecting rod $\mathrm{C}$, to a lever $\mathrm{H}$, mounted upon a stud I, and fixed outside the loom framing. Lever $\mathrm{H}$ is furnished with a bowl or runner $\mathrm{J}$, and is depressed and raised alternately by the combined actions of a doubleacting tappet $\mathrm{K}$ (fixed upon the picking shaft L), and a spring M respectively, which may be attached to any convenient part of the loom framing. With this shaking device, shaking may be timed to take place at exactly the right moment, by adjusting tappet $\mathrm{K}$ to operate the required healds so that their movement synchronises exactly with the movement of healds controlled by the dobby. This is indicated in the diagram by representing the dobby lifting-crank $\mathrm{N}$ in a horizontal position, when rising and falling healds meet midway (as indicated by the three positions of dobby jack-levers G). At the same time the front standard and doup healds have been raised to the centre of the shed, when the crossing of doup and standard warp threads commences. Also the loom cranks are half-way between the top and front centres, and therefore one-eighth of a pick (forty-five degrees) in advance of those represented in Fig. 466. 


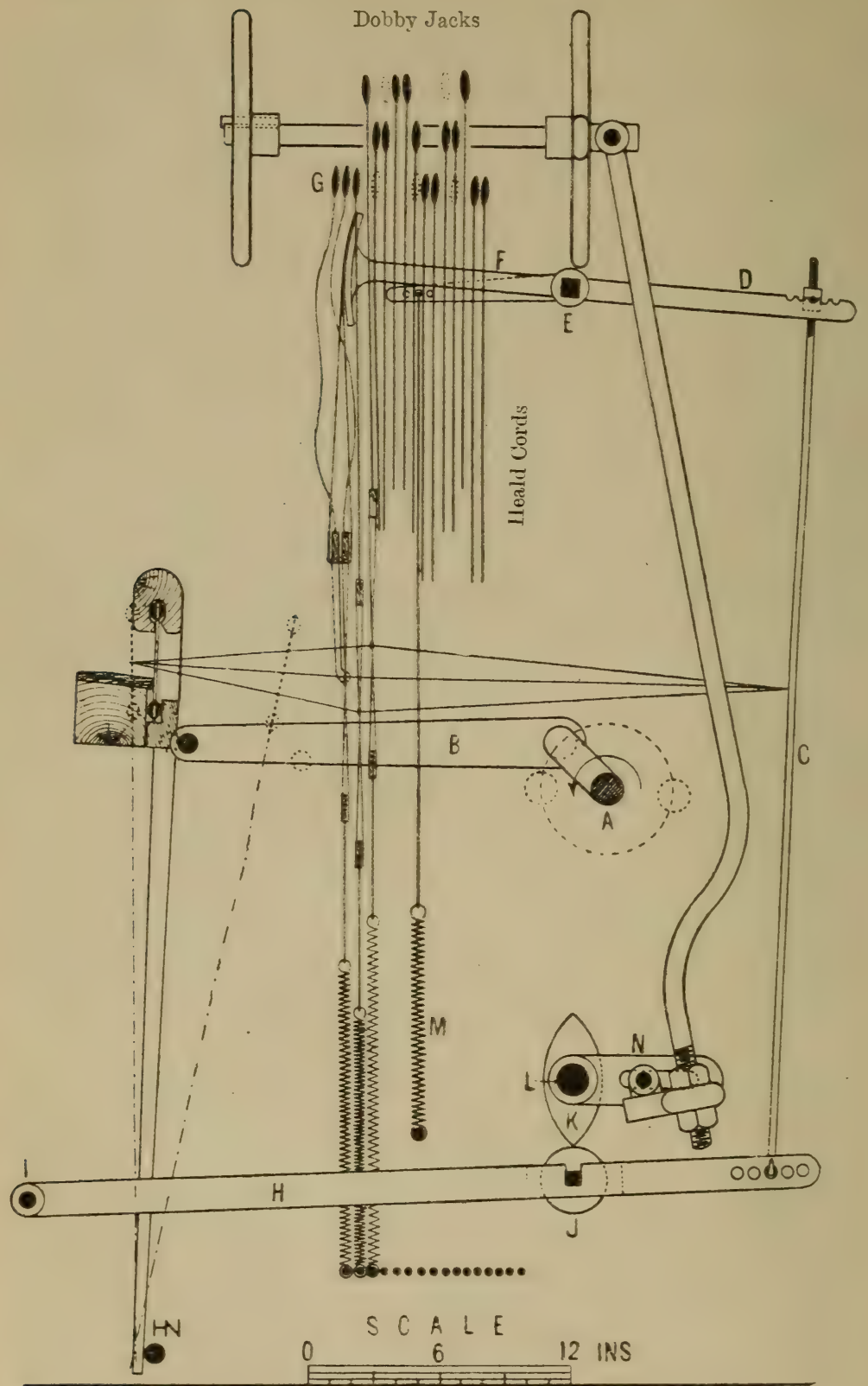

FIG. 467.-Another type of "shaking" device to operate in conjunction with open-shed dobbies employed for cross weaving, to facilitate the crossing of warp threads. 
$\$ 125$. When, however, shaking derices are adapted to openshed dobbies of the "Keighley" type, and not to the looms, they may only shake raised healds by first lowering them halfway and then raising them to the top again. Some dobby makers furnish their dobbies with a shaking device of some kind, and style them "leno" dobbies; but one of the most efficient and inexpensive methods of shaking with an open-shed double-acting dobby is to connect two contiguous jacks of the dobby to each heald that requires shaking, and operate them from the pattern lags as required. By this means a heald may be lowered by one jack-lever until it falls half-way, when it is caught and taken to the top again by the ascending fellow-jack. By governing the operation of shaking from the pattern lags, this arrangement is superior to all other shaking devices, as it enables shaking to be performed only when it is necessary, and at the exact moment when it is most effective; whereas other shaking devices operate for every pick, whether shaking is required or not; thereby vibrating healds and warp threads unnecessarily and detrimentally, as explained in the following section.

When adapted to a semi open-shed dobby, the function of shaking must be effected with depressed healds. This type of dobby is better adapted than an open-shed dobby for leno weaving, because it allows all healds to fall half-way after each pick; therefore, by raising half-way those healds that require shaking, standard and doup threads actually meet in the centre of the shed, thereby achieving the same result (in respect of crossing threads only) as that obtained by means of a closedshed dobby.

$\$ 126$. Before dismissing the subject of shaking, it will be of practical interest to indicate the circumstances under which it is quite inadvisable to employ any kind of shaking device to shake the front standard and doup healds (of a top-doup harness) after every pick; and also when it is more expedient to employ either a closed-shed dobby or else a shaking motion that may be made to operate only when required. For example, if a net leno design requires doup threads to remain down for several picks in succession, when on the open or normal side of their 
respective standard warp threads, the doup slips and their warp threads would rise half-way in the warp shed and return to the bottom again for every pick of weft; albeit those threads would be held down by the back standard heald. This would involve excessive straining and breaking of doup threads and rapid wearing out of doup slips. For these reasons, therefore, it would be better to employ either a closed-shed dobby, or else a shaking motion that could be controlled from the pattern lags in the manner described in $\$ 125$, whereby shaking may be effected by depressing standard warp threads only when it is absolutely necessary.

\section{Practical Details of Leno Weaving.}

$\$ 127$. The position of back standard healds, which govern doup threads, in relation to those governing standard or regular warp threads, is quite optional. Some advocate placing them to the rear of regular healds, as indicated in Figs. 433, 434 and 435 ; and others prefer to place them in front of regular healds, as represented in Fig. 439, and subsequent charts of drafts for leno designs. The advantage is probably in favour of the latter course ; for, whilst it reduces the distance between the "fell " of cloth and the back standard heald eyes (and thereby imparts a little greater tension upon doup threads during the formation of "open" sheds), it averts the chafing of doup threads against the leashes of the regular healds, around which they would bend when forming "cross" sheds.

$\$ 128$. In consequence of the additional tension imparted to doup threads during the formation of cross sheds, and the consequent rapid wear of doup slips and healds, it is expedient, for reasons of economy, to make designs that will require as few cross sheds as possible, consistent with the effect it is desired to produce in cloth. Also, when one or more than one doup thread crosses two or more standard warp threads, it will be better to raise (with a top-doup harness) all, in preference to a part, of those threads, when forming cross sheds, and thereby distribute the strain of shedding upon them equally.

$\$ 129$. In the production of some net leno fabrics in which the doup threads are abnormally thick, or which have to cross 
with a large number of standard warp threads, it is advisable in such cases to remove one or more than one reed wire, if necessary, to prevent excessive chafing of warp threads by being too confined and by bearing hard against the reed wires, as described in $\$ 107$. What are known as "flexible" reeds are well adapted for net leno fabrics. Such reeds are formed by wrapping the reed ribs on one side with pitched banding, in the usual manner; whereas those on the other side are wrapped with dry or unpitched banding. By this means considerable flexibility is obtained in the reed, which permits of the easier passage of knots or other obstructions, with less risk of breaking the warp threads. The dry banding allows a limited amount of vertical movement by the reed wires, which enables them to readily recover their original position after being bent out of a straight line. Also a deeper reed than those usually employed should be used for leno fabrics, to allow warp threads more freedom, and also to enable it to be more easily displaced in the event of a shuttle failing to pass safely through the warp shed.

$\$ 130$. Weavers who are engaged in the production of net leno fabrics, and other varieties of cloth containing thick "net" or corded warp threads of folded yarn, in which the presence of large knots would be objectionable or too large to pass through the eyes of the shedding harness, have sometimes to adopt special means of replacing broken warp threads without piecing them by tying their extremities together.

This may be effected by either of two alternative methods, by which the breach is repaired temporarily-namely, (a) either by substituting a similar thread withdrawn from a bobbin; or else (b) by attaching, in a special manner, to the broken thread on the warp-beam a "thrum" (short length) of similar yarn. The "thrum" is attached by means of a slip-knot to the original warp thread at a point about 18 to 24 in. from the severed end of that thread, and then passed through the harness eye and dent of the reed through which the original thread passed. In both of these cases the free end of the broken warp thread is allowed to droop backward, until sufficient length is unwound from the warp-beam to enable the severed end of the thread to extend well in front of the reed. When the broken warp thread 
is long enough, the temporary thread is removed, and the original warp thread is then passed in its proper place through the harness and reed as before the breach occurred.

\section{Tempered Steel-wire Doup Harnesses for Cross-weaving.}

131. The loops or slips, termed "doups," employed in conjunction either with heald or Jacquard harnesses for the purpose of effecting the crossing or douping of warp threads that characterises gauze and leno fabrics produced by cross-weaving, are usually made from worsted twine, which is more durable than cotton twine and more supple and elastic than linen twine. Albeit, the rapidity with which worsted doup slips wear out and break during weaving constitutes one of the chief difficulties rexperienced in the manufacture of fabrics constructed by their aid. The rapid wear of doup slips arises from the excessive abrasive friction to which they are subjected, during weaving, within their respective. front "standard" heald or mail eyes. Their breakage not only incurs the risk of causing imperfections in cloth by becoming entangled with and breaking warp threads, ibut the difficulty usually experienced of repairing defective doups, or replacing them with new ones, imposes a severe tax upon the duties of a weaver and, by involving loss of time, curtails production.

With the object of averting the disadvantages of worsted doup slips, and also securing greater efficiency and durability than they afford, tempered steel wire doup slips have been employed in lieu of twine doup slips in the construction of doup harnesses of various forms adapted to the special requirements of fabrics of different texture, and applicable either to heald or Jacquard harnesses for gauze or leno weaving. After being bent and shaped into the desired forms, the wires composing the respective units of a harness are neatly soldered where it is necessary to either fill up small interstices, or to effect a union between two hitherto separate portions, to increase their stability. The wires are subsequently tinned to prevent them from rusting, and also to give them a highly smooth finish, to reduce frictional resistance between them and warp threads to the minimum.

The simplest form of steel-wire doups referred to, as repre- 
sented by Fig. 468, differs very little in construction from doup harnesses made from twine. In this example, the upper halt only of the front standard $\mathrm{S}$ is open, to contain one side of the doup slip D, in which is formed an eye to receive a doup warp thread (as shown) by coiling the wire at the bend. A modified form of steel-wire doups suitable for finer warp yarn is represented by Fig. 469. In this example the doup slip D is a simple loop of the usual form, without an eye being formed at the bend. In the present case, however, the doup slip is inserted in both the upper half of the front standard $\mathrm{S}$ which is open, and also in loops formed at the bend of the lower half of the front standard, as indicated in the diagram. A third modification of wire doups is represented by Fig. 470, showing, at $A$, the constituent parts in their initial position which they occupy when the warp shed is closed; and at $\mathrm{B}$ and $\mathrm{C}$, the positions they occupy during the formation of open and cross sheds respectively. The special feature of this device consists of a doup slip D acting in conjunction with two separate front standards $\mathrm{S}^{1}$ and $\mathrm{S}^{2}$, each of which is formed with a long central loop or eye to contain the respective sides of the doup slip, as shown in the diagrams. The doup slip used in this modification, like that shown in Fig. 468 , has an eye formed at the bend for the reception of a doup warp thread, and is situated between the two front standards. With this device an open shed is formed by raising the doup slip along with either of the two front standards; whilst a cross shed is formed by raising the doup and the other front standard, whereby doup warp threads will be raised first on one side and then on the opposite side of their respective standard or regular. warp threads as indicated by Figs. $470 \mathrm{~B}$ and C. This arrangement therefore dispenses with the usual back standard heald.

As previously stated, the steel-wire doup harnesses under present notice are applicable to both heald and Jacquard harnesses. If they are to constitute a heald harness, their looped extremities are slid upon wires stretched along and secured to heald staves; but if they are to constitute a Jacquard harness. the wire units composing the harness are attached to mounting threads and lingoes, after the manner of ordinary twine couplings. It should be observed, however, that whatever form the 


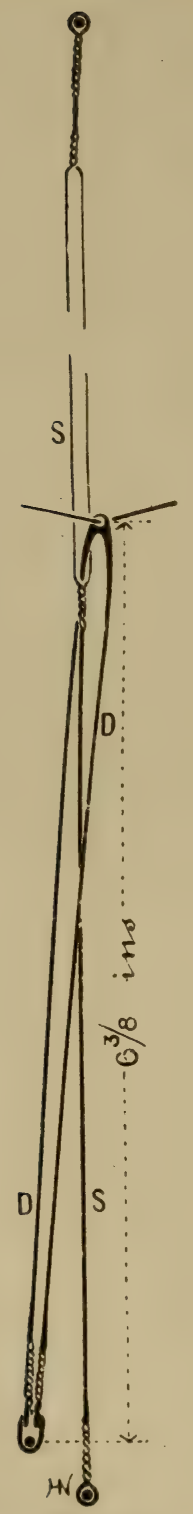

FIG. 468.
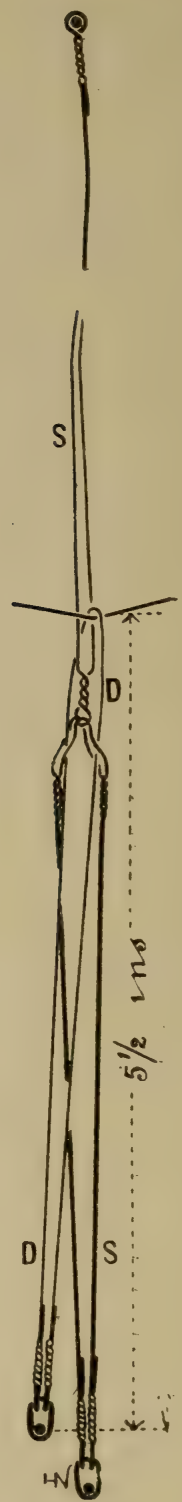

FIG. 469.

Units of two types of a steel wire doup harness for cross weaving: 
(HALZE ANI) NET LENO FABRICS.

267

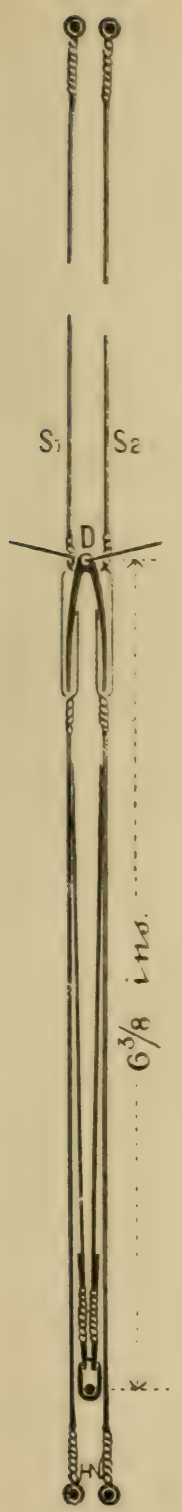

Fig. $470 \mathrm{~A}$.

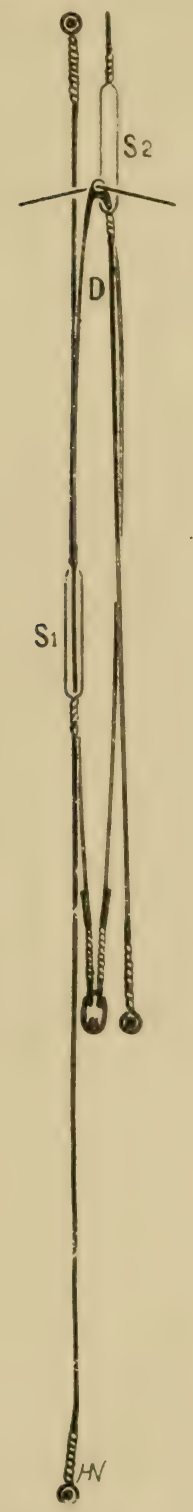

FIG. 470 B.

Formation of open shed.
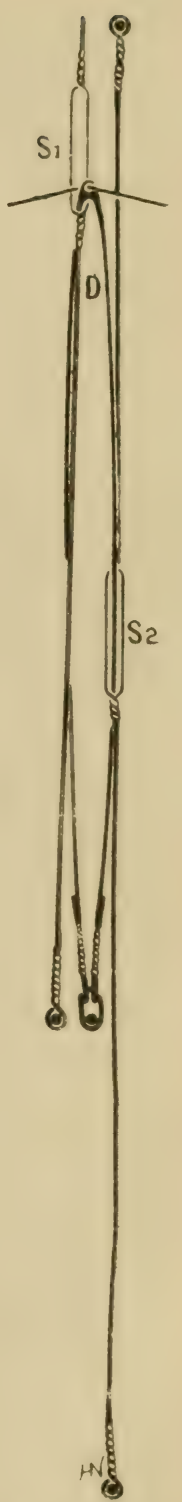

Fig. 470 C.

Formation of cross shed.

Units of a third type of a steel wire doup harness for cross weaving. 
harness may assume, the use of tempered steel-wire doups imposes certain restrictions and limitations in either the choice of shedding apparatus by which they are to be actuated, or else in the character of the designs that may be produced by their use, for reasons to be presently stated. The tempered steel wire, of which the units of the harnesses are made, is comparatively rigid, and lacks the suppleness and pliability of twine, which is capable of readily yielding and bending, and of freely adapting itself to the conditions imposed by shedding with a doup or leno harness. For this reason, steel-wire doups may only be employed in conjunction with single-acting Jacquard machines, single-acting dobbies, or other shedding appliances that bring all standard and doup warp threads parallel after each pick is inserted, to facilitate their crossing from their normal or open position to a crossed position or vice versâ : otherwise, if they are employed in conjunction with open-shed machines, designs produced by them will have to be restricted to such as may be developed without the necessity of bringing doup and standard warp threads parallel after each pick, unless that object is achieved by means of a "shaking" device. For the same reason, they may not be employed in the form of a half heald to operate in conjunction with either a leno brocade (Jacquard) harness, or a leno heald harness in which several back standard healds operate in conjunction with one doup and one front standard heald (as described in $\$ 108$ to 116). In either of these two circumstances, it frequently occurs that some doup slips are taut, whilst others are buckled, according to the different relative positions occupied by standard and doup warp threads in different parts of the harness. Hence, if wire doup harnesses (which are not so pliable as twine) are employed in such cases, each unit of the harness will require to be under separate control.

\section{Mock or Imitation Leno Fabrics.}

$\$ 132$. The term "mock" leno describes a variety of weaves of ordinary construction, in which the scheme of interweaving warp and weft is designed to produce open-work effects which simulate, in a very realistic manner, the genuine gauze and leno 
effects produced by the principle of cross weaving, as described in the previous sections of this chapter. Mock leno or openwork effects are sometimes produced alone, but more frequently in combination with the plain, a twill, satin or other simple weave, and sometimes with brocade figuring, to produce striped fabrics, which oftentimes bear a very close resemblance to true leno fabrics. They are also frequently used as ground fillings in brocade fabrics containing elaborately figured Jacquard designs, in imitation of leno brocade fabrics produced by a special gauze or leno harness.

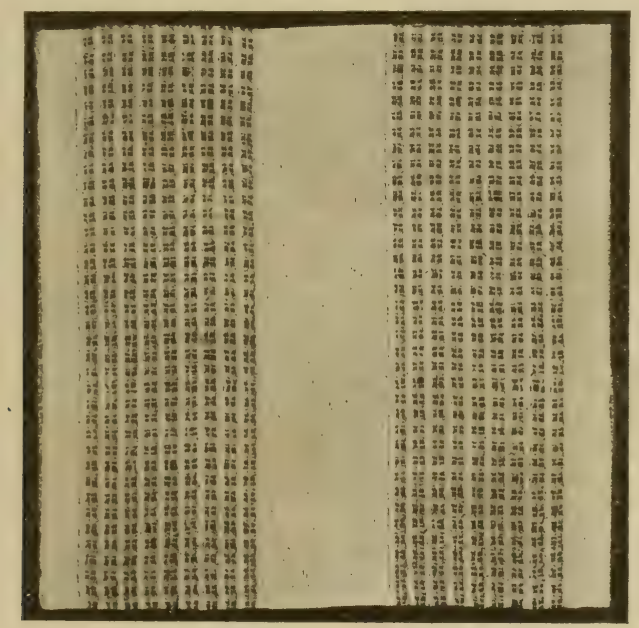

FIG. 471.-Fabric with mock leno stripes.

Fig. 471 is a photographic reproduction of an example of cloth woven with mock leno and warp satin stripes arranged alternately, and will serve to illustrate the realistic leno effects that may be obtained without employing a leno harness. Mock leno weaves are of very simple construction, and are chiefly dependent upon the frequent counterchanging of a suitable weave, to produce the desired effects. A few examples of these weaves are given in Figs. 472 to 475 . Fig. 472 is a simple pattern counterchanging on three warp threads and picks, and therefore repeats on six threads of warp and weft. The counter- 
change after the third and sixth warp threads and picks, combined with the particular method of interweaving them, produces distinct gaps or "frets" both lengthwise and crosswise of the fabric, and thereby creates a decided gauze or leno effect in cloth. The warp threads may be passed in pairs through each dent of the reed; but the leno effect will be emphasised by passing them in groups of three through each dent, commencing with the first three warp threads in the design.

Another good mock leno effect is produced by the "canvas" weave represented in Fig. 473, so extensively employed in the manufacture of the well-known canvas cloth, usually produced

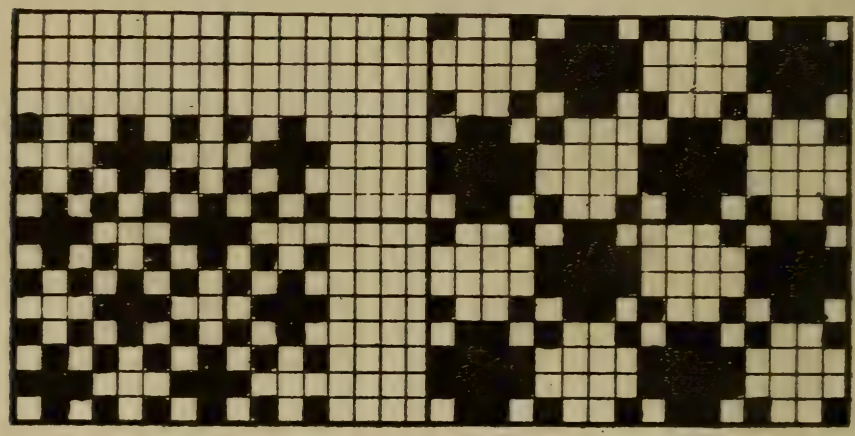

FIG. 472.

Fig. 473.

Mock leno designs.

in coarse textures from strong folded warp and weft, and chiefly used for the purpose of cross-stitching and other fancy needlework. This design is really a further development of that given in Fig. 472, and is made to counterchange after every four threads in both warp and weft, so that the pattern repeats on eight threads each way. The small perforations characteristic of this fabric, and through which the needle is inserted (when employed for fancy needlework), result entirely from the counterchange of the threads. The occurrence of the perforations is quite incidental to that weave, and therefore unavoidable. If it is required to introduce the canvas weave as a mock leno effect, in conjunction with another weave, to form stripes, a 
superior effect will result by passing warp threads in groups of four through each dent of the reed.

A third example of a mock leno effect, which is very extensively employed, is that illustrated by Fig. 471. The design for that example (as given in Fig. 474) repeats on six warp threads and six picks, and is counterchanged after the fifth and sixth threads in both directions, thereby causing the sixth thread of warp and weft to become quite isolated from adjacent threads, and so develop a leno effect of a very pronounced character. By.drawing. the first five warp threads in the first dent, and the sixth warp thread in the third dent, with the second and fourth dents left empty, a very realistic simulation of a genuine gauze or leno effect is developed in cloth. A modification of this

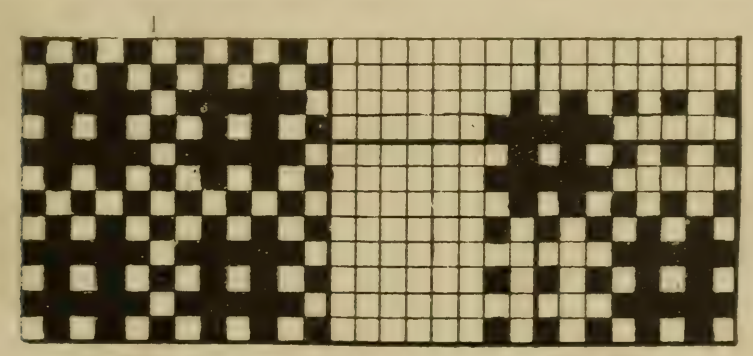

FIG. 474 .

Mock leno designs.

FIG, 475 .

weave is given in Fig. 475. With this weave warp and weft would be displayed in equal quantities on both sides of cloth. This circumstance is favourable to the effective introduction of coloured threads (say, the second, fourth, seventh and ninth warp threads and picks) to produce pleasing decorative effects. The mock leno designs given in Figs. 474 and 475 bear a close resemblance to the sponge design given in Fig. $237(\$ 43)$ and also to the huck-a-back weave, Fig. $245(\$ 44)$, to which they are closely allied; and if warp threads were suitably drawn through the reed, those weaves would also develop good mock leno effects. Many other varieties of mock leno designs could be given, but the present examples will suffice to demonstrate the general character of this useful class of imitation gauze and leno effects. 


\section{CHAPTER X.}

\section{LENO BROCADE FABRICS.}

$\S 133$. "Leno brocade" is a term descriptive of an interesting and important variety of textures that are characterised by the

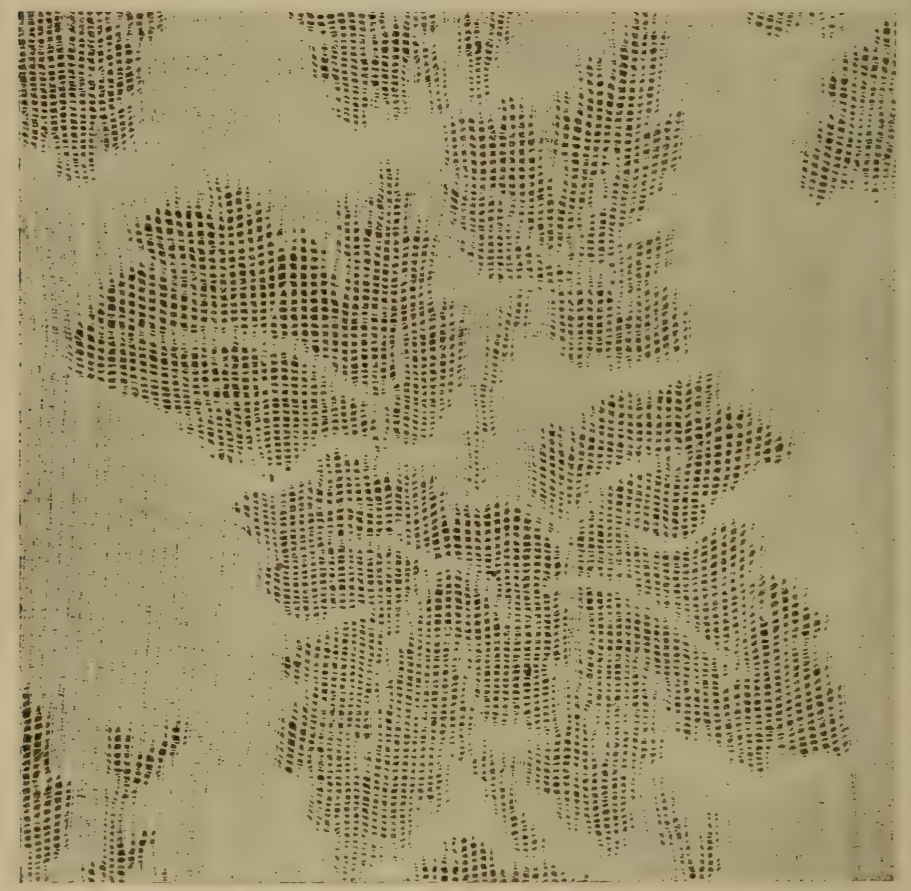

FIG. 476.-Jacquard leno brocade fabric.

gauze or leno principle of cross weaving introduced in combination with an ordinary brocade fabric consisting of one series each of warp and weft threads. They are produced in every variety 
of texture and material, according to the purpose for which they are intended. When manufactured of cotton, they are usually of light texture, for use as window curtains, light summer dress materials, and fancy aprons for domestic wear. They are usually constructed either with a leno figure surrounded byla ground filling of plain cloth, as exemplified in Fig. 476 ; or with

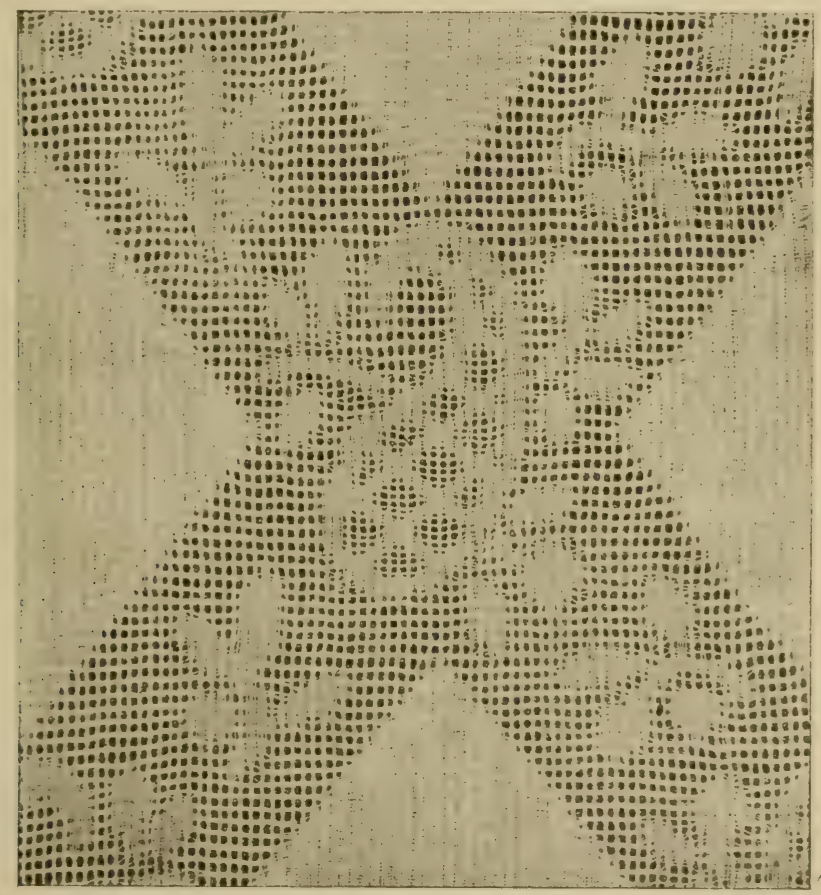

FIG. 477.-Jacquard leno brocade tabric.

a figure of the plain weave surrounded by a ground filling of leno, as in Fig. 477. In some examples of leno brocades, I warp, weft (or both) are allowed to float as required for the development of brocade or float figuring. In others, stripes or bands of net leno effects are developed by introducing coarse "corded" or "net" warp threads as an additional means of embellishment, as exemplified in Fig. 478; and also by means of swivel figuring, as illustrated in Figs. 479 and 480. 


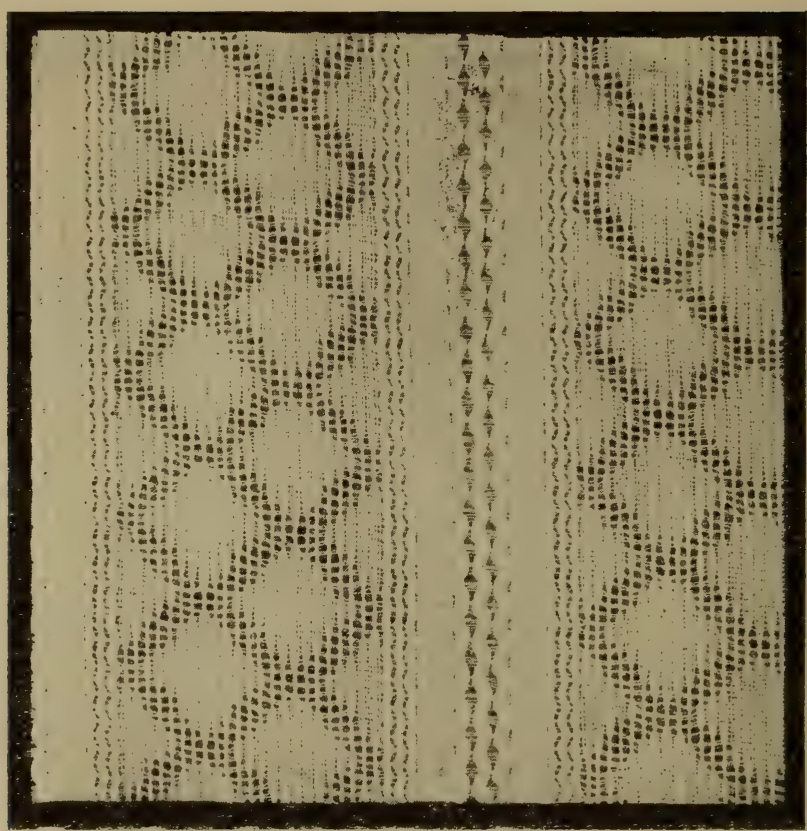

FIG. 478.-Fabric embellished with leno brocade and net leno effects.

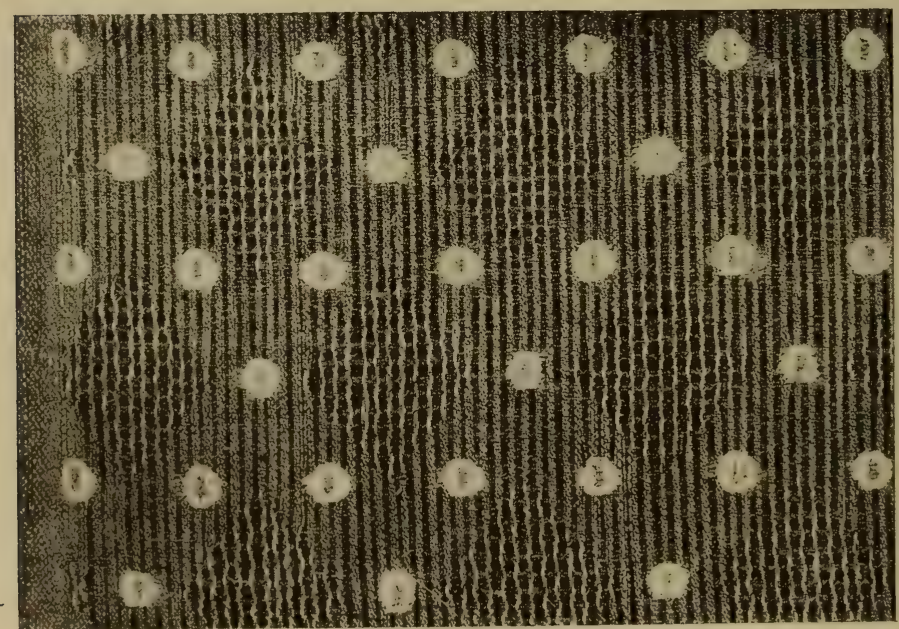

Frr. 479.-Fabric embellished with leno brocade and swivel figuring. 
Leno brocade fabrics offer practically unlimited scope to a designer in the production of decorative effects of both a floral and geometrical character, which are sometimes developed with a considerable amount of detail, especially when produced in silk and the finer cotton textures, some of which are admirable specimens of the art of weaving. The contrast afforded by the combination of a relatively close and compact texture of plain cloth with a leno texture of a more or less open character (which characterises these fabrics) produces a very pretty effect,

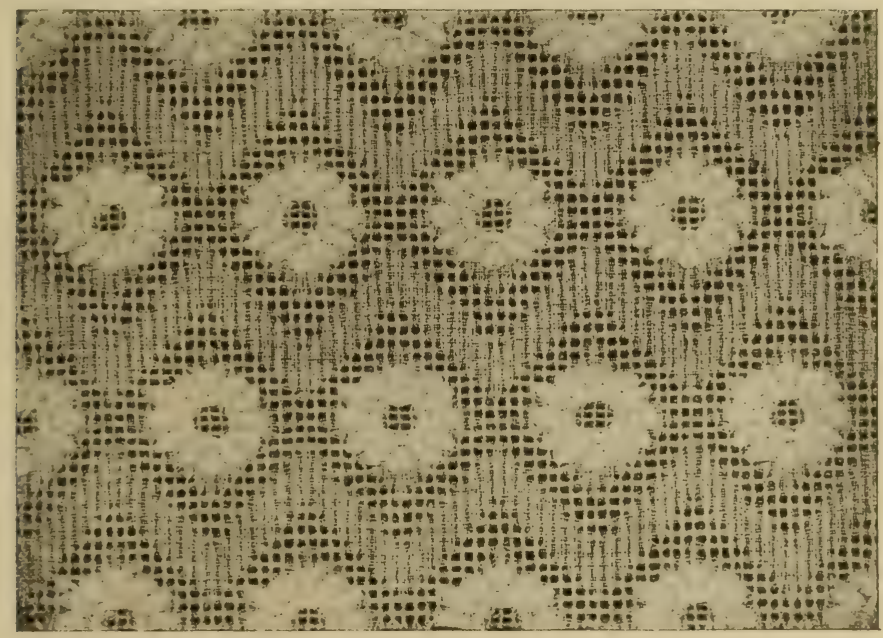

Frg. 480.-Fabric embellished with leno brocade and swivel figuring.

especially when they are lined with a fabric of an agreeably contrasting colour. Designs of a very simple character may be woven by means of an ordinary set of doup heald harnesses; but for the more elaborate designs it is necessary to employ a gauze or leno brocade Jacquard harness, which may be controlled either by a Jacquard machine of ordinary construction, or preferably by one that is specially adapted for the purpose, and as described in $\$ \$ 141$ and 142 .

The principles governing the construction of leno brocade fabrics are identical (so far as the leno structure is concerned) with those governing the construction of gauze and net leno 
fabrics that are produced by means of a heald leno harness. The scheme of douping or crossing the warp threads may either be of a uniform character in all parts, or else different schemes may be introduced to give greater variety of effect. Also doup and standard warp threads may either be arranged alternately with each other to form a "one-and-one" crossing, or else with one doup and two standard warp threads alternately: or, again, the threads may be arranged in alternate pairs, so as to form a "two-and-two" crossing. In the majority of leno brocade fabrics, the warp threads cross each other in pairs, with a uniform scheme of douping in the leno portions, in which either one pick or else two or more consecutive picks of weft are placed contiguously in each warp shed.

$\S 134$. Three graphic illustrations of a leno brocade Jacquard harness arranged on the "top doup" principle, and with both doup and standard warp threads crossing each other in pairs, are represented in Figs. 481, 482 and 483. Such a harness consists of three distinct parts-namely, the principal or brocade harness $A$, immediately in front of which is situated the doup harness proper $B$, both of which are drawn through the same comber-board $\mathrm{E}$; and $\mathrm{D}$, the "easer" harness situated about 12 to 15 ins. to the rear of the principal harness, and drawn through a separate comber-board G. The doup harness operates in conjunction with a set of doup slips C, which depend from a stave $F$ fastened on the front edge of the comber-board E. Each doup slip passes separately through a mail eye of the doup harness, and receives a pair of doup threads. The principal harness is precisely similar to that of an ordinary brocade harness, and may be used as such, quite independently of the other portions of the harness, for the development of brocade figuring, as required. The easer harness is usually furnished with large glass mails to receive doup warp threads, and is situated between two rods $H$, forming what is termed the "bridge," fixed underneath the warp threads, with the mails placed about 3 ins. below the bridge rods, whereby doup threads are deflected from a straight course so as to increase their length between the warp beam and "fell" of cloth, and thereby permit of those threads being "slackened" to relieve them of undue tension when they are required to form a cross shed. 


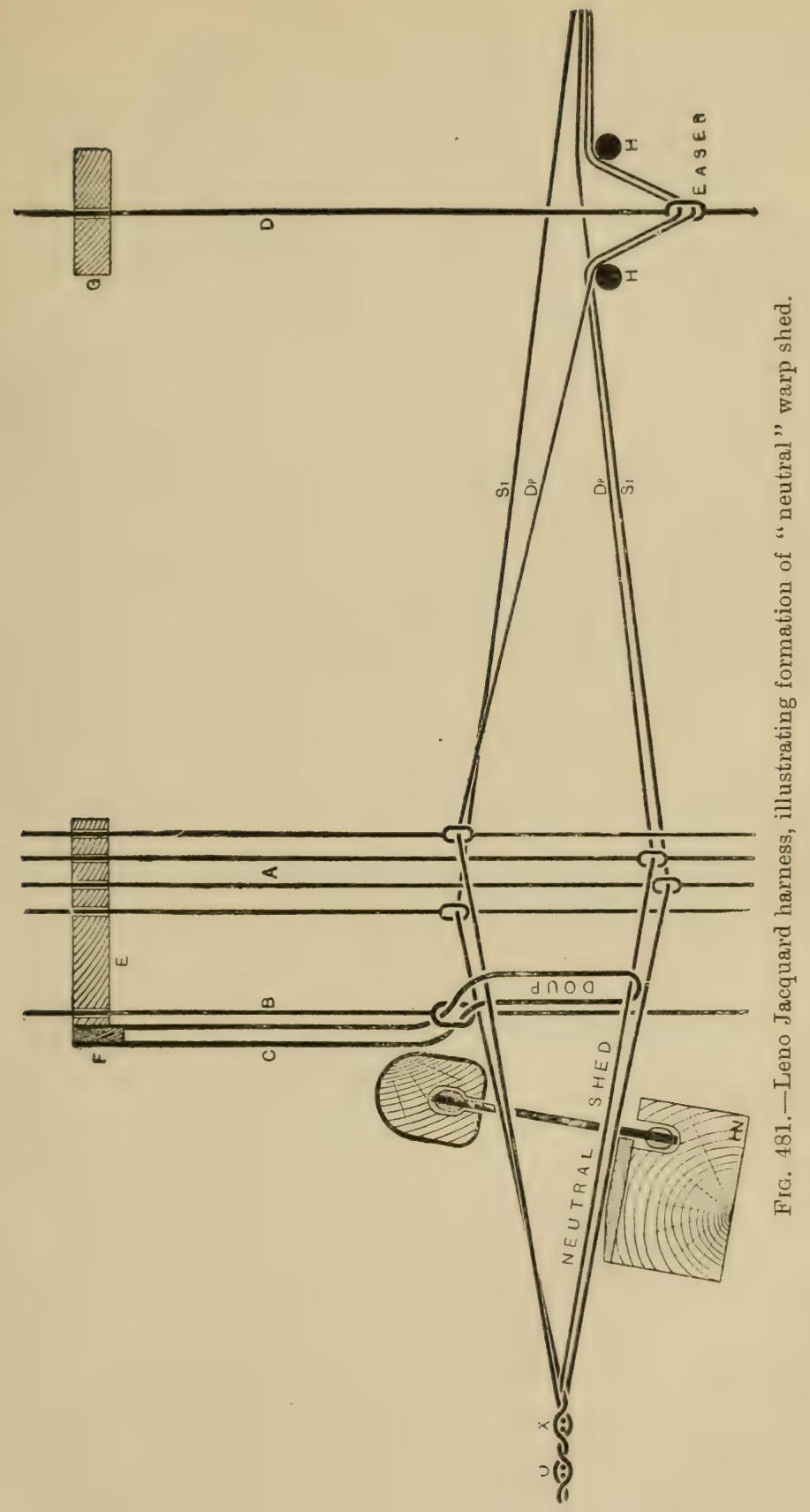




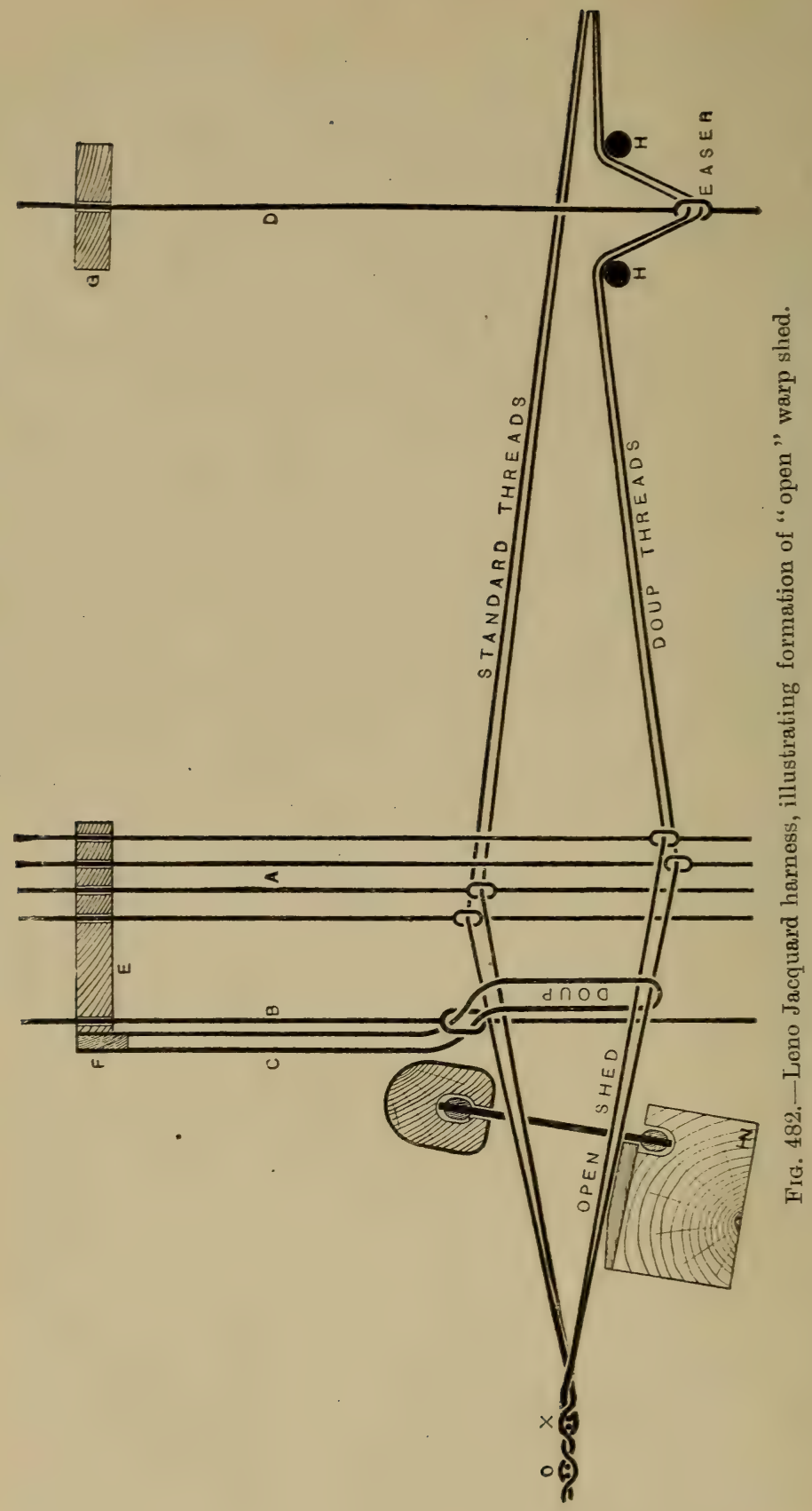




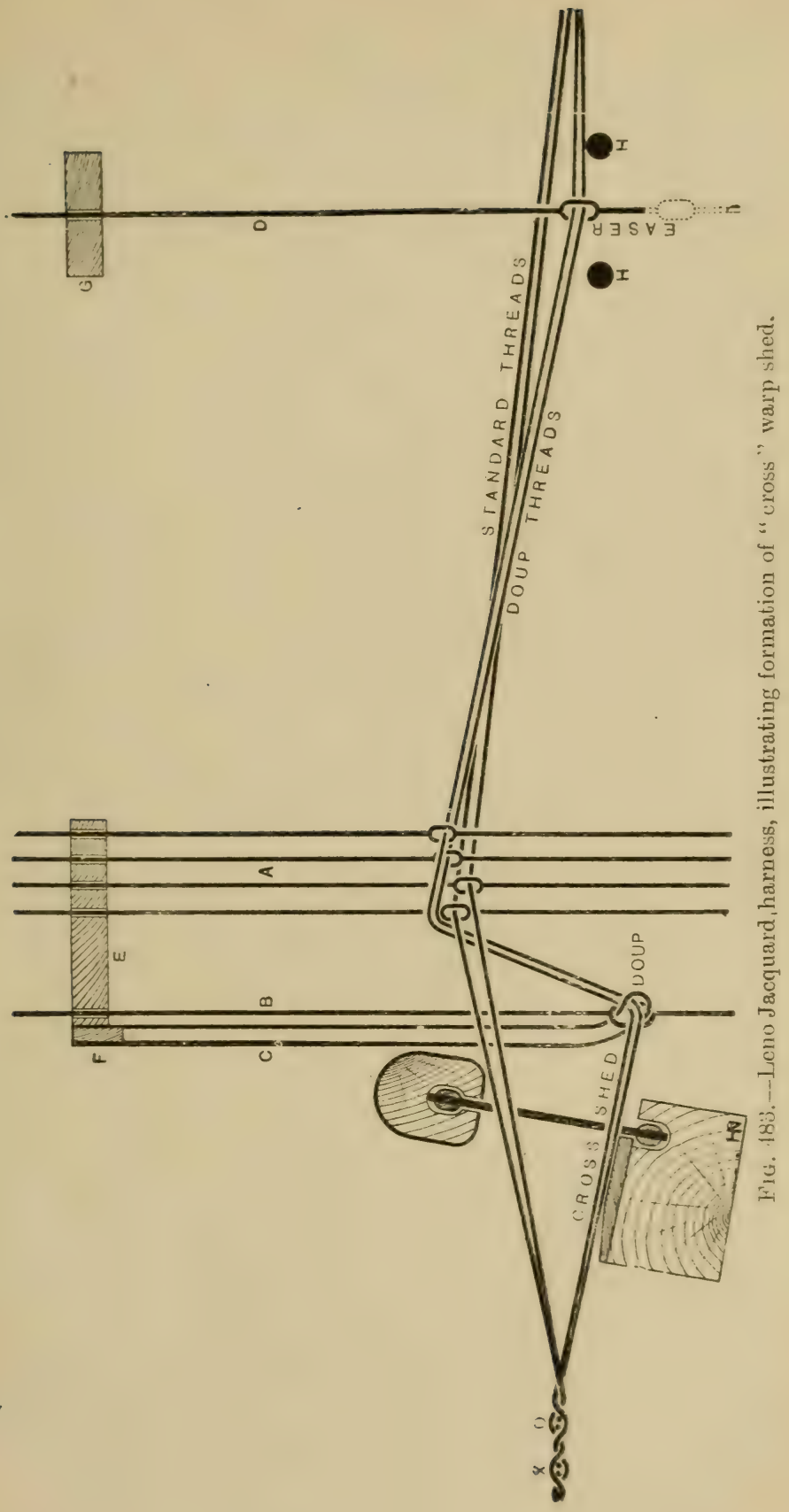


All warp threads are contained upon the same warp beam, excepting when net leno stripes are introduced, in which case, such number of extra warp beams are required (to contain the thick net doup threads) as corresponds with the different schemes of douping in the same fabric. If a two-and-two crossing of warp threads is required, alternate pairs of threads are passed together through the glass mails of the easer harness, to serve as "doup" warp threads, leaving intermediate pairs of threads for "standard" warp threads. Excepting for the deflection of doup threads by the easers, all warp threads pass perfectly parallel from the warp beam to their respective mail eyes of the principal harness, through which they are separately drawn in consecutive order.

After all warp threads have passed separately through the mails of the principal harness, each pair of doup threads is crossed in regular succession over a pair of standard threads, always in the same direction uniformly (say from right to left when stood in front of the loom, as represented in the diagrams), after which the doup threads only are passed together through their respective doup slips, when the two pairs of warp threads that cross each other are passed together through the same dent of the reed.

$\$ 135$. The method of drawing-in and crossing the warp threads in a leno brocade harness will be easily comprehended on referring to Figs. 481, 482 and 483, which illustrate the formation of a "neutral," an "open" and a "cross" shed respectively.

A "neutral" shed is one in which warp threads are raised in any desired manner (irrespective of whether they are standard or doup threads) for ordinary brocade weaving, in which no crossing of threads occurs. It is formed by raising the doup mails where required for the purpose of relieving the respective doup slips, in order to permit of doup warp threads separating within them to form a shed; whilst the corresponding easers remain down, as clearly indicated in Fig. 481.

An "open" shed (Fig. 482) is formed by raising standard warp threads and keeping doup threads down, by means of the principal harness; and at the same time raising the cor- 
responding doup mails so as to relieve the doup slips and permit of standard threads rising between them and the doup mounting threads, as indicated in the diagram, whilst the easers remain down.

A " cross" shed (Fig. 483) is formed by keeping doup threads down by means of the doup harness, and by raising standard and doup threads by means of the principal harness, and at the same time raising the corresponding easers to slacken doup threads, and so compensate for the short interval allowed them for the formation of a "cross" shed.

$\leqslant$ 136. As observed previously in $\$ 133$, the scheme of douping or crossing warp threads in leno brocade fabrics may be either of a uniform character, or else, several schemes may be introduced in different parts of a pattern to give variety of effect; though the usual practice is to employ only one scheme of douping, with warp threads crossing in pairs, and with either two, three or four consecutive picks inserted in each leno shed. It was further stated that, when drawing in warp threads through the harness, doup threads were crossed from the normal or open side to the crossed side of their respective standard threads uniformly in the same direction. It should at once be observed, however, that such circumstance does not make it imperative that all doup threads must cross either simultaneously or in the same direction, as it would under similar conditions if warp threads were drawn through a heald harness.

By employing a Jacquard machine to gorern warp threads independently, some may be made to form a cross shed, whilst others are forming an open shed for the same picks. Designers for leno brocade fabrics usually turn this opportunity to advantage by developing the leno portions of a design so that, when reproduced in cloth, those doup warp threads that were contained in alternate dents of the reed, when in the loom, will be on the crossed side, whilst those that were in the intermediate dents will be on the normal or open side, of their respective standard threads, for the same pick or picks of weft. Thus, a leno effect is produced in which successive pairs of doup threads cross their standard threads in reverse directions for 
the same picks, exactly as if they had been crossed in reverse directions in the harness, and made to form a cross and open shed alternately for consecutive picks or pairs of picks.

$\$ 137$. The foregoing observations are clearly demonstrated in Fig. 484, in which the arrangement of warp threads in the various sections of a "top doup" leno brocade harness and in the reed, and also a plan of cloth, are represented graphically. By carefully studying this diagram in conjunction with those given in Figs. 481 to 483 , the operation of a leno brocade harness will be more readily understood.

The plan of cloth represents a portion of a fabric in which a gauze figuring is developed by crossing warp threads in pairs, and inserting two picks in each leno shed; whilst warp threads and picks interweave as single threads to develop a ground texture of the plain calico weave. The scheme of douping is that in which warp threads contained in alternate dents of the reed cross in one direction whilst those in intermediate dents cross in the reverse direction for the same picks. For example, doup warp threads are held down and standard warp threads are raised, on their normal sides, in dents 3 and 5 , to form an "open shed" for the last pair of picks inserted; whilst, in dents 4 and 6, doup threads are down and standard threads are raised, on their crossed side to form a "cross shed ". for the same picks of weft. At the same time, warp threads in dents 1 and 2 are forming a "neutral" shed, to weave either plain cloth or brocade figuring, in which warp threads always occupy their normal relative position.

By a little closer observation of Fig. 484, it will be seen that a "neutral shed," as in dents 1 and 2, is formed by raising doup mails to relieve their doup slips and permit of warp threads being raised or left down in any order, to produce an ordinary brocade texture, as represented in Fig. 481. An "open shed," as in dents 3 and 5 , is formed by raising doup mails to relieve their corresponding doup slips and allow the doup threads to return to the normal side of their respective standard warp threads, which are raised, whilst doup threads and their easers remain down as represented in Fig. 482.

A "cross shed," as in dents 4 and 6 , is formed by raising 
both standard and doup warp threads by means of the principal harness, slackening the corresponding easers, and keeping doup

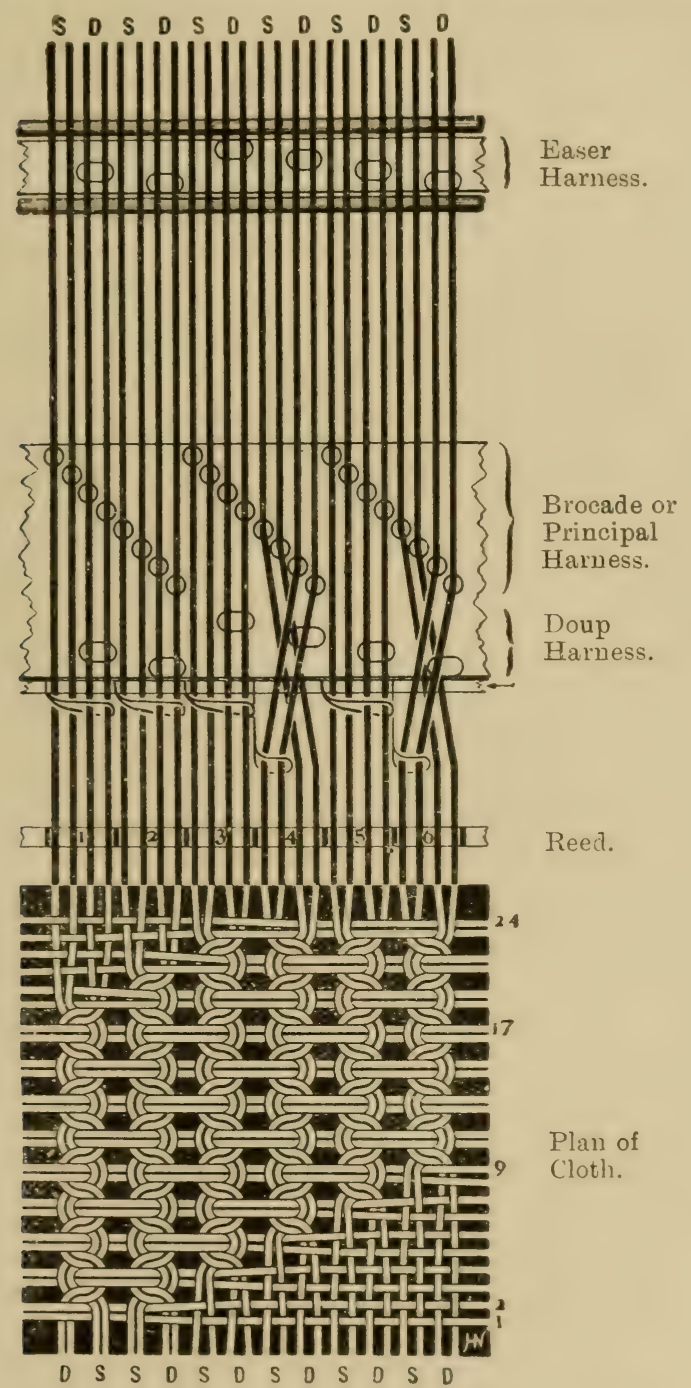

Fig. 484.-Plan of leno brocade harness and fabric. 
mails down to retain doup threads on the crossed side of their respective standard threads, as represented in Fig. 483.

$\S 138$. In $\$ 133$ it is stated that a leno Jacquard harness may be controlled either by means of a Jacquard machine of ordinary construction, or preferably by one that is specially adapted for such a harness. In either case precisely similar results are obtained, but an economical advantage is gained by the use of special leno Jacquard machines, inasmuch as they greatly facilitate and simplify both the preparation of designs to be reproduced by them, as well as the subsequent operation of card-cutting. If an ordinary Jacquard machine is employed for leno fabrics, it will be necessary to equip it with sundry auxiliary attachments to perform the functions of douping or lenoing and easing.

These supplementary appliances vary in character to suit the particular construction of the harness, of which there are various modifications, to be described presently. Such harnesses may be constructed on either the top doup or the bottomdoup principle as desired; although it appears to be the prevailing practice to employ a bottom-doup harness for the production of Jacquard leno fabrics. But whatever particular form a leno brocade harness may assume, it will be found to consist essentially of three distinct sections, as represented in Figs. 481,482 and 483 .

A simple and efficient adaptation of an ordinary singleacting Jacquard machine to govern a leno harness is represented in Fig. 485, which shows the connection of the harness to the machine. The harness is represented as a bottom-doup harness, with the doup slips C contained upon a stave $\mathrm{F}$ which is operated by spare hooks I of the machine, so that it is raised for every pick inserted. The depression of the doup stave is effected by means of a spring $J$ attached to the floor. When the stave containing the doup slips is placed above warp threads to constitute a top-doup harness (as represented in Figs. 481, 482 and 483), it is screwed to the front edge of the comberboard $\mathrm{E}$, and therefore becomes a fixture. The position of the doups, however, is quite optional provided the respective portions of the harness are governed in accordance with that position. 


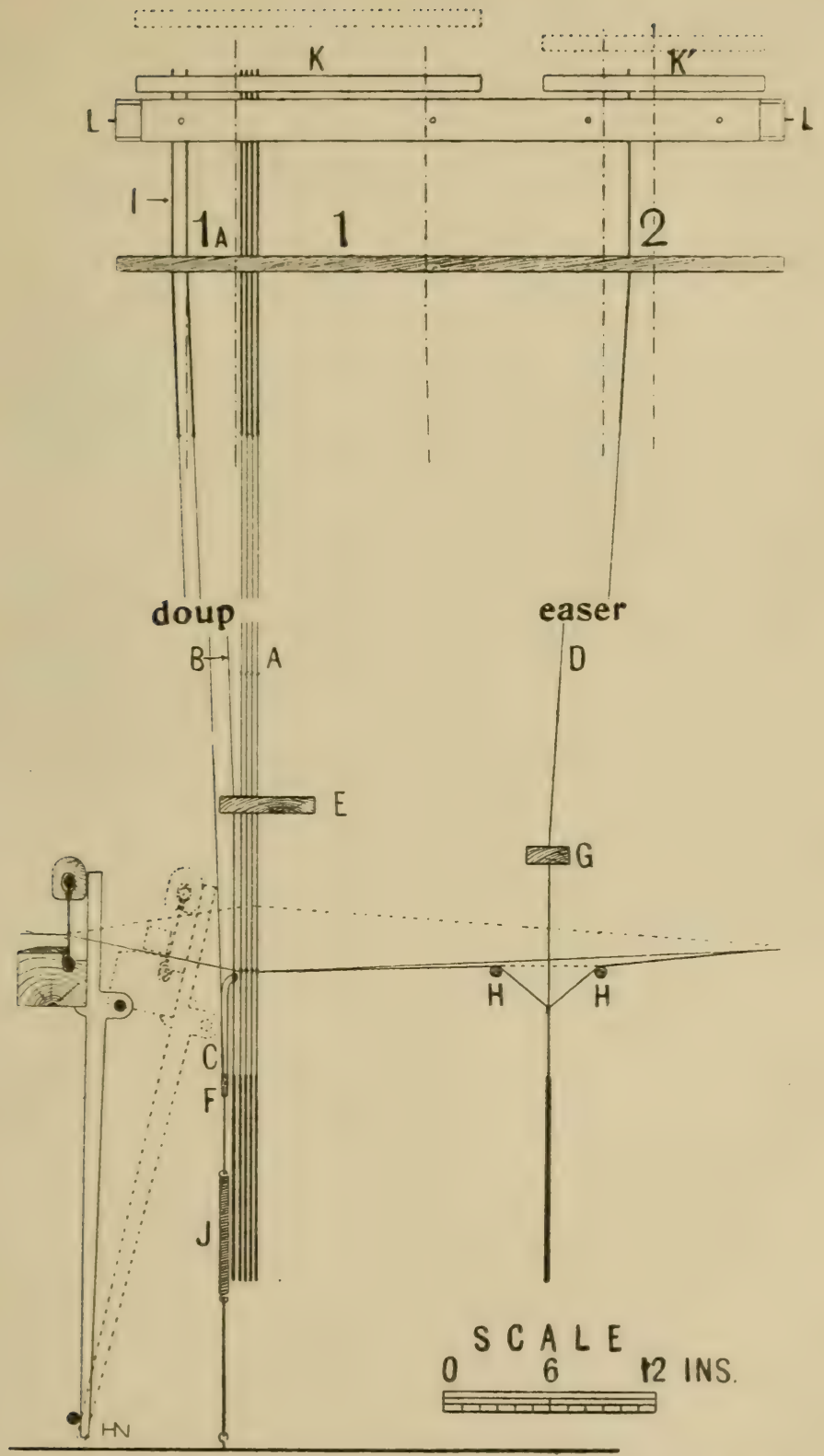

FIG. 485. -End elevation showing one type of a bottom-doup leno Jacquard harness governed by ordinary Jacquard machines. 
The Jacquard machine represented in Fig. 485 consists of two distinct sets of hooks and needles (1, $1_{\mathrm{A}}$ and 2) contained in the same framing, and actuated by two separate sets of pattern cards, which pass over the same cylinder $\mathrm{L}$, and therefore operate simultaneously for consecutive picks of weft. The larger division contains, say, 408 hooks and needles, with the "25-side" of the machine (which is on the left when facing the cylinder) over the front of the loom. This portion is subdivided into two parts, 1 and $1 \mathrm{~A}$, and governs both the principal and doup harnesses respectively. The smaller division (2) has a capacity for 208 hooks and needles to govern the easer harness $D$, though it need not contain more than one-quarter of the number contained in division 1 when warp threads are required to cross in pairs, in which case only one hook and needle is required for every four warp threads.

Thus, assuming a "tie-up" of 320 hooks, in part 1, for the brocade harness $\mathrm{A}$, and that warp threads are required to cross in pairs, 80 hooks would be required in part $1 \mathrm{~A}$ to govern the doup harness $B$, and 80 hooks in part 2 to govern the easer harness $\mathrm{D}$. This ratio is indicated in the diagram by 4 hooks in part 1 , and 1 hook each in parts $1_{\mathrm{A}}$ and 2 . It should be observed that when tying up a bottom-doup leno harness it is advisable to tie up the doup portion of the harness, so that the mails of that portion, which contain the doup slips, will be about $\frac{1}{8}$ in. lower than those of the principal harness, when the shed is closed, to facilitate the crossing of doup and standard warp threads. Also, doup mails should be a little larger, and the lingoes preferably slightly heavier than those of the principal harness; whilst the mails for the easer harness are usually of glass, and the lingoes for that harness should weigh about 10 per $\mathrm{lb}$. for warp threads of medium counts of yarn.

$\$ 139$. It is unnecessary, when slackening doup warp threads during the formation of cross sheds, to raise the mails of the easer harness through the same distance as those of the principal and doup harnesses. Some means, therefore, must be adopted whereby the easer mails will be raised a little shorter distance 
than those of the principal and doup harnesses. This object is effected in a variety of ways by different machines and harnesses, as' will be described presently. Thus, if the easer harness is governed by an independent set of hooks in the Jacquard

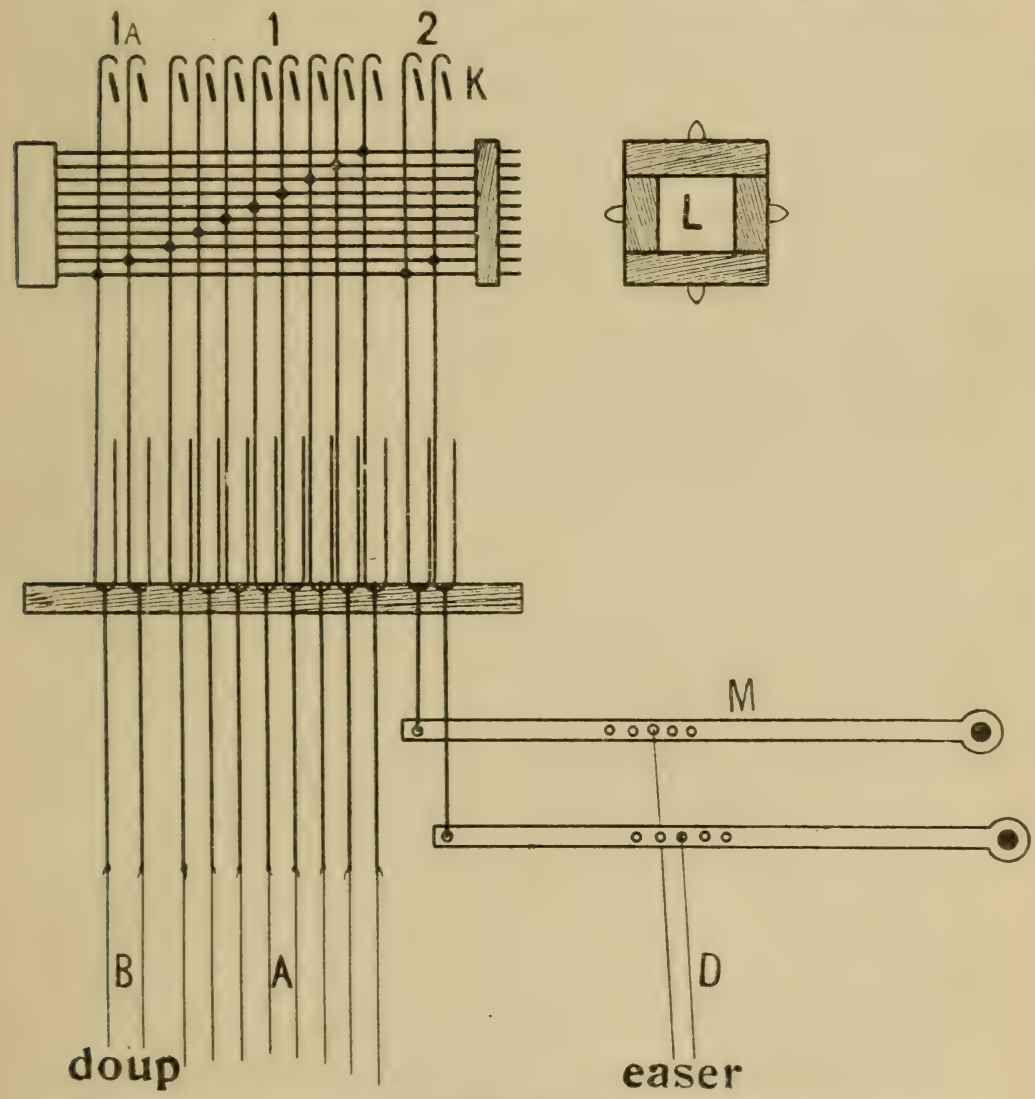

FIG. 486.-Jacquard machine adapted specially for governing a bottom-doup leno Jacquard harness.

machine, the function of easing may be accomplished by several optional methods, namely, by empioying two separate and distinct sets of griffe blades, one set $\mathrm{K}$ to raise doup and principal harness hooks, and another $\mathrm{K}^{1}$ to raise easer harness hooks. By this method both griffes are actuated from the crank shaft 
of the loom, and therefore act in unison; but the easer griffe is raised for a shorter distance than the principal griffe, as indicated by dotted lines.

By another method (illustrated in Fig. 486) only one set of griffes $\mathrm{K}$ is employed for all hooks, but the easer hooks (2) communicate with the easer harness $\mathrm{D}$ through the medium of a corresponding number of levers $\mathrm{M}$ of the second order, so as to reduce the lift of that harness.

By a third method, illustrated in Fig. 487, which also dispenses with the second set of griffes, the function of easing is effected by attaching the mounting threads of the easer harness $\mathrm{D}$ either to the corresponding mounting threads, or else to the neck cords of the doup harness $B$, so that the complementary doup and easer mails controlling the same doup thread or threads will always rise and fall simultaneously. This latter method of easing, however, may only be adopted with a bottomdoup harness, which forms cross sheds by raising doup warp threads by means of the doup harness, whilst standard warp threads remain down, at which periods it is necessary to slacken doup warp threads to relieve them of undue tension.

This harness also illustrates another departure from the leno harnesses described previously. Thus, instead of employing a stave containing all doup slips, these are attached to separate lingoes, and connected by means of mounting threads $\mathrm{B}^{1}$ to each of the two principal harness threads which also govern doup warp threads. By this means, the doup slips are raised independently by the principal harness threads, only when that is necessary, both for the formation of open sheds by particular groups of crossing threads, and also by doup harness threads for the formation of cross sheds. A disadvantage of this arrangement of a leno brocade harness, however, is that the connecting threads $\mathrm{B}^{1}$ become slack, as shown in the diagram, whenever their respective doup harness threads $B$ are raised to form cross sheds.

$\$ 140$. With the type of leno harness represented in Fig. 487 it would appear, at first sight, that, in consequence of the easer harness threads $D$ being attached directly to their complementary doup harness threads $B$, the easer harness would 


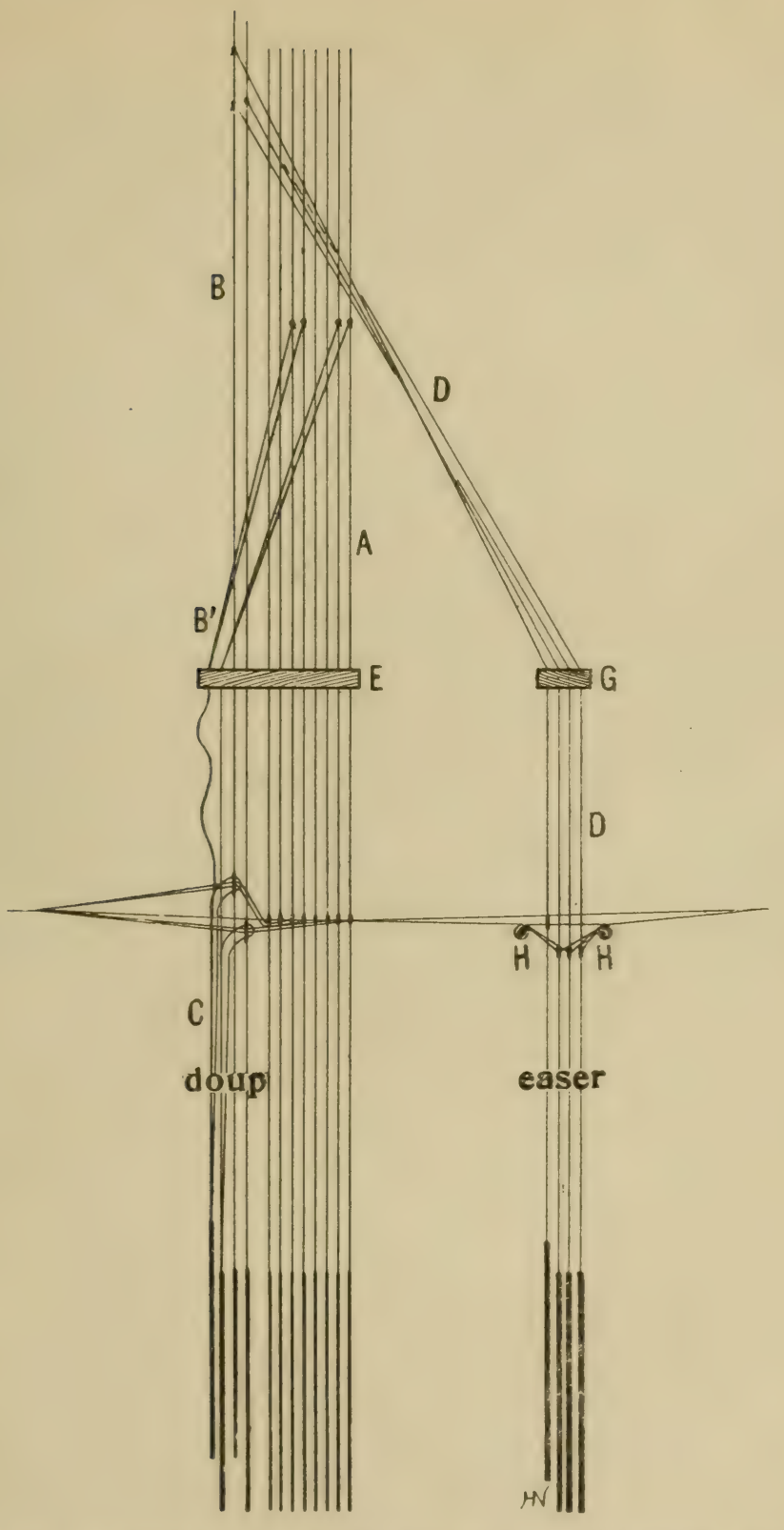

Fig. 487.-A second type of bottom-doup leno Jacquard harness gcverned by an ordinary Jacquard machine. 
be raised as high as the doup harness, which would be quite unnecessary. Such, however, is not the case, as a little reflection will show. In consequence of the divergence of easer mounting threads from their points of connection with the doup mounting threads (which are perpendicular) to the points where they pass through their comber-board G, the length of threads $\mathrm{D}$, between these points, will not increase in a measure equal to the lift of doup mounting threads; hence the shorter lift of easer mails, which may be increased or reduced by tying easer mounting threads to doup mounting threads at a higher or lower point respectively.

\section{Leno Jacquard Machines.}

$\$ 141$. A simple and efficient method of actuating a leno brocade mounting, with the object of raising the easer harness for a shorter distance than the principal and doup harnesses, is that provided by Devoge's special leno Jacquard machine as illustrated in Fig. 488, which represents a machine of this type adapted for governing a bottom-doup harness. In machines of this type, the hooks are disposed in three sections, namely, 1, 1A and 2 (as also represented in Fig. 486), to govern the principal, doup and easer harnesses respectively. The shorter lift of easer hooks constituting section 2, than that of the principal and doup hooks in sections 1 and $1 \mathrm{~A}$ respectively, is effected by employing two distinct sets of griffes $\mathrm{K}, \mathrm{K}^{1}$, each contained in a separate frame and actuated loy independent lifting levers. The frame containing griffes $\mathrm{K}$, which raise the principal and doup hooks, is connected by means of a link-rod $\mathrm{P}$ directly to the primary lifting lever $\mathrm{Q}$, and is raised for the full height of the shed; whereas the frame containing the easer griffes $\mathrm{K}^{1}$ is connected by means of a connecting link-rod $\mathrm{P}^{1}$ to a secondary lifting lever $\mathrm{N}$, fulcrumed on a pin $\mathrm{O}$, fixed in a bracket situated on the same side of the machine as that containing easer hooks, to ensure a shorter lift of those hooks. The free end of the secondary lifting lever $\mathrm{N}$ is furnished with a slot for the reception of a stud $R$ projecting from the connecting link-rod $P$, by means of which the lever $\mathrm{N}$ is oscillated. The primary lifting lever 


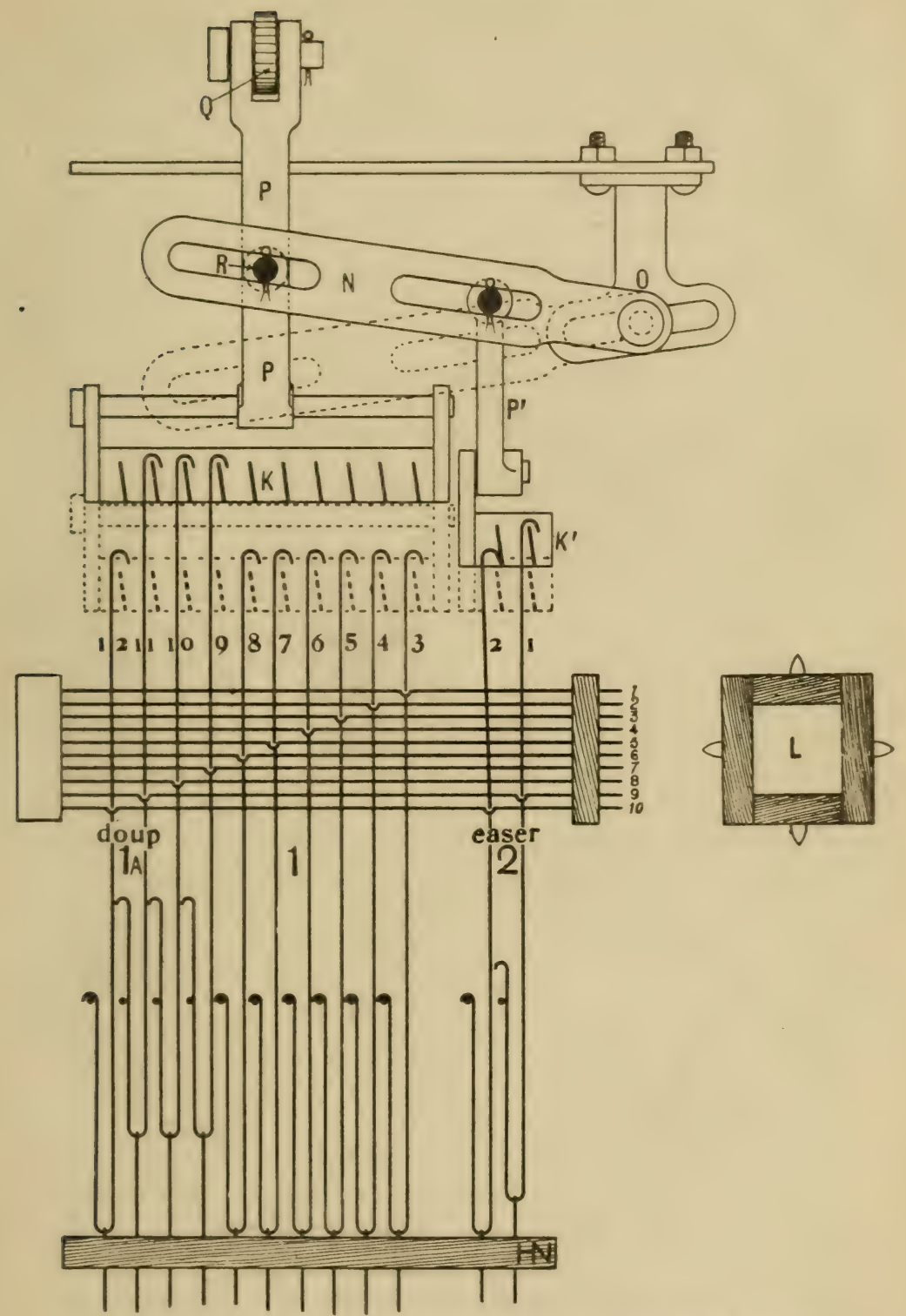

Fig. 488.-Devoge's leno Jacquard adapted for a bottom-doup harness. 
Q communicates, through the medium of a long connecting rod, to a crank fixed on one end of the crank-shaft of the loom. Hence both the primary and secondary lifting levers oscillate in unison with the rotation of the lifting crank.

In a 400's Jacquard machine of this type, the hooks are arranged in twelve rows containing 51 hooks in each row. These are controlled by ten horizontal rows of needles or lances, thereby requiring a card cylinder bored with ten long rows of holes in each side, to receive pattern cards of a corresponding index or gauge. The 408 hooks forming the middle eight rows (Nos. 3 to 10 inclusive) govern the principal or brocade section of the harness, and are controlled by the eight upper rows of needles. If the machine is mounted on a loom with the card cylinder at the back, the easer harness must be attached to, and governed by, the hooks forming the first and second rows; and the doup harness must be governed by the eleventh and twelfth rows of hooks. If, however, the machine is mounted with the cylinder towards the front of the loom, those conditions would be reversed by placing the easer hooks and griffes, in the rear part of the machine, to constitute the eleventh and twelfth rows, for governing the easer harness ; and by placing the doup hooks at the front, to constitute the first and second rows for governing the doup harness. Also, the secondary lifting lever $\mathrm{N}$ would have to be reversed, so as to place the fulcrum $\mathrm{O}$ on the same side as the easer hooks which require to be raised for a shorter distance, as described.

In the machine represented in Fig. 488, the first and last two rows of hooks, consisting of easer and doup hooks respectively, are controlled by only two rows of needles, namely, the ninth and tenth rows. Each needle in those rows is formed with two loops or cranks-one to receive an easer hook, and another to receive a doup hook, both of which hooks thereby become complementary to each other, and must, therefore, always respond in unison to the movement of the same controlling needle. Thus each needle in the ninth row simultaneously controls the two complementary easer and doup hooks that are contained in the first and eleventh rows respectively, whilst the complementary easer and doup hooks 
in the second and twelfth rows are controlled simultaneously by the same needle in the tenth row.

$\$ 142$. If a machine of this type is adapted for governing a bottom-doup harness, in which complementary doup and easer mounting threads must, of necessity, rise and fall simultaneously and in unison, the dual control of both a doup and an easer hook by the same needle will require those tivo series of hooks to be placed in the same direction, uniformly, as indicated in the diagram, Fig. 488. If, however, these machines are adapted for governing top-doup harnesses, in which complementary doup and easer mounting threads rise and fall in a contrary manner to each other simultaneously, the doup and

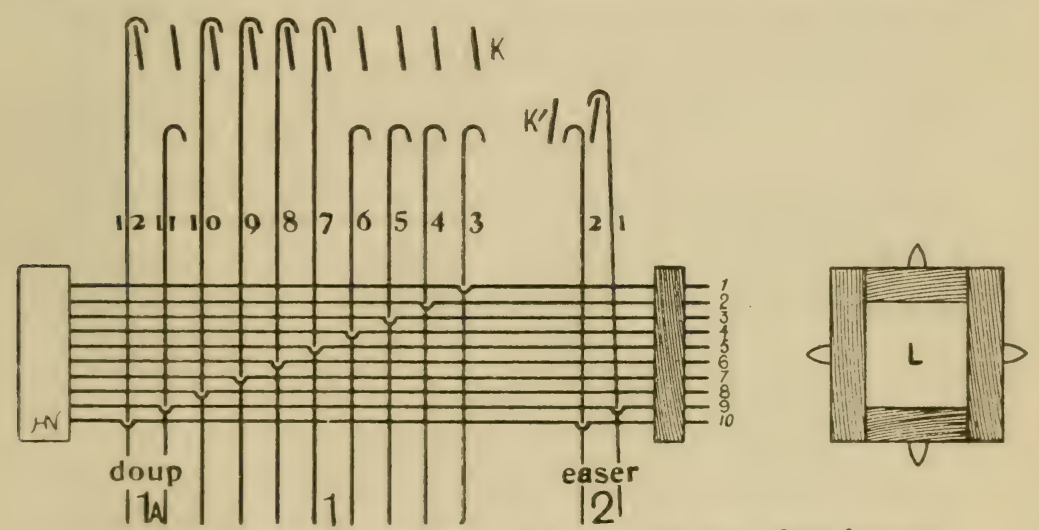

Fig. 489.-Devoge's leno Jacquard adapted for a top-doup harness.

easer hooks of the machine would, in that case, require to be placed in reverse directions with the easer hooks pointing away from the card cylinder and resting, normally, away from their griffes, as indicated in Fig. 489.

By adopting the simple expedient just described, and as indicated in Fig. 489, in the construction of a leno brocade Jacquard machine of this type, adapted for governing a topdoup harness, it thereby ensures the control of complementary doup and easer hooks always in a contrary manner; that is to say, if doup hooks remain down, for the formation of "crosssheds," the corresponding easer hooks are raised, at the same time, for easing the doup warp threads; and, per contra, if 
doup hooks are raised, for the formation of "open" and "neutral" sheds, the corresponding easer hooks remain down at the same time.

Leno Jacquard machines of the type under present notice
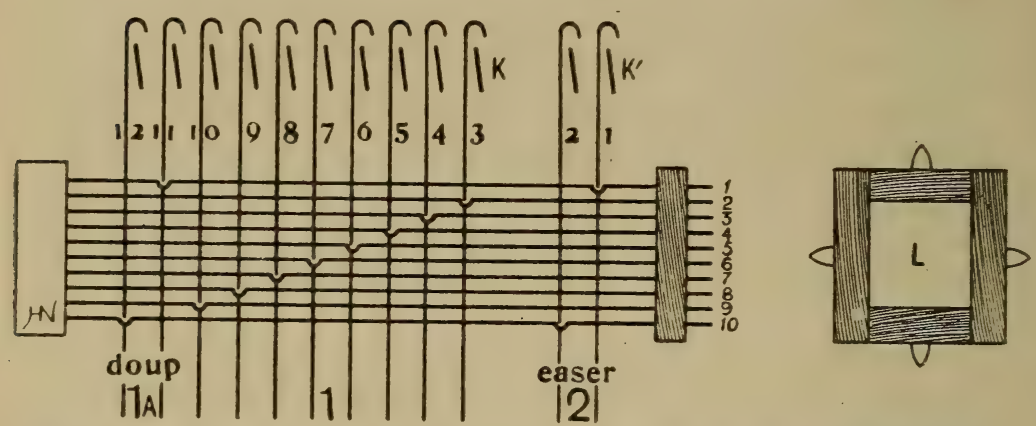

FIG. 490.-A second modification of Devoge's leno Jacquard adapted for a bottom-doup harness.

are sometimes made with minor and unimportant modifications to suit the personal preference of manufacturers. Thus, in some machines, the doup and easer hooks are controlled by
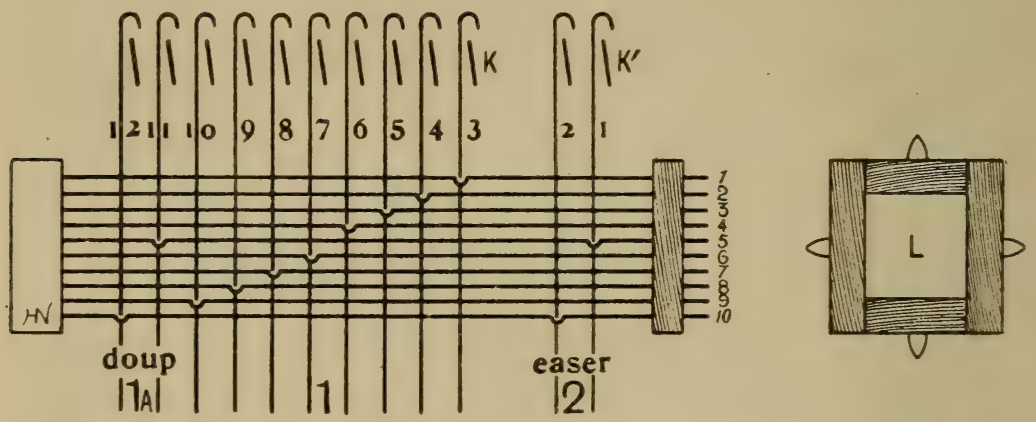

FIG. 491.-A third modification of Devoge's leno Jacquard adapted for a bottom-doup harness.

needles forming the first and tenth rows, as represented in Fig. 490. In others, those hooks are controlled by the fifth and tenth rows of needles, as represented in Fig. 491. These modifications, however, in no way affect either the operation of 
the machines or the preparation of designs for them. The choice of these arrangements is quite optional, and only affects the convenience, or prejudice, of the card cutter, who may favour either one or the other of the optional arrangements as described.

\section{Methods of Preparing Leno Brocade Designs.}

$\$ 143$. The method of indicating upon squared point paper an applied design, for a leno brocade fabric, varies according to the particular type of Jacquard harness and machine by which the design will be reproduced in the fabric, and of which there are several modifications that are designed specially for fabrics of this type. It is essential; therefore, that a designer for these fabrics should have some knowledge of the construction and operation of these special leno Jacquard machines and harnesses to enable him to prepare the applied designs with greater technical accuracy.

The small fragment of a leno brocade design, Fig. 492, will serve to demonstrate the general method of procedure in indicating, upon point paper, a design to be reproduced by means of a Jacquard machine in which the hooks and needles controlling the three sections of a leno brocade harness constitute three separate and distinct divisions, namely-A, for the principal or brocade harness, B, for the doup harness, and C, for the easer harness, gaited-up as a top-doup harness, as represented in Figs. 481, 482 and 483. The portion of the design indicated in Fig. 492 corresponds to the plan of cloth represented in Fig. 484 comprising twenty-four warp threads and twenty-four picks of weft; and, unlike most applied designs for fabrics of a simple brocade texture, which indicate the actual interlacing of warp and weft threads, the leno portions of a leno brocade design bear no resemblance whatever to the plan of inter-wveaving those threads, nor does it really indicate the order in which warp threads are raised or left down for the respective picks of weft.

What a leno brocade design actually does indicate is the operation of the harness threads in the respective sections. Thus, the operation of the principal harness threads is indicated 
at $\mathrm{A}$, whilst that for the doup and easer harness is indicated at $B$ and $C$ respectively. It is the relative positions of the warp threads, during shedding, that determines their relative positions in the cloth. Thus, in the leno portions of the design, standard warp threads are represented as being raised for every pick, whilst doup threads are represented up for alternate pairs of picks and down for intermediate pairs of picks. On examining the plan of cloth, however, as represented in Fig. 484, it will be seen that, although standard warp threads are raised in the leno portion for every pick, doup

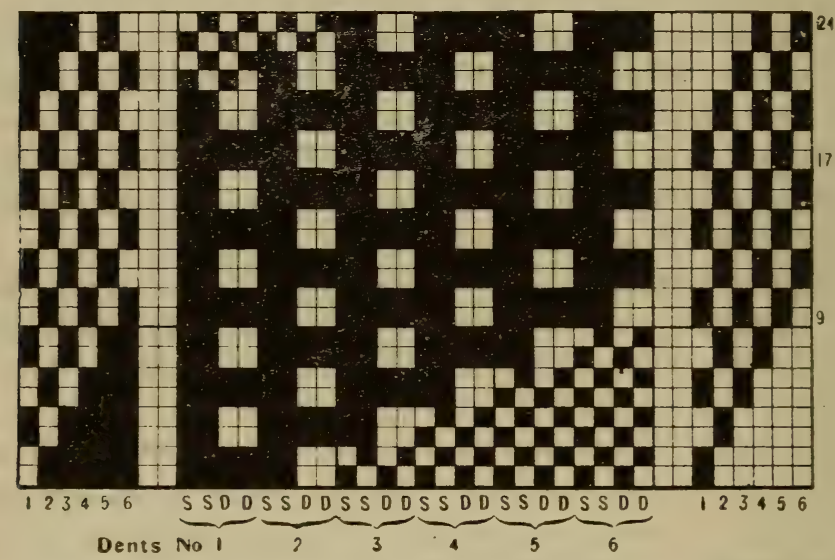

Fig. 492.-Method of preparing an applied design for a top-doup leno Jacquard harness governed by ordinary Jacquard machines, as repre. sented in Fig. 485.

threads are always down in that portion, because, although they are raised by the principal harness when forming a cross shed, they are held down on their crossed side by their respective doup mails and slips, as explained previously in 137 .

$\$ 144$. On examining this fragment of a design, it will be seen that whenever both standard and doup threads are raised together in the same dent of the reed, the corresponding doup harness thread governing those doup threads remains down, thereby retaining them on their crossed side to form a cross shed in that dent. At the same time, the corresponding easer harness thread is raised to slacken the doup threads, and thereby 
prevent excessive strain upon them. If, however, standard threads only are raised in a given dent of the reed, the corresponding doup harness thread also is raised to permit of the doup threads returning to their normal side in order to form an open shed, whilst the corresponding easer harness thread remains down.

The same operation of doup and easer harness threads takes place when it is required to form neutral sheds in the calico and brocade portions of the fabric. It will be seen, therefore, that corresponding doup and easer harness threads always work in a contrary manner to each other: thus, when one is up, the other is down.

It should also be observed that, when preparing a design, it is more expedient to allow warp threads to form an open shed for at least two picks of weft between a cross shed and the calico weave, as indicated in the plan of cloth and the design, Figs. 484 and 492 respectively, and thereby permit of warp threads opening out more gradually from their congested state in the leno portion of the fabric. By carefully comparing those figures, the analogy existing between them will be clearly manifest.

The leno effect represented in Fig. 484, in which warp threads in contiguous dents of the reed cross in reverse directions, has a far superior appearance to that in which they cross in the same direction uniformly. And although, as suggested preriously, an exactly similar effect would result by crossing contiguous pairs of doup threads in reverse directions in the harness, and forming cross and open sheds alternately for successive pairs of picks, that course would not be so practical as the plan adopted, owing to the greater strain imposed upon warp threads when forming a cross shed, and the consequent greater strain exerted upon the various parts of the loom.

For these reasons it is more expedient, when drawing-in the warp threads through the shedding harness, to cross all doup threads in the same direction uniformly, and to form cross and open sheds alternately in successive dents of the reed for the same picks of weft, than it is to cross doup threads in reverse directions, and form cross sheds only for some 
picks and open sheds only for others during weaving. By the former method, the power absorbed by shedding is of a more uniform value for every warp shed, thereby conducing to a more uniform velocity, and consequently less straining and wear and tear of the various parts of a loom. But if the latter course were adopted, the disparity between the greater power required to form a cross shed, and the lesser power required to form an open shed, would tend to cause a loom to work with a more or less jerky and irregular action, due to the excessive straining of the various parts during the formation of cross sheds.

$\$ 145$. The manner of setting out and developing an applied leno brocade design upon squared paper, ready for card-cut. ting, depends upon the relative disposition of the three divisions of hooks, and also the arrangement of needles controlling them, in the Jacquard machine by which the design will be reproduced in cloth. The method of preparing a design for a top-doup harness governed by a machine of the type represented in Fig. 485 is indicated in Fig. 492, whilst the same portion of design arranged for a bottom-doup harness to be governed by the same machine is indicated in Fig. 493. The only difference between the two designs is that they are counterchanged in respect of the principal and doup harnesses, to conform with the reverse conditions imposed by the respective arrangements of those harnesses, as described previously in $\$ 142$. Thus, in Fig. 492 (for a top-doup harness), the leno portion of the design is developed by raising standard warp threads for every pick of weft, and forming cross sheds by keeping the doup threads depressed by the doup harness, and raising them by the easer harness; whilst open sheds are formed by raising standard warp threads only. In Fig. 493, however (for a bottom-doup harness), those conditions are exactly reversed.

In leno Jacquard machines of the type for which those designs are prepared, the hooks commanding the doup and easer harnesses are situated respectively on each side of those commanding the principal harness; also doup and easer hooks are controlled by independent sets of needles that are in horizontal 
alignment with those controlling the principal division of hooks. The three divisions of hooks and needles may be contained either within the same framing (provided easer hooks have an independent griffe), and actuated by only one set of pattern cards; or else easer hooks and needles may be quite separate from the others, as represented in Fig. 485, and actuated by an auxiliary set of pattern cards. In either case, it is necessary to prepare a design for such a machine in three sections, as indicated in Figs. 492 and 493, to correspond with the three divisions of hooks and needles in those machines.

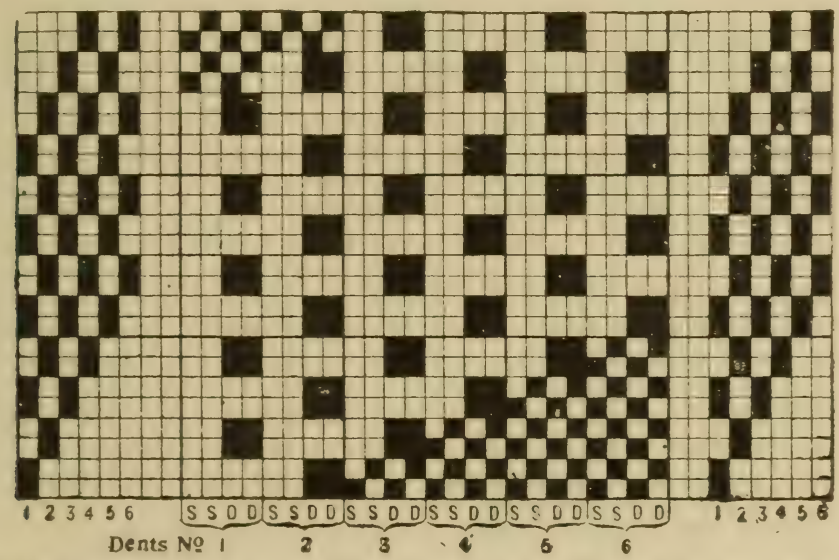

FIG. 493.-Nethod of preparing an applied design for a bottom-doup leno. Jacquard harness, governed by ordinary Jacquard machines, as represented in Fig. 485.

It is customary, however, for a designer to economise time and labour by indicating the operation of doup hooks only, on one side of the design, to be "read" by a card-cutter when cutting for both the doup and easer hooks. Thus, when cutting the easer set of pattern cards (for a top-doup harness) he would counter-change the doup chart of the design; but for a bottom-doup harness, the doup chart of the design would be "read" and transferred to the easer set of cards in exactly the same manner both for the doup and easer hooks.

$\$ 146$. The preparation of designs, and also the subsequent operation of card-cutting, is very greatly facilitated and simplified. 
by the use of leno Jacquard machines of the type represented in Figs. 486 to 491 ; as it is unnecessary, in these circumstances, for the designer to indicate, by means of separate charts on each side of the principal chart of the design, the actual operation of the doup and the easer hooks of the Jacquard machine, as is necessary when preparing applied. leno brocade designs that are to be reproduced by Jacquard machines of the ordinary type, as represented in Fig. 485. This advantage is afforded by both the easer and doup hooks, each being placed in two long rows situated in front and behind respectively those governing the principal harness, and also by the dual control of doup and easer hooks by only one set of needles that are placed in two separate horizontal long rows, but in vertical alignment with those controlling the principal figuring hooks. By such a disposition of hooks and needles the necdles controlling doup and easer hooks are contained in the same short vertical rows as the principal set of needles, and, therefore, come within the compass of the narrow width of a pattern card. This circumstance enables the operation of doup and easer hooks to be indicated on the design by using a distinctive colour of paint for that purpose. By this means a card-cutter is enabled to "read" and cut for both sections of needles simultaneously for successive "bars" on the design paper, in a manner to be explained presently.

The method of indicating a design upon squared paper, for machines of this type, to govern a bottom-doup harness, is indicated in Fig. 494. (It may be desirable, at this point, to observe that the type of leno harness in mind is that represented in Fig. 485, which requires the doup slips, constituting the doup or half heald, to be situated below warp threads, and controlled either by spare hooks in the Jacquard machine, or preferably by the griffe of the machine, to which it may be connected directly so that the doup heald stave will rise with the formation of every warp shed.)

Although Jacquard machines of this type contain ten rows of needles, the design paper requires to be ruled with only eight vertical divisions in each "bar," albeit they represent a row of ten needles, and are "read" as such by the card- 
cutter, as just stated. The portion of design given in Fig. $49 t$ is executed for a leno brocade fabric having a leno figure, with a ground of the plain calico weave. The scheme of douping or crossing indicated in the design is one in which warpthreads would cross in pairs, with three consecutive picks of

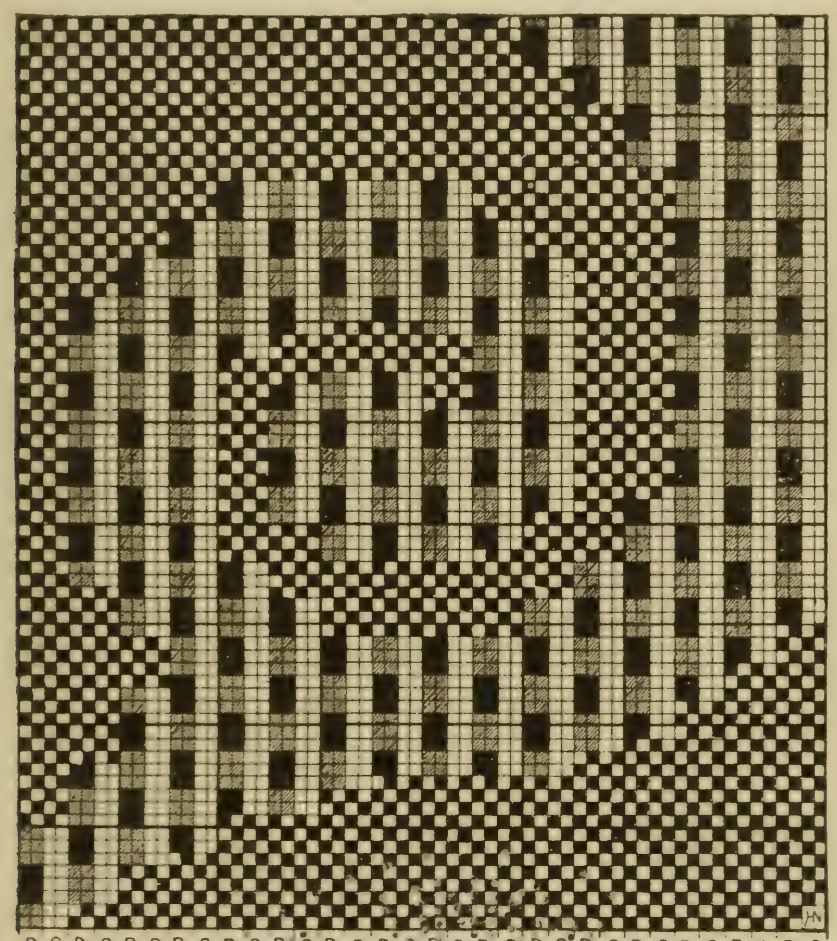

DSDSDSDSDSDSDSDSOSDSDSESDSDSDSDS

FIG. 491.-Method of prinaring an applied design for a bottom-doup harness governed; by'the leno Jacquard machines represented in Figs. 488, 490 and 491.

weft inserted togather in a cross and an open shed alternately, to produce the reverse cicssing af threads, as described previously in 136 and 54 , and represented graphically in Fig. 484. Black squares in the design indicate corresponding warp threads raised by the principal harness, and shaded squares (which would be indicated by another colour of paint) 
indicate corresponding doup warp threads only, raised by the doup and easer harnesses.

$\$ 147$. When cutting the pattern cards from a design to be reproduced by a Jacquard machine, in which the needles are arranged as represented in Figs. 488 and 489, the eight vertical divisions in each "bar" of the design paper correspond with the first eight rows of needles controlling the principal hooks, in respect of black squares only, whilst shaded squares placed in the third and fourth divisions of each "bar" (from the right) indicate that holes must be cut in a pattern card so that when this is presented to the needles, by the card cylinder, they will coincide with the corresponding needles in the ninth row of needles; also, shaded squares in the seventh and eighth divisions in each "bar" of the design paper, signify that holes must be cut opposite the corresponding needles in the tenth row. (It may be explained that, although it is really only necessary to indicate the operation of doup and easer hooks by painting the fourth and eighth divisions only in each "bar," wherever such indication is required, it is customary, in actual practice, to paint both divisions on the design paper, representing the two contiguous doup warp threads, as indicated in the fragment of the design, Fig. 494.)

If, therefore, a leno brocade design is prepared for a leno Jacquard machine which is specially adapted for governing a bottom-doup harness, and as represented in Fig. 488, the applied design will require to be developed in the manner indicated in the fragment of a designn represented in Fig. 494.

If, however, the drisign is prepared for a leno Jacquard

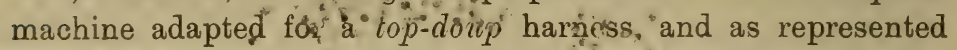
in Fig. 489, it will be necessary for the desigrer also to paint up, on the design paper, those divisions whioh represent the standard warp threadi, in the leno portions of the design, as indicated in Fig. 495 A This course is necessary by reason of raising both standard and dcup warp threads together, in the same dents of the reed, whenever these threads are required to form a cross shed, as described in $\$ \$ 135$ and 137, and illustrated in the graphic diagram, Fig. 483.

If the needles are arranged as in Fig. 490, the third and 
fourth divisions in each "bar" would correspond with the first row of needles, and the seventh and eighth divisions

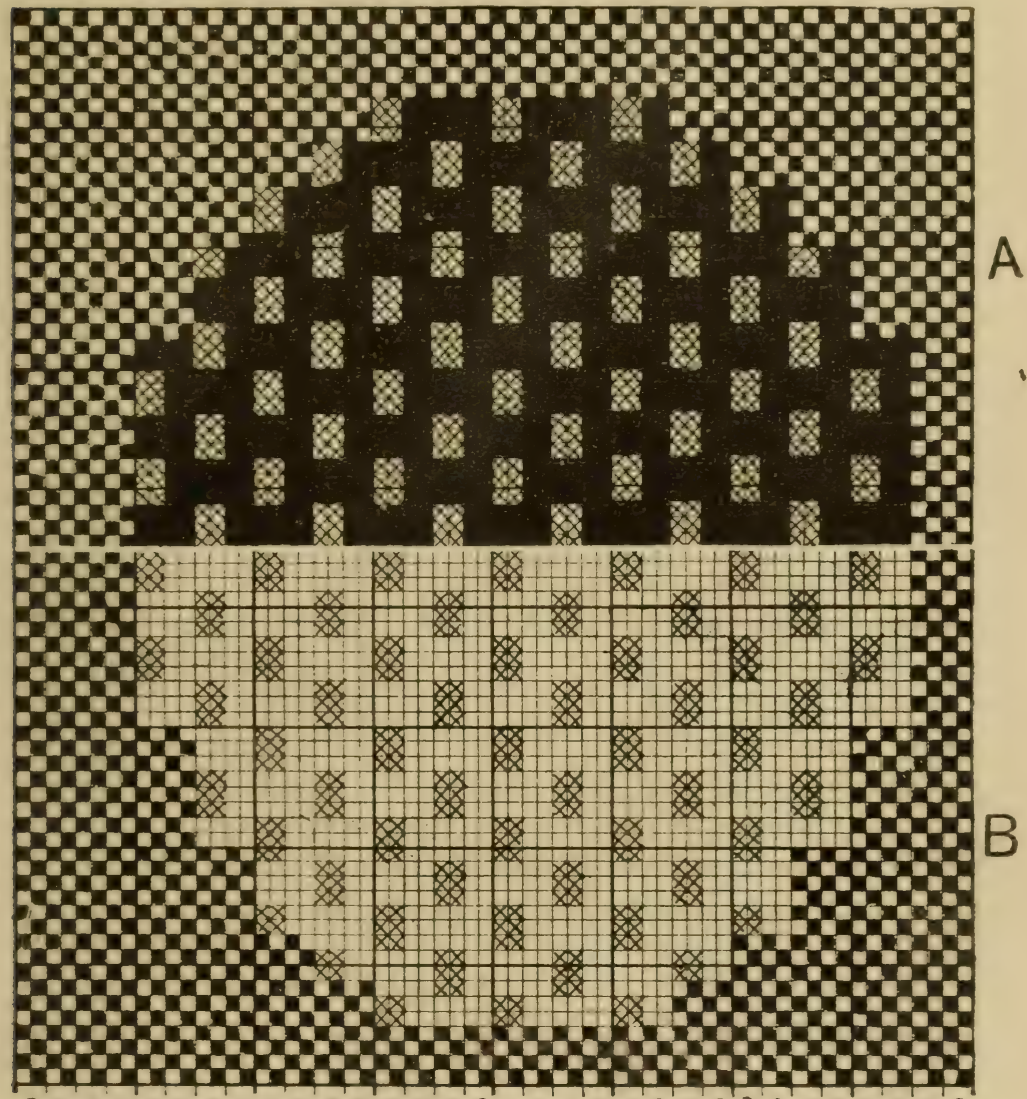

DSDSOSDSDSDSDSDSDSDSDSDSDSDSDSDS

Fig. 495.-Alternative methods (A and B) of preparing an applied design for a top-doup harness governed by the leno Jacquard machine represented in Fig. 489. By adopting the method as indicated at B the work of the designer is facilitated. Also, the card-cutter punches holes for blank squares, and misses filled squares, when "reading-off" the design.

would correspond with the tenth row, in respect of shaded squares only. This arrangement is probably the most convenient one for a card-cutter, inasmuch as he would have the 
advantage of employing both thumbs when cutting for the doup and easer harnesses. That circumstance would involve less risk of error than if the card-cutter employed the little finger and thumb of the left hand, as would be necessary when cutting for the ninth and tenth rows of needles for the arrangement shown in Figs. 488 and 489.

With the arrangement of needles as represented in Fig. 491, which is perhaps the least convenient one for a card-cutter, the third and fourth divisions in each "bar" of design paper correspond with the fifth row of needles, and the seventh and eighth divisions with the tenth row, when cutting for the shaded squares for doup and easer hooks.

$\$ 148$. When preparing an applied leno brocade design, it is necessary to exercise great care at the margin of the figure and ground to ensure a good and clear outline where they meet. This may be best achieved by adhering to a uniform system of allowing fellow standard and doup warp threads to form either an open or a cross shed, in the leno portions of the fabric, for not less than two picks of weft immediately before and after weaving plain calico cloth in the ground portions, as observed in Fig. 494.

For fabrics of coarse textures, it may suffice to allow only one pick of weft to be inserted in the cross and open sheds at the extreme margin of the leno portions of the fabric. By adopting this simple expedient, warp threads are partially relieved of the tensile strain that would arise by causing them to immediately and abruptly change from weaving in pairs in the leno portions, to the plain calico weave in other portions of the fabric. This precept is not always observed by designers for leno brocade fabrics, as an inspection of such fabrics sometimes reveals; still, it is advisable to obseive it as far as may be practicable. Also, if floating or brocade figuring is introduced as an additional means of embellishment in a leno brocade fabric, it should always be well separated from the leno portions by a good margin of the plain calico weave; otherwise the margin where the two portions meet will present a very straggling and unsatisfactory outline.

Another important consideration to observe in the prepara- 
tion of leno brocade designs, is the free and uniform distribution of the figure and ground portions of the design. This necessity arises in consequence of the greater rate of contraction by warp threads, and therefore a greater degree of tensile strain upon those threads, when weaving the leno portions than when weaving the brocade portions of the fabric. This disparity of contraction results from warp threads bending or deviating from a straight course in a greater measure in the leno than in the brocade portions of the fabric. Hence, it is necessary to distribute those portions as evenly as possible over the available surface, and so ensure a uniform rate of contraction by warp threads, and, therefore, a corresponding degree of tensile strain upon them uniformly. 


\section{CHAPTER XI.}

TISSUE, LAPPET AND SWIVEL FIGURED FABRICS; ALSO ONDULÉ FABRICS.

$\$ 149$. The terms "tissue," "lappet," "swivel," "ondulé " and "looped" fabrics are used to designate certain varieties of woven fabrics, each of which is distinguished by distinctive characteristic features. Fabrics of these descriptions do not, as a rule, embody any special feature of constructive design, but they consist chiefly of light and simple textures which, during weaving, are embellished with a scheme of figuring developed by one or other of the methods of figuring as specified. Since these fabrics, therefore, owe their chief interest to the special mechanical devices employed in their production, it is proposed to describe the salient features of those fabrics, in conjunction with such descriptions of the mechanical devices employed in their production as will enable readers the more readily and intelligently to comprehend how the scheme of figuring is developed upon them. The chief advantages of embellishing textile fabrics by means of tissue, lappet and swivel figuring are to produce decorative effects without materially increasing either the bulk or weight of the fabric, and also to produce such decorative effects with the minimum amount of material.

\section{Tissue-Figured Fabrics.}

Tissue figuring is a method of weaving employed chiefly in the production of light cotton muslin textures intended for use as window curtains, and of which an example is illustrated in Fig. 496. Fabrics of this class are frequently embellished with elaborate Jacquard designs of great beauty. These designs are developed by means of a series of extra picks of weft which 
TISSUE, LAPPET AND SWIVEL FIGLRED FABRIC'S. 307

interweave with a foundation texture either of the plain calico weave, or else, as in "Madras" muslin, upon a texture of gauze produced on a principle of cross weaving, whereby the extra figuring weft is firmly secured to the principal or foundation texture. The figuring weft is both softer and coarser than that employed for the foundation texture of the fabric, with the special object of imparting prominence to the figure. These two series of picks may be inserted in the order either of two ground and two figuring picks alternately; or else one pick

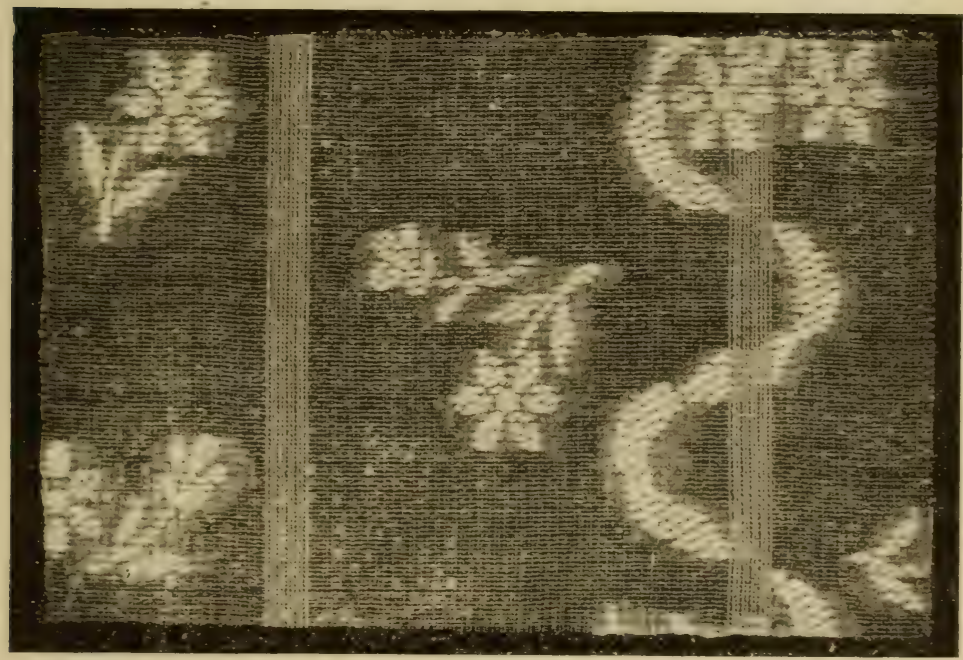

FrG. 496.-Light muslin fabric embellished by tissue figuring.

of each alternately; or with one ground pick and two figuring picks alternately. The two-and-two system of picking is, however, more economical, as it may be accomplished in a loom equipped with an ordinary picking motion, and with two shuttle boxes at only one end of the sley. If either of the other two systems of picking were adopted, they would involve the use of a loom provided with a special picking motion, to permit of picking two shuttles in succession from each end of the sley, which latter would therefore require to be furnished with two shuttle boxes at each end. 
The example of tissue weaving, illustrated in Fig. 496, has a foundation texture of muslin of the plain or tabby weave, woven with two ground and two figuring picks alternately. In fabrics of this class, the extra figuring weft interweaves only with warp threads where it is required to produce figure, and (when in the loom) floats loosely above all warp threads in the ground portion of the fabric, from which it is subsequently cut away as superfluous material. By weaving these fabrics face downward the work of shedding is made considerably easier, as all warp

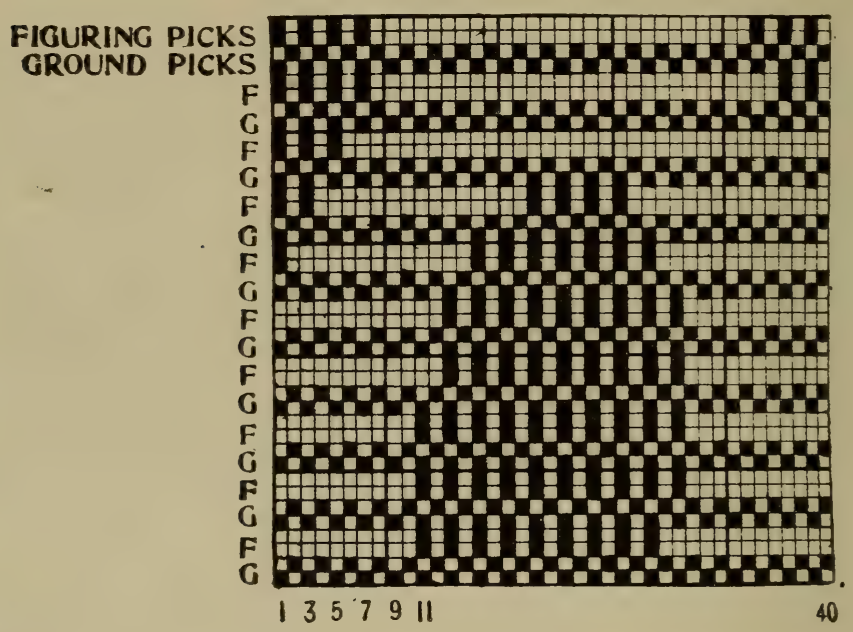

FIG. 497.-Method of preparing an applied design for tissue figuring, as represented in Fig. 496.

threads in the ground portion are left down en masse. A portion of the design showing the method of interweaving figuring weft with the foundation texture is given in Fig. 497, in which it will be seen that the first two and subsequent alternate pairs of picks (which are fine ground picks) interweave separately with warp threads on the tabby (plain calico) principle throughout, and thereby develop a perfect texture, irrespective of figuring weft; whereas, the third and fourth, and subsequent alternate pairs of picks (which are coarse figuring picks), interweave with warp threads, only where they are required to be 
retained in the fabric for figuring purposes, and float above intervening warp threads. In the figure portion, figuring picks lie together in pairs (although inserted separately during wearing) between odd-numbered and even-numbered warp threads by which they are firmly secured to the principal texture.

On examining the design it will be observed that figuring picks always lie between the same series of warp threads; that is, say, with odd-numbered threads above, and even-numbered threads below them. This circumstance permits of a more economical production of these fabrics, as alternate warp threads only require to be governed by means of a Jacquard machine, and intermediate warp threads by means of a heald. Thus a Jacquard machine with 408 hooks would serve to produce a design extending over any number of warp threads up to 816. Also, since two figuring picks are inserted between the same series of warp threads, only one pattern card would be required for four picks of weft, provided the card cylinder and griffes were controlled independently. For example, when the first ground pick is inserted, the heald only is raised; when the second ground pick is inserted, the griffes of the Jacquard machine are raised with the card cylinder out : and for the third and fourth picks, which are figuring picks, the griffes ascend and take up only such hooks as govern alternate warp threads in the figure portion of the fabric, in accordance with the selection made by the pattern card for those picks.

Not only does the foregoing system effect a considerable saring in the cost of harness threads, pattern cards and card cutting, but it also greatly facilitates the preparation of designs, as the latter may be prepared en bloc, instead of with the actual order of interlacing of each thread of warp and weft being indicated as in Fig. 497. Therefore, since only alternate warp threads are governed by the Jacquard machine, and only one pattern card is necessary for four picks, the counts of design paper required for a design is in the ratio of warp threads per inch, divided by two, to the total pichs per inch, divided by four. Thus, assuming there are to be forty-eight warp threads and eighty-four picks of wett per inch in the 
finished fabric, the required counts of design paper (for a 400's Jacquard machine with eight rows of hooks from front to back) would be in the ratio of $(48 \div 2)=24$ to $(84 \div 4)=21$, or ruled with eight squares by seven squares in each bar.

\section{"Madras" Muslin Fabrics.}

$\$ 150$. Fig. 498 is a diagram showing the structure of that variety of tissue-figured fabrics known as "Madras" muslin,

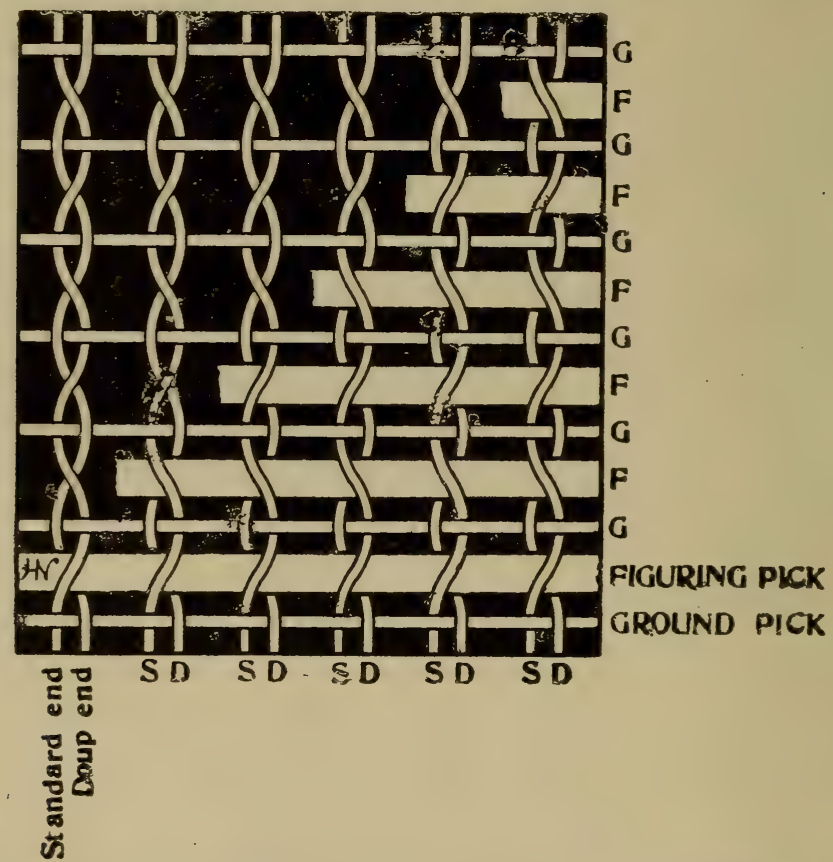

FIG. 498.-Graphic diagram illustrating the structure of "Madras" muslin fabrics.

of which the foundation texture is of a gauze or leno structure produced by the principle of cross weaving. The diagram represents a fabric in which ground and figuring picks are inserted alternately, thereby requiring for its production a loom having a "pick-and-pick" picking motion, and with two shuttle boxes at each end of the loom sley.

The peculiagr partial crossing of warp threads in these 
fabrics is obtained by the use of a special kind of reed known as a "gauze" reed, which is auxiliary to the ordinary beatingup reed carried by the sley. A gauze reed, as illustrated in Fig. 499, is constructed with wide dents or divisions $A$, in each of which there is fixed, centrally, a short pointed reed wire $\mathrm{B}$, secured to the bottom rib $\mathrm{C}$, and extending about halfway between the bottom and top ribs. The shorter reed wires
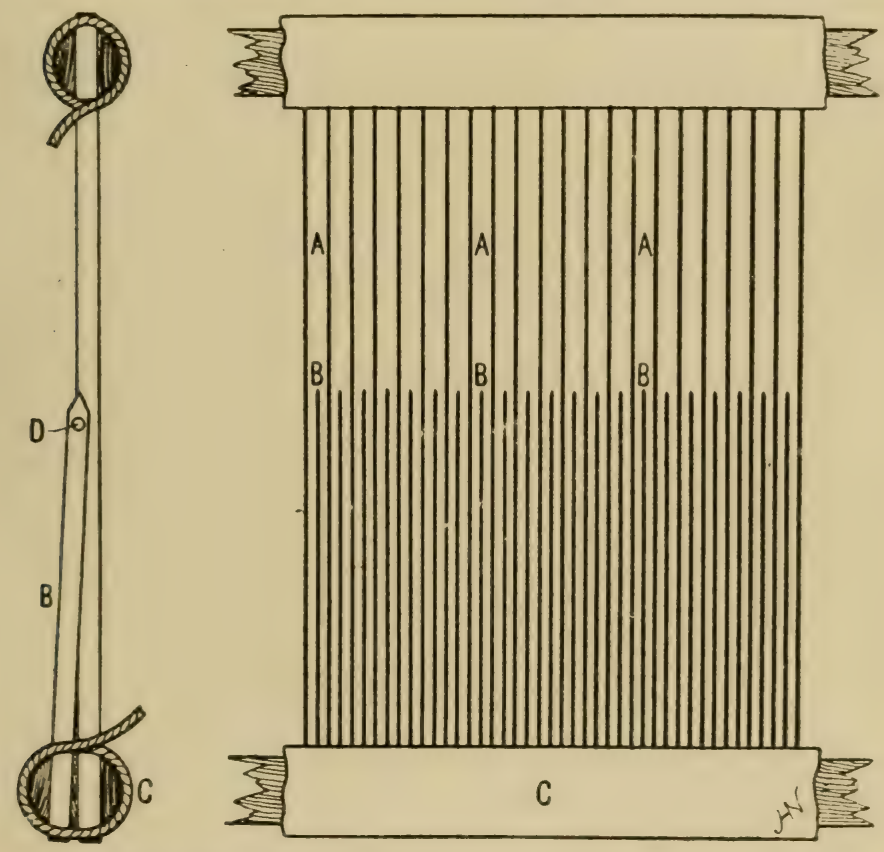

FrG. 499.-Gauze reed, employed in weaving "Madras" muslin fabrics.

are each provided with an eye $\mathrm{D}$, near the top, for the reception of alternate warp threads termed "doup" threads. The intermediate warp threads, termed "standard" threads, which are controlled by the Jacquard harness, pass separately between the wide dents of the reed. A "doup" and a "standard" thread, contained in the same dent of the gauze reed, are also passed together hetween the same dent of the 
ordinary reed to permit of their crossing each other. When in the loom, a gauze reed is placed a little in front of the Jacquard figuring harness, as shown at E (Fig. 500), and is raised to form a warp shed for the insertion of ground picks only. Its function, therefore, is analogous to that of a "doup" heald in an ordinary gauze or leno loom. Previous to the ascent of the gauze reed, the Jacquard harness is moved side-

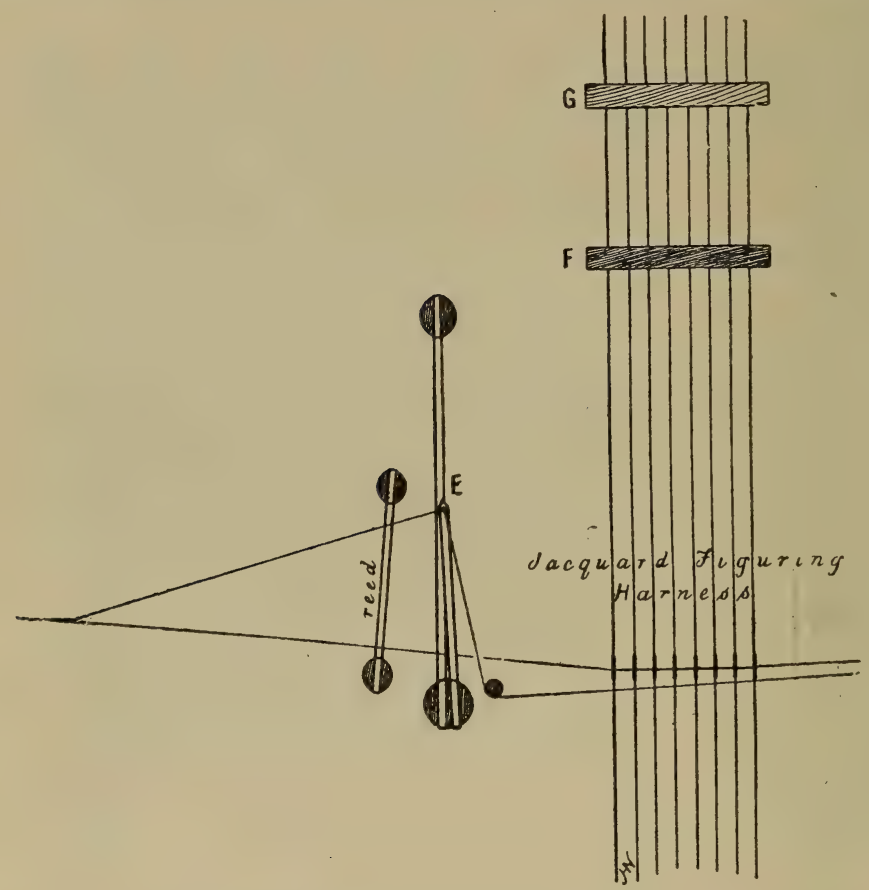

FIG. 500.-Part sectional end elevation of a loom for weaving

"Madras" muslin fabrics.

ways for a short distance, first to the right (when facing the loom) and then to the left, for consecutive ground picks, thereby placing "standard" warp threads on opposite sides of "doup" warp threads for the purpose of crossing and recrossing them. The lateral side movement of the harness threads, and the consequent movement of "standard" warp threads which they control, is accomplished by means of 
an auxiliary comber-board $F$, situated a few inches below the ordinary comber-board G. The auxiliary comber-board is rirtually a coarse wire comb of which the teeth are crossed at right angles by three or four wires, so as to form compartments for the reception of several mounting threads, to prevent the latter from swinging. Comber-board $\mathrm{F}$ receives its lateral movement in one direction by means of a lever connected to it at one end, and actuated by a cam; whilst its return movement is effected by means of a spring attached to the opposite end of the comber-board, and which is constantly pulling against the lever.

On referring to Fig. 498 it will be seen that all "doup" warp threads only are raised for the insertion of ground picks, and that "standard" warp threads are raised en masse in the figure portion only, and left down en masse in the ground portion for the insertion of figuring picks. Thus, figuring weft lies between "doup" and "standard" warp threads in the figure portion, and floats loosely above all warp threads in the ground portion of the fabric, from whence it is subsequently cut away as waste material.

\section{"Madras" Muslin Fabrics with Two and More Colours of Figuring Weft.}

$\$ 151$. "Madras" muslin fabrics are sometimes woven with two different colours of figuring weft to increase their decorative effect. In the production of this variety, three shuttles are required, namely, one to insert the fine ground picks, and one each to insert the respective figuring picks of coloured weft. The three shuttles are picked across the loom in succession; and although it may not at first appear to be practicable, it will, upon reflection, become manifest that a loom furnished with an ordinary picking motion, and two shuttle boxes at each end of the sley, will enable that order of picking to be adopted without haring recourse to a more complex and costly type of loom equipped with a "pick-and-pick" motion, and three shuttle boxes at each end of the sley.

A pick of each colour of figuring weft is inserted after every ground pick; and the different colours of weft may be 
displayed in any manner according to the desired scheme of decoration. For example, each colour of weft may be either displayed alone, or else the picks of each colour of weft may be inserted alternately with each other in the same part of the fabric, in order to produce a chintz or mingled effect by blending the two colours together. In the figure portions that are developed in such a manner, alternate standard warp threads only are raised in those parts for picks of one colour, and intermediate standard warp threads only for picks of the other colour. In all other respects, this variety of "Madras" muslin is similar to the two-shuttle variety described in $\$ 150$. Some "Madras" muslins contain as many as three and four different colours of figuring weft, which may be displayed either independently, or in any combination with each other, as desired.

\section{Lappet-Figured Fabrics.}

$\$ 152$. Lappet figuring is usually confined to the ornamentation of light muslin textures of cotton, and sometimes of silk, of the plain or calico weave; and less frequently it is employed in combination with gauze or leno and other woven effects. It consists of the development of figured effects produced by a more or less zigzag arrangement of extra warp threads, withdrawn from one or more than one auxiliary small warp beam. These extra warp threads are wrought into the foundation texture without interweaving with warp threads, and are permanently held in position by passing underneath picks of weft (when cloth is viewed obversely). The figuring warp threads, termed "whip" threads, are thereby made to lie in the same direction as picks of weft, which float quite freely on the face side of cloth only, between the points of their intersection, as clearly indicated in the accompanying photographic reproductions of lappet-figured fabrics. These characteristics are specially emphasised because they constitute the essential principles of lappet figuring, which sometimes bears a close resemblance to swivel figuring as described subsequently in $\$ 157$; and when once properly understood, they enable the difference between lappet and swivel figuring to be readily discriminated. 
Lappet figuring is confined to the production of comparatively simple decoration, as that system of figuring is incapable of producing elaborate designs such as are frequently met with in tissue-figured and swivel-figured fabrics that are usually produced by means of a Jacquard machine. The examples of cloth represented in Figs. 502 to 512 will serve, better than any verbal description, to indicate the general character and

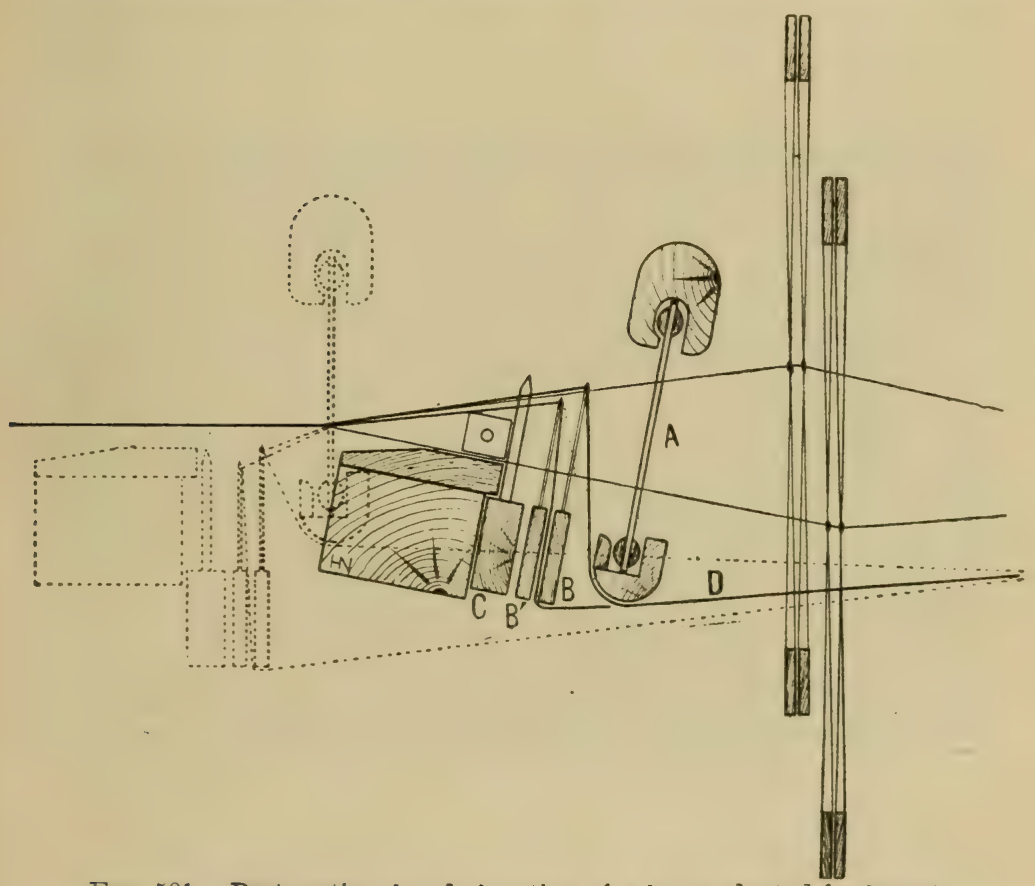

FIG. 501.-Part sectional end elevation of a loom adapted for lappet figuring.

scope of lappet figuring; whilst the following brief description of the essential features of a lappet loom will enable the production of these fabrics to be more easily comprehended.

$\$ 153$. Lappet looms differ in details of construction with different loom makers; but there are certain essential and incidental parts that are common to all looms of this type. These parts, which are represented in part sectional elevation 
in Fig. 501, comprise one or more needle-frames B, B1 situated between a reed $\mathrm{A}$ of ordinary construction, and a false reed or pin-stave C, all of which parts are supported by, and oscillate with, the loom sley, as indicated by representing those parts at their rear and forward extremities of their movement, by means of full and dotted lines respectively. The reed A, which is situated several inches to the rear of the position a reed usually occupies, serves the usual functions of maintaining an even distribution of warp threads over the required width of cloth, and of beating-up picks of weft. The pin-stave $\mathrm{C}$ is

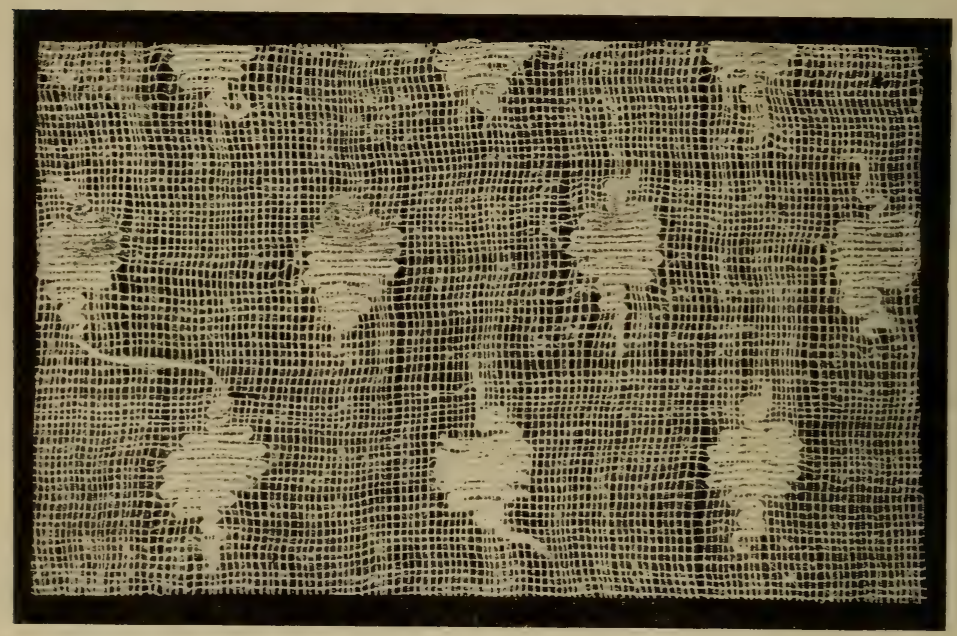

FIG. 502.- Simple spot lappet figuring developed by one needle-frame.

a stave containing a number of sharply-pointed pins, placed vertically"at intervals of about an inch to an inch and a quarter. This is placed immediately behind the rear edge of the shuttle race-board, and rises and falls alternately in unison with the backward and forward strokes of the sley. Its function is to serve as a guide for the shuttle in its passage through the warp sheds, after which it disappears below warp threads and cloth, as the sley advances to beat up the picks of weft.

The needle-frames $\mathrm{B}, \mathrm{B}^{1}$ are narrow staves, each containing a series of sharply-pointed needles placed vertically, and having 
eyes formed near the top, for the reception of whip or figuring threads, which they control. In addition to their oscillation with the sley, needle-frames receive a reciprocal compound movement both vertically and laterally. These movements synchronise with the backward and forward strokes of the sley respectively. Thus, as the sley recedes, and just before picking takes place, needles are raised to insert their whip threads between the ordinary warp threads, to take their place with the upper half of the warp shed. Then, after each pick of
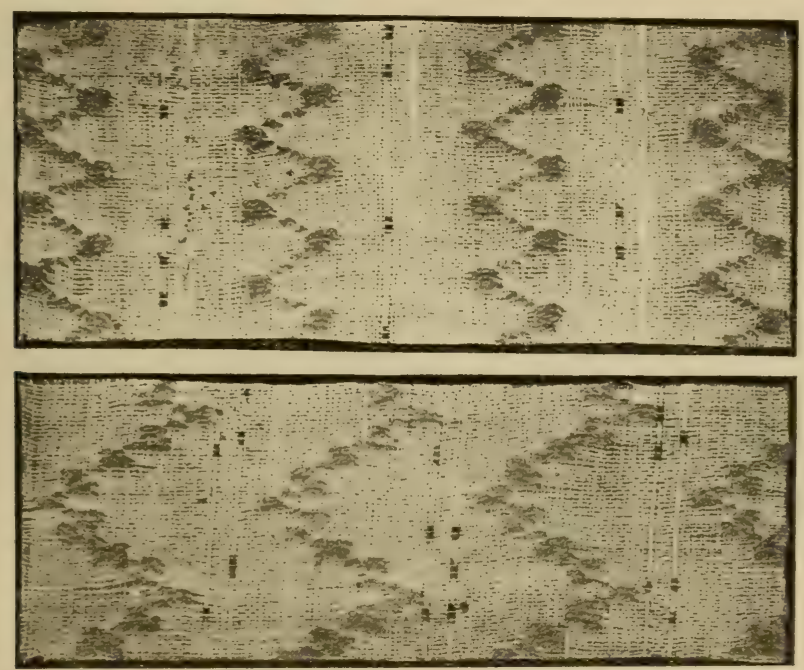

FiG. 503.-Lappet figuring developed by one needle-frame.

weft is inserted in the shed, the needles descend, as the sley advances to beat up the picks of weft, which, by passing underneath whip threads, prevent the withdrawal of these as needles descend, and retain them at the points at which they were inserted between ordinary warp threads. When the needles have descended a sufficient distance to be quite clear of warp threads and cloth, they may be moved laterally, in either direction, for the purpose of passing figuring threads from side to side of the figure, and placing them in the required positions, 
according to the pattern, ready for insertion into the warp shed for the next pick of weft.

The lateral movement of needle-frames may be effected in various ways, either by means of lattices furnished with pegs of different lengths, varying according to the amount of movement required; or by means of shaped pattern or "lappet" wheels, of which there are several varieties. The device known as the "Scotch" lappet motion is that which is more generally adapted to lappet looms. This motion consists essentially of a wooden pattern wheel or disc, mounted freely

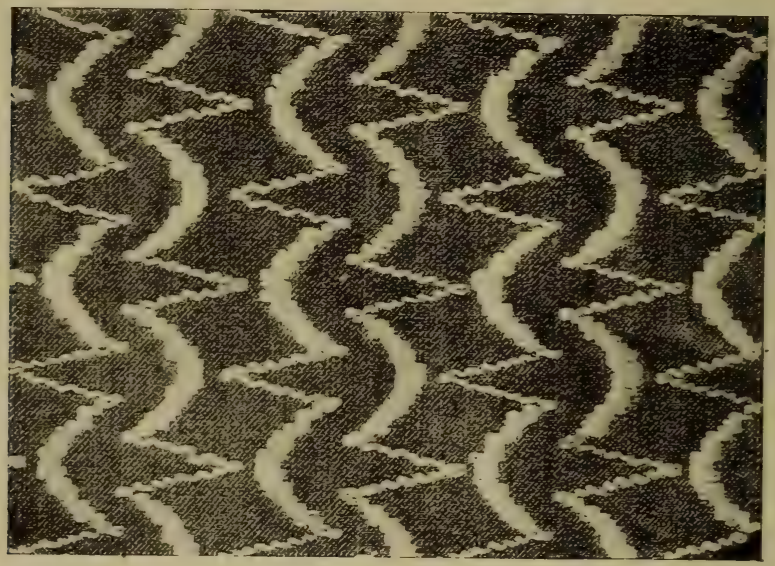

FIG. 504.-Lappet figuring dereloped by two needle-frames.

on a stud at one end of the loom, and having such number of irregularly stepped concentric grooves of uniform depth cut into the face side as corresponds with the number of needleframes to be actuated by it. The configuration of each groove is in accordance with the particular movement to be imparted to the respective needle-frames, for the development of the required pattern. Each groove receives and acts upon a smail bowl or runner mounted upon a short pin or stud fixed in an extension of each needle-frame. These extensions pass horizontally in front of the wheel, so as to place the axes of the runners and pattern wheel in exactly the same horizontal 
plane. The rim of the pattern wheel is also formed with such number of saw or ratchet teeth as corresponds with the number of picks (or half that number, according to special circumstances) to be inserted in each repeat of the pattern. It will now become manifest that by rotating the pattern wheel intermittently one tooth for each pick (or for every two picks) the needle-frames will be moved sideways in accordance with the configuration of the respective grooves, and thereby cause the whip threads to assume a more or less zigzag course, and float freely between the extreme edges of figure without inter-

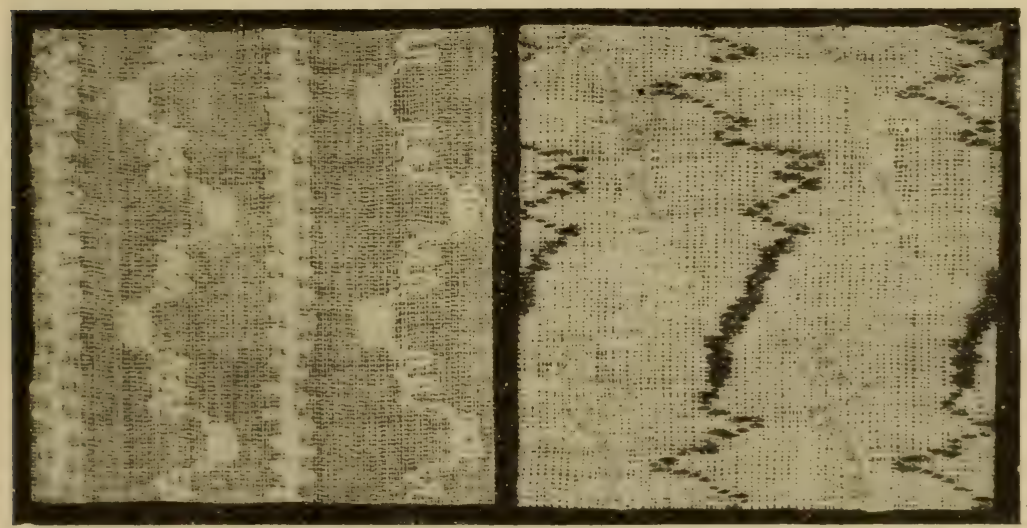

FIG. 505.-Two-frame lappet figurhng FIG. 507.-Two-frame lappet figuring with design, Fig. 506. with design, Fig. 508.

mediate intersections. A separate needle-frame is required for each distinct order of interwearing the figuring threads. If all figuring threads are required to interweave in the same manner, only one needle-frame, operated by one groove in the lappet wheel, is required; but, if figuring threads are required to interweave in two or more different orders, then a corresponding number of needle-frames operated by a figuring wheel constructed with the same number of grooves will be required. It is rarely, howerer, that more than three needleframes are employed in the same loom.

Since needle-frames are situated below warp threads, it 
follows that the cloth will be woven face downward, and that the pattern is thereby obscured from the observation of a weaver. This circumstance is obviously to the disadvantage of a weaver, who is unable to readily detect any imperfection in the pattern that may arise during weaving: hence, in some lappet looms needle-frames are situated above warp threads, with the needles inverted, so as to weave the cloth face upward, and with the pattern in full view of a weaver. With this arrangement, however, the small warp beams containing the figuring threads are conveniently placed above the healds; and as those threads descend in front to their respective needle eyes, they form an obstruction to a weaver when piecing and drawing in broken warp threads. Also, in consequence of inserting "whip" threads from above, instead of from below, regular warp threads, during shedding, they are more liable to cause the lower half of the warp shed to become uneven, and thereby impede the free passage of a shuttle during picking.

$\$$ 154. Fig. 502 illustrates an example of a simple spot lappet figuring produced by means of one needle-frame which is governed in such a manner that, after inserting one horizontal row of spots, it becomes inoperative for an interval equal to ten picks of weft, and then inserts the spots of the next row so that they occupy an intermediate position in relation to those of the preceding and succeeding rows of spots, as indicated by the unsevered trailing whip thread observed in the photograph. Two other examples of lappet figuring, developed by only one needle-frame, are indicated in Fig. 503, which illustrate two simple zigzag or wavy stripe effects produced by the continuous operation of the respective needleframes by which they were obtained.

Three examples of two-frame lappet figuring are illustrated in Figs. 504, 505 and 507. The ingenious effect represented in Fig. 504 is obtained simply by reversing two similar stripes in order to produce a "drop turn-over" design having the appearance of an "all-over" pattern. The neat effect represented in Fig. 505 is obtained by alternating a straight and a zigzag stripe, as indicated; whilst that illustrated in Fig. 507 is produced by reversing and dropping two irregular wavy 
TISS(F, TAPPET ANI) SWTVEL FIGURE1) FABRI(S. 321

stripes that are developed by white and gold-coloured silk whip threads respectively, to impart additional attractiveness to the fabric.

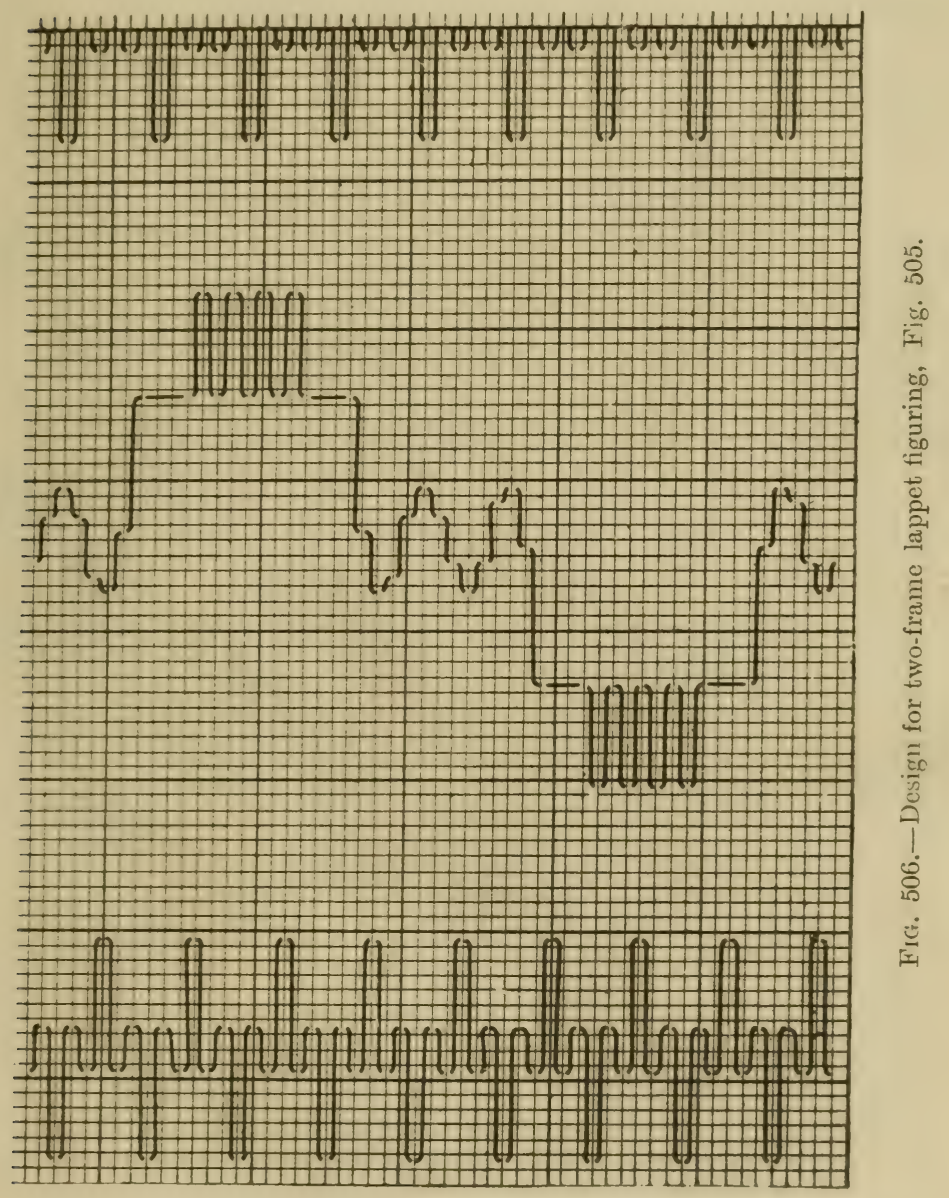

A good method of indicating, on squared paper, a design for lappet figuring, is demonstrated in Figs. 506 and 1508, which are the designs for the lappet fabrics illustrated in Figs. 505 and 507 respectively, and of which designs the vertical and 


\section{$32: 2$}

GRAMMAR OF TEXTILE DESIGN.

horizontal lines on the squared design paper, and not the spaces between those lines, represent the warp and weft threads

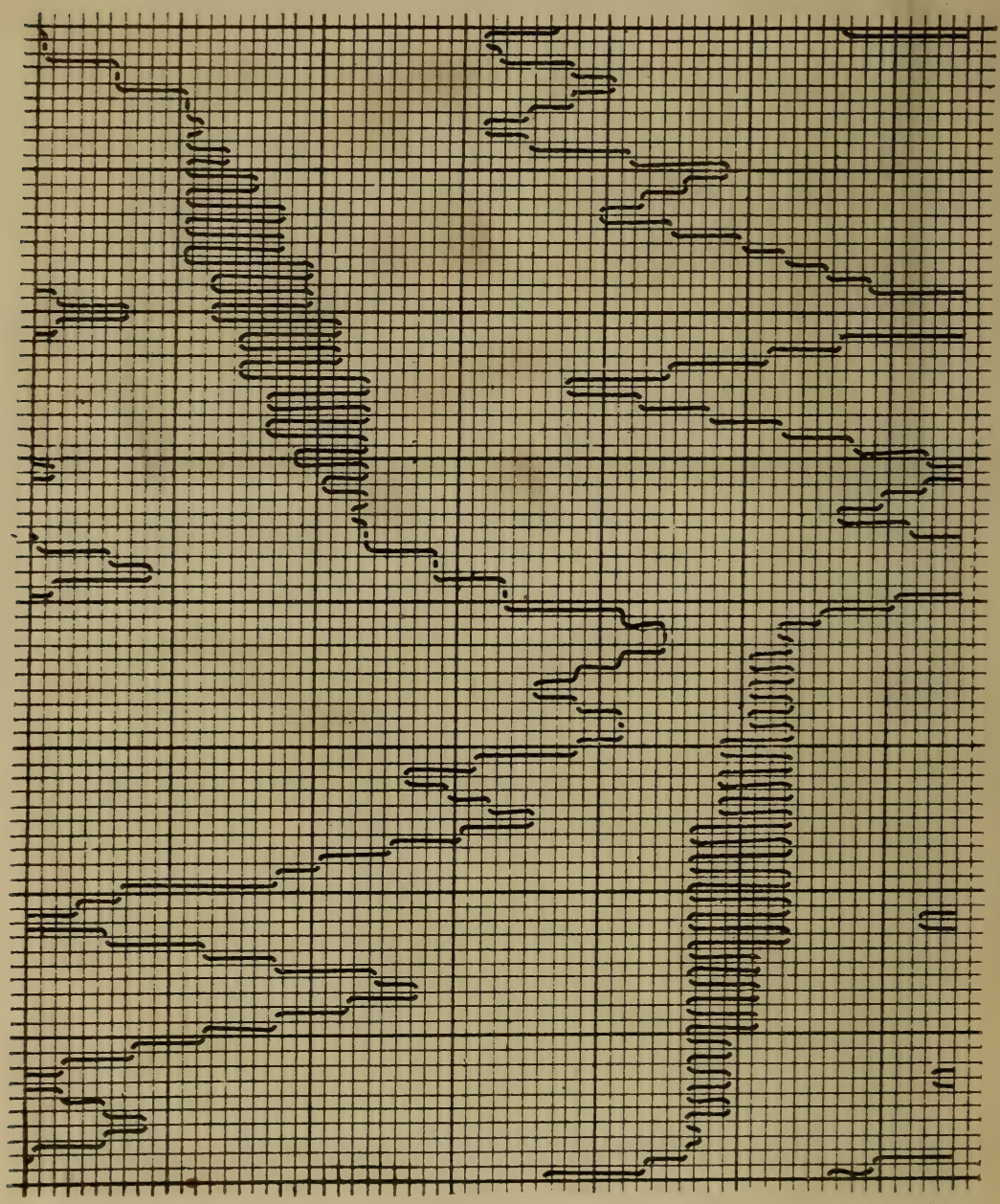

FrG. 508.-Design for two-frame lappet figuring, Fig. 507.

respectively of the foundation texture on which the lappet figuring is developed. Instead of indicating the respective whip threads by means of thick lines, however, as observed in 


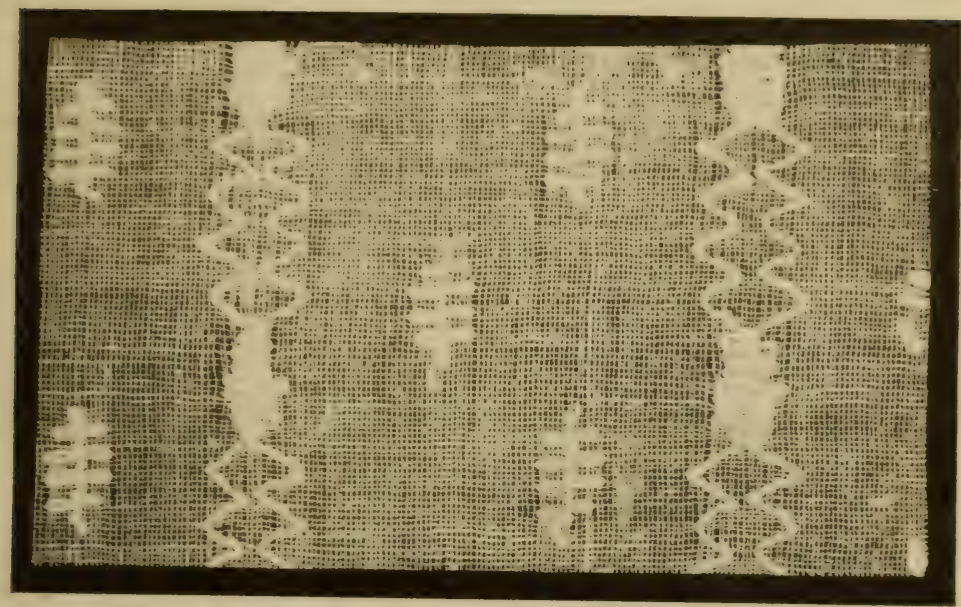

FIG. 509.-Good example of three-frame lappet figuring.

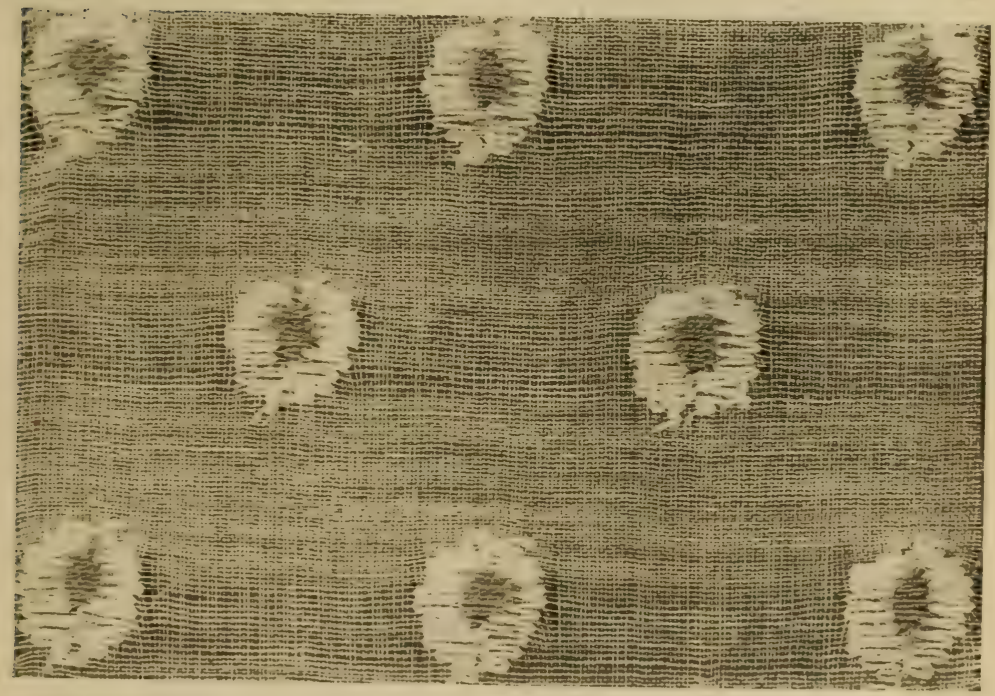

FIG. 510.-Three-frame spot lappet figuring, with centre of spots of yarn of different colour from that of outer portion. 
the accompanying designs, a simpler and easier method is to - fill in the squares of the ruled paper solidly, and thus indicate the lappet figuring as a block pattern in the usual manner of preparing an applied textile design.

Two good examples of lappet figuring produced by three needle-frames are represented in Figs. 509 and 510. The stripes in Fig. 509 are developed by means of two needleframes operated in a similar manner, but in reverse directions

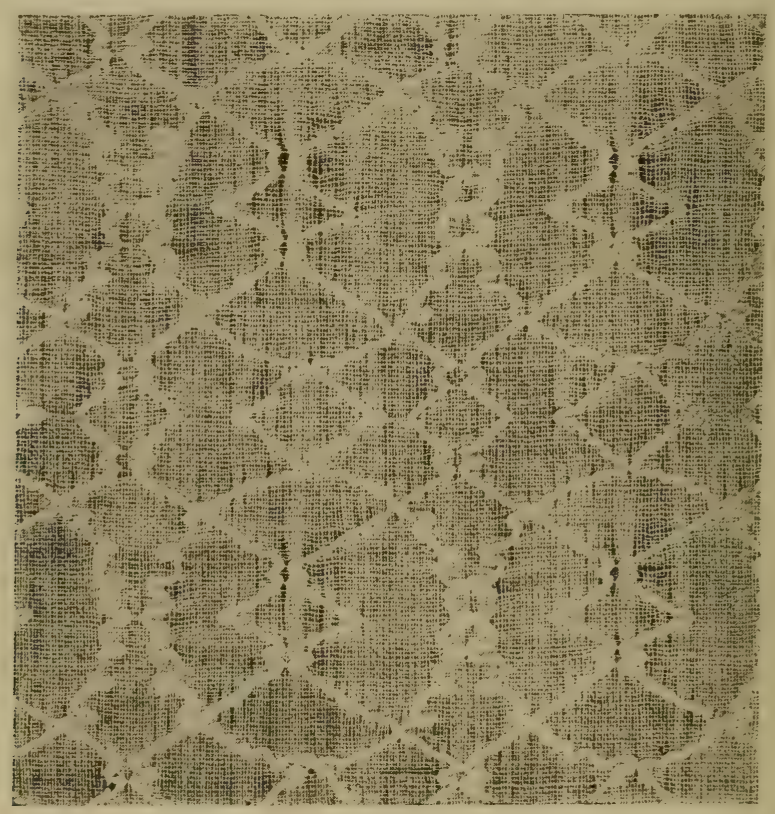

FIG. 511.-Four-frame lappet figuring.

simultaneously and continuously, to produce symmetrical stripes, whereas the detached figures between the stripes are produced by one needle-frame which disposes those figures alternately, as exemplified in spot lappet figuring, Fig. 502.

The example of lappet figuring illustrated in Fig. 510 is of an unusual character, and consists of a white spot enclosing a pale blue spot, with an alternate disposition. The right and left halves of the white spots are developed by means of two separate needle-frames operated in reverse directions simul- 
taneously, whilst the coloured whip threads are controlled by it third needle-frame to develop the inner spots, as indicated.

\section{Cross-thread Lappet Figuring.}

$\$ 155$. A specimen of lappet figuring of a novel and ingenious character is illustrated by Fig. 512. In this example, figuring threads are caused to actually cross each other in reverse directions, and thereby derelop an effect closely resembling that of a net leno produced by the principle of gauze or cross wearing,

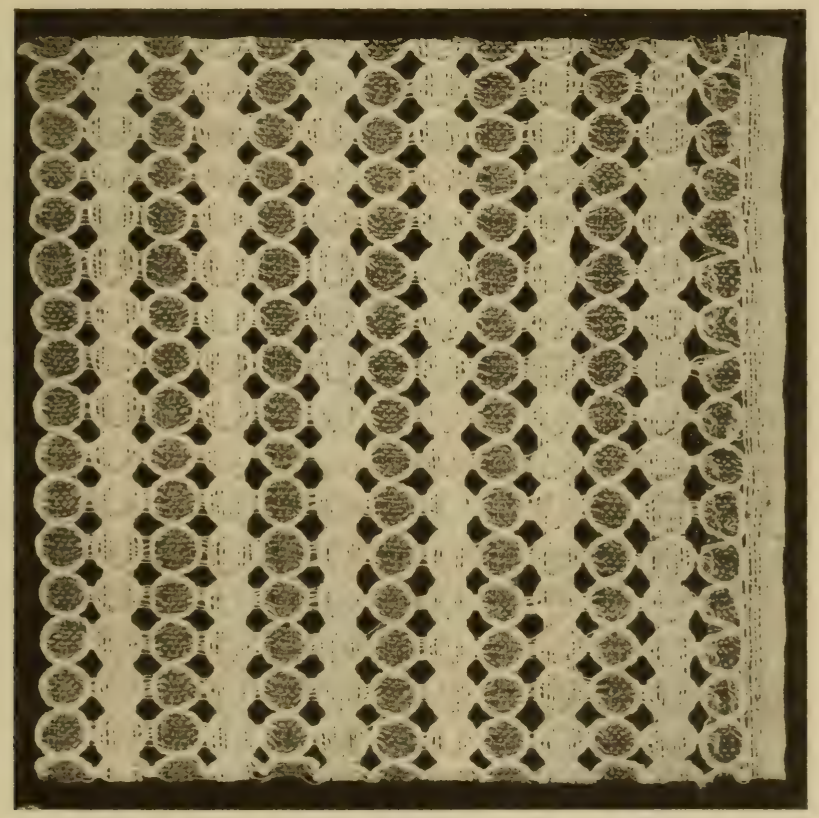

FiG. 512.—Cross-thread lappet figuring developed by two needle-frames

for which it might easily be mistaken. For the production of such effects, it is only necessary to pass the figuring threads of one or more than one needle-frame entirely underneath the needle-frame or frames that are in the rear of those, and thus permit of figuring threads passing or crossing each other. For example, if two needle-frames are employed, the figuring threads 
of the first frame must pass entirely underneath the second frame before passing through their respective needle eyes, as represented in the diagram, Fig. 501. The usual method of passing whip threads through the needle eyes is to take them upward immediately after passing underneath the lower reed case, and then insert them through their respective needle eyes, as indicated in the diagram by means of a dotted line extending from the lower reed case to a needle in the front frame $B^{1}$.

\section{Spot Lappet Figuring.}

$\$ 156$. Some lappet fabrics are woven with detached figures arranged alternately, or otherwise, to distribute them evenly over the surface of cloth, as exemplified in Fig. 502. In the production of such examples it is the usual practice, after weaving each horizontal row of figures, to cause the needleframe or frames to automatically become inoperative until they are required for the next row of figures, when they are "shunted" sideways for the required distance, so as to dispose the figures of alternate rows either midway between those of intermediate rows, or otherwise, in a manner similar to the swivel-figured spots illustrated in Figs. 513 (A), and 513 (B). Now, seeing that lappet fabrics are usually woven face downward (as explained at the end of $\$ 153$ ), it follows that the figuring whip threads will trail loosely between the intervals separating the detached figures produced by the same whip thread. These loose threads are subsequently cut away, thereby leaving a short remnant or tail of thread exposed on the face side of cloth, at both the initial and final extremities of each figure, and causing blemishes of an objectionable character. This disfigurement, however, may be averted by causing the needles to rise in exactly the same position for all picks inserted between the end of one row of figures and the beginning of those in the next row, thereby inserting the respective whip threads uniformly between the same warp threads for those picks. The object of this procedure is to cause the whip threads to trail or lie above the picks of weft between the horizontal rows of figures (when in the loom) and therefore to be on the reverse side or back of cloth, whence they are subsequently cut away, leaving the 
severed tail ends exposed on that side, and thus keeping the face of cloth free from such blemishes. Lappet figures or spots developed in accordance with this practice, and which are known in the trade as "tailless" lappet spots, constitute a superior style of lappet figuring as compared with those constructed in the usual manner.

\section{Swivel-Figured Fabrics.}

$\$ 157$. Swivel figuring is a system of ornamentation by means of extra weft inserted by auxiliary shuttles that are designed expressly for that purpose. This method of figuring is adopted extensively for the decoration of silk fabrics, book-marks, ties, ribbons, ladies' dress materials, and sometimes of light cotton fabrics for similar uses. Being of a more refined and elegant. character than either "tissue" or "lappet" figuring, it is capable of producing decorative designs, figures and pictoriai representations of a floral and scenic description, in a very effective manner. The extra figuring weft is usually of silk, and, unlike "lappet" figuring, it may interweave with the warp threads in any conceivable manner, instead of floating loosely and quite freely between the extreme edges of the figures. Swivel figuring is usually developed upon a foundation texture either of the plain calico or tabby weave, or else that of a simple three-end or four-end twill weave. An example of swivel figuring of a very simple character is illustrated in Fig. 513 which shows both the obverse and reverse of the same fabric at $\mathrm{A}$ and $\mathrm{B}$ respectively.

Swivel figuring is very easily distinguished from "lappet" figuring, by the interlacement of the extra figuring weft with warp threads, between the extreme edges of figure; and also by that weft bending around warp threads when returning at the edges of figure, and not around picks of weft, as in "lappet" figuring. During the operation of weaving, swivel-figured fabrics, like lappet-figured fabrics (produced by means of bottom needle-frames) are produced face downward, as represented in Fig. 513 (B). The swivel shuttles containing the figuring weft are of a very different form to those of ordinary loom shuttles: and, unlike the latter, they are not propelled separately and 
independently across the entire width of the loom; but in the prevailing type of swivel loom several of these special shuttles are carried simultaneously and positively through a corre-

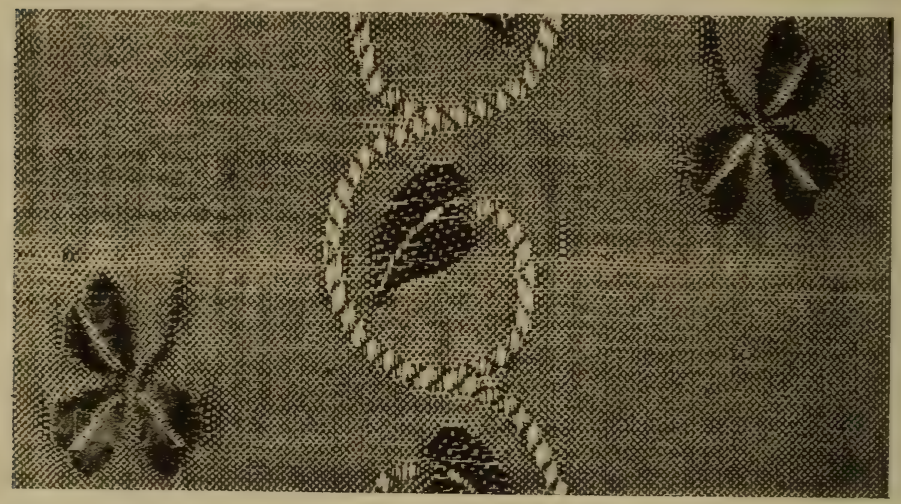

FIG. 513 (A).-Swirel figuring (showing the obrerse of Fig. 513 (B)).

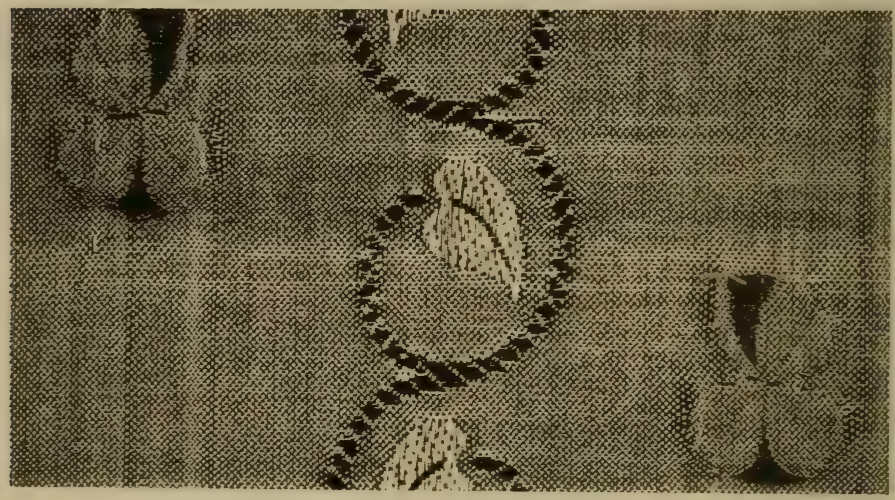

FIG. 513 (B).-Swirel figuring (showing the reverse of Fig. 513 (A)).

sponding number of sectional warp sheds formed at regular intervals apart across the width of cloth, for the purpose of inserting the extra figuring weft for the development of figure.

The swivel shuttles, termed "poppets," are carried by, and move to and fro with, the sley, as the latter oscillates; and 
they mar be arranged in one, two or more horizontal rows, or tiers laceording to the number of colours of figuring weft required for the same finnre) above the warp threads. Each tier mar contain any practicable number of "poppet " shuttles. but with a corresponding number in each row ; and they are supported at regular intervals apart, in a frame termed the "poppet rack," as illustrated in Fig. 514, which represents front and end elerations and a plan of the swivel shuttles, at A, B and C respectively. The poppet rack is depressed and
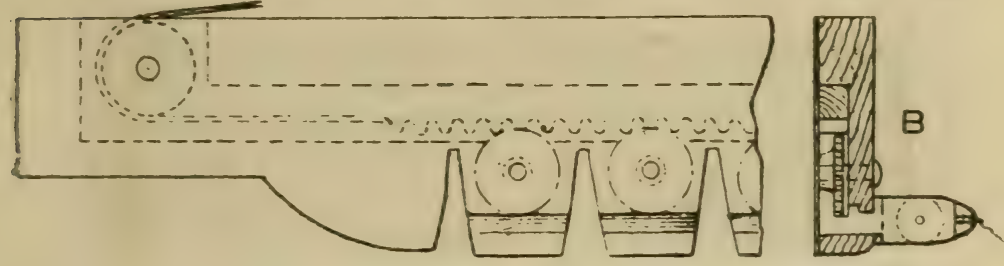

A

B

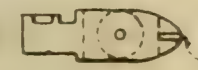

Fig. 511.- " Poppet "rach and shuttles for inserting picks of extra figuring weft in the derelopment of swivel figuring.

raised in a prescribed manner, in order to place any particular tier of "poppets" in position for the latter to enter the sectional warp sheds, and insert picks of figuring weft. The "poppet" shuttles are then passed simultaneously through the sectional warp sheds, and then raised quickly above the cloth to permit of the reed advancing to beat up the short picks of weft. A warp shed is then formed all across the entire warp for the passage of the ordinary shuttle to insert an ordinary pick of weft for the foundation texture; after which, the sectional figuring sheds are again formed for the reception of figuring 
weft from the same or another tier of "poppets," according to the colour of weft required.

\section{Ondulé Fabrics.}

$\$ 153$. The term ondulé is used to distinguish an unusual and interesting variety of woven fabrics in which either warp threads or picks of weft are caused to assume undulating, wavy, or sinuous lines. An example of warp ondulé is represented by Fig. 515, which is reproduced from a specimen of cloth of this:

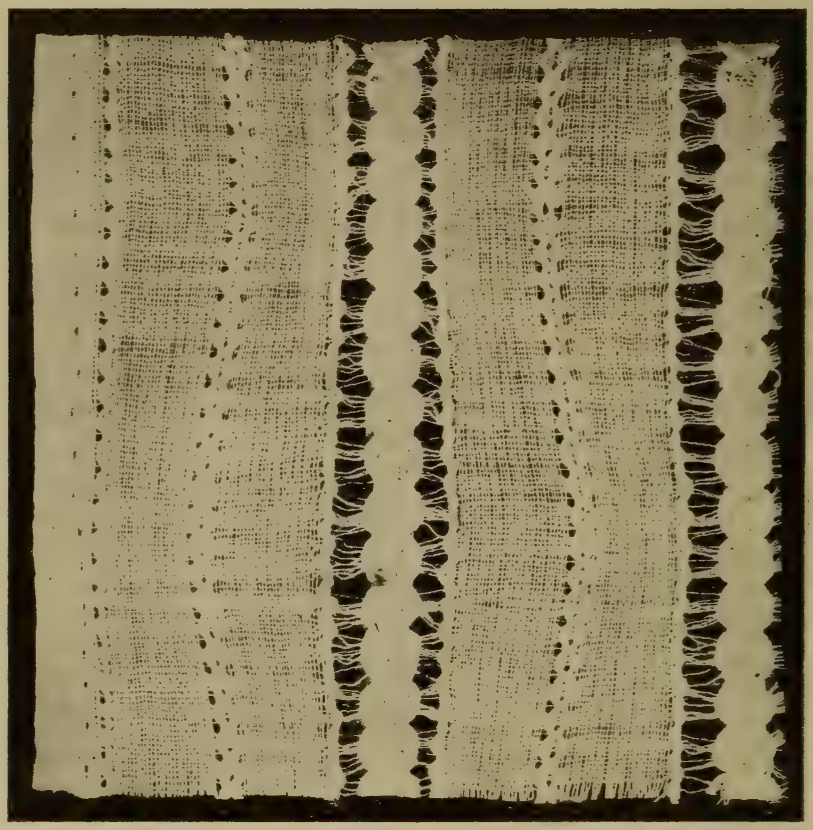

FIG. 515.-Warp ondulé fabric, with net leno and cross-thread lappet figuring.

description. (It may be observed, incidentally, that the specimen here represented also embodies the principles of cross weaving, in combination with lappet figuring, of the special cross-thread variety described in $\$ 155$, and illustrated in Fig. 512 , in which the whip or figuring threads cross each other in reverse direc- 
tions.) Apart from the features just indicated in parentheses, there is nothing of an unusual structural character in the class of fabrics under present consideration, which are generally of simple construction. Their chief interest, therefore, lies in the special mechanical devices required for their production, which are sometimes of a novel and very ingenious character. When the undulations are required in the direction of warp threads, these special devices consist essentially of some means whereby the war'p threads are gradually spread apart and then closed in alternately, to produce contrary sinuous lines running lengthwise of the fabric, which object may be achieved by either of two distinct methods. One method is to employ a reed, of which the dents may be contracted in some parts and expanded in others according to the effect desired; but a more approved

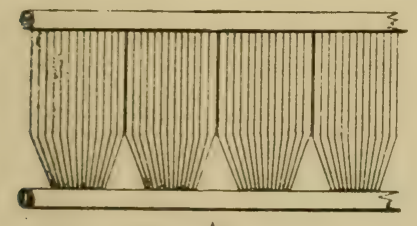

A

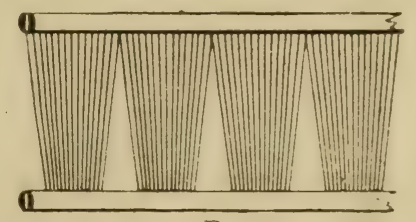

B

FIG. 516.-Ondulé, paquet or fan reeds, for developing warp ondulé effects.

and practical method of obtaining warp ondulé effects, however, is by means of what are variously termed "paquet," "ondule" and inverted "fan" reeds, in which some of the reed wires are permanently inclined at gradually varying angles, and in opposite directions; albeit, they are all in the same vertical plane when viewed from the ends of the reed.

Ondulé or paquet reeds are made in a great variety of forms according to the particular effect desired in cloth; and, during wearing, they are operated by auxiliary mechanism which slowly raises and depresses them alternately, thereby causing warp threads to gradually deviate from their normal straight course, and assume the characteristic undulating or sinuous lines. Three typical varieties of ondulé reeds are represented at A, B, Fig. 516, and at R, Fig. 517. That shown in Fig. 517 is a common variety termed a double or inverted fan reed, for- 


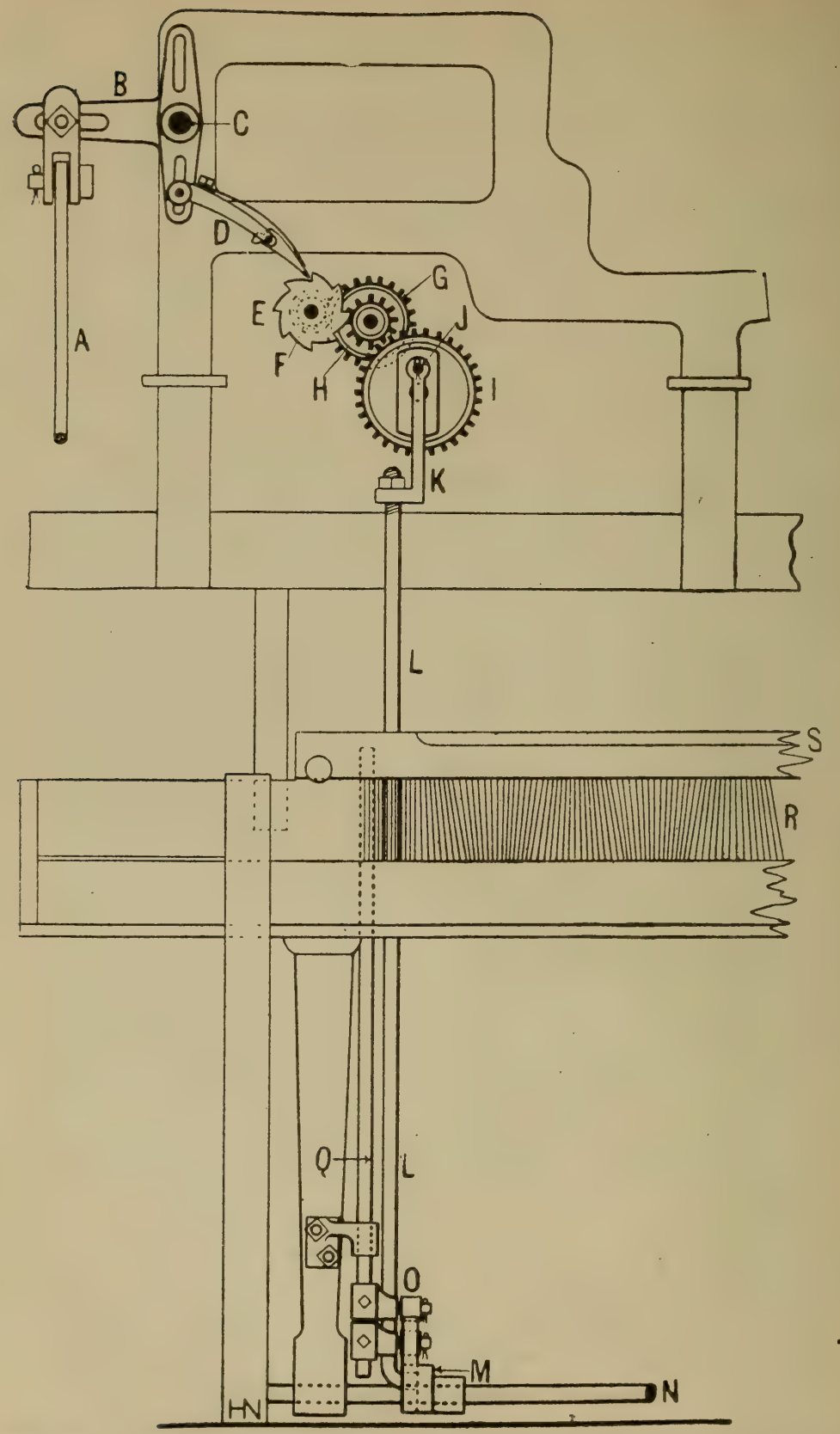

FIG. 517.-Part front elevation of a loom for developing warp ondulé effects by means of reeds of a special type, of which an example is shown at R. (See also Fig. 518.) 


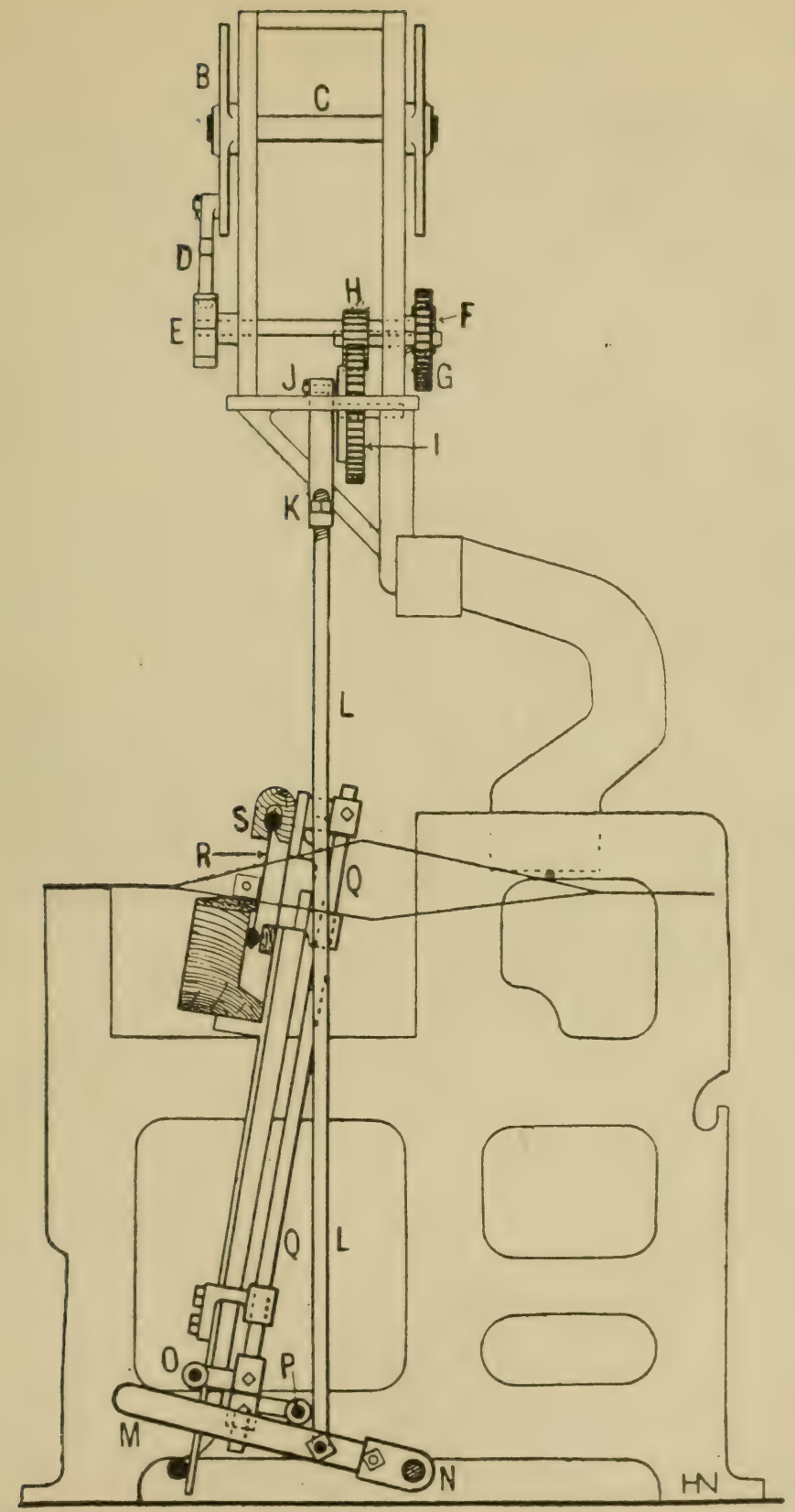

FIG. 518.--End elevation of a loom for developing warp ondulé effects. (See also Fig. 517.) 
the production of regular and contrary sinuous lines; whilst those represented at A, B, Fig. 516, are designed to produce perforations in cloth. The intervals at which the perforations will occur both horizontally and vertically, in cloth, is determined by the distance between the gaps in the reed, and the velocity with which it is reciprocated respectively. Some ondulé or paquet reeds are of a variegated character, with vertical dents alternated with dents inclined at various angles to the right and left, on each side of the vertical dents. They are also made in a variety of other forms according to the effects desired.

$\$ 159$. A loom equipped with a device (invented by Mr. R. Foulds) for the development of ondule effects, is represented by front and end elevations in Figs. 517 and 518 respectively. In this loom, an inverted fan reed $R$ is contained in a frame to which is imparted a slow reciprocal vertical and intermittent movement by means of a dobby acting through the medium of a train of wheels and suitable connections of rods and levers, as indicated in the diagrams. Mounted freely upon a stud or pin $J$, fixed in a wheel $\mathrm{I}$, is a pendant arm $\mathrm{K}$, from which is suspended a long rod $\mathrm{L}$, with its lower end attached to a lever $\mathrm{M}$, fixed to a cross bar $\mathrm{N}$, on the opposite end of which is a duplicate lever M. Resting upon the respective levers M are two rods $Q$, which slide freely in brackets bolted to the sley swords, and therefore oscillate with the sley. The sley cap S, containing the upper ribs of the reed, is secured by means of brackets to the upper ends of rods $Q$, which are supported (at different times) by means of one or other of two arms, secured to their lower ends, and furnished with bowls or runners $\mathrm{O}$, $\mathrm{P}$, that rest upon their respective levers $\mathrm{M}$. It will now be seen, therefore, that as wheel I is slowly and intermittently rotated, levers M will alternately rise and fall with a corresponding velocity, whereby different parts of the reed wires are brought opposite the "fell" of cloth when beating up the weft. Thus, in consequence of the angular disposition of reed wires, warp threads are congested or expanded in width, according as they pass through the congested or expanded extremities of the reed dents respectively. 
The object of employing two runners $\mathrm{O}, \mathrm{P}$, placed at the ends of separate arms extending both forward and rearward of the respective rods $Q$ to which they are secured, is to cause the reed to assume a position midway between its highest and lowest elevations, during the formation of warp sheds, and thereby prevent excessive chafing of warp threads that would otherwise be caused by their bearing hard and rubbing against the reed wires at the upper and lower extremities of a fan reed. This object is achieved in the following manner: As the sley oscillates, runners $\mathrm{O}$ and $\mathrm{P}$ are alternately brought to bear upon different parts of levers M, thereby causing the reed to either descend or ascend to a neutral position as the sley recedes, according to the upward or downward inclination (from the cross bar $\mathrm{N}$ ) of levers $\mathrm{M}$ respectively.

\section{Weft Ondulé Fabrics.}

160. An exceptional and interesting specimen of cloth of the ondule variety is photographically represented by Fig. 463. This example may be described as a weft, or cross-over ondulé effect, since they are picks of weft and not warp threads that assume a wavy character (as indicated by the selvedge, on the left of the fabric). This example is produced from organzine silk warp picked with genapped worsted, to produce a light muslin texture of the plain or calico weave, suitable for a summer dress material.

A weft ondule effect may be developed in a variety of ways, either by means of shaped reeds expressly designed to produce the required effect in cloth; or by applying a constantly varying degree of tension to alternate groups of warp threads. Reeds, styled "Erdmann" reeds (after their inventor), are either constructed of shaped wires placed vertically, so as to appear curved on their front edge when the reed is riewed end-wise; or the wires may be straight and arranged at varying angles, so that if the reed were viewed end-wise, they would appear to cross each other like the letters $\mathrm{V}$ or $\mathbf{X}$; and if riewed in plan, they would form a serpentine or undulating line from end to end of the reed. By slowly and alternately. raising and depressing such reeds, the picks of weft will assume 
varying degrees of undulation according to the velocity with which the reed is vertically reciprocated. By keeping the reed stationary at any given point, picks may be inserted uniformly parallel with each other, either straight, or in more or less undulating lines, as required.

$\leqslant 161$. Weft ondulé effects may be produced in looms furnished with ordinary reeds, by dividing warp threads (im-

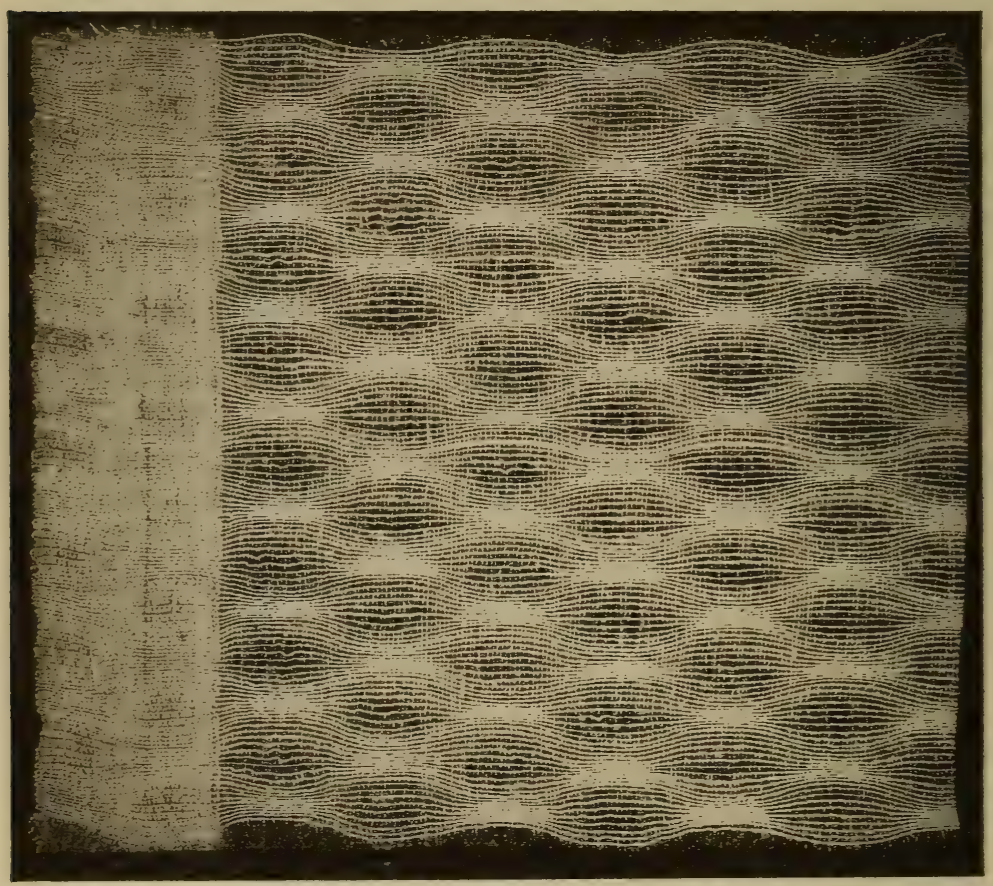

FIG. 519.-Weft ondulé fabric.

mediately after leaving the warp beam) into groups according to the length of wave required, and by passing alternate groups of threads over one bar, and intermediate groups over a second bar. By slowly oscillating both bars in contrary directions (by means of cams, cranks or eccentrics) a gradually varying degree of tension will be imparted to warp threads, whereby the two divisions of threads will be alternately tautened and slackened. This will cause the picks of weft to 
assume more or less wavy lines, according to the disparity between the tension of the two divisions of threads. An alternative, though less practical method of obtaining a similar result would be to wind the two divisions of threads upon separate warp beams, and, by any suitable means, apply varying degrees of resistance to the withdrawal of yarn from them, and thereby alternately increase and diminish the tension of the two divisions of warp threads in a contrary manner. 


\section{CHAPTER XII.}

\section{BROCADE FABRICS.}

$\S 162$. The term "brocade" is commonly employed as a generic term virtually comprising all varieties of woven fabrics of simple texture consisting of only one series each of warp and weft threads, and as distinct from compound types of fabrics of more complex construction. In its more limited application, however, the term "brocade" refers more particularly to the lighter and medium weight textures of silk, linen and cotton fabrics of simple structure and embellished by more or less elaborate Jacquard figuring which may be developed by displaying either warp threads or picks of weft only, or both series of threads, more or less freely upon a ground texture usually consisting either of the plain calico or "tabby" weave or other simple and neutral weave such as the smaller twill, satin and matt or dice weaves, according to the particular effect desired in the fabric.

\section{Weft Figuring,}

A typical example of cotton brocade fabric of medium quality and intended for bleaching, or dyeing, is that illustrated in Fig. 520 , taken from a specimen of grey cloth produced with weft figuring only, on a ground texture of the plain calico weave and containing seventy-six warp threads per inch, of ordinary 30 's T, with two warp threads in each dent of the reed; and ninety-eight picks per inch, of 20's soft-spun weft.

In consequence of the great difference in the firmness of texture that usually exists between the ground weave and the figure portion of a brocade fabric, the figure is liable to be somewhat weak and flimsy, more especially if a ground weave of plain cloth-the firmest and most compact of all (338) 
weaves-is employed. By reason of the threads being loosely interwoven in the figure portion, they crowd together ; whereas in the ground portion they are so compacted that they tend to open out and encroach upon the figure where they have

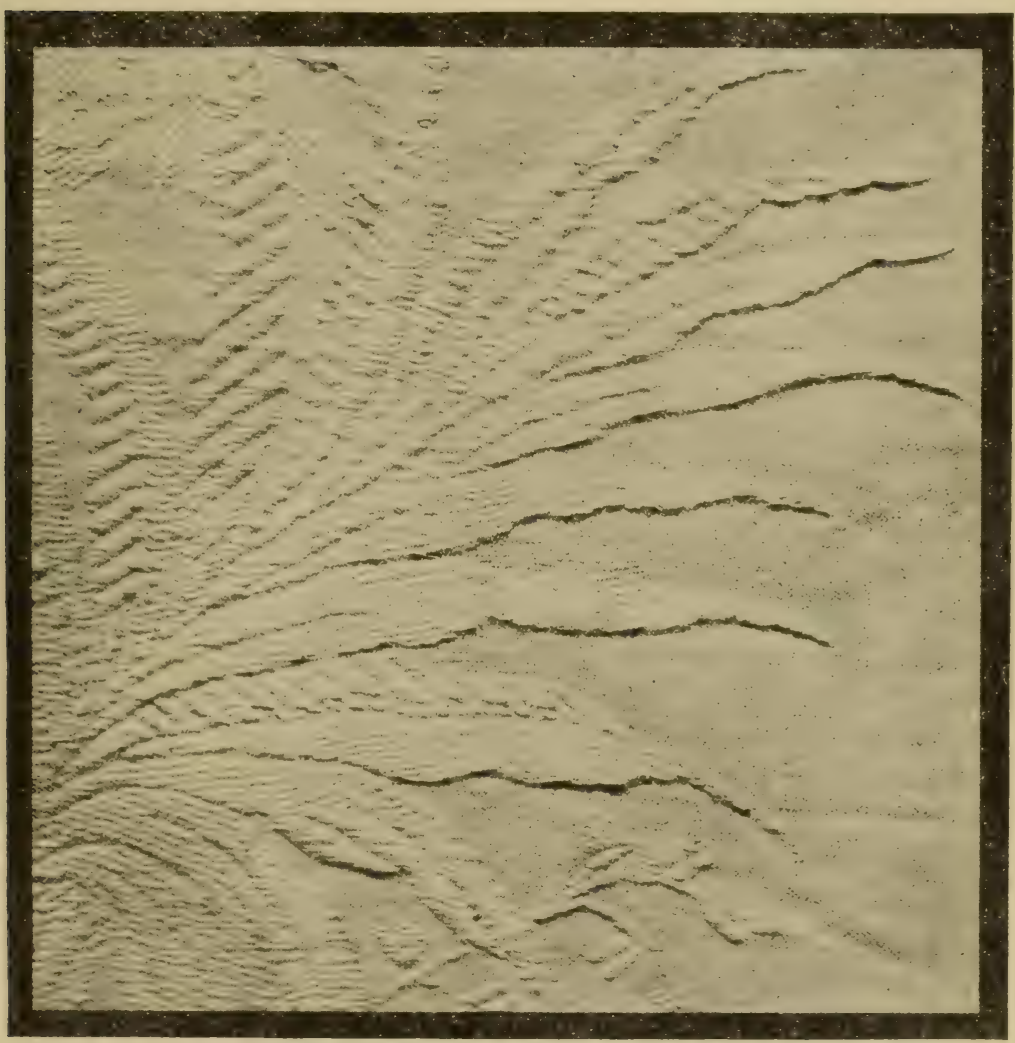

FIG. 520.-Cotton brocade fabric with weft figuring and a ground weare of plain calico.

more room. The tendency of the warp and weft threads to leave their proper place is more pronounced in respect of the weft than the warp threads, as the picks of weft are being constantly acted upon by the reed, whereas the war'p threads are held taut during weaving. The result is that the former system of threads, instead of lying in a perfectly straight line 
across the cloth, assume a decidedly wavy appearance, and cause thin open places near the edges of the figure, especially those edges that lie in a more or less horizontal direction of the picks of weft, as may be clearly observed in the photographic reproduction, Fig. 520.

Although the defects just indicated are, in a greater or a lesser degree, inherent to brocade fabrics generally, and cannot, therefore, be prevented entirely, they may, however, to some extent, be minimised by the textile designer exercising certain very simple precautions, when preparing the applied designs for the card-cutter, and as demonstrated subsequently in $\$ 167$. The defects referred to may also be minimised by ensuring a proper ratio between both the counts of warp and weft yarn, and also between the number of warp and weft threads per inch in the fabric, according to the particular character of the ground weave employed; and since the ground portion of a fabric, and not the figured portion, should constitute the firmest and strongest texture of cloth, that part should be taken as the foundation on which to base the particulars indicated above according to the science of cloth structure and practical experience; for unless a firm and well-balanced ground structure is obtained the entire fabric will be faulty. For example, if the yarn is too coarse, or if there are too many warp and weft threads per inch in the fabric, the ground portion will be harsh and boardy, and will tend to wrinkle. On the other hand, if the reverse conditions prevail the whole fabric, and especially the figured portion, will be flimsy and weak.

$\S 163$. Designs of almost any style or character may be developed in brocade fabrics, but those having fulness of figure give the best result. Unless the cloth is of very fine texture and of good quality, any minute detail of the pattern would be lost; also, the design should not consist of heavy masses of figure, but the various elements composing it should be disposed evenly over the whole surface. This is not merely to obtain any artistic effect, but with a view to causing all warp threads to bear an equal strain. If the design is of a decidedly stripy character, with the stripes running in the direction of the warp, the warp threads weaving the ground 
stripe will bear the greater strain during weaving; and when the cloth is cut out of the loom it will tend to wrinkle or buckle in those places, through contraction, on being relieved of such strain.

As stated in the previous section, brocade figuring may display either warp or weft threads only, or both series of threads, on the obverse of the fabric. The latter course offers considerably more scope to a designer in the development of a greater variety of textural effects, more especially if the warp and weft threads are of different colour. Also, the character of the warp and weft must not differ materially from each other either in respect of the counts or quality of the yarn, otherwise those figures in the design which are developed from the inferior material will suffer by comparison with those of the superior material, and thereby impart to the entire fabric the appearance of one of an inferior quality.

The manner of developing applied designs for brocade fabrics of the variety illustrated in Fig. 520 is indicated in Fig. 521, which represents a small fragment of the design for that fabric. After sketching in the design on squared or point paper, the designer proceeds to indicate the margin of the figure and ground portions by means of a single dotted outline consisting of single dots that are produced on the same "tab" or "shed" which requires alternate warp threads only to be raised for alternate picks of weft, and intermediate warp threads only for intermediate picks of weft, whereby two or more contiguous warp threads are never raised at the same time, nor is a warp thread ever raised for two or more consecutive picks of weft, although any odd number of consecutive warp threads may be left down at the same time; also warp threads may remain down for any odd number of consecutive picks of weft. When the outline of the ground and figure is completed in the manner described, the designer then proceeds to bind the floating weft figures with such elementary binding weaves as will ensure the desired effects in the cloth and, at the same time, prevent too long floats of weft on the face side of cloth, and too long floats of warp threads at the back. 
It should be observed, however, for reasons to be stated presently, that the binding weaves selected for this purpose must consist of single binding points only, and with those points or dots always on the same "tab" or "shed," as explained previously. Also, when selecting the binding weaves to employ for this purpose, care should be exercised to employ

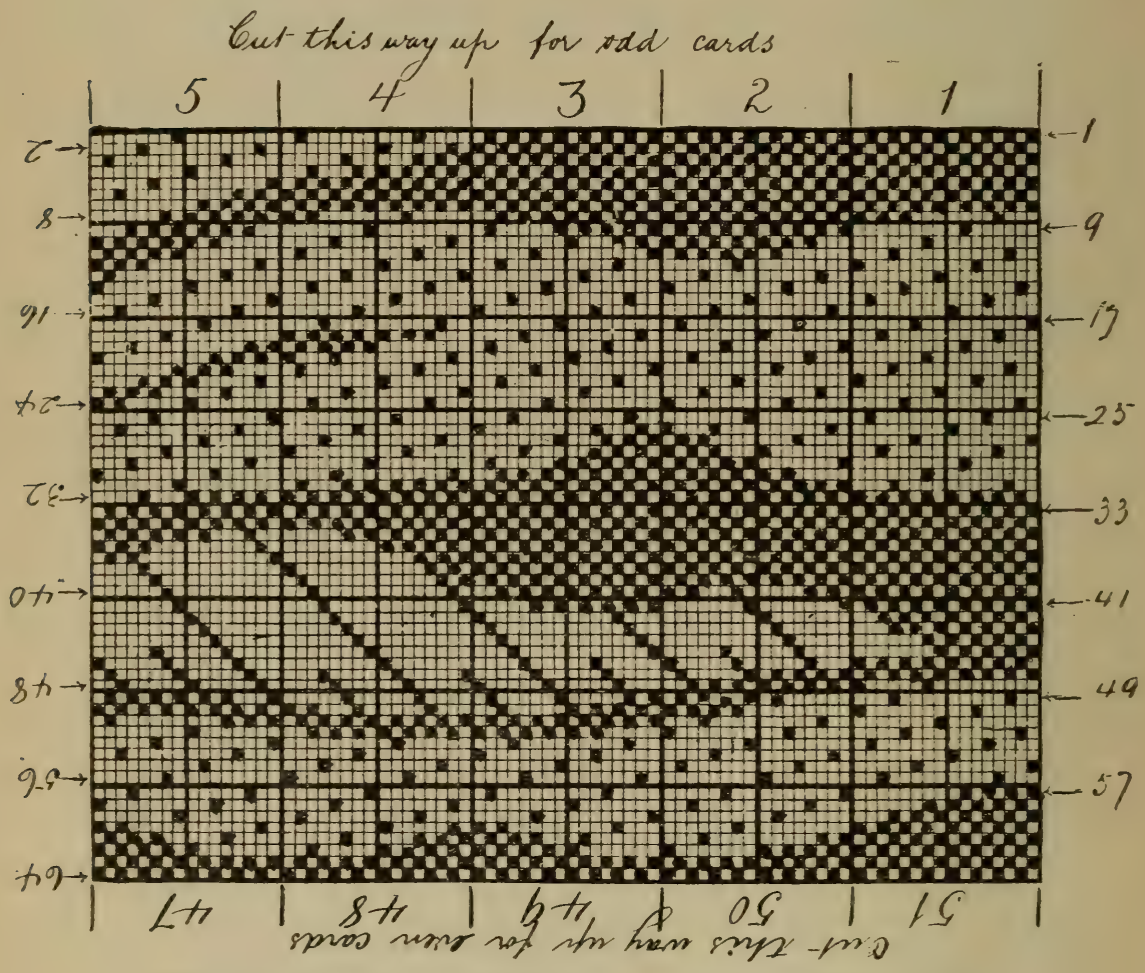

FIG. 521. - Portion of applied design for brocade fabric, Fig. 520.

such as will give due prominence to the figures without leaving too long floats that will weaken the fabric; and fabrics of relatively finer texture may be bound with weaves of a looser character than those of coarser textures, without detriment to the stability of the fabric. The binding weaves employed in the portion of design, Fig. 521, are the 10-end satin and the 12-end twill weaves. 
It is unnecessary to fill in the ground portion of the design by indicating the plain tabby weave in the manner represented in Fig. 521, but with the object of saving both time and labour. on the part of the designer, it is the usual practice to fill in the ground portion with a thin wash of green or yellow paint to distinguish it from the figure portion, which is left blank. In this case the green or yellow paint signifies the plain calico weave, and must be "read" accordingly by the card-cutter when cutting the pattern cards.

$\S 164$. Although the raising of warp threads uniformly on the tabby principle of weaving in the manner described, and as indicated in the portion of a brocade design, Fig. 521, imposes certain limitations upon a designer both in the choice of a design and also in the choice of suitable binding weaves, the practice incidentally affords an opportunity of effecting economy in the production of this variety of brocade fabrics by adopting what is termed the "split-shed" method of tying up the Jacquard figuring harness or mounting threads to the separate hooks of an ordinary double-acting two-cylinder Jacquard machine which, in this case, virtually consists of two separate and distinct sets of hooks and lances constituting two machines in the same framing. Thus, by operating the two sets of hooks in alternate succession for the odd and evennumbered pattern cards and picks of weft respectively, only one-half the number of pattern cards will be required as compared with the number that would be necessary if warp threads of both the odd and even series were raised together for the same picks of weft, thereby saving the cost of onehalf the number of pattern cards, card-cutting, lacing and wiring.

For example, a double-acting two-cylinder Jacquard machine tied-up with a "split-shed" harness may control a given number of warp threads and employ a pattern card for each pick of weft only, whereas if the same number of warp threads were controlled by an ordinary "straight-tie" harness, it would be necessary to employ either a Jacquard machine of double the capacity of the former, or else two separate machines of equal capacity, and to operate them simultaneously for each pick 
of weft, thereby requiring twice as many pattern cards. With a "split-shed" tie-up, however, it is only possible to weave fabrics either with all-weft or else all-warp figuring, according to which is to constitute the face and back of the fabric. But in either case the fabrics must of necessity be woven with the weft figuring uppermost, seeing that odd and even warp threads cannot be raised together, although they may remain down at the same time as described previously. Also, in consequence of the margin of the figures ending abruptly against a ground weave of plain cloth, they assume a somewhat broken and irregular outline, more especially in fabrics of coarser texture and poor quality.

\section{Warp and Weft Figuring,}

$\$ 165$. The embellishment of brocade fabrics with both warp and weft figuring affords considerably greater scope, to a textile designer, than is possible either with weft or warp figuring only, but the development of warp and weft figuring in the same design requires a much higher degree both of artistic and technical ability on the part of the designer because of the greater variety of textural effects that are thereby afforded, and also of the increased danger of faulty construction arising from a much greater freedom of interlacement of warp and weft threads.

Unlike many other types of fabric, the brocade type generally will permit of the successful development of designs of almost any kind, whether of a geometrical or floral character, provided there is a free distribution of figure over the surface of the fabric in order to ensure uniform tension upon the warp threads. Those in which both warp and weft threads are employed in the development of figure are rendered much more effective by having each of a different colour, but such as will give an agreeable contrast, and at the same time produce a harmonious combination. Each colour may be employed separately in its full tone, or both may be combined to produce any intermediate tone.

It is usual to employ the full tones of each colour for the figure portion and a neutral tone for the ground portion of the 
fabric. There is then no danger either of warp or weft figure being overpowered or lost by contrast with the ground, such as would be liable to occur by contrast with a ground weave in

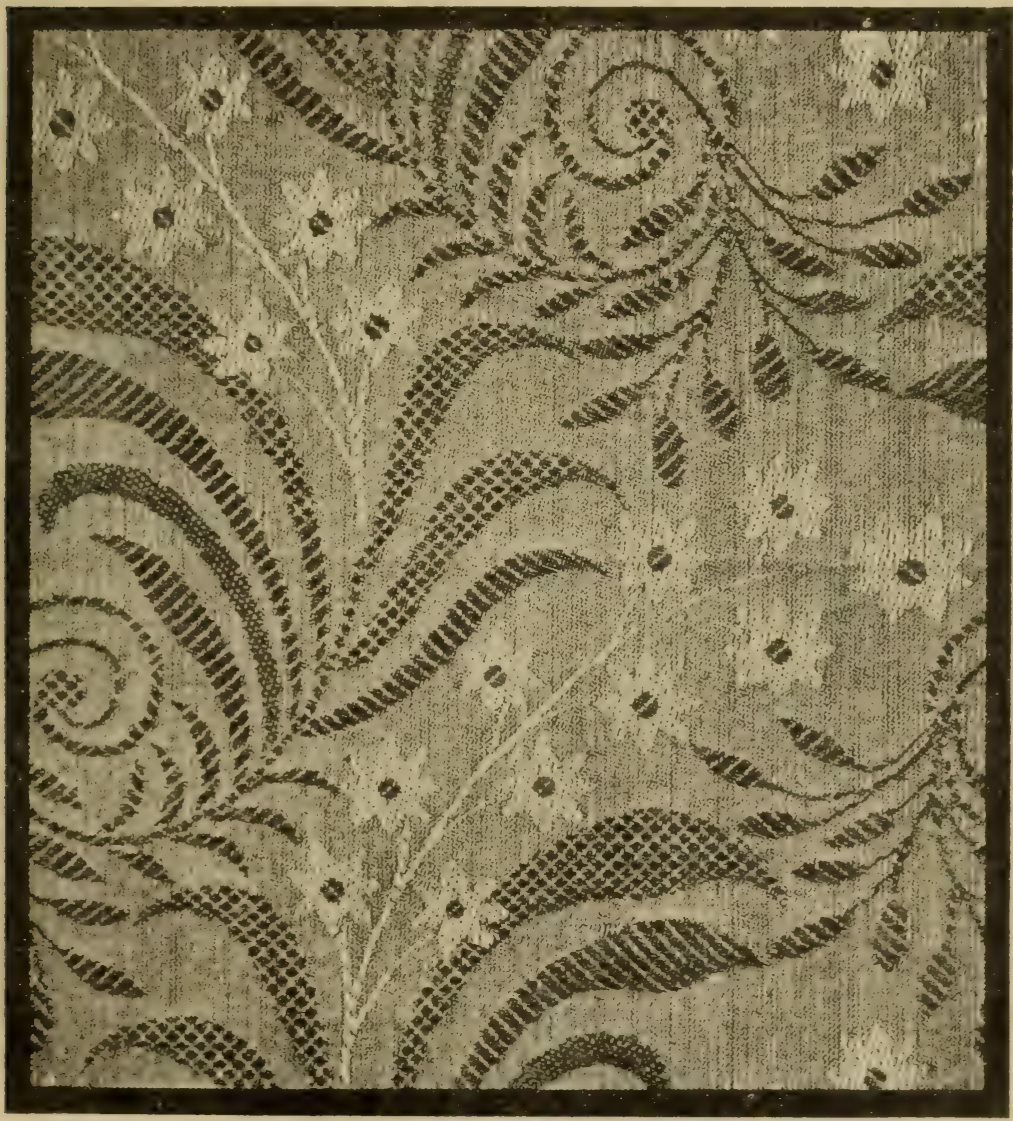

Fir. 522,-Cotton brocade fabric with both warp and weft figuring and a ground weave of plain calico.

which either warp or weft were allowed to preponderate on the face. Portions of figure that occupy a central position in a large object, or those that require subduing, may also be developed in a neutral weare, or other suitable intermediate tone, inclining to that either of the warp or the weft. 
A very simple example of warp and weft brocade figuring is that illustrated in Fig. 522, which is a photograph of a cotton brocade fabric containing 84 warp threads per inch of $2 / 80$ 's bleached yarn, and 78 picks per inch of 30's indigo weft. And although the design is of a plain character, its effectiveness is enhanced by the employment of a variety of simple binding weaves. Thus, the white warp flowers are bound with the 8-end satin weave, with the central weft spot bound with a single diagonal twill cutting; whereas the weft leaves are variously bound with the 8 -end satin and 8 -end twill weaves; whilst the ground consists of the plain calico weave which produces the best of all neutral effects by reason of the warp and weft being displayed in exactly equal proportions, and with the least possible exposure of the surface of the threads.

The choice of suitable binding weaves in the development of an applied textile design is of the greatest importance; for what may, in all other respects, constitute a suitable design, this may be marred by unsuitable or unskilful binding. As a general rule, only such binding weaves should be employed for brocade fabrics as will produce a plain and even surface. Honeycomb and similar rough-looking weaves should, therefore, be avoided, unless such effects are, for some special reason, desirable. For ordinary purposes, elementary weaves such as simple twill, satin, "diamond" and "rice" weaves, will be found to meet most requirements; but if these are inadequate, recourse may be had to weaves of a more elaborate character such as small matt, rib, cord, corkscrew, hopsack, granite and suchlike effects.

Experience, combined with judgment, can alone guide a designer in the choice of suitable weaves; but it will generally be found that the boldest effects are produced by those weaves in which the intersections of warp and weft are least distributed, and also by those containing few intersections; and that the more subdued effects are produced by those weaves in which the intersections are most uniformly distributed, and also by those containing frequent intersections. For example, a twill weave gives a much bolder effect than a satin weave, or than the plain or calico weave. 
Designers would frequently dispense with the use of binding weaves in some portions of a design, were it not an absolute necessity to employ them to ensure the stability of the fabric. The employment of these binding weaves often mars what would otherwise have produced a far more pleasing effect;

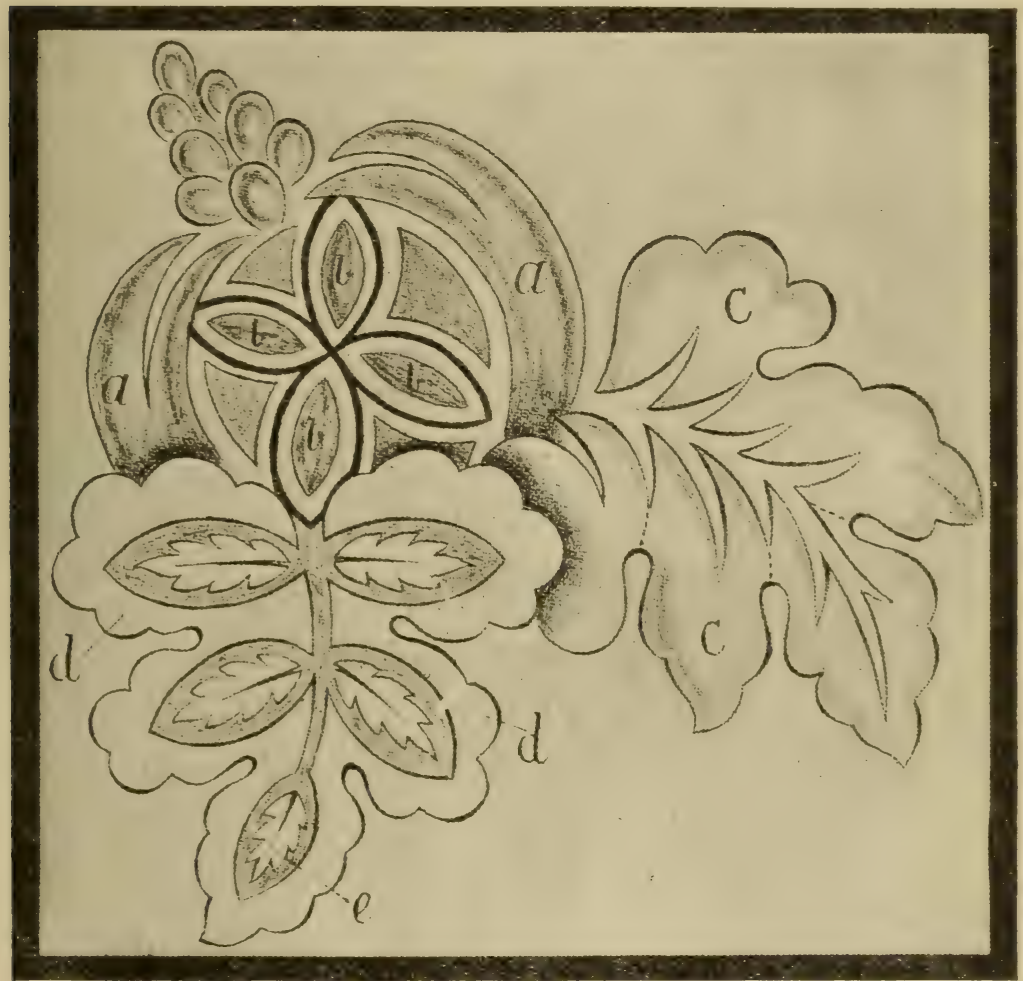

FIG. 523.-Part of sketch design for a brocade fabric with warp and weft figuring developed as in applied design, Fig. 524.

but seeing that they are a technical necessity, they should be turned to the best possible advantage by imparting the most agreeable effect of which they are capable. On the other hand. however, portions of the figure that do not require binding are sometimes treated as if they did, in order to harmonise 
those parts with others that require binding for structural purposes. Also, unimportant and obscure portions of a design may be bound with the intersections disposed irregularly, in order to prevent, in the most effective manner, too long floats of warp or weft.

A skilful designer will rarely employ more binding points in a design than are essential to ensure structural stability; but he will avail himself of every opportunity of combining structure and design so as to obviate any necessity for binding. For example, when developing a leaf, he will take advantage of the venation, marking and edging which, by extending or increasing them in a suitable manner, may serve the additional purpose

$\$ 166$. A portion of a sketch which is eminently suited for development as a brocade design containing both warp and weft figuring is illustrated in Fig. 523. From the varied character and the scope which this sketch offers to a designer for skilful treatment, it is selected as a suitable theme for demonstrating the practical application as a textile design of which a portion is indicated in Fig. 524.

The group consists of a conventional fruit $a$ of the dehiscent kind which open to allow the seeds $b$ to escape, in which act it is represented in the sketch; and two simple five-lobed leaves $c, d$, with sinuate margins, upon the lobes of which latter leaf $d$, five small serrate-edged leaves $e$ are superimposed.

By carefully studying the design, Fig. 524, much useful information respecting the development of a brocade design will be obtained. Black squares on'design paper represent warp of one colour, and white squares weft of another colour. The surface of the fruit is bound with a 10-end warp satin weave to impart smoothness, whilst the seeds are developed with a 6 -end diamond weave to give them due prominence. The interior of the fruit is treated in a subdued manner to cause it to recede. The upper half of the leaf $c$ may be bound with an 8-end warp twill weave, and the lower half with an 8-end warp satin weave, or vice versâ.

Or, another effective plan would be to develop alternate lobes with a twill, and the others with a satin weave, care 


\section{5}

-

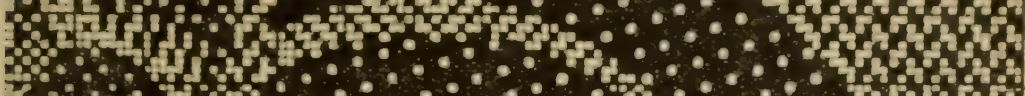

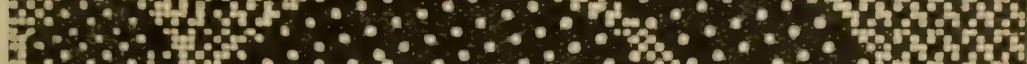

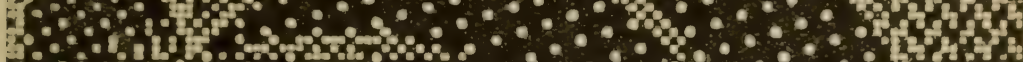

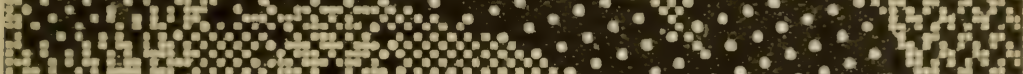

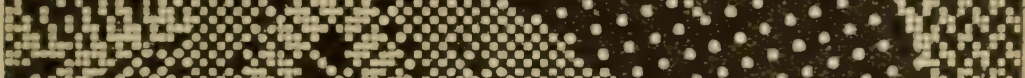

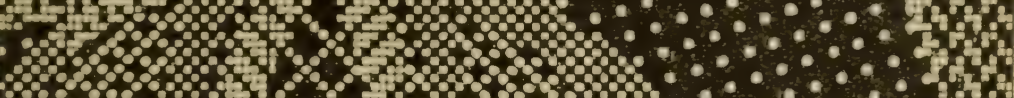

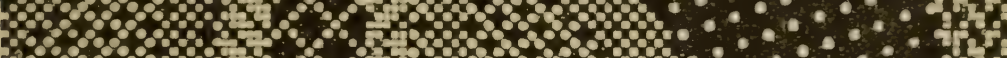

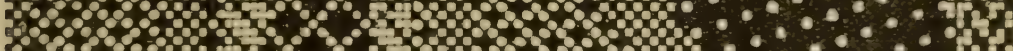

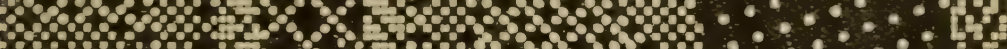

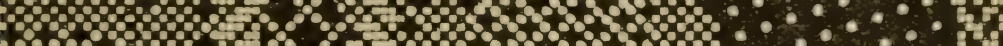

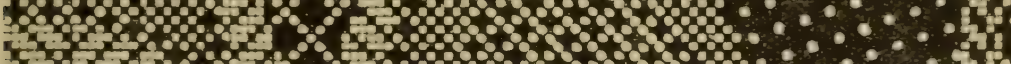

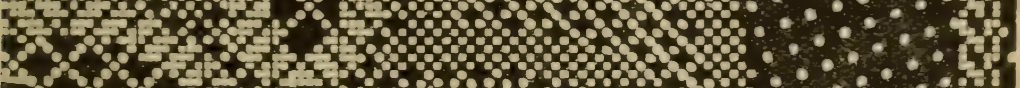

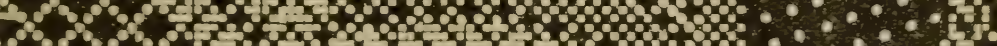

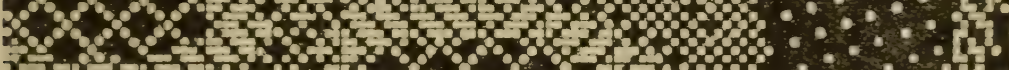
2.3.

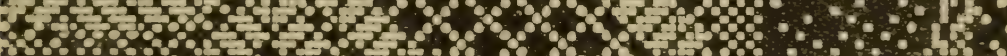

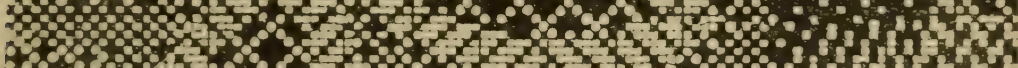
W6\% $6 \%$ \%

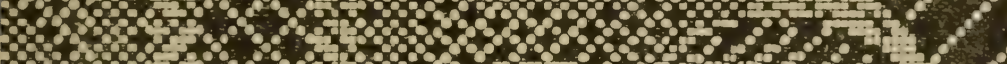

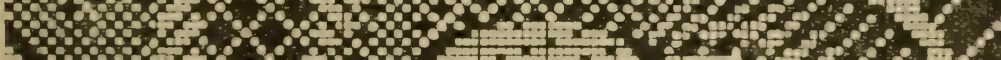

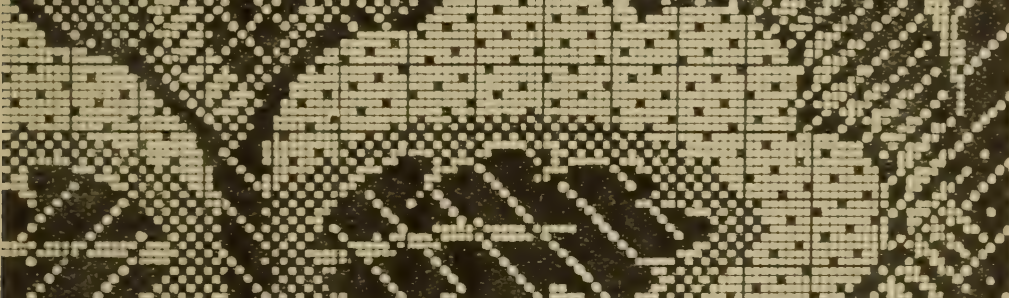

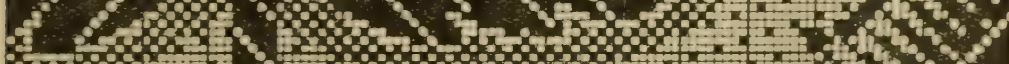

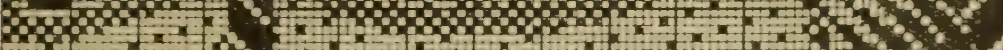

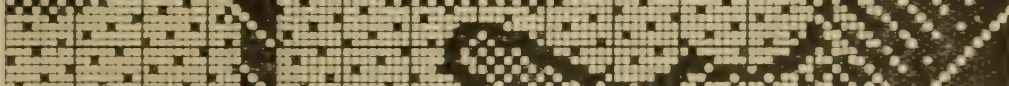

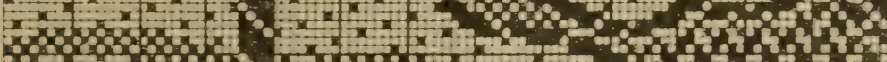

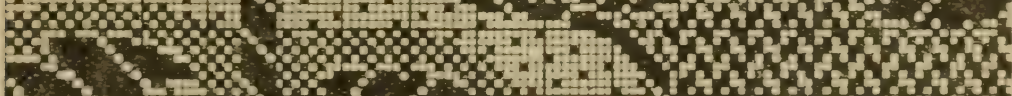

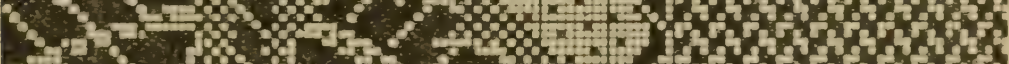

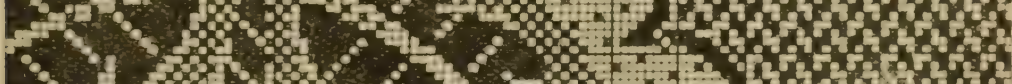

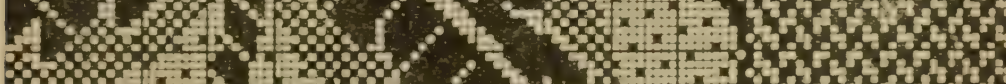

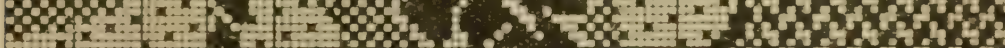

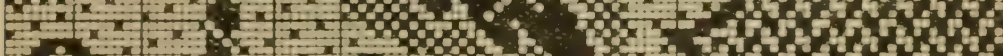

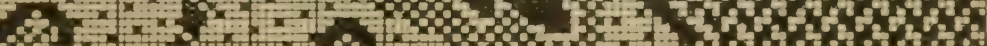

FIg. 524.-Part of an applied design for a brocade fabric with both warp and weft figuring developed in several different weaves to obtain variety of effect as in sketch design, Fig. 523. 
being taken to prevent long floats of yarn where the two weares meet. If the latter plan were adopted, it would perhaps be better to entirely separate the lobes from each other by a single cutting line made by slightly extending the veins, as indicated by dotted lines in the sketch, Fig. 523. The lobes of the leaf $d$ are developed with a 10 -end weft satin weave, and edged with a double warp line; and the small leaves $e$ with an 8-end warp twill weave to give them boldness. These smaller leaves are separated from the lobes of $d$ by a neutral weave of plain cloth which will cause both to stand well up. Minor portions of the design are treated in such a manner as will prevent too long floats of yarn; and the whole stands upon a neutral ground weave of the broken "Harvard" $(2-2)$ twill.

$\S 167$. In addition to binding both the figure and ground portions of a brocade fabric for the purpose of preventing too long floats of warp and weft, it is essential to properly "lock" or "check" the binding points which occur on the extreme margin of the figure and ground, especially where the margin lies more or less in either a horizontal or a vertical direction. The object of "checking" or "locking" these marginal binding points in a horizontal direction is to prevent picks of weft from sliding along the warp threads where a weft margin lies horizontally against a warp margin ; and also, in a like manner, to prevent warp threads from sliding along picks of weft where a warp margin lies vertically against a weft margin. If this precaution were neglected, warp threads and picks of weft would slide out of their proper straight lines, and thereby cause the parts where this occurs to assume a more or less broken and straggling appearance in the fabric.

The tendency of the warp and weft threads to slide out of their proper place in the manner just described arises, in respect of warp threads, from picks of weft extending beyond their fellows and encroaching upon one or more warp threads which are thereby free to slide underneath those picks of weft and thus assume a more or less wavy and irregular line. Also, in a like manner, picks of weft slide underneath encroaching warp threads from a similar cause. This objectionable 
tendency, however, may, in a very large measure, be prevented or minimised by the practice of "checking" or "locking" the marginal binding points in the manner indicated in Figs. 525 (A) and 526 (A), which indicate the incorrect and the correct methods of binding a design, and the resultant effects in cloth, at Figs. 525 (B) and 526 (B) respectively.

Thus, Fig. 525 (B) illustrates the effect that would be produced in cloth if the marginal binding points. were left unchecked as indicated in the portion of a design, Fig. 525 (A); whilst the superior results obtained by the system of checking,
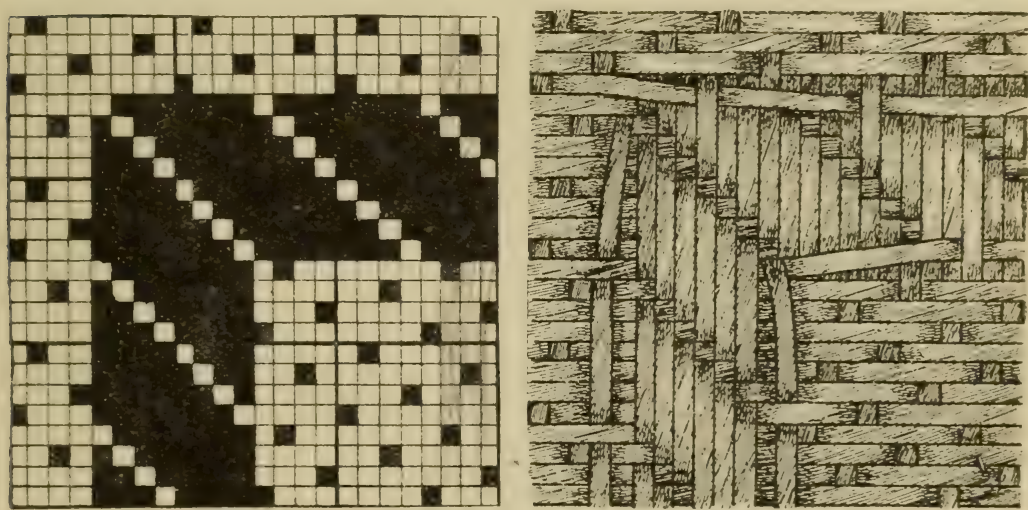

A

B

by inserting the adoitional marginal binding points indicated by black and wite crosses, in Fig. 526 (A), are illustrated in Fig. 526 (B), 60 which there is imparted a much smarter appearance by keeping the marginnl threads quite straight and in their proper places. The presence of these additional binding points, howerey, freauently constitute in themselves an objectionable featuite in' a textile fabric, as they sometimes tend, unless they are employed with discretion, to impair, instead of enhancing, the good appearance of a design by over-binding. Still, of the two evils it is perhaps the least objectionable, and the safer one to adopt, as it conduces to increase the stability of the fabric. 
By exercising a little ingenuity in the selection and arrangement of binding weaves, a designer may frequently avoid the necessity of "checking" in many parts of a design, by the simple expedient of causing the marginal binding weaves of the figure and ground portions to check or lock each other automatically. This object is more easily accomplished where the margins of figure and ground are perfectly horizontal and vertical, as in. counterchange designs of a geometrical char-

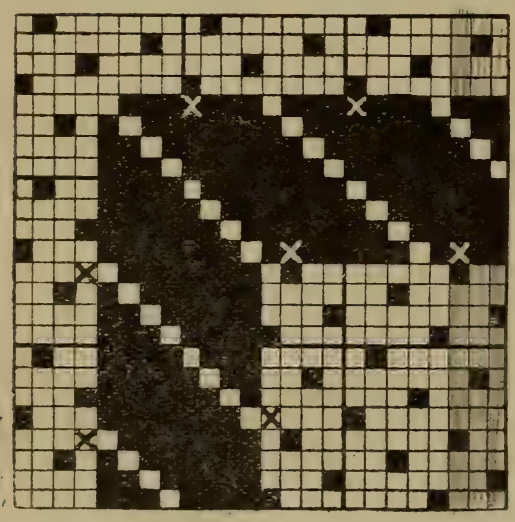

A

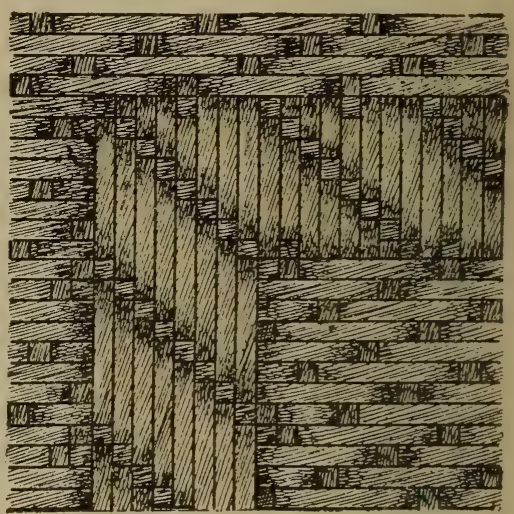

B

FIG. 526.

acter, than in designs of a foliated character where only short lengths of yarn are exposed at the margins. A good plan to adopt in binding a desìgm, when weaves "with single binding points that are all on the same "tab" are uşej, its to have the weave of the figure portion on the opposite " the weave of the ground portion. By adopting this course the binding points will, in mary places, check each other automatically, and fewer additional binding points will be required.

\section{Brocade Figuring with Extra Warp Threads and Extra Picks of Weft.}

$\$ 168$. Brocade fabrics may be rendered additionally attractive by the employment of extra warp threads or of extra picks of weft, of a different colour to the ordinary warp and 
weft. Such extra coloured threads do not replace ordinary threads of the corresponding series, so as to form bands of colour up or across the piece, but are wrought into the fabric as additional threads in such manner as is predetermined in

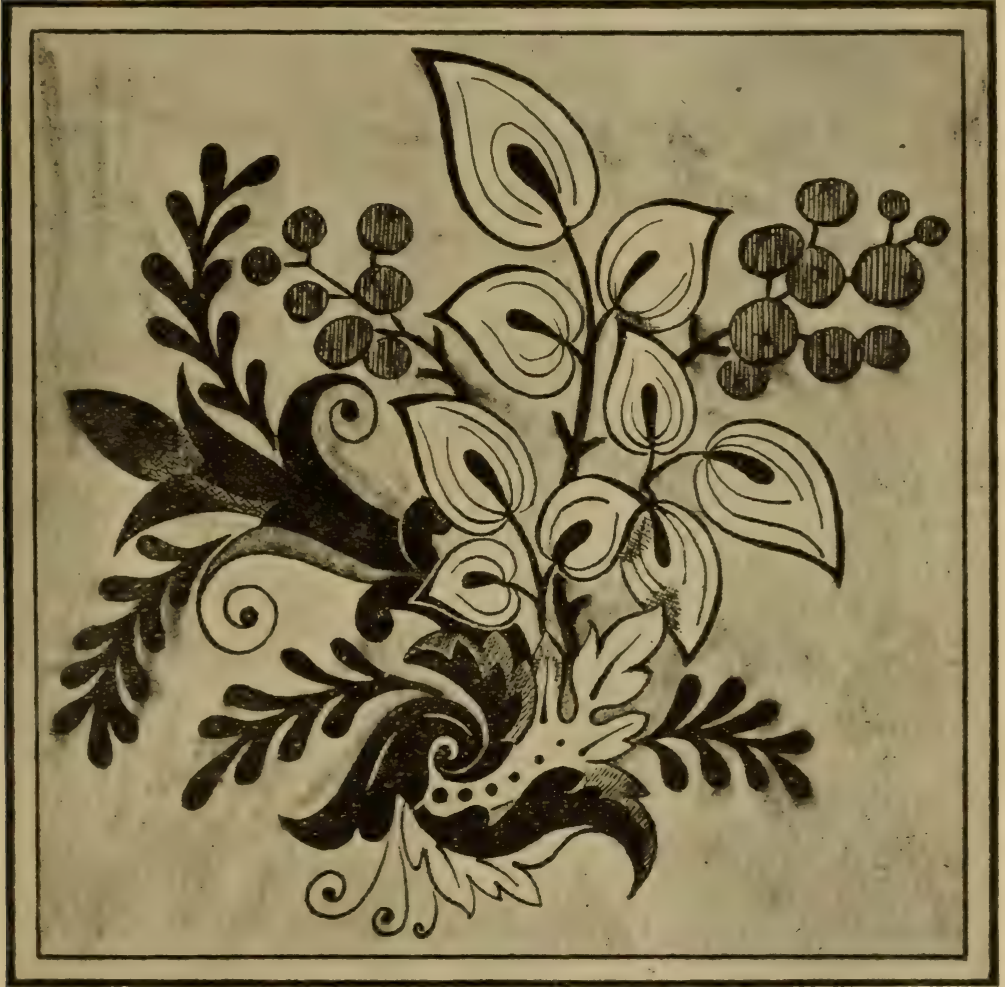

FIG. 527.-Part of a sketch design for a brocade fabric, with both warp and weft figuring, in which extra warp threads, of a different colour, are introduced in certain parts only, to develop the small berries.

the design. This extra material may either float loosely at the back of the fabric when not required on the face for figuring purposes, and be afterwards sheared off, or it may be interwoven with the ground portion of the fabric.

In the latter event, however, care must be taken to select 
such ground weave as will enable the binding points to be properly obscured, so as to prevent them showing through on the face side of the cloth. It is obvious, therefore, that a ground weare of plain cloth would not answer such a requirement, in consequence of that weave exposing the threads on both sides of the cloth for the least possible interval, and thereby not providing any covering for the binding points.

The conventional group of foliage, Fig. 527, is a good motif for a design in which either extra warp or extra weft may be effectively employed. The two bunches of berries could be developed with the extra material, and the remaining portions with the ordinary warp and weft. A neutral weare of some kind should be used for the ground filling, so as to display both warp and weft figure with equal effectiveness.

\section{Extra Warp Figuring.}

Extra warp threads should be governed, by preference, by spare hooks in the same Jacquard machine as that which gorerns the ordinary warp threads. By this method only one set of pattern cards is necessary, and less attention is required of the weaver than when an extra machine and set of cards are employed.

Since extra warp threads do not interlace with weft with anything like the same frequency as do the ordinary warp threads their contraction during weaving is considerably less than that of the latter. They should, therefore, be wound upon a separate warp beam, when their delivery can be suitably regulated.

The development of a design for a brocade fabric in which extra warp threads are employed, is little different from that of an ordinary design. The designer must know the number and disposition of extra warp threads at his command, if he is making a design to an existing tie-up; but if he creates a new tie-up, he may dispose the extra warp threads to suit his requirements, and hare the Jacquard harness tied up accordingly.

$\$ 169$. When preparing a design on point paper, the portions of figure to be developed with the extra warp threads should be 
painted in a different colour from that representing the ordinary warp. Also, if the extra warp threads are to be interwoven to prevent them floating loosely at the back, a third colour should be used to indicate the binding points.

A very simple example that will serve to demonstrate the development of such a design is represented in Fig. 528 which consists of two square cones arranged alternately upon a 4 -end basket or matt-weave for ground filling. The centre of each base is developed with the extra warp threads (represented by shaded squares) to form a diamond. This is surrounded by a weft figure (represented by blank squares), and the sides of the cones are developed with the ordinary warp (represented by black squares). Round white dots indicate both warp threads lifted to bind one of them into the fabric at the back.

The design repeats on 48 warp threads and 90 picks of weft, - with 22 extra warp threads, i.e., 11 for each figure.

Assuming the design to be woven by means of a 400 's Jacquard machine (408 hooks) with a straight-through tie-up on 384 hooks, the card-cutter would repeat the design eight times orer on each pattern card, because $384 \div 48=8$. As there are 408 hooks in the machine, this would leave $408-384=24$ spare hooks of which $11 \times 2=22$ are required to operate the extra warp threads, leaving 2 hooks for selvedge warp threads. If the 24 spare hooks are left on the 26 -side of the machine, the tie-up will be as follows: 384 hooks (counting from the 1st hook in the 51st bar on the 25-side of the machine) for ordinary warp threads; 22 hooks for extra warp threads; and the last 2 hooks (on the 26-side) for selredge threads; making a total of 408 hooks.

The design indicated in Fig. 528 is set out for this tie-up, and wili be contained in it eight times. The extra warp threads must be indicated in the design at $\mathrm{A}$ where they are required to appear in the cloth; and also (in another colour of paint) where they must be lifted for binding purposes. Their order of lifting must then be transferred to the hooks B set apart for them. Selvedge hooks $C^{\prime}$ are working a plain weave.

Harness threads from extra figuring hooks must pass through the comber-board behind the ordinary harness so that their 


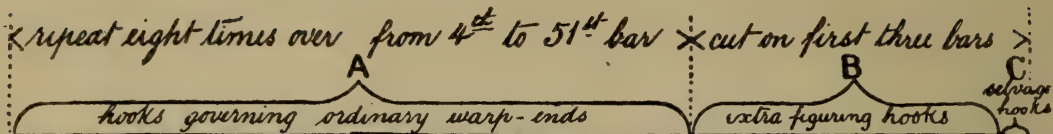

PH"

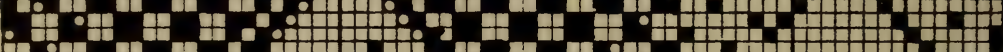

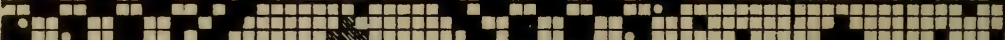
HP

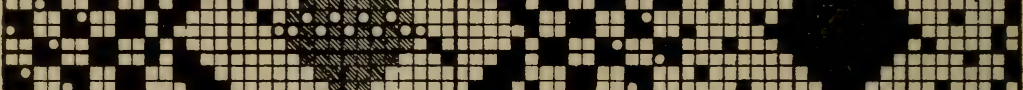
늘 :

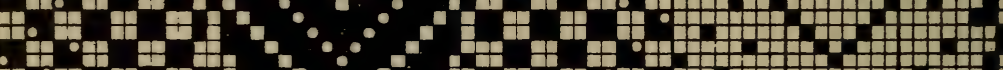

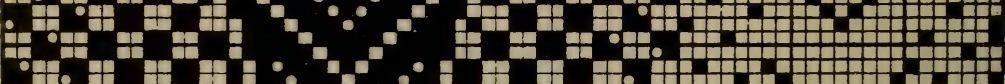

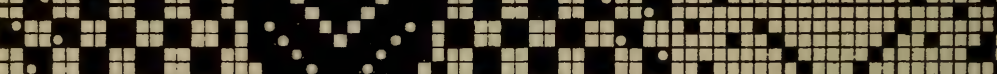
Ho H

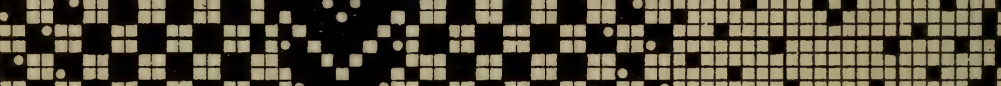
Ho H $\mathrm{H}^{\mathrm{H}} \mathrm{H}$

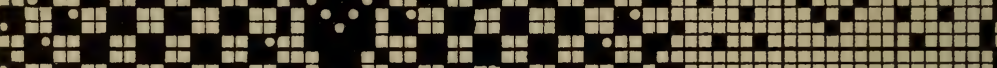
:

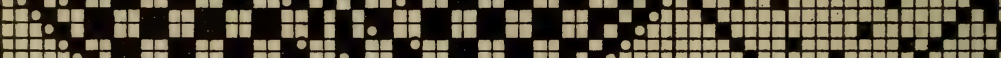
H.7.

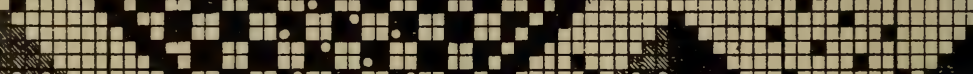
사 \# \# 2

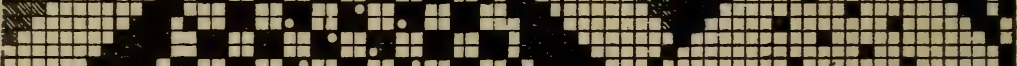

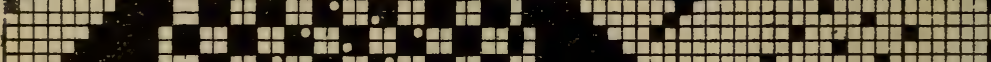

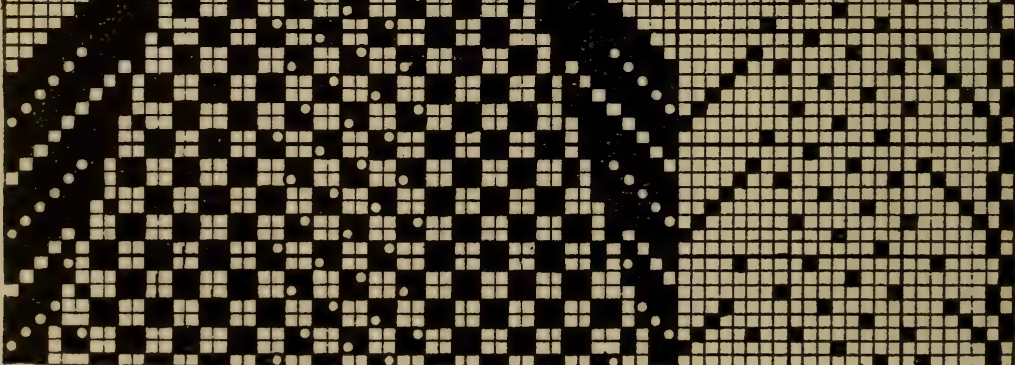

Fig. 528.-Applied design for a brocade fabric illustrating the method of introducing extra figuring warp threads as represented in sketch design, Fig. 527. 
maiis will fall exactly where the extra warp threads will lie in cloth.

The methods of binding warp and weft are indicated in Figs. 529 to 534 inclusive. The binding of the figure is effected in the usual manner. Thus, the weft figure is bound with the ordinary warp, and both warps are bound with weft where required, in the manner indicated at $a, a^{1}$, Fig. 529. Warp threads should not be bound in the manner indicated at $b, b^{1}$, Fig. 530, by sinking one and lifting its fellow on the same pick. This puts undue strain upon those threads, and gives a "pitted" appearance in the cloth, due to their pulling against each other. Although both series of warp threads lie together at the back of the cloth when the weft is on the face, care
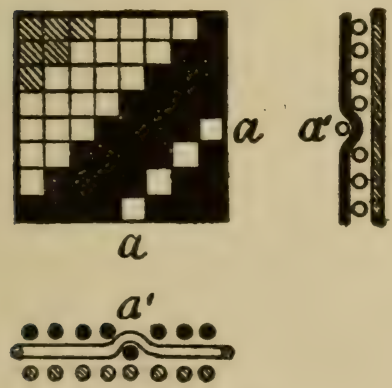

FIG. 529.

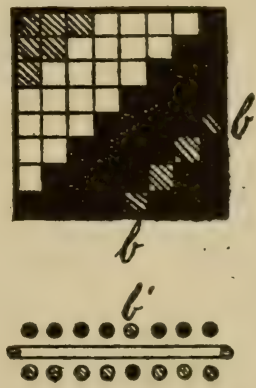

Fig. 530.

must be taken to prevent them being together on the face at the same time: not only to avoid their confusion, but because a warp effect requires a weft foundation, and vice versâ The two series of warp threads must, therefore, be separated by picks of weft, as indicated at $c, c^{1}$, Fig. 531, when one of the two series of warp threads is required on the face. The method of binding the picks of weft when these pass over both series of warp threads is indicated at $d, d^{1}$, Fig. 532.

The binding points for the extra figuring warp threads must be inserted in such positions as will effectually obscure them when the fabric is viewed obversely. This object may only be effected by raising the extra warp threads over the picks of weft when these are at the back of the cloth, in which case 
the binding points will be covered by the regular warp threads, as indicated at $e, e^{1}$, Fig. 533. Where there is a mass of warp threads on thel face of the fabric, as represented on each side of the cones, in the design, Fig. 528, there is no difficulty

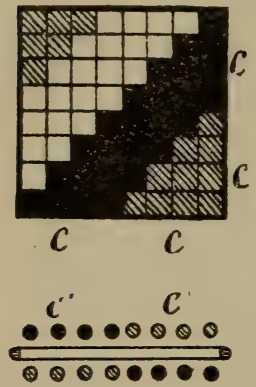

Fig. 531.
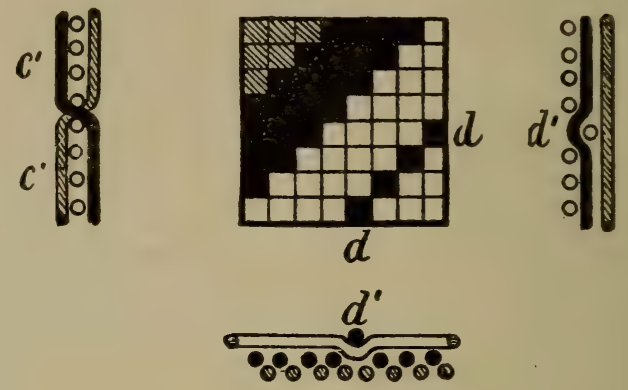

FiG. 532.

in selecting suitable binding points; but in a ground weave similar to that employed, there is greater risk of such points not being effectually:obscured.

These observations are also applicable to the binding of ordinary warp threads to prevent their floating too far at the
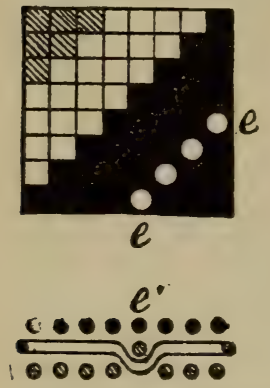

F1G. 533.
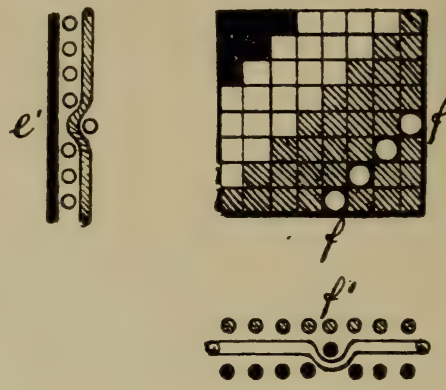

FIG. $53 \therefore$

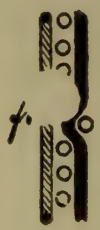

back when covered with extra warp threads on the face, as indicated at $f, f^{1}$, Fig. 534. To ensure the foregoing conditions, the card-cutter must be instructed to punch holes in the pattern cards where there is paint on design paper represented by solid squares and round white dots; and to leave the pattern cards blank where the design paper is bare, and also where 
there is paint represented, in the present case, by means of shaded squares.

\section{Extra Weft Figuring.}

$\leqslant$ 170. Instead of employing an extra series of warp threads in order to produce additional embellishment in a fabric, this object may be effected by inserting an extra series of picks of weft. The latter method of introducing the extra figuring threads is, of course, a more costly procedure than the previous method, because it involves the use of a loom equipped with a "checking" or shuttle-box motion. Also, additional pattern cards are required for the extra picks of weft. If, in the fore-

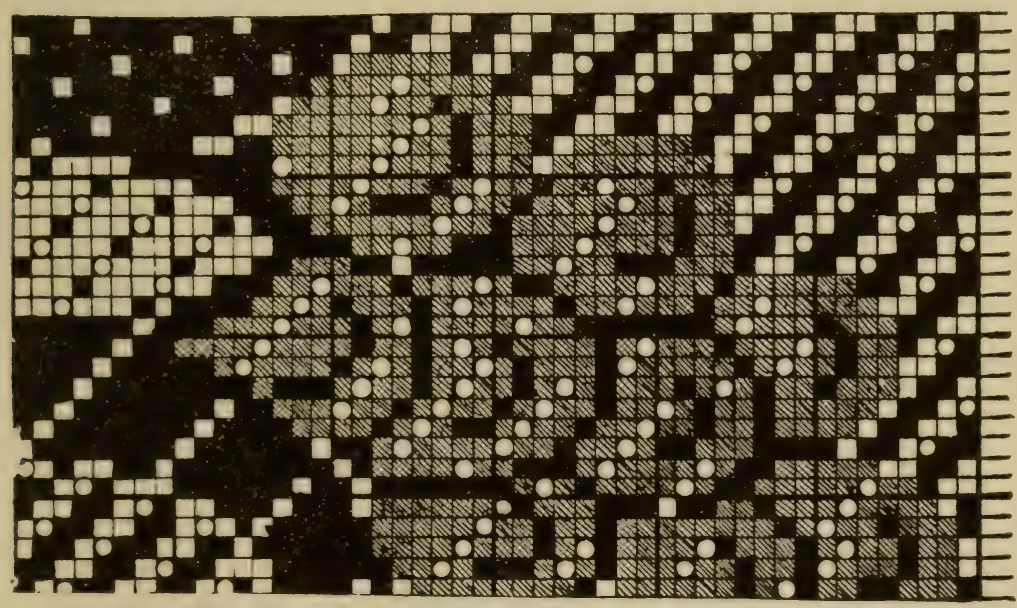

going description of extra warp figuring, $\$ 168$ and 169 , the terms "warp" and "weft" are read as "weft" and "warp" respectively, the method of figuring with extra weft will be clearly understood.

The method of developing an applied design for an extra weft-figured effect is indicated in the portion of a design, Fig. 535. The fruit is developed with the extra material, and is indicated on design paper by paint represented by shaded squares. Long floats of weft in either series must be bound by 
warp, and not by taking one pick to the back and lifting the other to the face, for similar reasons to those stated with reference to extra-warp figuring, and illustrated at $b, b^{1}$, Fig. 530. Either series of weft is prevented from-floating too far at the back of the cloth by binding over one series of warp threads when the other series is on the face as indicated in the design by paint represented by round white dots.

For the ordinary picks the card-cutter must be instructed to cut paint represented by black and shaded squares; and for the extra picks to cut that represented by black squares, and also the design paper. Paint represented by round white dots must always be missed, for these indicate the binding points of the lower weft, and will, therefore, not be visible on the face of the fabric.

Where the extra weft is indicated in the design, each horizontal space on design paper represents two pattern cardsone for a pick of the ordinary weft, and one for a pick of the extra weft, both of which should bear the same cardinal number; but pattern cards for extra picks should be distinguished from those for ordinary picks by affixing to the cardinal numbers some distinguishing mark, as the letter X.

If the cloth is produced in a loom equipped with a double shuttle box at one end of the sley only, the pattern cards will require to be laced for an "alternate" picking motion to insert two picks of the ordinary weft and two picks of the extra figuring weft alternately. But a superior result is obtained by employing a loom with two shuttle boxes at each end of the sley and equipped with a "pick-and-pick" picking motion, in order that an ordinary pick of weft and an extra pick of weft may be inserted separately in alternate succession, instead of in pairs.

If the loom is equipped with a double box at one end of the sley only, the pattern cards for Fig. 535 must be laced in the following order, namely: $1,2,3,3 \mathrm{x}, 4 \mathrm{x}, 4,5,5 \mathrm{x}, 6 \mathrm{x}, 6,7$, $7 \mathrm{x}, 8 \mathrm{x}, 8$, and so on, but if weft is inserted pick-and-pick, cards must be laced as follows: $1,2,3,3 \mathrm{x}, 4,4 \mathrm{x}, 5,5 \mathrm{x}$, and so on.

Some method should also be devised whereby the cloth is taken up at half the usual rate of speed whilst the extrg figure 
portion is weaving, because the extra picks of weft do not add to the length of the fabric. This object may be accomplished by connecting the stop-catch of the take-up motion to a spare hook in the Jacquard machine (or if all are engaged, an extra hook may be inserted) and holes punched in the extra pattern cards to cause the stop-catch to be raised whenever those pattern cards operate. 


\section{CHAPTER XIII.}

\section{DAMASK FABRICS.}

$\$$ 171. Damask fabries comprise two chief modifications, namely: (1) "Simple" damasks, containing only one series each of warp and weft threads, as exemplified both in worsted and linen table covers, table linen, light curtains and hangings; and (2) "Compound" damasks, containing one series of warp threads and two series of weft threads, namely, "face" and "back" picks respectively, as exemplified in some varieties of furniture upholstering fabrics, curtains and hangings.

Damask fabrics in general are characterised either by warp satin figuring upon a weft satin ground, or vice versâ, and usually with satin weaves of the same denomination as the 5,8 or 10 -end weaves. Or, instead of reversed satin weaves for the figure and ground portions of the fabric, these are sometimes developed with simple warp and weft twill weaves respectively. And again, a twill weave may be employed for the figure and a satin weave for the ground, as in the example illustrated in Fig. 536 which is taken from a sample of damask fabric containing 39 groups of silk warp threads per inch, with 7 threads in each group, or $39 \times 7=273$ threads per inch, and 100 picks of worsted weft per inch. The figuring is developed with a simple 8-end weft twill on a ground texture of 8-end warp satin.

In addition to the reversing of the same weave or else two nearly similar weaves, for the development of the figure and ground portions of the fabric, in the manner just described, and thereby obtaining a true counterchange of weaves which virtually results in a reversible fabric, those fabrics that are constructed on the true damask principle of weaving are further characterised by a more or less coarsely-stepped 
margin of the figure and ground resulting from the method of controlling, by means of the figuring harness, the warp threads in groups of two or more contiguous threads simultaneously, and also of inserting two or more consecutive picks of weft during the operation of each pattern card for the development of the design; although both the warp threads and picks of

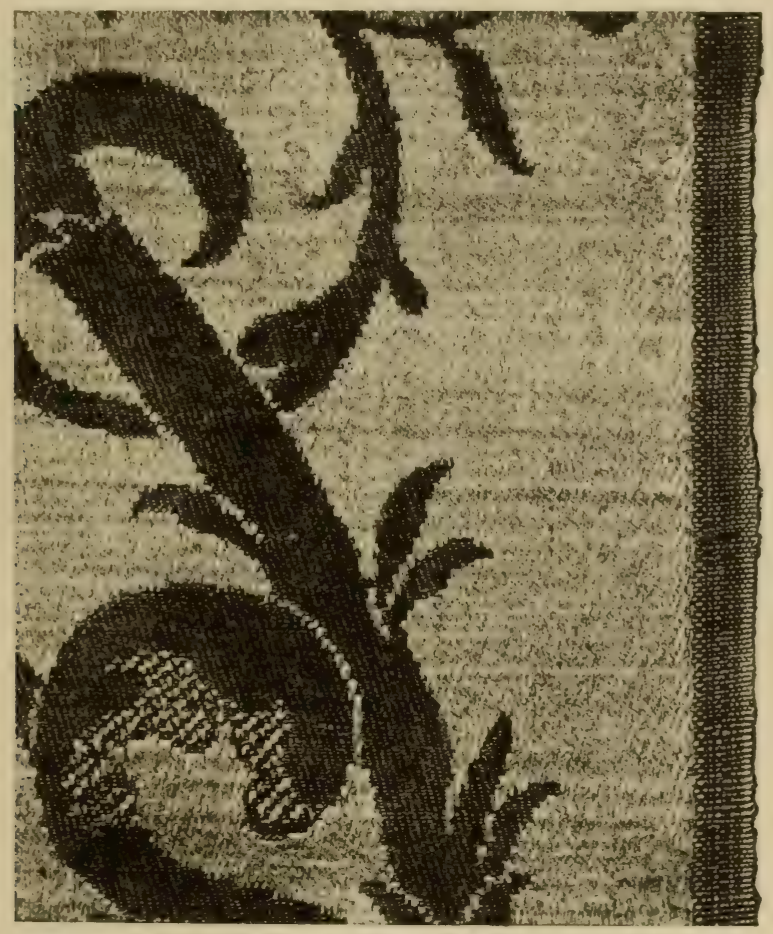

FIG. 536.-Compound damask fabric.

weft each interweave independently in some definite order for the purpose of binding both the figure and ground portions of the fabric in the manner described.

It will be seen, therefore, from the foregoing, that damask fabrics differ from brocade fabrics not so much in the general principle of their construction, as in the manner of developing 
figure upon them. In brocade fabrics a variety of different binding weaves may be employed both in the figure and ground portions of the fabric. Also, warp and weft may exchange places in any desired manner (excepting when woven with a "split tie-up" as described in $\$ 164)$, whereas in true damask fabrics a simple satin or twill weave only is employed both for the figure and ground.

Of course, when designing for "brocade-damask" fabrics, in which individual warp and weft threads may interweave in any order, as required, it is a common practice to introduce a variety of different binding weaves in the development of the figure in order to impart additional attractiveness to the design ; but these fabrics partake more of the character of brocades than of damasks, and more correctly belong to the former class of fabrics.

$\$ 172$. It is in the special mechanism employed in the production of damask fabrics rather than their construction, that the greatest interest attaches. The extreme fineness of texture that usually obtains in these fabrics, especially those of silk or fine linen containing sometimes as many as 400 threads per inch, places them far beyond the range of the ordinary type of Jacquard machines of reasonable capacity, even for designs repeating on a few inches only, in the fabric. And to equip a loom with a sufficient number of ordinary machines would not only incur enormous expense, but that course would also oftentimes be quite impracticable.

In order, therefore, to overcome these practical difficulties and still enable true damask fabrics to be produced economically, there have been devised many very ingenious methods whereby a considerable number of warp threads may be commanded by a comparatively small number of hooks, needles, or both, in the Jacquard machine. Also, by using each pattern card for several picks in succession, a pattern repeating on a large number of picks may be woven with one-quarter, one-third or one-half that number of pattern cards, according as each of the latter is used for four, three or two picks respectively.

Although each of these several optional methods operate in a different manner in attaining the same result, they are all 
based upon the same principle, namely, that of governing the warp threads en masse for the formation of the figure; and individually for the purpose of binding the warp and weft threads in some definite order or sequence.

\section{The Compound Presser Harness.}

$\$ 173$. The best known of these special damask equipments, and the one most suited for fabrics of very fine texture, is that known as the "compound presser harness" of which an end elevation and a plan are represented in Figs. 537 and 538 respectively, and of which there are two chief modifications. The prevailing type of "presser" harness, as represented in Figs. 537 and 538, consists of an ordinary Jacquard figuring harness $\mathrm{C}$ that operates in conjunction with a heald harness $\mathrm{G}$ for binding purposes, and comprising any reasonable number of healds (in the present case-five) according to the binding weave selected. Both harnesses control the same warp threads, but in a different manner. Thus, after passing through the mails $\mathrm{H}$ of the Jacquard harness in groups of two, three or more threads together, they are then passed separately through heald eyes G1 to G5 so as to form a five-shaft "straight-through" draft, as indicated in the plan, Fig. 538. The heald eyes are made much longer than usual in order to permit of a full Jacquard shed being formed within them.

After a shed is formed, the healds occupy three different positions, namely, a "top" position as No. 1; a "neutral" position as Nos. 2, 3 and 4 ; and a "bottom" position as No. 5, and may be actuated either by means of tappets, a dobby, or Jacquard hooks B1, which are raised by a griffe A1 operated independently from the griffe $A$ that lifts the figuring hooks B. Each heald is controlled by two hooks which are connected by a cord E supporting a small pulley and hook F. The healds are suspended by cords passing from the pulley-hooks and are pulled downwards, after being raised by means of weights or springs. By lifting two hooks B1 that are connected, a heald is raised to the "top" position; by lifting one hook only it is raised to a "neutral" position; and it remains at the "bottom" if both hooks are left down. 


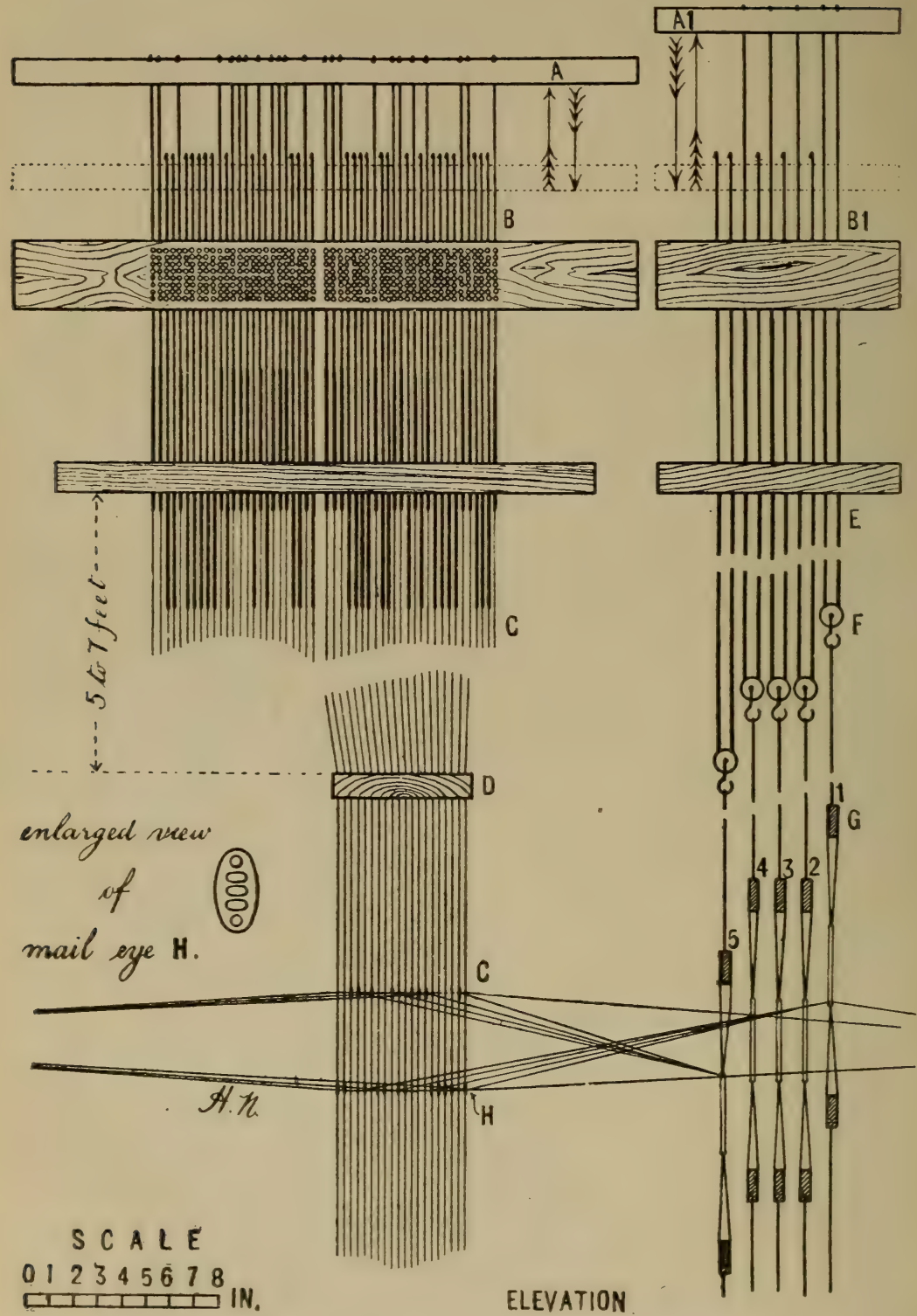

Fig. 537.-Compound presser harness, for damask weaving. 
It is the function of the Jacquard figuring harness to form the pattern by raising warp threads en masse for the figure, and by leaving them down en masse for ground; and it is the function of the healds to produce the binding weave throughout the entire fabric, by raising some of the warp threads that

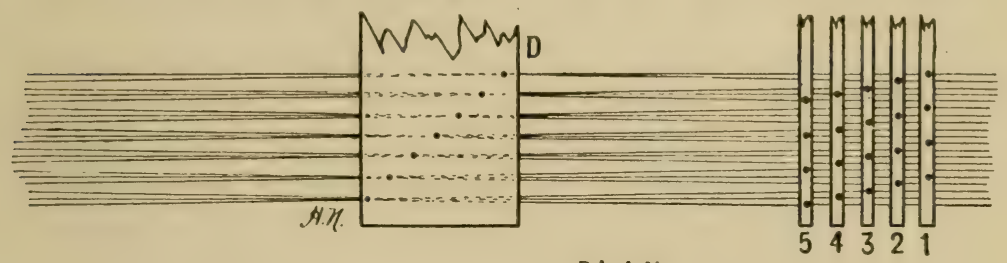

\section{PLAN}

Fig. 538.-Part plan of Fig. 537.

are left down, and by keeping down others that are raised by the figuring harness. This object is accomplished by governing the healds in a manner according to the binding weave desired as a twill or satin weave. At least one heald must be raised and one left down for each pick, whilst the remainder occupy a "neutral" position in order to prevent any obstruction to the figuring shed.

The shedding plans indicating the manner of operating the

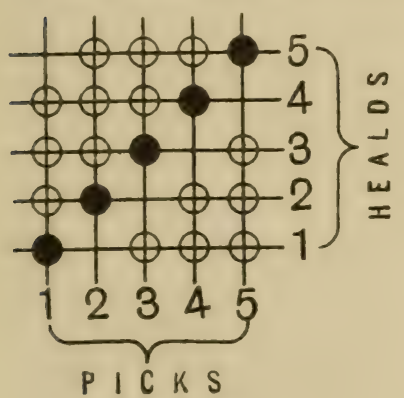

FIG. 539.

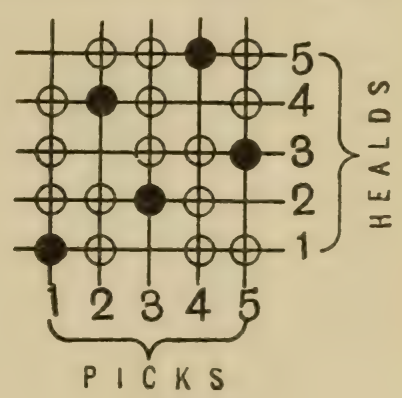

Fig. 540 .

healds so as to produce a 5 -end twill and 5 -end satin weare are indicated in Figs. 539 and 540 respectively. Horizontal lines represent healds, and vertical lines picks of weft. A black spot placed on an intersection of two such lines signifies that the heald must be raised to the "top" position for the 
corresponding pick of weft. Also, a circle indicates that a heald must be raised to a "neutral" position; and a blank indicates a heald left down. For example, in Figs. 539 and 540 a black spot is placed upon the intersection of horizontal line No. 1 with vertical line No. 1 ; and circles are placed upon horizontal lines 2,3 and 4 ; whilst 5 is blank on the same vertical line. Therefore, heald No. 1 would be raised to the "top"; healds Nos. 2, 3 and 4 would be raised half-way; and heald No. 5 would remain down on the first pick, and so on.

$\$ 174$. As stated previously in $\$ 171$, each pattern card may serve for two or more picks of weft in succession. This is accomplished by keeping the griffes of the Jacquard machine raised for such number of picks as are required to be inserted for each pattern card, and then changing the card for the next series of picks : and as the griffes must be raised for every pick of weft, the changing of pattern cards must be accomplished quickly between the last pick of one pattern card and the first pick of the succeeding card. The lifting cam governing the Jacquard machine should be constructed to effect the change as easily and smoothly as possible, otherwise it will cause considerable vibration to the harness, and consequently bad weaving.

The construction of a cam to change the shed after every third pick is demonstrated in Fig. 541. The cam is divided into three equal parts, 1, 2 and 3, each representing one pick of weft. Two-thirds of the third division are employed in closing the shed after the third pick is inserted, and also in forming a new shed for the next three picks. This allows onethird of a pick for the passage of the shuttle through the shed. The cam is constructed to the following data: Centre of cam A, to lowest point $\mathrm{L}$, equals 2 ins.; distance from lowest to highest point $\mathrm{C}$, equals 3 ins.; diameter of treadle bowl J, equals 2 ins. B and D are imaginary lines between which the pin of the treadle bowl moves when the latter is passing from the highest to the lowest point, and vice versâ.

The portion $\mathrm{F} \mathrm{J}$ of the third division, equal to the two-thirds of a pick, is divided as follows: $F$ G, equal to five-twelfths of a pick, is employed for closing the shed; $G \mathrm{H}$, equal to one- 
twelfth of a pick, for a pause or dwell; and $\mathrm{H} . J$, equal to sixtwelfths or one-half of a pick is employed for opening the shed. A little more time is allowed for opening than for closing a shed, in order to make it easier for lifting. The sectors F G,

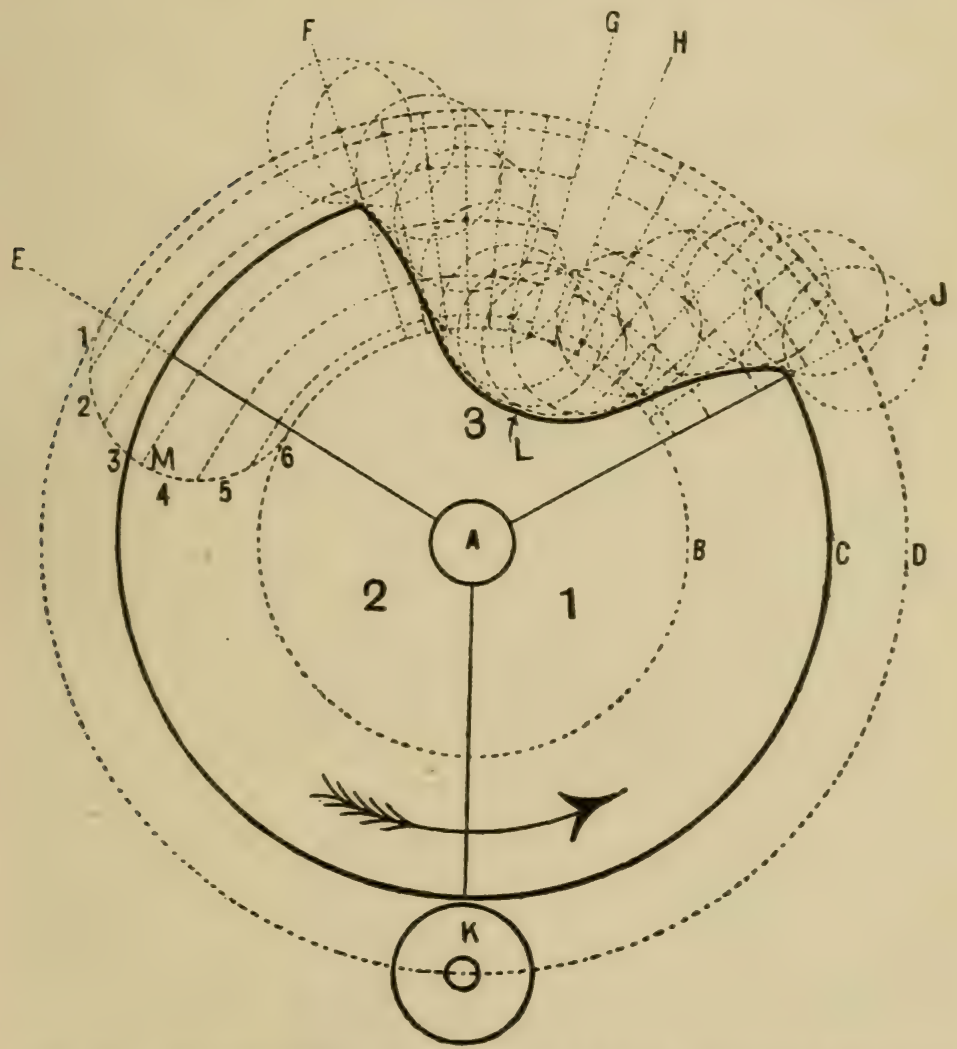

FIG. 541.- Showing method of constructing a 3-pick Jacquard lifting cam or tappet.

and $\mathrm{H} \cdot \mathrm{J}$, are each subdivided into any number of equal parts (in this example, six), by radial lines. These are intersected by concentric lines to form a corresponding number of unequal divisions between the lines $\mathrm{B}$ and $\mathrm{D}$. These unequal divisions may be readily obtained by describing a semicircle $\mathrm{M}$ on any 
radial line between circles $\mathrm{B}$ and $\mathrm{D}$, and dividing that semicircle into, say, six equal parts, $1,2,3,4,5$ and 6 . By projecting lines from each intersection of the arc to cut the diameter at right angles, the six unequal divisions are obtained. The intersections of consecutive radial and concentric linescommencing on the outer circle $\mathrm{D}$ at $\mathrm{F}$ where the shed begins to close-indicate different points in the path of the centre of treadle bowl as it moves between its extreme positions. By describing circles from each of these points equal in diameter to that of the treadle bowl, and then drawing curves tangent to them and connecting the highest and lowest points of the cam, the outline of the latter is completed.

A separate set of pattern cards is employed to operate the hooks B1, Fig. 537, which control the healds; and as this set of cards passes over a separate cylinder that works independently from that of the figuring set, very few pattern cards are required. Also, the binding weave of the fabric may be readily changed with little cost, by replacing one set of pattern cards by another set, in order to operate the healds in a different order.

As the griffe blade A1 must rise and fall, and the card cylinder move outward and inward for each pick of weft, both may be operated from the crank shaft, if employed on a powerloom, since that shaft makes one revolution for each pick; but if employed on a hand-loom, they should be operated independently by a treadle from that which controls the principal machine.

$\$ 175$. Another modification of the "presser" harness is that represented by a part elevation and a plan in Figs. 542 and 543 respectively. Thus, instead of employing a single set of healds formed with very long eyes, as in the modification described previously, there is employed in the present modification a double set of clasped healds, arranged in two tiers, as indicated in the diagram. Thus one set A has warp threads drawn above the clasp to form a "straight-through" draft, and is used to raise those threads singly to bind the ground portion; and the other set B has warp threads drawn below the clasp in the same order, and is used to depress the threads singly, to 


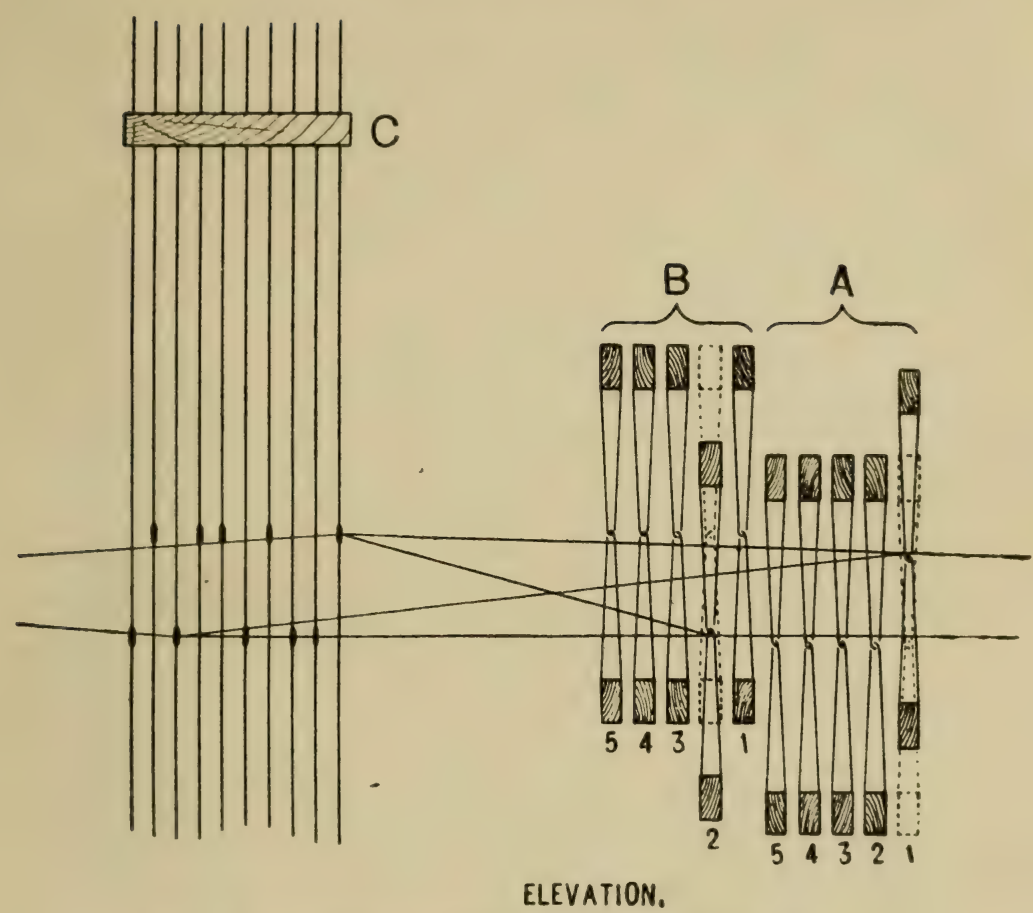

F'rG. 542.-Compound presser harness for damask wearing.

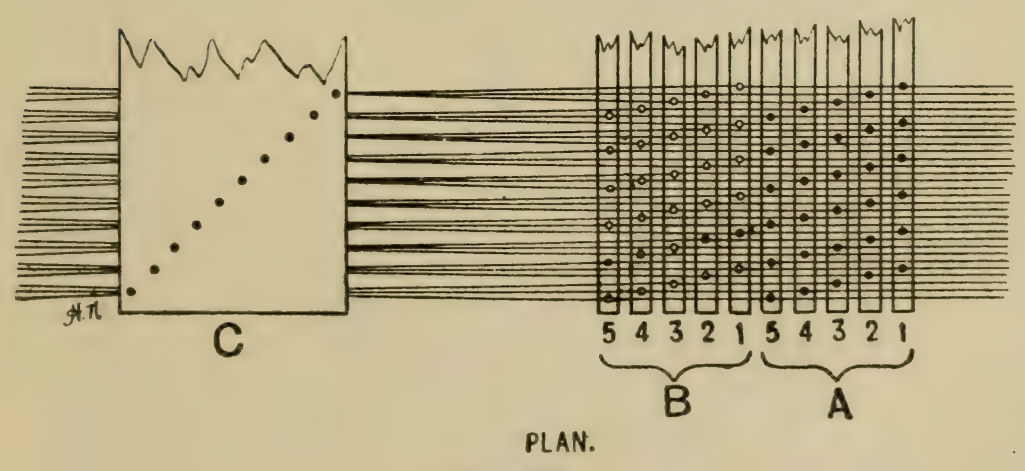

FIG. 543. 
bind the figured portion of the fabric. The drafting chart for this arrangement of harness is represented in the plan, Fig. 543 , in which black dots indicate warp threads drawn above, and circles indicate them drawn below the clasps. The manner in which the healds must be operated for a 5 -end satin binding weave is represented in the shedding plan, Fig. 544, in which black dots indicate healds raised, and circles healds depressed.

One of the most objectionable features of the compound

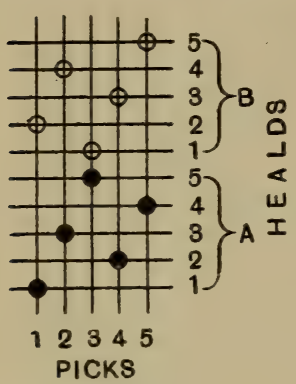

FiG. 544 . "presser" harness is that it puts enormous strain upon warp threads at such times as they are either held down or else raised by the healds; whereby they are deflected very acutely within a comparatively short interval of space. This evil, however, may be minimised by setting the figuring harness well back from the healds, and making a small warp shed. The latter condition involves the use of shuttles having a smaller weft-holding capacity, and a consequent frequent stoppage of the loom to replenish the shuttle with fresh supplies of weft as this becomes exhausted. Also, the heald harness, especially when employed in two sets, as represented in Figs. 542 and 543 , is cumbrous and takes up an inordinate amount of space which, in the economics of present-day manufacturing, is an important consideration.

For these reasons the "presser" harness is not nearly so well adapted to the exigencies of power-loom weaving (by which the warp is subjected to more or less severe treatment) as it is to hand-loom weaving, in which all operations are under the control of the weaver.

\section{Damask or Twilling Jacquard Machines.}

$\$ 176$. These circumstances, combined with the relatively greater expense necessarily involved in the slower production of damask fabrics by means of hand-looms, have given rise to numerous devices to enable these fabrics to be produced both more expeditiously and economically, by means of power- 
looms, and without the attendant disadvantages of the "presser" type of harness. These devices consist of a distinctive type of Jacquard machines of special construction, and known as "damask" or "twilling" Jacquard machines, of which there are several different modifications, for each of which their respective inventors claim some special merit over other machines of the same type. But whatever may be the relative merits of these respective machines, none are capable of producing damask fabrics of the extreme fineness of texture or general excellence that is possible with the "presser" type of harness which permits of any reasonable number of contiguous warp threads being controlled by each Jacquard hook and harness thread; whereas single warp threads only are governed by each hook and harness thread of a damask or twilling Jacquard machine of the type indicated.

Iany of these special Jacquard machines display a considerable amount of technical knowledge and mechanical ingenuity of a very high order; and although these devices differ in respect of minor structural details, they all embody the same general principles of construction and perform their respective functions in a similar manner. The cardinal features of Jacquard machines of this type consist essentially of (1) the controlling of two or more consecutive hooks by each needle ; (2) the controlling of those hooks from two independent sources in such a manner that they may either be raised or left down en masse, for figuring purposes, and, at the same time, individual hooks may either be raised or left down independently and in some prescribed order or sequence, for the purpose of binding both the figure and ground portions of the fabric, according to the particular binding weave selected, and quite irrespective of the manner in which the pattern cards have been cut; and (3) the employment of each pattern card for two or more picks in succession.

\section{The Bessbrook Damask or Twilling Machine.}

$\$ 177$. Of the numerous attempts that have been made by inventors and manufacturers to bring the power-loom into successful competition with the hand-loom in the production 
of damask fabrics of the correct type, none have met with the same measure of success (in Britain, at least) as that attained by Barcroft's invention, in 1869, of what is generally known as the "Bessbrook" damask machine, which is named after its birthplace in Ireland.

The essential features of this ingenious machine are indicated in Figs. 545 to 547, in which there are represented two needles $\mathrm{B}$, each controlling three consecutive figuring hooks $\mathrm{C}$. In a "straight-over tie-up," each hook C commands a separate warp thread in each repeat of the design; and the hooks controlled by the same needle B command consecutive warp threads. Very long loops $\mathrm{D}$ are formed at the bottom of hooks $\mathrm{C}$ to receive the twilling bars $\mathrm{G}$, each of which passes through and supports one long row of hooks C. Strong hooks $\mathrm{E}$ are attached, one at each end, to the twilling bars $\mathrm{G}$, by which the latter may be raised in order to raise all the figuring hooks $\mathrm{C}$ resting upon those bars, although some of the figuring hooks $\mathrm{C}$ may have escaped their respective griffe blades $\mathrm{F}$, which are capable of swivelling from their normal inclined position to a vertical one as indicated at F4. The griffe blades $\mathrm{F}$ are also provided at each end with a shaped piece of metal I, situated immediately behind the strong hooks E. Thus, when a griffe blade $\mathrm{F}$ assumes a vertical position, it has the double effect of leaving down its own row of figuring hooks $\mathrm{C}$, and of pushing the strong hooks $\mathrm{E}$ over the griffe blade immediately in front, thereby causing all the hooks in that row to be raised by the corresponding twilling bar, even although some of the hooks $\mathrm{C}$ may have been pushed off their own griffe blades by the pattern cards.

The griffe blades $\mathrm{F}$ are oscillated from their normal inclined position to a vertical one by means of a series of bars 5, Fig. 547 , which bars are provided with several notches $J$ that are cut at regular intervals apart, and which take over the ends of the griffe blades $\mathrm{F}$. These bars 5 are fixed above, and at one end of the griffe blades $\mathrm{F}$, and are operated by means of a series of iron pegs fixed in the barrel $\mathrm{N}$, in order to move the griffe blades in such a manner as will cause the figuring books $\mathrm{C}$ to be raised or else left down according to the binding 


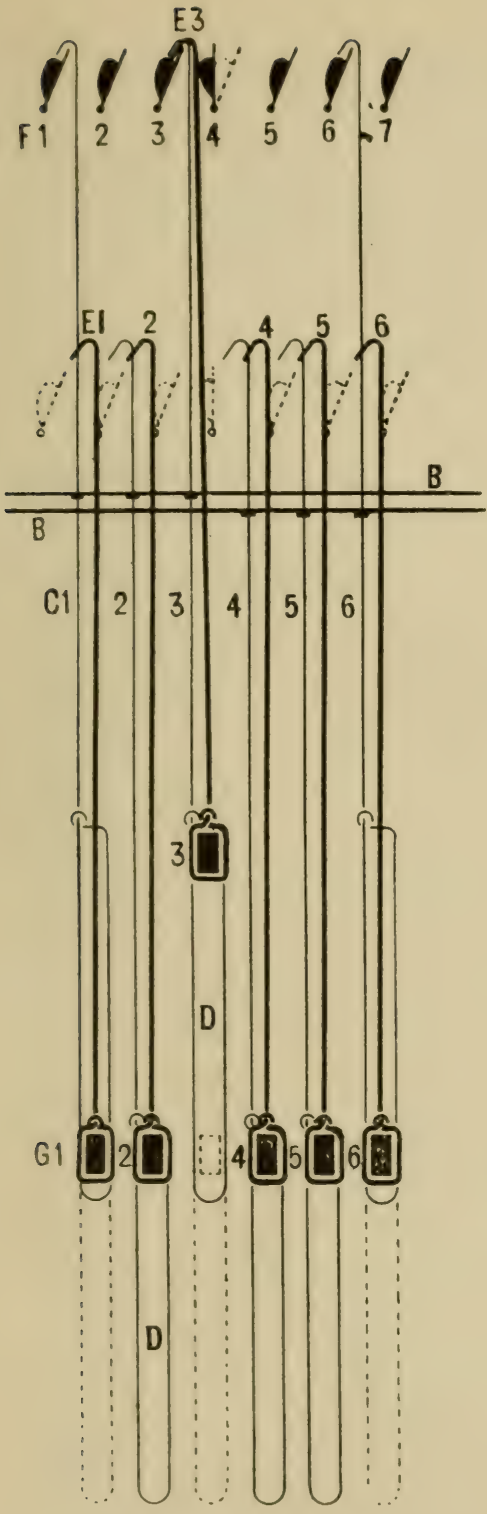

END ELEVATION

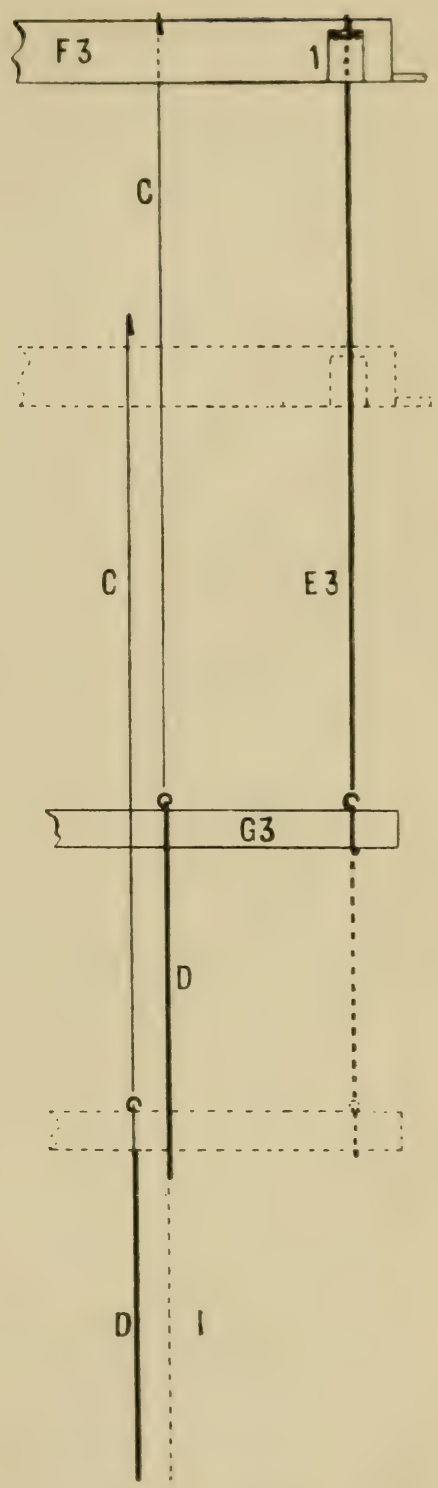

FRONT ELEVATION

FIG. 546 .

FIG. 545 .

Showing construction of Barcroft's (Bessbrook) damask twilling Jacquard machine. 
weave desired. The interval-measured in rows of hooksbetween the notches $J$, and also the number of bars used, determines the number of warp threads and picks upon which the binding weave may repeat. Thus, for a 5-end twill or satin weave, each bar 5 commands one griffe blade out of five; therefore, five, or a multiple of five, bars will be required.

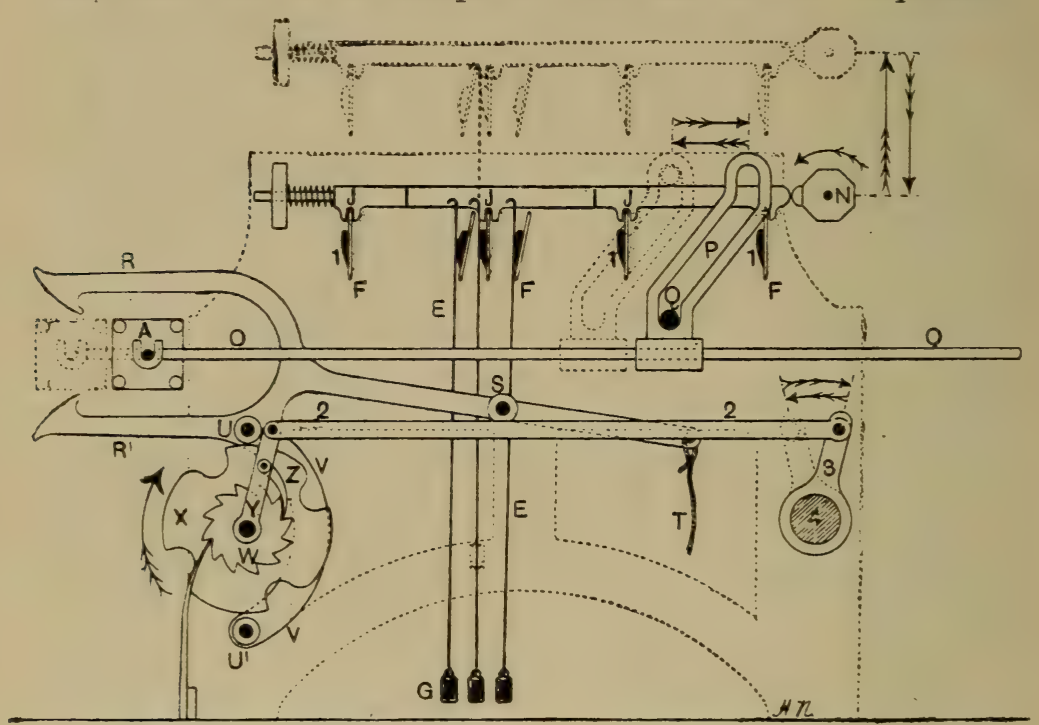

FIG. 547,-End elevation of Barcroft's (Bessbrook) damask twilling Jacquard machine.

Also, for an 8-end weave eight bars will be required, with each bar commanding every eighth griffe blade.

Only such binding weaves may be employed as will repeat on the number of warp threads that corresponds with the number of rows of hooks $\mathrm{C}$, or any measure of such number. For example, if there are 24 rows of hooks-as usually exists in Jacquard machines employed in the linen damask trade-in that case binding weaves repeating on $3,4,6,8,12$ and 24 warp threads may be employed. The number of griffe blades $\mathrm{F}$ must exceed the number of rows of hooks $\mathrm{C}$ by one blade, which is an extra blade, introduced solely for the purpose of pushing the strong hooks $\mathrm{E}$, in the last row, over the last 
griffe blade proper, whenever the first griffe blade is vertical; whereby all the hooks $\mathrm{C}$ in the last row are raised, and all those in the first row are left down. Thus, if it is required to make an 8-end weave with a Jacquard machine containing 24 rows of hooks, the first bar 5 must command griffe blades Nos. $1,9,17$, and also the extra blade 25 .

As the Bessbrook Jacquard machine is single-acting, the griffe blades $\mathrm{F}$, along with the bars 5 and the barrel $\mathrm{N}$, rise and fall, also the card cylinder A moves inward and outward for every pick of weft inserted. At each ascent of the griffe blades, the barrel $\mathrm{N}$ is turned so as to bring a fresh set of pegs to act upon the ends of the bars 5 , whereby, as the griffes ascend, other rows of hooks $\mathrm{C}$ are raised and left down respectively, in order to produce the binding weave; although each pattern card may continue to serve for two or more picks of weft in succession.

The card cylinder is moved outward and inward by means of bowls $Q$ acting, as they ascend with the griffe, upon swan necks $\mathrm{P}$, which latter are secured to rods $\mathrm{O}$ carrying the card cylinder.

$\mathrm{By}$ a very ingenious arrangement, the card cylinder may more outward and inward any number of times before it is turned to bring a fresh pattern card to operate upon the needles. This is accomplished by causing a tappet $\mathrm{X}$ to move turning catches $R, R 1$, fulcrumed at $\mathrm{S}$, so that the cylinder escapes them as it moves outward. But on the last pick of each pattern card, either the upper or else the lower catch is brought into action, according as the cylinder is required to turn forward or backward respectively. Two bowls U, U1 are also carried, one above, on the hook $R$, and one below the tappet $\mathrm{X}$, at the end of arm $\mathrm{V}$.

Thus, if the card cylinder is required to turn forward, the bowl $\mathrm{C}$ rests quite freely upon the tappet $\mathrm{X}$; but if the cylinder is required to turn backward, the bowl U1 is held upward aysainst the tappet by pulling down a cord $\mathrm{T}$ by means of a spring. The tappet represented in Fig. 547 is constructed to change the pattern eards after three picks of weft have been inserted. The elerations put the catches out of action for 
two picks, and the depressions put a catch into action for one pick, to change the pattern card. The tappet $\mathrm{X}$ makes one revolution for every four cards, or twelve picks; and is turned by means of a ratchet wheel $\mathrm{W}$ which is moved one tooth for each pick of weft by means of a pawl $\mathrm{Z}$ which is carried by an arm $Y$ fulcrumed on the tappet pin, and oscillated by means of a shaft 4 , operating through the medium of an arm 3 and a link rod 2.

\section{Method of Preparing Applied Damask Designs.}

$\$ 178$. The preparation of designs to be reproduced either by means of a compound "presser" harness, or a damask twilling machine, is extremely simple, and demands very little technical knowledge combined with good draughtsmanship. This is due to the fact that a designer for such fabrics has to consider only the ornamentation of these fabrics, apart from their structural features, which are predetermined, and therefore entirely dependent upon the mechanism governing the warp threads, which circumstance greatly simplifies and expedites the work both of the designer and the card-cutter.

Each vertical space on the design paper represents such number of warp threads as are grouped together: also each horizontal space represents a pattern card and, consequently, such number of picks as may be inserted for each card.

When preparing an applied damask design, the designer does not indicate the actual interlacement of the individual warp and weft threads, as when preparing a design for a brocade or other fabric of simple structure, and in which the warp and weft threads may be interwoven in any desired order; but he merely indicates the manner in which the Jacquard hooks are to be raised and left down respectively, by painting up the design en bloc and without indicating any binding weaves whatever. Such a design, therefore, is not a plan of the fabric, indicating the manner in which warp and weft threads are interlaced, but simply a block plan of the general scheme of decoration.

The method of preparing a damask "block" design is demonstrated in Fig. 548, whilst the actual order in which the warp and 
weft threads interweave in the fabric is indicated in Fig. 549, which represents the war'p threads governed in groups of three, and with three picks of weft inserted for each pattern card for figuring purposes; but with a reversed 5-end satin binding weave for the figure and ground portions of the design.

As a consequence of controlling the warp threads in groups of three and also inserting three picks of weft for each pattern card, a somewhat coarsely "stepped" margin is imparted to the figure and ground. This "stepping" is relatively more or less pronounced according to the number of warp threads controlled by each hook of the Jacquard machine, and also according to the number of picks inserted for each pattern card.

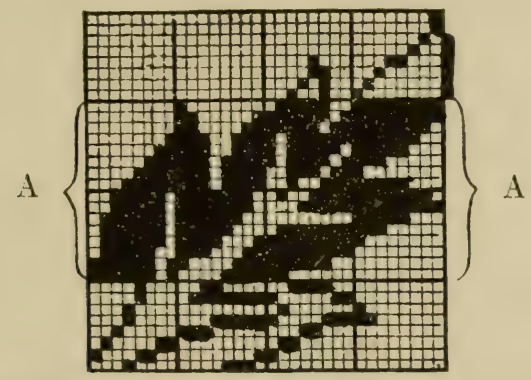

Fig. 549.- Part of an applied design en bloc for a damask fabric.

Care should be taken to obtain a suitable ratio between the number of threads controlled by each hook and the number of threads per inch; and also between the number of picks inserted to each card and the number of picks per inch in the fabric, in order to avoid causing the margin of the figure from appearing too coarsely stepped. The means adopted for binding the separate threads of warp and weft in a damask fabric is also detrimental to the formation of a clearly-cut and sharplydefined outline of the figure, particularly when the margins are not exactly parallel with the warp threads or picks of weft. This difficulty arises in consequence of the binding points occurring with such perfect regularity over the whole of the fabric that many of the hinding points encroach upon the extreme margins both of the figure and the ground in many places 
where they are quite unnecessary, thereby causing the figure and ground to blend one into the other, and thus producing an indistinct marginal outline.

"As it is impossible, however, excepting with diaper patterns, to ensure the "checking" or "locking" of marginal binding points uniformly, the warp and weft threads are therefore liable to slide out of their proper place from causes previously explained in $\$ 167$, and illustrated in Figs. 525 and 526.

The character of these defects will be clearly comprehended by a parailel reference to Figs. 549 and 550 ; and also by care-

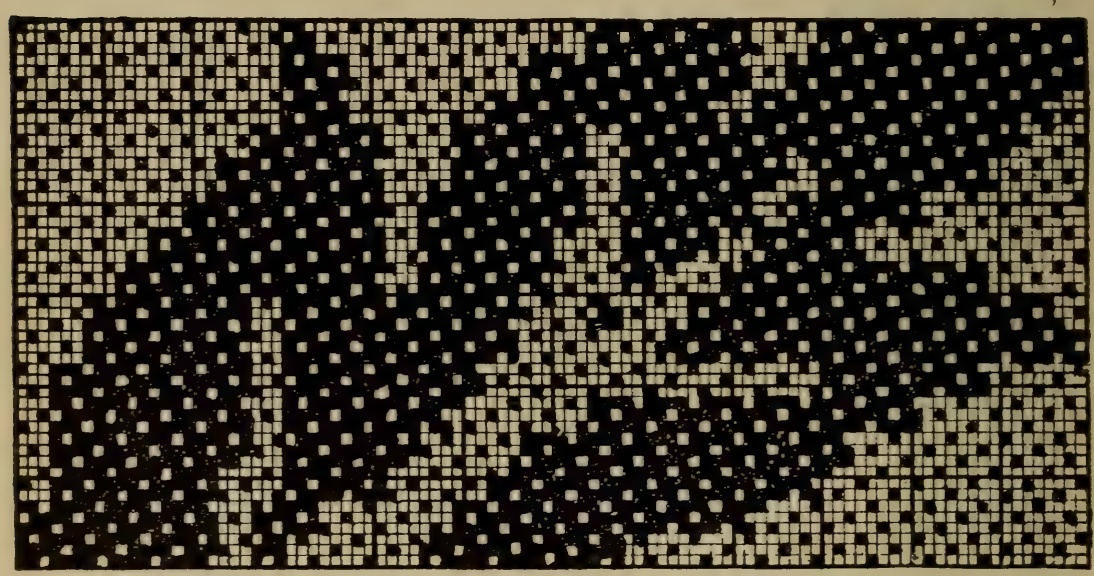

FIG. 549. - Showing structure of damask fabric with figuring as at A, Fig. 548.

fuily examining a damask fabric. The design represented in Fig. 550 indicates the manner in which the same portion of design, as that represented en bloc in Fig. 548, would be developed if it were to be woven by means of an ordinary Jacquard harness, having independent control of each warp thread and if a pattern card were employed for each pick of weft inserted, and also provided that the design were required to occupy the the same number of warp threads and picks of weft as that represented in Fig. 549. And although these designs are exactly similar in other respects, there is a marked contrast in their respective margins of the figure and ground of which the outlines in Fig. 550 have a much finer stepping and smarter 
appearance than those of Fig. 549 . It will also be observed in Fig. 550 that the binding points have not been allowed to encroach upon the margins of the figure and ground, excepting where they were absolutely necessary in order to prevent too long floats. Also opportunity has been taken to "check" wherever it was necessary, in order to preserve a clear outline by preventing a jagged margin.

$\$ 179$. The proper counts of design paper to employ for an applied damask design is not determined according to the ratio of warp threads and picks of weft per inch in cloth, as is the

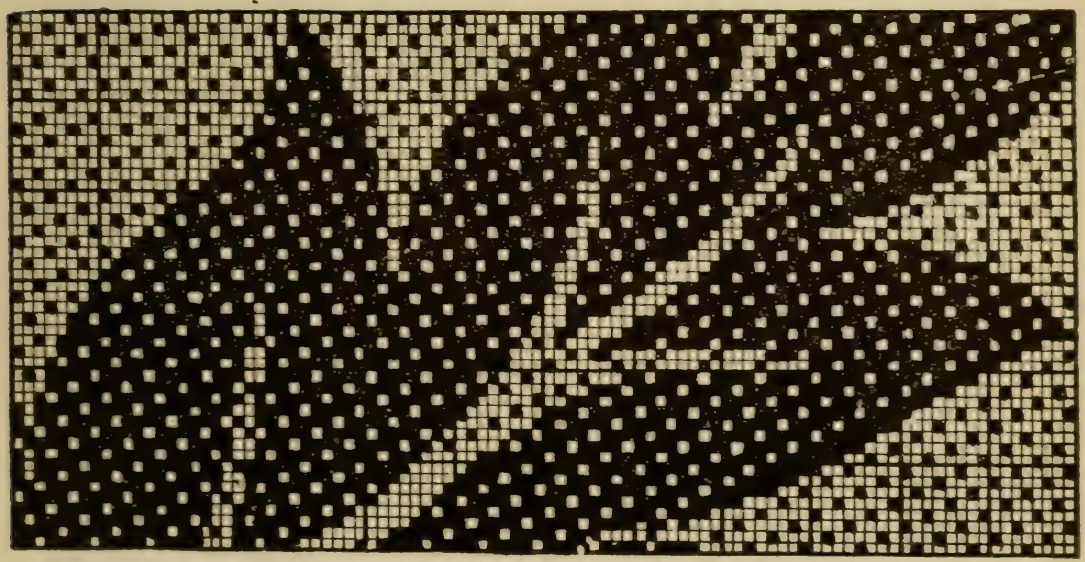

FrG. 550.-Showing structure of ordinary simple brocade fabric with figuring as at A, Fig. 548.

case for simple tabrics generally; but according to the number of groups of warp threads and the number of pattern cards representing one inch of cloth. Thus, assuming a design is to be made for a fabric containing 114 war'p threads per inch, with 3 threads grouped together, and 100 picks per inch, with 3 picks to each pattern card, the ratio of hooks and pattern cards per inch would be in the ratio of 38 to 33 respectively. Therefore, if the needles of the Jacquard, by which the design is to be woven, are eight rows deep, then-38 (hooks per inch) : 8 (hooks in a bar) : : 33 (cards per inch) : 7 (cards in a bar) nearly. In this case, therefore, the proper counts of 
design paper to use would be that ruled with $8 \times 7$ squares in each bar.

When damask fabrics are woven by means of the compound "presser" harness, and with more than two warp threads drawn through each mail eye, it is usual to employ what are termed "decked" mails, one form of which is illustrated (detached) in Fig. 537. Each warp thread passes through a separate division in the mail, whereby they are prevented from twisting around each other between the back rest of the loom and the heald eyes; and shedding by the healds is facilitated.

The weight of lingoes attached to the bottom of harness threads must be suited to the counts and strength of warp threads, and also to the number of threads drawn through each mail eye. Strong or coarse yarn, also mails controlling a greater number of warp threads, will require relatively heavier lingoes than are required for fine yarn, or for mails controlling a lesser number of warp threads.

With a compound "presser" harness, the same set of pattern cards may be employed to weave fabrics having a different number of warp threads and picks of weft per inch from that for which the pattern was originally intended. This is accomplished by increasing or reducing the number of threads in some or all of the harness mails, and also by inserting more or less picks for some or all the pattern cards. Care must be taken, however, if any of these courses are adopted, to maintain the same ratio between the number of Jacquard hooks and pattern cards per inch as that for which the design was originally set out; otherwise the pattern will be distorted. These advantages, howerer, are not possible with special damask Jacquard machines of the type described in $\$ §$ 176 and 177 .

$\$ 180$. Unlike that of brocade and similar fabrics of simple structure the stability of damask fabrics is entirely unaffected by the character of the design, by reason of the absolute uniformity of texture both in the figure and ground portions. This structural feature permits of the successful reproduction of almost any kind of pattern, whether of a geometrical or a floral character. 
In most fabrics of simple texture it is important that large masses of figure should be aroided with the object of preventing structural weakness; and also that the figure should be distributed over the surface as uniformly as possible to ensure uniform tension upon warp threads; but these considerations do not apply to damask fabries for reasons just stated.

The following is a comparison of the number of pattern cards and Jacquard machines required to produce a design repeating on the same number of warp threads and picks, to be woven (1) either by means of the compound "presser" harness or a damask machine; and (2) by means of an ordinary singlethread brocade harness.

Assuming, in the first case, that a Jacquard machine with 408 needles is employed-all of which are used in a full repeat of the pattern-and that each needle corresponds to five warp threads; also that the pattern repeats on 408 pattern cards, each of which represents three consecutive picks of weft, the total number of warp threads on which the design will repeat is $408 \times 5=2040$; and the total number of picks is $408 \times 3=1224$.

Now, in the second case, five similar Jacquard machines would be required, each with three times as many pattern cards as in the first case. Therefore, the number of pattern cards required would be $5 \times 3=15$ times as many, or a set of $408 \times 3=1224$ cards for each machine and a total of $1224 \times 5=6120$ cards, with a corresponding amount of time, labour and expense incurred in designing, cost of pattern cards, card-cutting, lacing and wiring. Over against these items, however, if a "presser" harness is employed, there is the extra cost of the heald harness and other incidental accessories : or, if a damask machine is adopted, the extra cost of this must be taken into consideration.

\section{Compound Damask Fabrics.}

$\$$ 181. Compound damask fabrics comprise two distinct varieties, namely, (1) those produced with an additional series of weft threads, and (2) those constructed with two distinct 
series both of warp and weft threads with the object of producing textures of greater strength, bulk and weight, whereby they are adapted more suitably for the specific purposes for which they are chiefly intended to be applied as, for example, curtains, hangings and furniture upholstering fabrics of that variety sometimes popularly described as "French tapestry". In both of these varieties the extra series of weft threads constitute "backing" picks only, with the primary object of imparting to the fabrics additional firmness and stability. These extra picks of weft play no part whatever in the scheme of decoration, as they consist of strong and full-bodied threads of linen with the object of ensuring a firm foundation texture which is not so liable to stretch.

In the second variety of these fabrics the extra series of warp threads is employed for binding or "stitching" purposes only, and, like the extra picks of weft, they play no part whatever in the development of the design. In fact, when viewed obversely, compound damask fabrics are, to all outward appearance, exactly similar to those of simple construction that are produced from only one series each of warp and weft threads : and it is only by close inspection that their compound structure is revealed. This close similarity of the two varieties of damask fabrics arises in consequence of the "backing" picks of weft being concealed so effectually at the back of the fabric, and also by being dyed the same colour as that of the "face" warp threads.

The warp threads of compound damask fabrics usually consist either of organzine silk, or else spun silk, of one colour; whereas the "face" picks of weft consist of "tram" silk of a different colour, to produce an agreeable contrast between the figure and ground portions of the fabric. Also, if an extra binding warp is employed, it should be of exactly similar material and of the same colour as the "face" or figuring warp threads; and each series of warp threads requires to be wound upon a separate warp beam in order to allow for the different rates of contraction by each series during weaving, and which are in the ratio of about 8 of the "face" warp to 9 of the binding warp, or 100 to ${ }^{1 \top} 2.5$ respectively. 
The warp threads are controlled in groups for the development of the pattern, and individually for the purpose of interweaving them into the body of the fabric in some definite order. Also, two picks of weft are inserted for each pattern card, namely, one face and one back pick alternately; thereby requiring a loom provided with two shuttie boxes at each end of the sley, a shuttle-box motion, and also a pick-and-pick picking motion.

$\$ 182$. The structure of a typical example of the first-named variety of compound damask fabrics is demonstrated by the

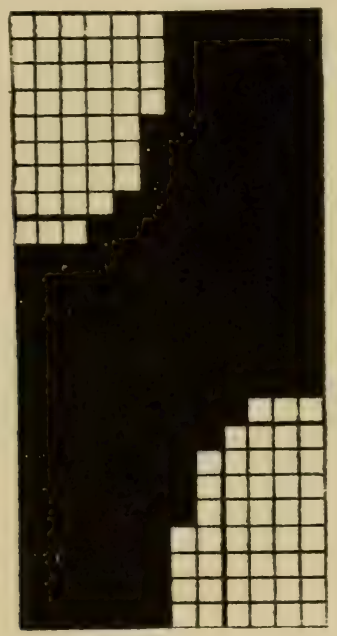

Fig. 551.-Part of block design for compound damask fabric, Fig. 536.

aid of designs and sectional diagrams of cloth as represented in Figs. 551 to 553, and of which Fig. 551 indicates a portion of a design prepared en bloc in the usual manner. Each vertical space on the design paper represents four warp threads, and each horizontal space, two picks of weft, namely, a face and a back pick.

The actual order of interweaving the warp and weft threads is represented in Fig. 553 (A) in which the black squares indicate warp threads raised over face picks, and shaded squares warp threads raised over back picks. In the figured portion of the 
fabric the warp is raised en masse on the face picks; but one warp thread in sixteen is left down in the manner indicated in Fig. 552, in order to bind the weft at the back of the fabric and thereby prevent it from floating too freely on that side.

In the ground portion of the fabric one warp thread in every eight is raised for the same picks, in order to produce an 8 -end weft satin binding weave on the face side. The warp threads are raised en masse over the back picks throughout; but one warp thread in eight is left down in order to produce an 8 -end weft satin binding weave on the reverse side of the fabric.

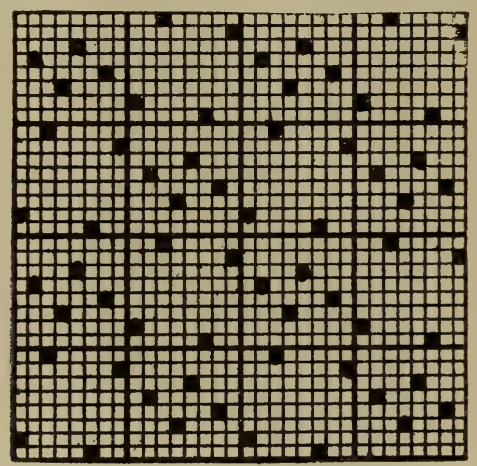

FiG. 552.-Plan of binding weave employed for binding the coarse back picks of weft in compound damask fabrics, Fig. 536.

The face weft may be bound in any desired manner when at the back; but care should be taken to select some method of binding that will ensure a uniform rate of contraction of warp threads; otherwise more tensile strain will be imposed upon some warp threads than upon others during weaving. Also, the binding points should be inserted only where the warp threads have been left down on the corresponding back picks, as indicated by the letters $\mathrm{H}$, in Figs. 553 (A) to 553 (D). A diagram representing a longitudinal section of the fabric as it would occur at E, Fig. $553(\mathrm{~A})$, is indicated in Fig. $553(\mathrm{~B})$; whilst transverse sections at F and G, Fig. 553 (A), are indicated in Figs. 553 (C) and 553 (D) respectively.

Compound damask fabrics of the second-named variety are 
scarcely distinguishable from those belonging to the firstnamed variety, in consequence of figuring warp threads and binding warp threads being exactly similar. On removing the
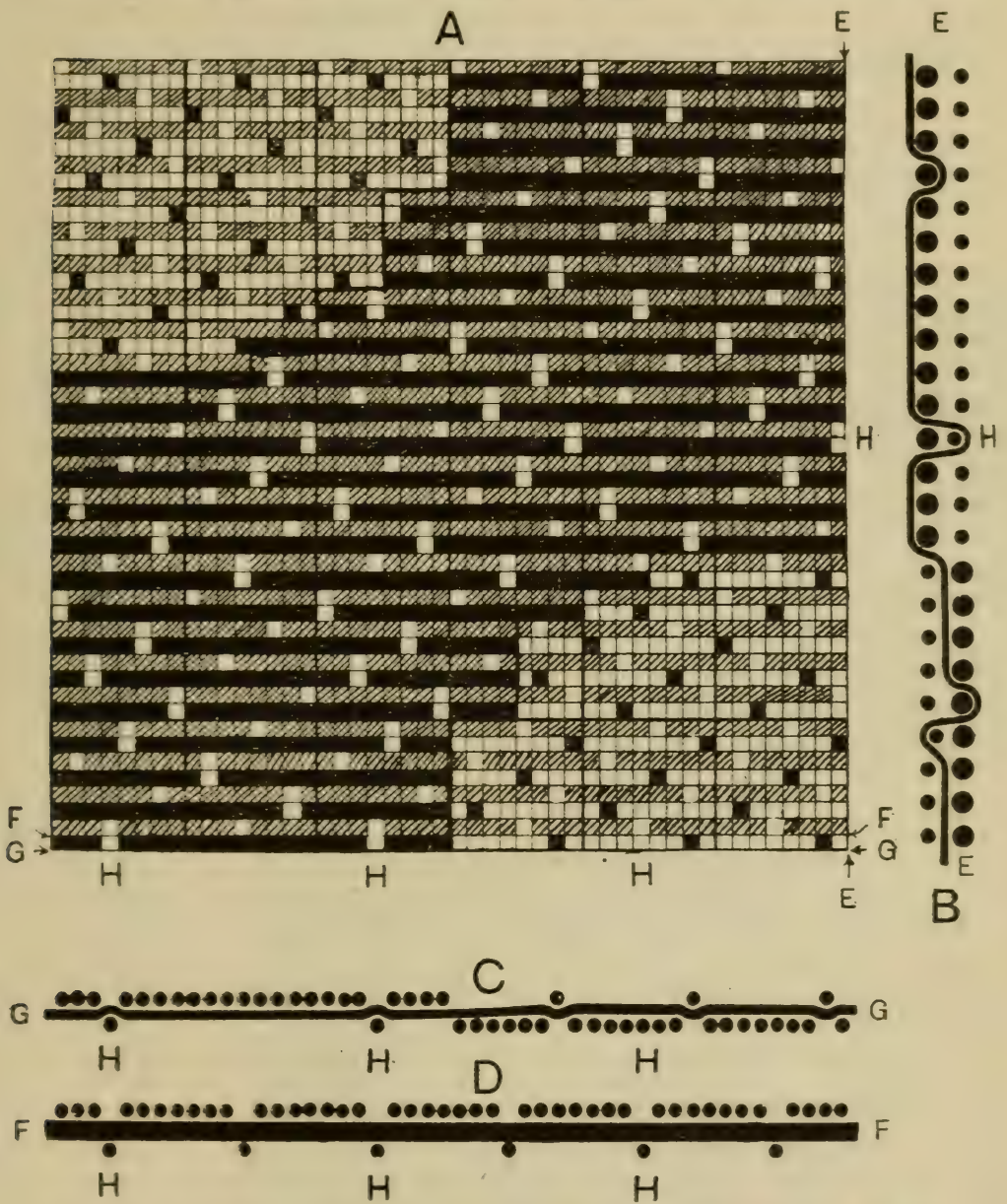

Fic. 553.- Showing actual structure of compound damask fabrics, Fig. 536.

threads from the actual fabric, however, the binding warp threads reveal a more wavy appearance than that of the figuring threads, due to their greater rate of contraction during weaving. In the example selected for description, there are four 
figuring warp threads, controlled by the same hook of the Jacquard machine, to one binding warp thread. These are drawn

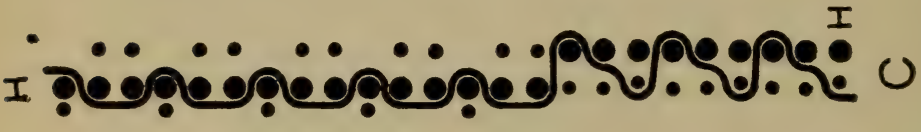 \\ 0

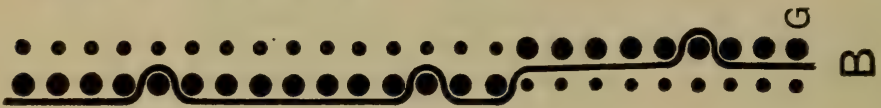
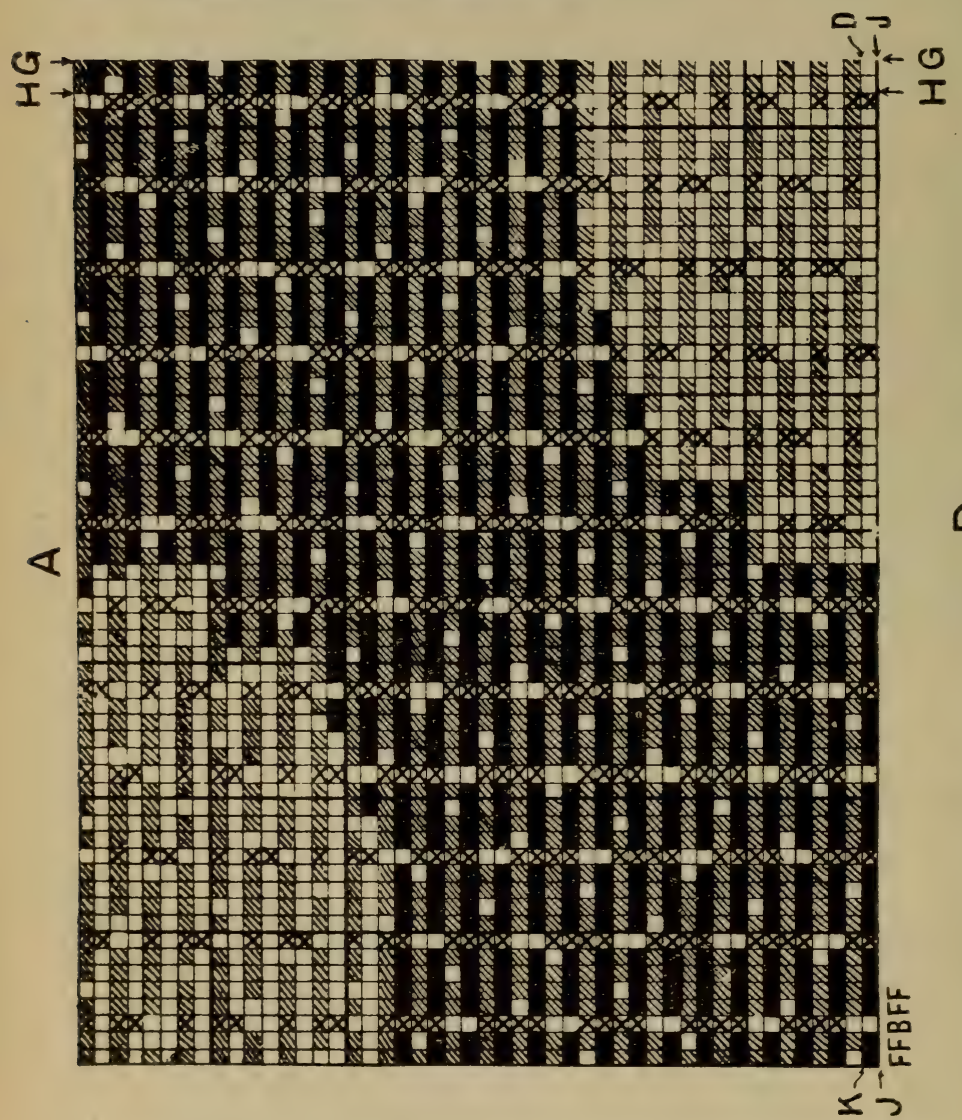

through the mail eyes of the shedding harness and between the dents of the reed, in the order of two face, one back and two 
face threads uniformly, as indicated by the letters FFBFF in Fig. 554 (A), which indicates the actual order in which the warp and weft threads interweave in the fabric for the portion of the block design represented in Fig. 551. Black squares in the design, Fig. 554 (A), indicate figuring warp threads raised orer face picks, and shaded squares indicate those threads raised orer back picks; whilst crosses indicate binding warp threads raised over both series of weft threads. Figuring warp threads are raised or left down, en masse, according to the design, for the face picks, which never interweave with figuring warp threads, as observed in the sectional diagrams, Figs. 554 (B and D).

When the back picks are inserted, all the figuring warp threads are raised en masse, excepting one in every eight which is left down in order to produce an 8-end binding weave with those picks. The binding warp threads and the face picks produce a 3 -end weft $\left(\frac{1}{2}\right)$ twill for the ground portion, and a 3 -end warp $\left(\frac{2}{1}\right)$ twill for the figure portion of the fabric. This object is effected by raising every third binding warp thread in the ground portion, and leaving down every third warp thread in the figure portion, in respect of the face picks only. But in order to prevent the risk of two separate and distinct cloths being formed in either portion of the fabric, the binding warp threads also interweave with the back picks in order to produce a 3 -end $\left({ }^{2}\right)$ twill throughout the fabric, as indicated in the design, Fig. 554 (A) and the sectional diagrams Figs. 554 (B, C, D and E). 


\section{CHAPTER XIV.}

\section{ALHAMBRA AND KINDRED FABRICS.}

$\S 183$. The term "Alhambra" is a descriptive trade name employed to distinguish a well-known type of textile fabrics comprising several varieties that are chiefly produced in relatively coarse and heavy cotton textures of an inferior and cheap class that are sold under a variety of fanciful names, and employed extensively as counterpanes or bed-quilts. Fabrics of this type assume various modifications, but they are all characterised by certain essential and distinctive features by which they may be distinguished from other types. As a general rule, they are characterised by a somewhat coarse and heavy texture of uniform structure, based upon the plain tabby or other elementary weave, to constitute a firm foundation texture with which is freely interwoven a supplementary series of figuring warp threads of coloured yarn, introduced for the sole purpose of embellishment. Thus, if the figuring.warp threads were removed entirely from an Alhambra fabric, there would still remain a perfect and substantial foundation fabric of simple texture devoid of decoration.

The foundation texture in Alhambra and similar fabrics is usually composed of relatively fine warp threads of bleached yarn, termed the binding or ground warp, which interweaves in a specific and uniform manner with relatively coarse and soft bleached weft, to give body and weight to the fabric. Figuring warp threads vary in counts and character in different fabrics, and are distributed uniformly either alternately with ground warp threads or else in pairs or groups of three or more in alternation with single ground warp threads, according to the particular style and quality of cloth. In the production of the more common variety of Alhambra fabrics, of which a (390) 
typical example is represented in Fig. 555, figuring warp threads of relatively fine yarn are passed together in pairs

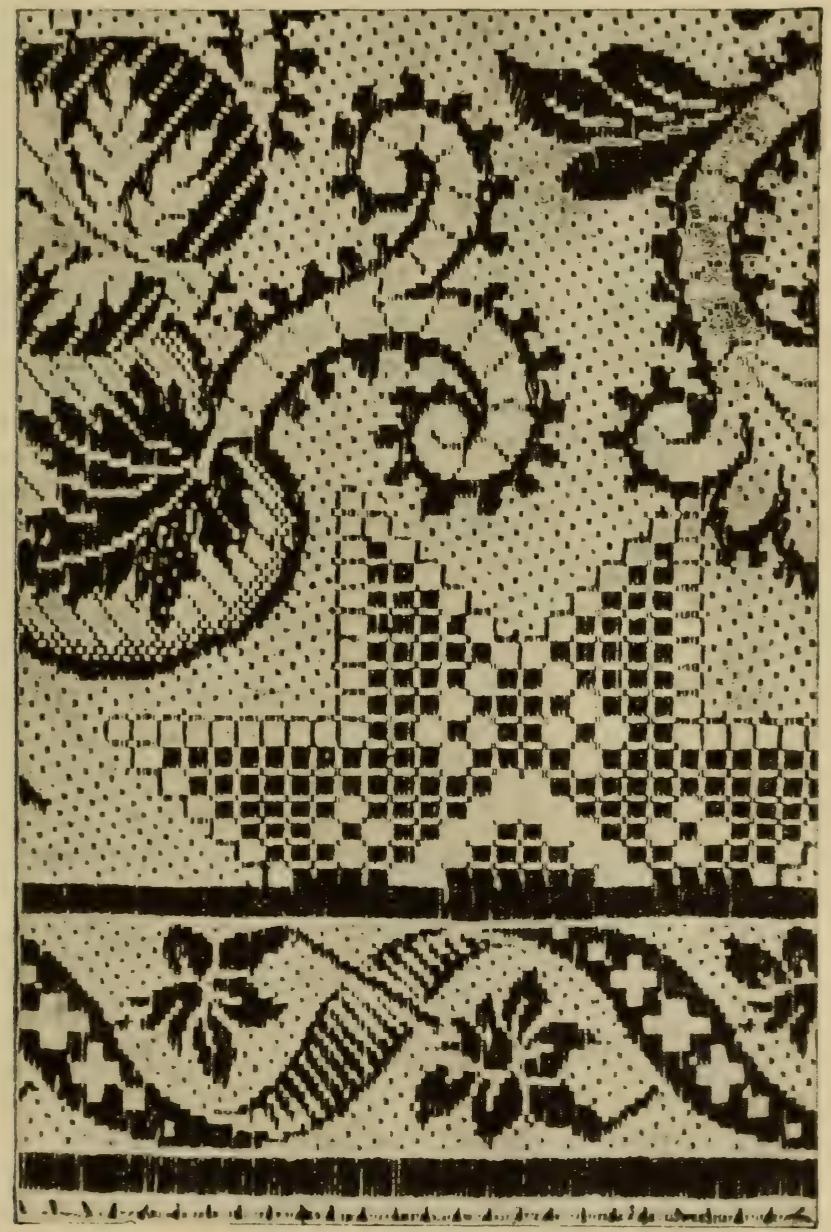

FIG. 555.-Alhambra counterpane fabric-fine texture.

through the respective mail eyes of a Jacquard harness by which they are governed; after which the respective pairs of threads are passed, along with a ground warp thread, between successive dents of the reed. The object of passing two or 
more figuring threads of finer yarn, instead of one thread of coarser yarn, through each mail eye of the figuring harness is to develop a more solid and compact figure, by the spreading of the several threads which more effectually cover the weft and thereby produce a fabric having a finer and superior appearance. Figuring and ground warp threads each require to be wound upon separate warp beams to permit of their different rates of contraction during weaving.

The interlacement of ground warp threads and picks of weft in a specific and uniform manner, in order to develop a foundation texture of simple construction, is a circumstance favourable to the economical production of Alhambra fabrics, as it enables ground warp threads to be governed by means of healds which, by operating in conjunction with a Jacquard harness, constitute a compound harness. The number of healds required is determined by the particular weave constituting the foundation texture, and may be of any practicable number which may be situated either before or behind the Jacquard harness, and operated by means of tappets.

When preparing an applied or working design on squared paper ready for card cutting, for an Alhambra fabric of the usual variety, as represented in Fig. 555, it is only necessary for the designer to consider the figuring warp without paying any attention whatever to the ground warp which interlaces with weft in a systematic manner. The development of an Alhambra design is demonstrated in Fig. 556, which represents an actual portion of the design for the example of cloth represented in Fig. 555. After painting up the design according to the required scheme of decoration, the figure and ground portions are then developed with suitable binding weaves of tabby, twill, satin, diamond or other appropriate weave, primarily with the object of stitching or binding the figuring warp threads to the foundation texture, in order to prevent them from floating too freely on both the face and back of the fabric ; and incidentally to impart character and interest to the design. By skilful treatment and a judicious selection of binding weaves suited to the different parts of a design, in order to emphasise its principal features and to subordinate others according to 
their relative importance, a design that would otherwise be insipid and characterless may be greatly improved.

$\$ 184$. With the object of conveying to readers who are unfamiliar with Alhambra fabrics a conception of their character

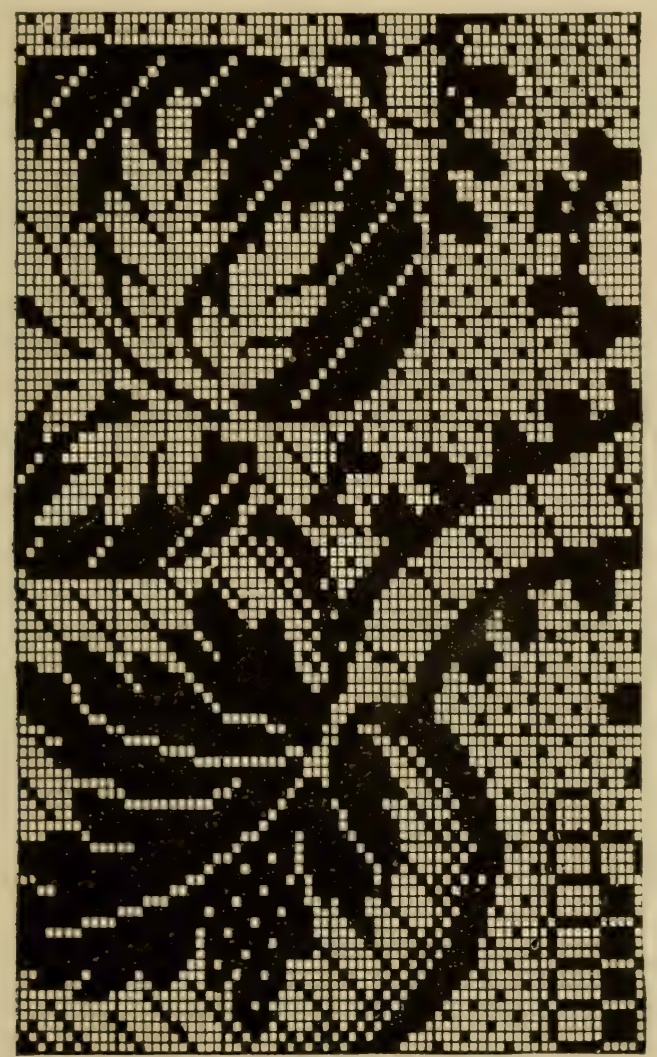

Frg. 556.-Part of an applied design for Alhambra fabric, Fig. 555.

and texture, the accompanying data and other particulars relating to the construction of the several examples of these fabrics will be helpful. Thus, the sample of cloth represented in Fig. 555 contains 29 pairs (58 single) of coloured figuring warp threads of 16 's ordinary twist; 29 white ground warp 
threads of $2 / 40$ 's yarn; and 24 picks of $2 \frac{1}{4}$ 's soft bleached weft per inch. During weaving, the contraction of the figuring warp is 10 per cent. and of the ground warp $22 \frac{1}{2}$ per cent. approximately, of the original warp length-i.e., 111 and 129 yds. of the respective warps would contract to $100 \mathrm{yds}$. in cloth.

The counts of design paper required for an Alhambra design is determined by the ratio of mail eyes contained in, say, one inch width of the Jacquard harness, to the number of picks per inch in the fabric. Example: The design for the cloth represented in Fig. 555 is to be reproduced in cloth by means of a 600's Jacquard machine, with hooks and needles each disposed in twelve long rows. The figuring harness contains 29 mail eyes per inch, and the cloth is to contain 24 picks per inch. The correct counts of design paper, therefore, is that ruled with 12 by 10 squares, as indicated in Fig. 556.

It is also important, at this stage, to direct attention to a disfigurement which is liable to occur in Alhambra fabrics. during weaving, and one that may be prevented if the necessary precaution is observed when preparing the design. The disfigurement referred to is that caused by binding warp threads lying athwart figuring threads, or else separating them (if two or more figuring warp threads pass through each mail eye of the Jacquard harness) instead of bing covered by them, when the latter are raised or depressed singly for binding purposes. This tendency arises in consequence of the figuring and ground warp threads that pass through the same dent of the reed being raised or depressed together, instead of oppositely, at the points where these blemishes occur.

This evil may be avoided by disposing, as far as practicable, all single binding points of the various binding weaves, both in the figure and ground portions of the design, so that all those in the figure portion will be disposed on the same "tab" or "shed" uniformly, and all those in the ground portion disposed on the opposite "tab" to that selected for the figure portion. That is to say, if warp threads are required to be raised singly, over only one pick at a time, those over alternate picks of weft should be, say, odd-numbered warp 
threads only; while those over intermediate picks should be even-numbered warp threads. Also, those threads that are left down for the alternate picks should be even-numbered threads, whilst those left down for the intermediate picks should be odd-numbered threads. The observance of this simple precaution, when preparing a design, permits of the operation of pattern cards being adjusted in relation to the shedding by the healds, to ensure that when figuring warp threads are either raised or depressed singly, for binding purposes, in the manner just described, they will interweave on the opposite "tab" or "shed" to that of their respective fellow-binding warp threads that are in the same dent of the reed, and so remove the cause of the evil consequences just indicated.

Vote.-In the technical phraseology of textile designers, the word "tab" (an abbreviation of "tabby," signifying the plain or calico weave) is used to denote both the odd series and the even series of a group of threads (when applied to either series separately), as the "odd tab" and "even tab"; which terms indicate that one line of a warp shed is formed by oddnumbered warp threads only for alternate picks of weft, and by even-numbered warp threads only for the intermediate picks of weft.

$\$$ 185. An example of another variety of thambra fabric which illustrates a slight departure from the more common variety described previously is that represented in Fig. 557. The only difference between these two varieties arises from tiguring warp threads of relatively coarse folded yarn being passed separately through each mail eye of the figuring harness, thereby causing the figure to appear coarser and in greater relief than if several figuring threads of finer yarn were passed together through each mail eye. This fabric contains 17 figuring warp threads of $3 / 14$ 's yarn; 17 ground warp threads of 20's T.; and 2.3 picks of $5 \frac{1}{2}$ 's bleached soft weft per inch; producing a comparatively light, open and soft texture, suitable for the summer season.

$\$ 186$. Another variety of Alhambra counterpanes, sold under the trade-name of "Trellis" quilts, is represented in Fig. 55.8. 
These constitute a distinct modification of that type of fabric, as they differ in several respects from those of ordinary construction. Fabrics of this class are characterised by a lattice or chequered figure, developed by means of a series of distinctive warp threads and picks of weft disposed separately at regular

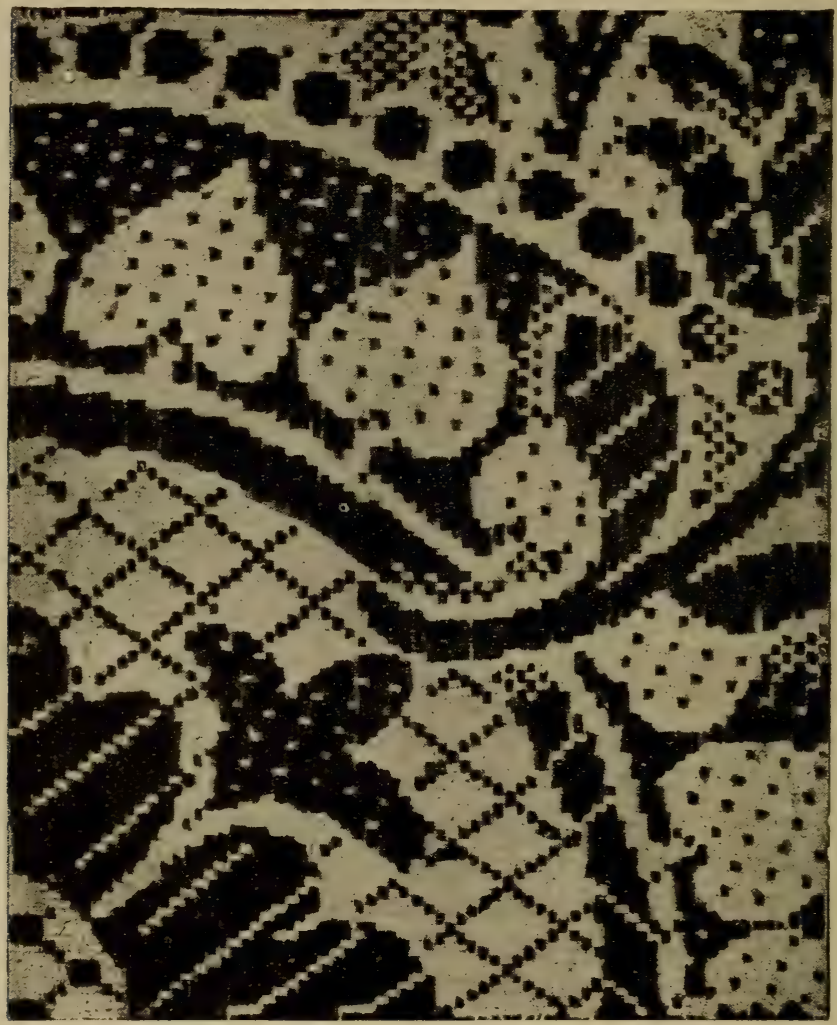

FLG. 557.-Alhambra fabric-coarse texture.

intervals apart to form squares; and, further, by the margin of figure and ground stepping in unison with those squares. In the present example of cloth the squares occur at intervals corresponding to the space occupied by six dents of the reed (and, therefore, six mail eyes of the figuring harness) and seven picks of weft. 
This object is attained by disposing coloured warp threads, of relatively fine yarn, in groups of three threads, through tive out of every six consecutive mail eyes; whilst a bleached folded thread is passed through every sixth mail eye, uniformly, to mark the vertical divisions; and also by inserting a distinctive pick of weft at intervals of seven picks, uniformly, to mark the horizontal divisions. Both kinds of figuring warp threads are delivered from the same warp beam; and only one kind of weft is employed. The foundation texture of "Trellis" quilts also differs slightly from that of ordinary Alhambra fabrics by having two fine binding warp threads situated between each group (or mail eye) of figuring warp threads, and contained in the same dent of the reed, in order to constitute one pair of threads. These warp threads are governed by means of four healds, and interweave in pairs for six out of seven consecutive picks of weft uniformly (in order to produce a foundation texture based on what is termed the double plain or tabby weave), but for every seventh pick of weft a warp shed is formed by raising all alternate ground threads and depressing all intermediate ones uniformly across the fabric. Therefore, the tappets by which the healds are actuated will not require to be constructed with more than seven picks to the circuit.

The pattern is developed by raising or leaving down figuring warp threads en masse, as required, in groups comprised by six or a multiple of six mail eyes, always either commencing or else ending with a thick white folded warp thread. When a figuring warp shed is formed, it remains open for six consecutive picks of weft, but for the seventh pick, uniformly, all figuring warp threads that were raised for the six previous picks are depressed, whilst those that were depressed are raised for that pick of weft, and so on continuously-hence the development of the trellis or lattice effect characteristic of this variety of Alhambra fabrics.

These circumstances not only greatly facilitate the preparation of designs for these fabrics, by dispensing with the necessity of introducing binding weares to prevent long floats of figuring warp threads, but they also provide an opportunity whereby 
considerable economy may be effected in the manufacture of such fabrics. Thus, by employing a Jacquard lifting cam constructed to keep the griffes raised, and the card cylinder out, for six consecutive picks, and then to change cards for the seventh pick, two pattern cards only could be made to serve for seven picks of weft.

Or again, still further economy could be effected by employing a special "twin-hook" or "terry" Jacquard machine of the type illustrated in Fig. 422, or its equivalent, constructed with two complete sets of hooks (with one set reversed, or turned away from the card cylinder) controlled by one set of needles, but with each set of hooks commanded by a separate set of griffe blades. The griffe blades would be operated in a contrary manner, so that one set would be raised for six picks and left down for one pick of weft uniformly. By adopting this course, only one pattern card would be required for every seven picks of weft.

The example of trellis cloth represented in Fig. 558 has required a harness containing 26 mails per inch to produce it. Five-sixths of the mails each contain a group of three coloured warp threads of 16 's T., and one-sixth of the mail eyes each contain one thread of $3 / 20$ 's bleached yarn. There are 52 ground warp threads (26 pairs) of $2 / 40$ 's bleached yarn, and 38 picks of 5 's soft bleached weft per inch. The contraction of figuring warp threads during weaving is 5 per cent., and of ground warp threads $22 \frac{1}{2}$ per cent. approximately of the warp length.

$\$ 187$. By means to be described presently, several efforts have been made to modify Alhambra fabrics so as to develop the pattern by dense masses of figure and ground, unbroken by binding weaves of any description : but in consequence of the additional cost of production, and structural imperfections, to which the modification gives rise, they are not successful. This modification is effected by inserting, at regular intervals, supplementary picks of fine weft of the same colour as figuring warp threads, and causing them to interweave with those threads on the tabby principle, to prevent them floating too freely. The extra picks of weft may be inserted separately at intervals of, say, 
four coarse picks, which would require the use of a loom furnished with a pick-and-pick picking motion, and a loom sley with two shuttle boxes at each end.

A more economical, though less satisfactory, method, howerer, would be to insert two consecutive fine binding picks in opposite tabby sheds of the figuring warp threads, at intervals of eight

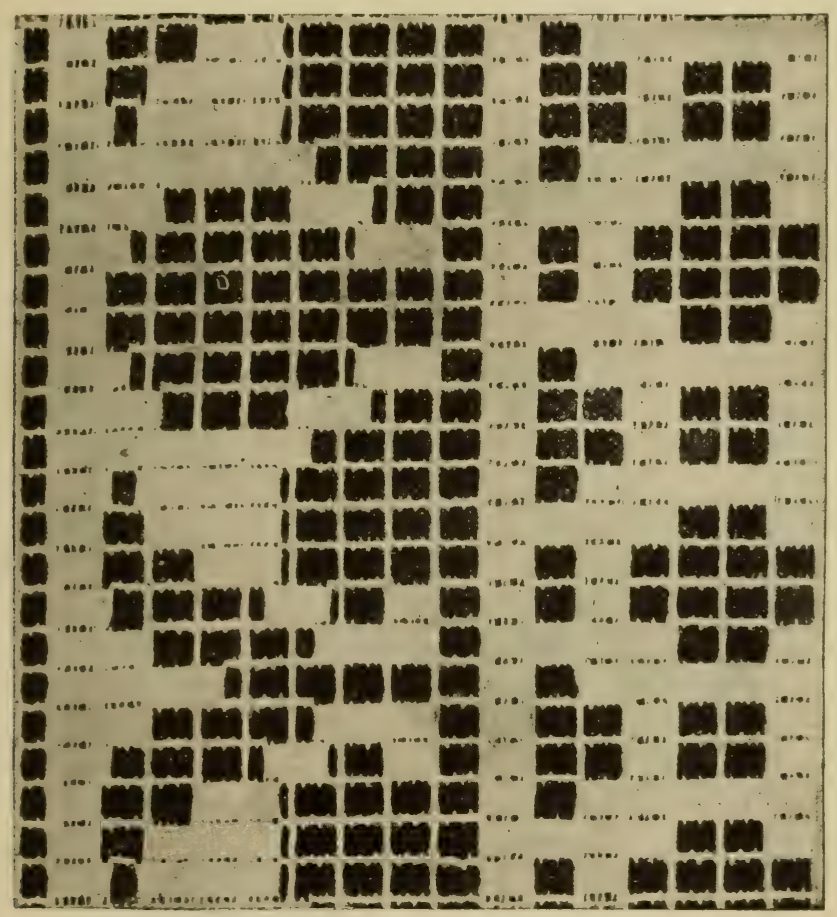

Frg. 558.-Modified structure of Alhambra fabric.

coarse picks; thereby requiring a loom with two shuttle boxes at one end of the sley only, and an ordinary picking motion. Whichever of these alternative methods is adopted, the shedding of figuring warp threads, for the fine binding picks only, may be more economically effected by a form of "presser harness," in which odd and eren-numbered harness threads respectively pass through two separate comber-boards. By forming knots on the harness threads, immediately above the comber-boards, 
and raising these alternately for consecutive picks of fine weft, the latter become interlaced with figuring warp threads on the tabby or calico principle, whereby the binding of those threads is effected mechanically, without having recourse to binding weaves, which frequently disfigure a design, and so tend to detract considerably from its artistic merit.

\section{Figured Repp Fabrics.}

$\S 188$. A variety of fabric bearing a somewhat similar char- acter both in appearance and construction to those of Alhambra fabrics, and also belonging to the same class of domestic goods, is sold under the description of "repp" quilts or counterpanes, of which an example is represented, photographically, in Fig. 559. These fabrics are characterised by two distinct series of figuring warp threads of different colours, which may either be of the same or different counts of yarn; and either one or two series of picks of weft: but they have no series of binding warp threads, as in true Alhambra fabrics. For this reason the degree of firmness of texture is entirely dependent upon the scheme of interweaving as predetermined by the design. Repp fabrics are further characterised by a freely floating figure, developed by warp threads only, and surrounded by a repp or transversely ribbed ground. The threads of both series of warp threads are arranged alternately with each other, and may be wound either upon the same, or upon separate warp beams. If wound upon the same beam, care should be taken, when preparing a design, to distribute the masses of figure and ground as evenly as possible, in order to ensure a uniform degree of contraction by both series of warp threads during weaving. Both series of threads may be displayed either on the face or back of cloth; but it is usual, and for structural reasons more economical, to display them separately, and not simultaneously, in the same parts of the fabric. By adopting this course, two fellow warp threads that are in the same dent of the reed will always interweave with weft in an exactly opposite manner, and therefore occupy reverse positions in cloth.

This circumstance is favourable to a more economical pro- 
duction of these fabrics by using a "twin-hook" or "terry" Jacquard machine of the type illustrated in Fig. 422, in which two reversed hooks, controlled by one needle, govern two fellow warp threads (one of each series) contained in the same dent of the reed. Thus, if a needle is pushed backward, one hook

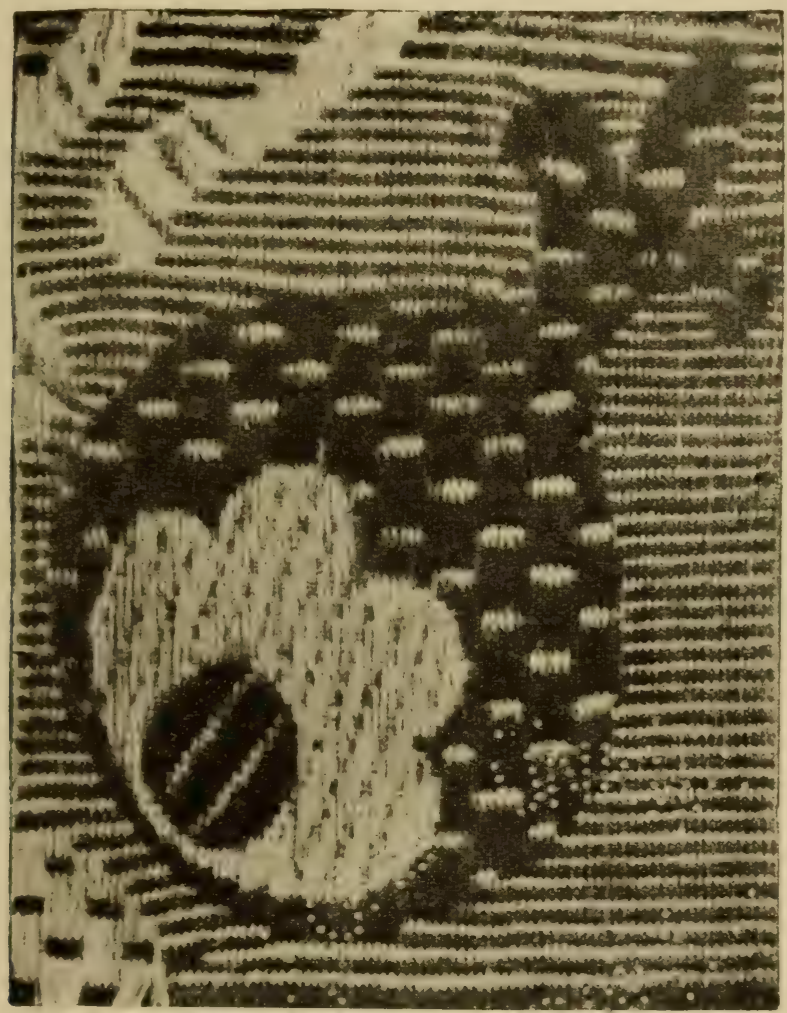

FiG. 559. - Compound figuied resp fabric.

is pushed off, and the other over, its respective griffe blade, and, per contra, if a needle remains in its normal position, the first hook will remain over, and the second hook off, its griffe, and thereby cause their respective warp threads to be governed in a contrary manner to each other in the manner described. From this it will be manifest that repp fabrics of the type 
under present notice display only warp threads on both sides of the fabric; whereas picks of weft are never exposed, but lie concealed between the two series of warp threads, thereby producing a perfectly reversible fabric in which the two different colours of figuring warp threads are exactly counterchanged.

In some fabrics of this class the repp ground filling surrounding the figure is developed by raising, in that part, warp threads of one series only for alternate picks of weft; and those of the other series only for intermediate picks of weft. In others, two consecutive picks are placed contiguously in alternate warp sheds, and a single pick in the intermediate sheds, to produce a coarse and fine rib alternately. A superior repp effect, however, is obtained by employing two different kinds of weft of considerable disparity in counts, and by inserting a fine and a coarse pick alternately with each other in reverse warp sheds, in the ground portion of the fabric. This course, however, would increase the cost of production, as it would require the use of a pick-and-pick loom to enable two shuttles to be propelled in succession from each end of the loom sley, besides incurring a higher rate of wages paid for weaving on such a loom.

The example of repp cloth represented in Fig. 559 contains 23 groups (69 threads) of warp threads of 20's coloured yarn; 23 warp threids of $3 / 16$ s bleached yarn; and 18 picks of 3 's bleached soit weft per inch. Each series of warp threads is wound upon a separate beam. The fne coloured warp threads contract 10 por cent., and the folved white threads 15 per cent., of the warp length, during weaving: 


\section{CHAPTER XV.}

\section{PIQUÉS OR TOILET WELTS: ALSO MATELASSE FABRICS.}

$\$ 189$. Piqué and toilet welt are optional terms that are variously employed to signify a compound type of fabric of which there are several modifications. But they are all char-

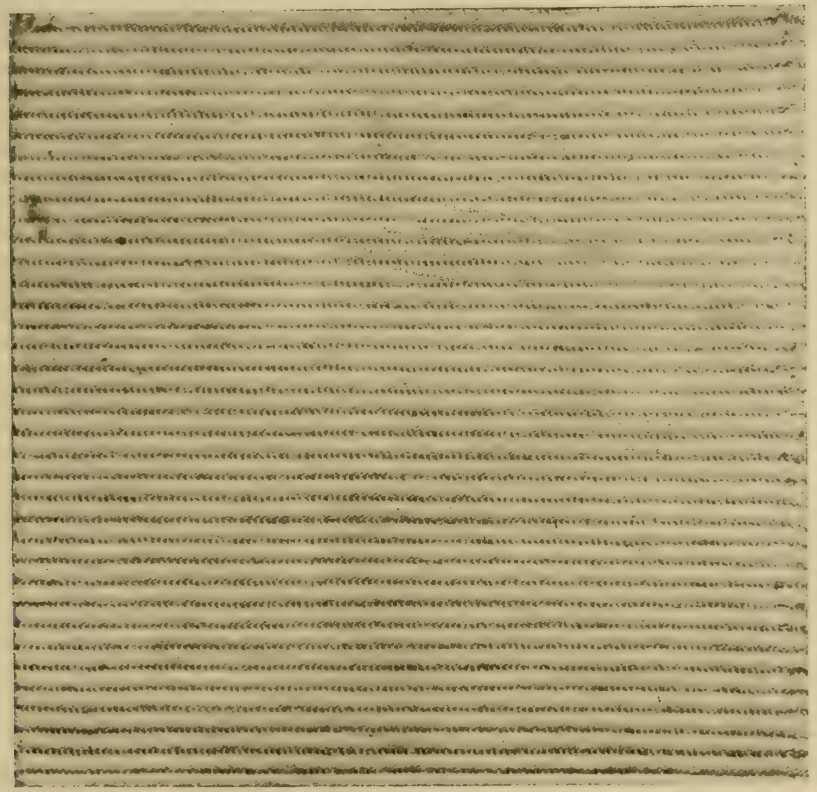

FrG. 560.-Piqué or toilet welt (note selvedge on one side).

acterised by more or less pronounced ridges and furrows producing a series of ribs, welts or cords with a surface tissue of the plain calico weave, and extending in parallel lines trans- 
versely across the width of the fabric, that is, in the direction of weft threads, as illustrated in Fig. 560 , which is a full-scale photograph of an example of a plain piqué fabric containing twelve ribs or welts per inch. In their general outward appearance, piqué fabrics bear a very close resemblance to Bedford cord fabrics as described in Chapter V., and for which they are often mistaken by those who are unfamiliar with the structure of these respective types of fabrics, which differ very materially in their structural features, but which may be easily distinguished when their distinctive characteristics are known. But even without such knowledge they may be identified unmistakably, if any portion of the selvedge is present, by remembering that the ribs or cords in a pique fabric extend transversely and therefore at right angles to the selvedges, whereas the ribs in a Bedford cord fabric extend lengthwise, in the direction of warp threads and, therefore, parallel with the selvedges.

$\$ 190$. Piqué fabrics are chiefly manufactured entirely of cotton woven in the grey or natural state and afterwards bleached. They comprise a variety of modifications in their structural details, and are also produced in a variety of different textures, chiefly according to the purpose for which they are intended as, for example, ladies' light summer holiday and yachting costumes, men's fancy vests, neckties and many other articles of personal attire and also for domestic purposes. They may be either of a perfectly plain or simple character, that is, with the ribs or welts of uniform width, between the furrows or " cutting lines," throughout: or they may be produced with variegated ribs of two or more different widths in order to obtain variety of decorative effect.

In fact, piqué fabrics admit of decorative treatment by almost any of the usual means of embellishment that are possible in weaving, namely: (1) by substituting coloured threads of warp or weft, or of both series of threads, in place of the ordinary threads of either series; (2) by introducing coloured threads of either series as extra or "crammed" threads which may either be interwoven into the fabric, or else obscured, in those parts where they are not required to be displayed on 
the surface for decorative effect. Or, again, the extra threads may be allowed to float quite freely, at the back of the cloth, when not required on the face, and afterwards to be cut away as waste material ; (3) by the development of ordinary brocade or floating figures of warp or weft threads; (4) by the development of what is technically described as "toilet" figuring or "quilting," in which the calico surface' tissue of the fabric is "quilted" and left somewhat free or bag-like, in the figured portions of the fabric, in order to develop a more or less raised or embossed figuring as exemplified in "toilet" quilting fabrics of the type described in the following chapter; and (5) by introducing gauze or "net-leno" stripes of crossweaving; as: well as by the combination of any two or more of the several methods just indicated.

The peculiar construction of pique fabrics, however, imposes certain restrictions in the choice of suitable designs that may be successfully executed in fabrics of that type which permit of embellishment of a very simple character only. Such designs should, therefore, consist essentially of rectilinear figures only, as piqué fabrics are entirely unsuited for the successful development of curved lines, floral or other elaborate decoration.

$\$ 191$. Piqué fabrics are composed essentially of two separate and distinct series of warp threads in combination with two and, in some examples, three series of weft threads, according to their special modification. The two series of warp threads are technically termed "face" and "back" threads respectively, according to their respective functions and the relative positions which they normally occupy in cloth. They are employed in the proportion of two "face" warp threads to one "back" warp thread, and are drawn through the eyes of the shedding harness and between the dents of the reed in the order of one "face," one "back" and one "face" warp thread, uniformly. Face war'p threads are of finer counts of yarn and contract more, during weaving, than back war'p threads, which latter require to be of greater strength than face threads since they are submitted to a higher degree of tension when in the loom. For this reason, therefore, and also because of the different rate of contraction between the face and back warp threads, 
during weaving, each series of threads require to be wound upon a separate warp beam.

Piqué fabrics of lighter texture and inferior quality are usually woven with weft of the same counts of yarn throughout; but those of superior quality and stronger and heavier texture are produced from weft of two different kinds: one being of relatively fine counts, and another of coarse counts of yarn. The different series of weft threads are technically termed "face," "cutting," "wadding" and "back" picks, according to their relative disposition in the cloth. Face picks, of relatively fine counts of yarn, interweave with face warp threads on the plain or tabby principle, to produce the surface tissue of plain calico cloth. Cutting picks, of the same kind of weft as the face picks, produce the "cuttings" or furrows between the ridges of the ribs or welts. Wadding picks are sometimes inserted for the purpose of increasing the prominence of the ribs and also to produce a fabric of greater strength, bulk and warmth; whilst "back" picks are sometimes employed to increase the weight, strength and firmness of texture; but these picks are never employed excepting in conjunction with "wadding" picks also.

Face picks and "cutting" picks invariably consist of the same kind of weft inserted by the same shuttle. Wadding picks also are sometimes of the same kind of weft as the face and cutting picks, thereby requiring a single shuttle-box loom only to produce piqué fabrics of that variety. For piqué fabrics of superior quality, however, it is usual to insert wadding picks of coarser and softer spun yarn than that which is employed for the face and cutting picks, in order to impart more body and stability to the fabric. This procedure, however, necessitates the employment of a check loom having at least two shuttle boxes at one end of the sley, thereby increasing the relative cost of production, not only in respect of capital outlay, but also in the higher rate of wages paid for weaving on multiple shuttle-box looms. If backing picks also are inserted in these fabrics, they are usually of the same kind of weft (and inserted by the same shuttle) as the wadding picks, consisting of soft spun roving weft of coarser counts of yarn than that of the face and cutting picks. 
Some piqué fabries of very inferior quality and light texture have only one cutting pick inserted to form the furrows of the ribs, and are entirely devoid both of wadding and back picks. Most piqué fabries, however, and especially those of superior structure and quality, are constructed with two cutting picks to form the furrows, and with one, two or more wadding picks inserted in each rib, whilst other varieties are constructed with backing picks also.

\section{Plain Piqué Fabrics.}

$\$$ 192. Plain piqué fabrics may be woven in any ordinary type of loom furnished with a heald harness which may be controlled either by means of tappets or a dobby; but if their embellishment exceeds the practicable limitations of a heald harness, it will be necessary to employ a Jacquard harness to control the back warp threads only, for figuring purposes; whilst the face warp threads may be controlled by healds, as in the production of toilet quilting fabrics of the type described in Chapter XVI. Or else it may be necessary to govern both the face and also the back series of warp threads, by means of a Jacquard harness, according to the particular character of the designs. If a heald harness is employed, this usually consists of a set of six healds, namely, four healds at the front, coupled together to constitute two pairs, as in a plain calico loom, to control the face warp threads; and two healds at the back of those to control the back warp threads, with both series of warp threads drafted through the eyes of the healds and between the dents of the reed in the order of one face, one back and one face warp thread, and passed together through the same dent of the reed uniformly, in the manner indicated in the drafting chart, Fig. 561.

The structure of the most elementary example of a plain one-shuttle piqué fabric, devoid both of wadding and backing picks, is indicated in the design, Fig. 562, whilst a longitudinal section of the cloth reproduced from that design is represented in Fig. 563. The design for this example is complete on three war'p threads only (i.e., one face, one back and one face thread) 
and four picks of weft, of which three constitute face picks to produce the crown of each rib or welt, and one, a cutting pick,

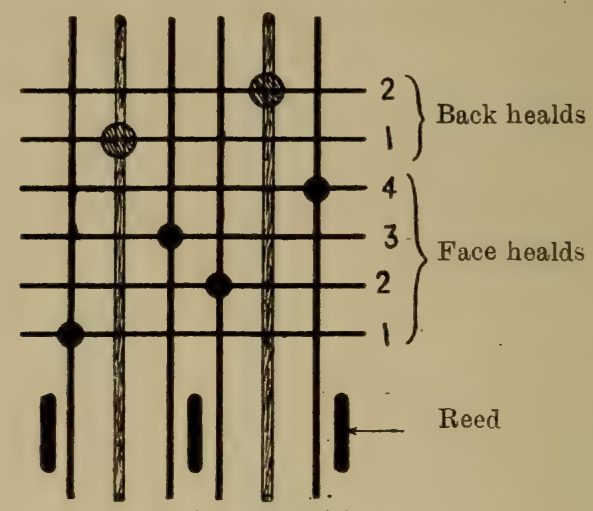

Frg. 561.-Draft for piqué fabrics.

to form the furrow between two ribs. It will be observed that only the face warp threads interweave with face picks of weft,

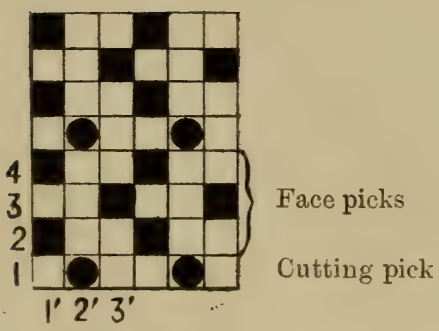

- Frg. 562 .

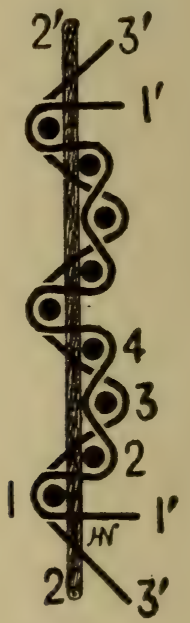

FIG. 563.

to produce a surface texture of the plain calico weave, whilst all back warp threads remain down, for those picks. But when a cutting pick is inserted, all face warp threads are depressed 
and all back warp threads are raised, whereby the greater tension of the atter causes the cutting picks to be pulled down into a lower plane than the face picks, and thereby create the transverse furrows or channels in the fabric between the ridges forming the ribs. Another example of a piqué fabric of simple structure contains four face picks, to produce relatively wider ribs or welts, as indicated in the design, Fig. 564, and of which Fig. 565 represents a longitudinal section.

$\leqslant$ 193. The next further development in the structure of

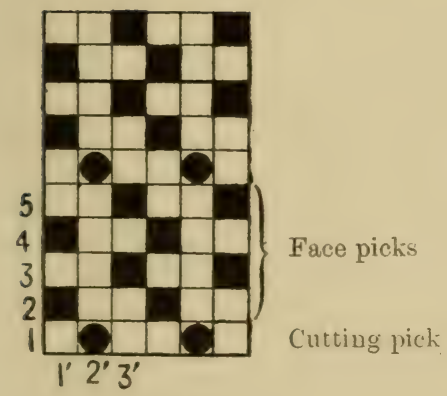

FIG. 564.

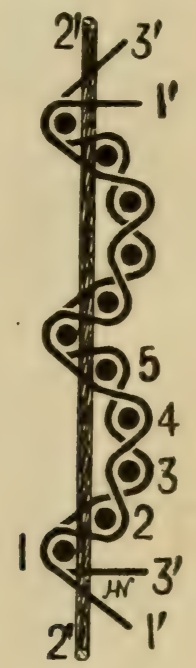

FIG. 565.

plain pique fabrics is effected by introducing one, two or more wadding picks of weft which never interweave with any warp threads, but simply lie quite straight and free between the surface tissue and the back warp threads, thereby increasing the relative prominence of the ribs and also the weight and bulk of the fabric. Any number of wadding picks may be inserted in each rib according to the prominence which it is desired to give to the ribs. A design for a pique tabric containing only one wadding pick in each rib is given in Fig. 566, which repeats on six picks, namely, one cutting pick, four face picks and one wadding pick, as represented in the cloth section, 

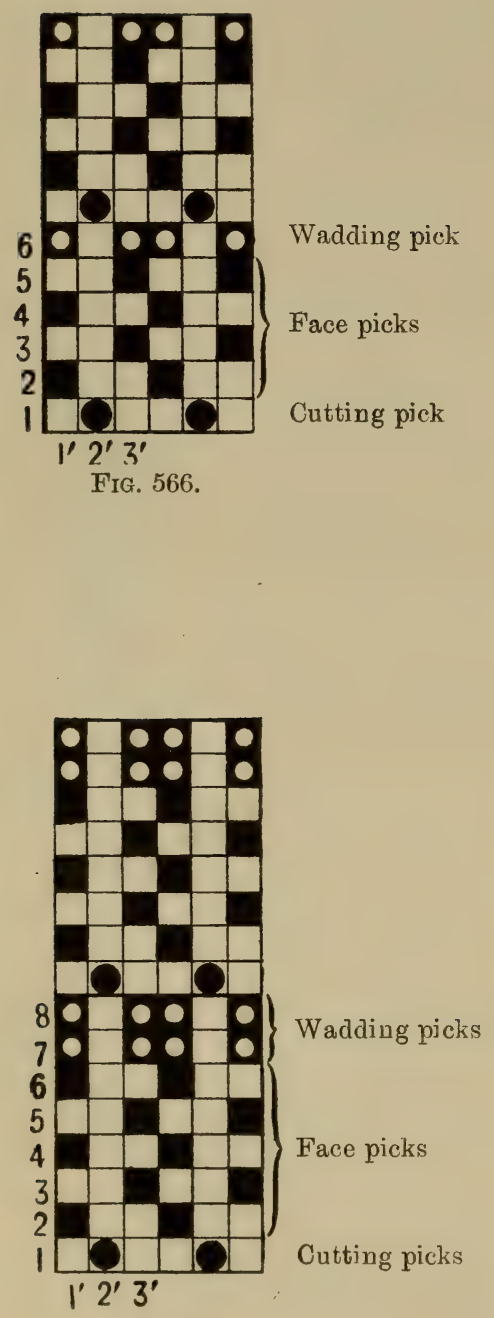

FIG. 568.

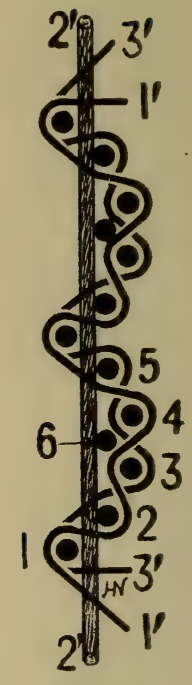

FIG. 567.

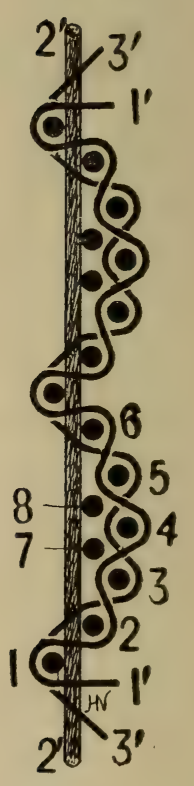

Frg. 569. 
Fig. 567. In all other respects this example is similar to the previous one, whilst a fourth example of a piqué fabric, of which the design and cloth section are given in Figs. 568 and 569, repeats on eight picks inserted as follows: one cutting pick, five face pioks and two wadding picks, all inserted by the same shuttle.

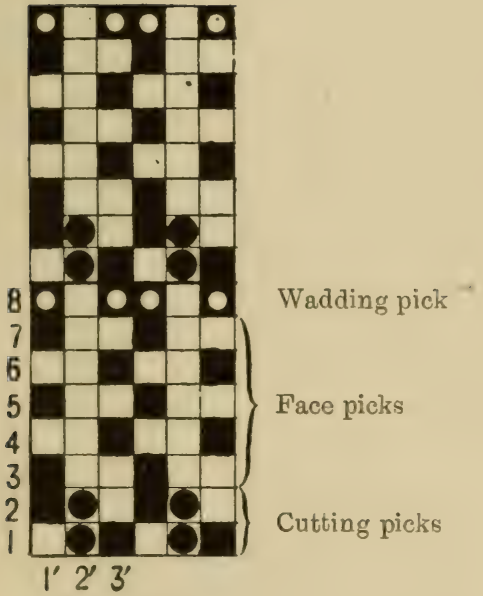

Fig. 570.

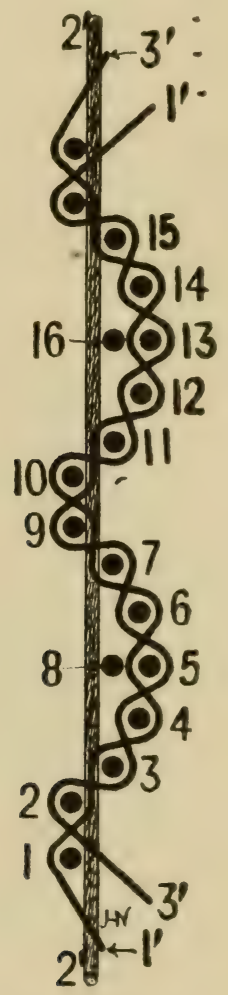

FIG. 571.

The four examples of pique fabrics just described contain only one cutting pick each to form the furrows between the ribs, but the majority of piqué fabries have two eutting picks inserted, in order to produce deeper and better defined furrows. Fig. 570 is a design for a piqué tabric containing eight picks to each rib, with two cutting picks, five face picks and ove 
wadding pick, as represented in the cloth section, Fig. 571, from which it will be seen that the continuity of the plain calico weave is broken by inserting the second cutting pick and first face pick of each rib in the same face warp shed. By adopting that procedure the repeat of the pattern is confined to eight picks only; but by continuing the plain weave through-

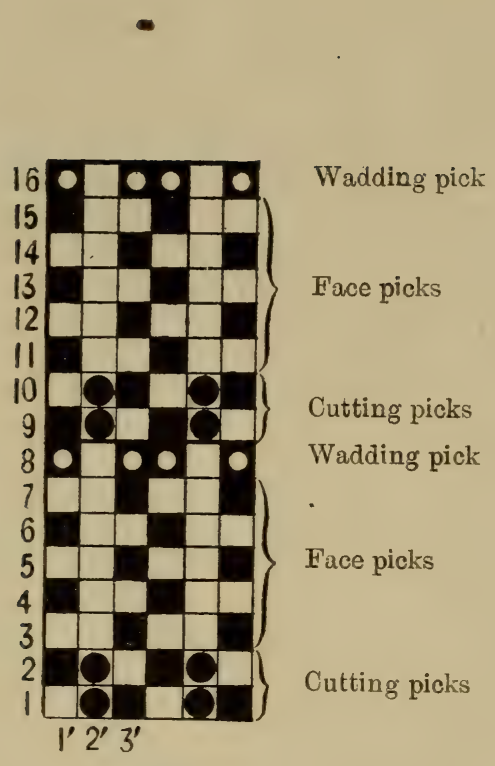

FIG. 572.

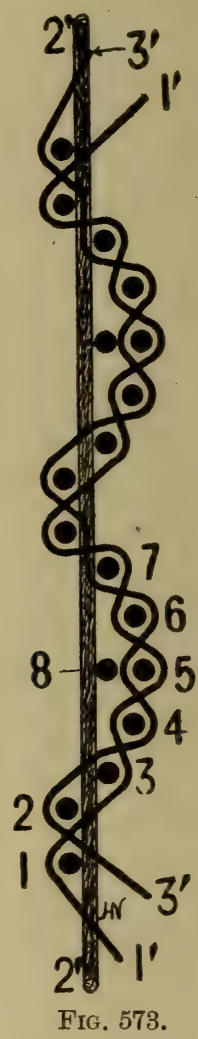

out, the complete repeat of the working design would comprise two ribs and, therefore, sixteen picks, as indicated in the design, Fig. 572, and also in the sectional diagram of the cloth, Fig. 573.

$\$ 194$. An important feature in the construction of piqué fabrics is the particular manner in which the cuttings or 
furrows, between the ribs or welts, are produced. Thus, if the furrows are produced by inserting two cutting picks, these may be interwoven according to two optional and distinct methods in order to develop what are described as "coarse"

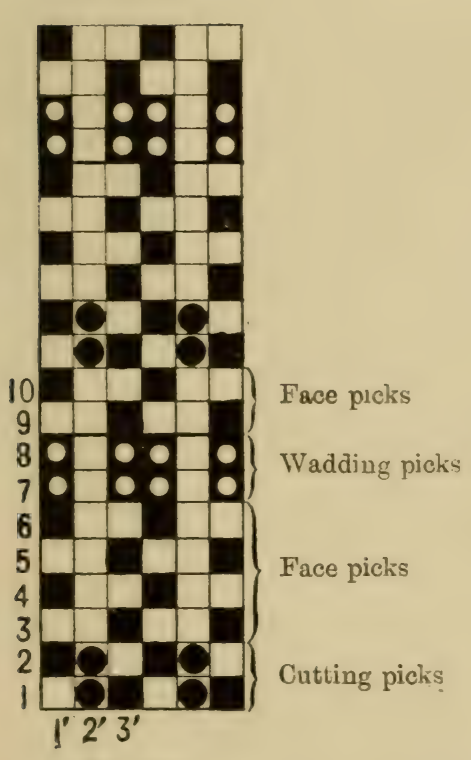

Fig. 574.

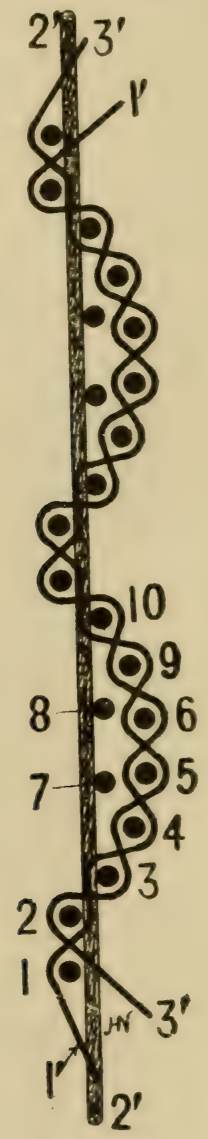

FIg. 575.

cuttings and "fine" cuttings respectively. If "coarse" cuttings are adopted, the furrows are relatively broad and shallow ; whilst "fine" cuttings produce furrows of deeper and sharper delineation which, for reasons to be stated presently, imparts to the fabric a very superior effect. 
A design for a piqué fabric with "coarse" cuttings is given in Fig. 574, whilst a longitudinal section of the cloth produced from it is represented in Fig. 575. In this example, each rib or welt contains ten picks of weft inserted in the following rotation, as indicated both in the design and sectional diagram,

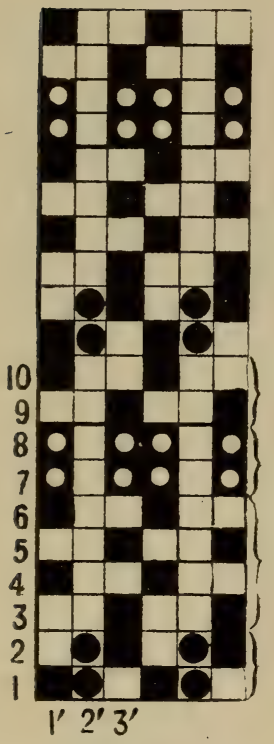

FIG. 576.
Face picks

Wadding picks

Face picks

Cutting picks

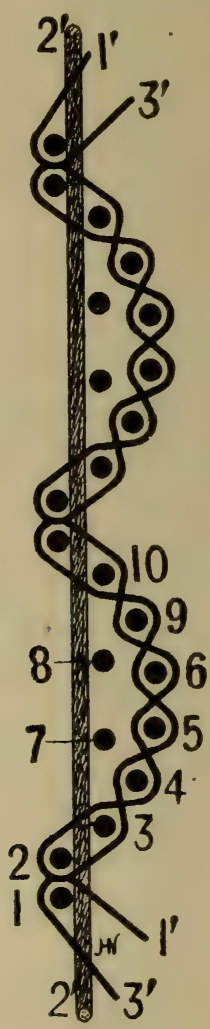

FIG. 577.

namely: two cutting picks, four face picks; two wadding picks and two face picks. (It should be observed at this stage, for the general guidance of students, that wadding picks should not be inserted until at least four face picks have followed the cutting picks of each rib. Otherwise, the wadding picks would prevent those face picks that follow them from approaching 
near enough to the preceding cutting picks, thereby involving the risk of producing "cracks" or "frets" immediately after each furrow or cutting.) On examining the sectional diagrams, Figs. 573 and 575 , it will be observed that the cutting picks simply form a continuation of the plain calico weave which forms the entire surface tissue of the fabric. Hence, if the back warp threads were severed and withdrawn entirely away from the fabric, the surface tissue in both of these examples would still remain a perfect piece of plain calico fabric just as if it had been produced as such, independently, in a plain calico loom.

The construction of a piqué fabric produced with "fine" cuttings is indicated in the design, Fig. 576, whilst a section of the cloth produced from it is represented in the diagram, Fig. 577. The only difference between this and the previous example is in the relation of the cutting picks and the contiguous face picks on each side of them. Thus, instead of continuing the plain calico weave unbroken, throughout the entire surface tissue of the fabric, the two cutting picks in the present example are, respectively, inserted in the same face warp sheds as the contiguous face picks, albeit they are each inserted in reverse face warp sheds to each other, as indicated both in the design and diagram. By adopting this simple expedient, the ribs or welts are thereby more free to close together and thus produce narrower, deeper and finer cuttings or furrows between them.

\section{Backed Piqués.}

$\$ 195$. The next stage in the development of plain piqués is to insert backing picks of weft which, as stated in $\$ 191$, are only employed in conjunction with wadding picks for the purpose of increasing the weight, strength and firmness or stability of the fabric. As these back picks are visible only on the back of the cloth, and bear no evidence whatever, on the face, of their presence, a backed piqué may only be distinguished from the simple "loose back" variety by its distinctly different appearance when viewed on the reverse side, as observed in Fig. 578, which shows the obverse and reverse sides of the 
same fabric, at A and B respectively, after bleaching. This fabric contains 16 picks in each rib, with 10 face and 2 cutting picks of fine weft; 2 wadding and 2 back picks of coarser weft. During weaving, these are inserted in the following rotation: 2 cutting, 4 face, 1 wadding, 1 back, 6 face, 1 wadding and

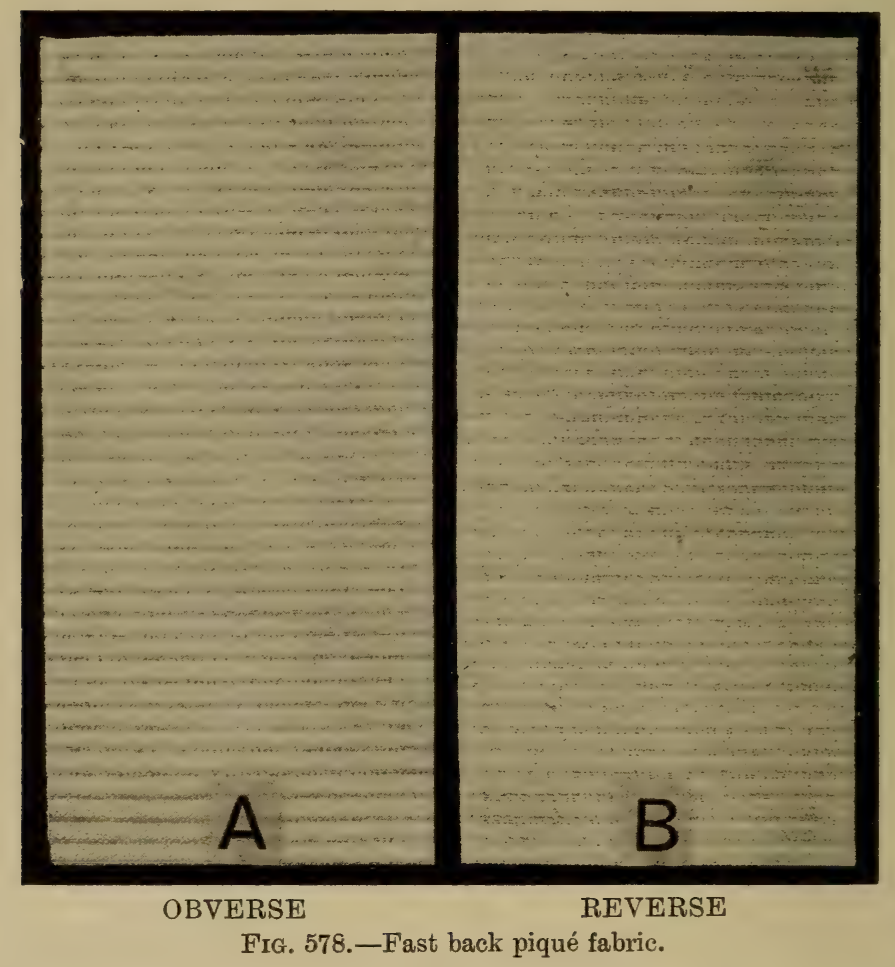

1 back pick, as indicated in the design, Fig. 579, and the section of cloth, Fig. 580, which demonstrate the construction of this variety of piques. Instead, however, of inserting a wadding and a back pick in immediate succession, as indicated in the design and section of this fabric, both of the wadding picks and also both back picks may, respectively, be inserted in succession during weaving; but the method indicated effects a better distribution of those picks in the fabric. 


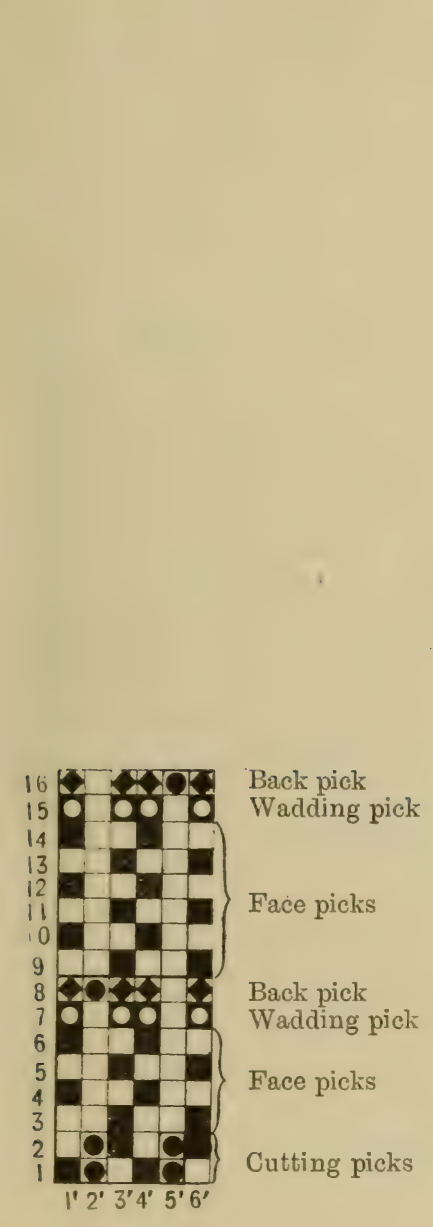

FIG. 579 .

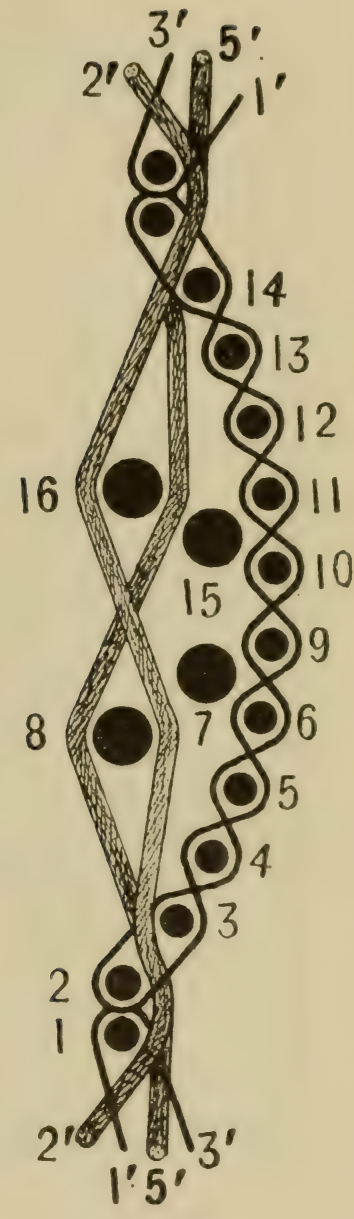

Fig. 580 .

\section{Decorative Effects in Piqué Fabrics.}

$\$ 196$. The simplest departure from the plain-ribbed piqué, with the object of producing decorative effects, is exemplified in the development of variegated ribs of two or more different widths, as illustrated in Fig. 581. The variegation of the 
ribs may be carried to any degree without departing from the general principles governing the construction of piqués, but simply by increasing or reducing the number of picks according to the relative width of ribs desired.

Other means of decoration, as briefly indicated in $\$ 190$, are illustrated in Figs. 582 to 593. These examples will serve to demonstrate the wide and almost unlimited scope which

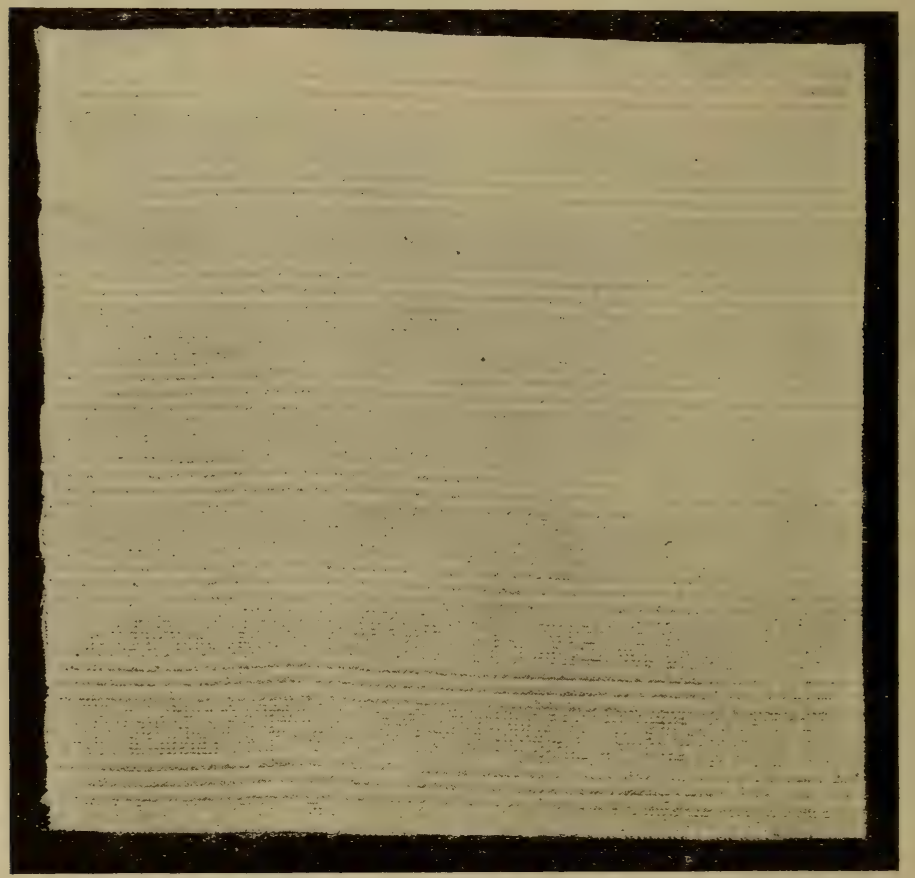

Fra. 581.-Piqué fabric with variegated welts or ribs.

the decoration of piques affords to designers in the creation of very beautiful, though simple effects, with comparatively little cost or artistic effort. Thus, Fig. 582 is an example of a simple piqué decoration obtained by interweaving two fine picks, of blue and red weft, in the crown or ridge of six ribs in succession and then leaving an interval of six plain ribs. Thus, two picks of red weft are inserted in succession, but in the same face warp shed, in three alternate ribs; whilst 


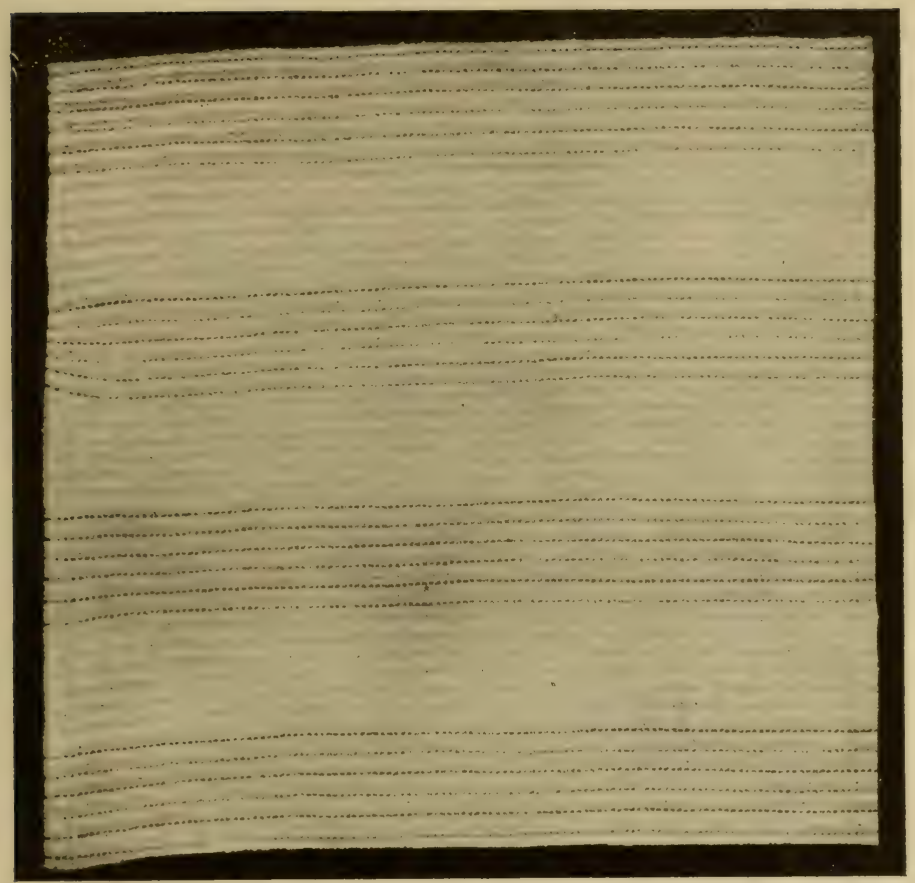

FrG. 582.-Piqué fabric embellished with coloured "face" picks of weft.

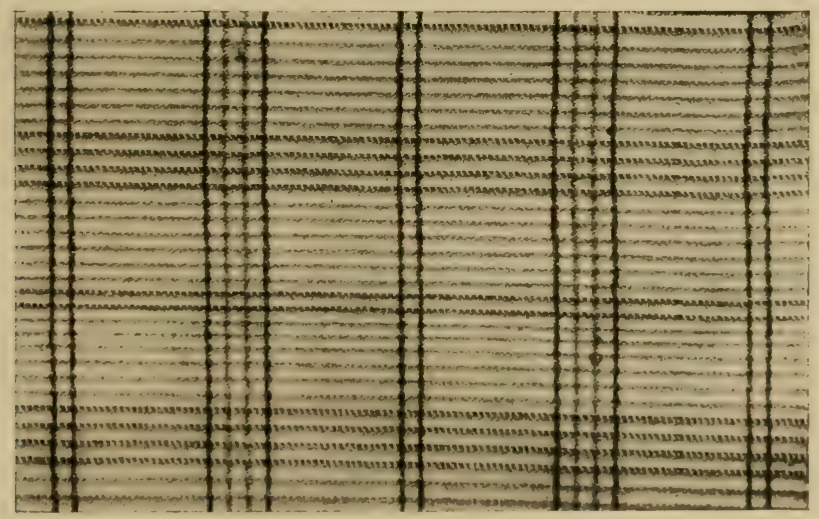

F'rg. 583.-Piqué fabric embellished both with coloured face warp threads and "cutting" picks of weft. 
two picks of blue weft are inserted, in the same manner, in the three intermediate ribs, thereby requiring a loom furnished with four shuttle boxes on one side, namely, one for the face and cutting picks; one for the wadding and back picks; and one each for the red and blue picks of weft.

Fig. 583 illustrates the development of a check pattern by

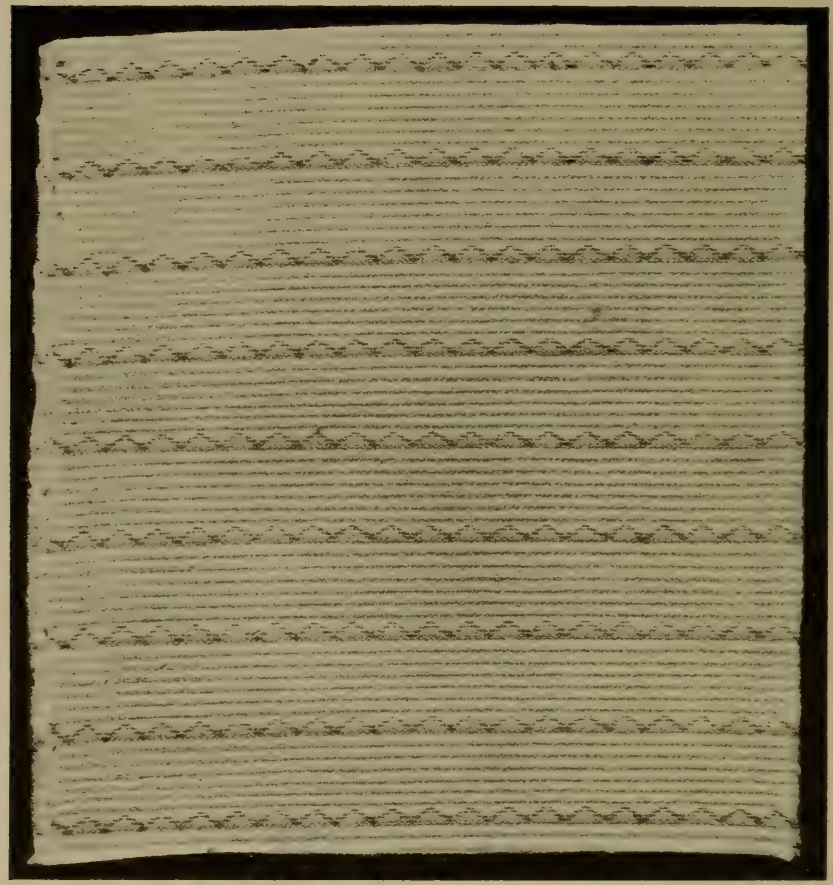

FIG. 584.-Piqué fabric embellished with extra picks of coloured weft.

substituting coloured threads both of warp and weft in place of the regular face warp threads and cutting picks. Where these occur, they are in pairs of the same colour, that is, two blue and two red threads of warp and weft, of which the latter are interwoven as cutting picks.

In Fig. 584 a simple wave effect is produced by introducing extra picks of navy blue weft to interweave in the crown of a wider rib. The coloured picks are disposed in eight pairs, 
alternately with pairs of regular face picks, and float more or less freely, on the surface, to produce the effect desired.

Fig. 585 illustrates a neat figured effect obtained by means of extra coloured warp threads arranged in pairs that are raised on the surface to float over one rib only at the same time, and then depressed to pass underneath the cutting picks, in order to develop a series of square spots or figures that are disposed alternately.

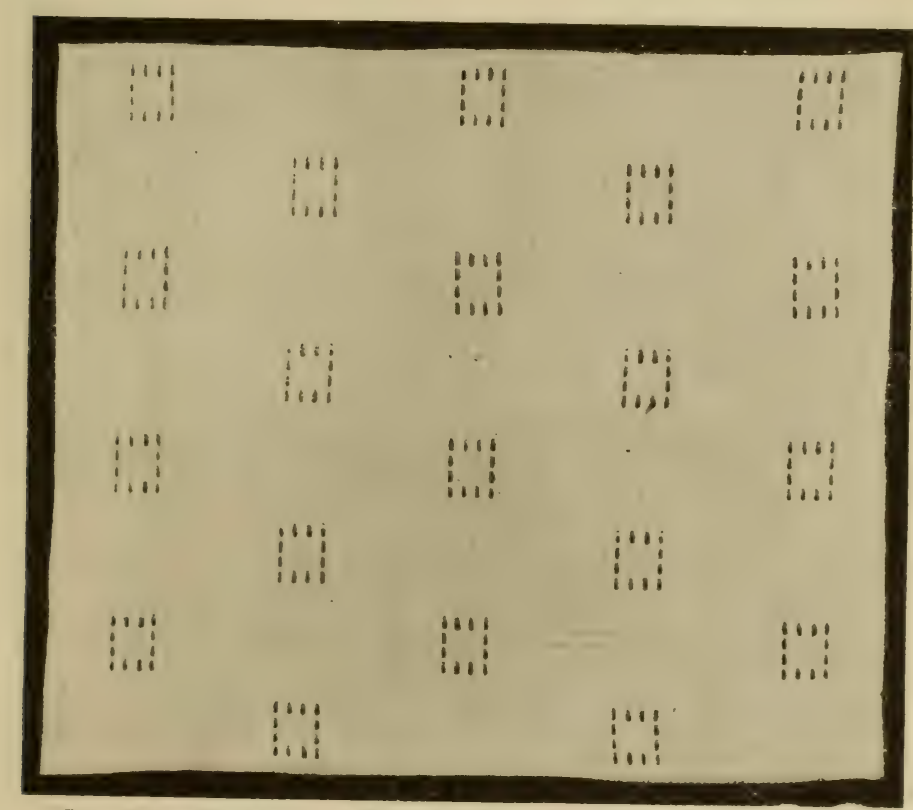

FIG. 585.-Piqué fabric figured with extra coloured warp threads.

In Fig. 586 a check pattern is evolved by means of extra "crammed" warp and weft threads employed to develop coloured figured stripes in each direction at Tregular intervals of $1 \frac{1}{4} \mathrm{in}$. In this case the scheme of figuring would require the use of a Jacquard machine and harness to control both the face and the back warp threads, as the figured effect is beyond the practical range of a dobby and heald harness.

Fig. 587 is a neat spot pattern developed by causing the 


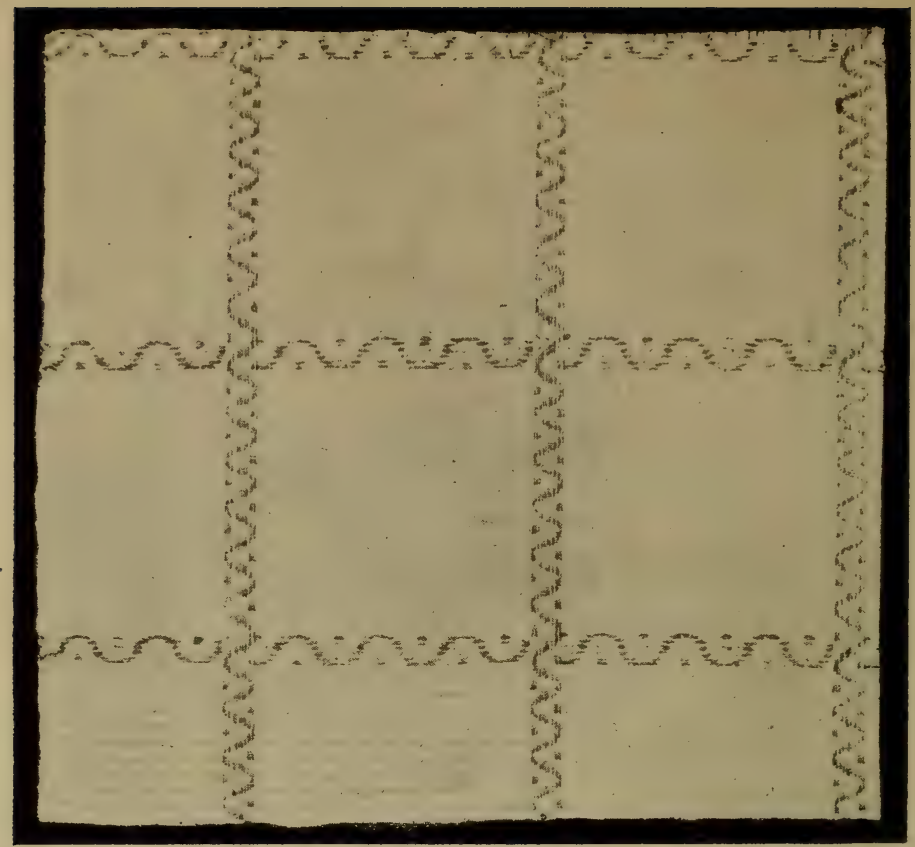

FIG. 586-Piqué fabric figured both with extra coloured warp threads and picks of weft.

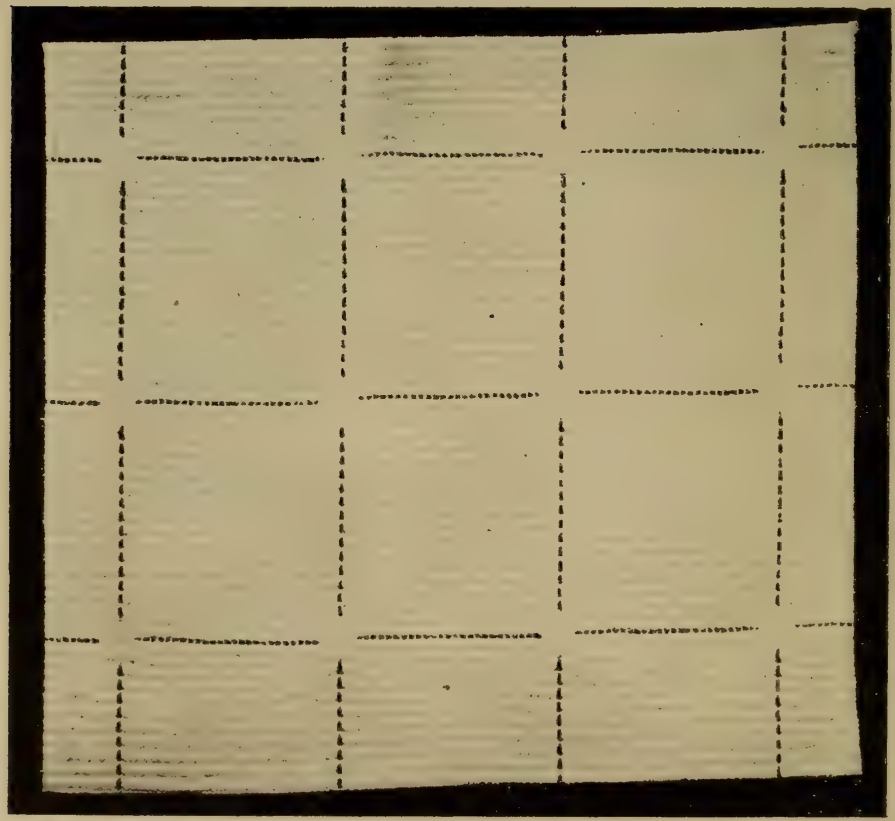

FIG. 587.-Piqué fabric figured by "floating" face warp threads; also by coloured warp and weft threads. 
face warp threads to float freely on the surface over four ribs; whilst additional embellishment is obtained by means of coloured threads of warp and weft to produce a hair-line check.

In Fig. 588 the figuring is obtained by allowing the weft to float freely on the surface to develop a series of diamondshaped forms enclosing a lozenge of plain "toilet quilting,"

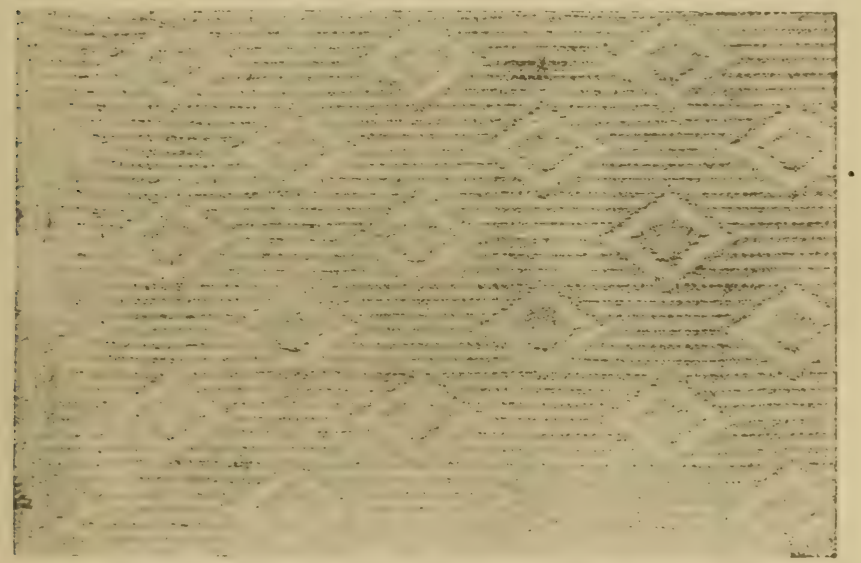

FIG. 588.-Piqué fabric figured by floating all picks of weft.

as described in Chapter XVI. This idea is still further developed in Figs. 589 and 590, in which the true principle of "toilèt quilting" is observed. In Fig. 589 the figures are disposed alternately on a plain broad-ribbed piqué; whereas, in Fig. 590, a series of diamond figures are arranged horizontally on a fine-ribbed piqué groundwork also containing bands of broader ribs.

\section{The "Float" Mounting,}

$\$ 197$. The type of Jacquard harness employed for these fabrics is a "sectional" or "divisional" harness constructed, or " tied-up," on the principle commonly termed a "float " hax"ness which is divided, both in the Jacquard machine and the comber-board, into two separate and distinct sections, termed the "face" and "hack" harness, to control the "face" and 


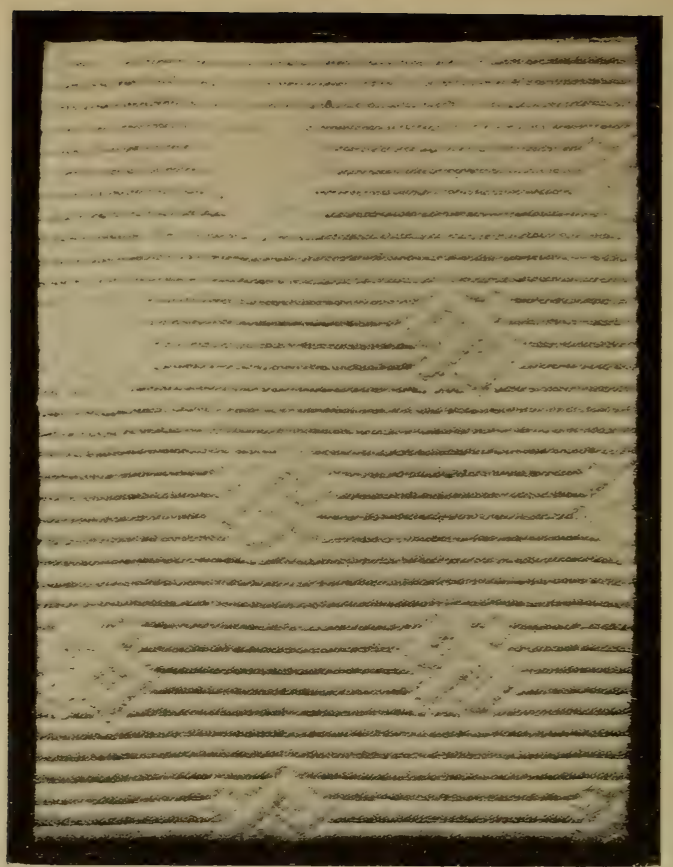

FIG. 5-9.-Piqué fabric with "toilet" or "quilted" figuring.

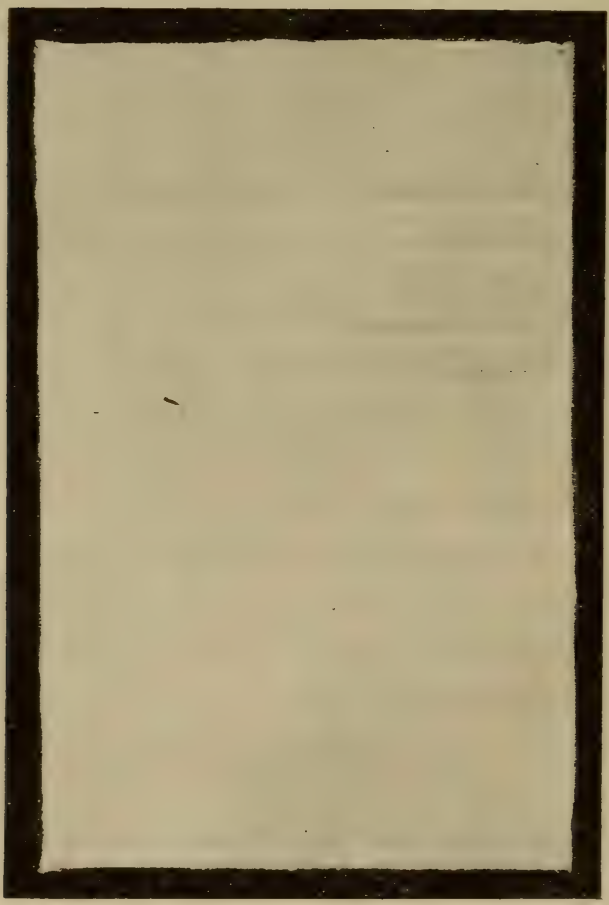

FIG. 590.-Piqué fabric with "toilet" or "quilted" figuring and variegated ribs or welts and of which Fig. 591 is the applied design. 
"back" warp threads respectively, and of which the harness or mounting threads, and mail eyes, are in the ratio of two face warp threads to one back warp thread. For example, if a 400 's Jacquard machine ( $\$ 08$ hooks) with a "tie-up" of 240 hooks, "face," and 120 "back" $=360$, is mounted on the loom with the cylinder on the left, when facing the loom, the mounting threads from the last 240 hooks-i.e., over the front part of the loom and on the right or "26-side" of the machine, when facing the cylinder-will pass through the holes in the front part of the comber-board, to govern "face" warp threads: whilst the mounting threads from the 120 hooks immediately preceding the 240 face hooks and on the left or "25-side" of the machine-orer the back of the loom-pass through holes in the rear part of the comber-board, to control "back" warp threads, thus leaving the first 48 hooks on the "25-side" idle, unless they mar, if required, be employed as "spare" hooks to gorern "extra" figuring warp threads for the development of simple effects, as exemplified in Figs. 585 and 586.

\$ 198. The method of preparing an applied or working design for a "float" harness of this type is demonstrated in Fig. 591, which is a portion of the actual design for the fabric represented in Fig. 590. The "face" and "back" sections of the harness are indicated on the design paper separately, side by side, to correspond with the division of hooks in the Jacquard machine. The present pattern repeats on 48 "face," and 24 "back" warp threads; but the design is set out for a "tie-up" of 240 "face," and 120 "back" hooks, in a 400 's machine. When cutting the design, therefore, the card-cutter will, in the present instance, first "read off" the "face" hooks five times orer in succession, and then proceed to "read off" the "back" hooks for a corresponding number of times. The fine ribs in this fabric each contain 5 picks of the same counts of weft, namely, 1 cutting pick, 3 face picks, and one wadding pick, inserted in the order named. The wider ribs contain 14 face and $1: 3$ wadding picks inserted alternately, and in order to prevent the wadding picks from crowding too closely together, in the fabric, they are very loosely interwoven 


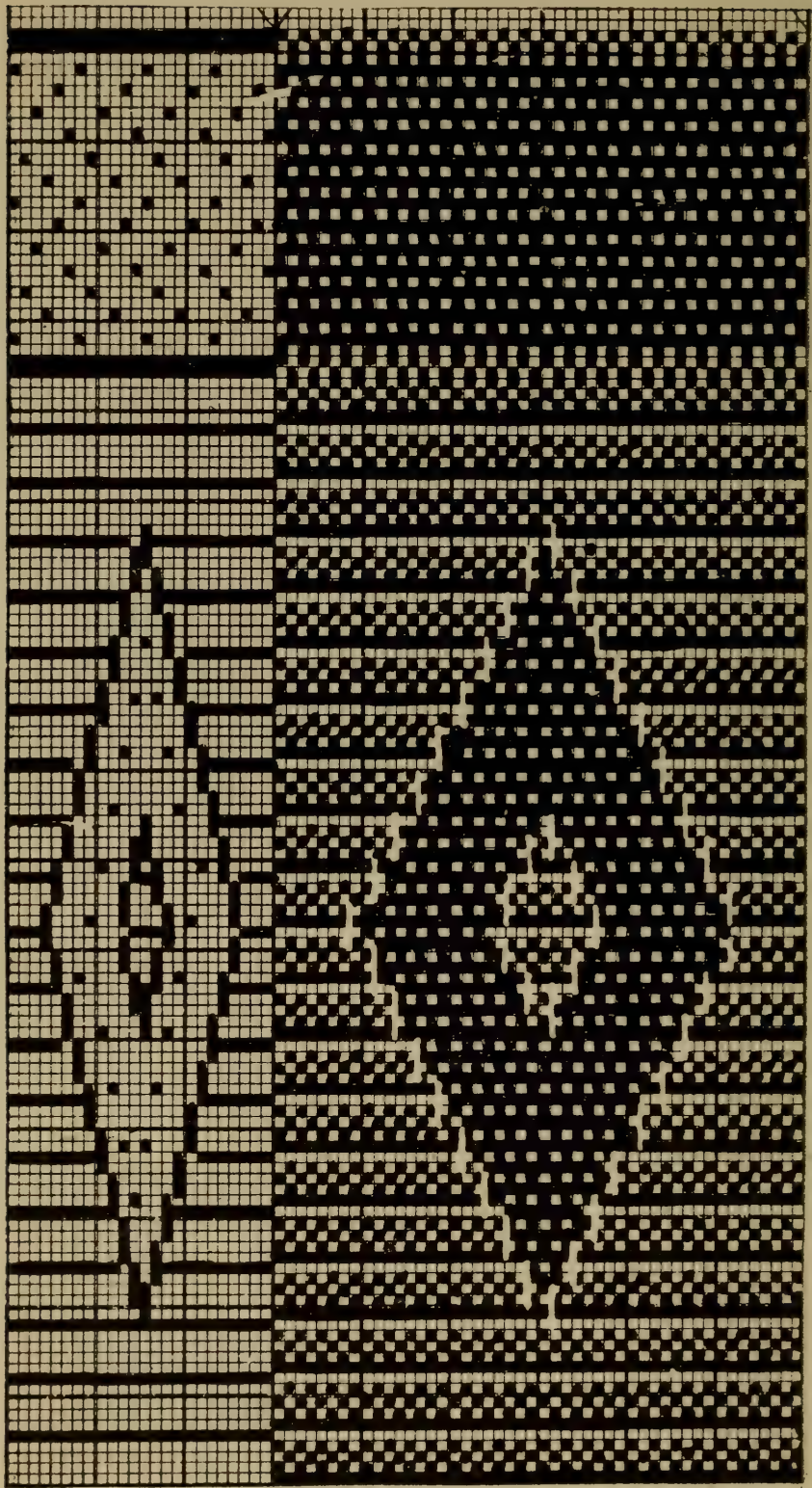

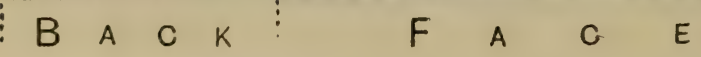

Frg. 591.-Applied design for figured piqué fabric, Fig. 590. 
with the back warp,threads in the manner indicated in the design. The diamond-shaped "toilet" figures are also developed in a similar manner, with a marginal "stitching" by raising single "back" warp threads over four consecutive picks of weft, to "stitch" or bind down the surface tissue of plain cloth. The points at which the "back" warp threads appear on the face of the cloth are indicated by means of short

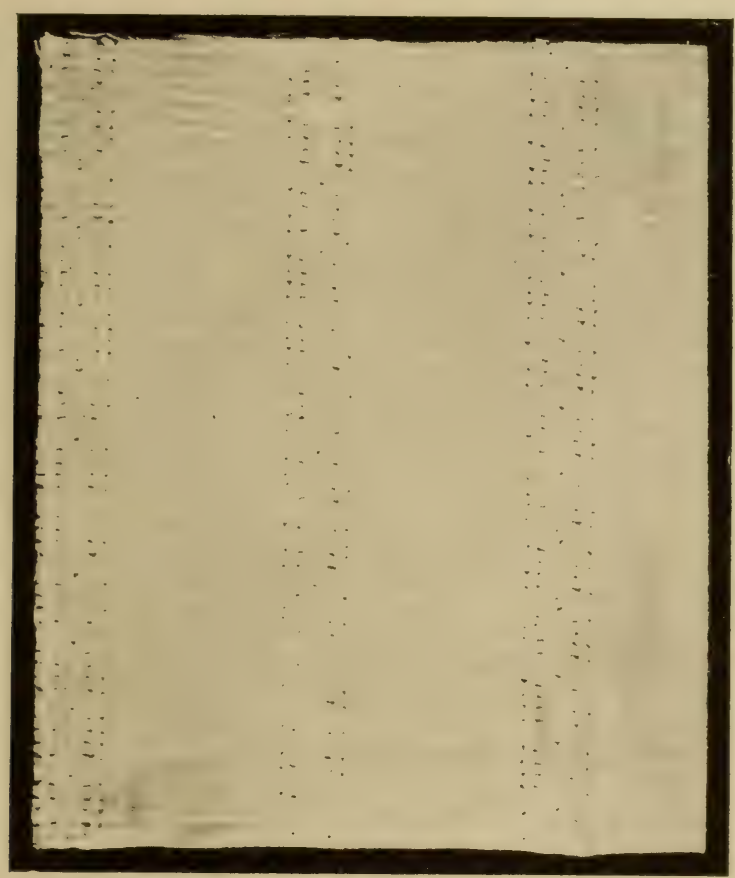

FIG. 532.-Piqué fabric with net leno stripes.

white lines in the "face" section of the design, but this procedure is not necessary in actual practice.

$\$ 199$. Two examples illustrating the embellishment of piqué fabrics by means of "net leno" figuring are represented in Figs. 592 and 593. The first of these is an example of a "two-doup" open leno stripe effect with the stripes disposed at regular intervals apart on a plain pique ground with the 
ribs of uniform width; whereas the second of these examples illustrates a "one-doup" "net leno" cord effect forming a single wave line separating piqué stripes containing ribs of two different wiaths respectively. Also, in the hollow of each wave is a single spot of coloured yarn, displaying two "taped" extra warp threads passing over four picks of weft at intervals corresponding to the width of the wider ribs, or two

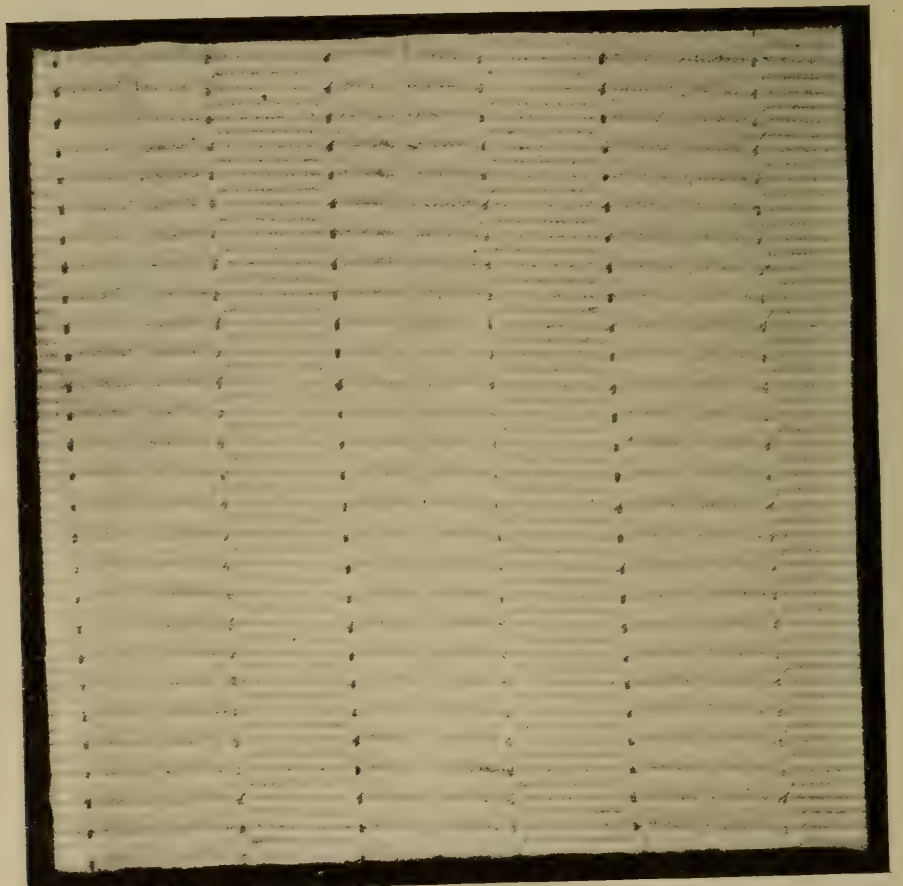

FIG. 593.-Piqué fabric with net leno stripes.

of the narrower ribs, as indicated. Many other examples could be produced in illustration of the various methods of embellishing piqué fabrics, but those given will serve to indicate the general procedure adopted, and the numerous modifications of which these are capable.

$\$ 200$. Piqué fabrics, both plain and figured, are produced in many different qualities, ranging from a very low to a very high 
grade. The data obtained from tive different examples are given in the following table :-

DATA RELATING TO PIQUÉ FABRICS.

\begin{tabular}{|c|c|c|c|c|c|c|}
\hline \multirow{2}{*}{$\begin{array}{l}\text { Dents } \\
\text { per In. } \\
\text { in Reed. }\end{array}$} & \multirow{2}{*}{$\begin{array}{l}\text { Ribs } \\
\text { per In. }\end{array}$} & \multirow{2}{*}{$\begin{array}{c}\text { Number of Picks } \\
\text { per Rils. }\end{array}$} & \multicolumn{2}{|c|}{$\begin{array}{l}\text { Counts } \\
\text { of Warp. }\end{array}$} & \multicolumn{2}{|c|}{$\begin{array}{l}\text { Counts } \\
\text { of Weft. }\end{array}$} \\
\hline & & & Face. & Back. & Fine. & Coarse. \\
\hline $\begin{array}{l}30 \\
32 \\
40 \\
50 \\
60\end{array}$ & $\begin{array}{l}16 \\
12 \\
14 \\
30 \\
44\end{array}$ & $\begin{array}{l}\text { 1C. } ; 5 \mathrm{~F} . ; 1 \mathrm{IV} .=7 \\
2 \mathrm{C} . ; 8 \mathrm{~F} . ; 2 \mathrm{WV} ; 2 \mathrm{~B} .=14 \\
2 \mathrm{C} . ; 10 \mathrm{~F} . ; 2 \mathrm{~W} . ; 2 \mathrm{~B} .=16 \\
2 \mathrm{C} . ; 8 \mathrm{~F} . ; 6 \mathrm{~W} .=16 \\
2 \mathrm{C} . ; 4 \mathrm{~F} . ; 4 \mathrm{~W} .=10\end{array}$ & $\begin{array}{l}30 \text { 's T. } \\
40 \text { 's T. } \\
40 \text { 's T. } \\
100 \text { 's T. } \\
100 \text { 's T. }\end{array}$ & $\begin{array}{l}20 \text { 's T. } \\
30 \text { 's T. } \\
30 \text { 's T. } \\
2 / 90 \text { 's } \\
2 / 90 \text { 's }\end{array}$ & $\begin{array}{r}30 \text { 's } \\
40 \text { 's } \\
50 \text { 's } \\
110 \text { 's } \\
110 \text { 's }\end{array}$ & $\begin{array}{l}\overline{-} \\
20 \text { 's } \\
30 \text { 's } \\
40 \text { 's }\end{array}$ \\
\hline
\end{tabular}

\section{Matelasse Fabrics.}

$\$ 201$. "Matelasse" is a term which designates an interesting variety of fabrics that are largely manufactured in worsted material for ladies' mantle cloths; also in worsted and cotton materials, respectively, for men's fancy vestings, and of which two typical examples are illustrated in Figs. 594 and 595, which represent a cotton vesting and a wor'sted mantle cloth, respectively. These fabrics are essentially compound textures of a complex structure evolved by interweaving two distinct series each of warp and weft threads, termed "face" and "back" warp threads and picks respectively, according to the respective positions which those threads occupy in the fabric. Each series of warp threads requires to be wound on to a separate weaver's beam to allow of their different rates of contraction during weaving. Face war'p threads are generally of finer counts of yarn than back warp threads and are in the ratio of two to one respectively. When drawing-in the warp threads these are passed through the shedding harness and reed in the order of one face, one back and one face thread, uniformly, with all three warp threads together in the same dent of the reed. In some very superior qualities of cotton matelasse vesting fabrics, however, both face and back warp threads are passed with an alternate or "end-and-end" 


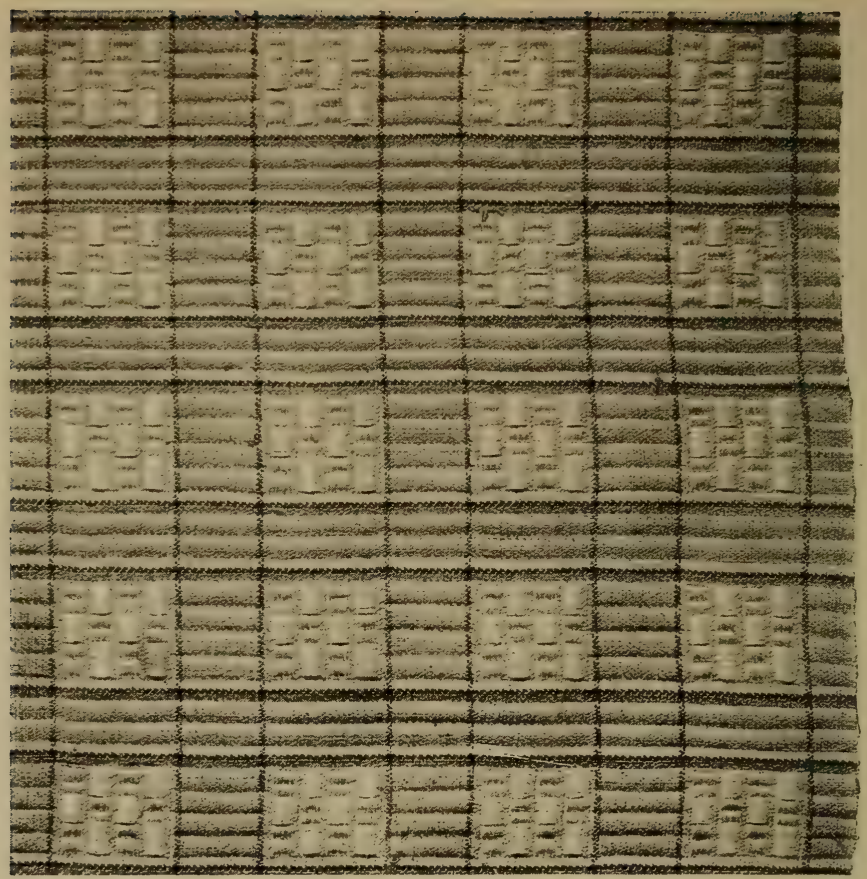

Fiq. 594.-Matelasse fabric.

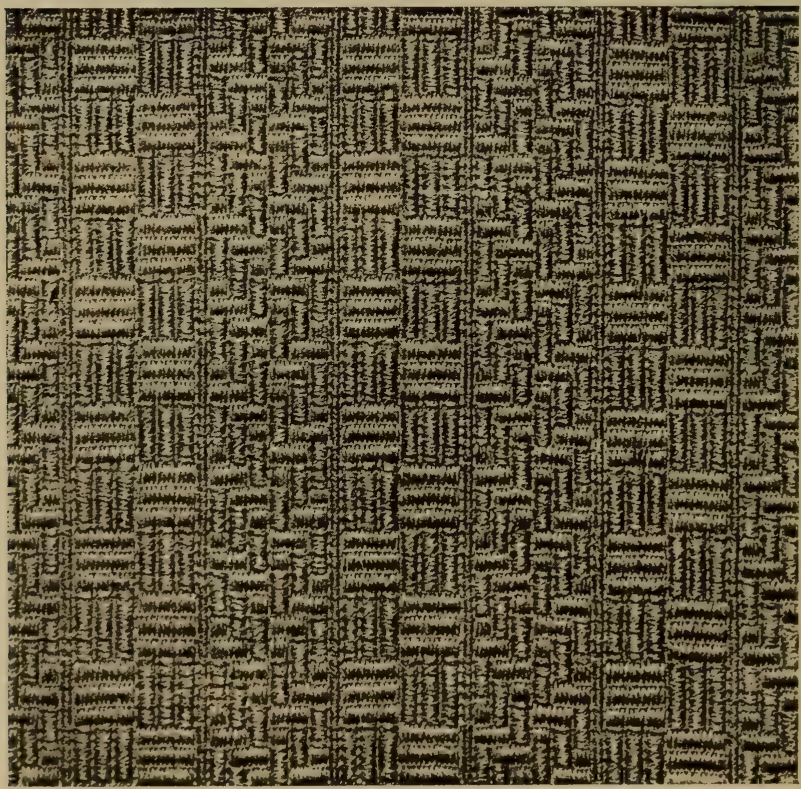

Fic. 595.-Matelasse fabric. 
disposition through the shedding harness, and with four warp threads in each dent of the reed. It is usual, however, to enıploy only one counts of weft, both for face and back picks, in these fabrics, and to insert them according to the disposition of the warp threads, i.e., either two face and one back pick, or else alternately.

Matelasse fabrics are characterised by well-divided and sharply-defined geometrical figures of a chequered or diaper

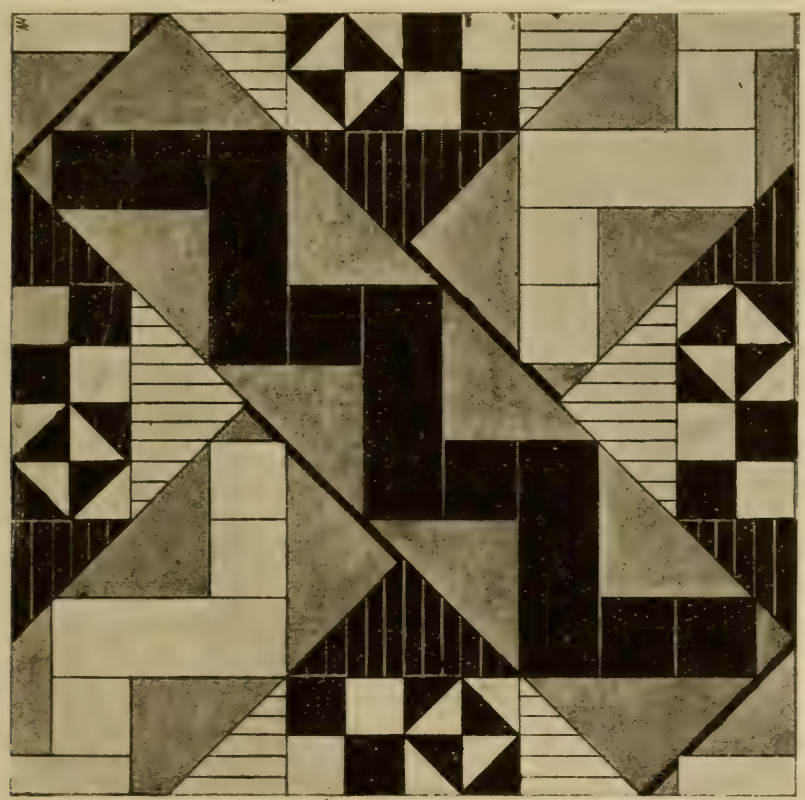

Fici. 596.-Sketch design for matelasse fabric.

character developed by floating both face warp and weft threads either more or less freely on the surface, or by interweaving them in some elementary order, as in a simple brocade fabric, to constitute the surface tissue of the fabric; whilst the back of the fabric consists of a texture of the plain calico weave produced by interweaving back warp and weft threads on that principle. These fabrics are woven from yarn in the "grey" or natural colour, and afterwards bleached, when they present a very rich and pleasing effect. 
Two typical sketch designs suitable for this type of fabric are given in Figs. 596 and 597. These are counter-change patterns evolved by straight lines only, as the peculiar structure of matelasse fabrics is not favourable to the development of curvilinear patterns. Black, in the sketches, represents the face warp threads floating freely on the surface: white indicates the face picks of weft: and grey indicates any neutral

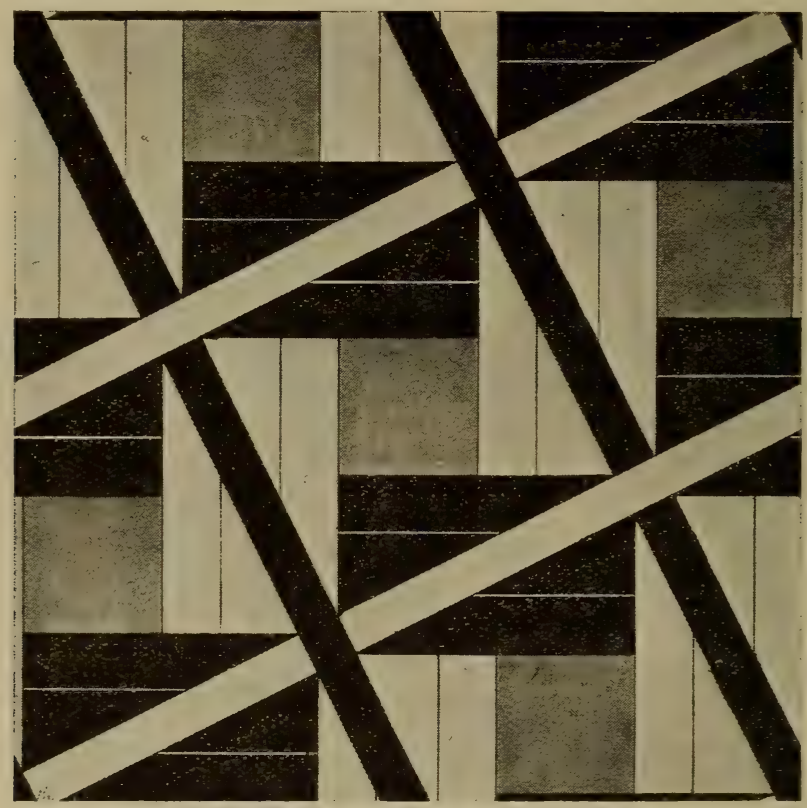

FIG. 597.--Sketch design for matelasse fabric.

simple weave as the "tabby" or plain calico weave; a "twoand-two" dice; a matt weave, or other similar effect.

$\$ 202$. The development of applied designs, on point paper, for matelasse fabrics requires expert technical knowledge of fabric structure, inasmuch as the effects produced are not simply mechanical, but depend entirely on the creative ability of the designer. The type of shedding harness employed for matelasse fabrics is the "float" harness, as employed for figured piqué fabrics, and described in $\$ 197$. The method of 
preparing a matelasse design, for the card-cutter, is demonstrated in the applied design, Fig. 599: but before this can be accomplished, it is necessary to prepare a block pattern, as represented in Fig. 598, and afterwards set this out on design paper in two separate divisions corresponding with the "face" and "back" series of hooks in the Jacquard machine, as ex-

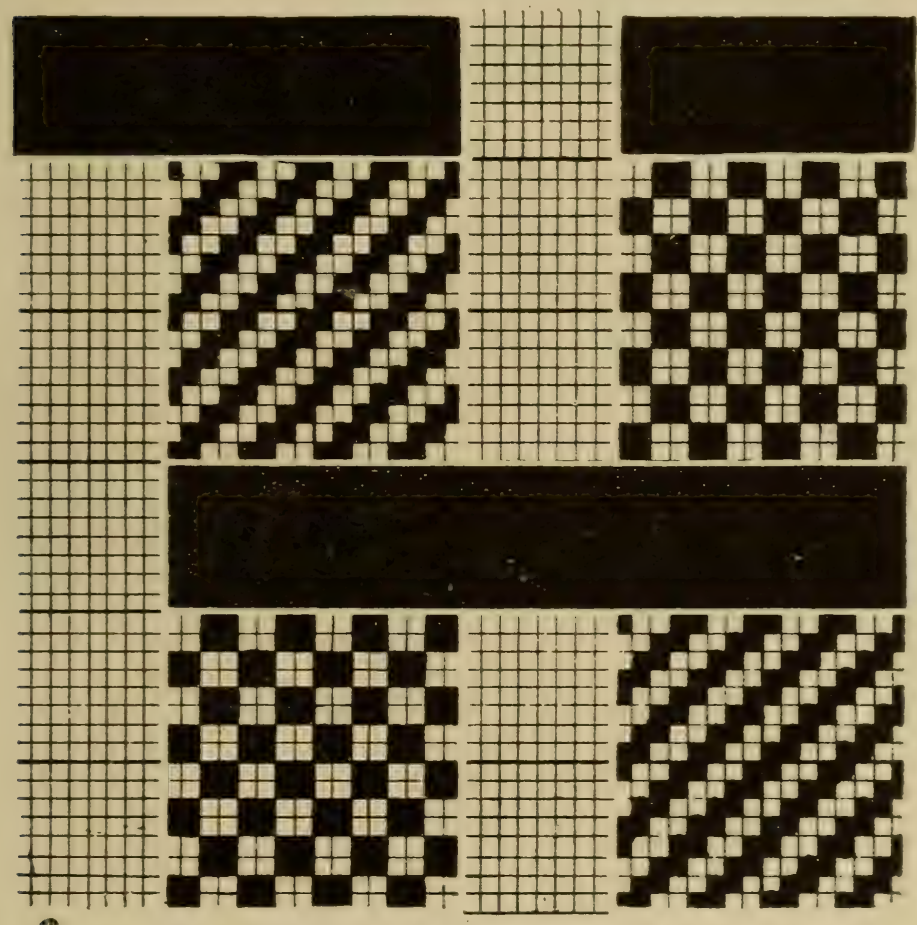

Frg. 598.-Block design for matelasse fabric, of which Fig. 599 is the applied design.

plained in $\$ 197$. The pattern selected is a simple counterchange effect repeating on forty-eight face warp threads and picks with a disposition of two face and one back thread in each series. Before proceeding to transfer this to design paper, for the card-cutter, however, it is better to distinguish the back picks from the face picks by faint lead pencil lines drawn across both the face and back sections of the design. The features of 
the pattern may then be set out, in lead pencil, in the "face" section of the applied design, after which the "cutting" lines marking the well-defined edges or other distinctive lines of the figures are indicated, in pencil, in the "back" section of

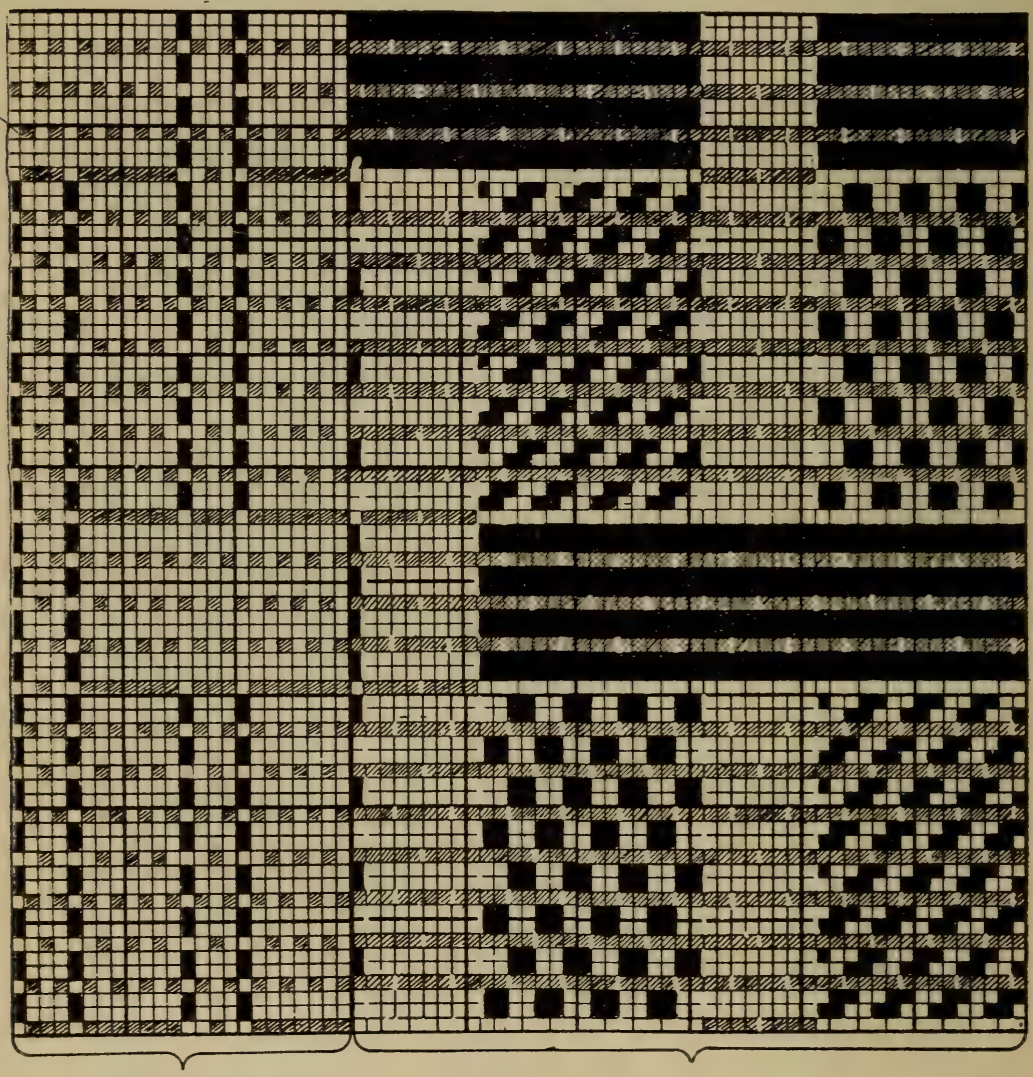

Back hooks

Face hooks

Fig. 593.-Applied design for matelasse fabric with pattern as in block design, Fig. 598.

the design. Thus, the delineation of pattern in an upward direction, or warp-way, is obtained by causing a back warp thread to pass over the face picks and underneath the back picks, as far as may be necessary, and as indicated by longi- 
tudinal white lines in the block pattern, Fig. 598. The delineation of pattern transversely, or weft-way, is obtained by causing a back pick of weft to pass over face warp threads, and underneath back warp threads, as indicated by transverse white lines, in the block pattern. The face section of the design may then be painted up in order to develop the features in accordance with the block pattern. In this section, care should be taken to raise all face warp threads over the back picks excepting where it is necessary to produce a transverse cutting, when face warp threads are left down, and back warp threads raised in those parts. Finally; the plain tissue, at the back of the cloth, is developed by interweaving the back warp and weft threads with the plain calico or tabby weave, excepting when those threads are raised to the surface for the delineation of pattern in the manner just described.

Additional embellishment may be imparted to these fabrics by introducing coloured threads either of warp, weft or both series, and which may either supplant or else supplement, as extra threads, the regular warp and weft threads, according to the particular effect desired. Also, matelasse figuring may also be produced in combination with a plain pique ground, as exemplified in Fig. 594, which also displays a simple coloured check pattern.

A good quality of cotton matelasse is produced from 80 face warp threads of $2 / 70$ 's, and 40 back threads per inch, of $2 / 50$ 's yarn: and 80 face and 40 back picks per inch, of $2 / 60$ 's weft. 


\section{CHAPTER XVI.}

\section{TOILET QUILTING FABRICS.}

$\$ 203$. Although the term "toilet" fabric is the general description of all kinds of cotton fabrics that are used for toilet purposes, such as, for example, counterpanes or bedcovers, dressing table covers, and mats, quite irrespective of the particular class or type of fabric to which they belong, that term, "toilet," is also employed as a technical term descriptive of a particular well-known type of cotton fabric known as "toilet quilting," which is essentially a compound texture comprising several modifications, all of which are characterised by an embossed or raised figure portion of the plain calico weave surrounded by a comparatively rough ground portion also consisting of plain cloth, but which is quite flat and depressed. All these different modifications of "toilet quilting," however, bear a close resemblance in their general appearance when viewed obversely, but they differ essentially in the minor details of construction, and more especially in the manner in which the reverse side of the fabric is constructed as explained hereafter, at the end of $\$ 205$.

Toilet quilting fabrics of the type under present notice, however, are produced from "grey" warp and weft, and afterwards bleached, are strictly one-sided and not reversible fabrics, when in use, and are described, technically, as "two," "three," "four," "five" and "six-pick" toilet quilting, according to the number of picks of weft inserted during the interval between the formation of successive "figuring" warp sheds : or, in other wordsif a Jacquard machine is employed for figuring purposesaccording to the number of picks that are inserted during the operation of each pattern card of that machine. They contain at least two series of warp threads and either one or else two 
series of picks of weft, according to the particular variety of fabric required, and are constructed with what are termed "loose backs," "half-fast" or "stocking backs," and "fast backs". The warp threads consist of what are termed $(a)$ "face" threads, and (b) "back" threads, which latter are also variously described as "figuring," "stitching" and "binding" warp threads.

$\$ 204$. "Face" warp threads are invariably of finer counts of yarn than "back" or "stitching" warp threads which are held at much greater tension than the former series of threads, during weaving, and therefore require to be considerably stronger. Also, since the rate of contraction, during weaving, is much greater with "face" warp threads than with "back" warp threads, it is, therefore, necessary to wind each series of threads on separate warp beams.

Face warp threads are always controlled by a heald harness governed by means of tappets to produce the plain "tabby" or calico weave for the face or obverse side of the fabric; whereas the back or figuring warp threads may be controlled either by means of healds governed by a dobby, or else by means of a Jacquard figuring harness for more elaborate schemes of figuring. Face and back warp threads are invariably employed in the proportion of two face threads to one back thread, and are drawn through the shedding harness and reed in the order of one face, one back and one face thread between each successive dent of the reed uniformly always with a back warp thread between the two face threads in the same dent, and a reed wire always separating two contiguous face threads, as indicated in the drafting chart, Fig. 602, which is the method of drawingin the warp threads through a heald harness consisting of eight healds, namely, four coupled healds to govern face warp threads, and four healds to govern back warp threads that are drawn in with a "V" or pointed diaft, although any other number of healds and scheme of drafting the figuring warp threads may be adopted according to the character of the design to be produced.

Some toilet quilting fabrics are constructed with only one series of picks of weft termed "face" picks, which interweave with "face" warp threads only to produce a plain calico texture 
of cloth to constitute the entire "face" or surface tissue of the fabric. Other varieties contain two series of weft consisting of "face" picks and "wadding" or "filling". ("padding") picks sometimes of the same kind and counts of weft, but usually with wadding picks of coarser and softer spun "roving" weft which lies midway between the surface tissue and the back of the fabric to impart additional bulk, weight and warmth to the fabric. Superior qualities of toilet quilting, however, contain three series of weft, namely, "face" picks, "wadding" picks and also "back" picks ; either with the same or different counts of weft both for "face" and "wadding" picks, but with the same kind and counts of weft for both the "face" and the "back" picks, of which the latter lie entirely at the back of the cloth and interweave only with "back" or figuring warp threads, when these are not required for "stitching" over the "face" tissue of the fabric, and in a manner to be explained subsequently in $\$ \$ 210$ and 213 .

$\$ 205$. Before describing minutely the details of the construction of the several modifications of toilet quilting fabrics, it is advisable, at this stage, to define clearly what constitutes the cardinal and essential characteristics of this important type of fabric, as illustrated by an obverse and reverse view, in Figs. 600 and 601 respectively, of a specimen of "five-pick" "fastback" toilet quilting of medium quality, containing 52 "face" warp threads of 24 's T.; 26 " back" warp threads of 18's T; 52 "face" picks and 26 "back" picks, both of 18 's weft; and "wadding" picks of 8's soft weft per inch, when out of the loom and in the "grey" or unbleached state. As stated in the previous paragraph, the entire surface tissue of these fabrics consists of a perfect sheet of the plain calico weave produced by interweaving "face" warp threads and "face" picks of weft only on the "tabby" or plain weave principle of weaving, and over which surface tissue of plain cloth the "back" or "stitching" warp threads are raised singly (that is, never with two or more consecutive threads together) over two consecutive "face" picks only, at the same time, along the margin of the figure portion for figuring purposes and also in the ground portion of the fabric; but the "stitching" threads remain at the 
back of the fabric immediately behind the figured portions, thereby leaving the surface calico tissue in these parts quite free and loose from the back of the fabric and therefore causing the figure portions to appear somewhat baggy and to stand out more or less prominently in bold relief from the subdued or depressed ground portion of the fabric. The embossed figuring is emphasised by the insertion of "wadding" picks of coarser and softer weft which lie quite loosely between the "face" texture and the back of the fabric, in the figure portions only; but these picks interweave with the "back" warp threads at the back of the fabric in the ground portion, in a manner to be described subsequently.

In "loose-back" toilet quilting fabrics, "back" warp threads do not interweave with any picks of weft, behind the figure portions, but they float quite freely and loosely in those parts, thereby constituting a very objectionable feature of this particular variety, as the stragging threads are liable to be caught and pulled, accidentally, and thus impair both the appearance and stability of those fabrics. This objection, however, is partly averted in the "half-fast" or "stocking-" back variety of toilet quilting fabrics, and prevented entirely in the "fast-" back variety, as seen in Fig. 601. Thus, in a "half-fast" or "stocking-" back toilet quilting, a "back" pick is inserted either at regular intervals of three pattern cards, uniformly, or else a "back" pick is inserted for each of two consecutive pattern cards out of every four cards, uniformly. In both cases the object is to interlace the "back" picks with "back" warp threads, and thus prevent these from floating entirely and quite freely at the back of the cloth, behind the figure portions, as described. The most perfect examples of toilet quilting fabrics, however, are the "five-pick" and the "six-pick" varieties, in which a "back" pick is inserted for each pattern card, thereby constituting a perfect open and light muslin tissue of plain calico at the back of the fabric, as seen in Fig. 601, and produced by combining the "back" picks of weft and the "back" or figuring warp threads on the "tabby" or plain calico principle of weaving, as stated in $\$ 204$, and in a manner described in $\$ 210$ and 213 . 
$\$ 206$. Another characteristic feature of true toilet quilting fabrics is observed in the figuring or "stitching" warp threads of, say, the odd series only being raised during the operation of, say, odd-numbered pattern cards, and even figuring threads only are raised for even-numbered cards. Under these circumstances, therefore, consecutive figuring warp threads are never raised simultaneously for the same pattern card, although any odd number of consecutive figuring threads may be left down simultaneously. In like manner, figuring warp threads of the

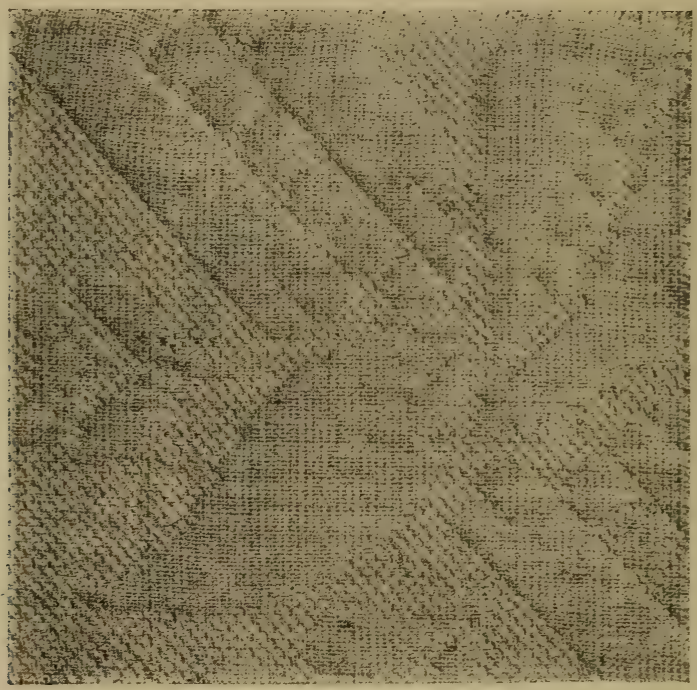

FIG. 600.-Toilet quilting fabric-obverse.

same series, whether odd or even, are never raised for consecutive pattern cards, although they may be left down for any odd number of those cards in succession. Warp threads that are raised in this manner are said to be on the "tab" or "shed"-the term "tab" being an abbreviation of "tabby," a term which is commonly employed by textile designers to indicate the plain calico weave. This disposition of figuring warp threads causes a somewhat rough and broken outline at the margin of the figure and ground portions of the fabric, but that defect is inherent to this disposition of threads in fabrics of 
any description and is, therefore, unavoidable. If, in the production of toilet quilting fabrics, the figuring threads were allowed to float freely above the surface tissue during the operation of more than one pattern card in immediate succession, they would lose their binding or stitching power over the face of the cloth in the ground portion instead of holding it down firmly in those parts. Also, if contiguous figuring threads were raised above the face of the fabric, the wadding and back picks of weft, wherever those threads were so raised,

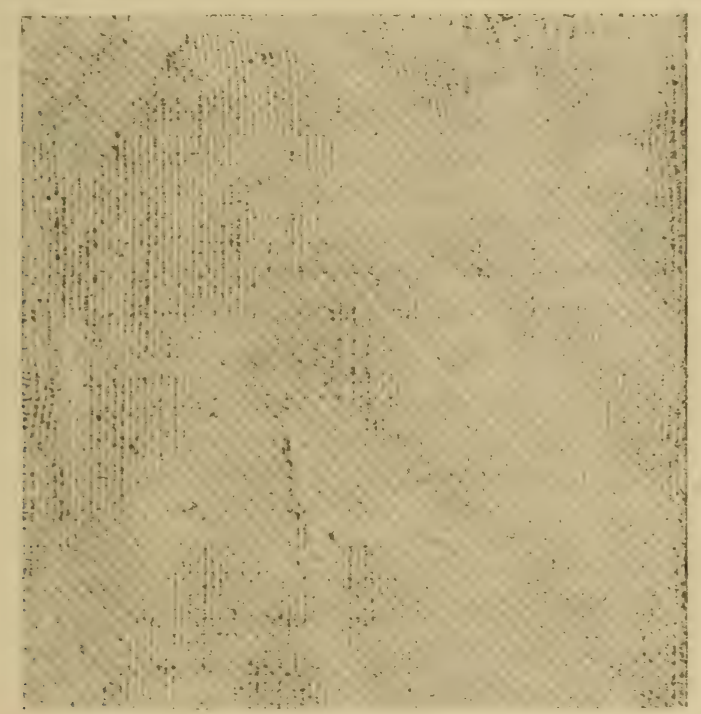

FIG. 601.-Toilet quilting fabric-reverse.

would float quite freely at the back, and thereby weaken the structure of the fabric, especially in those parts where such floats occurred. For these reasons, therefore, it is expedient, in the construction of toilet quilting fabrics, to raise or dispose the figuring warp threads on the "tab" or "shed" in the manner described, and as indicated in Fig. 623, which illustrates the method of preparing an applied design for toilet quilting, although in certain varieties of fabrics of this type, described as "toiletings," and which are usually embellished with small figured designs of a simple character, the general practice of 
raising the figuring threads on the "tab" is not always strictly observed, but may be departed from in some instances, according to the discretion of the designer and the particular effects which he wishes to produce in cloth.

$\$ 207$. From the foregoing general description of the chief characteristics of toilet quilting fabrics, the following detailed description of the various modifications of this type of fabric, and of the accompanying charts indicating the drafts and shedding plans for their production, will be more easily comprehended by those who are unfamiliar with their manufacture. A modification of this type of fabric embodying certain characteristic features of the true "toilet" principle of fabric structure is that formerly described as "dandy toileting," of fine texture and employed chiefly for making fancy white summer vests and neckties for men's wear. This variety of toilet fabric is constructed with two series each of both warp and weft. threads, namely, face threads of fine counts and "back" threads of coarser counts of yarn, but without any "wadding " picks. Warp threads are drawn through the shedding harness and reed in the order of one "face" F, one "back" B and one "face" thread between each successive,dent of the reed and as indicated in the drafting chart, Fig. 602; whilst the weft series of threads are inserted in the order of two "face" and two "back" picks, in alternate succession and as indicated in the "shedding plan," Fig. 603, which draft and shedding plan are adapted to produce a simple figured effect consisting of the simple six-end diamond weave represented in Fig. 199. Face warp threads are drawn through a set of four plain healds that are coupled together virtually to constitute only two healds with the usual calico or plain drafting in the order technically indicated as " $1,3,2,4$," which signifies that consecutive "face" warp threads are passed through the eyes of the 1st, $3 \mathrm{rd}, 2 \mathrm{nd}$ and 4 th heald in regular succession and as indicated in Fig. 602; whereas "back" warp threads are drawn through four healds, placed in the rear of the "face" healds, and with a "V" or pointed draft repeating on six warp threads, as indicated. Therefore, by raising the respective healds in the manner indicated in the shedding plan, Fig. 603, the back warp threads 
will be raised to stitch over only one "face" pick and one "back" pick, and thus bind down the plain surface tissue to produce the six-end diamond. In the shedding plan, Fig. 603, for the "dandy" toileting, it will be observed that the front four face healds are all raised together, thereby raising all the "face" warp threads en masse when the "back" picks are inserted, in order to place those picks entirely at the back of the cloth where they interweave on the "tabby" principle with the "back" warp threads only, thus constituting a two-pick, two-shuttle, "fast-back" toileting.

$\$ 208$. Another example of a toileting fabric of similar surface appearance to the "dandy" toileting just described but of relatively lighter and inferior texture, is that described as "runup" toileting containing only "face" picks all of the same kind of weft, and therefore requiring the use of only one shuttle for its production. The picks of weft are inserted separately but with two picks in each "face" warp shed uniformly, and with "back" warp threads raised over two consecutive picks at a time to produce a six-end diamond weave with those warp threads passing over two picks that are inserted in opposite or contrary "face" warp sheds, and therefore intersecting the two consecutive picks that are inserted in the same "face" warp sheds, as indicated in the shedding plan, Fig. 604. This variety of toileting fabric, which is produced with a "loose" back and without wadding picks, is a cheaper imitation o "dandy" cloth and used for similar purposes. Also, unlike that example, the construction of "run-up" cloth involves a slight variation from the true character of typical "toilet quilting" fabrics, by departing from the strictly plain calico weave for the surface tissue which should contain single picks of weft only, in each " face" warp shed. Otherwise if that method of picking were observed in the production of "run-up" toileting, it would produce the most elementary example of the true toilet quilting fabric of the "two-pick" variety containing only "face" picks of the same kind of weft, and therefore devoid both of "wadding" and "back" picks.

By studying the shedding plans, Figs. 603 and 604, in conjunction with the drafting chart, Fig. 602, and then indicating 


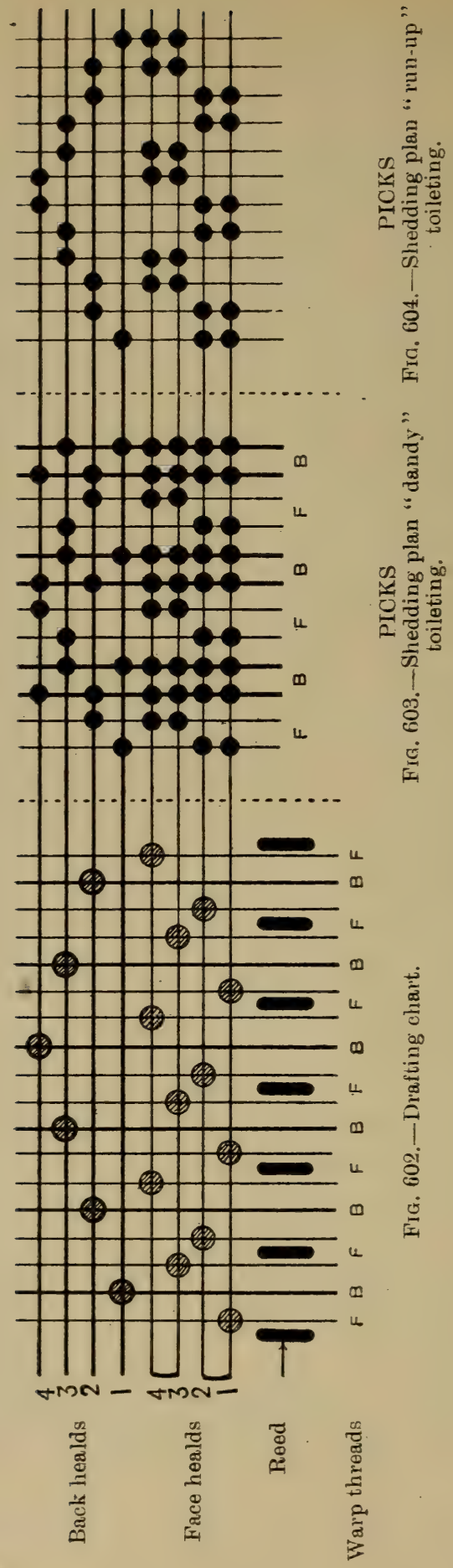


on design or point paper the manner in which both the "face" and "back" warp threads are raised and depressed for consecutive picks of weft, the structure of the respective varieties of toiletings just described will be the better comprehended.

\section{Varieties of Toilet Fabrics.}

$\$ 209$. For convenience of reference, toilet fabrics may be classified under six chief divisions, each comprising the following varieties, namely :-

1. A : "Dandy"-Two-PICk, two-shuttle, fast-back toileting, containing "face" and "back" picks only, but no "wadding" picks-Fig. 603.

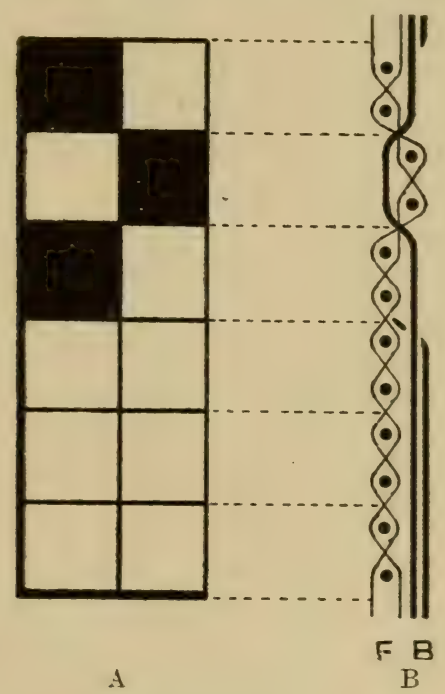

Fig. 60 ร.

B: "Pun-up "-Two-Prck, one-shuttle, loose-back toileting, containing "face" picks only-Fig. 604.

2. Two-PICK, one-shuttle, loose-back toilet quilting, containing "face" pick only, as represented by a longitudinal section of cloth, in Fig. 605 B.

3. A: Three-Pick, one-shuttle, loose-back toilet quilting, containing "face" and "wadding" picks only. 
B : Three-Pick, one-shuttle, "half-fast" or "stocking-" back toilet quilting, containing "face," "wadding". and "back" picks, with a "back" pick inserted for every third pattern card.

C : " " two-shuttle, loose-back toilet quilting, containing "face" and "wadding" picks only, as represented in Fig. $606 \mathrm{~B}$.

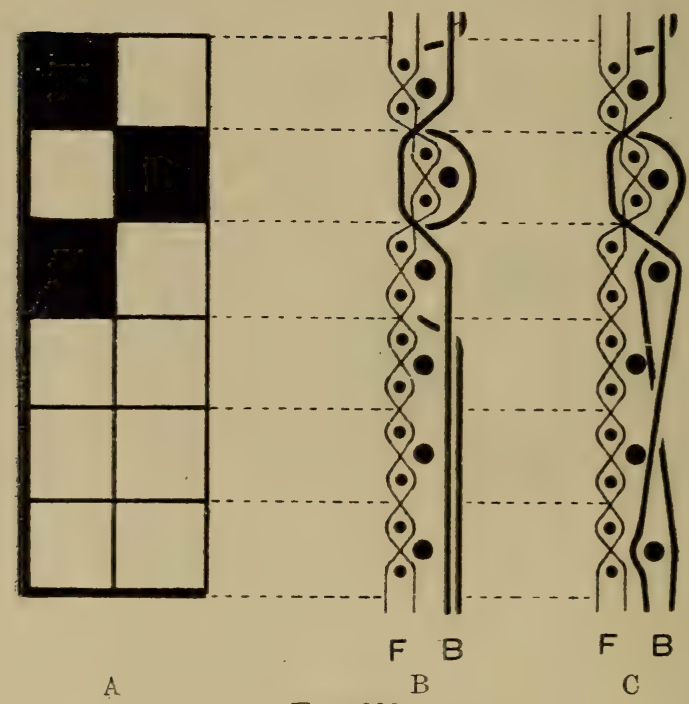

FIG. 606.

D : Three-Pick, two-shuttle, "half-fast" back toilet quilting, containing "face," "wadding" and "back" picks, with a "back" pick inserted for every third pattern card, as represented in Fig. $606 \mathrm{C}$.

4. A : Four-pick, one-shuttle, loose-back toilet quilting, containing "face" and "wadding" picks only.

B : , , , one-shuttle, "half-fast" back toilet quilting, containing "face," "wadding" and "back" picks, with a "back" pick inserted for each of two consecutive pattern cards, out of every four cards. 
C: Four-picik, as 4 B, but with a "back" pick inserted for every third pattern card.

D : . . . one-shuttle, fast-back toilet quilting, containing "face," "wadding" and "back" picks, with a "back" pick inserted for every pattern card.

E : ,. ,. two-shuttle-otherwise as $4 \mathrm{~A}$, and as represented in Fig. $607 \mathrm{~B}$.

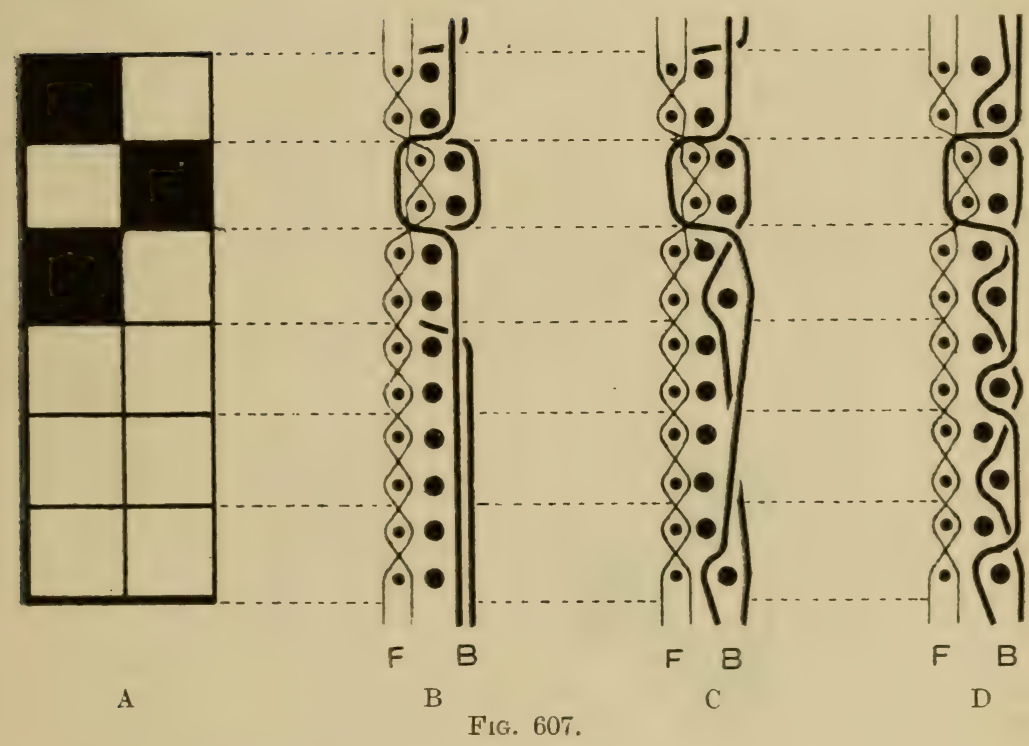

F : Four-pick, two-shuttle-otherwise as $4 \mathrm{~B}$.

G: " , " , -otherwise as $4 \mathrm{C}$, and as represented in Fig. $607 \mathrm{C}$.

$\mathrm{H}$ : " , " , -otherwise as 4 D, and as represented in Fig. 607 D.

5. Five-PICK, two-shuttle, fast-back toilet quilting, as represented in Fig. 608 B.

6. Six-ріск, two-shuttle, fast-back toilet quilting, as represented in Fig. 609 B.

The structure of toilet quilting fabrics is better demonstrated 


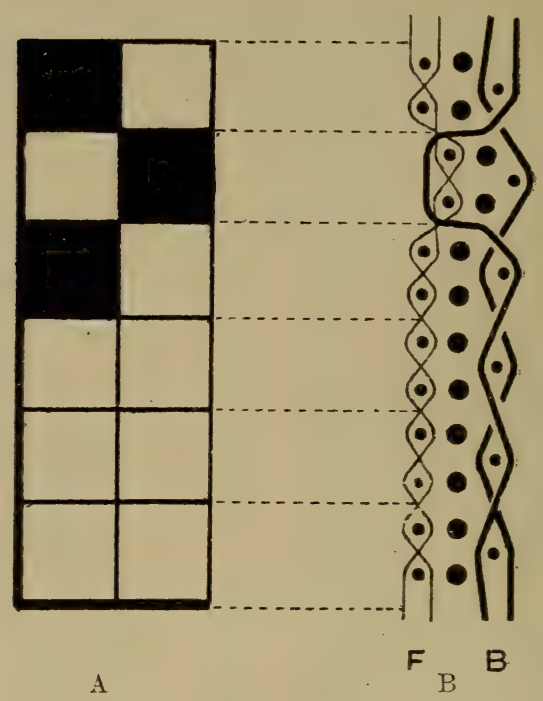

Fig. 609.

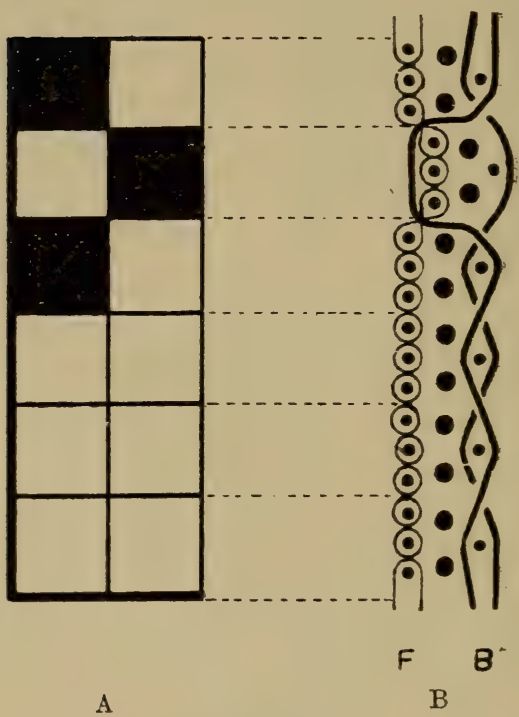

Fig. 609. 
by the aid of the following graphic diagrams, Figs. 605 to 609 , representing longitudinal sections of several varieties of those fabrics. These sectional diagrams are placed by the side of the corresponding portion of an applied toilet design of which only two vertical and eight horizontal spaces on design papercorresponding to two hooks of the Jacquard machine and eight pattern cards respectively-are shown. The sectional diagrams are also accompanied by the respective shedding plans indicating the manner in which the Jacquard harness, comber-boards and healds are raised during the operation of each pattern card, according to the particular variety of fabric produced.

$\$ 210$. A toilet quilting harness consists of a Jacquard figuring harness to govern the "back" or "stitching" warp threads, and operates in conjunction with a set of four coupled healds, placed in front of the figuring harness, to control the "face" warp threads. For "loose-back" toilet quilting the harness threads pass through the holes of a single fixed comber-board; but for " half-fast" and "fast-back" toilet quilting, harness threads are passed through two separate comber-boards to receive odd-numbered and even-numbered harness threads respectively. In this case, a large knot is formed on each mounting or harness thread immediately above their respective comber-boards, which are raised in alternate succession in order to raise first the alternate and then the intermediate "back" warp threads, alternately, across the entire width of the fabric for the insertion of "back" picks only, as indicated in the shedding plans for those varieties. Excepting for this difference between one fixed comber-board and two operative comber-boards controlling odd and even mounting threads, respectively, the drafting chart for all varieties of toilet quilting fabrics is the same, as indicated in Fig. 610. The Jacquard machine is governed by means of a lifting cam which makes one complete revolution during the operation of each pattern card, corresponding to such number of picks as are inserted for each card ; whilst the comberboards and healds are governed by means of positive box-plateside tappets, as represented in Fig. 615. The production of one-shuttle toilet quilting requires the use of an ordinary 
single-box loom only; but the two-shuttle varieties require the use of a loom with two shuttle boxes at one end of the sley, and with a "checking" or box motion to operate those boxes for the two kinds of weft employed. Also, an ordinary alter-

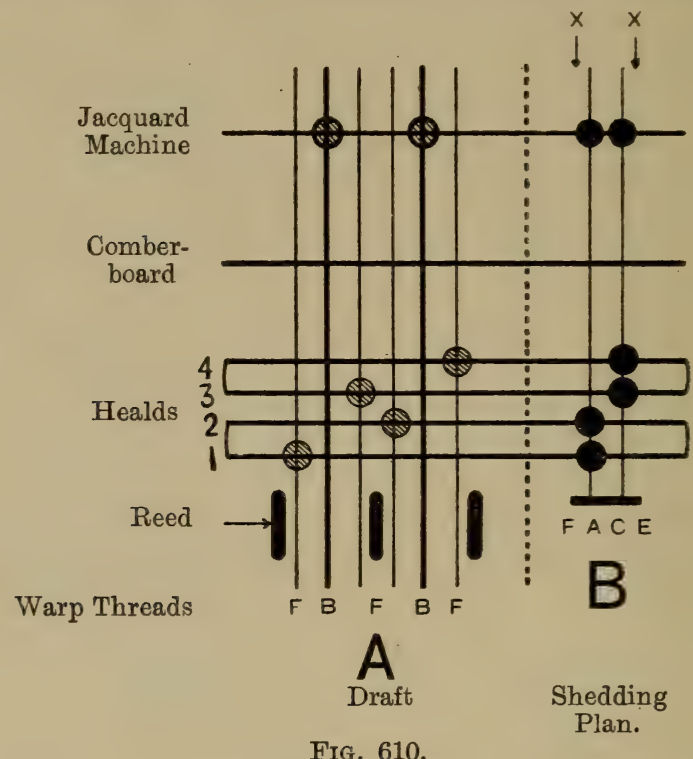

nate picking motion only is necessary for these looms; but this requires both the fine and coarse picks of weft to be inserted in two or a multiple of two picks of each kind of weft in succession, and as indicated in the shedding plans of those varieties.

\section{Two-pick, One-shuttle Toilet Quilting.}

$\$ 211$. The structure of two-pick, one-shuttle toilet quilting is represented by a longitudinal section of cloth, in Fig. 605 B, whilst the drafting chart and shedding plan for that variety are indicated in Fig. $610 \mathrm{~A}$ and $\mathrm{B}$ respectively. This variety contains only two picks of weft, both of which are "face" picks $\mathrm{F}$, for each pattern card; during the operation of which the Jacquard machine is raised and remains up for both picks in succession, and then changes very quickly to form the next 
figuring warp-shed, as indicated by small arrows $\mathrm{X}$, in the shedding plan, Fig. $610 \mathrm{~B}$. At the same time the healds governing "face" warp threads rise and fall in alternate succession for consecutive picks of weft and in exactly the same manner as in an ordinary plain calico loom, to produce the surface tissue of plain cloth as represented in the sectional diagram, Fig. 605 B, and also in the shedding plan, Fig. 610 B. Therefore, all figuring warp threads that are raised "stitch" over two "face" picks of weft in succession, whilst those threads that remain down float quite freely at the back of the cloth for any odd number of pattern cards in succession, thereby producing a "loose-back" toilet quilting devoid both of "wadding" and "back" picks, and one which is, therefore, the simplest and relatively the lightest texture of this type of fabric.

\section{Three-pick Toilet Quilting,}

$\$ 212$. In a three-pick toilet quilting, three picks are inserted for each pattern card, namely, two "face" picks and

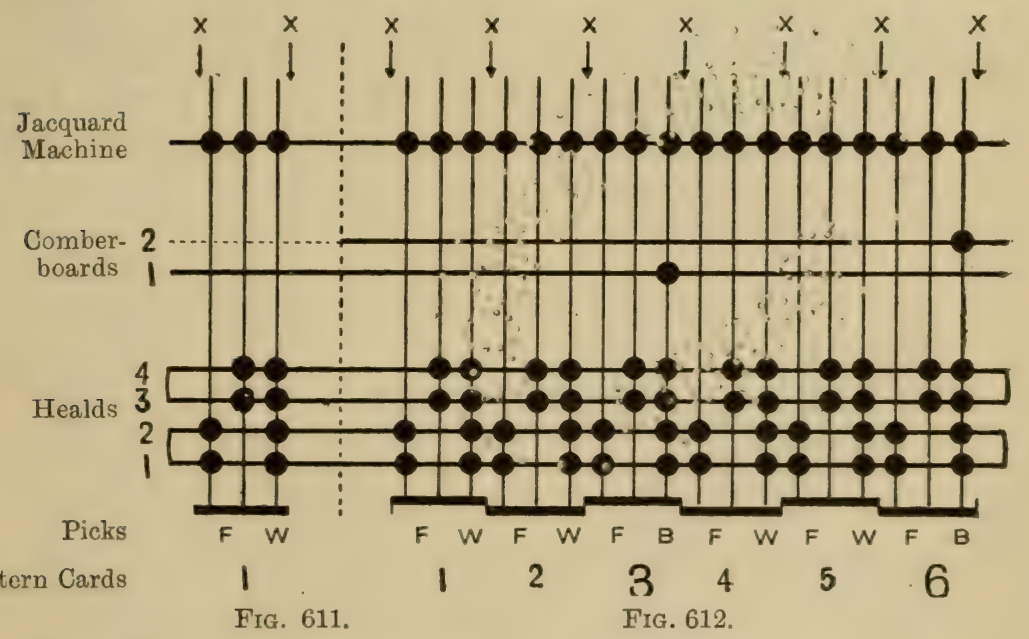

one "wadding" pick W, either of the same or different counts of weft. Also, they are usually constructed with a "loose back" and sometimes with a "half-fast" or "stocking- 
back," as represented by sectional diagrams, Figs. $606 \mathrm{~B}$ and $\mathrm{C}$ respectively, and of which the shedding plans for four different varieties of three-pick toilet quilting are indicated in Figs. 611 to 614 inclusive. Thus, if the "loose-back" variety is produced from the same kind of weft both for face and wadding picks, it may be woven in a single-box loom with the weft inserted in the order of two face picks and one wadding pick for each pattern card, uniformly, as indicated in the shedding plan, Fig. 611, for that variety. If, however, wadding picks are of coarser counts of weft than the face picks, the picks of weft

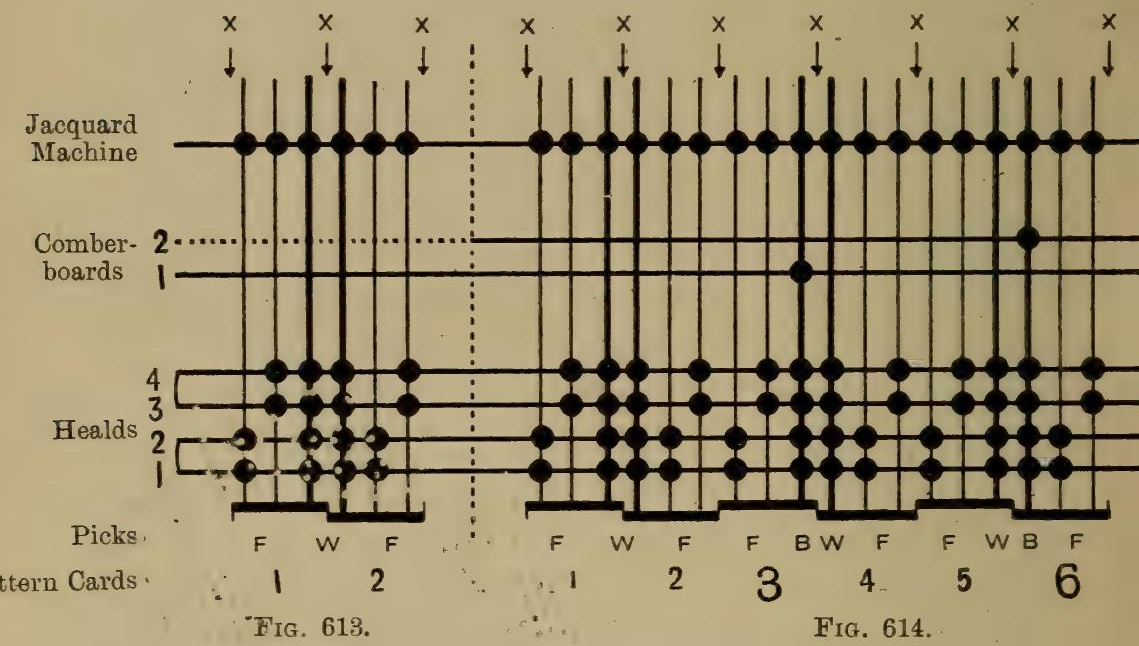

are inserted in the order of twc face picks and one wadding pick for alternate psticern cards, and one wadding pick and two face picks for interinediat cards as indicated in the shedding plan, Fig. 613, and in order to conform to the restriction imposed by a loom sley constructed with a double shuttle-box at one end, and a single shuttle-box at the other end, and also a loom equipped with an alternate picking motion, as explained in $\$ 210$.

In weaving this and all other varieties of "loose-back" and " half-fast" back-toilet quilting the Jacquard machine remains up for all the picks that are inserted for each pattern card, and must, therefore, change quickly between the last pick of one 
eard: and the first pick of the next card, as indicated by the arrows X.

$\$ 213$. If a toilet quilting fabric is to be produced either with a "half-fast" or a "fast back," the odd and even series of mounting or harness threads will require to be passed respectively through two separate comber-boards, and with the harness threads knotted immediately above those boards that will be raised in alternate succession for the " back " picks only, in order to raise first the odd series and then the even series of figuring or stitching warp threads for consecutive "back" picks respectively, and as indicated in the several shedding plans for those varieties of toilet quilting. The comber-boards are governed by means of box-plate side tappets and "jacklevers". Each of these boards may be controlled by a separate tappet constructed according to the particular order of shedding. required, and by connecting-rods secured to the ends of the tappet treadles and their respective "jack-levers". By an alternative method which is indicated in Fig. 615, both comberboards $\mathrm{F}$ may be controlled by the same tappet $\mathrm{A}$. This is effected by attaching to the tappet treadle $\mathrm{B}$ two long hooked rods $C$ that are connected respectively, by means of cords $G$, to two separate spare or extra hooks $\mathrm{H}$ of the Jacquard machine, as represented in the diagram. Therefore, whenever the tappet treadle B is depressed, both of the hooked liftingrods $\mathrm{C}$ will also descend together. When these rods are in their normal position, they are held, by means of spiral springs $\mathrm{D}$, quite clear away from the ends of the " jack-levers," $\mathrm{E}$, until they are pulled over the ends of those levers by the ascent of the corresponding Jacquard hooks $H$. Thus, by constructing the tappet $\mathrm{A}$ in such a manner that it will depress the treadle-lever $\mathrm{B}$ always for the last pick of each pattern card in succession, uniformly, and also by cutting holes in those cards according both to a pre-arranged plan as determined by the particular variety of toilet quilting to be woven, and to the order in which the "back" picks are inserted, the hooked lifting-rods $\mathrm{C}$ will be pulled over the ends of their respective "jack-levers" $\mathrm{E}$ in alternate succession, to raise the corresponding comber-boards $\mathrm{F}$ for consecutive 
"back" picks, and quite irrespective of whether these are to constitute "half-fast" or "stocking-back," or a "fast-back"

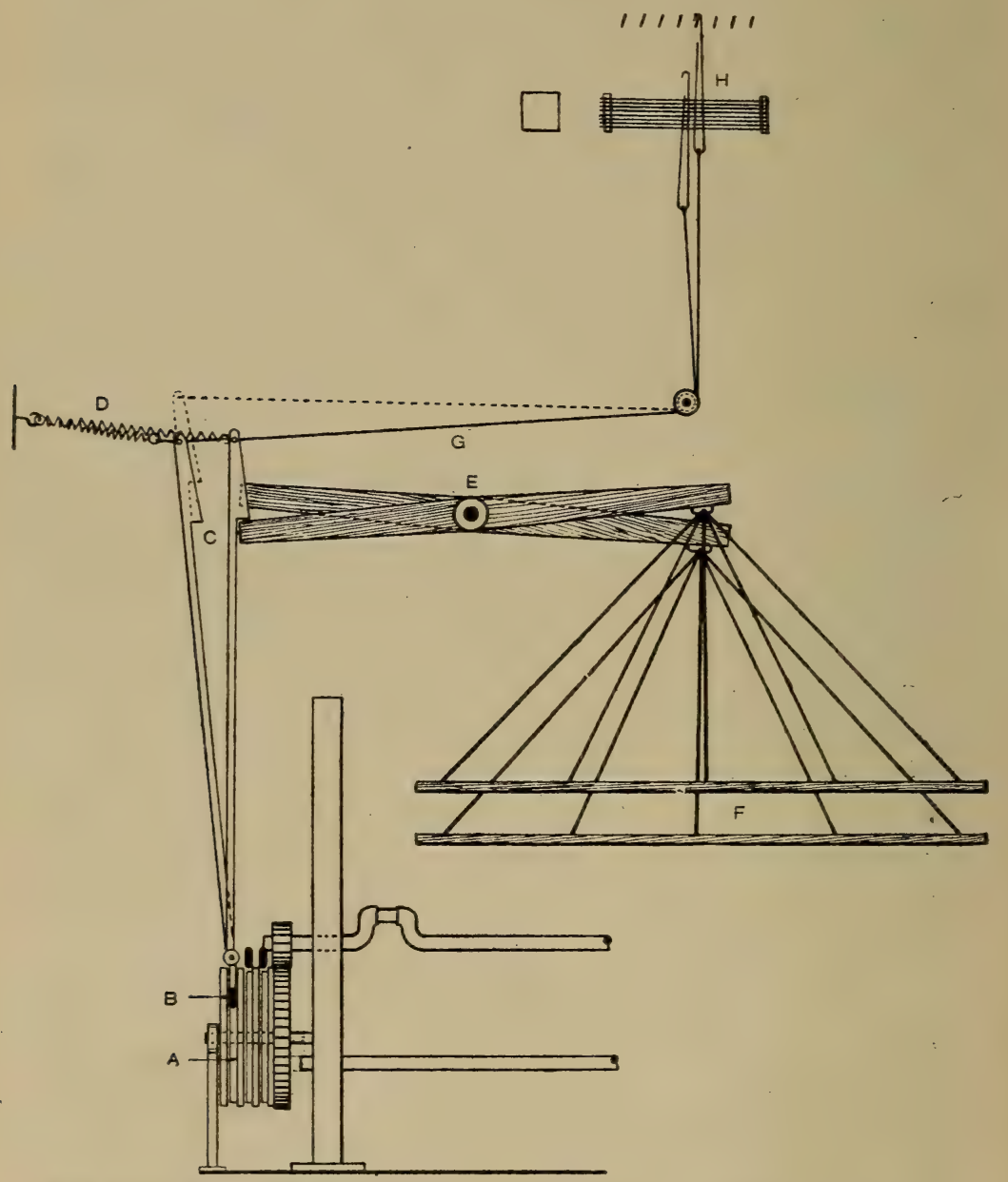

FIG. 615.-A method of operating comber-boards in a toilet quilting loom. toilet quilting fabrics. It should be observed, however, that this method of governing the action of the comber-boards, that is, by means of spare Jacquard hooks that are controlled by. 
the pattern cards, is not applicable to the two-shuttle "halffast" or "stocking-back" variety of three-pick toilet quilting, of which the shedding plan is indicated in Fig. 613, for the simple reason that, in this example, two consecutive "back" picks are inserted as the last pick of one pattern card and the first pick of the next card respectively, and not as the last pick of those pattern cards, uniformly.

\section{Four-pick Toilet Quilting.}

$\S 214$. The structure of the three principal varieties of fourpick toilet quilting is illustrated by sectional diagrams, Figs. $607, \mathrm{~B}, \mathrm{C}$ and D, which represent the "loose-back," the " halffast" or "stocking-back" and the "fast-back" varieties, for which the shedding plans are indicated in Figs. 616 to 619

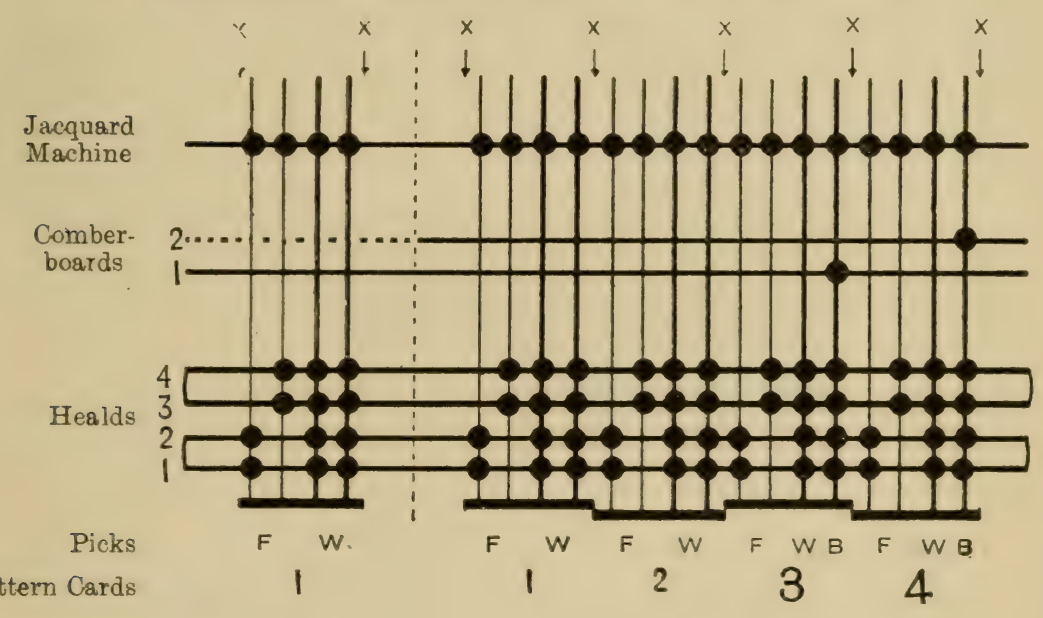

FIG. 616.

FrG. 618.

respectively. The sectional diagram, Fig. $607 \mathrm{C}$, and the shedding plan, Fig. 617, relate to the "stocking-back" variety produced by inserting a "back" pick for every third pattern card, uniformly; whereas the shedding plan, Fig. 618, is for the alternative method of which a "back" pick is inserted for two consecutive pattern cards in every four cards, as indicated. The shedding plan for the four-pick "fast-back" 
toilet is indicated in Fig. 620, which, in this and also in the shedding plans for the "five-pick" and "six-pick" toilet quiltings,

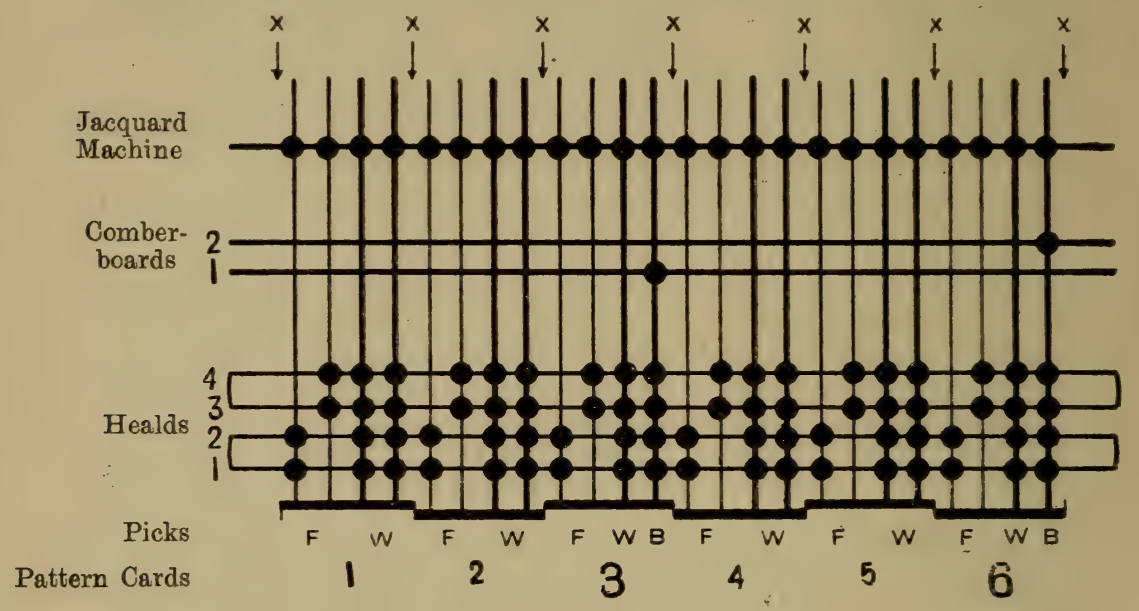

FIG. 617.

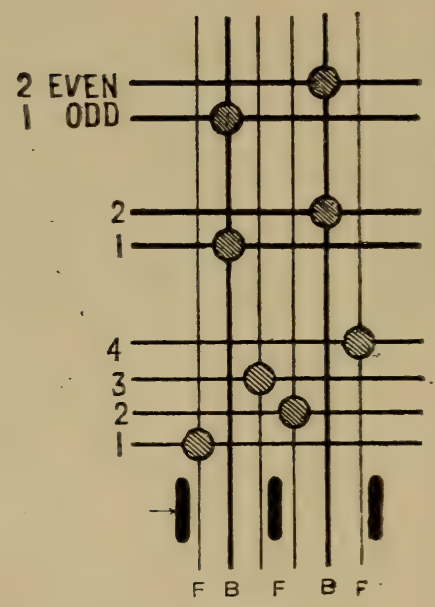

Fig. 619.

as indicated in Figs. 621 and 622 respectively, is arranged for what is known in the trade as a "split-shed" "tie-up" 
of the Jacquard figuring harness, in which the odd and the even series of mounting threads, respectively, are each controlled independently by two separate Jacquard machines, as indicated in those shedding plans, and also as represented in the drafting chart, Fig. 619. This course is only possible by reason of raising figuring warp threads first of the odd and then of the even series only, during the operation of alternate and intermediate pattern cards respectively, as explained previously in $\$ 206$. With a "split-shed" harness, each Jacquard machine controls all figuring warp threads of one series

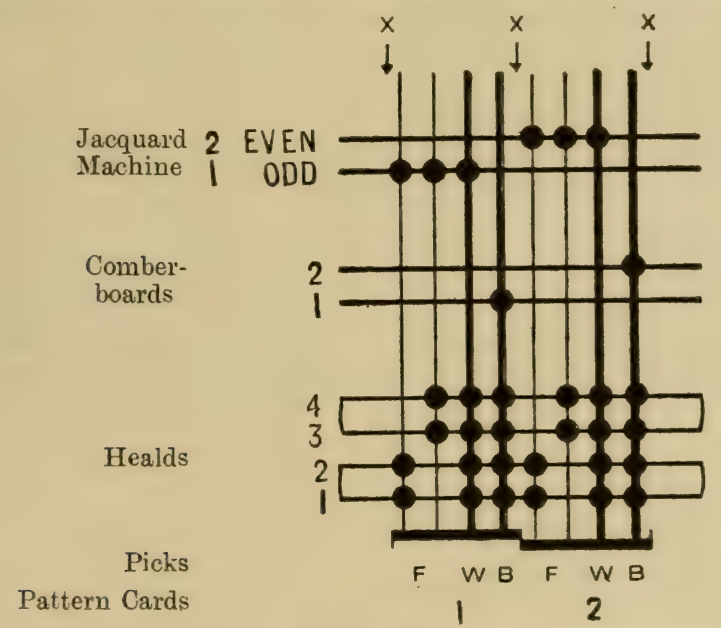

Fig. 620 .

only, respectively, across the entire width of the fabric, and only one of the two machines operates for each pattern card employed, thereby requiring only one-half the usual number of those cards and therefore only one-half the amount of cardcutting that would otherwise be required if the Jacquard machines each controlled all figuring warp threads in half the full width of the "back" warp respectively, in which case both machines would operate simultaneously for every pattern card employed, instead of rising in alternate succession in the manner described and indicated in the shedding plans. 
Another practical detail concerning the construction of "fast-back" toilet quilting fabrics relates to the manner of operating the Jacquard machines. It was observed in $\$ 212$ that, in the production of "loose-back" and "half-fast back" toilets, the Jacquard machine requires to change quickly between the last pick of one pattern card and the first pick of the succeeding card, in order to keep the figuring warp

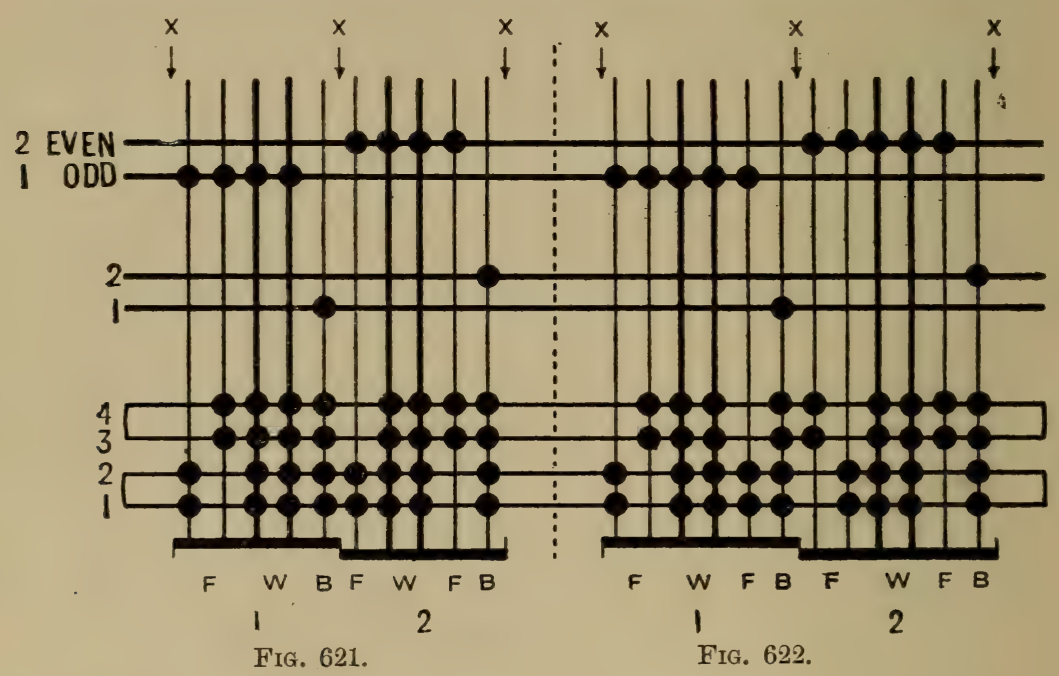

shed open during all the picks of weft inserted for each pattern card. If, however, the last pick of each successive pattern card is a "back" pick uniformly, as in all " fast-back" toilets, the Jacquard machine may, under these circumstances, descend immediately after the previous pick of weft is inserted, and remain down entirely for the "back" picks, as indicated in the shedding plans, Figs. 620, 621 and 622.

\section{Five-pick Toilet Quilting.}

$\$ 215$. The five-pick variety of toilet quilting fabrics is invariably produced from weft of two different counts and with a "fast back," as represented in the sectional diagram, Fig. 608 $\mathrm{B}$, of that variety of which the shedding plan is indicated in Fig. 621. The "face" and "back" picks are both of the same 
counts of fine weft, inserted by the same shuttle, whilst the "wadding" picks are of coarser counts of soft spun or roving weft. In order to conform to the conditions imposed by a loom equipped with an alternate picking motion, and with a loom sley constructed with a double shuttle-box at one end only, and a single box at the other end, the order in which the picks are inserted in a five-pick toilet fabric is not the same for each of any two consecutive pattern cards, by reason of "five" being an odd number, whereas only an even number of picks of the same kind of weft may be inserted under the conditions just specified, for reasons stated in $\$ 210$. For these reasons, therefore, the picks of weft are inserted in the order of two "face" (F), two "wadding" (W) and one "back" (B) pick, for, say, the odd-numbered pattern cards only; and in the order of one "face," two "wadding," one "face" and one "back" pick for even cards only, as indicated in the shedding plan, Fig. 621, thereby requiring the tappets governing the healds and also the pattern chain governing the action of the shuttle-boxes to be constructed ten picks to the revolution.

\section{Six-pick Toilet Quilting.}

$\$ 216$. The six-pick toilet quilting is a very uncommon variety which differs in construction from the five-pick toilet only in the detail of having three instead of two "face" picks inserted for each pattern card uniformly, as represented by the sectional diagram, Fig. 609 B, of that variety, and also as indicated in the shedding plan, Fig. 622.

In addition to the foregoing examples of toilet quilting fabrics, there are several other varieties of a kindred type, but which do not conform strictly to the essential conditions governing the construction of the more typical examples of this class of fabrics. For example, in one of these varieties which is of the "loose-back" structure, a coloured "back" or figuring warp is employed, of which any number of consecutive warp threads are displayed for any number of consecutive pattern cards, quite freely above the plain surface tissue of the fabric in a manner exactly similar to that observed in respect of the figuring warp threads in Alhambra fabrics, 
as described in Chapter XII. Excepting for the coloured figuring warp threads which require to be dyed with "fast" dyes, these fabrics, like other "toilet" fabrics, are woven in the "grey" state and afterwards bleached.

$\S 217$. Another variety of this class of fabrics is produced as a typical five-pick "fast-back" toilet quilting with the additional embellishment afforded by the employment of an extra coloured figuring warp, of which the threads are displayed in any desired manner and quite freely over the plain surface tissue, but which float loosely at the back of the fabric whence they are subsequently cut away as waste material previous to bleaching.

\section{Preparation of Applied Designs for Toilet Quilting Fabrics.}

$\S 218$. Unlike applied designs for textile fabrics of simple structure produced from only one series each of warp and weft threads, and which indicate the actual manner in which those threads are interlaced in the woven fabric, an applied toilet quilting design merely serves to indicate the manner in which the "back" or "figuring" warp threads or hooks of the Jacquard machine are raised for the respective pattern cards. Hence, the vertical spaces on the squared design paper represent figuring warp threads, or Jacquard hooks, and the horizontal spaces represent Jacquard cards. Therefore, the proper counts of design paper to employ for a toilet quilting design is determined according to the ratio of figuring warp threads and pattern cards employed in a given unit of space, say one inch, in the finished fabric. Thus, if a design to be prepared for any variety of toilet quilting fabric containing 32 back warp threads, and requiring 40 pattern cards per inch, is to be woven in a loom mounted with a 400 's Jacquard machine ( 8 hooks deep), the required counts of design paper will be ruled $8 \times 10$ squares to a bar; but if a 600 's machine ( 12 hooks deep) is employed, the design paper should be ruled with $12 \times 15$ squares to a bar.

The development of an applied toilet quilting design is indicated in Fig. 623, which represents a small portion of such a design, prepared on paper ruled with $8 \times 8$ squares to a bar, 
showing how the figuring warp threads are raised singly on the "tabby" principle of weaving, as explained in $\$ 206$. After painting in the margin of the ground and figure in the manner indicated, it is usual, in order to economise time and labour, to fill in the ground portion with a thin wash either of green or yellow paint, as indicated by shaded squares, and which

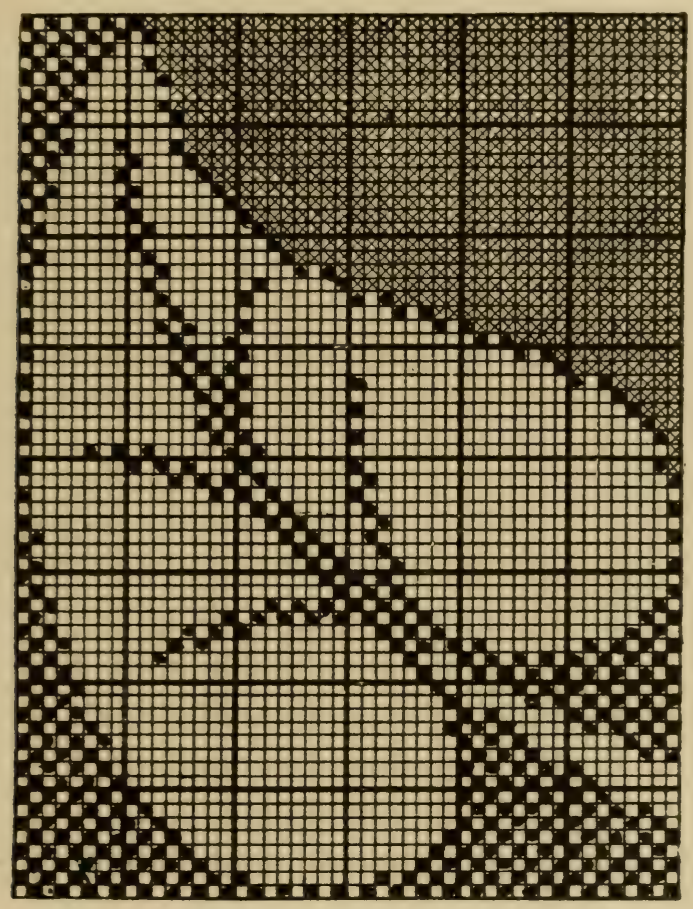

FIG. 623.-Method of developing applied designs for toilet quilting fabrics.

is understood by the card-cutter to signify that this colour must be "read off" and cut as "tabby".

$\$ 219$. It may, at this juncture, be observed that applied toilet quilting designs for all the different varieties both as regards the number of picks inserted for each pattern card and also the character of the reverse side of the fabric, are all developed in exactly the same manner, so that the same design is equally applicable to any one of those varieties of which the 
particular character is determined entirely by the mechanical equipment of the loom.

It should further be observed that if pattern cards for a toilet quilting design are being cut for a "split-shed " "tie-up" of the figuring harness, of which the odd and the even series of mounting threads are each controlled independently by separate Jacquard machines, as described in $\$ 214$, the card-cutter will, in these circumstances, "read" two bars of the design paper as representing only one short row, either of 8 or else 12 hooks, according to the index of those machines. In this case, therefore, alternate dots, representing the "tabby" weave, on design paper, will be cut as if they were placed consecutively. Thus, the vertical spaces corresponding to $1,3,5$ and 7 in each of the first two and succeeding pairs of bars on design paper, ruled with eight vertical spaces in each bar, represent hooks $1,2,3$, $4,5,6,7$ and 8 respectively, in each short row, of the "odd" Jacquard machine, and, in like manner, the vertical spaces, 2 , 4, 6 and 8 in the same pairs of bars represent hooks 1 to 8 in each short row, of the "even " machine, albeit consecutive hooks in those machines govern only the odd and the even series of figuring warp threads, respectively, as described.

$\$ 220$. Toilet quilting fabrics are produced in a variety of different grades of texture and quality of which the following table gives the particulars of ten actual examples of these fabrics in the "grey" or unbleached state, namely :- 
TOILET QUILTING FABRICS.

\begin{tabular}{|c|c|c|c|c|c|c|c|c|c|c|}
\hline \multirow{2}{*}{ 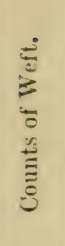 } & 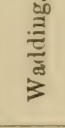 & $i=\infty$ & 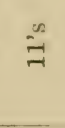 & $\dot{\sigma}$ & $i^{n}$ & $\dot{\omega}$ & लै & $\stackrel{\infty}{\Rightarrow}$ & $\stackrel{\infty}{\mathrm{g}}$ & ڤે \\
\hline & 迎 & {$\left[\begin{array}{l}\infty \\
i=1 \\
\infty \\
\infty\end{array}\right.$} & $\stackrel{\infty}{=}$ & $\hat{\sigma}^{\infty}$ & ஷิ & $\begin{array}{l}\infty \\
\infty \\
\infty \\
c\end{array}$ & in & is & in & $\check{c}_{\infty}^{\infty}$ \\
\hline \multirow{2}{*}{ 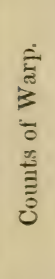 } & 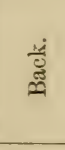 & $\begin{array}{ll}E & E \\
-1 & 1 \\
\infty & \infty \\
\infty & \infty \\
-\infty & \infty \\
-1\end{array}$ & $\begin{array}{l}E \\
\dot{0} \\
\dot{\alpha} \\
\dot{\alpha}\end{array}$ & $\begin{array}{l}E \dot{~} \\
0 \\
\infty \\
\infty \\
-1\end{array}$ & 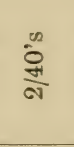 & $\begin{array}{l}E \rightarrow \\
\text { on } \\
0 \\
0 \\
-1\end{array}$ & $\begin{array}{l}\infty \\
\frac{i}{a} \\
\text { के }\end{array}$ & $\begin{array}{l}i^{\infty} \\
\frac{i}{a} \\
\frac{i}{d}\end{array}$ & $\frac{c_{2}}{a}$ & $\frac{\delta_{0}^{20}}{\text { के }}$ \\
\hline & 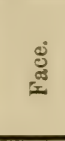 & 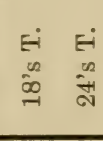 & $\begin{array}{l}\text { Ei } \\
\text { co } \\
\text { Сे }\end{array}$ & $\begin{array}{c}E- \\
\infty \\
\infty \\
\infty \\
-1 \\
\end{array}$ & $\frac{\tilde{D}^{2}}{\frac{\omega^{2}}{\sigma}}$ & $\begin{array}{l}E+ \\
\infty \\
0 \\
0 \\
0\end{array}$ & $\begin{array}{l}\omega^{n} \\
\frac{\infty}{a} \\
\cdots\end{array}$ & $\begin{array}{l}E- \\
\text { on } \\
\text { in } \\
\text { TH } \\
\end{array}$ & $\frac{d}{\frac{d}{a}}$ & $\frac{8}{\sigma}$ \\
\hline \multicolumn{2}{|c|}{ 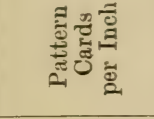 } & ฉి สి & ஓి & के & 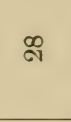 & के & 아 & is & 과 & छ艹 \\
\hline \multicolumn{2}{|c|}{ 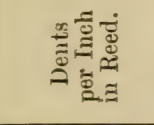 } & สิ นั จั & జ్ & $\stackrel{\infty}{\sim}$ & ๑ి & ๙ొ & శ్లి & F & 와 궁 & 과 \\
\hline \multicolumn{2}{|c|}{ 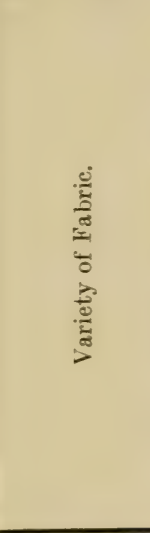 } & 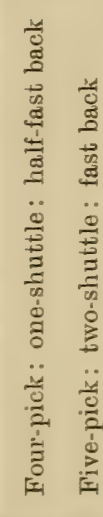 & 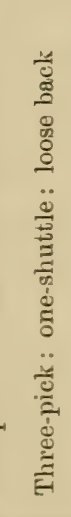 & 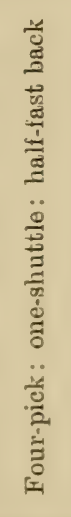 & 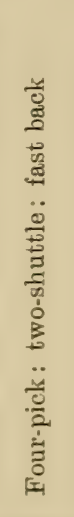 & 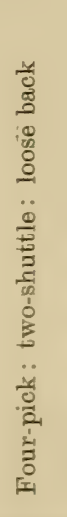 & 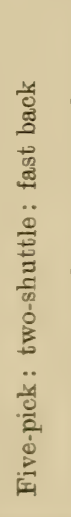 & 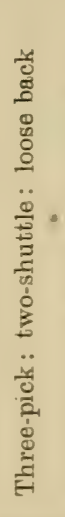 & 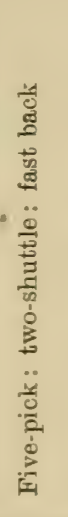 & 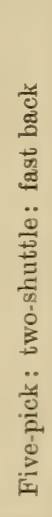 \\
\hline & & $\begin{array}{ll}-1 & \infty \\
\dot{z} & =\end{array}$ & $=$ & 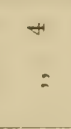 & $=$ & $\begin{array}{l}0 \\
=\end{array}$ & $=$ & & & $\stackrel{1}{=}$ \\
\hline
\end{tabular}




\section{CHAPTER XVII.}

\section{PATENT SATIN OR MITCHELINE FABRICS.}

$\$ 221$. "Mitcheline" and "patent satin" are the trade names of the first two varieties of a distinctive type of cotton fabric of which there are several modifications that differ from each other in minor details only. They are employed chiefly as counterpanes or bed-quilts, toilet covers and mats, antimacassars and similar articles, and are usually woven in the grey state and afterwards bleached. Some are woven with a white or coloured figuring on a coloured ground, and they may be embellished with coloured threads to produce stripes or checks, by embroidery, printing and painting.

The prototype of this class of fabrics was patented in 1868 by D. Mitchell-hence the term "Mitcheline"-and, in 1881, an improved structure of this fabric was patented by T. Taylor and J. Warburton, and registered as "patent satin," by which trade description it is known universally, and of which an example is illustrated in Fig. 624. The term "satin," however, is used in this connection merely as a fanciful description, as there is pothing of the satin or "sateen" weave in the construction of a "patent satin" fabric. This is essentially a compound structure produced by an ingenious combination of two series each of warp and weft threads to develop two distinctly different tissues of the plain "tabby" or calico weave, but which are so united, by interweaving, that the two tissues virtually constitute a single fabric in which the scheme of figuring is evolved simply by interchanging their respective positions.

The two series of warp threads consist of figuring threads of relatively coarse yarn and binding threads of finer yarn, and are in the ratio of two to one respectively: whilst the picks of weft 
consist of coarse figuring weft and fine ground weft in equal proportions. The respective series of warp threads are wound upon separate weavers' beams to allow of the different rate at which those threads contract during weaving. The coarse figuring warp and weft are interwoven on the plain tabby weave

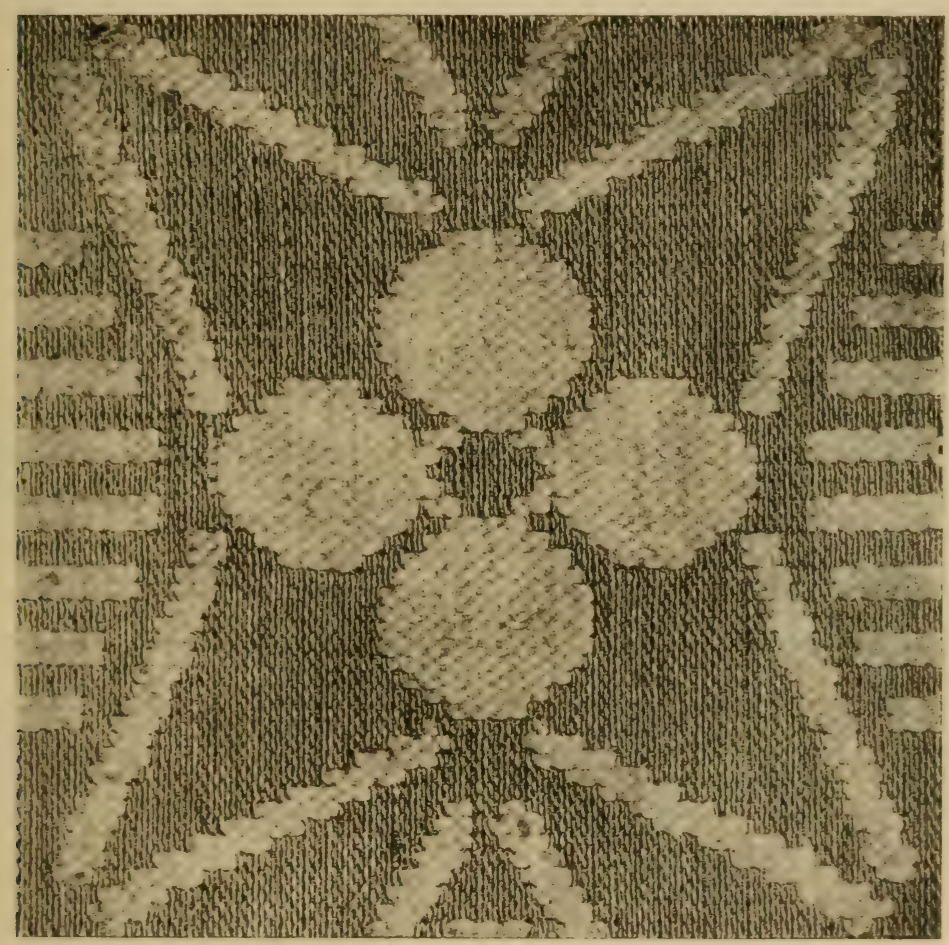

Fra. 624.-Patent satin fabric (Mitcheline) of coarse texture.

to produce a relatively coarse tissue for figuring purposes, whilst the finer warp and weft produce a finer calico tissue for the ground. At the same time, the fine binding or stitching warp threads interlace with the fine picks of weft in such a manner that the two independent tissues are so firmly stitched together that they virtually become one compact and firm fabric.

$\$ 222$. The figuring warp threads are governed by a Jacquard 
harness and two comber-boards, operating in conjunction with a pair of healds to control the binding warp threads, and of the same type as that of a "toilet quilting" harness, described in $\$ 210$, Chapter XVI. The harness threads are also divided into two equal sections, each passing through a separate comberboard, to constitute an "odd" and an "even " series respectively, and with the mounting threads "knotted" immediately above the comber-boards. Each hook of the Jacquard machine controls two harness threads and two consecutive figuring warp

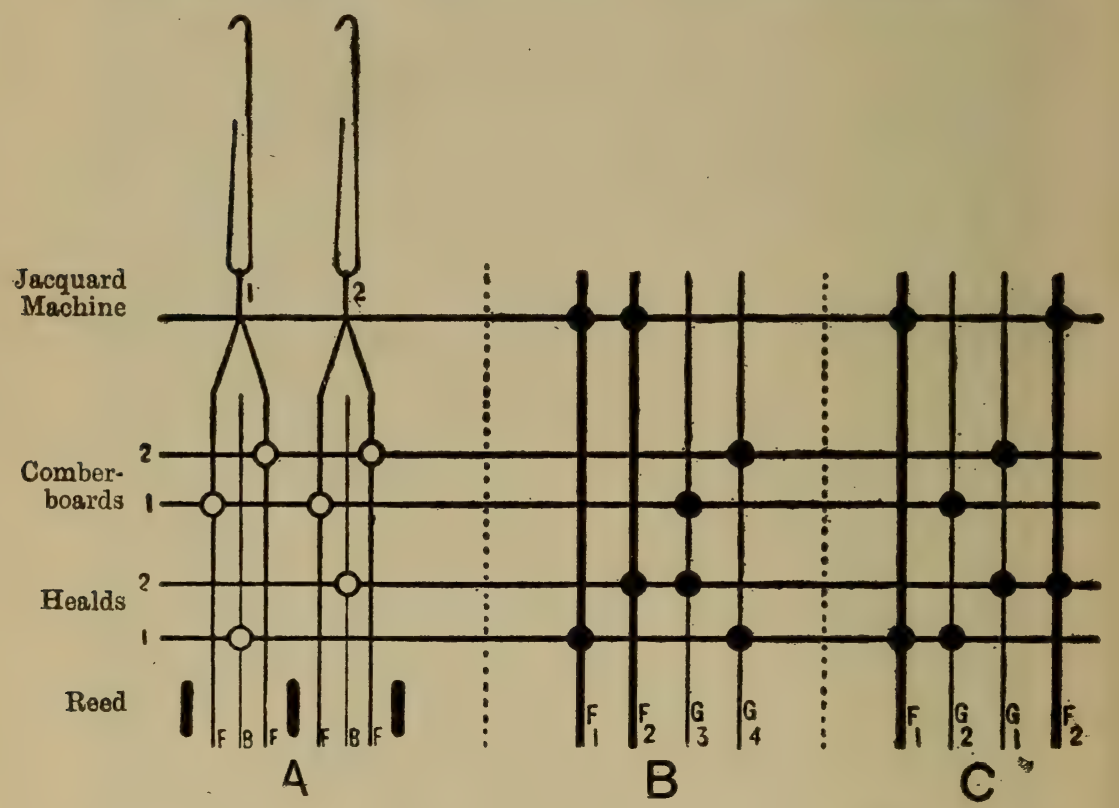

FIG. 625.-Draft and shedding plans for patent satin fabrics.

threads, with each of the two complementary harness threads, that are attached to the same hook, passing through a separate comber-board. Thus, the first hook commands the first and second figuring warp threads by means of two separate mount. ing threads that pass through the first and second comberboard respectively; the second hook commands the third and fourth warp threads in a similar manner, and so on, as indicated in the drafting plan A, Fig. 625, which also indicates the shedding plan at $\mathrm{B}$. 
When "drawing-in" the warp threads, these are passed through the harness eyes and reed in the order of one figuring thread through the first mail eye of the Jacquard harness of the first comber-board; one binding thread through the first eye of the first heald; and one figuring thread through the first mail eye of the second comber-board. These three warp threads are passed together through the same dent of the reed, and the operation is repeated, with consecutive warp threads, harness eyes and dents of the reed following in their proper rotation, as indicated in the "draft," in which it will be observed that a fine binding warp thread always intercepts two complementary figuring warp threads that are controlled by the same hook of the Jacquard machine, and which are also passed together through the same dent of the reed. With this arrangement of shedding harness and method of drafting, the figuring warp threads are raised and left down en masse by the Jacquard machine for the development of the design, whilst they may be raised in "odd" and "even" series, respectively, by raising the comber-boards in alternate succession to produce the fine calico ground tissue of the fabric, and at the same time operating the healds alternately to produce the figuring tissue, and also to bind both tissues firmly together in a manner to be described presently, and as indicated in the shedding plan B, Fig. 625.

In the production of these fabrics, four picks of weft, namely, two coarse and two fine picks are inserted during the operation of each pattern card, and one complete action of the Jacquard machine which is operated by a lifting cam that makes one revolution in four picks, and is constructed to raise the griffes for the two coarse figuring picks and to depress them for the two fine ground picks. At the same time one heald is raised and the other depressed whilst the first figuring pick is inserted. Hence, that pick extends entirely above depressed figuring warp threads and below raised warp threads, in accordance with the design; but it lies between the odd and even series of binding warp threads. Whilst the griffes are still raised, the healds reverse their relative positions for the insertion of the second coarse pick. The griffes then descend, the first comber-board is raised, and the healds remain in their present position, when the 
first fine ground pick is inserted. For the second fine ground pick the griffes remain down, whilst the first comber-board descends as the second one rises, and the healds also change their relative positions (as indicated in the shedding plan), after which this cycle of operations is again repeated for each successive pattern card, uniformly. The healds and comber-boards are operated by means of box-plate positive side tappets that raise and depress the healds for two consecutive picks in alternate succession, but in a contrary manner, thus, $\frac{2}{2}$, whilst the two comber-boards rise for the third and fourth (fine) picks respectively, thus $\frac{1}{2}$. The healds change their relative positions between the two coarse figuring picks, and again between the two fine ground picks of weft. Hence, a coarse and a fine pick are inserted side by side in each binding warp shed, as indicated in the sectional diagram B, Fig. 626, which represents at $A$ the method of preparing an applied design for the card cutter for a "patent satin" fabric, and also a longitudinal and a transverse section of the cloth at $B$ and $C$ respectively.

$\S 223$. A modification of the normal structure of "patent satin" fabrics, as just described, is effected by controlling the figuring warp threads separately by the Jacquard machine instead of in pairs, and also by inserting only two picks of weft for each pattern card, namely, one coarse and one fine pick as indicated in the draft and shedding plan A and B respectively, Fig. 627. But, in order to effect this object without the necessity of employing a loom adapted with two shuttle boxes at each end of the sley, and a "pick-and-pick" picking motion, the picks of weft, in this modified structure, are inserted in pairs of coarse and fine picks respectively, but in the following rotation, thus : for alternate pattern cards, first a coarse and then a fine pick is inserted; and for intermediate cards the picks are inserted in the reverse order of a fine and a coarse pick respectively, as indicated in the shedding plan B, Fig. 627. By adopting this course of inserting the picks in pairs of the same counts of weft, a loom with two shuttle boxes at one end and an alternate pioking motion may be employed.

The special advantage of this modification of structure is that it produces a relatively finer margin or edging of the figures, and 
thus imparts to the fabric a smarter and superior appearance by stepping the margin of the figure in single threads, instead of in steps of two threads of warp and weft for each figuring hook and pattern card. This superior effect, however, is obtained only at much greater cost in requiring twice as many figuring

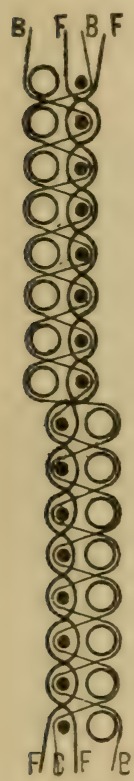

B
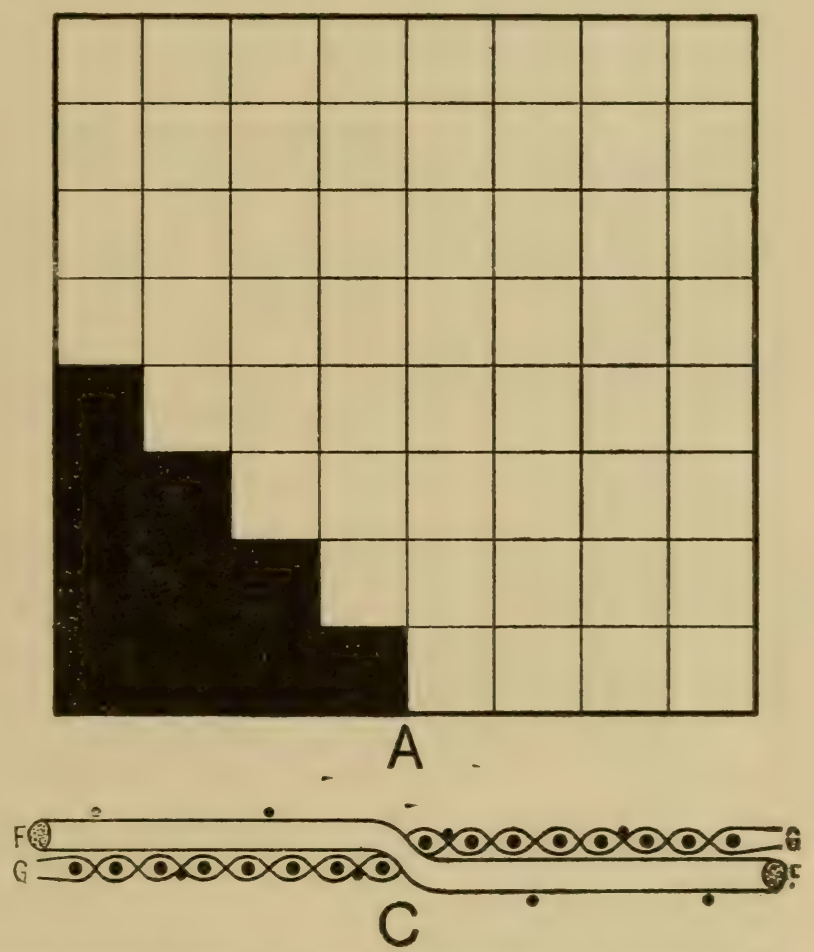

Fig. 626.-Part of applied design (A) with corresponding cloth sections (B, C) of patent satin fabric, Fig. 624.

hooks, and, therefore, four times as many pattern cards, for fabrics of similar texture, and designs of equal dimensions.

\$224. An applied design for a "patent satin" fabric is prepared en bloc in the manner indicated at A, Fig. 626. Each vertical division on design paper represents one hook of the Jacquard machine, and each horizontal division corresponds to one pattern card, irrespective of the number of figuring warp 
threads controlled by each hook, and of the number of picks inserted for each card. The correct counts or ruling of the design paper is determined by the ratio of hooks and pattern cards representing a given unit of measurement in each direction. When painting up a design, it is usual to paint the figure and leave the ground bare, in which case the card-cutter punches holes in the pattern cards for blank squares, and leaves them blank for filled squares of design paper.

$\S 225$. Patent satin fabrics are produced in a wide range of qualities, of which the actual data relating to ten different examples is specified in the following table:-

DATA RELATING TO PATENT SATIN FABRICS.

\begin{tabular}{|c|c|c|c|c|c|c|}
\hline \multirow{2}{*}{ Example. } & \multirow{2}{*}{$\begin{array}{c}\text { Dents } \\
\text { per In. } \\
\text { in Reed. }\end{array}$} & \multirow{2}{*}{$\begin{array}{l}\text { Cards } \\
\text { per In. }\end{array}$} & \multicolumn{2}{|c|}{ Counts of Warp. } & \multicolumn{2}{|c|}{ Counts of Weft. } \\
\hline & & & Figuring. & Binding. & Figuring. & Ground. \\
\hline 1 & 18 & 11 & 12 's T. & 26's T. & $2 t$ 's & 20 's \\
\hline 2 & 19 & 12 & 12's T. & 28 's T. & $3 \frac{11}{4}$ 's & 20 's \\
\hline 3 & 19 & 13 & 12 's T. & 28 's T. & $3 \frac{4}{2}$ 's & 20 's \\
\hline 4 & $21 \frac{1}{2}$ & 17 & 14's T. & 28 's T. & $3 \frac{1}{2}$ 's & 30 's \\
\hline 5 & $21 \frac{2}{2}$ & 17 & 14 's T. & 28's T. & 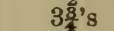 & 40 's \\
\hline 6 & $21 \frac{2}{7}$ & 18 & 16 's T. & 36 's T. & $4 \frac{1}{2}$ 's & 30's \\
\hline 7 & $21 \frac{\pi}{2}$ & 24 & $2 / 30$ 's & $2 / 50$ 's & 6 's & 14 's \\
\hline 8 & $30^{2}$ & 32 & 20 's T. & 36's T. & 8's & 60 's \\
\hline $9^{1}$ & 33 & 40 & 30 's T. & 45 's T. & 18 's & 110 's \\
\hline $10^{2}$ & 38 & 40 & 35 's T. & 45 's T. & 18 's & 120 's \\
\hline
\end{tabular}

${ }^{1}$ Examples 9 and 10 are of a very superior quality of the modified structure of "patent satin" fabrics in which the figuring warp threads are each controlled by separate hooks, and also with only two picks inserted for each pattern card, as described in $\S 223$. 


\section{('HAPTER XVIII.}

TAPESTHY FABRICS: ALSO KIDDERMINSTER OR SCOTCH CARPET FABRICS.

$\$ 226$. Tapestry fabrics that are produced by power-loom weaving comprise numerous distinctive types and modifications that differ essentially, both in their structural features and in the character of texture, chiefly according to the particular use for which they are specially adapted-as, for example, window curtains or hangings, door-hangings, furniture upholsterings, wall-coverings, table-covers, and counterpanes. Many of these fabrics display considerable artistic merit both in design and colour combination, but the mechanical restrictions and commercial considerations imposed in their manufacture are obstacles both to the free development of designs and to the employment of such a considerable variety and free distribution of colours that are possible of achievement in the true specimens of tapestries that are produced by hand-loom weaving, and as exemplified in Gobelin, Beauvais, and several other famous tapestry creations that have been evolved entirely by the handicraft and artistic skill of the weaver, and in the production of which there are virtually no mechanical or artistic limitations or restrictions, but only such restraint as may be imposed by human possibilities.

It is not, however, the purpose of this treatise to describe the construction either of the true hand-loom varieties of tapestry productions, nor those of the numerous varieties of so-called modern power-loom tapestries, of which there are many admirable examples possessing considerable technical and artistic merit. It is proposed, therefore, only to describe briefly the construction and manufacture of a certain type of power-loom tapestry fabrics, of which Figs. 627 and 628 are photographic reproductions of two typical examples. 


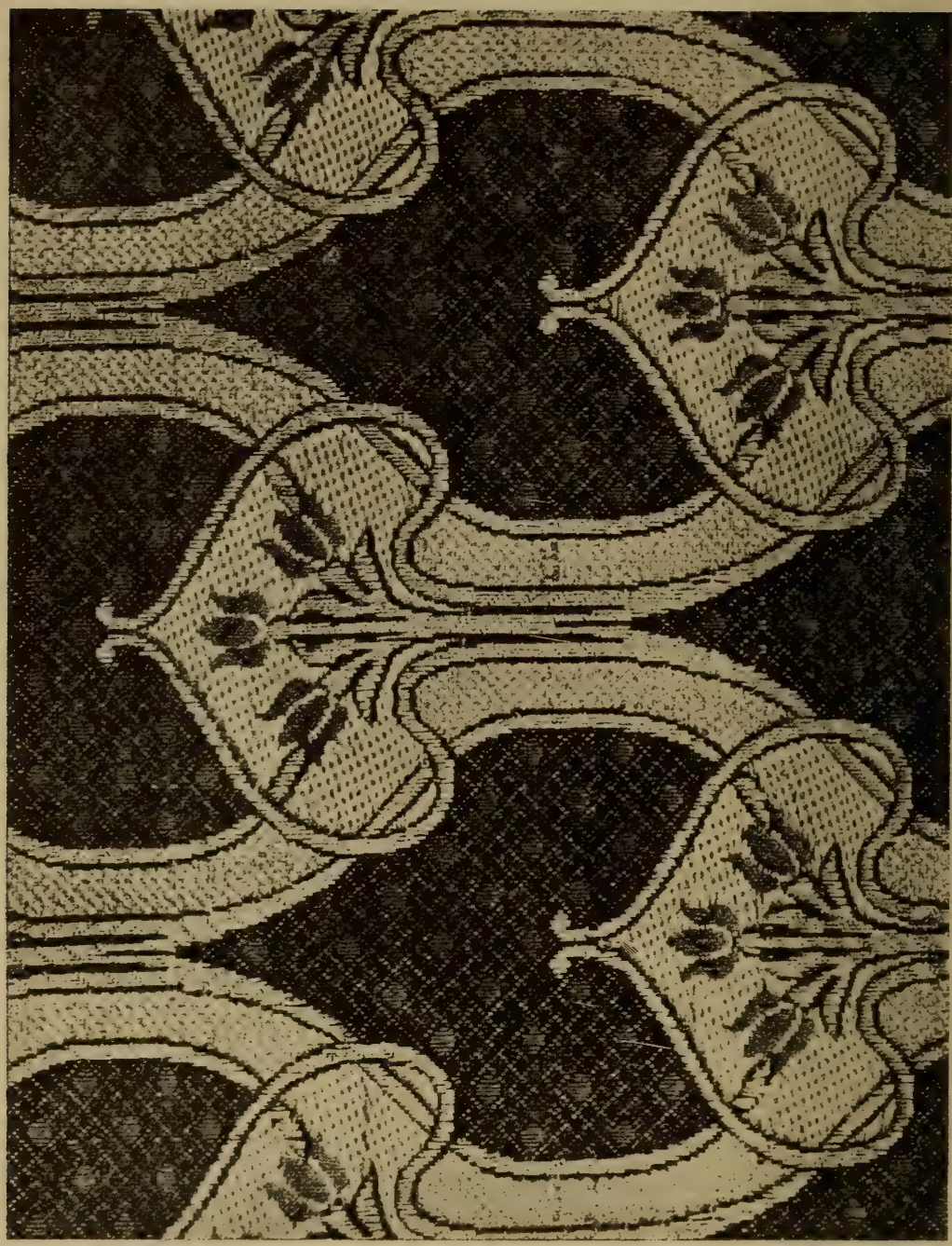

D. 


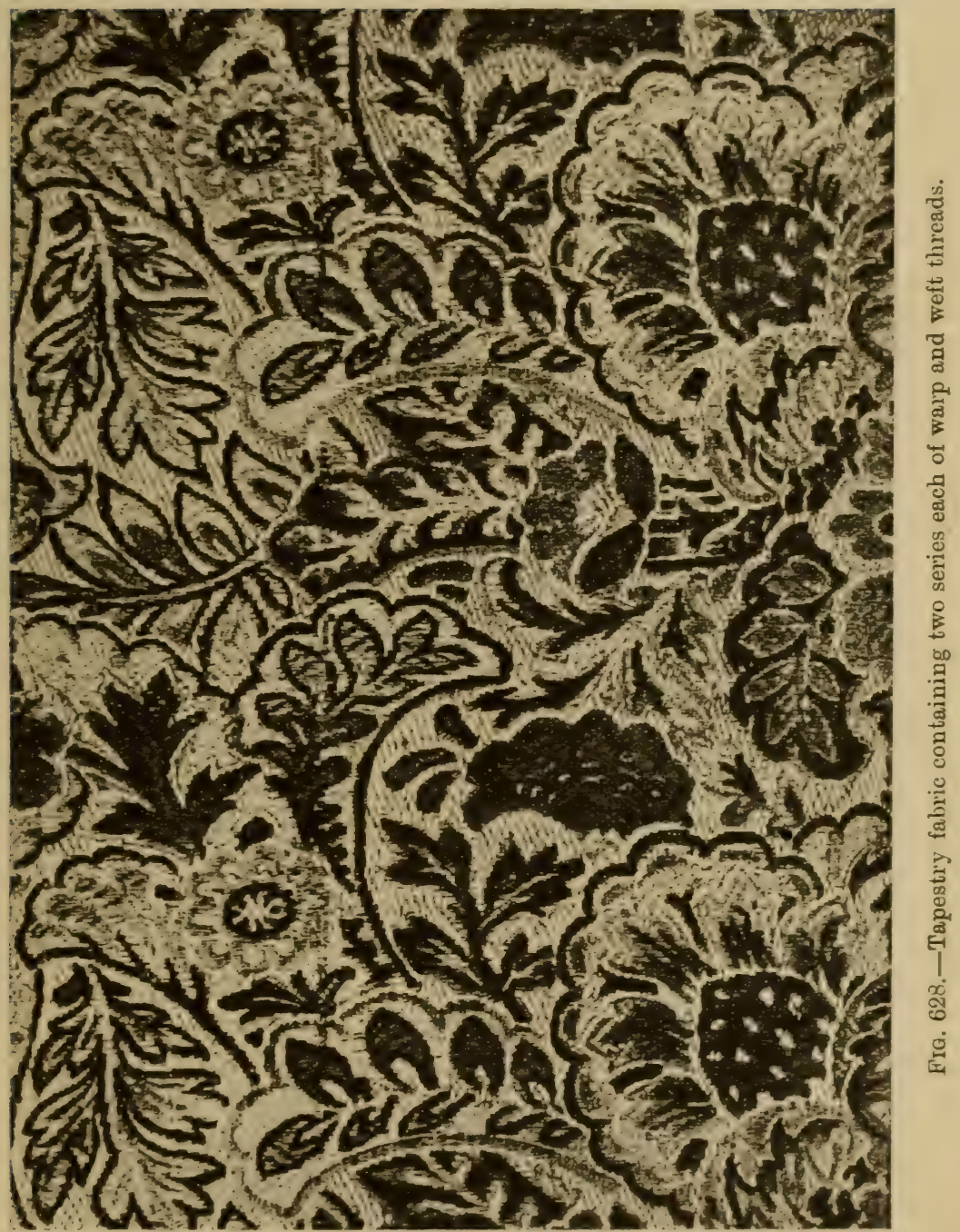


In this type of tapestry fabric there are several modifications that differ chiefly in the number of distinct series of warp and weft threads employed, and in all of which modifications the entire series of warp and weft threads interweave quite freely in any desired combination. There are no extra "binding" or "stitching" threads either in the warp or the weft, and the warp or weft threads of each series may be displayed either on the face or the back of the fabric as desired.

$\$ 227$. The various modifications of this type of tapestries are constructed with one or more distinct series of warp threads in combination with one or more distinct series of weft threads of different colour. Or, instead of any one of the warp or weft series of threads being of the same colour, uniformly, some of the threads of either or of both series may be supplanted by threads of other colours to produce a greater variety of colour effect without in any way affecting the structure of the fabric, since the threads which supplant the others (and which are usually said to be "planted") are not introduced as "extra" or additional threads, but are substituted only in the place of others.

The special object of "planting" with different colours of threads is to display those colours locally, either to accentuate certain features of the design or else to endow it with additional embellishment, without increasing the price of the fabric or cost of production, as would be incurred if the additional colours were obtained by the employment of supplementary threads. The several colours are "disposed" in accordance with a prearranged scheme which, when once adopted, remains fixed, so far as "planted" warp threads are concerned, until the warp, prepared according to that scheme of "planting," is finished. If, however, this method of "planting" is adopted in respect of the weft series of threads, the scheme of "colouring" may very easily be varied, simply by controlling the shuttle boxes of the loom according to the particular colours of weft required.

Tapestry fabrics of this type are usually manufactured entirely from cotton yarn for both warp and weft, as exemplified in Fig. 627 , which represents a comparatively heavy texture, composed entirely of cotton yarn and intended for table-covers or counterpanes. Sometimes, however, worsted yarn is employed in the 
heavier textures for one or more series of weft threads. Also, in some tapestries of lighter texture that are more suitable for window curtains or door-hangings, silk is sometimes employed for one of the series of weft threads as an additional embellishment, and also to impart to the fabric a brighter and richer appearance, as exemplified in Fig. 628.

$\$ 228$. It is proposed, however, to describe the construction of the three following varieties of tapestry fabrics, only :-

1. With one warp and two series of weft threads, with weft only displayed on both sides.

2. With two warps and one series of weft threads, with warp only displayed on both sides.

3. With two series each of warp and weft threads.

\section{Tapestry Fabrics with One Warp and Two Series of Weft, with All-weft Surfaces.}

In this variety of tapestry fabrics, two different colours of weft are employed. These are inserted in alternate succession or " pick-and-pick," thereby requiring a loom equipped with a special picking motion and with two shuttle boxes at each end of the sley. Only the picks of weft are displayed on both the
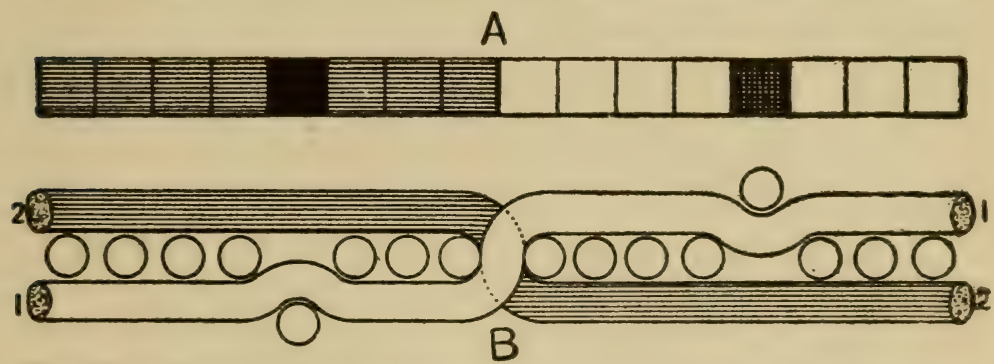

Fig. 629.--Transverse section of tapestry fabric containing one series of warp threads and two series of picks of weft.

obverse and reverse sides of the fabric which is, therefore, reversible, with the figure and ground portions developed by reversing the relative positions of the two kinds of weft, whilst the warp threads simply lie between, excepting when they are employed for binding or stitching over and under the picks of weft to prevent too long floats, as indicated in the diagram B, 
Fig. 629, which represents a transverse section of the fabric as it would appear if woven according to the strip of the design as represented at $A$, which represents sixteen warp threads and two picks of weft, i.e., one pick of each colour.

An applied design for this fabric would require to be prepared with three colours of paint, whilst the design paper would serve for a fourth colour, namely, one each to represent the respective colours of weft; and two for the binding or stitching points. Therefore, assuming red and white (paper) for the two colours of weft, and yellow and black for the face and back binding points respectively, the instructions to the card-cutter would be as follows:-

1. Red pick-cut white (paper) and yellow.

2. White pick-cut red and yellow.

Yellow dots are always cut, and black dots always missed. Thus, where a yellow dot occurs, the warp thread is raised at those points for both picks, to bind the weft which is, for the time being, floating on the face of the fabric; whereas a black dot indicates that the warp thread must be left down for both picks, to bind the weft at the back of the fabric, when in the loom. When binding the design, care should be taken to dispose the binding points for the back, as nearly as possible midway between those of the face.

\section{Tapestry Fabrics with Two Warps and One Series of Weft, with All-warp Surfaces.}

$\$ 229$. The structure of this variety of tapestry is virtually similar to that described in the previous section, excepting that the warp and weft of the former variety are transformed, as it were, into the threads of the other series, that is, warp threads become as weft threads, and vice versâ. The two series of warp threads may be wound either upon the same weaver's beam, or on two separate beams. They may also be controlled either by two equal divisions of the same Jacquard machine, to govern two sections of the harness threads-one for each series of warp threads-or each section of the harness may be governed by a separate machine, according to their capacity and other con- 
ditions. The two series of warp threads, if equal in number, are disposed through the harness eyes and reed with an "endand-end" or alternate disposition. Also, as there is only one kind of weft, an ordinary single shuttle-box loom only is required.

In preparing an applied design for this variety of tapestry fabric, as for the previous variety, three colours of paint are required in addition to the design paper, which represents a fourth colour, namely, one each to represent the respective colours of waip threads; and one each for the face and back binding or stitching points respectively, as observed in the strip of design, Fig. 630 A, which represents thirty-two warp threads,

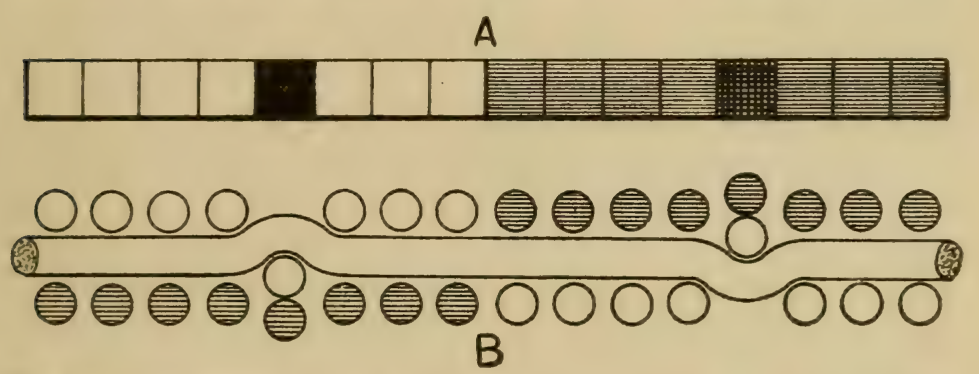

FIG. 630.-Transverse section of tapestry fabric containing two series of warp threads and one series of picks of weft.

i.e., sixteen of each colour, and one pick of weft, of which a transverse section is indicated at B. Thus; assuming the warps are indicated by red and white, with yellow and black dots for the face and back binding points respectively, the card-cutting instructions would be as follows:-

1. Red warp harness-cut red and yellow.

2. White warp harness-cut white (paper) and yellow.

Yellow dots are always cut, in order to raise at those points a warp thread of each colour to bind the face; whereas black dots are always missed, in order to leave down a warp thread of each colour, to bind the back of the fabric when in the loom, and as indicated in the sectional diagram $B$. 


\section{Tapestry Fabrics with Two Series Each of Warp and Weft Threads.}

$\$ 230$. Tapestry fabries produced from two series of warp threads and two series of weft threads are much more interesting than the varieties described previously, inasmuch as the additional series of threads introduces a fourth colour which increases very considerably the scope of a designer both in the choice of a subject for a design and also in the wider range which the additional colour affords in the development of different schemes of colouring, as exemplified in the specimens of this variety of tapestry fabrics illustrated in Figs. 627 and 628.

Both of these examples of tapestry fabrics are composed of two series, each of warp and weft threads of different colour, and they each embody precisely the same principles in their construction, although they are quite dissimilar both in texture and in weight, according to the different purposes for which they are constructed. Fig. 627 illustrates an ordinary two-warp, two-weft tapestry; whilst Fig. 628, which is of a similar structure, also exemplifies the method of "planting" threads of different colour, in one series of warp threads only, as described in $\S 227$.

The warp threads may be arranged in the fabric, and therefore through both the shedding harness and reed of the loom, either with an end-and-end or alternate disposition-that is, with a thread from one series and then one from the other series of warp threads drawn in alternate succession through separate mail eyes of the harness, whence they pass both together through the same dent of the reed, as observed in the construction of Fig. 628. Or else, the threads from the respective warps may be taped in pairs, uniformly with a two-and-two thread disposition-that is, with two contiguous threads from one warp, and then two from the other warp, drawn in alternate pairs through separate mail eyes, whence both pairs of threads from the respective warps are passed, all four together, through the same dent of the reed, as observed in the construction of Fig. 627 , of heavier texture, in which the colours of the two 
series of warp threads are green and orange respectively. By thus employing twice the number of warp threads of finer counts of yarn, and passing them in pairs through the harness eyes, and with four threads through each dent of the reed, it thereby ensures a much better distribution of the warp threads. which therefore cover up the weft more effectively, and also conduces to the development of a relatively much finer texture of superior quality than if only one-half that number of warp threads of coarser counts of yarn were passed separately through the harness eyes, and with only two threads through each dent of a reed of corresponding counts.

Both series of warp threads may optionally be wound together, either with an end-and-end or a two-and-two disposition, on the same weavers' beam; or else they may each be wound upon separate beams. But both for technical and practical considerations, however, the former course is preferable, inasmuch as both the degree of tension and the rate of contraction during weaving should be exactly the same for both series of war'p threads. Also, the weaver will have the weighting of only one beam to regulate, thereby avoiding the risk of obtaining unequal tension upon the two series of warp threads, as would be liable to occur if they were each wound upon separate warp beams. On the other hand, however, if two separate warp beams are employed, each carrying only one-half the aggregate number of threads, the length of the respective warps may then be doubled, thereby reducing by one-half the amount of time and labour spent in gaiting and looming, and also incurring less waste of material which those operations involve.

$\$ 231$. The warp threads of both series are controlled entirely by a Jacquard harness, which may be governed, optionally, either by means of single-acting or else double-acting Jacquard machines of ordinary construction, without any special mechanism. Also the Jacquard harness is constructed preferably in two separate and distinct sections-one for each of the two series of warp threads. The two sections of the harness pass through the front and rear portions of the same comber-board respectively, and are governed either by separate sections of one machine only, or else by two separate machines, according to 
the planning of the harness tie-up and the capacity of the Jacquard machines. If only one machine is employed, this is divided equally into two similar sections, virtually to constitute two smaller machines placed side by side, and mounted preferably with the card cylinder (or cylinders) on one side (or both sides) of the loom, with the object of keeping both sections of the harness quite separated for their entire length. If, however, a separate single-acting machine is employed for each section of the harness, both sections may be kept quite separate by mounting the two machines back to back, and operating them simultaneously, as if they constituted two equal sections of one Jacquard machine only.

As stated previously, these two examples of tapestries each contain two series of weft threads of different colour. These are inserted in the fabrics with a pick-and-pick or alternate disposition, thereby requiring each end of the loom sley to be constructed with two shuttle boxes controlled by a shuttle box or checking motion, and also a loom provided with a pick-at-will picking motion adapted for picking twice in succession from each side of the loom; but in all other respects the loom em. ployed may be of the usual type of construction. Also, for practical considerations only, it is expedient to govern the operation of both the shuttle box and picking motions by means of spare hooks in the Jacquard machine to ensure the operation of those motions, and also of those with the pattern cards, in unison with each other. Otherwise, if these several parts are each controlled independently, they are liable to get out of harmony with each other, and thus create difficulties.

$\$$ 232. Method of Preparing Tapestry Designs.-When preparing an applied design upon squared or point paper, to be read off by the card-cutter, for tapestry fabrics of the type under present consideration, it is necessary to indicate the respective colours of warp and weft threads by means of a corresponding number of distinctive colours of paint, which may be either of the same colour as the respective series of threads which they represent, or of different colour. In actual practice, however, the design paper itself usually represents one series of weft threads, and thus saves the time and trouble of painting it on the design. 
The counts or ruling of design or point paper required must be in accordance with the index of the Jacquard machine employed, and also with the relative number of warp threads and picks of weft per inch in respect of only one series each of those threads, and not in respect of the aggregate number of threads contained in one inch.

An applied design for each of the examples of tapestry fabrics represented in Figs. 627 and 628, is planned on such number of vertical and horizontal divisions of the design paper as corresponds with the nominal number of warp and weft threads-but in respect of only one of each series of those threads-constituting one complete repeat of the design, which must repeat either on the full tie-up or any measure of that. The design may be developed in any suitable colours of paint to represent the respective colours of warp and weft threads which may be interlaced and combined in any conceivable practical manner to produce the desired effects in cloth. Binding weaves of any description may be employed to prevent too long floats of threads, but for technical reasons it is not advisable to insert single binding points of one series of warp threads on a warp surface composed of warp threads of the other series, nor those of one series of weft threads on a weft surface composed of the other series; for, although such a course may be practicable, it would impart to those threads an abnormal degree of tensile strain, and also develop structural defects in the woven fabric. For these reasons, therefore, it is more expedient to bind any one series of threads with those of either of the transverse series of threads. Also, some designers prefer to separate the figure and ground with an outline consisting of one or two threads of the darkest colour, to impart to the margin of the figure a sharper and clearer definition.

\$233. Instructions for Card Cutting.-From the foregoing description it will be manifest that each vertical division on the design paper represents two separate and distinct warp threads, that is, one thread of each series; and also, in like manner, that each horizontal division represents two picks of weft-one of each series, as observed in the diagram B, Fig. 631, which represents a transverse section of cloth as it would appear if 
woven according to the strip of the design shown at A. Hence it is necessary for the card-cutter to read off and cut the design four times over-once for each pick of each colour of weft for each section of the Jacquard harness, whether these are controlled by one machine only, or by two separate machines.

Therefore, assuming a design is developed with colours of paint corresponding with those of the respective colours of warp and weft threads composing the specimen of coarse tapestry, Fig. 627, the instructions for card cutting will be as follows :-
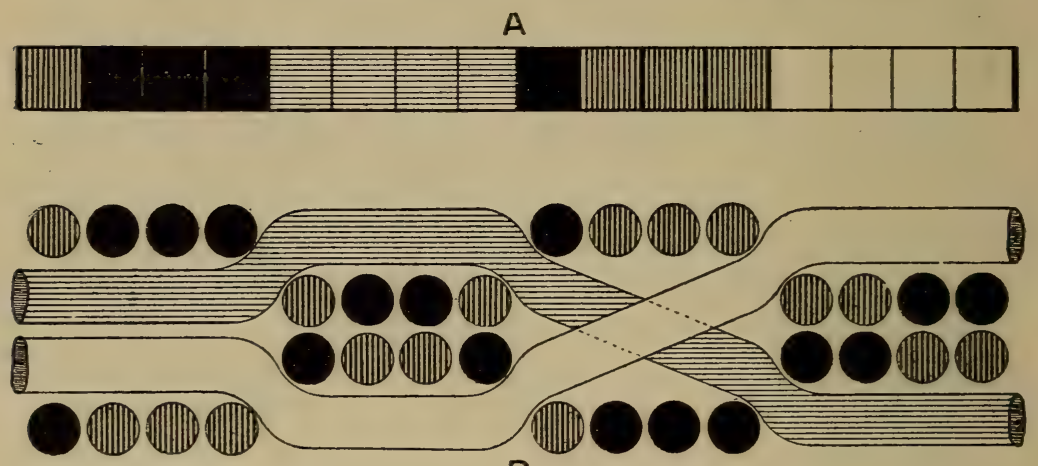

B

FIG. 631.-Sectional diagram showing construction of a tapestry fabric containing two series each of warp and weft threads as Figs. 627 and 628.

1. For Section of Harness Controlling Green Warp. Threads.

(A) Black weft . . . Cut green and pale blue.

(B) Pale blue weft . . , " , black.

2. For Section of Harness Controlling Orange Warp Threads.

(A) Black weft . . Cut orange and pale blue.

(B) Pale blue weft . . , , , , black.

It will be observed that when cutting for a specified section of harness and colour of weft, the colour of warp threads controlled by that particular section, and also the other colour of weft, only, are cut, whereby the respective series of warp and weft threads are disposed in the fabric in the manner indicated in the sectional diagram B, Fig. 631. A longitudinal section of 
the fabric would also reveal an exactly similar structure, which is essentially of a compound character of texture in which the respective series of warp and weft threads are disposed each in such a manner that whicherer of the two series either of warp or weft threads is displayed on one side of the fabric, the complementary threads of the same series are displayed on the reverse side in the corresponding part of the fabric; or else both groups of the same series of threads are disposed midway between the two outer series of threads simultaneously, thereby producing a reversible fabric, but one in which the scheme of colour distribution is different on each side, as indicated in the diagram B, Fig. 631.

$\$ 234$. The counts of design paper required for the applied designs of the accompanying specimens of tapestries, and other data relating to their construction, are specified as follows :-

Fig. 627: Tapestry Fabric of Coarse Texture; composed of $3 / 48$ 's green and orange mercerised cotton warp threads taped in pairs uniformly, and containing 64 actual (or 32 nominal) threads of each colour per inch in the cloth, with 30 dents per inch in the reed, and 4 separate warp threads (two of each colour) in each dent; also of 6 's black and pale blue soft-spun cotton weft, with 32 picks of each colour of weft per inch.

The shedding harness, shuttle boxes, and picking mechanism are all controlled by a 400 's double-acting one-cylinder Jacquard machine with a straight tie-up repeating on 160 hooks for each section of the harness, which are governed respectively by separate sections of the machine. Therefore, since the nominal number of threads per inch is the same for both warp and weft, the correct counts of design paper to employ for this example is that ruled with $8 \times 8$ divisions in each bar, because a 400 's Jacquard machine contains eight rows of hooks and lances or needles.

Fig. 628: Tapestry Fabric of Fine Texture; composed of $2 / 60$ 's cotton yarn for both series of warp threads, one of which is dyed blue uniformly, whilst the other is composed of both maroon and light brown threads planted in stripes of different width, and containing 72 threads of each warp per inch in the cloth, with 68 dents per inch in the reed, and two separate 
warp threads (one from each warp) in each:dent; also of fivedram light gold floss silk weft (equivalent to a little finer than 60 's cotton weft), and 16 's black soft-spun cotton weft, with 60 picks of each kind of weft per inch.

The shedding harness is tied-up with a straight tie-up repeating on 300 hooks, and may therefore be controlled either by one 600's Jacquard machine, or else two 300's machines. In either case the counts of design paper required for this example is in the proportion of $72: 60$, so that with 12 hooks in a row the correct counts would be $12 \times 10$ vertical and horizontal divisions respectively.

If, instead of employing one 600's Jacquard machine, there are two 300's machines mounted back to back, it is important to observe that when cutting the pattern cards for the rear machine the design will require to be inverted, and read off upside down, because the hooks on the 26 side (on the right when facing the card cylinder) of that machine control the harness threads in the rear section, corresponding to those in the front section that are controlled by the hooks on the 25 side (on the left of the cylinder) of the front machine. Also, it will be necessary, when drawing in the warp threads through the harness mail eyes, to reverse the direction of those governed by the rear section, because the two complementary warp threads of different colour that pass through the same dent of the reed are governed respectively by two hooks that are situated in corresponding rows of both Jacquard machines. This will be understood by reference to the following plan, which indicates the order in which the harness threads pass through the holes in the front and of the rear sections comber-board, thus :-

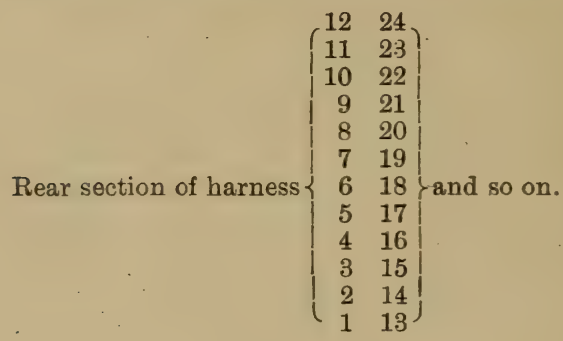




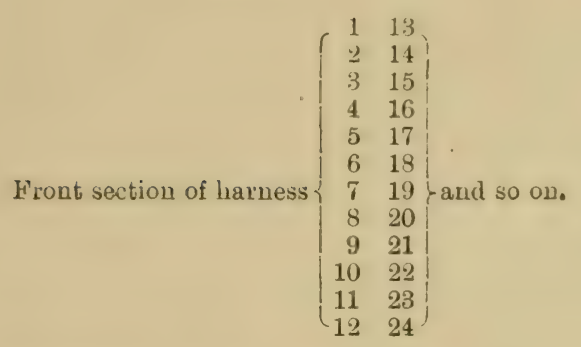

\section{Kidderminster or Scotch Carpet Fabrics,}

$\$ 235$. "Kidderminster" and "Scotch carpet" are optional terms that are variously employed to designate a distinctive

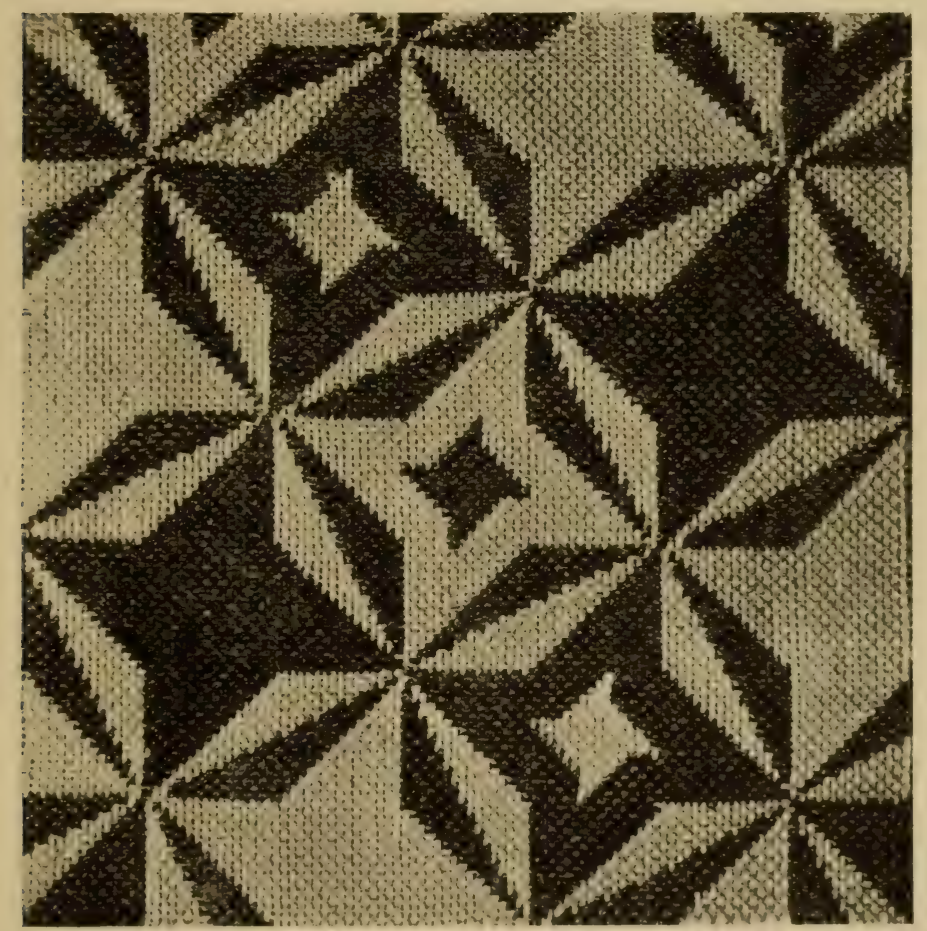

FiG. 632.-Scotch or Kidderminster carpet fabric.

type of compound fabric structure embodied in a wide range of textures varying from heavy and coarse, to light and fine grades. 
chiefly according to the character of the textile material from which they are produced and also the purpose which they are intended to serve, as, for example, carpets, rugs, curtains, hangings, coverlets and mats, counterpanes and such-like articles for domestic use. An example of this type of fabric, which is essentially a compound structure evolved from two series each of both warp and weft threads, is illustrated in Fig. 632, and contains 64 warp threads (taped in pairs) of each series of threads, i.e., $64 \times 2=128$ warp threads and 32 picks of weft of each series, i.e., $32 \times 2=64$ picks per inch of $3 / 48$ 's cotton yarn for both warp and weft, which produces a very substantial fabric of medium texture and one, therefore, suitable for use as hangings and antimacassars.

\section{A}
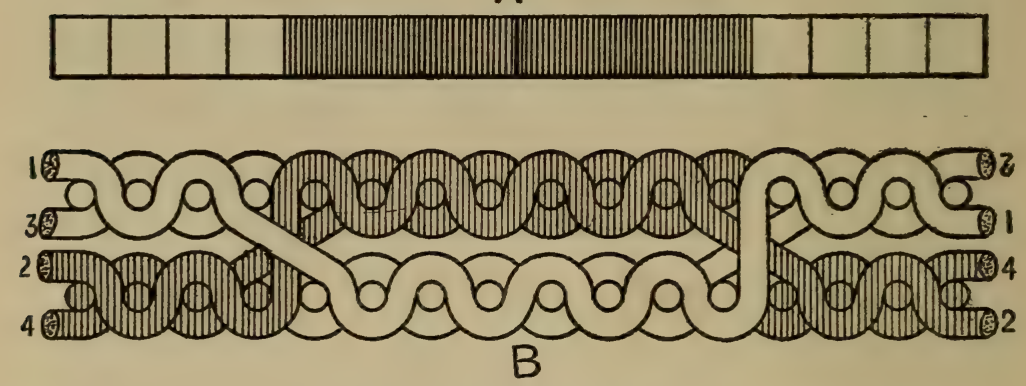

FIG. 633.-Sectional diagram showing construction of Kidderminster or Scotch carpet, Fig. 632.

Fabrics of this type embody the true double cloth or two-ply principle of construction, as they comprise two separate and distinct tissues of difierent colour and of similar or dissimilar texture, usually of the plain calico or other simple weave. In the scheme of decoration, however, the two tissues interchange their relative positions and thus become united only at the margin of the figure and ground, where they penetrate each other and thus constitute a true reversible fabric as illustrated in Fig. $633 \mathrm{~B}$, which represents a sectional diagram of this fabric, whether viewed transversely or lengthwise, as it would be presented if woven according to the strip of the design $A$, each square of which represents two warp threads, i.e., one of each colour, and two picks of weft, i.e., one of each colour also. 
The two series of threads of the same colour are interwoven so as to develop the figure and ground in monotones of the respective colours employed.

$\$ 236$. Both series of warp threads are, preferably, wound together on the same weaver's beam, to ensure a uniform degree of tension on each series, and they are passed through the harness eyes and dents of the reed with an "end-and-end" or

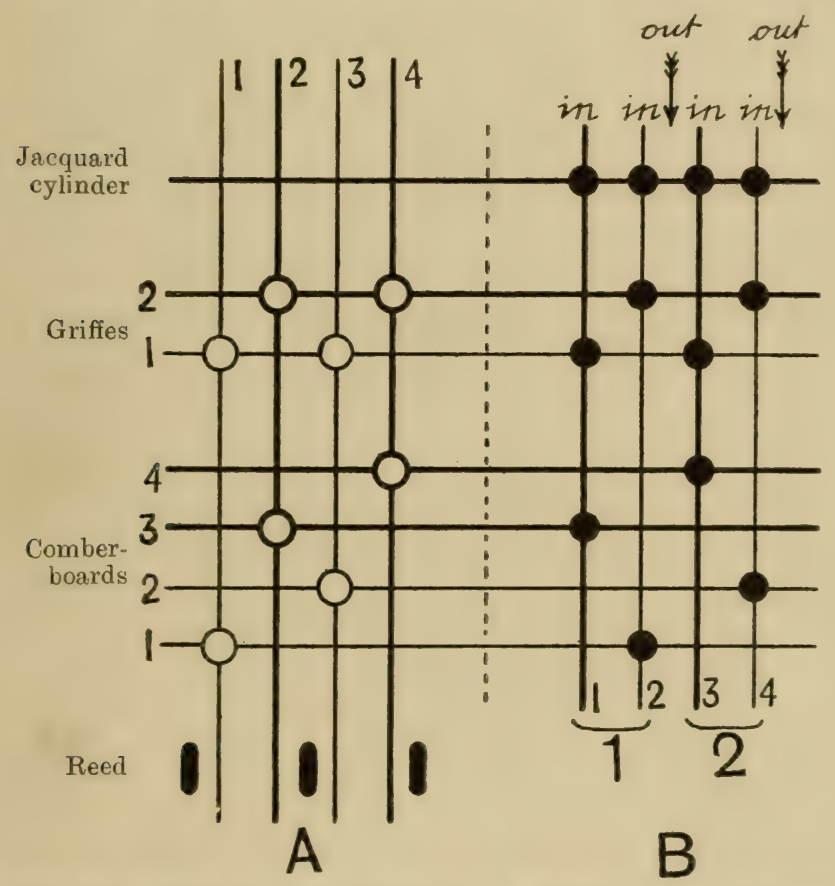

Fig. 634.-Draft (A) and shedding plan (B) for Scotch or Kidderminster carpet.

alternate disposition, as indicated in the drafting chart A, Fig. 634, which also indicates the shedding plan at $B$, as these would be adapted to the special type of Jacquard machine and shedding harness which are employed in the manufacture of these fabrics. This type of machine and harness, as represented in Fig. 635, is designed to control the two series of warp threads from two independent sources, namely, by the Jacquard machine $\mathrm{H}$, which controls the warp threads en masse according to the 


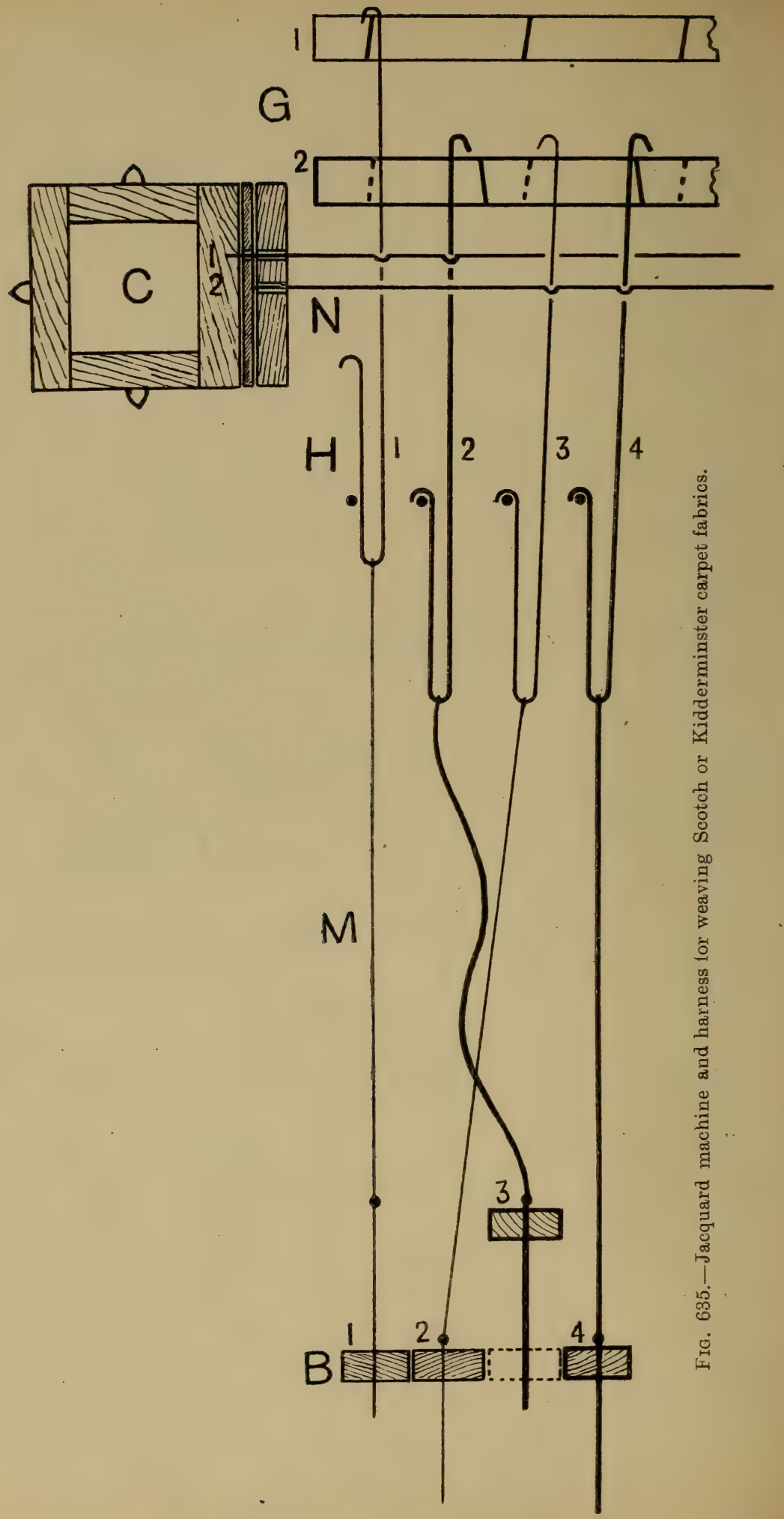


design; and also by means of comber-boards $B$, by which the warp threads are raised in a prescribed order according to the method of interweaving the warp and weft for the creation of texture, as distinct from the scheme of decoration.

The special advantages of this type of machine and harness are that they simplify the preparation of designs which are developed en bloc, i.e., in solid masses of figure and ground; they also facilitate the operation of card-cutting and effect considerable economy in pattern cards, each of which serves for two consecutive picks, one of each colour of weft.

$\$ 237$. The Jacquard machine indicated is constructed with two distinct sets of hooks, H 1-4, of which only the first four rows from the card cylinder are represented in the diagram. The alternate rows of hooks, 1,3 , 5, etc., constitute one set which faces the cylinder $\mathrm{C}$, with their beaks or nebs normally over their respective griffe blades $G 1$, of which there are also two sets; whilst the hooks in the intermediate rows, $2,4,6$, etc., constitute the second set, with their nebs pointing axay from the cylinder, and also normaily away from their respective griffe blades G 2, as indicated by the hook H 2. Both sets of hooks, however, are controlled by only one set of needles $\mathrm{N}$, each of which commands two hooks in opposite rows that stand back to back, i.e., with their nebs pointing away from each other, as for example, $\mathrm{H} 1-2$, and $\mathrm{H} 3-4$ respectively.

Each set of hooks controls a separate section of the harness threads M, one for each series of warp threads; whilst each section of harness threads is again subdirided into an "odd" and an "even" series that pass through two separate comberboards B, immediately above which the mounting threads $M$ are knotted, whereby the corresponding division of warp threads of either series may be raised collectively to form a warp shed with that series, for the insertion of a pick of weft of the corresponding colour of yarn. Thus, the mounting threads governed by hooks contained in rows $\mathrm{H} 1,3,5$, etc., which face the cylinder, constitute the front section of the harness, of which the odd and even series of threads pass through comber-boards B 1-2 respectively; whilst the odd and even series of harness threads, which constitute the rear section and is governed by hooks contained in rows $\mathrm{H} 2,4$, 6 , ete. 
pass through comber-boards B 3-4 respectively, as indicated in the diagram, Fig. 635, and also in the drafting chart A, Fig. 634 .

$\S 238$. The card cylinder C, griffe frames G 1-2, and comberboards B 1-4 are each operated independently and as indicated in the shedding plan B, Fig. 634. Thus, the cylinder is governed by a cam that makes one revolution for two picks and is designed to hold the cylinder against the needles for both picks. The cylinder then changes the card quickly and returns before the warp shed is formed for the first pick of the next following pattern card, as indicated by the small arrows in the shedding plan. The griffe frames containing the two sets of griffes G 1-2 are operated by a double crank fixed on one end of the picking tappet-shaft of the loom, whereby the respective griffe frames are raised in alternate succession for the two consecutive picks of weft inserted for each pattern card: whilst the four comber-boards B 1-4 are operated by side tappets and are each raised in a prescribed order, as indicated in the shedding plan, once during the operation of two pattern cards, corresponding to four picks of weft, which represent one complete cycle of movements by the card-cylinder $\mathrm{C}$, griffes G 1-2, and the comber-boards B 1-4, namely:-

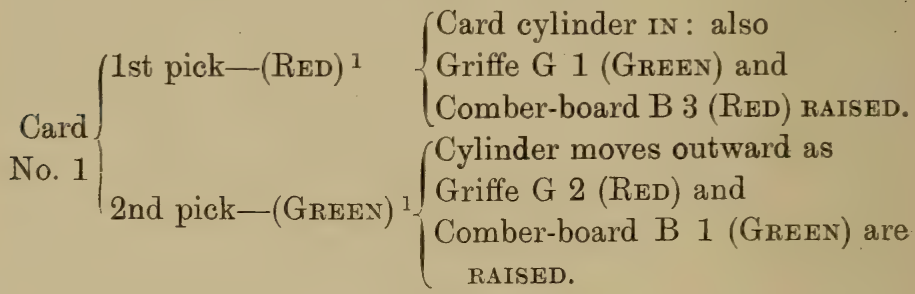

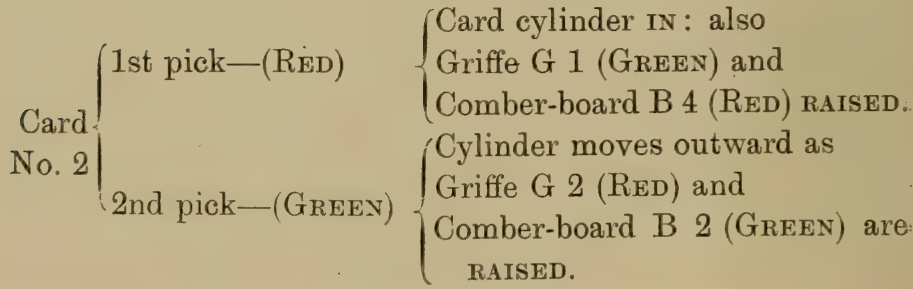

\footnotetext{
${ }^{1}$ The two colours red and green are represented in Figs. 634 and 635 by thick and thin lines respectively.
} 
It should be observed that each pair of hooks that are controlled by the same needle command two contiguous warp threads, one of each colour, that are contained in the same dent of the reed, and that only one of each pair of hooks and, therefore, warp threads of one series and colour only may be raised, by the griffes, at the same time and for the same pick of weft. Thus, for the first pick of each card, which is a red pick, only green warp threads are raised or left down en masse by the Jacquard hooks for the development of the pattern, whilst at the same time the odd and even series of red warp threads are raised for the odd and even pattern cards respectively, by the comber-boards B 3, 4, for the creation of the red tissue of cloth. Likewise, for the second pick of each card, which is a green pick, only red warp threads are raised or left down en masse for the pattern, and, at the same time, odd and evennumbered green warp threads are raised by the comber-board B 1, 2 for the odd and even pattern cards respectively, and as indicated in the shedding plan B, Fig. 634, for the creation of the green tissue of the fabric. 



\section{INDEX.}

A.

Action of the loose reed in terry looms, 171.

Alhambra fabrics, 390.

- "Trellis" quilts, 395.

Alternative dispositions of pile and ground warp threads in terry pile

fabrics, relative merits of, $170,188,207$.

Angle of twill, 30 .

B.

Back standard healds, 218.

- - position of, in relation to regular healds in leno looms, 262.

Backed fabrics, 126.

- - reversible, 132.

- - warp, 130.

- - weft, 128.

- - what to bear in mind when preparing designs for, 127.

Beaverteen fabrics, 139.

Bedford cord fabrics, 110 .

- - alternative methods of introducing extra coloured warp threads into, for figuring purposes, 120.

- - - detailed specifications of all the examples of, herein described 125.

- - plain-ribbed, 112.

- - twill.ribbed, 115.

_ - - usual means of embellishing, 111.

- - variegated, 114.

_ - - with Jacquard figuring, 122

Bessbrook damask or twilling machine, 373.

Bottom-doup harness for cross-weaving, 213.

"Brighton" weaves, 86.

- - construction of, 87.

Brocade fabrics, characteristics of, 338.

- - figured with extra warp, 354.

- $-\ldots$ warp and weft, 352, 359.

(493) 
Brocade fabrics, leno, 211, 272.

- - preparation of designs for, 346.

- - warp and weft figured, 344.

— - weft figured, 338.

Broken twills, 67.

Brussels carpets, formation of the looped pile in, 170 .

C.

Calico or plain weave, construction of the, 6 .

- - - definition of the, 6,9 .

- $-\ldots$ methods of embellishing the, 15.

- - - modifications of the, 7 .

- $\ldots$ - variety of form in the, 9.

- $-\ldots$ - - texture in the, 8.

Cantoon or " diagonal " fabrics, 137.

Canvas cloth, 270.

Card cutting for a "float" harness, 425.

- _ - "split-shed tie-up," 462.

- - from brocade designs, 343.

- _ - leno brocade designs, 302.

- _ - tapestry designs, 481.

"Cassimere " or "Harvard" ( $\left(\frac{2}{2}\right)$ twill, 27.

Catch-cord, the function of a, 192.

"Cellular" gauze fabrics, structure of, 220.

Classification of twill weaves, 24.

Comber-boards in toilet quilting looms, operation of, 453.

Combination of two twill weaves, end-and-end, 62.

- - - - pick-and-pick, 65.

Combined twills, 60.

Compound net leno fabric, 231.

- "presser" harness for damask fabrics, 365.

- shedding harness for figured terry fabrics, 199.

Corded and ribbed fabrics, simple, 10.

_ _ _ - with variegated ribs, 19.

- or ribbed velveteen fabrics, 152 .

Cords, velvet, 153, 160, 163.

Corduroy fabrics, 160.

- - figured, 163.

- - machines for cutting, to form pile, 164.

- - - - (circular knife), 165.

- _ - - (straight knife), 167.

- _ " thickset," 162.

- - with variegated cords, 160.

Corkscrew twills, 48.

Counterpanes, Alhambra quilts or, 390 . 
Counterpanes, toilet quilts or, 436 .

_- "Trellis" quilts or, 395.

"Cover" in cloth, definition of, 32.

Crêpe or "oatmeal" weaves, 103.

Cross shed, formation of a, with a bottom-doup harness, 219.

- _ - - with a top-doup harness, 224, 227, 257.

Cross-thread lappet figuring, 325.

Cross-weaving, different types of shedding harnesses for, 213.

- - steel-wire doup harnesses for, 264.

\section{D.}

Damask fabrics, 362 .

- - compound, 383.

- - - "presser" harness for, 365.

- - preparation of designs for, 378.

- or twilling Jacquard machines, 372.

- _ - - Bessbroak, 373.

Density of pile in terry pile fabrics, circumstances affecting the, 190.

Design, chief divisions of textile, 3.

- definition of woven, 3.

- Grammar of Textile, definition of, 3.

- or point paper, counts of 4 .

- - - use of, 4.

Designs, leno, what to bear in mind when preparing, 262.

Details of leno weaving, practical, 262.

"Diagonal" or cantoon fabrics, 137.

Diamond weaves, 78.

Direction of $t$ wist in yarn, and the influence it exercises upon the relative prominence of twills, the, 32 .

Dispositions of pile and ground warp threads in terry pile fabrics, relative merits of alternative, $170,188,207$.

Dobbies for gauze and leno weaving, relative merits of different types of, 256.

Double-faced or reversible fabrics, 132.

Double reeds for terry pile weaving, 208.

Doup harnesses, relative merits of top and of bottom, 254.

- - steel-wire, for cross-weaving, 264.

- healds, 218.

- - steel wire, disadvantages of, 268.

- - worsted, disadvantages of, 264.

- warp threads, 213.

Doups, definition of, 213.

Draft and shedding plans for figured terry fabrics, 200.

Dugdale's terry pile motion, 178. 
E.

Easers, slackeners, or vibrators in leno looms, the function of, $218,219$.

Embossed designs in velveteen fabrics, 159.

"Erdmann" reeds, 335.

Essential factors in terry pile weaving, 1๕0.

- parts of a gauze or leno harness for cross-weaving, 213.

$\mathbf{F}$.

Fan, ondulé or paquet reeds, 331.

Figured Bedford cord fabrics, Jacquard, 122.

- corduroy fabrics, 163.

- or ornamented twills, 73.

- terry pile fabrics, 196.

- velveteen fabrics, Jacquard figured, 154.

Firmness of texture, influences affecting the, 6 .

Flexible reeds, advantages of, 263.

"Float" mounting, the, 423.

Formula for the construction of satin weaves, 45 .

Front standard healds in leno looms, 213.

Full-cross leno fabrics, 253.

Fustian fabrics, the chief varieties of, 133.

- - (beaverteen), 139.

- - (cantoon or "diagonal "), 137.

- - (corduroy, figured), 163.

- - (corduroy, plain), 160.

- - ("imperial," reversible), 136.

- — ("imperial " sateen), 136.

- - ("imperial " or swansdown), 135.

- (lambskin), 136.

- - (moleskin), 137.

- - (moleskin, printed), 138.

- - (velveteen, Jacquard figured), 154.

- - (velveteen, plain), 140.

- - (velveteen, ribbed or corded), 152.

- - cutting, 133, 140, 142, 164.

- - by hand, 142.

- - - machinery, 164.

\section{G.}

Gauze fabrics, structure of "cellular," 220 .

- or leuo fabrics, 211.

_ - _ - different types of shedding harnesses for weaving, 213.

- - heald harness, essential parts of a, 213.

- plain, 213. 
Gauze reed, construction of a, 311 .

- - function of a, 312.

Grammar of Textile Design, definition of, 3.

"Grecian" weaves, 94.

H.

Hacking's terry pile motion, 182.

Harness, essential parts of a gauze or leno heald, 213.

Harnesses, relative merits of top and of bottom doup, 254.

- steel-wire doup, for cross-weaving, 264.

"Harvard" or "Cassimere" twill, 27.

Herring-bone twills, 73.

Holden's terry pile motion, 174.

Hollow-cut or ribbed velveteen fabrics, 153.

Honeycomb effects, how they are produced, 86 .

- weaves, 79.

- - characteristics of, 79 .

Huck-a-back weaves, 91.

I.

"Imperial" or swansdown fabrics, 135.

- reversible, 136.

- sateen, 136.

Influence exercised by the direction of twist in yarn upon the relative prominence of twills, 32 .

Intervals of selection, for the construction of satin weaves, 48 .

K.

Kidderminster or Scotch carpet fabrics, 485 .

- - - - draft and shedding plan for, 487.

- - - - preparation of designs for, 489.

- - - - special type of Jacquard machine for, 487.

Lambskin fabrics, 136.

L.

Lappet figuring, 306, 314.

- cross-thread, 325.

- - of a novel character, 325 .

- spot, 326.

- loom, essential parts of a, 315 .

- looms, disadvantages of needle frames being situated below warp threads in, 320 .

- motion, Scotch, 318.

- wheel, description of a, 318.

Leno brocade fabrics, characteristics of, 272. 
Leno brocade harness for a bottom doup, 284, 288.

- - - top doup, 276.

- designs, what to bear in mind when preparing, 262.

- device for douping or crossing warp threads in front of the reed, Whitehead and Wood's, 248.

- effects, special, 247.

- fabric, compound net, 231.

- fabrics, full-cross, 253.

- mock or imitation, 268.

- Jacquard machines, 284, 287, 290.

- - Devoge's special types of, 290.

- looms, position of back standard healds in relation to regular healds in, 262.

- or gauze fabrics, 211.

- - heald harness, essential parts of a, 213.

- weaving, practical details of, 262.

- - relative merits of different types of dobbies for, 256 .

Linear zigzag or "spider" weaves, 97.

Lister and Carter's terry pile motion, 176.

Loop pile fabrics, 209.

Loose reod action in terry looms, 171.

- motion in relation to shedding, in terry looms, 189, 190.

M.

Madras muslin fabrics, 307, 310, 313.

_ - loom for weaving, 310.

_ — with two or more colours of figuring weft, 313.

Matelasse fabrics, 429.

- preparation of designs for, 432 .

Matt weaves, simple. 20.

- - variegated, 21.

Mitcheline or patent satin fabrics, 464 .

_ _ _ _ detailed specifications of, 470

_ - - - draft and shedding plans for, 466.

_ - _ - preparation of designs for, 469.

Mock or imitation leno fabrics, 268.

Moleskin fabries, 137.

- - printed, 138.

Moquette fabrics, formation of the looped pile in, 170.

Mounting, the "float," 423.

N.

Net leno fabric, compound, 231.

- - figuring by means of several back standard healds to each doup heald, 231.

- or gauze fabrics, $211,222$. 
O.

"Oatmeal" or crêpe weaves, 103.

Ondulé fabric, weft, 335.

- fabrics, 306, 330.

- warp, 330.

- loom, 334.

- paquet, or fan reeds, 331 .

Open shed, formation of an, with a bottom-doup harness, 218 .

- - - - - with a top-doup harness, 224, 227, 257.

Ornamented twills, figured or, 73.

"Osman " Turkish terry towels, 194.

P.

Paquet, ondulé, or fan reeds, 331.

Patent satin or Mitcheline fabrics, 464.

_ - _ - detailed specifications of, 470.

- - - - draft and shedding plans for, 466 .

_ - - - preparation of designs for, 469.

Perching, to produce a "nap" or downy surface, 136.

Pick-and-pick motion, definition of $\mathrm{a}, 14$.

Picks of weft, definition of, 1.

Pile fabrics, terry and loop, 169.

Piqué or toilet welts, 403.

- - - backed, 415.

- - - decorative effects in, 417.

_ _ _ detailed specifications of, 429.

- _ - plain, 407.

Plain or calico weave, and its modifications, the, 7 .

_ $\ldots$ - methods of embellishing the, 15.

_ _ - variety of form in the, 9 .

$\therefore-\ldots$ variety of texture in the, 8 .

Plain-ribbed Bedford cord fabrics, 112.

"Planting" warp threads in tapestry fabrics, 478.

Plush fabrics, weft, 152.

"Poppet" rack, in swivel looms, 328.

- shuttles, in swivel looms, 328.

Position of back standard healds in relation to regular healhs, in leno looms, 262.

Practical details of leno weaving, 262.

"Presser" harness for damask fabrics, compound, 365.

Principle of fabric structure, the general, 1.

Principles of fabric structure, minor, 2.

Prominence of twills, influences affecting the relative, 31. 
Q.

Quilting fabrics, toilet, 436.

- - cardinal features of, 438.

- - - detailed specifications of, 463.

- - - distinctive varieties of, 445 .

_ - figuring harness for, 449.

_ - preparation of designs for, 460 .

Quilts. Alhambra, 390.

— "Trellis," 395.

R.

Rearranged twills, 44.

Rearrangement of twills by alternation of threads, 58 .

- _ on a satin basis, 55 .

Reed, gauze, construction of a, 311 .

- - function of a, 312 .

- loose, action, in terry looms, 171.

- wires, removal of, to obtain wider dents, 229, 263.

Reeds, double, for terry pile weaving, 208.

- " Erdmann," 335.

- flexible, advantages of, 263.

- ondulé, paquet or fan, 331.

Relative merits of alternative methods of disposing pile and ground warp threads in terry pile fabrics, 170, 188, 207.

_ - - different types of dobbies for gauze and leno weaving, 256.

- _ - top and of bottom-doup harnesses, 254.

Repp fabrics, figured, 400.

- plain, 14.

Reversible or double-faced fabrics, 132.

- " imperial,", 136.

Ribbed and corded fabrics, simple, 10.

— _ — variegated, 19.

- fabrics, warp, 10.

- weft, 10.

- or corded velveteen, 152.

- hollow-cut velveteen fabrics, 153.

Rice weaves, 70 .

S.

Satin basis, rearrangement of twills on a, 55 .

- patent, or Mitcheline fabrics, 464.

_ _ _ - detailed specifications of, 470.

_ _ - _ draft and shedding plans for, 466 .

_ $\ldots$ - - preparation of designs for, 469 .

- weares, characteristics of, 44. 
Satin weaves, construction of, 45 .

_... formula for the construction of 4.4 .

- - imperfect, 45.

Scotch lappet motion, 318 .

- or Kidderminster carpet fabrics, 485. ',

_ _ - draft and shedding plwn for, 487.

_ - _ - preparation of designs for, 489.

- - - - special type of Jacquard machize for, 487.

Selvedge motions, when necessary, 18.

Selvedges (self-edges), 1.

Shaking devices, the function of, in leno looms, 256.

- - when necessary, 257.

- in leno looms, different methods of, 257.

Shed of warp (warp-shed), 1.

"Shed" or "tab," definition of, 341, 395, 441.

Slackeners, easers, or ribrator's in leno looms, the function of, $218,219$.

Smith's terry pile motion, 180.

Specifications of Bedford cord fabrics, table of, 125.

"Spider" or linear zigzag weaves, 97.

"Split-shed tie-up" for brocade fabrics, 343 .

- - toilet quilting fabries, 456.

Sponge weaves, 88 .

- - characteristics of, 89.

Spot lappet figuring, 326.

Standard or regular warp threads, 213.

Steel-wire doup harnesses for cross-weaving, 264.

- - - disadvantages of, 268.

swansdown or "imperial " fabrics, 135.

Swivel figuring, 327.

- how to distinguish, from lappet figuring, 327.

- shuttles or "poppets," 328.

T.

"Tab" or "shed," definition of, $\$ 341,395,441$.

Tabby or plain calico weave, 6,9 .

Tapestry designs, instructions for card cutting from, 481.

- - preparation of, 480 .

- fabrics, 471.

- - "planting" (supplanting) warp threads in warps for, 478.

- - with all warp surfaces, 476.

- - - weft surfaces, 475.

- - two series both of warp and weft threads, 478.

- pile carpets, formation of looped pile in, 170.

Terry looms, loose reed motion in, 171.

- - timing of the loose reed motion in relation in shedding iu, 189, 190. 
Terry pile fabrics, 169.

- - - circumetences affecting the relative density of pile in, 190.

- - compound Ehedding harness for figured, 199.

_- - draft arĩ słtedding plans for figured, 200.

- - figured, 196.

_ - five-pick figured, 196, 200, 202, 20 \%.

- - - plain, 192:

_ _ four-pick figured, 200, 202.

- _ - plain, 190.

_ - _ in " fast-reed" looms, method of weaving, 204.

_ - method of designing for, 202.

- - relative merits of alternative methods of disposing pile and ground warp threads in, 170, 180, 207.

_ - - six-pick plain, 194.

— - - three-pick figured, 196, 200.

- - - - plain, 187,

- motion, Dugdale's, 178.

- - Hacking's, 182.

- - - Holden's, 174.

_ - Lister and Carter's, 176.

- - Smith's, 180.

- motions, 169, 171.

- or "twin" Jacquard machines, 198.

- towels, "Osman" Turkish, 194.

- weaving, practical details relating to, 207.

Texture, definition of, 8.

- influences affecting the firmness of, 6 .

- variety of, 8.

"Thickset" corduroy fabric, 162.

Tissue figuring, 306.

Toileting, " dandy," 442.

— "run-up," 443.

Toilet quilting fabrics, 436 .

- - cardinal features of, 438.

_ - - detailed specifications of, 463.

_- - distinctive varieties of, 445 .

_ - - figuring harness for, 449.

_ - - preparation of designs for, 460 .

- - five-pick variety of, 458 .

- - four-pick variety of, 455 .

_ - six-pick variety of, 459.

- - three-pick variety of, 451 .

-

- welt or piqué fabrics, 403.

- _ - - backed, 415.

_ _ _ - decorative effects in, 417 . 
Toilet weit or piqué, detailed specifications of, $42 ! 9$.

- - - - plain-ribbed, 407.

Top-doup harness for cross-weaving, 179.

"Trellis" quilts or counterpanes, 395.

Turkish "Osman" terry towels, 194.

Twill, angle of, 30 .

- "Harvard" or "Cassimere," 27.

- "wale" of, definition of, 24.

Twill-ribbed Bedford cords, 115.

- weaves, classification of, 24.

- end-and-end combination of, 62.

- pick-and-pick combination of, 65 .

Twilling Jacquard machines, Bessbrook damask or, 373.

- - - damask or, 372 .

Twills, broken, 67 .

- combined, 60 .

.... continuous, 24.

- corkscrew, modifications of, 53.

- - simple, 48.

- warp-face, 51.

- weft-face, 52.

- figured or ornamented, 73.

- herring-bone, 73.

- influence exercised by the direction of twist in yarn, upon the relative prominence of, 32.

- influences affecting the relative prominence of. 31 .

- rearranged, 44.

- rearrangement of, by alternation of threads, 58 .

- - on a satin basis, 55 .

- "wale" of, definition of, 24.

- warp and weft-face, 26.

- warp-face, 24.

- wary or zigzag, 40.

- weft-face, 25.

"Twin" or terry Jacquard machine, 198.

Twist in yarn, influence of the direction of, upon the relative prominence of twills, 32 .

\section{V.}

Pariegated Bedford cord fabrics, 114.

- corduroy fabrics, 160.

Variety of form in the plain or calico weave, 9.

- - texture in the plain or calico weave, 8.

Velvet cords, 153, 160, 163.

- fabrics, 133. 
Velvet ribbed, 153.

Velveteen fabrics, 140.

- - definition of, 140.

- - embellished with embossed designs, 159.

_- forming the pile in, 133, 140, 142.

- - hollow-cut or ribbed, 153.

- how to distinguish between embossed and woven designs in, 159.

- - Jacquard figured, 154.

- - preparation of designs for figured, 155.

- - ribbed or corded, 152.

- tabby-backed, 141.

- twill-backed, 151.

_- with "lashed" or fast pile, 140, 147.

Vibrators, easers, or slackeners in leno looms, the function of, 218, 219

W.

"Wale" of twill weaves, definition of, 24.

Warp, definition of, 1.

Warp-ribbed fabries, 10.

Warp shed, definition of, 1.

Wavy or zigzag twills, 40 .

Weft, definition of, 1.

Weft-ribbed fabrics, 10.

Welts, piqué or toilet, 403.

- - - backed, 415 .

- - - decorative effects in, 417.

_ _ - - detailed specifications of, 429.

- - - - plain, 407.

"Whip" threads for lappet figuring, 314.

Whitehead and Wood's special leno device for douping or crossing warp

threads in front of the reed, 248.

Worsted doup healds, disadvantages of, 264.

Woven design, definition of, 3.

\section{Y.}

Yarn twist, its influence upon the relative prominence of twills, 32.

Z.

Zigzag or wavy twills, 40.

- or "spider" weaves, linear, 97. 


JUL 24.940 
